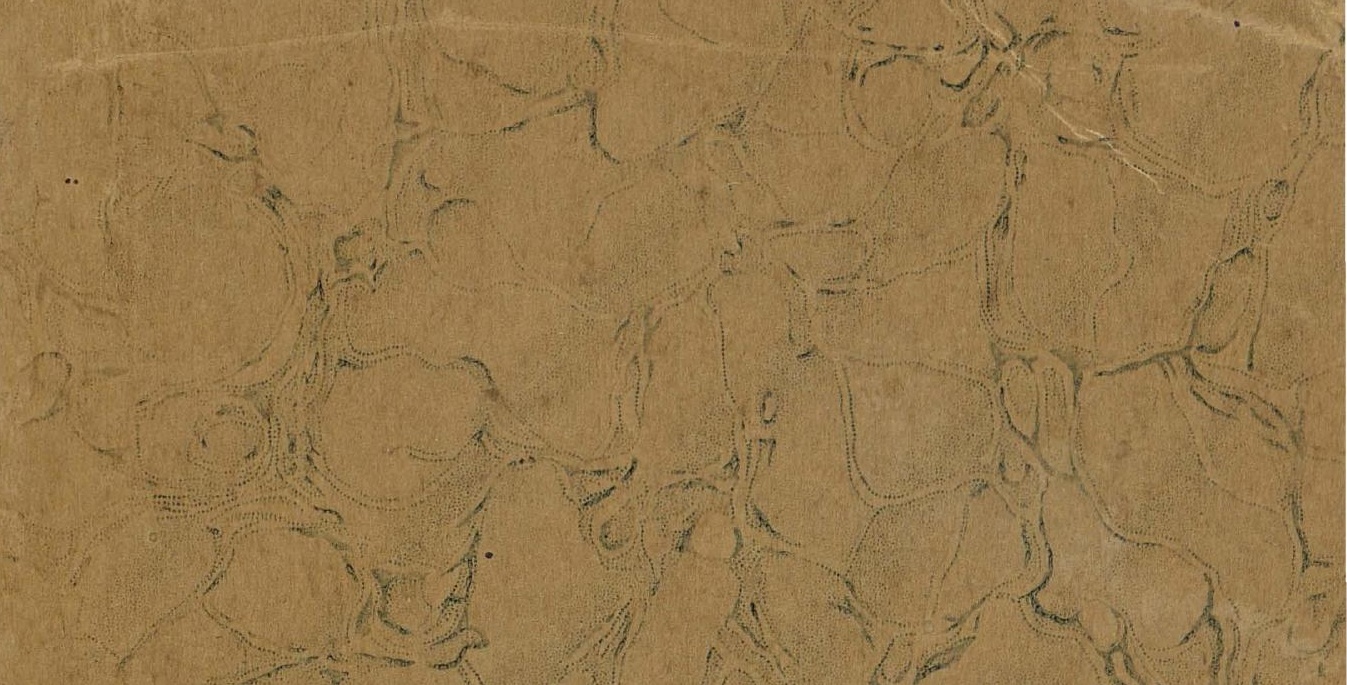

\title{
A DICTIONARY OF ALTITUDES IN THE UNITED STATES
}

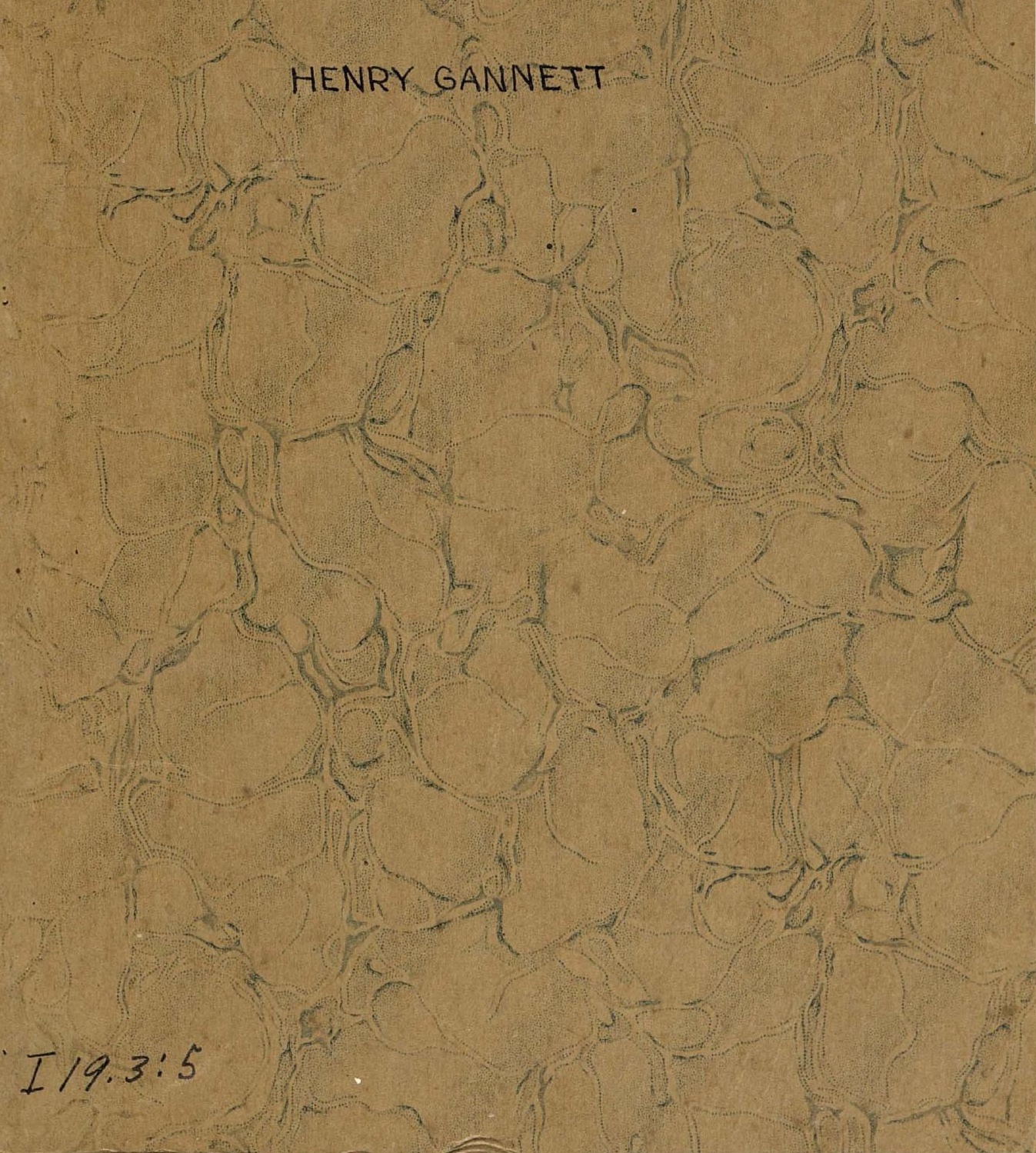





\title{
B ULLETIN
}

OF THE

\author{
UNITED STATES
}

\section{GEOLOGICAL SURVEY}

No. 5

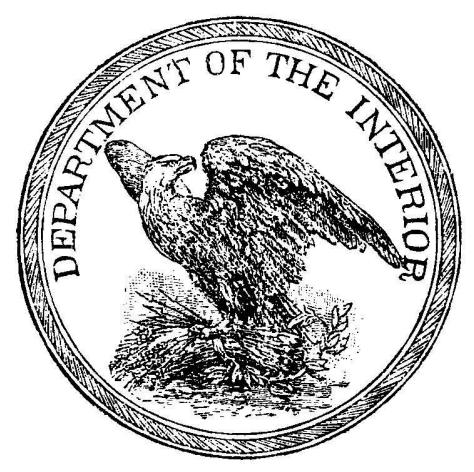

WASHINGTON

GOVERTIENT PIINTING OFFIJE

1884 



\section{UNITED STATES GEOLOGIOAL SURVEY}

J. W. POWELL DIRECTOR

A

\section{DICTIONARY OF ALTITUDES}

IN

\section{THE UNITED STATES}

COMPILED BY

\section{HENRY GANNETT \\ CHIEF GEOGRAPHER}

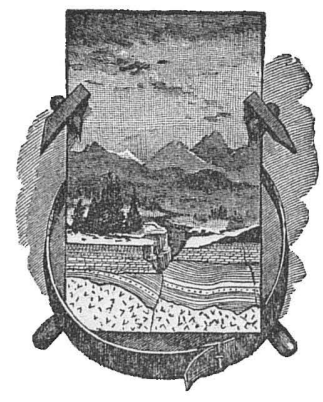

WASHINGTON

GOVERNMENT PRINTING OFFIOE 1884 



\title{
LETTER OF TRANSMITAL.
}

\author{
Department OF THE INTERIOR, \\ United States Geological Strvet; \\ Washington, D. C., November 1, 1883.
}

SIR: I have the honor to transmit herewith the manuscript of a Dictionary of Altitudes. The work of making a compilation of measurements of altitude was commenced by me under the anspices of the Geological Survey of the Territories, by which organization three different editions of the results were published, under the title of "Lists of Elevations," in the years 1873, 1875, and 1877, respectively. These earlier editions related principally to that portion of the country west of the Mississippi River. The present work embraces within its scope the whole country. The elevations are tabulated by States and Territories, alphabetic arrangement being observed throughont.

Very respectfully, yours,

Hon. J. W. Powela,

$$
\begin{aligned}
& \text { HENRY GANNETT, } \\
& \text { Chief Geographer. }
\end{aligned}
$$

Director United States Geological Survey. 



\section{CONTENTS.}

Letter of transmittal ...............................................

Paga.

Table of contents....................................................

Discussion of authorities.............................................

Abbreviations of names of railroads...................................

Tables:

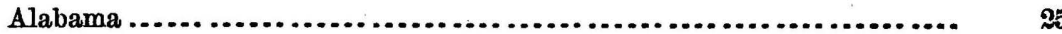

Alaska ........................................................ 29

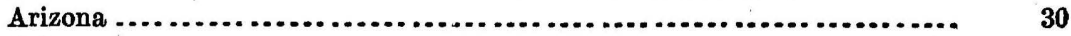

Arkansas...................................................... 35

California .................................................... 37

Colorado........................................................ 55

Connecticut .................................................. 71

Dakota ..................................................... 73

Delaware ................................................... 76

District of Columbia .......................................... 77

Florida ...................................................... 78

Georgia ................................................... $\quad 89$

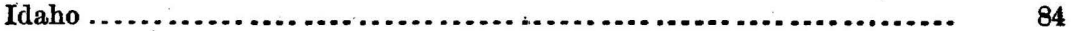

nlinois ...................................................... 87

Indiana................................................... 95

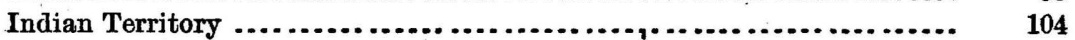

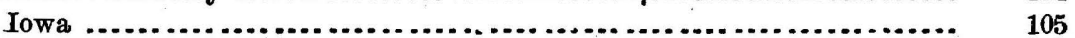

Kansas .................................................... 113

Kentucky................................................ 120

Louisiana .................................................. 125

Maine ......................................................., 126

Maryland ...................................................... 129

Massachusetts ................................................. 133

Michigan .................................................... 138

Minnesota ................................................... 147

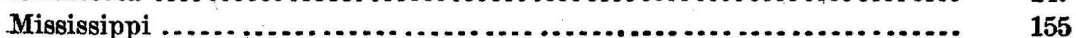

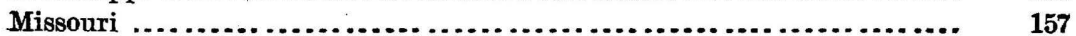

Montana ...................................................... 165

Nebraska ................................................... 169

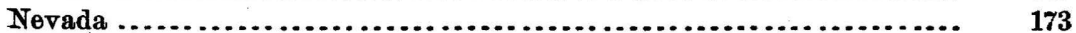

New Hampshire .............................................. 182

New Jersey .................................................... 187

New Mexico ................................................... 192

New York................................................... 203

North Carolina................................................... 223

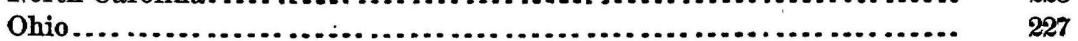

Oregon .......................................................... 241

Pennsylvania ..................................................... 245

Rhode Island .................................................. 275

South Carolina............................................. 276 
Tables-Continued. Paga

Tennessee.................................................... 279

Texas....................................................... 283

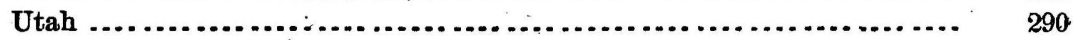

Vermont .................................................. 301

Virginia ....................................................... 304

Washington ................................................. 312

West Virginia................................................. 314

Wisconsin ................................................... 317

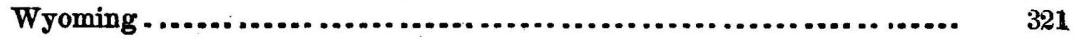

(132) 


\section{DISCUSSION OF AUTHORITIES.}

ADIRONDACK SURVEY (ADIR. SURVEY), OF NEW YORK.

Most of these determinations are barometric. Some, however, have been determined by trigonometric methods and some by level.

APPALACHIAN OLUB (APPAL. OLUB).

Most of the determinations credited to this source are barometric, or at least depend upon barometric bases.

\section{CITY WNGINEER.}

These figures are the results of levelings courteously furnished me by the city engineers of the principal railway centers of the country.

$$
\text { DALL, WM. H., ASST. U. S. O. AND G. } s \text {. }
$$

Heights derived from this source were determined by angles meas. nred with the sextant, from a base line measured by means of sextant latitudes and chronometric longitudes, from observations taken on board ship. (Vide Coast Survey Report for 1875.)

DE LACY, W. W.

All heights were measured by aneroid.

\section{EASTMAN.}

These results are from barometric work and from observations upon the boiling point of water.

$$
\text { EMORY, W. H. }
$$

Barometric determinations abstracted from a "Report of Explorations from Fort Leavenworth to San Diego," 1846. [30th Congress, 1st. session, Senate Document.]

\section{FOSTER AND WHITNEY.}

Barometric determinations from the report on Lake Superior mining . region.

$$
\text { FRÉMONT, CAPT. J. C. }
$$

Barometric determinations from reports of various expeditions in the West. Owing to the want of a barometric base, they are, in general, of poor quality.

$$
\text { GARDINER, J. T. }
$$

The heights credited to this anthority were deduced from railroad levels, and were published in the report of the United States Geological Survey of the Territories for the year 1873. 


\section{GEOLOGICAL SURVEX OF MAINE.}

Most of these results are barometric.

GEOLOGICAL SURVEY OF NEW HAMPSHIRE.

Elevations of towns credited to this source are, in the main, from lev. els; those of mountain summits are from barometric work.

GEOLOGICAL SURVEY OF NEW YORK.

Elevations were determined barometrically.

GEOLOGICAI SURVEY OF VERMONT.

All elevations under this authority are from barometric determinations.

GODDARD.

Determinations by level from preliminary railroad surveys made by the Central Pacific R. R. Co., of California. Like all preliminary work, these figures are not in the highest degree reliable.

$$
\text { GUYOT, PROF. ARNOLD. }
$$

Nearly all the elevations given under this authority are in the Appalachian region, and nearly all were determined by barometer. They are of the highest character of this class of work. Most of the figures are copied from Professor Guyot's articles in the "American Journal of Science and Arts."

$$
\text { HAYDEN, DR. F. V. }
$$

Under this authority is given all work done by the United States Geological Survey of the Territories. All determinations are barometric, or trigonometric resting on barometric bases. In most of this work effective measures were taken to avoid the well-known periodic errors incident to barometric work. The base-stations were placed, as nearly as possible, at the average height of the country to be surveyed. The high peaks were connected by a system of trigonometric leveling, by means of which, barometric observations, taken on different summits, were reduced to a common point. The mean results thus obtained were referred to base-stations located upon high mountain peaks.

$$
\text { HIND, PROF. H. Y. }
$$

Assinaboine and Saskatchewan Exploring Expedition, 1858.

Heights were measured by barometer.

IVES.

Report of Colorado Exploring Expedition [Thirty-sixth Congress, first session, Senate Document].

All determinations are barometric. 
JENNEY, PROF. WILLIAM P.

Heights were abstracted from the map of the Black Hills.

All determinations are barometric.

$$
\text { JONES, CAPT. W. A. }
$$

Report of Expedition to Northwestern Wyoming and the Yellowstone Park, 1873.

All determinations are barometric.

KING, CIJARENCE.

This reference is mainly to the work of the Geological Exploration of the 40th Parallel.

All determinations are barometric, or depend upon barometric bases. LANDER.

Heights are taken from "Report on a Railroad Route from Puget Sound to South Pass," 1854. [House Document 129.]

Elevations were determined barometrically.

$$
\text { LAND-OFFICE REPORTS. (L. O. REPORTS.) }
$$

Most of the heights given in these reports are the merest approximations.

\section{LA PEROUSE.}

"Voyage to the Northwestern Coast of America," 1786.

Heights were determined by trigonometric measurements from sea bases.

$$
\text { LUDLOW, CAPT. WILLIAM. }
$$

Under this authority are given elevations determined by his "Expedition to the Black Hills of Dakota," 1874, and his "Expedition to the Head of the Yellowstone and the Judith Basin," 1875.

Heights were measured by the barometer.

MALESPINA.

"Voyage to the Northwestern Coast of America."

Heights were measured trigonometrically from bases at sea.

MEDICAL DEPARTMENT, UNITED STATES ARMY (MED. DEPT. U. S. A).

Nearly all heights given under this authority were determined barometrically.

$$
\text { MEXICAN BOUNDARY SURVEY. }
$$

Elevations were determined barometrically.

MULLAN, CAPT. JOHN.

"Explorations for a Wagon Road from Walla Walla to Fort Benton," 1862.

All heights were determined barometrically. 
NEW YORK STATE SURVEY.

These results are from refined trigonometric work.

\section{NICOLAY.}

"Explorations in the Upper Mississippi Region," 1836 to 1840.

All determinations were barometric.

PACIFIC RAILROAD REPORTS (P. R. R. REPORTS).

Under this authority is included all work done by the War Department, between 1850 and 1855 , for the purpose of finding a practicable railroad route from the Missouri River to the Pacific.

All the work is barometric, and, taken as a whole, is poor, owing mainly to the fact that, practically, there was no barometric base station. The hypsometric work of the expedition near the northern boundary, under Governor Stevens, was particularly bad. These remarks concerving the quality of the work apply equally well to all the earlier expeditions to the West, owing, doubtless, to the same cause.

$$
\text { PARRY, PROF. b. C. }
$$

Elevations were barometrically determined.

$$
\text { PETERMANN, DR. A. }
$$

Elevations were taken from his map of the United States.

$$
\text { POWELL, MaJ. J. W. }
$$

To this authority is credited the hypsometric work of the United States Geological and Geographical Surrey of the Rocky Mountain Region.

$$
\text { PRELIMINARY RAILROAD LEVELS. (PRE. R. R. LEVELS). }
$$

These are distinguished from other railroad levels, because, as a rule, the preliminary levels are of a far less degree of accuracy; the results can therefore be accepted only with caution.

\section{RAILROADS.}

Elevations determined by railroad levels are credited to the roads by which they were furnished. On page - et seq. will be found a list of these railroads, with the abbreviations used for them, arranged alphabetically in the order of the abbreviations. The height given is that of the track at the station. In cases where two or more railroads meet or cross at the same point on the same grade, the elevation by only one of them, and that the best known or best determined road, is given. The collection and adjustment of these levels has formed by far the greater part of the work of this compilation.

The profiles of most of the roads west of the Mississippi were col- 
lected and published in "Lists of Elevations," 4th edition, in 1877. Since that time, circumstances have conspired to assist me greatly in the collection of this material in all parts of the country. In 1880, while I was connected with the Census Office, the late Chief Signal Officer, General A. J. Myer, courteously placed at the disposal of that office copies of all the material of this kind which had been collected by the Signal Office. Further, in the railroad schedule of census was inserted a request for an abstract of the profile of each road. This request was acceded to in all cases when possible, and through this means a great number of profiles from the most recent levels were received. Through the courtesy of the Census Office I have been permitted to use them in the present publication. Moreover, I have received within the past year profiles of a number of the most important roads in the country, which close up gaps in connections, and establish strong connecting links in chains of levels. In addition to the abore, there were in print, principally in the reports of State geological surveys, a large number of profiles. Those of New Hampshire have been adjusted admirably and published in the report of the State Geological Survey. The same is the case with the large and complex system of Pennsylvania. The collection of profiles of this is exceptionally complete and admirably adjusted, and renders the portion of the Dictionary relating to this State by far the fullest and most satisfactory. The Geological Survey of New Jersey has published profiles of many of the roads of that State. The profiles of most of the roads of Virginia and West Virginia have been published by Maj. Jed. Hotchkiss, in his "Summary of Virginia" and the "Virginias." "Profiles of most of the North Carolina roads have been published in the report of the State Geological Survey by Prof. W. C. Kerr; many of those of Alabama in the reports of Prof. E. A. Smith; of Ohio, in the State Geological Survey reports; of Indiana, in the reports of the Bureau of Statistics and Geology; of Wisconsin, Minnesota, and Iowa, in the reports of the geological surveys of those States. In this connection I desire to express my obligations to Prof. Warren Upham, of the geological and natural history survey of Minnesota, for his kind co-operation in the collection and adjustment of the railroad material in his State.

Much interest in this work has been manifested by railroad engineers, many of whom have contributed liberally of their time for the correction and proper connection of the profiles of their roads or systems of roads. Among them I should mention Mr. H. V. Hinckley, assistant engineer A., T. and S. F. R. R., who has, at great expenditure of time and labor, prepared a correct profile of his road, with its numerous branches; Mr. George H. Nettleton, president of the system of roads in southeastern Kansas; S. S. Montague, chief engineer of the Central Pacific system; and Mr. George Nealley, formerly assistant engineer on the Union Pacific Railroad, to whom I am indebted, in addition to other favors, for a correct profile of the Union Pacific Railroad. 
While a great degree of success has attended my efforts for obtaining railroad profiles, there are still remaining a large number of roads, some of them of great importance, which have thus far failed to respond, owing in most cases to the fact that their records of lerels have been destroyed.

The adjustment of these levels has been a most perplexing task. The errors in the compilation of the profiles themselves, and the uncertainties in the connections and crossings of different roads, together with the legitimate errors of leveling, conspire to make this work of adjustment one of the most difficult and unsatisfactory undertakings imaginable. I cannot claim that the result is by any means what could be desired, but I believe that, with the material and resources at hand, fairly good results have been obtained. The work of the United States Lake Survey in determining the heights of the Great Lakes, and of the Mississippi River Commission in determining the elevation of points along the Mississippi River, and of the transcontinental levels of the Coast and Geodetic Surver, have been accepted as starting-points.

The adjustment of the railroad levels of New Hampshire and of Pennsylrania has also been accepted, after subjecting it to tests for accuracy. The adjustment of railroad levels by Mr. James $\mathbf{T}$. Gardiner, the results of which were published in the annual report of the United States Geological Survey of the Territories for 1873, was examined critically in the light of the determinations of heights made by the Coast and Lake Surveys, of the Mississippi River Commission, and the more recent and presumably more correct profiles reseived since the time of $\mathrm{Mr}$. Gardiner's work. The changes in the heights of the Great Lakes, amounting to 7 feet (lower) in the cases of Michigan, Huron, and Superior, necessitated considerable changes in the States of Michigan, Wisconsin, and Minnesota, and the Territory of Dakota. The levels of the Mississippi River Commission lowered Cairo 12 feet, and the Saint Louis Directrix 15 feet. These corrections were even more wide-spread in their results. They induced changes throughout the whole of that portion of the country west of the Mississippi River as far as the Rocky Mountains. Eastward their effect was felt as far as Indianapolis, Ind., and the Falls of the Ohio. Aside from these changes in Mr. Gardiner's adjustment, his work has borne the tests admirably, and the results, with perhaps slight modification in certain cases, have been accepted and used for further adjustment.

It has been suggested that the method of least squares might be used to advantage in the adjustment of this material. This method does not seem to me to be at all applicable to the class of errors here encountered. These are, in the main, what may be classed as abnormal errors, due, not to want of accuracy of observations, but to mistakes, blunders, and uncertainties, a class of errors from which the method of least squares would deduce only false results. 
RAYNOLDS, CAPT. W. F.

Under this authority are given the hypsometric results of the expedition of Captain Raynolds to the country about the sources of the Snake and Missouri Rivers in 1860-'61.

All the work is barometric.

RUFFNER.

"Reconnaissance in the Ute Country," 1873.

All determinations are barometric.

SIMPSON, OAPT. J. H.

"Reconnaissance in Navajo Country," 1849 [Thirty-first Congress, first session, Senate Doc. 6.]; and "Explorations in the Great Basin of Utah," 1859, published in 1876.

All determinations are barometric.

SMITHSONIAN INSTITUTION.

Most, if not all, of these elevations have been determined by barometer.

$$
\text { STUART, GRANVILLE. }
$$

All determinations are barometric.

$$
\text { TONER, DR. J. M. }
$$

Under this authority are given all heights taken from his "Dictionary of Elevations."

UNITED STATES COAST AND GEODETIC SURVEY (U. S. C. AND G. S.).

Heights have been determined by the most refined geodetic methods. UNITED STATES ENGINEER CORPS (U. S. ENGR. CORPS).

The figures given under this authority are the results of exact levels. UNITED STATES GEOLOGICAL AND GEOGRAPHICAL SURVEY OF THE ROCKY MOUNTAIN REGION.

All, or practically all, heights were determined by barometer.

UNITED STATES LAKE SURVEY.

These results are from exact levels or refined trigonometric work. UNITED STATES SIGNAL OFFICE.

The character of these determinations was misstated in "Lists of Elevations, 4th Edition," published in 1877. In nearly all cases these elevations were determined from railway levels and from levels from railway bench-marks. They were determined by barometer only in cases where exact methods could not be employed. 
WALLEN.

"Explorations for a Wagon Road from the Dalles of the Columbia to Great Salt Lake, via Harney's Lake."

All determinations are barometric.

WATER-POWER OF MAINE:

Most of the heights were obtained by level.

WHEELER, OAPT. GEORGE M.

To this authority are credited all elevations determined by the United States Geographical Surveys West of the 100th Meridian.

Nearly all the work is barometric or trigonometric, depending upon barometric bases.

$$
\text { WHITNEY, PROF. J. D. }
$$

Under this authority are given elevations determined by the Geological Survey of the State of Oalifornia, and the "Geological and Topographical Reconnaissance of the South Park, Oolorado."

All heights are barometrically determined.

WILKES, CAPT. OHARLES.

"Voyage of Exploration to the Pacifie."

WILLIAMSON.

Elevations are all barometrically determined. 


\section{ABBREVIATIONS OF NAMES OF RAILROADS, GIVEN AS AUTHORITIES.}

Adir. R. R..............Adirondack Railroad.

A. C. R. R. ............ Alabama Central Railroad.

A. \& D. R. R........... Adrian and Detroit Railroad.

A. \& F.B. V. R. R....... Atlantic and French Broad Valley Railroad.

Ala. G. S. R. R .........Alabama Great Southern Railroad.

A. \& G. W. R. R........Atlantic and Great Western Railroad.

A., G. \& W. I. T. R. R. . Atlantic, Gulf and West India Transit Railroad.

A. \& L. R. R ...........Albany and Lebanon Railroad.

A., M \& O. R. R........Atlantic, Mississippi and Ohio Railroad.

A. \& N. R.R............Atchison and Nebraska Railiroad.

A. \& N. I. R. R........ Atlantic and North Carolina Railroad.

A. \& P. R. R ............ Atlantic and Pacific Railroad.

Ash. \& P. R. R.......... Ashtabula and Pittsburgh Railroad.

A. \& R. A. L. R. R......Atlanta and Richmond Air Line Railroad.

A.\& S. R. R. ...........Albany and Susquehanna Railroad.

A. \& St. L. R. R ........ Atlantic and St. Lawrence Railroad.

A., T. \& S. F. R. R.... Atchison, Topeka and Santa F'́ Railroad.

A. V. R. R............. Allegheny Valley Railroad.

A. \& W. P. R. R........Atlantic and West Point Railroad.

B.R. R............... Barclay Railroad.

B. \& A. R. R........... Brunswick and Albany Railroad.

Boston \& Albany R. R.Boston and Albany Railroad.

Bed. \& Bridge. R. R... Bedford and Bridgeport Railroad.

Bost.,Con.\& Mont. R.R.Boston, Concord and Montreal Railroad.

B., C. \& P. R. R....... Buffalo, Corry and Pittsburgh Railroad.

B.,.C. R. \& N. R. R ...... Burlington, Cedar Rapids and Northern Railroad.

B. \& E. R. R.......... Belleville and Eldorado Railroad.

B. E. Valley R. R ....... Bald Eagle Valley Railroad.

B. G. R. R............. Bell's Gap Railroad.

B. \& H. R. R...... .... Baltimore and Hanover Railroad.

B. \& M. R. R. R....... Burlington and Missouri River Railroad.

B. \& N. R. R. ..........Burlington and Northwestern Railroad.

Bost. \& N. Y. R. R.....Boston and New York Railroad.

B., N. Y. \& P. R. R.... Boston, New York and Philadelphia Railroad.

B. \& O. R. R........... Baltimore and Ohio Railroad.

B. \& P. R. R. ............ Baltimore and Potomac Railroad.

Bost. \& Prov. R. R.... Boston and Providence Railroad.

B., P. \& C. R. R....... Baltimore, Pittsburgh and Chicago Railroad.

B. \& S. R. R........... Burlington and Southwestern Railroad.

B. \& S. Ill. R. R........ Belleville and Southern Illinois Railroad.

B. \& S. S. R. R.... ..... Bellefont and Snow Shoe Railroad.

B. \& S. W. R. R....... Bellaire and South Western Railroad.

Buff. \& S. W. R. R. ... Buffalo and South Western Railroad,

B. V. R. R............ Buffalo Valley Railroad.

Cent. R. R............. Central Railroad (Ga.).

Chat. R. R ........ ... Chateangay Railroad.

Bull, 5-2

(141) 
Camden \& A. R. R..... Camden and Amboy Railroad.

C. \& A. R. R. .......... Chicago and Alton Railroad.

C. B. \& Q. R. R....... Chicago, Burlington and Quiney Railroad.

C. C. R. R. ............ Colorado Central Railroad.

Car. C. R. R...... ...... Carolina Central Railroad.

Charlotte, C. \& A. R. R. Charlotte, Columbia and Augusta Railroad.

Corning, C. \& A. R. R . Corning, Cowanesque and Antrim Railroad.

C., C., C. \& Y. R. R ....Cleveland, Columbus, Cincinnati and Indianapolis Railroad.

C. C. \& De R. R. R.... Cazenovia, Canastota and De Ruyter Railroad.

C. D. \& C. G.T. J. R.R.Chicago, Detroit and Canada Grand Trunk Junction Railroad.

C. \& E. IIl. R. R....... Chicago and Eastern Illinois Railroad.

C. \& F. R. R........... Catasauqua and Fogelsville Railroad.

C. F. \& M. R. R....... Cedar Falls and Minnesota Railroad.

C. \& G. R. R............ Columbia and Greenville Railroad.

C. G. \& S. L. R. R....... Cape Girardeau and State Line Railroad.

C. \& G. T. R. R ......... Chicago and Grand Trunk Railroad.

C. H. \& D. R. R....... Cincinnati, Hamilton and Dayton Railroad.

C. \& H. V. R. R....... Columbus and Hocking Valley Railroad.

Cent. Iowa R. R........ Central Iowa Railroad.

C. \& I. R. R .......... Chicago and Iowa Railroad.

C., I., St. L. \& C. R. R . Cincinnati, Indianapolis; Saint Louis and Chicago Railroad.

C. \& L. N. G. R. R. .... Chester and Lenoir. Narrow Guage Railroad.

C. \& M. L. S. R. R .... Chicago and Michigan Lake Shoro Railroad.

C. M. \& St. P. R. R.... Chicago, Milwaukce and Saint Paul Railroad.

C., Mt. V.\& D. R. R.... Cleveland, Mount Vernon and Delawaro Railroad.

C. N. R. R............. Cincinnati Northern Railroad.

C. \& N. W. R. R........ . Chicago and North Western Railroad.

C.N. O.\& T.P.R.R.... Cincinnati, New Orleans and Texas Pacific Ruilroad.

C. \& O. R. R ........... Cbesapeake and Obio Raïlroad.

Cal. P. R. R........... California Pacific Railroad.

C. P. R. R.............. Central Pacific Railroad.

Cin. \& P. R. R .......... Cincinnati and Portsmouth Railroad.

Conn. \& Pass. R. R. .... . Connecticnt and Passumpsic Railroad.

Cleve. \& P. R. R........ Cleveland and Pittsburgh Railroad.

C. \& P.D. R. R......... Columbia and Port Deposit Railroad.

C. \& R. R. R........... Columbus and Rome Railroad.

Conn. River R. R....... Connecticut River Railroad.

C. R. \& Ft. Ẃ. R. R..... Cincinnati, Richmonll and Fort Wayne Railroad.

C., R. I. \& P. R. R....... Chicago, Rock Island and Pacific Railroad:

C. S. R. R.............. Cincinnati Southern Railroad.

C. \& S. R. R........... Cayuga and Susquehanna Railroad.

C. \& St. L. R. R ......... Cairo and Saint Louis Railroad:

C., St. P., M. \& O. R. R.Chicago, Saint Panl, Minneapolis and Omaha Railroad.

C., T. V. \& W. R. R .... Cleveland, Tuscarawas Valley and Wheeling Railroad.

C. \& V. R. R........... Cairo and Vincennes Railroad.

Cent. Vt. R.R........... Central Vermont Railroad.

Conn. Western R. R.... Connecticut Western Railroad.

C. \& W. R. R.......... Catawissa and Williamsport Railroad.

C.,W. \& M. R. R......... Cincinnati, Wabäsh and Michigan Railroad.

C. \& Z. R.R............ Cincinnati and Zanesville Railroad.

D. R. R.............. Delaw̌are Railroad.

-D., A. V. \& P. R. R...... Dunkirk, Allegheny Valley and Pittsburgh Railroad.

D. \& B. C. R. R......... Detroit and Bay City Railroad.

D. \& B. V.R.R......... Denver and Boulder Valley Railroad.

D. \& D. R. R............. Dorchester and Delawaie Railroad. 
D., G., H. \& M. R. R...Detroit, Grand Haven and Milwaukee Railroad.

D., H. \& W. R. R... .... Danville, Hazelton and Western Railroad.

D., L. \& N. R. R ....... . Detroit, Lansing and Northern Railroad.

Del., L. \& W.R. R ..... Delaware, Lackawanna and Western Railroad.

D. M. \& Ft. D. R. R..... Des Moines and Fort Dodge Railroad.

D., M. \& M. R.R ........ Detroit, Mackinac and Marquette Railroad.

D. M. \& N. W. R. R..... Des Moines and North Western Railroad.

D. P. R. R. ............ Denrer Pacific Railroad.

D. \& R. Canal.......... Delaware and Raritar Canal.

D. \& R. G. R. R .......... Denver and Rio Grande Railroad.

D. R. V. R. R........... Duck River Valley Railroad.

D. \& S.E.R.R ......... Dayton and South Eastern Railroad.

D. \& S. P. R. R......... Denver and South Park Railroad.

D. \& St. P. R. R........ Davenport and Saint Paul Railroad.

D.\& S. W. R. R .........Danville and South Western Railroad.

E. A. L. R. R ........... Elberton Air Line Railroad.

E. Broadtop R. R........East Broadtop Railroad.

E. B. \& W. R. R.........East Brandy wine and Waynesburg Railroad.

E. \& C. R. R ........... Ebensburg and Cresson Railroad.

E., H. \& N. R. R..........Evansville, Henderson and Nashville Railroad.

E. L., \& B. S. R. R ........ Elizabethtown, Lexington and Big Sandy Railroad.

E. L. \& R. R. R. R'.......East Line and Red River Railroad.

E. M. R. R .............East Mahanoy Railroad.

European \& N. A. R. R.European and North American Railroad.

E. Pa. R. R ............ East Pennsylvania Railroad.

E. \& P.R. R........... Erie and Pittsburgh Railroad.

Eureka \& P. R. R.......Eureka and Palisade Railroad.

Eel R.R. R.............Eel River Railroad.

E. S. R. R.............Eastern Shore Railroad.

E. \& T. H. R. R ........ Evansville and Terre Haute Railroad.

E., T. H. \& C. R. R ......Evansville, Terre Haute and Chicago Railroad.

E. T., V. \& G. R. R....... East Tennessee, Virginia and Georgia Railroad.

Ft.D. \& Ft. R. R. R .... Fort Dodge and Fort Ridgeley Railroad.

F., J. \& G. R. R ......... Fonda, Johnstown and Gloversville Railroad.

F. \& N. Y.R. R..........Freehold and New York Railroad.

F., O.\& C. R. R.........Fredericksburg, Orange and Charlottesville Railroad.

Ft. M. \& N. W.R. R ....Fort Madison and North Western Railroad.

Ft. S., S. E. \& M. R. R .. Fort Scott, South Eastern and Memphis Railroad.

Ft. W., J. \& S. R. R ....Fort Wayne, Jackson and Saginaw Railroad.

Ga. R. R ..............Georgia Railroad.

G. B. R. R ............ Green Bay Railroad.

G., B. \& C. R. R....... . . Golden, Boulder and Caribou Railroad.

G. B. \& Minn. R. R ..... Green Bay and Minnesota Railroad.

G., C.\& S. F. R. R...... Gulf, Colorado and Santa F' 6 Railroad.

G., H. \& S. A. R. R ..... Galveston, Harrisburg and San Antonio Railroad.

G., I. \& S. R. R .........Geneva, Ithaca and Sayre Railroad.

G., L. \& S. J. R. R..... Georgetown, Leadville and San Juan Railroad.

G. R. \& Ind. R. R ....... Grand Rapids and Indiana Railroad.

G. R. V. R. R .......... Grand River Valley Railroad.

G. \& S. P. R. R........ Golden and South Platte Railroad.

G., W. T. \& P. R. R ... Gulf, Western Texas and Pacific Railroad.

H. \& B. T. R. R ....... Huntington and Broad Top Railroad.

H. \& C. M. R. R....... Hannibal and Central Missouri Railroad.

H. \& N. R. R.......... Hannibal and Naples Railroad.

H., P. \& F. R. R........Hartford, Providence and Fishkill Railroad. 
H. \& St. J. R. R .......Hannibal and Saint Joseph Railroad.

H. \& T. C. R. R ........ Houston and Texas Central Railroad.

I., B. \& W. R. R....... Indiana, Bloomington and Western Railroad.

Ill. Cent. R. R . ......... Illinois Central Railroad.

I., D. \& S. R. R........ Indianapolis, Decatur and Springfield Railroad.

I. \& G. N. R. R ......... International and Great Northern Railroad.

Inds. \& La F. R. R .... Indianapolis and La Fayette Railroad.

IIl. '\& St. L. R. R ....... Illinois and Saint Louis Railroad.

Inds. \& V. R. R........ Indianapolis and Vỉncennes Railroad.

J. \& B. R. R ...........Junction and Breakwater Railroad.

J. C. \& Ft. K. R. R . .... Junction City and Fort Kearney Railroad.

J., L. \& S. R. R.... .... Jackson, Lansing and Saginaw Railroad.

J., M. \& I. R. R......... Jeffersonville, Madison and Indianapolis Railroad.

J. \& N. Ind. R. R ....... Joliet and Northern Indiana Railroad.

J. R. \& K. Canal ....... James River and Kanawha Canal.

J. S. E. R. R............ Jacksouville South Eastern Railroad.

J. S., P. C. \& B. R. R ... Jersey Shore, Pine Creek and Buffalo Railroad.

K. C. R. R.............. Kansas Central Railroad.

K. C., Ft. S. \& G. R. R .. Kansas City, Fort Scott and Gulf Railroad.

K. C., L. \& S. R. R .... Kansas City, Lawrence and Southern Railroad.

K. C., St. J. \& C. B. R. R.Kansas City, Saint Joseph and Council Bluffs Railroad.

K. \& N. W. R. R.......Keokuk and North Western Railroad.

K. P. R. R.............. Kansas Pacific Railroad.

K. \& S. H. R. R........Kalamazoo and South Haven Railroad.

K. U. R. R............ Kentucky Union Railroad.

L. R. R................ Lackawanna Railroad.

L. \& A. R. R .......... Lowell and Andover Railroad.

L. A. \& I. R. R........ Los Angeles and Independent Railroad.

L. B. R. R............. Lawrenceville Branch Railroad.

L., C. \& L. R. R...... Louisville, Cincinnati and Lexington Railroad.

L. C. \& S. C. R. R.... .Lewisburg Center and Spruce Creek Railroad.

L. E. \& W. R. R.......Lake Erie and Western Railroad.

L. \& L. R. R ........... Lehigh and Lackawanna Railroad.

L. \& M. R. R. R....... Louisville and Missouri River Railroad.

L., N. A. \& C. R. R....Lonisville, New Albany and Chicago Railroad.

L., N., S. \& N. A. R. R.Louisville, Nashville, South and North Alabama Railroad.

L. \& Q. R. R.......... Lancaster and Quarryville Railroad.

L. R. \& Ft. S. R. R.... Little Rock and Fort Smith Railroad.

L. R. V. \& Ark. R. R .... Little River Valley and Arkansas Railroad.

L. \& S. R. R........... Lehigh and Susquehanna Railroad.

L. Schuyl. R. R........ Little Schuylkill Railroad.

L. S. \& M. R. R. R . . . . . .Lake Superior and Mississippi River Railroad.

L. S. \& M. S. R. R ... . . . . Lake Shore and Michigan Southern Railroad.

L. \& S. V.R. R.......... Longview and Sabine Valley Railroad.

L. \& S. W. R. R.........Lawrence and South Western Railroad,

L. \& T. R. R........... Lebanon and Tremont Railroad,

M. R.R................ Montrose Railroad.

Mt. A. R. R ............. Mont Alto Railroad.

M. \& A. G. T. R. R.......Mobile and Alabama Grand Trunk Railroad.

M. \& B. R. R...... .... Macon and Brunswick Railroad.

Me. Cent. R. R..........Maine Central Railroad.

Mass. Cent. R. R........Massachusetts Central Railroad.

Mich. A. L. R. R.........Michigan Air Line Railroad.

Mich. Cent. R. R....... Michigan Central Railroad.

Mill C. R. R............ Mill Creek Railroad. 
Muncy C. R.R.......... Muncy Creek Railroad.

Memphis \& C. R. R .... Memphis and Charleston Railroad.

M. \& C. R. R............ Marietta and Cincinnati Railroad.

M. \& C. Co. R. R........ Mifflin and Center County Railroar.

M. C. \& S. B. R. R....... Mauch Chunk and Switch Back Railroad.

M. C.\& T. R. R......... Mississippi Central and Tennessee Railroad.

M. \& D.R.R........... Mechanicsburg and Dillsburg Railrond.

M. \& E.R. R........... Montgomery and Eufaula Railroad.

M. \& G. R. R.......... Millville and Glassboro' Railroad.

M., H. \& O. R. R....... Marquette, Houghton and Ontonagon Railroad.

M. H. \& S. H. R. R.... Mine Hill and Schuylkill Haven Railroad.

M. \& I. Canal.......... Michigan and Illinois Canal.

Mo., Iowa \& Neb. R. R.Missouri, Iowa and Nebraska Railroarl.

McK. \& B. R. R........McKean and Buffalo Railroad.

M., K. \& C. R. R...... Memphis, Kansas and Colorado Railrohd.

Mo., Kans. \& Tex. R. R. Missouri, Kansas and Texas Railroad.

M., K. \& T. E. R. R... Missouri, Kansas and Texas Extension Railroad.

Man. \& Lawrence R. R..Manchester and Lawrence Railroad.

M. \& L. R. R. R........Memphis and Little Rock Railroad.

M.,L. S. \& W. R. R.... Milwaukee, Lake Shore and Western Railroad.

Morgan's I. \& T. R. R.Morgan's Louisiana and Texas Railroad.

Minn. Mid. R. R.......Minnesota Midland Railroad.

M. \& M. R. R......... Mobile and Montgomery Railroad.

Mil. \& N. R. R.R .... Milwaukeo and Northern Railroad.

M. \& N. G. R. R. ....... . Marietta and North Georgia Railroad.

Man. \& N. Weare R. R. . Manchester and North Weare Railroad.

M. \& N.W.R. R........Mobile and North Western Railroad.

M. \& O.R. R. ........... Mobile and Ohio Railroad.

M. \& P. R. R . .......... Marietta and Pittsburgh Railroad.

M. R. R. R ............. Mineral Range Railroad.

M. \& S. R. R ............ Mahanoy and Shamokin Railroad.

M. \& St. L. R. R........ Minneapolis and Saint Louis Railroad.

M. \& W.R. R ...........Macon and Western Railroad.

M. \& W.R.R. R......... Montpelier and Wells River Railroad.

N. Carolina R. R ........ North Carolina Railroad.

N. C. R. R............. Northern Central Railroad.

N. C. \& B. V.R. R ...... New Castle and Beaver Valley Railroad.

N.C. \& F. R. R......... New Castle and Franklin Railroad.

Nev. Co. N. G. R. R . ..... Nevada Connt.y Narrow Guage Railroad.

N.D.\&. C. R. R ........ Newburgh, Dutchess and Connecticut Railroad.

N. E. R. R............ North Eastern Railroad.

N. E. Pa. R. R .......... North East Pennsylvania Railroad.

N. H. \& Northam. R. R.New Haven and Northampton Railroad.

N. J. C. R. R ............ New Jersey Central Railroad.

N. M. \& S. P. R. R ....... New Mexico and Southern Pacific Railroad.

N.\& N.R.R ............ Nashville and Northwestern Railroad.

N. O.M. \& T. R. R...... New Orleans, Mobile and Texas Railroad.

N. O.\& N.E.R. R...... Now Orleans and North Eastern Railroad.

N.P.R.R ............. Northern Pacific Railroad.

N. Pa. R. R ............ North Pennsylvania Railroad.

N. Valley R. R . .......... Nesquehoning Valley Railroad.

N.\& W.R.R ........... Norfolk and Western Railroad.

N. W.N.C. R. R......... North Western North Carolina Railroad.

N.W. U. R. R ........... North Western Union Railroad.

N. Y., B. \& M. R. R.... . New York, Boston and Montreal Railroad. 
N. Y. C. \& H. R. R. R ... New York Central and Hudson River Railroad.

N. Y. C. \& N. R: R...... Neiw York City and Northern Railroad.

N. Y.\& H. R. R . ........ New York and Harlem Räilroad.

N. Y., K: \& S: R. R ..... New York, Kingston and Syracuse Railroad.

N. Y.; L. E: \& W: R. R . : New York, Lake Erie and Western Railroad.

N. Y. \& N. H. R. R....... New York and New Haven Railroad.

N.Y., N. H.\& Htfd. R.R.New York, New Haven and Hartford Railroad.

N. Y.\& O. M. R. R....... New York and Oswego Midland Railroad.

N.Y., Prov.\& Bost. R.R.New York, Providence and Boston Railroad.

N. Y., Pa. \& O. R. R .... New York, Pennsylvania and Ohio Railroad.

N. Y., R. \& P. R. R....... New York, Ridgeway and Petersburg Railroad

O.\& E. Canal ......... Ohio and Erie Canal.

O.\& M. R. R... ........ Ohio and Mississippi Railroad.

O., N. \& B. H. R. R ..... Omaha, Niobrara and Black Hills Railroad

O. \& R. V.R. R......... Omaha and Republican Valley Railroad.

Oreg. R. R. \& Nav. Co .. Oregon Railroad and Navigation Company.

O.\& R. R. R ........... Oswego and Rome,Railroad.

Oregon \& Cal. R. R .... Oregon and California Railroad.

O. \& S. R. R .......... Oswego and Syracuse Railroad.

P. R.R ................ Perkiomen Railroad.

Pa. R. R .............. Pennsylvania Railroad.

Peters. R. R............ Petersburgh Railroad.

P. A. L. R. R . ............ Piedmont Air Line Railroad.

P. B. R. R.............. Peach Bottom Railroad.

P. \& B. C. R. R......... Philadelphia and Baltimore Central Railroad.

P. \& C.R. R . ..........Pittsburgh and Connellsville Railroad.

P., C. \& St. L. R. R .....Pittsburgh, Cincinnati and St. Lonis Railroad.

P. \& D. R. R........... Passaic and Delaware Railroad.

Pa. \& Del. R. R ........Pennsylvania and Delaware Railroad.

P., D. \& E. R. R ...... Peoria, Decatur and Evansville Railroad.

P. \& E. R. R. .......... Philadelphia and Erie Railroad.

Paducah \& E. R. R .... Paducah and Elizabethtown Railroad.

P., Ft. W. \& C. R. R...Pittsburgh, Fort Wayne and Chicago Railroad.

P., G. F. \& C. R. R ....Portsmouth, Great Falls and Conway Railroad.

P., H. \& B. R. R........Poughkeepsie, Hartford and Boston Railroad.

P. H. \& N. W. R. R .....Port Huron and North western Railroad.

P. \& K. C. R. R ......... Parker and Kansas City Railroad.

P., K. \& P. R. R .......Plymouth, Kankakee and Pacific Railroad.

Port. \& Ogden. R. R ...Portland and Ogdensburg Railroad.

Phil. \& N, R. R.........Philadelphia and New town Railroad.

P.\& P. R. R ........... Pennsylvania and Petroleum Railroad.

P., P. \& J. R. R . ....... Peoria, Pekin and Jacksonville Railroad.

P. R.R. R .............Port Royal Railroad.

Phil. \& R. R. R ......... Philadelphia and Reading Railroad.

P. \& R. I. R. R .......... Peoria and Rock Island Railroad.

Prov. \& Spring. R. R. .. Providence and Springfield Railroad.

Phil. \& T. R. R ........ Philadelphia and Trenton Railroad.

P., T.\& B. R. R......... Pittsburgh, Titusville and Buffalo Railroad.

P., V.\& C. R. R ........ Pittsburgh, Virginia and Charleston Railroad.

Prov. \& Worces. R. R ...Providence and Worcester Railroad.

P., W. \& B. R. R........ Philadelphia, Wilmington and Baltimore Railroad

Prov., Warren \& Bris-

tol R. R ..............Providence, Warren and Bristol Railroad.

R. \& A. R. R........... Richmond and Alleghany Railroad.

R. \& A. A. L. R. R ....... Raleigh and Augusta Air Line Railroad. 
R. \& C. R. R ........... Reading and Columbia Railroad.

Rome \& C. R. R........ Rome and Clinton Railroad.

R. \& D. R. R ........... Richmond and Danville Railroad.

R., F. \& P. R. R .........Richmond, Fredericksburgh and Potomac Railroad.

R. G. R. R............ Rio Grande Railroad.

R. \& G. R. R.......... Raleigh and Gaston Railroad.

R. H. R. R ............. Rich Hill Railroad.

R., N.\& P. R. R........ Rochester, Nunda and Pennsylvania Railroad.

R. \& P.R. R .......... Richmond and Petersburgh Railroad.

R. \& S. L. R. R.......... Rochester and State Line Railroad.

R. \& S. R. R ............Rensselaer: and Saratoga Railroad.

R. V.\& B. \& C. R. R ... Republican Valley and Burlington and Colorado Railroad.

R., W.\& O. R. R ....... Rome, Watertown and Ogdensburgh Railroad.

R., Y. R.\& C. R. R...... Richmond, York River and Chesapeake Railroad.

S. R. R ................ Salisbury Railroad.

S. \& A. R. R ........... Sheuango and Allegheny Railroad.

Spring, Athol \& North-

ern R. R ............. Springfield, Athol and Northern Railroad.

S. B. R. R ............. Summit Branch Railroad.

S. \& B. R. R............ Syracuse and Binghamton Railroadi.

S. C. R. R ............. Southern Central Railroad.

S. Carolina R. R ....... South Carolina Railroad.

S. \& C. R. R........... Savannah and Charleston Railroad.

S. C.\& D.R.R......... Sioux City and Dakota Railroad.

S. C. \& J. R. R ......... Short Creek and Joplin Railroad.

S. C.\& P. R. R ......... Sioux City and Pacific Railroad.

S. \& C. V.R. R........ . Syracuse and Chenango Valley, Railroad.

S., G. \& C. R. R ......... Syracuse, Geneva and Corning Railroad.

St. J. \& D. C. R. R ..... . Saint Joseph and Denver City Railroad.

S. K.\&W.R. R........ Southern Kansas and Western Railroad.

S. \& L. R. R ........... Sunbury and Lewiston Railroad.

St. L., I. Mt. \& S. R. R. Saint Louis, Iron Mountain and Southern Railroad.

St. L., K. C. \& N. R. R . Saint Louis, Kansas City and Northern Railroad.

S. L. \& S. R. R......... State Line and Sullivan Railroad.

St. L. \& S. E. R. R..... Saint Louis and South Eastern Railruad.

St. L. \& S. F. R. R...... Saint Louis and San Francisco Railroad.

St. L., S. \& L. R. R. R .. Saint Louis, Salem and Little Rock Railroad.

St. L., V. \& T. H. R. R ...Saint Louis, Vandalia and Terre Haute Railroad.

S. \& M. R. R .......... Savannah and Memphis Railroad.

S. \& M. P.R. R......... Somerset and Mineral Point Railroad.

S. \& N. B. R. R......... Seliusgrove and North Branch Railroad.

S. P. R. R .............. Southern Pacific Railroad.

S. \& P. R. R . .......... Sacramento and Placerville Railroad.

St. P. \& P. R. R ....... Saint Paul and Pacific Railroal.

St. P.\& S. C. R. R...... Saint Paul and Sioux City Railroad.

S. P., S., \& T. F. R. R .. Saint Paul, Stillwater and Taylor's Falls Railroad.

S., R.\& D. R. R ........ Selma, Rome and Dalton Railroad.

S. \& R. R. R ........... Seaboard and Roanoke Railroad.

S. \& S. R. R.......... . Schuykill and Susquehanna Railroad.

S.\& S. W. R. R ........ Salina and South Western Railroad.

S. W. R.R ............. South Western Railroad.

S. W. Pa. R. R ......... South West Pennsylvania Railroad.

T.C. R. R.............. Texas Central Railroad.

T. \& C. R. R ............ Tyrone and Clearfield Railroad.

T., E. \& S. L. R. R ...... Tioga, Elmira and State Line Railroad. 
T. H. \& Inds. R. R ..... Terre Haute and Indianapolis Railroad.

T. H. \& S. R. R.......... Terre Haute and Southeastern Railroad.

T.\&. N. O. R. R ......... Texas and New Orleans Railroad.

T.\& P. R. R ............ Texas and Pacific Railroad.

T. P. \& W. R. R.........Toledo, Peoria and Warsaw Railroad.

T. W. \& W.R. R .......Toledo, Wabash and Western Railroad.

U. \& B. R. R. R ….... Utica and Black River Railroad.

U. C.R. R ............. Utah Central Railroad.

U., C. \& B. R. R....... Utica, Clinton and Binghamton Railroad.

U., C. \& S. R. R ....... Utica, Chenaugo and Susquehanna Valley Railroad.

U. \& D. R. R.......... Ulster and Delaware Railroad.

U., I. \& E. R. R........ Utica, Ithaca and Elmira Railroad.

U. \& N. R. R.......... Utah and Northern Railroad.

U. \& N. F. R. R ........ Ursina and North Fork Railroad.

U. P. R. R ............ Union Pacific Railroad.

U. S. R. R . ............ Utah Southern Railroad.

U. \& T. R. R......... Union and Titusville Railroad.

U. T. Co. R. R......... Union Transit Company Railroarl.

U. W. R. R............Utah Western Railroad.

V.R. R ............... Valley Railroad.

Vt. \& Mass. R. R …... Vermont and Massachusetts Railroad.

V., M., T. \& G. R. R .... Vermont, Massachusetts, Troy and Greenfield Railroad.

V. \& N. R. R .......... Vicksburg and Nashville Railroad.

Vt. Valley R. R ......... Vermont Valley Railroad.

W. R. R..............Western Railroad (Ala.).

W. \& A. R.R .......... Western and Atlantic Railroad.

W. C. R. R ............ West Chester Railroad.

Wis. Cent. R. R ......... Wisconsin Central Railroad.

W. C. \& P. R. R ....... West Chester and Philadelphia Rail road. .

W.C.,Va.M.\&G.S.R.R Washington City, Virginia Midland and Great Sonthern

Railroad.

W. \& E. R. R..........Williamsport and Elmira Railroad.

W.\& E. Canal......... Wabash and Erie Canal.

W. J. R. R..............West Jersey Railroad.

W. N. C. R. R ........ Western North Carolina Railroad.

W. Md. R. R ............ Western Maryland Railroad.

W. \& O.R.R..........Washington and Ohio Railroad.

W. \& O.\& O. C. R. R. . Western and Oregon, and Oregon Central Railroad.

W. Pa. R. R ............ West Pennsylvania Railroad.

W. \& R. R. R..........Wilmington and Reading Railroad.

W., St. L. \& P. R. R .... Wabash, Saint Louis and Pacific Railroar.

W. V.R.R...........Walkill Valley Railroad.

Wis. Val. R. R ......... Wisconsin Valley Railroad

Warwick V. R. R....... Warwick Valley Railroad.

W. Va. C. \& P. R. R. . . West Virginia Central and Pittsburgh Railroad.

W.\&W.R.R.......... Wilmington and Western Railroad.

Yough. R. R ........... Youghiogheny Railroad.

Y. Br. Pa. R. .......... York Branch Pennsylvania Railroad. 
ALABAMA.

\begin{tabular}{|c|c|c|}
\hline Station. & Authority. & Elevation. \\
\hline . & . & \\
\hline Acron $\ldots . . . . . . . . .$. & Ala. G. S. R. R & 149 \\
\hline 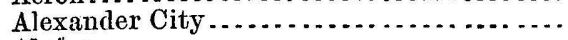 & S. \& M. R. R & 742 \\
\hline 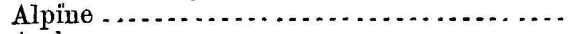 & S. R.\&.D. R. R. & 470 \\
\hline 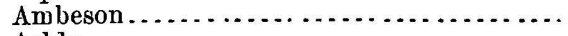 & S.R.\& D.R.R. & 702 \\
\hline Ashly $\ldots \ldots \ldots \ldots \ldots \ldots \ldots \ldots \ldots \ldots$ & S. R. \& D.R.R. & 456 \\
\hline 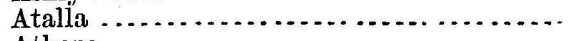 & Ala. G. S. R. R - & 602 \\
\hline . & L.N.S. \& N.A. T & 707 \\
\hline 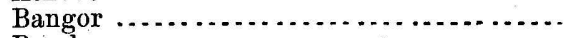 & L. N.S. \& N. A. R. R & 466 \\
\hline 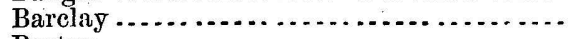 & S. R. \& D.R.R.... & 509 \\
\hline 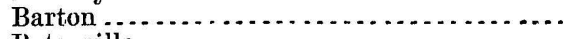 & Memplis \& C. R. R & 498 \\
\hline 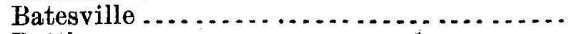 & M. \&.E. R. R. . . . . & 280 \\
\hline 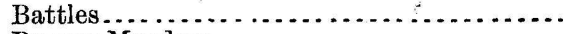 & Ala. G. S. R. R . & 152 \\
\hline 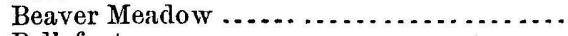 & M. \& O.R.R... & 136 \\
\hline 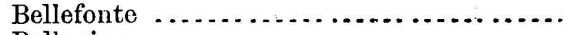 & Memphis \& C.R.R & 639 \\
\hline Belleriew . . . . . . . . . . . . . . . . . . . . & A. C.R. R. . . . . . . . . & 160 \\
\hline Bibb Mills . . . . . . . . . . . . . . . . . . . . . . & S.R. \& D. R. R. . . . . . . . & 446 \\
\hline 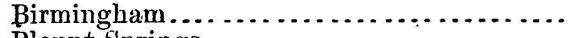 & Ala. G. S. R. R....... & 596 \\
\hline Blount Springs $\ldots \ldots \ldots$ & L. N.S.\& N.A.R & 432 \\
\hline 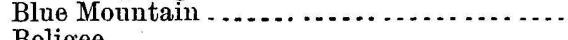 & S.R. \& D.R.R. . & 791 \\
\hline Boligee .................. & Ala. G. S. R.R R & 123 \\
\hline $\begin{array}{l}\text { Boyle....... } \\
\text { Brandon ... }\end{array}$ & $\begin{array}{l}\text { L. N.S. A N.A. H } \\
\text { Ala.G.S.R.R... }\end{array}$ & $\begin{array}{l}5 \% 2 \\
896\end{array}$ \\
\hline & S.R.\&D.R.R. & $\begin{array}{l}896 \\
388\end{array}$ \\
\hline & 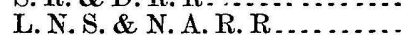 & $\begin{array}{l}388 \\
562\end{array}$ \\
\hline 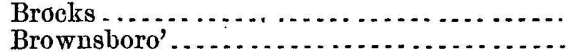 & Memphis \& C. R. R .......... & $\begin{array}{l}562 \\
631\end{array}$ \\
\hline 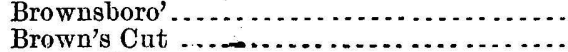 & Ala. G. S. R. R ..... & $\begin{array}{l}631 \\
244\end{array}$ \\
\hline 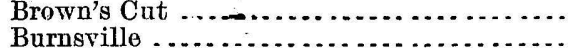 & S.R \& D.R.R. & 244 \\
\hline $\begin{array}{l}\text { Burnsville } \ldots \ldots \ldots \\
\text { Cahabamines....... }\end{array}$ & 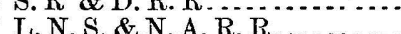 & 182 \\
\hline 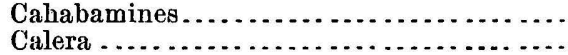 & L. N.S.\& N.A.R.R & 598 \\
\hline $\begin{array}{l}\text { Calera } \ldots \ldots \ldots \ldots \ldots \ldots \\
\text { Calvert } \ldots \ldots \ldots \ldots \ldots\end{array}$ & L. N. S. \& N. A. R. R & 500 \\
\hline 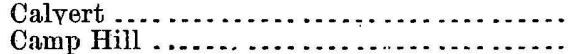 & M. \& A. G. T. R. R. . & 57 \\
\hline Camp Hill $\ldots \ldots \ldots \ldots \ldots \ldots \ldots$ & S. \& M. R. R ..... & 733 \\
\hline 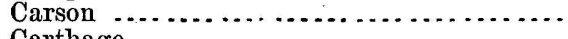 & M. \& A. G. T. R. R. & 54 \\
\hline 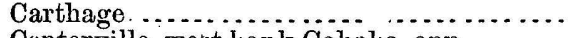 & Ala. G. S. R. R.... & 176 \\
\hline Centerville, west bank Cahaba, opp....... & M. \& A. G. R. R. & 252 \\
\hline 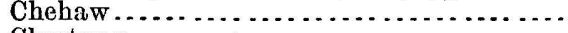 & W.R.R., of Ala... & 252 \\
\hline 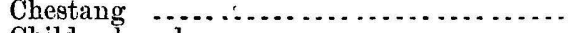 & M.\& A.G.T.R. R.. & 43 \\
\hline 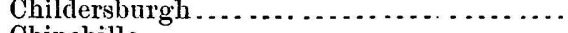 & S. R. \& D.R. R.... & 416 \\
\hline Chinchilla & M. \& O.R.R.... & 78 \\
\hline 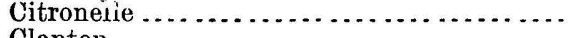 & M. \& O.R.R. . . & 317 \\
\hline 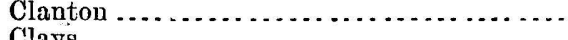 & L.N.S. \& N.A.R.R & 594 \\
\hline 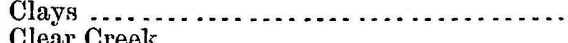 & S.R. \& D.R.R. R. & 193 \\
\hline$\ldots \ldots \ldots \ldots \ldots \ldots \ldots \ldots \ldots \ldots$ & L.N.S.\& N.A. & 538 \\
\hline (n. & $\ldots \ldots \ldots$ & 419 \\
\hline$\ldots \ldots \ldots \ldots \ldots$ & $\cdots \cdot$ & $\begin{array}{r}15 \\
183\end{array}$ \\
\hline Cold Creek. & M.\&A.G.T.R.R. & $\begin{array}{r}183 \\
34\end{array}$ \\
\hline Collinsville $\ldots \ldots \ldots \ldots \ldots \ldots \ldots$ & Ala. G. S. R. R ... & 738 \\
\hline Columbiana $\ldots \ldots \ldots \ldots \ldots \ldots \ldots \ldots$ & S.R. \& D.R.R. & 535 \\
\hline mbus $\ldots \ldots \ldots \ldots \ldots \ldots \ldots \ldots \ldots \ldots$ & W.R.R . . . . . . . . & 262 \\
\hline ( & L.N.S.\& N.A.R.R........ & 456 \\
\hline Coosa Station ....... & S.R.\& D.R. R........... & 447 \\
\hline Cottondale ... & Ala. G. S. R. R ..... & 273 \\
\hline Courtland..... & Memphis \& C. R. R ........ & 560 \\
\hline Coxe's........ & S.R. \& D. R. R........... & 373 \\
\hline Creola _... & M.\& A. G. T. R. R.......... & 23 \\
\hline Cross Plains,$\ldots \ldots \ldots \ldots \ldots \ldots \ldots$ & S. R.\& D.R.R........... & 697 \\
\hline 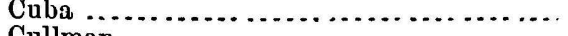 & Ala. G. S. R. R ... & 219 \\
\hline 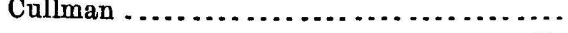 & L. N. S. \& N. A. R. & 800 \\
\hline
\end{tabular}




\begin{tabular}{|c|c|c|}
\hline Station. & Authority. & Elevation. \\
\hline & & Feet. \\
\hline Yunninghs & L. N.S. \& N. A & 438 \\
\hline & S.R. \& D.R. R & 540 \\
\hline & S. \& M.R.R. & 755 \\
\hline teville. & L. N. S. \& N. A & 298 \\
\hline atur... & L. N.S.\& N. A & 575 \\
\hline r Park. & M. \& O. R. R. & 148 \\
\hline Dickson... & Memphis \& C. & 488 \\
\hline Dixio ..... & S. R. \& D. R. R & 282 \\
\hline$\ldots \ldots \ldots \ldots \ldots . . . .$. & L. N. S. \& N. A & 796 \\
\hline . & L. N. S. \& N. A & 197 \\
\hline ................. & Ala. G. S. R. R. & 129 \\
\hline . & M. \& E.R.R. & 200 \\
\hline ... & Ala. G. S. R. R & 979 \\
\hline Eutaw .... & Ala. G. S. R. R & 189 \\
\hline Falkville..... & L. N. S. \& N. A & 601 \\
\hline Fitzpatrick's . . . . & M. \& E.R.R. & 262 \\
\hline Flint ............. & L. N. S. \& N. A. & 566 \\
\hline Foots ................ & L. N. S. \& N.A. & 646 \\
\hline Fort Deposit ..... & M. \& M. R. R . & $5 \% 20$ \\
\hline Payne....... & Ala. G. S. R. R & 883 \\
\hline - . & S. R. \& D.R. & כ42 \\
\hline$\ldots \ldots \ldots \ldots$ & L. N. S. \& N. A & 536 \\
\hline - $\ldots \ldots \ldots$ & S. \& M.R. R & 65 \\
\hline$\ldots \ldots \ldots \ldots$ & A. C. R. R... & 183 \\
\hline er . . . . . . . . & S. \& M. R. R . & 867 \\
\hline ad........ & Ala. G. S: R. R & 491 \\
\hline ood......... & Ala. G. S. R.R. & 691 \\
\hline Griffiths' Mills ... & S. R. \& D. R. R & 691 \\
\hline . & M. \& A. G. T. R. & 180 \\
\hline 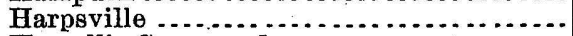 & Ala. G.S.R.R. & 186 \\
\hline l's Cross-roads $\ldots \ldots \ldots \ldots \ldots \ldots$ & A. C. R. R $\ldots$ & 192 \\
\hline$\ldots \ldots \ldots \ldots \ldots \ldots \ldots \ldots$ & L. N. S. \& N. & 562 \\
\hline . & L. N. S. \& N. & 671 \\
\hline - . & L. N. S. \& N. A & 751 \\
\hline Hele & L. N. S. \& N.A. & 398 \\
\hline Hollimans .. & Ala. G. S. R. R. & 937 \\
\hline 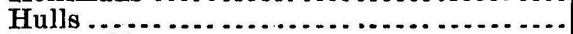 & Ala. & 141 \\
\hline ................ & ......... & 612 \\
\hline . & L. N.S. \& N. A. R & 650 \\
\hline$\ldots \ldots \ldots \ldots \ldots$ & M. C.\& T.R.R. & 459 \\
\hline n's Gap..... & S. \& M.R.R. & 690 \\
\hline ville $\ldots . .$. & $\ldots \ldots$ & 628 \\
\hline$\ldots \ldots \ldots$ & $\ldots \ldots \ldots$ & 704 \\
\hline 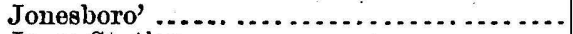 & la. G.S. R & 527 \\
\hline 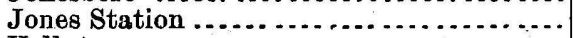 & A. C. R. R... & 209 \\
\hline - & S. \& M. R. R & 800 \\
\hline lga & S. R. \& & 426 \\
\hline La & & 671 \\
\hline noville ..... & $\ldots \ldots$ & 620 \\
\hline ............... & & 160 \\
\hline iton ......... & $\ldots$ & 563 \\
\hline$\ldots \ldots \ldots$ & $\therefore \ldots . .$. & 54 \\
\hline gston ...... & $\ldots . . . .$. & 144 \\
\hline$\ldots \ldots \ldots$ & ........ & 134 \\
\hline$\ldots \ldots$ & $\mathbf{R} \ldots \ldots \ldots$ & 623 \\
\hline & S. K. K.. & 485 \\
\hline ald & \& N.A.R.R... & 683 \\
\hline & his \& C.R.R.... & 573 \\
\hline iville ...... . & S. R. \& D. R. R ....... & 356 \\
\hline & M. \& A.G.T.R.R.... & 253 \\
\hline tthews ........ & M. \& F. R.R....... & 262 \\
\hline$\ldots \ldots \ldots \ldots \ldots \ldots$ & Ala. G. S. R. R.... & 176 \\
\hline Midway.... & & 506 \\
\hline
\end{tabular}




\begin{tabular}{|c|c|c|}
\hline Station. & Aut] & Elevation. \\
\hline & & Feet \\
\hline Milner . . . . . . & L. N. S. \& N. A. & 838 \\
\hline . & A. C. R. R... & 119 \\
\hline (n) & M. \& E. R. R. & 252 \\
\hline Mobile................ & M. \& O.R.R. & 6 \\
\hline " Signal Station ............ & U. S. Signal C & 69 \\
\hline$\ldots \ldots \ldots \ldots \ldots \ldots$ & S. R. & 469 \\
\hline Montgomery & L. N. & 162 \\
\hline 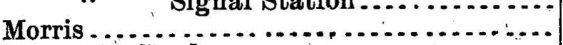 & L. N. S. \& N. A. & $\begin{array}{r}219 \\
406\end{array}$ \\
\hline 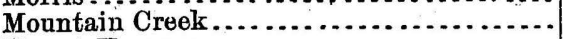 & L.N.S.\&.N.A. & 540 \\
\hline 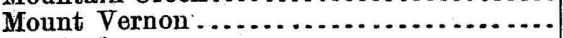 & M. \& A. G. & 49 \\
\hline Munford .......... & S. R. \& D. I & 621 \\
\hline Nebo Mount, tunvel at grade...... & M. \& A. G. I & 330 \\
\hline " $\quad$ summit of mount ... & M. \& A. G.T. & 449 \\
\hline Oak Grove. & M. \& E. R. R. & 226 \\
\hline Olmstead ........ & Ala. G. S. H & 288 \\
\hline Opelika......... & W:R.R., of A & 812 \\
\hline Oxford $\ldots \ldots \ldots \ldots \ldots \ldots \ldots \ldots \ldots$ & S.R. \& D.R. & 653 \\
\hline Patona..................... & S. R. & 689 \\
\hline$\ldots \ldots \ldots \ldots \ldots$ & S. R. & 213 \\
\hline Pelham $\ldots \ldots \ldots \ldots \ldots \ldots \ldots \ldots$ & L.N.S. N & 425 \\
\hline acola Junction . . . . . . . . . . . . & M. \& M. R. & 68 \\
\hline$\ldots \ldots \ldots$ & L. N. S. \& N & 790 \\
\hline 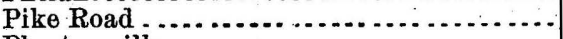 & M. \& E.R. R & $\quad 295$ \\
\hline . & S. R. \& D. R & 241 \\
\hline Plateau........ & M. \& A. G. T & 37 \\
\hline Pollard ......... & M. \& M. R. R & 67 \\
\hline …............. & S. R. \&. D. R. R & 548 \\
\hline …............. & Ala. & 599 \\
\hline . & \& N.A & 591 \\
\hline . & Memphis \& C & 652 \\
\hline ; & W. R. R...... & $12 \%$ \\
\hline k Alabama River ............ & A. C. R. R.... & 110 \\
\hline “ Junction, Western R. R........ & A. C. R. R. & 108 \\
\hline “ crossing, S. R.\& D.R. R .......... & A. C. R. R. & 112 \\
\hline " Depot, Broad street. $\ldots . . . . . . . . . .$. & A. C. & 122 \\
\hline "6 crossing, N.O.\& S.R.R.... & A. C. R. R & 121 \\
\hline Sepulga..... & M. \& & 200 \\
\hline Shade's C & L. N. S. \& N. & 610 \\
\hline 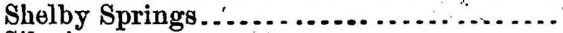 & & 5\%9 \\
\hline , $\ldots \ldots \ldots \ldots \ldots \ldots \ldots$ & L. N. S. \& N. A. & 462 \\
\hline 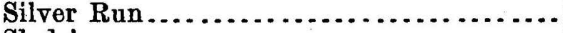 & S. R. \& D. R. R. & 630 \\
\hline lade's.... & M. \& A. G. T. $\mathbf{R}$ & 54 \\
\hline allwoo & R. I & 429 \\
\hline & & 312 \\
\hline 西, & S. R. R. & $7 \cdot 27$ \\
\hline - & S. R. R & 574 \\
\hline l's & S. R. R. & 610 \\
\hline & is \& C. R. R... & 602 \\
\hline art's . & S. R. R & 159 \\
\hline ( & S. \& M. R. R. & 497 \\
\hline 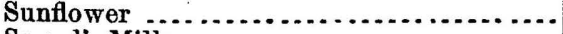 & M. \& A. G.T. R. R & 28 \\
\hline$\ldots \ldots \ldots \ldots$ & S. R. \& D. R. R ... & 741 \\
\hline ladega.......... & S. R. \& D. R. R & 561 \\
\hline$\ldots \ldots \ldots$ & Ala. G. S. R. R . & 485 \\
\hline Taylor's .... . & A. C. R. R.... & 173 \\
\hline$\ldots \ldots \ldots$ & & \\
\hline Thompson's ....... . & & 289 \\
\hline Three-Notch Road. & M. \& E. R. R. . . . $\ldots \ldots \ldots$ & 492 \\
\hline Wn Creek ....... & Memphis \& C. R. R......... & 560 \\
\hline ity ............. & M. \& C. R. R..... & 634 \\
\hline ssville....... & Ala. G. S. R. R & 702 \\
\hline к & & 181 \\
\hline
\end{tabular}




\begin{tabular}{|c|c|c|}
\hline Station. & Authority. & Elevation. \\
\hline 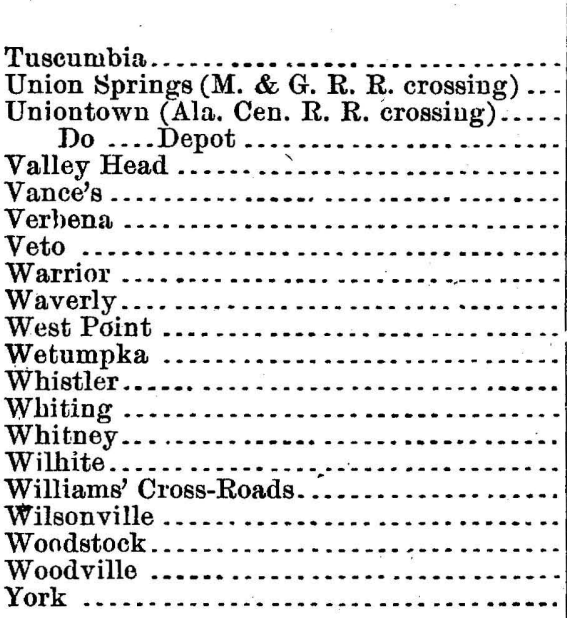 & 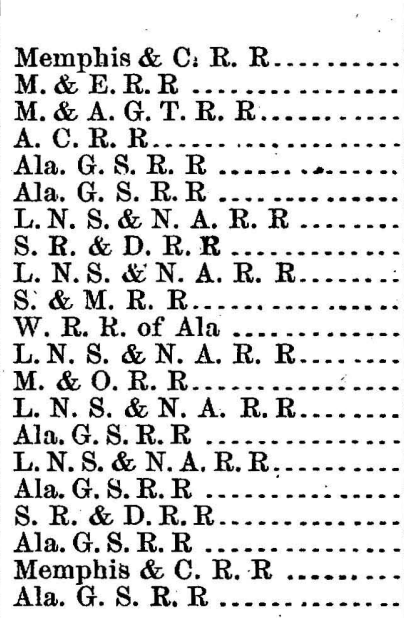 & $\begin{array}{r}\text { F'eet. } \\
488 \\
494 \\
274 \\
282 \\
1,031 \\
.514 \\
448 \\
160 \\
547 \\
805 \\
417 \\
183 \\
41 \\
553 \\
613 \\
606 \\
514 \\
427 \\
519 \\
596 \\
159\end{array}$ \\
\hline
\end{tabular}


ALASKA.

\begin{tabular}{|c|c|c|}
\hline Station. & Authority. & Elevation. \\
\hline$\cdots$ & & Feet. \\
\hline Adakh .. & U. S. C. \& G. S. & 5,678 \\
\hline 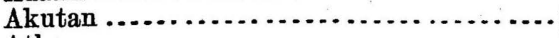 & U. S. C. \& G. S. & 3,888 \\
\hline 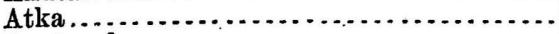 & U. S. C. \& G. S.............. & 4,988 \\
\hline 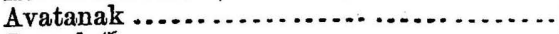 & U. S. C. \& G. S. . . . . . . . & 1,207 \\
\hline Bogosloff. & U. S. C. \& G. S? & 844 \\
\hline 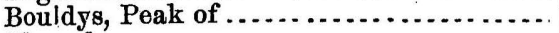 & U. S. C. \& G. S. & 1,145 \\
\hline Chuqul ............. & U. S. C. \& G. S. & $-3,109$ \\
\hline Cook, Mt...... & Dall ............ & 16,000 \\
\hline . & Dall ............... & 15,900 \\
\hline . & 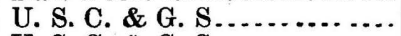 & 5,525 \\
\hline$\ldots \ldots \ldots \ldots \ldots \ldots$ & U. S. C. \& G. S.............. & 2,855 \\
\hline Fairweather, Mt . . . . . . . . . . . . . . . . . & Malespina .................. & 14,589 \\
\hline (n. & Tebenkoff . . . . . . & 14,000 \\
\hline - $\ldots \ldots \ldots \ldots \ldots$ & Vasilieff $\ldots \ldots \ldots \ldots \ldots \ldots$ & 13,946 \\
\hline . $\ldots \ldots \ldots \ldots \ldots$ & Russian hydro. chart ........ & 14,708 \\
\hline$\ldots \ldots \ldots \ldots \ldots . . . . .$. & English Admiralty chart..... & 14,708 \\
\hline Do. & Tebenkoff, chart iii .......... & 13,864 \\
\hline Do.. & 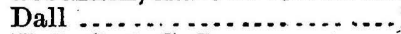 & 15,500 \\
\hline Gareloi ... & U. S. C. \& G. S. & 5,334 \\
\hline$\ldots \ldots \ldots \ldots \ldots \ldots \ldots$ & U. S. C. \& G. S......... & 11,270 \\
\hline$\ldots \ldots \ldots \ldots \ldots \ldots \ldots$ & U. S. C. \& G. S............ & 12,066 \\
\hline Kyska, North Peak & U. S. C. \&. G. S........... & 4,085 \\
\hline Macushin ........ & U. S. C. \& G. S............ & 5,961 \\
\hline St. Elias, Mt .... . & Malespiua.................. & 17,854 \\
\hline Do........ & Tebenkoff. & 16,938 \\
\hline Do... & La Perouse............... & 12,661 \\
\hline & English hydro. chart ........ & 14,970 \\
\hline Do... & Russian hydro. chart ........ & 17,854 \\
\hline Do..... & Dall $\ldots \ldots \ldots \ldots \ldots \ldots \ldots \ldots$ & 19,500 \\
\hline Shishaldin ....... & 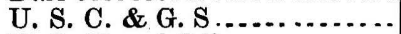 & 8,683 \\
\hline 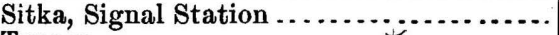 & U. S. Sigual Office............ & \\
\hline$\ldots \ldots \ldots \ldots \ldots \ldots \ldots$ & U. S. C. \&. G. S........... & 7,108 \\
\hline .................. & U. S. C. \& G. S.. & 5,961 \\
\hline . & U. S. C. \& G. S. & 8,954 \\
\hline , $\ldots \ldots \ldots \ldots$ & U. S. C. \& G. S. . . . . . . . & 3,374 \\
\hline 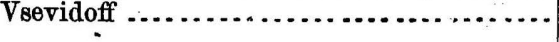 & 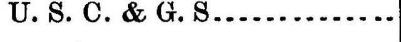 & 8,868 \\
\hline
\end{tabular}




\section{ARIZON.A.}

\begin{tabular}{|c|c|c|}
\hline Sta & Authority. & Elevation. \\
\hline & & \\
\hline corn Spring & Powell ... & 6,360 \\
\hline $\begin{array}{l}\text { Adonde } \\
\text { Agna Prieta.... }\end{array}$ & S.P.R.R. & $\begin{array}{r}214 \\
4,017\end{array}$ \\
\hline Allentown .... & A.\& P.R. & 6,026 \\
\hline $1 \ldots . . .$. & A.\& P.R. & 5,879 \\
\hline ope Springs & Wheeler. & 8,065 \\
\hline nvil Rock ..... & Wheeler . & 5,354 \\
\hline Apache Camp & $\begin{array}{l}\text { Wheeler } \\
\text { U. S. Signal Office }\end{array}$ & $\begin{array}{l}5,000 \\
5,004\end{array}$ \\
\hline Apache Spring ... & Wheeler......... & 6,124 \\
\hline . & Toner .... & 6,350 \\
\hline$\cdots$ & A. \& P. R. & $\begin{array}{l}5,105 \\
7,381\end{array}$ \\
\hline Iabrey $\ldots . . .$. & A.\&P.R.R & $\begin{array}{l}, 381 \\
5,128\end{array}$ \\
\hline Cliffs. & Powell ... & 7,330 \\
\hline Natic & Toner ........ & 5,156 \\
\hline$\cdots$ & A. \& P.R.R. & 3,472 \\
\hline $\begin{array}{l}\cdots \\
\cdots . .\end{array}$ & R.P.R.R. & 2,127 \\
\hline $\begin{array}{l}\text { Bear } \\
\text { Belle }\end{array}$ & $\ddot{A} \& \mathrm{P} . \mathrm{R} . \overline{1}$ & $\begin{array}{l}4,264 \\
7,099\end{array}$ \\
\hline Bent & S.P.R.R. & 3,580 \\
\hline Junction... & A.T. \& S. F. R. & 3,521 \\
\hline on $\ldots . . . .$. & $\mathrm{A} \& \mathrm{p} \mathrm{p}$ & 1,426 \\
\hline inm....... & A. \& P.R.R .. & 5,372 \\
\hline $\begin{array}{l}\text { Bill } \\
\text { Bitte }\end{array}$ & Pow & $\begin{array}{l}6,620 \\
4,890\end{array}$ \\
\hline Peak & Pow & 6,700 \\
\hline & Whe & 7,796 \\
\hline & Wh & 4,412 \\
\hline & S. $\bar{P}$. & 3,761 \\
\hline & Whe & 4,872 \\
\hline & S. F. R & 4,462 \\
\hline & pt. U.S.A & 5,330 \\
\hline $\mathbf{T}$ & Whee & 7,704 \\
\hline & & \\
\hline & Med. Dept., U. S. & 6,500 \\
\hline & A.T.\& S.F.R.R & 3,618 \\
\hline & & \\
\hline & Haj & 9,280 \\
\hline & $R, R \ldots$ & 5,199 \\
\hline ....... & R. of Ariz & 1,398 \\
\hline & Wheeler.......... & 6,031 \\
\hline ......... & & 5,868 \\
\hline ( & A. 8 & \\
\hline & R. R . & $5,2 \% 4$ \\
\hline & er.......... & 4,201 \\
\hline & Whe & 7,476 \\
\hline $\operatorname{San} \mathrm{Sim}$ & Whe & 3,855 \\
\hline o Chiquito Bridge ...... & Wheeler................ & 5,638 \\
\hline o Plateau. ............................... & Powell $\ldots \ldots \ldots \ldots \ldots \ldots, 6,0$ & 08,000 \\
\hline Ranch..... & Wheeler & 5,367 \\
\hline tion & A.T.\& S.F.R.R . & 3,771 \\
\hline 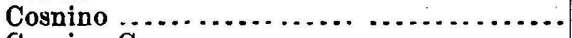 & A. \& P.R. R ...... & 6,434 \\
\hline S & Wheeler ........... & 6,244 \\
\hline & Wheeler.......................... & 4,170 \\
\hline & & \\
\hline
\end{tabular}




\begin{tabular}{|c|c|c|}
\hline Station: & Authority. & Elevation. \\
\hline & & \\
\hline Criswell's Ranch................... & Wheeler $\ldots \ldots \ldots \ldots \ldots$ & \\
\hline 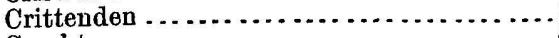 & A. T. \& S. F. R. R........... & 4,172 \\
\hline Crookton $\ldots \ldots \ldots \ldots \ldots \ldots \ldots \ldots \ldots$ & A. \& P.R. R . . . . . . . . . & 5,657 \\
\hline Cross Mt $\ldots \ldots \ldots \ldots \ldots$ & Petermann............. & 5,182 \\
\hline Crown Point $\ldots \ldots \ldots \ldots \ldots \ldots$ & Powall ...................... & 7,140 \\
\hline Date Creek, Camp & Toner.. & 3,726 \\
\hline Deer Spring...... & Wheeler & 5,981 \\
\hline$\ldots \ldots \ldots \ldots$ & U. S. Geol. Survey .... . . . . . . . . & 6,862 \\
\hline 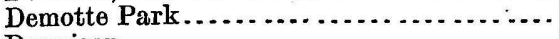 & Powell $\ldots \ldots \ldots \ldots \ldots \ldots \ldots$ & $5,800-6,000$ \\
\hline 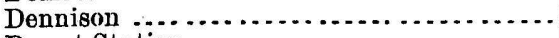 & A. \& P. R. R . . . . . . . . & 4,979 \\
\hline$\ldots . . .6 . . .6$ & Wheeler .... ............. & 2,135 \\
\hline 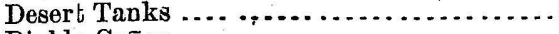 & Wheeler .................... & 5,192 \\
\hline$\ldots \ldots \ldots \ldots$ & $\ldots \ldots \ldots \ldots$ & 4,765 \\
\hline 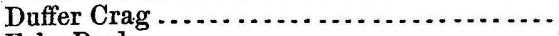 & Powell. & 6,500 \\
\hline 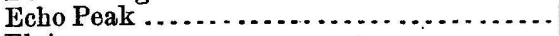 & Powell . & 5,360 \\
\hline Elgin... & A.T. \& S. F. R. R ..... & 4,690 \\
\hline El Puerto del Dado Pass ..... . ............ & Petermann ............ & 5,433 \\
\hline 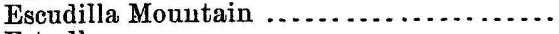 & 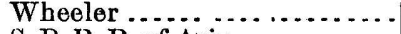 & 10,691 \\
\hline$\ldots \ldots \ldots \ldots$ & 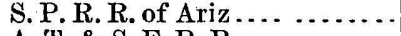 & 1,523 \\
\hline Fairbank Junction ....................... & A.'T. \& S. F. R. R ........... & $3,8: 8$ \\
\hline . & A. \& P. R. R . . . . . . . . . . & 5,909 \\
\hline$\ldots \ldots \ldots . . . . . . . . .$. & A.P.R.R $\ldots \ldots \ldots \ldots \ldots \ldots$ & 6,862 \\
\hline nce, Sigual Station .................. & U. S. Signal Office ............ & 1,553 \\
\hline 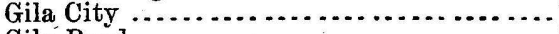 & 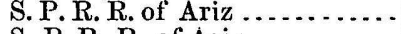 & 175 \\
\hline$\cdots \cdots \cdots$ & S. P. R. R. of Ariz ............. & 739 \\
\hline t (Wainupits Pikavu)..- & $\ldots \ldots \ldots \ldots$ & 5,390 \\
\hline rin, Camp (old) $\ldots \ldots \ldots \ldots$ & $\ldots . . . . . . . . .$. & 2,816 \\
\hline 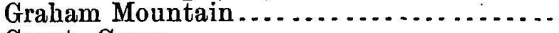 & (n............... & 10,516 \\
\hline Grant, Camp...... & Wheeler. & 4,833 \\
\hline .................... & Wheeler. & 2,118 \\
\hline 1al Station $\ldots \ldots \ldots \ldots \ldots \ldots \ldots$ & Office............. & 4,737 \\
\hline 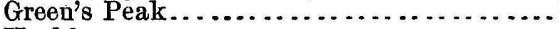 & Wbeeler................... & 10,093 \\
\hline$\because \ldots \ldots \ldots \ldots \ldots \ldots$ & A. \& P.R.R. . . . . . . . . . & 3,522 \\
\hline 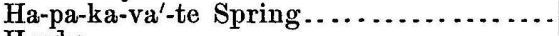 & Powell .: & 6,840 \\
\hline & A. \& P. R. R. & 4,910 \\
\hline Ranch .... & Powell .. & 8,970 \\
\hline Wells. & .......... & 5,290 \\
\hline 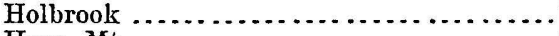 & A. \& P. R. R.. & 5,047 . \\
\hline$\ldots \ldots \ldots \ldots \ldots$ & Poterson $\ldots . . . . . . . . . . .$. & 6,321 \\
\hline Rock Spring.... & Powell .................. & 5,730 \\
\hline$\ldots \ldots \ldots \ldots$ & A. T. \& S. F. R. R........ & 4,253 \\
\hline ai $\ldots \ldots \ldots \ldots \ldots \ldots$ & A. \& P. R. R.................. & 3,277 \\
\hline 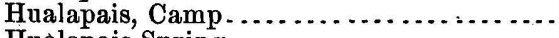 & 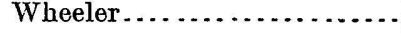 & 5,322 \\
\hline is Spring $\ldots \ldots \ldots \ldots \ldots \ldots \ldots$ & (1) & 4,258 \\
\hline areys' Peak . . . . . . . . . . . . . . . . . & $\ldots \ldots \ldots \ldots$ & 12,562 \\
\hline o's Pool Point. ... . & Pow & 6,890 \\
\hline 's Pools. . & Po & 5,260 \\
\hline Jacob's Well. & Pacific R. R. Reports .... & 5,973 \\
\hline & Wheeler................ & 6,065 \\
\hline$\ldots \ldots \ldots$ & Wheeler............... & 6,814 \\
\hline 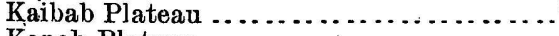 & & 8,000 \\
\hline Plateau... & Pow & $000-6,400$ \\
\hline 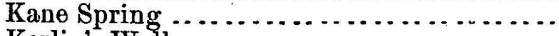 & Powell .. & 3,686 \\
\hline Well & Powell.......... & 5,502 \\
\hline 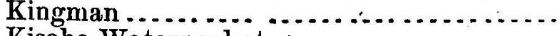 & 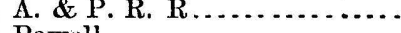 & 3,303 \\
\hline Kisa & Pow & 5,980 \\
\hline La Pilla.... . & Wheeler.. & 5,850 \\
\hline Law's Spring . . . . . & Powell... & 6,870 \\
\hline Lee's Ferry . . . . . . . . & Powell .... & 3,170 \\
\hline x Springs ... & Pacific R. R. Reports ........ & 7,378 \\
\hline$\ldots \ldots \ldots \ldots \ldots \ldots$ & & 5,680 \\
\hline tone Tanks. . . . . . . . . . . . . . . . . & Powell .. & 5,040 \\
\hline stone Water-pocket. $\ldots \ldots \ldots \ldots$ & 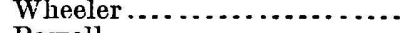 & 5,405 \\
\hline Little Colorado River (mouth of) .......... & Powell ..................... & $\mathfrak{2}, 690$ \\
\hline
\end{tabular}




\begin{tabular}{|c|c|c|}
\hline Station. & Authority. & Elevation. \\
\hline & & Feet. \\
\hline 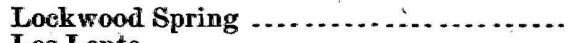 & Wheeler & 526 \\
\hline $\cos _{2}$ & Wheeler. & 542 \\
\hline (1) & …. & 836 \\
\hline$\ldots$ & Wheeler.. & 2,000 \\
\hline n.............. & $\begin{array}{l}\text { Med. Dept., L. S. A ........ } \\
\text { S. P. R. R. of Ariz. ......... }\end{array}$ & 3,726 \\
\hline (n....... & Pacific IR. R. Reports ...... & 1,188 \\
\hline$\ldots .$. & S. P. R. & 4,036 \\
\hline (c) & Wheeler & 6,670 \\
\hline of) $\ldots \ldots \ldots \ldots$ & Powell & , \\
\hline ...................... & Powell & 705 \\
\hline (2................ & Powell. & 4,710 \\
\hline$\ldots \ldots \ldots$ & S. P. R. R. & 543 \\
\hline ttlement .......... & Powell ... & 4,700 \\
\hline 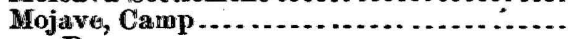 & Wheeler- & 755 \\
\hline Do. & Med. Dept., U. S. A....... & 604 \\
\hline . & Pacific R. R. Reports..... & 605 \\
\hline Logollo & 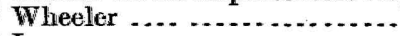 & 7,535 \\
\hline$\ldots . . . .$. & Ives............... & 5,890 \\
\hline$\ldots \ldots \ldots$ & & 5,500 \\
\hline (n.......................... & Powell ...... & 3,198 \\
\hline Iusha Lake . . . . . . . . . . . . . . . . . . . . . . & Powell ...................... & 5,380 \\
\hline tt $\ldots \ldots \ldots \ldots \ldots \ldots$ & $\ldots \ldots \ldots$ & 6,580 \\
\hline 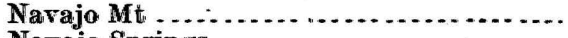 & Powell $\ldots \ldots \ldots \ldots \ldots$ & 10,038 \\
\hline 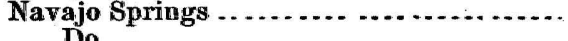 & 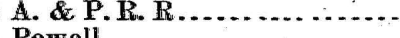 & 5,605 \\
\hline (n) & $\ldots . . .6$ & 4,155 \\
\hline ө & 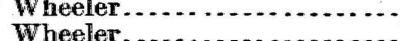 & 4,101 \\
\hline (1) & $\begin{array}{l}\text { Wheeler. } \\
\text { Pacifie R. R. Reports }\end{array}$ & 866 \\
\hline & Powell ....................... & $\begin{array}{l}5,020 \\
5,800\end{array}$ \\
\hline (n) & Whoeler & 6,216 \\
\hline Spring ................... & Ives. . & 739 \\
\hline 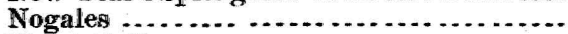 & $\mathbf{F} . \mathbf{R} \ldots \ldots$ & 3,813 \\
\hline$\ldots \ldots \ldots \ldots$ & R. sarveys...... & 4,686 \\
\hline & R. surveys ...... & 4,820 \\
\hline Spring & Powell. & 6,359 \\
\hline$\ldots \ldots$ & S. P. R.R ...... & 4,104 \\
\hline 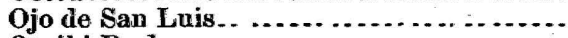 & - - & 5,044 \\
\hline Peak ............................. & $\ldots \ldots \ldots \ldots \ldots$ & 6,700 \\
\hline 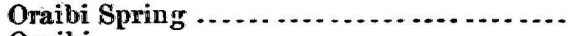 & rell $\ldots . . . . . . . . . . . . . .$. & 5,950 \\
\hline 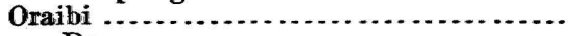 & ler....................... & 4,757 \\
\hline Do. & $\ldots \ldots$ & 009 \\
\hline 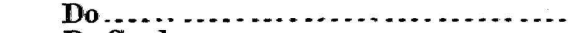 & $\ldots . . . .$. & 316 \\
\hline Do Garden & ........ & 395 \\
\hline ......................... & 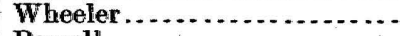 & 10,265 \\
\hline 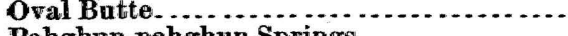 & Powell ...................... & \\
\hline rings .......... & & \\
\hline nted Cañon, entrance $\ldots \ldots \ldots \ldots \ldots$ & $\ldots . . .$. & 746 \\
\hline ited Rock ... & 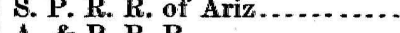 & 728 \\
\hline - n. & A. de $\mathbf{P} \cdot \mathbf{R} \cdot \mathbf{R}, \ldots$ & 1,065 \\
\hline & S, P. P & $\begin{array}{l}3,538 \\
3,012\end{array}$ \\
\hline 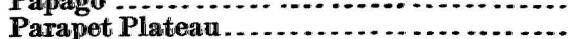 & Arrz & \\
\hline edge) & Pow & 7,276 \\
\hline & & $, 000-7,300$ \\
\hline & ............. & 6,896 \\
\hline & & 7,19 \\
\hline $\mathbf{N}$ & Wh & 6,272 \\
\hline & A. \& $\mathbf{P}$. & $4 ; 759$ \\
\hline & well .. & 4,110 \\
\hline .... & Tone & 3,174 \\
\hline$\ldots \ldots \ldots$ & 'to & 315 \\
\hline & & \\
\hline
\end{tabular}




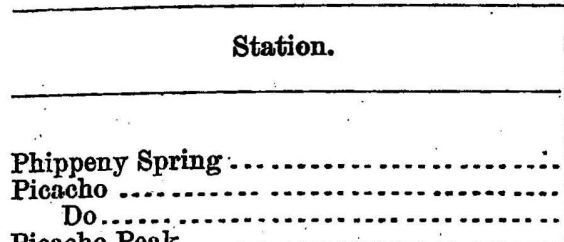

Picacho Peak

Picacho Spring

Picket Post

Pimas Villages

Pine Tanks

Pinneveta.

Pipe Spring Point

Pipo Springs

Playa de los Pimas

Polliwiog Spring

Pottery Hill.

Powell

Prescott

Prieto Crossing

Puello Colorado

Pueblo Viega

Quercns Cañon.

Rachel Butte.

Railroad Pass

$$
\text { Do. }
$$

$$
\text { Do. }
$$

Railroad Pass Station

Rawlins, Camp (old)

Red Butte.

Red Mesa

Red Rock

Relief Springs

Rillito

Rock Spring

Rock Spring, Fort

Runk's Pass

Russell Point.

Russell's Tank

Saccaton

Sacramento

Safford

Saint John's

Saint Joseph

Salt Spring

San Carlos Agency

Sanders

Sanford's

San F'rancisco, $\mathrm{Mt}$

Do $\ldots \ldots$
Do $\ldots \ldots \ldots \ldots$

San Francisco Spring

San Simon

Sentinel

Shi-wits Platean

Do

Shungo-pah-we

Sichoamavi

Sierra Blanca, (timber lirio on)

Signal Peak.

Silver Spring

Sitgreaves Pass

Skull Valley, Camp

Sonoita

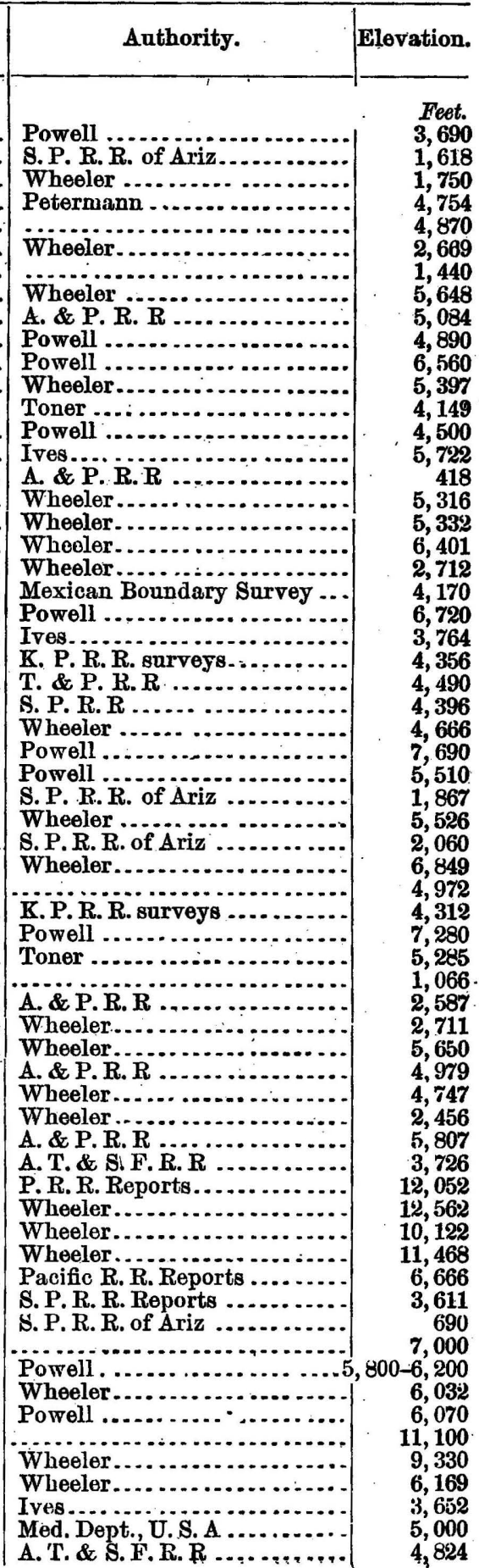

(157)

Bull. 5-3 


\begin{tabular}{|c|c|c|}
\hline Station. & Authority. & Elevation. \\
\hline & & Feet. \\
\hline $\begin{array}{l}\text { Spears' Ranch... } \\
\text { Springerville. }\end{array}$ & $\begin{array}{l}\text { Wheeler. } \\
\text { Wheeler. }\end{array}$ & $\begin{array}{r}681 \\
6,862\end{array}$ \\
\hline 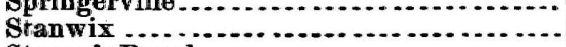 & S.P.R.R. of Ariz & $\begin{array}{r}0,002 \\
567\end{array}$ \\
\hline Steven's Ranch .. & Powell .............. & 4,650 \\
\hline $\begin{array}{l}\text { Summit Spring .. } \\
\text { Sunset Crassing. }\end{array}$ & $\begin{array}{l}\text { Wheeler. } \\
\text { Wheeler. }\end{array}$ & $\begin{array}{l}7,867 \\
4,891\end{array}$ \\
\hline $\begin{array}{l}\text { Sunset Crossing .. } \\
\text { Snnset Gap...... }\end{array}$ & Wheeler.. & 5,755 \\
\hline Sunset Tanks.... & Wheeler.. & 5,797 \\
\hline Supai ............. & $\begin{array}{l}\text { A.\&.P.R.R } \\
\text { S.P.R.R of Ariz }\end{array}$ & $\begin{array}{l}6,917 \\
1,298\end{array}$ \\
\hline $\begin{array}{l}\text { Sweet W ater ........ } \\
\text { Taylor, Mt ........ }\end{array}$ & 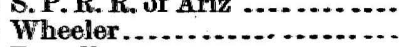 & $\begin{aligned} & 1, 296 \\
& 11,200\end{aligned}$ \\
\hline Tegua ................ & Powell ..... & \\
\hline Do.............. & Wheeler.... & 6,299 \\
\hline Terra & Powell & 6,070 \\
\hline $\begin{array}{l}\text { Texas Hill ...... } \\
\text { Thomas Peak ... }\end{array}$ & $\begin{array}{l}\text { S.P.R.R. of A } \\
\text { Wheeler...... }\end{array}$ & $\begin{array}{r}355 \\
11,496\end{array}$ \\
\hline Thompson's Sprir & Powell ............... & 4,980 \\
\hline Tho & Pow & \\
\hline Tipton Mesa ..... & Powell ... & 6,680 \\
\hline Tipt & Wheeler....... & 7,364 \\
\hline Tonto Pass...... & A.\&. P. R.R. surve & 7,529 \\
\hline Toroweap Valley & Powell ..... & $\begin{array}{l}4,550 \\
6,760\end{array}$ \\
\hline $\begin{array}{l}\text { Trident Point ...... } \\
\text { Trumball, Mt ..... }\end{array}$ & $\begin{array}{l}\text { Powell .... } \\
\text { Powell ... }\end{array}$ & $\begin{array}{l}6,760 \\
8,360\end{array}$ \\
\hline $\begin{array}{l}\text { Trumball, Mt ...... } \\
\text { Truxton ........... }\end{array}$ & $\begin{array}{l}\text { Powell } \\
\text { A.\& P.R.R }\end{array}$ & $\begin{array}{l}5,172 \\
4,172\end{array}$ \\
\hline Truxton Springs, & Wheeler... & 3,885 \\
\hline Tubace - & Toner .... & 3,000 \\
\hline Tucson & S.P.R.R. & 2,392 \\
\hline & Whe & 2,538 \\
\hline Do. Si & U. S. Signal & 2,404 \\
\hline Tull Spri & Wheeler.- & 5,861 \\
\hline atean & & $400-6,100$ \\
\hline Union Pass.... & Wheeler & 3,600 \\
\hline Val & R.R. Rep & 5,754 \\
\hline Verd & Wheeler... & 3,160 \\
\hline Vigil's Ranch & Wheeler. ........................ & 5,875 \\
\hline 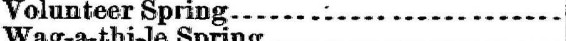 & r. & 7,106 \\
\hline thile Spring & Pow & 6,040 \\
\hline Wallipi Pass ....... & K.P.R. R. surveys .......... & 3,473 \\
\hline Pags......... & 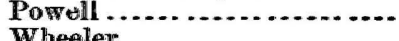 & 6,070 \\
\hline $\begin{array}{l}\text { Washing } \\
\text { Washi-pa }\end{array}$ & Wheoler............ & $\begin{array}{l}8,825 \\
4,421\end{array}$ \\
\hline Whipple, Fo & Wh & 5,318 \\
\hline & Pacifie R. R. Reports .. & 5,335 \\
\hline Whipple Pass & K.P.R.R. surveys .... & 7,206 \\
\hline Sprin & Wheeler............. & 6,301 \\
\hline$\cdots \cdot$ & & 1,202 \\
\hline k's cien & S.P.R.R.. & $\begin{array}{l}4,166 \\
4,300\end{array}$ \\
\hline & A. \& P.R.R. & 6,727 \\
\hline Ranch & Wheeler............. & 7,487 \\
\hline & & 4,170 \\
\hline Willow Spring - & Wheeler.: & 7,294 \\
\hline Winslow, & A.\& P.R. 1 & 4,825 \\
\hline Wonsit's Plain ..... & Powell .............. & $4,500-5,000$ \\
\hline Junetion......... & A.\& P.R.R ...... & $\begin{array}{l}5,552 \\
5,369\end{array}$ \\
\hline Yucei & A.\& P.R.R ......... & 1,774 \\
\hline $\begin{array}{l}\text { Yuma } \\
\text { Do. Signal Sta }\end{array}$ & S.P.R.R R & 142 \\
\hline Do. Signal Sta & U. S. Signal Office............... & \\
\hline
\end{tabular}


ARKANSAS.

\begin{tabular}{|c|c|c|}
\hline Sta & Authority. & Elevation. \\
\hline & & Feet. \\
\hline $\begin{array}{l}\text { Alexander... } \\
\text { Alicia........ }\end{array}$ & $\begin{array}{l}\text { St. L., I. Mt. \& S, R. R } \\
\text { Toner.............. }\end{array}$ & \\
\hline ma $\ldots . . . .$. & L. R. \& Ft. S.R.R. & $\begin{array}{l}244 \\
477\end{array}$ \\
\hline tus ...... & L. R. \& Ft. S. R. R . & 581 \\
\hline renta...... & M. \& L. R.R.R....... & 301 \\
\hline zadelphia. & $\begin{array}{l}\text { St. L., I. Mt. \& S. R. H } \\
\text { L. R. \& Ft. S. R. R.. }\end{array}$ & $\begin{array}{l}191 \\
399\end{array}$ \\
\hline stin .......... & St. L., I. Mt. \& S. R. R & 258 \\
\hline cing Cross. & Toner & 246 \\
\hline$\cdots \cdots$ & St. L., I. Mt. \& S. R. R & $\begin{array}{l}250 \\
283\end{array}$ \\
\hline ville .. & 'Smithsonian Inst ...... & 1,790 \\
\hline & St. L., I. Mt. \& S. R. R & 246 \\
\hline ink & M. \& L.R. R.R...... & \\
\hline thorn & Smithsonian Inst $\ldots \ldots$ & 650 \\
\hline C & \& Ft. S. R. R... & $\begin{array}{l}449 \\
279\end{array}$ \\
\hline & Toner ...................... & $\begin{array}{l}279 \\
123\end{array}$ \\
\hline & (n. & 223 \\
\hline & To & 1,412 \\
\hline & $\& \mathrm{Ft}$ & 409 \\
\hline & L. R. \& Ft. S. R. R... & 51,0 \\
\hline …...... & L. R. \& Ft. S. R. R & $\begin{array}{l}361 \\
294\end{array}$ \\
\hline & $\begin{array}{l}\text { St. L., I. N } \\
\text { Toner .... }\end{array}$ & $\begin{array}{l}294 \\
268\end{array}$ \\
\hline & M. \& L.R.R.R...... & 181 \\
\hline & Toner ...... & 1,350 \\
\hline ins. & soni & 1,000 \\
\hline Fori & \& $\mathrm{L} . \mathrm{R}$ & 281 \\
\hline$\cdots$ & L. R. \& Ft. S. R. R... & 467 \\
\hline ver & St. L., I. Mt. \& S. R. R & $\begin{array}{l}272 \\
149\end{array}$ \\
\hline & Toner ............. & $\begin{array}{l}149 \\
500\end{array}$ \\
\hline & & 211 \\
\hline & L. 1 & 451 \\
\hline & L. R. \& Ft. S. R. R... & 353 \\
\hline $\mathrm{G}$ & St. L , I. Mt. \& S. R. R & 226 \\
\hline & L., I. Mt. \& S. R. R & $\begin{array}{l}213 \\
210\end{array}$ \\
\hline Hol & & 243 \\
\hline & St. L., I. Mt. \& S. R. R & 357 \\
\hline Hopefic & 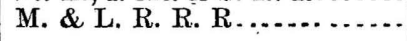 & 218 \\
\hline n Mississ & R R & $\begin{array}{l}217 \\
404\end{array}$ \\
\hline & & $\begin{array}{l}404 \\
718\end{array}$ \\
\hline & & 214 \\
\hline & St. L., I. M & 287 \\
\hline & L. R.\& Ft. S. R. R ... & 330 \\
\hline & St. L., I. Mt. \&. S. R. R. & 222 \\
\hline & $\mathrm{L}, \mathrm{I} . \mathrm{I}$ & $\begin{array}{l}229 \\
271\end{array}$ \\
\hline & L. R.\& Ft. S.R.R. & 437 \\
\hline & M. \& L.R.R.R.... & 210 \\
\hline & Pacitic R. R. Reports..... & 632 \\
\hline $\begin{array}{c}\text { Little Rock ..... } \\
\text { Do......Signa }\end{array}$ & $\begin{array}{l}\text { St. L., I. Mt. \& S. R. R. . } \\
\text { UJ. S. Sirual Office. }\end{array}$ & $\begin{array}{l}263 \\
298\end{array}$ \\
\hline Lockbart's ....... & 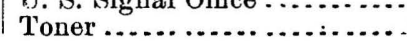 & 242 \\
\hline
\end{tabular}




\begin{tabular}{|c|c|c|}
\hline Station. & Authority. & Elevation. \\
\hline & & \\
\hline London ... & L. R. \& Ft. S. R. R. & 406 \\
\hline McAlmont & Toner ................ & 263 \\
\hline McLean's Landing ... & Toner & 360 \\
\hline $\begin{array}{l}\text { Madison, on St. Franci } \\
\text { Do ..town proper }\end{array}$ & M. \& L. R. R. R.. & 213 \\
\hline Malvern ............. & St. L., I. Mt. \& S. R. I & 277 \\
\hline Mineral Spring. & Toner ................. & \\
\hline Minturn Spring & Tomer ... & 251 \\
\hline Moar & Tone & 287 \\
\hline Montana .. & L. R.\& Ft. S. R. R. & 432 \\
\hline Morrilton . & L.R.\& Ft. S. R. R. & 393 \\
\hline Mull & L. R. \& Ft, S. R. R. & 439 \\
\hline Murta ....... & Toner .................. & 261 \\
\hline Napo & Pacific R. R. Rep & 142 \\
\hline $\mathrm{Ner}$ & St.L., I. M & 232 \\
\hline O'Koan ... & St. L., I. Mt. \& S. R. R & 276 \\
\hline ant & Toner ..... & 215 \\
\hline & L. R. & 386 \\
\hline & L. R. & 424 \\
\hline & Ft. & 311 \\
\hline $\mathrm{Pe}$ & , I. Mt. \& S.R.R. & 290 \\
\hline & Tone & 418 \\
\hline $\mathbf{P} 1$ & L.R.\& Ft. & 333 \\
\hline Pre & St. L., I. Mt. \& S. R. R & 328 \\
\hline$\ldots, \ldots, \cdot$ & & \\
\hline & & 264 \\
\hline & L. & 391 \\
\hline$\cdots .$. & Med. Dept., U.S. A . & 460 \\
\hline & L.R.\& Ft. S.R.R.. & 422 \\
\hline Hill . & Smithsonian Inst. & 188 \\
\hline ..... & St. L., I. Mt. \& S. R. R. . & 253 \\
\hline Texark & St. L., I. Mt. \& S. R. R.. & 303 \\
\hline ..... (Junc. T. \& J. Div. T' & T. \& P.R.R ........... & 358 \\
\hline Van Buren .......... & L.R. \& Ft. S. R. R. & 449 \\
\hline Do - & L. R. \& Ft. S. R. R. & 450 \\
\hline Do ..... (l. w. Arkansas River, 1878). & L. R. \& Ft. S. R. R.... & 423 \\
\hline t Ridge $\ldots \ldots \ldots \ldots \ldots \ldots$ & St. L., I. Mt. \& S. R. R.. & 275 \\
\hline & Toner.... & 231 \\
\hline & L. R. \& & 331 \\
\hline (.......... & Smithsonian Inst $\ldots . .$. & 660 \\
\hline Wl & L.R.\& Ft. S. R. R..... & $43 \tilde{5}$ \\
\hline
\end{tabular}


CALIFORNIA.

\begin{tabular}{|c|c|c|}
\hline & Authority. & Elevation. \\
\hline & & Feet. \\
\hline bbey Hill . . . . . . & $\begin{array}{l}\text { U.S.C. \& G.S } \\
\text { U.S.C. \& G.S }\end{array}$ & $\begin{array}{r}1,232 \\
375\end{array}$ \\
\hline campo............ & & \\
\hline $\begin{array}{l}\text { dalante....... } \\
\text { dams, Mt..... }\end{array}$ & Cal. P. R. R . & $\begin{array}{r}76 \\
8,31\end{array}$ \\
\hline dams, Mt.... . & $\begin{array}{l}\text { Wheeler.... } \\
\text { Wheeler... }\end{array}$ & $\begin{array}{r}8,431 \\
282\end{array}$ \\
\hline dobe Meadows. & Wheeler.... & 6,594 \\
\hline Agua Caliente... & Emory ..... & 3,013 \\
\hline $\begin{array}{l}\text { Do } \\
\text { Do................... }\end{array}$ & $\begin{array}{l}\text { Wheeler....... } \\
\text { Wheeler....... }\end{array}$ & $\begin{array}{r}725 \\
3,617\end{array}$ \\
\hline lamo Mocho .... & P.R.R. Reports & -70 \\
\hline lcatraz Island. & U.S.C.\& C & 143 \\
\hline lgodones....... & P. R. R. Reports. & 46 \\
\hline lpine $\ldots \ldots \ldots \ldots$ & C.P.R.R..... & $\begin{array}{l}2,822 \\
3,607\end{array}$ \\
\hline Alta ............. & C.P.R.R. & 740 \\
\hline Alturas ... & Wheeler.. & 4,365 \\
\hline Altur & & 4,459 \\
\hline Ranc & Wheeler.. & 1,494 \\
\hline Anaheim. & C.P.R.R. & 130 \\
\hline & & \\
\hline , Mt.... & Wh & 9,000 \\
\hline N. & U.S.C.\& G & 159 \\
\hline Pea & U. & 782 \\
\hline Anet & Ton & 161 \\
\hline Antel & C.P.R.R. & 154 \\
\hline & & 359 \\
\hline ring. & Wheeler.... & 4,272 \\
\hline Aral & Wh & 5,697 \\
\hline & C.P.R.R.. & \\
\hline Bridge & Wheeler... & 3,375 \\
\hline$\therefore .$. & $\therefore$. & 1,81 \\
\hline Auburn .... & C.P.R.R.......... & 1,360 \\
\hline$\cdots \cdot \cdot$ & Smithsonian Inst .... & 1,176 \\
\hline Point & $\operatorname{ler} \ldots \ldots \ldots$ & 7,449 \\
\hline a, Foint & $\begin{array}{l}\text { U. S. C. \&. G.S } \\
\text { Wheeler............. }\end{array}$ & 594 \\
\hline Camp ... & & 384 \\
\hline Bache, $\mathrm{Mt}$...... & U. S. C & 3,793 \\
\hline D & $\operatorname{ann} . \ldots \ldots \ldots \ldots \ldots$ & 3,790 \\
\hline $\operatorname{ch} \ldots$ & Wheeler........ & 4,076 \\
\hline , & $\begin{array}{l}\text { Wheeler.... } \\
\text { Wheeler }\end{array}$ & $\begin{array}{l}5,387 \\
6,284\end{array}$ \\
\hline & Wheeler....... & $\begin{array}{r}0,284 \\
432\end{array}$ \\
\hline Bakes & Toner..... & 3,285 \\
\hline Bald I & ler........ & 5,829 \\
\hline & Wheeler................ & 8,295 \\
\hline Bald Rock . & Wheeler .......... & 7,825 \\
\hline Mt... & $\begin{array}{l}\text { Whitney } \\
\mathrm{L} \text { A \& } \mathrm{R}\end{array}$ & $\begin{array}{r}6,357 \\
103\end{array}$ \\
\hline $\begin{array}{l}\text { Ballon } \\
\text { Banta }\end{array}$ & 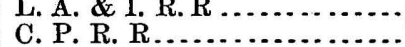 & $\begin{array}{r}103 \\
30\end{array}$ \\
\hline & Monterey $\mathrm{R} . \mathrm{R} . . .$. & \\
\hline & Wheeler............... & 6,039 \\
\hline Bares' Ranch, Surprise Valley ........ & Wheeler........................... & 4,680 \\
\hline Barker's Ranch.$\ldots \ldots \ldots \ldots \ldots \ldots \ldots \ldots$ & Wheeler...................... & 594 \\
\hline $\begin{array}{l}\text { Barnard's Hotel ........................................ } \\
\text { Batavia.... }\end{array}$ & Wheeler $\ldots \ldots \ldots \ldots \ldots \ldots$ & 3,851 \\
\hline 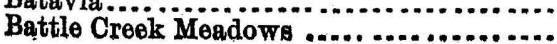 & 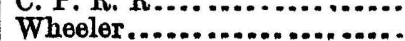 & 4,700 \\
\hline
\end{tabular}




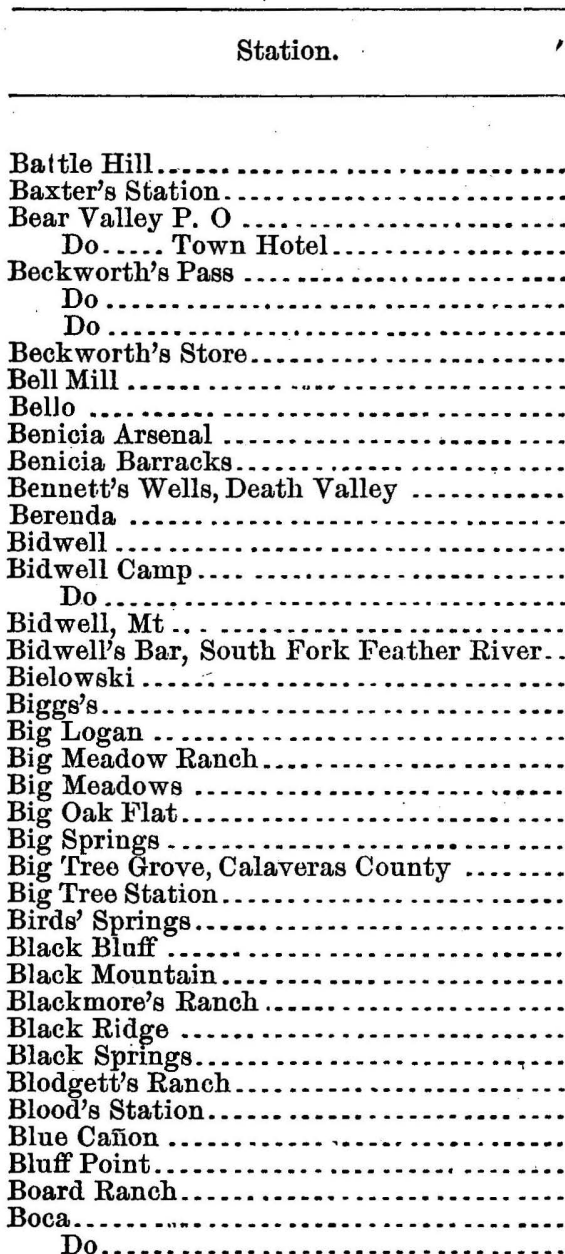

Bodega Head

Bold's Ranch

Bonita, Point

Boneyard Ranch

Boot-jack Ranch

Borden

Boston Peak

Bower Cave.

Box Elder.

Bozeman's Ranch.

Brandy City

Breccia Pass

Breckinridge, $\mathrm{Mt}$

Breckenridge, Mt.

Brewer, Mt

Brewery

Bridgeport

Bridgeport $\mathrm{P} .0$

Brighton

Brighton, Cross S..$\ddot{\text { R. R }}$

Bronoho

.

Wheeler.

Wheeler.

Wheeler.

Wheeler

R. R. survejs

Whitney

Wheeler.

Wheeler.

Cal. P. R. R

U. S. C. \& G. S

Med. Dept., U. S. A

Wheeler

Toner ..

Wheeler.

Wheeler.

Med. Dept., U. S. A

Wheeler.

Wheeler.

Whitney

C. P. R. R.

Toner..

Wheeler.

Wheeler.

Wheeler.

Wheeler.

Wheeler.

Wheeler.

Wheeler.

U. S. C. \& G. S

U. S. C. \& G. S

Wheeler.

U. S. C. \& G. S

Wheeler .

Wheeler.

Wheeler.

C. P. R. R

U. S. C. \& G. S

Wheeler.

Wheeler.

C. P. R. R

U. S. C. \& G. S

Wheeler........

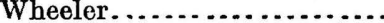

Wheeler.

Toner.

Wheeler.

Wheeler.

Wheeler.

Wheeler.

Goddard

Wheeler.

Wheeler

Whitney

Wheeler

Wheeler

Wheeler

S. \& P.R. R ...............

C. P. R. R

Wheeler.
Elevation.

Feet.

2,389

4,115

2,087

6,592

5,193

4,682

5,327

4,887

3,681

203

6

64

256

4,612

4,647

4,680

8,551

342

3,269

124

70

6,464

4,234

2,823

4,553

4,794

3,925

3,949

208

2,811

2,230

756

6,485

216

6,979

4,693

177

4, 639

5,230

5,531

241

141

283

2,450

2,107

172

6,519

2,360

1,430

622

3,157

3,592

10,150

5,693

7, 418

13,886

2, 838

1, 357

6,423

42

54

5,310 


\begin{tabular}{|c|c|c|}
\hline Station. & Authority. & Elevation. \\
\hline & & \\
\hline Brown's Flat ........... & $\ldots \ldots \ldots \ldots$ & 1,964 \\
\hline Brown's Peak ........... & (.................. & 5,392 \\
\hline Brown's Ranch.... & (n) & 1,759 \\
\hline Buckeye .......... & . . . & 4,938 \\
\hline Buckhorn Ranch (or Warren Station) ....... & $\ldots \ldots . . . .$. & 693 \\
\hline 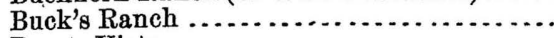 & Wheeler. & $5,11 \%$ \\
\hline 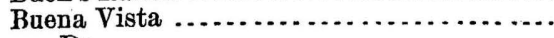 & Wheeler & 323 \\
\hline Do $\ldots \ldots$ & Nev. Co. N. G. R. R ........... & 2,618 \\
\hline 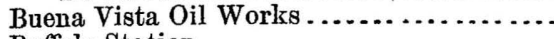 & & 790 \\
\hline Buffalo Station...$\ldots \ldots \ldots \ldots \ldots \ldots \ldots \ldots \ldots \ldots$ & (n.................. & 4,378 \\
\hline Burrows, Mt $\ldots \ldots \ldots \ldots \ldots \ldots \ldots \ldots \ldots$ & 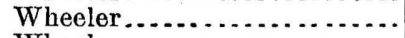 & 4,267 \\
\hline 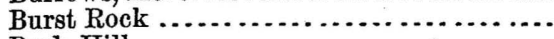 & 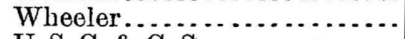 & 9,157 \\
\hline$\ldots \ldots \ldots \ldots$ & 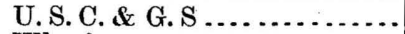 & 482 \\
\hline Butte Creek Bridge .... & 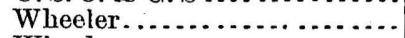 & 4,692 \\
\hline Butte Creek House ...... & Wheeler.. & 5,758 \\
\hline Butt, Mt $\ldots \ldots \ldots \ldots \ldots \ldots \ldots \ldots \ldots \ldots$ & Wheeler.... & 7,830 \\
\hline Byrnes' Ferry...$\ldots \ldots \ldots \ldots \ldots \ldots \ldots \ldots \ldots$ & $\ldots \ldots \ldots \ldots . . .6$ & 380 \\
\hline 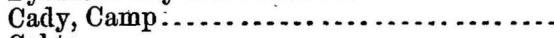 & 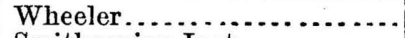 & 1,894 \\
\hline ................... & Smithsonian Inst ............ & 2,000 \\
\hline Cahuenga Pass...$\ldots \ldots \ldots \ldots \ldots \ldots \ldots$ & Wheeler....................... & 750 \\
\hline 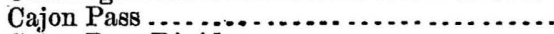 & Pacific R. R. Reports......... & 4,676 \\
\hline$\ldots \ldots \ldots \ldots \ldots$ & Wheeler................... & 4,195 \\
\hline Cajon Ranch.... & Pacific R. R. Reports ......... & 412 \\
\hline Calaveras Grove. & 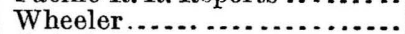 & 4,730 \\
\hline Caliente......... & C. P.R.R. & 1,290 \\
\hline Do........ & Wheeler. . & 1,314 \\
\hline Caliente Springs ... & Wheeler.................. & 3,688 \\
\hline California City Point................... & U.S. C. \& G.S ............ & 75 \\
\hline$\ldots \ldots \ldots \ldots . . . . . . .$. & C. P. R. R................. & 331 \\
\hline 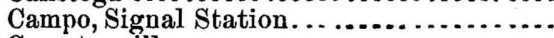 & U. S. Signal Office............ & 2,527 \\
\hline Camptonville $\ldots \ldots \ldots \ldots \ldots \ldots \ldots \ldots \ldots$ & ................ & 2,388 \\
\hline Camp Weldon (Mountain) .............. & Wheeler.. & 2,716 \\
\hline 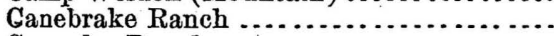 & Wheeler................... & 3,904 \\
\hline 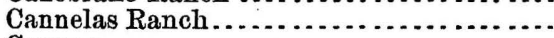 & 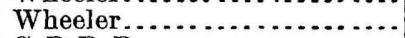 & 799 \\
\hline 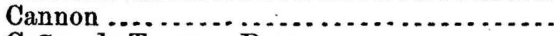 & C. P.R.R. & 90 \\
\hline Cañon de Turruco Pass . . . . . . . . . . . . . . . & & 4,256 \\
\hline 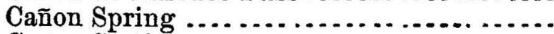 & Wheeler. & 1,238 \\
\hline$\ldots \ldots \ldots \ldots . . . . . .$. & 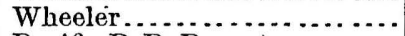 & 2,650 \\
\hline Capitan Grande & Pacific R. R. Reports......... & 730 \\
\hline Caples Ranch. & 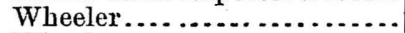 & 7,780 \\
\hline$\ldots . . . . . . . .$. & 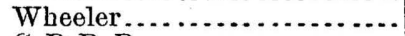 & 5,512 \\
\hline Carbondale $\ldots \ldots \ldots \ldots \ldots \ldots \ldots \ldots$ & 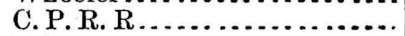 & 222 \\
\hline Caribou Bridge, North Fork .............. & Wheeler.................... & 2,843 \\
\hline 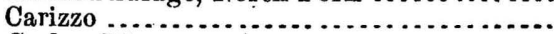 & & 431 \\
\hline Carlos, Mt ... & Whitney . & 4,977 \\
\hline Carmel, Mt ............... & U.S.C.\& G.S ........... & 4,415 \\
\hline Carnelian Hot Springs .......................... & Wheeler.................... & 6,237 \\
\hline Carson Cañon Toll-house ................. & $\ldots \ldots \ldots \ldots$ & 6,596 \\
\hline Carson Pass.................................. & $\ldots \ldots \ldots \ldots \ldots . . . . .$. & 7,972 \\
\hline Do...... & 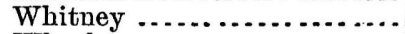 & 8,759 \\
\hline$\ldots \ldots \ldots . . . . . . .$. & Wheeler................... & 8,634 \\
\hline Cartago... & nen & 3,589 \\
\hline Carthage, on $\mathrm{Ow}$ & $\ldots \ldots \ldots . . . . . . .$. & 3,589 \\
\hline 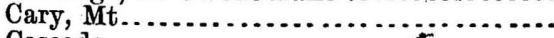 & ................... & 9,970 \\
\hline$\ldots \ldots \ldots$ & C. P. R.R. & 6,538 \\
\hline Castle, Mt .. & Wheeler... & 9,013 \\
\hline Castle Peak. & Whitney.. & 12,500 \\
\hline Castle Rock... ...................... & Wheeler................. & 9,872 \\
\hline Castroville, on line of S.P.R.R.......... & Monterey R. R............ & 19 \\
\hline Catharines...$\ldots \ldots \ldots \ldots \ldots \ldots \ldots \ldots$ & S. P. R. R . . . . . . . . . . & 512 \\
\hline Cathedral Rock (lower) & 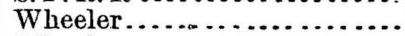 & 6,430 \\
\hline 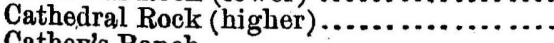 & Wheelor.................. & 6,529 \\
\hline 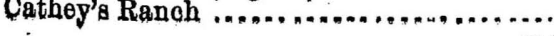 & Wheeler.................... & 1,260 \\
\hline
\end{tabular}




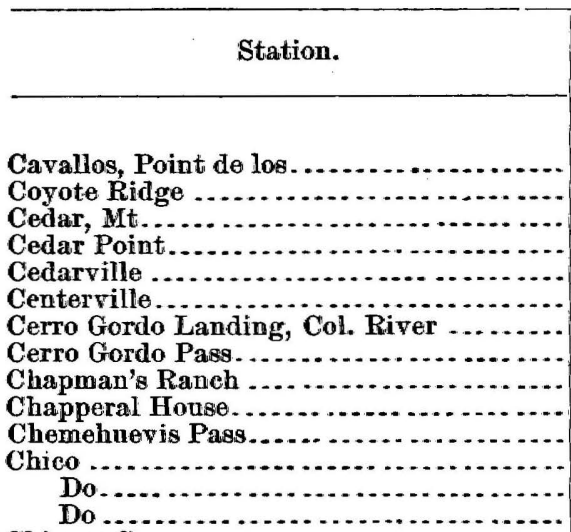

Chinese Camp

Chiquita Peak

Cicero

Cienega

Cisco

South Fork Yuba River

Clark Peak

Clark's.

(a)

Clayton

Clipper Gap

Cloud Rest.

Clover Valley

Cohen's Ranch

Cohuilla Village

Colby's Ranch.

Cold Spring

Cold Spring Ranch

Cole's Ranch

Coleville (blacksmith shop)

Colfax

Do

Colfax Jumetion, with Nevada Co. R. $\mathbf{R}$

Colona

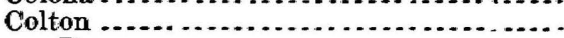

Do

Columbia

Columbia P. O

Columbia Rock (above valley)

Conejos

Conejos Ranch

Conness Peak

Contra Costa.

Cook's Point (Mountain)

Cook's Wells .

Coomb's Station

Cooper's Ranch

Copperopolis $\mathrm{P}$. O

Corbett's Rauch

Coreoran, Mt

Cordelia

Do

Cory's Peak

Costa

Cottonwood

Cottonwood Island

\begin{tabular}{|l|l}
\hline Authority. Elevation. & \\
\hline
\end{tabular}

U. S. C. \& G.S

U.S. C.\& G.S ............... 1,034

Wheeler................... $\quad 8,308$

Tower.................... 5,614

Wheeler.................. $\quad 4,675$

Wheeler................... 503

Wheeler..................... 3,656

Wheeler................... 8,874

Wheeler..................... 4, 4992

Wheeler................. 5,076

K.P. R. R. surveys ........... $\quad 675$

C. P. R. R................ 193

Smithsonion Inst ............. 150

Wheeler................... 177

Wheeler ..................... $\quad 1,299$

Wheeler................... 8,136

Wheeler.................. $\quad 2,095$

C.P.R.R.............. 90

L. A.\& I. R. R ............. 121

C. P. R. R................ 5,934

Wheeler.................... 5,654

Whe ler.................... 11, 295

Wheeler.................... $\quad 3,925$

Wheeler................... 4,677

Smithsonian Inst ........... 76

Wheeler.................. $\quad 5,808$

C. P. R. R............... 1,759

Wheeler................... $\quad \mathbf{9}, 772$

Wheeler.................... 3,464

Wheeler ................... 281

$\begin{array}{lr}\text { Pacific R. R. Reports ......... } & 85 \\ \text { Wheeler................... } & 4,990\end{array}$

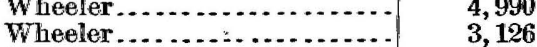

Wheeler.....................

Wheeler...................... 565

Wheeler.................... 1,221

Wheeler .................... $\quad 5,190$

New. Co. N. G. R. R .......... $\quad 2,422$

Wheeler................... 3,022

C. P.R.R................ $\quad 2,422$

............................. 835

Wheeler................... 808

C.P.R.R. . . . . . . . . . . . 56 ...

Toner ............

Wheeler................... $\quad 2,157$

Wheeler.................. 5,005

Wheeler.................... 2,565

Wheeler.................. 579

Wheeler.................. 12,518

U. S. C. \& G. S . . . . . . . . . .

Wheeler..................... 6,336

Pacific R. R. Reports ........

Wheeler.................... $\quad 2,886$

Wheeler.................... 8,406

Wheeler.................... $\quad 1,015$

Wheeler.................... 1,075

Wheeler..................... 14, 093

C.P.R. R ............... 11

Pacific R. R. Reports .......... 69

Wheeler ................... 11, 326

C. P. R. R............... 85

Toner ..................... 423

Wheeler..................... 787 


\begin{tabular}{|c|c|c|}
\hline Station. & Authority. & Elevation. \\
\hline & & Feet. \\
\hline 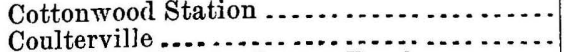 & & $\begin{array}{l}2,488 \\
1,665\end{array}$ \\
\hline Cow Creek Ranch, Sonora Road ........... & (n) & $\begin{array}{l}1,000 \\
5,905\end{array}$ \\
\hline 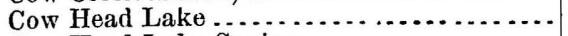 & . & 6,041 \\
\hline Cow Head Lake Spring . ................... & $\ldots \ldots \ldots \ldots$ & 5,329 \\
\hline Cow Spring & $\ldots \ldots \ldots \ldots$ & 3,876 \\
\hline 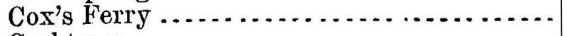 & $\ldots \ldots \ldots \ldots \ldots \ldots$ & 250 \\
\hline 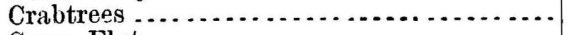 & $\ldots \ldots \ldots . . . . . . . .$. & 934 \\
\hline Crane Flat $\ldots \ldots \ldots \ldots$ & ................ & 6,054 \\
\hline Crane Valley.................... & ................... & 3,185 \\
\hline 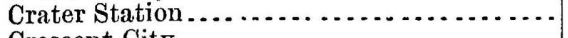 & & 1,000 \\
\hline Crescent City $\ldots . . . . . . . . . . . . .$. & Inst $\ldots \ldots \ldots$ & 12 \\
\hline 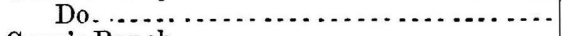 & Wh & 3,306 \\
\hline$\ldots \ldots \ldots \ldots \ldots$ & ler. & 5,157 \\
\hline$\ldots \ldots \ldots \ldots \ldots \ldots$ & C.P.R.R & 313 \\
\hline$\ldots \ldots \ldots \ldots \ldots \ldots \ldots \ldots$ & Wheeler. & 1,221 \\
\hline , Fort $\ldots \ldots \ldots \ldots \ldots$ & U.S.A........... & 3,390 \\
\hline Crow's Ranch, Clover Valley ............... & $\ldots \ldots \ldots \ldots \ldots$ & 5,464 \\
\hline Crystal Lake..................... & 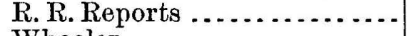 & 5,907 \\
\hline 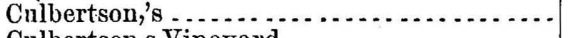 & $\ldots \ldots \ldots$ & 980 \\
\hline 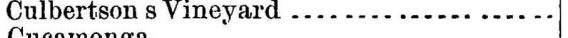 & eeler. & 981 \\
\hline Cucamonga $\quad \ldots$ & $\beta_{1}$ & 952 \\
\hline a Peak & n............ & 1,328 \\
\hline Ranch $\ldots \ldots \ldots \ldots \ldots \ldots$ & $\begin{array}{l}\text { Wheeler... } \\
\text { Wheeler... }\end{array}$ & $\begin{array}{l}8,5 \% 9 \\
1,168\end{array}$ \\
\hline 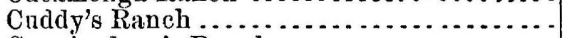 & Wheeler... & $\begin{array}{l}1,108 \\
5,278\end{array}$ \\
\hline Ranch .................. & Wh & 387 \\
\hline 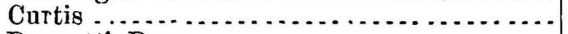 & C.P.I & $\begin{array}{r}307 \\
39\end{array}$ \\
\hline 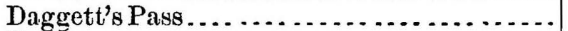 & (n) & 6,824 \\
\hline . & Wheeler. & 2,162 \\
\hline$\ldots \ldots \ldots \ldots \ldots \ldots \ldots \ldots \ldots$ & eler.. & 568 \\
\hline$\ldots \ldots \ldots \ldots \ldots \ldots$ & $\cdots \ldots \ldots \ldots$ & 13,227 \\
\hline 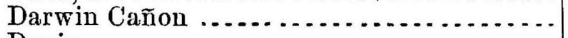 & Wl & 3,143 \\
\hline 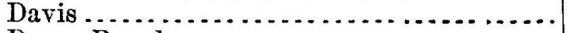 & R & 54 \\
\hline n.......... & $\ldots .$. & 451 \\
\hline$\ldots \ldots \ldots \ldots$ & $\ldots \ldots \ldots$ & 3,426 \\
\hline$\ldots \ldots \ldots . . . .$. & ...... & 4,451 \\
\hline 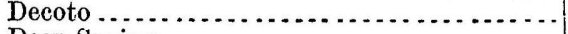 & $\ldots . . .$. & \\
\hline (n. & 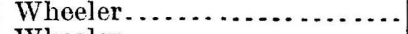 & 4,957 \\
\hline (n) & $\cdots \ldots \ldots \ldots \ldots \ldots$ & 4,518 \\
\hline . & $\ldots \ldots \ldots$ & 4,840 \\
\hline$\ldots \ldots \ldots \ldots \ldots \ldots \ldots$ & 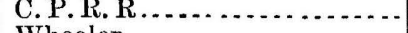 & 313 \\
\hline$\ldots \ldots \ldots \ldots$ & .......... & 1,989 \\
\hline Der & Wh & 2,922 \\
\hline $\begin{array}{l}\text { De } \\
\text { Di }\end{array}$ & (n) & 6,910 \\
\hline n. & $\cdots$ & .202 \\
\hline (n) & S & $\begin{array}{l}2,327 \\
3,849\end{array}$ \\
\hline $\begin{array}{ll} \\
\ldots \ldots \ldots \ldots \ldots \ldots \ldots \ldots \ldots\end{array}$ & (1) & $\begin{array}{r}3,849 \\
65\end{array}$ \\
\hline - & (......... & \\
\hline & (1) & 5,88 \\
\hline Donner Pass.. & Surveys .......... & 7,90 \\
\hline & $\ldots \ldots \ldots \ldots \ldots . . . .6$ & 7,05 \\
\hline Doon's Saw Mill . & $\mathrm{Wl}$ & 3,420 \\
\hline Dos Palmas & Wh & 103 \\
\hline & C.P.R.R & 114 \\
\hline 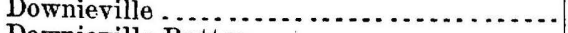 & Smithsonian Inst ........... & 2,200 \\
\hline Downieville Buttes . . ..................... & 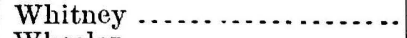 & 8,400 \\
\hline 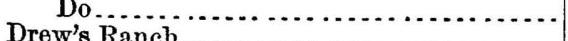 & Wheeler. & 8,541 \\
\hline & Wheeler........ & 1,090 \\
\hline $\begin{array}{l}\text { Dribblesby's Ferry . } \\
\text { Drune Barracks }\end{array}$ & Pacific R. R. Reports ....... & 954 \\
\hline $\begin{array}{l}\text { Drune Barracks ..... } \\
\text { Dudley's }\end{array}$ & Med. Dept., U. S. A............ & \\
\hline & & \\
\hline
\end{tabular}




\begin{tabular}{|c|c|c|}
\hline Station. & Authority. & Elevation: \\
\hline & & Feot. \\
\hline Duxbnry .. & U.S.C. \& & 797 \\
\hline Dugan's......... & S. \& P. R. & 1,106 \\
\hline Dunderberg Pea & Wheeler. & 12,289 \\
\hline Dutch Flat ........ & C.P.R. R & 3,395 \\
\hline Dutch Henry's Ra & Wheeler. . & 1,195 \\
\hline Duteh Hill Mining & Wheeler ... & 4, 692 \\
\hline Dyer Mountain, n & Wheeler... & 7,369 \\
\hline Eagle Lake . . . . . . & Wheeler. . & 5,115 \\
\hline Eagle Mountain & Wheeler- . & 9,933 \\
\hline Eagleville........ & Wheeler.... & 4,632 \\
\hline Ebbitt's Pass ..... & P.R.R. Rep & 8,793 \\
\hline Echo Peak .... & Wheeler... & 11,231 \\
\hline Edgar's Spring. & Wheeler. - & 4,061 \\
\hline Eisen Vineyard. & Wheeler.. & , 35.3 \\
\hline Eldorado Mill .. & Wheeler... & 863 \\
\hline Elephant, Mt. $\ldots \ldots \ldots \ldots \ldots$ & Wheeler....... & 10,418 \\
\hline Eleren Mile Station, Mariposa Road ....... & Wheeler ...... & $\therefore \quad 5,567$ \\
\hline Eliot's Ranch, on Little Truckee River .... & Wheeler... & 6,233 \\
\hline Elizabeth Lake. .. & Wheeler.. & 3,317 \\
\hline Elk Grove....... & C.P.R.R. & \\
\hline Elk Horn ....... & ports ........... & 89 \\
\hline Elkhorn Ranch. & Wheeler. & 1,049 \\
\hline Elk Valley ..... & & 3,751 \\
\hline Ellis ........ & C.P.R. R & 76 \\
\hline Ellis Mountain . & Wheeler. & 8,675 \\
\hline Elmira......... & C.P.R. R & 75 \\
\hline El Monte.... & Wheeler.. & 329 \\
\hline El Paso Mines...... & $\ldots \ldots \ldots \ldots$ & 4,113 \\
\hline Emigrants' Gap .... & ................. & 5,221 \\
\hline Do $\ldots . . . . .$. & ports .......... & 5,221 \\
\hline Eureka „......... & 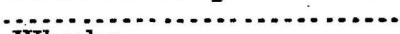 & 5,223 \\
\hline Eureka Valley .. & . $\ldots \ldots \ldots \ldots$ & 5,957 \\
\hline Excelsior Hotel & $\ldots \ldots$ & 4,570 \\
\hline Fandango Peak & Wheeler.. & 849 \\
\hline Far West, Camp & Med. De & 175 \\
\hline Fears' Station... & Wheeler & $\mathbf{3}, 393$ \\
\hline son's Mill. . & Wheeler. & 389 \\
\hline Mile Creek & $\ldots \ldots$ & 1,267 \\
\hline Fisherman's Peak & $\ldots \ldots \ldots$ & 14,448 \\
\hline nd Station... & Toner... & 1,900 \\
\hline cen $\ldots . .$. & C.P.R. R & \\
\hline$\ldots$. & Tor & \\
\hline Folson & S. & 182 \\
\hline t Ranch. & $\ldots \ldots$ & 2,217 \\
\hline y's Ranch & Wheeler. & 3,381 \\
\hline Fornis' Ranch.. & Wheeler. & 4,225 \\
\hline ee's Ranch. & Whe & 3,587 \\
\hline Point ............ & G. S. & 186 \\
\hline y-nine," Cañor & $\ldots \ldots \ldots \ldots$ & 6,306 \\
\hline s Bar........ & . & \\
\hline 's Station ... & - . & \\
\hline r's Peak.... & Wheeler. & \\
\hline 8' Spring... & $\ldots \ldots \ldots$ & \\
\hline Lagoon .. & & \\
\hline Peak. & & \\
\hline ntain. & Wh & 10,84 \\
\hline Fr & C.P.R. R & \\
\hline Frenchman's Cove & Wheeler. & 5,565 \\
\hline Fresno .............. & C.P.R.R. & 294 \\
\hline Do............ & Wheeler. & 314 \\
\hline Fresno Flat .... . & Wheeler. & 2,192 \\
\hline Fryes' $\ldots \ldots$ & Wheeler. & 2,181 \\
\hline & & \\
\hline
\end{tabular}




\begin{tabular}{|c|c|c|}
\hline Station. & Authority. & Elevation. \\
\hline & & Feet. \\
\hline Farnace Creek ..... & Wheeler. & 405 \\
\hline Furnace Springs.... & Wheeler. & 337 \\
\hline 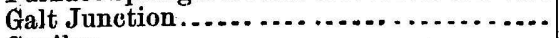 & C.P.R.R & 49 \\
\hline 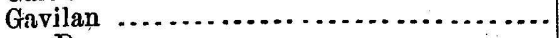 & U.S. C. \& & 2,816 \\
\hline 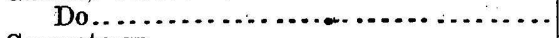 & $\ldots \ldots \ldots \ldots$ & 3,381 \\
\hline Georgetown $\ldots \ldots \ldots \ldots \ldots \ldots \ldots$ & Toner ... & 2,433 \\
\hline 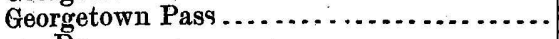 & Whitney & 7,119 \\
\hline 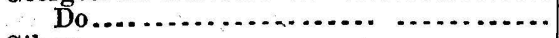 & Surveys ........... & 7,154 \\
\hline Gilroy $\ldots \ldots \ldots \ldots \ldots$ & $\ldots \ldots \ldots \ldots \ldots \ldots \ldots$ & 193 \\
\hline 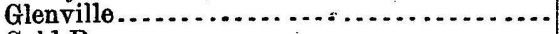 & Wheeler. & 3,094 \\
\hline 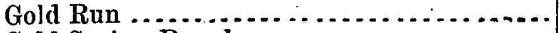 & C.P.R.R & 3,220 \\
\hline Gold Spring Ranch .. & Wheeler. & 2,014 \\
\hline Goodrich's Ranch ................ & Wheeler. & 4,883 \\
\hline Goose Lake . . . . . . . . . . . . . . . . & Wheeler. & 4,697 \\
\hline Gordon's Ranch ................... & 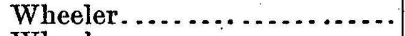 & 737 \\
\hline Gorman's Ranch .................... & Wheeler & 3,838 \\
\hline Goshen $\ldots \ldots \ldots \ldots$ & C.P.R. R & 278 \\
\hline Goshen Junction, with S. P. R. R. & C.P.R.R & $2 \& 0$ \\
\hline Guano Island & $\ldots \ldots \ldots$ & 28 \\
\hline Granite Spring & 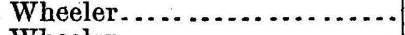 & 1,435 \\
\hline Granite Springs & Wheeler......................... & 4,115 \\
\hline Granite Station $\quad . \ldots \ldots \ldots \ldots$ & Wheeler....... & 1,744 \\
\hline 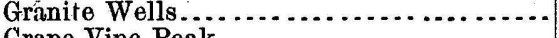 & Wheeler. . & 2,080 \\
\hline 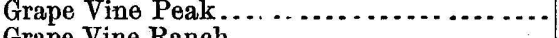 & Wh & 8,528 \\
\hline Grape Vine Ranch... & Wh & 2,247 \\
\hline Grape Vine Spring .. & (n) & 2,432 \\
\hline 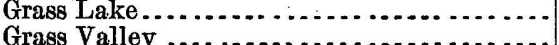 & $\cdots$ & $\begin{array}{l}8,564 \\
2,090\end{array}$ \\
\hline $\begin{array}{r}\text { Grass Valley }{ }_{1} \\
\text { Do } \ldots \ldots \ldots\end{array}$ & 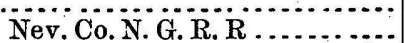 & $\begin{array}{l}2,090 \\
2,454\end{array}$ \\
\hline Gravel Range & 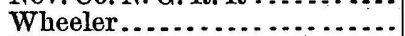 & 2,987 \\
\hline 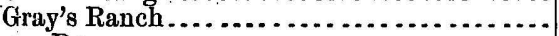 & Wheeler... & 307 \\
\hline 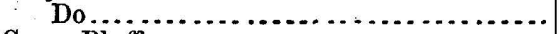 & Wheeler.. & 1,100 \\
\hline Green Bluff ... & U.S. C. \& G.S & 486 \\
\hline Green Mountain & Wheeler...... & 1,351 \\
\hline Do.......... & Wheeler. & 1,352 \\
\hline Green's Ranch ... & Wheeler....... & 4,479 \\
\hline Greenville ....... & Wheeler...... & 3,544 \\
\hline$\ldots \ldots \ldots \ldots \ldots \ldots$ & ............ & 97 \\
\hline$\ldots \ldots \ldots \ldots \ldots \ldots \ldots$ & $\ldots \ldots \ldots$ & 473 \\
\hline Grizzly Giant, Mariposa Grove ............ & . & 5,838 \\
\hline Grizzly Hill..................... & Wheeler (Theod) ............. & 5,709 \\
\hline Grizzly Peak......... & Wheeler......................... & 11,723 \\
\hline Do............. & Wheeler... & 10,369 \\
\hline Groveland & Wheeler... & 2,828 \\
\hline 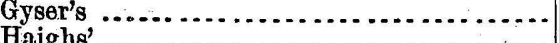 & Wheeler........ & 5,864 \\
\hline . & Wheeler........ & 1,807 \\
\hline . & eler. & $\begin{array}{l}739 \\
359\end{array}$ \\
\hline $\begin{array}{l}\text { Halfway ho } \\
\text { Halloran } \mathrm{Sn}\end{array}$ & Wh & 359 \\
\hline$\cdots$ & Wh & 272 \\
\hline 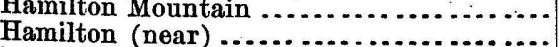 & (nts & $\begin{array}{r}4,440 \\
260\end{array}$ \\
\hline 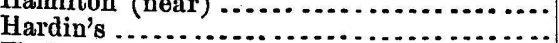 & 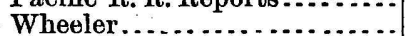 & 3,39 \\
\hline Harkness Mountain, near Big Meadows.... & ler (Theod) & 8,875 \\
\hline Harris' Ranch, Madeline Plains............ & ler.................. & 5,339 \\
\hline Harris' Station, Amander Road............. & Whe & 5,439 \\
\hline s Ranch......... & Wheeler. & 242 \\
\hline Haskell's Peak $\ldots \ldots \ldots \ldots \ldots \ldots \ldots \ldots \ldots$ & Wheeler. . & 8,126 \\
\hline 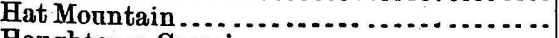 & Wheeler.. & 7,676 \\
\hline own Crossing ........................ & 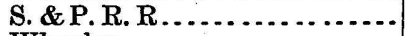 & \\
\hline lah Town...$\ldots \ldots \ldots$ & Wheeler......................... & 3,150 \\
\hline s' Station $\ldots \ldots \ldots \ldots \ldots \ldots \ldots \ldots \ldots \ldots \ldots$ & Wheeler.......... & 5,957 \\
\hline 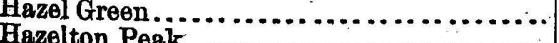 & Wheeler................................. & 5,550 \\
\hline & Wheeler,..................... & 2,960 \\
\hline
\end{tabular}




\begin{tabular}{|c|c|c|}
\hline Station. & Authority. & Elevation. \\
\hline [azel Valler & & Feet. \\
\hline Helena, Mount... & $\begin{array}{l}\text { Wheeler... } \\
\text { Whitney. }\end{array}$ & $\begin{array}{l}3,404 \\
4,343\end{array}$ \\
\hline Tenness Pass.... & C.P.R.R.Su & \\
\hline Do & Whitney ... & 6,996 \\
\hline ennessy's Br & Wheeler.. & 1,821 \\
\hline enry, Mount. & Whitney . & 2,398 \\
\hline Hermit Valley & Wheeler.... & 7,039 \\
\hline $\begin{array}{l}\text { Hickman's Ra } \\
\text { High Bluff ... }\end{array}$ & $\begin{array}{l}\text { Wheeler....... } \\
\text { U. S.C.\& G.S.. }\end{array}$ & $\begin{array}{r}1,907 . \\
533\end{array}$ \\
\hline High Hill ..... & U. S. C.\& G.S. & 490 \\
\hline d Peak. & Wheeler... & 10,956 \\
\hline Hill's $\mathrm{I}$ & Pacific R. & 4,131 \\
\hline ite's & Wheeler. & 1,601 \\
\hline don's ....... & Wheeler. & 4,506 \\
\hline Hoffimann Peak & Wheeler.. & 10,872 \\
\hline$\ldots \ldots \ldots$ & C.P.R.R. & 76 \\
\hline er.......... & $\begin{array}{l}\text { Toner .... } \\
\text { S. \& P.R. }\end{array}$ & $\begin{array}{r}284 \\
32\end{array}$ \\
\hline Lake ... & Wheeler.. & 3,949 \\
\hline .... & & 543 \\
\hline$\cdots \cdot$ & Willi & 7,072 \\
\hline$\cdots$ & Wheeler.. & $\begin{array}{r}847 \\
5.477\end{array}$ \\
\hline …... & Wheeler.. & $\begin{array}{l}5,477 \\
3,860\end{array}$ \\
\hline $\begin{array}{ll}\cdots \\
\cdots \ldots . .\end{array}$ & $\begin{array}{l}\text { Whe } \\
\text { Whe }\end{array}$ & $\begin{array}{l}3,860 \\
3,635\end{array}$ \\
\hline Hotc. & Wheeler... & 2,931 \\
\hline Hot S & & 6,080 \\
\hline & Whe & 7,384 \\
\hline Do. & Wheeler... & 7,692 \\
\hline Hougl & $\mathrm{Wh}$ & \\
\hline & WI & 3,860 \\
\hline & Ton & 980 \\
\hline & & 3,122 \\
\hline & $\mathrm{Me}$ & 50 \\
\hline $\mathrm{Hu}$ & Wh & 4,847 \\
\hline $\mathrm{Hu}$ & $\mathrm{P}$. & 5,351 \\
\hline Hunte & Wh & 6,274 \\
\hline & & 6,275 \\
\hline obave ] & & 2,899 \\
\hline$\cdots$ & & 2,667 \\
\hline $\mathrm{Hyc}$ & Wh & 5,288 \\
\hline & Whee & 1,759 \\
\hline & Tor & 2,234 \\
\hline dence, Camp ............. & ler....$\ldots \ldots \ldots \ldots$ & $\begin{array}{l}3,957 \\
4,800\end{array}$ \\
\hline 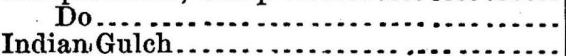 & $\begin{array}{l}\text { Smithsonian Inst .......... } \\
\text { Wheeler }\end{array}$ & $\begin{array}{r}4,800 \\
951\end{array}$ \\
\hline $\begin{array}{l}\cdots \cdot \\
\cdots \cdot .\end{array}$ & $\begin{array}{l}\text { Wheeler } \\
\text { Toner } . .1\end{array}$ & 3,280 \\
\hline Indian Wells ...... & C.P.R.R.... & -20 \\
\hline & & 2,608 \\
\hline Ingalls, Mount . .... & Whes & $\begin{array}{l}8,471 \\
4,808\end{array}$ \\
\hline $\begin{array}{l}\text { Inskip Toll-gate..... } \\
\text { Ione ............... }\end{array}$ & $\mathrm{R}$ & 287 \\
\hline$[\ldots \ldots \ldots \ldots$ & Wheeler................................ & 4,238 \\
\hline & & 934 \\
\hline Jacksonville .. & Wheeler.......................... & 602 \\
\hline Jelly's Ranch .. & Wheeler............... & \\
\hline $\begin{array}{l}\text { Joe's Peak ...... } \\
\text { John's, Mount . }\end{array}$ & 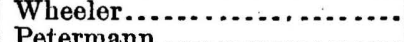 & 9,712 \\
\hline & Goddard & 6,752 \\
\hline & C.P.R.R. Surveys & 7,374 \\
\hline $\begin{array}{l}\text { Do........ } \\
\text { Do...... }\end{array}$ & $\begin{array}{l}\text { Simpson } \ldots \ldots \\
\text { Whitney } . . \ldots \ldots \\
\end{array}$ & $\begin{array}{l}7,222 \\
7,339\end{array}$ \\
\hline
\end{tabular}




\begin{tabular}{|c|c|c|}
\hline Station. & Authority. & Elevation. \\
\hline & & Feet. \\
\hline Johnson's Ranch $\ldots \ldots \ldots \ldots \ldots \ldots$ & $\ldots \ldots \ldots$ & 3,460 \\
\hline Jobuson's Ranch, Bressor Creek. ........... & sian. & 5,643 \\
\hline Jones, Fort..... & J.S. A ............. & $\begin{array}{l}2,570 \\
3,416\end{array}$ \\
\hline 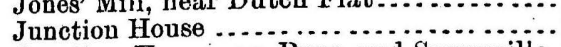 & 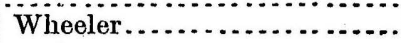 & 3,562 \\
\hline $\begin{array}{l}\text { Junction House, on Reno and Susanville } \\
\text { road, near Beckwith's Pass .............. }\end{array}$ & & 4,639 \\
\hline Junction with Oregon Branch ............ & C. P. R. R & 163 \\
\hline Kaweah Peak $\ldots . . . . . . . . . . . . . . . . . . .$. & Whitney . & 14,000 \\
\hline Keg Spring, Willow Creek..................... & Wheeler .... & 5,757 \\
\hline Kern Laka & Pacific R. R. Reports ......... & 398 \\
\hline 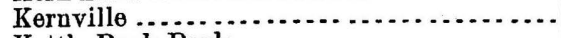 & Wheeler ........................... & 2,551 \\
\hline$\ldots \ldots \ldots \ldots \ldots$ & Wheeler $. . . \ldots \ldots \ldots \ldots . . . . . . .$. & 7,843 \\
\hline 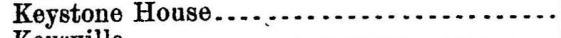 & Wheeler ..... & $\begin{array}{l}1,093 \\
0,58\end{array}$ \\
\hline Keysville & wheoke. & 2,558 \\
\hline Kincaid's Flat. $\ldots \ldots \ldots \ldots \ldots$ & eler .. & 1,589 \\
\hline Kincaid's Ranch & Wheeler ....... & 1,771 \\
\hline King's Springs, Death Valley .................. & Wheeler ....... & -225 \\
\hline Kingston & $\begin{array}{l}\text { Petermann ...... } \\
\text { Wheeler ....... }\end{array}$ & 1,118 \\
\hline 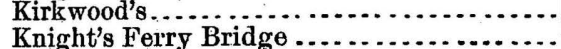 & $\begin{array}{l}\text { Wheeler } \ldots \ldots \ldots \\
\text { Wheeler } \ldots \ldots \ldots\end{array}$ & $\begin{array}{r}780 \\
180\end{array}$ \\
\hline 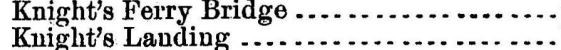 & C. P.R.R.. & $\begin{array}{r}180 \\
43\end{array}$ \\
\hline 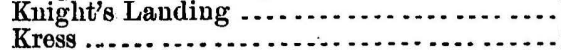 & Nev. Co. N. G. R. R........ & 2,857 \\
\hline $\begin{array}{l}\text { Kress } \\
\text { La Bayonn }\end{array}$ & Wheeler ................... & $\begin{array}{r}2, \infty 7 \\
16\end{array}$ \\
\hline (n) & Wheeler ........ & 222 \\
\hline 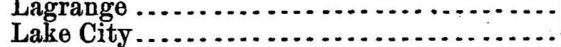 & Wheeler .... & 4,624 \\
\hline Lake City Pass... & Wheeler ...... & 7,035 \\
\hline Lakeview .......... & Wheeler ...... & 4,851 \\
\hline La Laguna Ranch . - & Wheeler .... & 129 \\
\hline Lambert's Soda Spring ... & Wheeler .... & 8,558 \\
\hline La Motte's . . . . . . & Wheeler ... & 6,491 \\
\hline Lane's Crossing, Mojave River............ & Wheeler ... & 2,819 \\
\hline 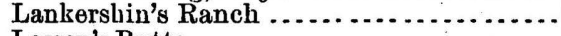 & Wheeler ... & 563 \\
\hline Lassen's Butte. . . . . . . . . . . . . . . . . . . . . & Wheeler . & 10,437 \\
\hline$\cdots$ & Whitney. & 10,577 \\
\hline Lathrop Junction, with Visalia Division .. & C. P.R.R. & 26 \\
\hline . & S. P. R. R..... & 782 \\
\hline Lava Bed Station. $\ldots \ldots \ldots \ldots$ & Whoeler ......... & 446 \\
\hline$\ldots \ldots \ldots \ldots$ & Toner ..... & 66 \\
\hline Leach's Point . & Wheeler .. & 3,409 \\
\hline Leek Spring . & Wheeler ... & 7,242 \\
\hline Lewis' Ranch .................. & Wheeler ....... & 966 \\
\hline Lewis' Ranch, near Loyalton. & Wheeler . & 4,949 \\
\hline ............ & Wheeler & 3,756 \\
\hline$\ldots . . .$. & Wheeler. & 3,647 \\
\hline e Point Bluff & U. S. C. \& G. S. & 495 \\
\hline Lincoln ....... & C. P.R.R... & 161 \\
\hline n's Head .... & Wheeler .. & 1,693 \\
\hline$\ldots \ldots \ldots$ & Wheeler -. & 6,442 \\
\hline 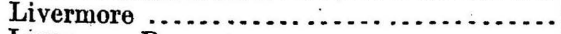 & C. P. R. R. & 485 \\
\hline re Pass... & Whitney . & 686 \\
\hline ool Landing, Colorado River ..... & Whoeler .. & 606 \\
\hline Point........................... & U. S. C. \&. G. S. & 326 \\
\hline & U. S. C. \& G & 378 \\
\hline Lodi . & C. P. R. R.. & 55 \\
\hline Lomo ...... & Wheeler .. & 3,848 \\
\hline Lone Pine. & 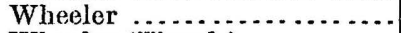 & 3,810 \\
\hline Longville ....... & Wheeler ('Theod.) ......... & 4,309 \\
\hline Lookout Hill. . . . . . & 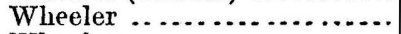 & 4,214 \\
\hline Lookout Mountain.$\ldots \ldots \ldots \ldots \ldots \ldots \ldots$ & Wheel $\cdot \mathbf{r} \ldots \ldots \ldots \ldots \ldots$ & 9,670 \\
\hline Loomis' Ranch .... & 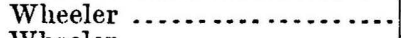 & 4,357 \\
\hline$\ldots \ldots \ldots \ldots \ldots$ & Wheeler .... & 3,248 \\
\hline & C. P. R. R. & \\
\hline
\end{tabular}




\begin{tabular}{|c|c|c|}
\hline Station. & Authority. & Elevation. \\
\hline & & \\
\hline Los Augeles.... & Wheeler..... & \\
\hline Do........... & Pacific R. R. Repo & 250 \\
\hline Do........San & L. A. \& I. R. R... & 260 \\
\hline Do........ Sig & U. S. Signal Offi & 350 \\
\hline os Encinos Ran & Wheeler ....... & 772 \\
\hline Los Pozos Ranch & Whoeler ...... & \\
\hline Los Toros..... & Wheoler. & 203 \\
\hline ott's Diggins & Whetior ..... & 6,310 \\
\hline uther's Pass . & Goddard ..... & \\
\hline & Simpson. & 7,505 \\
\hline Lyell, Mt ....... & Wheeler ..... & 13,190 \\
\hline Do.............. & Whitney ....... & 13,217 \\
\hline yon's Ranch & Wheeler .. & 1,397 \\
\hline MdBride's ..... . & Wheeler ..... & 5,561 \\
\hline McBride's Peak . & Wheeler ..... & 13,441 \\
\hline MeConnahas' & Wheeler ........ & 3,981 \\
\hline IcCum & Pacitic R. R. Repo & 3,491 \\
\hline AcDor & Wheeler.......... & 7,954 \\
\hline Ran & Wheeler....... & 5,297 \\
\hline eG & er (Theod. & 9,214 \\
\hline IcKesick's Pf & Whesler........ & 7,083 \\
\hline k's $\mathrm{R}$ & Wheeler....... & 4,469 \\
\hline lo's ... & Wheeler........ & 1,888 \\
\hline laco & Toner........... & 450 \\
\hline Hat & er.......... & 7,676 \\
\hline adeline Pass. & P. R. R. Reports & 5,667 \\
\hline falaga ............ & Wheeler......... & 2,320 \\
\hline & Wheeler....... & 5,030 \\
\hline 1 & \& G.S.. & 283 \\
\hline and, N. W & \&.G.S... & 101 \\
\hline & U.S & \\
\hline Island .... & \& G.S. & 74 \\
\hline 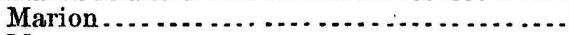 & \& G.S. & \\
\hline Maripo & Wh & 1,962 \\
\hline Do. & Wh & 1,971 \\
\hline Do.. & ......... & 1,942 \\
\hline Maripos & & 3,700 \\
\hline ark & Wh & 5,525 \\
\hline & Wh & 7,750 \\
\hline & Wh & 8,631 \\
\hline ach. & Wh & $8,0 \pi 4$ \\
\hline & R. Reports.. & 3,793 \\
\hline & & 187 \\
\hline C. H. & U.S. & \\
\hline Martin's & R. R... & \\
\hline Do & y R.R... & 1,982 \\
\hline Ranch. & ex............ & 2,055 \\
\hline arys & C. P.R. R............ & 67 \\
\hline .Do & Suithsonian Inst .......... & 80 \\
\hline Hill ........ & $\&$ G. S ......... & 2,445 \\
\hline Ranch... & & 6,294 \\
\hline so, Mt. & Wheeler (Theod.).. & 8,844 \\
\hline & $\therefore \ldots \ldots$ & 34 \\
\hline & P. R. R...... & 58 \\
\hline & Wh & 10,540 \\
\hline dow Mountain.... & & 11,734 \\
\hline dow Valley........ & Wheeler.. & 3,757 \\
\hline$\cdots, \ldots \ldots, \ldots, \ldots$ & C. P. R. R.. & \\
\hline ey Valley .................... & Wheeler & 9,503 \\
\hline ….............................. & C. P.R. R............... & 173 \\
\hline Falls ....................... & 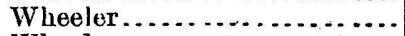 & 360 \\
\hline & Wheeler..................... & 11,413 \\
\hline
\end{tabular}




\begin{tabular}{|c|c|c|}
\hline Station. & Anthority. & Elevation. \\
\hline & & \\
\hline Merritt's ......... & 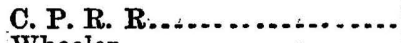 & 54 \\
\hline Mesquite Spring - & 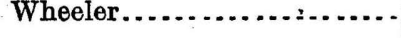 & 2,010 \\
\hline Mesquite Wells.. & 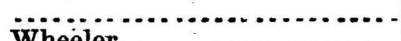 & 3,674 \\
\hline Middle Lake, Su & Wheeler... & 4,551 \\
\hline Midway & C. P. R. R & $\begin{array}{r}356 \\
-76\end{array}$ \\
\hline Mill Creek, Sonora road.... & Wheeler... & 7,076 \\
\hline Iiller, Fort ..... & Med. Dep & 402 \\
\hline $\begin{array}{l}\text { Miller's Ranch } \\
\text { Mills of Madera Flume \& Trading Co }\end{array}$ & Wheeler... & $\begin{array}{l}4,0 \text { b5 } \\
4,499\end{array}$ \\
\hline 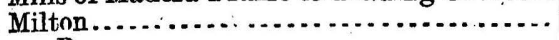 & Wheeler. & 376 \\
\hline Do $\ldots . . .$. & Wheeler. & 5,845 \\
\hline Mineral Bar... & .................. & 1,121 \\
\hline Mitchell's Ranch .......... & Wheeler. & 4,285 \\
\hline Moccasin, Mt.... & Wheeler & 2,791 \\
\hline Modesto ...... & C. P. R. 1 & 93 \\
\hline Mojavo ....... & C. P. R. R & 2,751 \\
\hline umne $\ldots$ & Toner .... & 5,523 \\
\hline Mokelumne, Mt & Wheeler. & 9,467 \\
\hline Molate Island .. & $\mathbf{S} \ldots \ldots \ldots \ldots$ & 169 \\
\hline Molate Point ... & 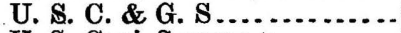 & 133 \\
\hline Mono Lake..... . & Survey ............ & 6,730 \\
\hline Mono Pass .... & . & 10,765 \\
\hline$\ldots \ldots$ & Toner .... & 354 \\
\hline Monte Diablo & Whi & 3,856 \\
\hline Monterey ... & Monterey $\mathbf{R}$. & 7 \\
\hline Do $\ldots$. & Med. Dept., & 140 \\
\hline Moonlight Valley & Wheeler ... & 5,433 \\
\hline Moquelumne Hil & Smithsonian Inst ............. & 1,502 \\
\hline Moran's Ranch......... & 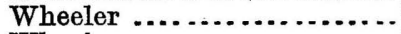 & 3,984 \\
\hline Mormon Bar .... & Whe & 1,630 \\
\hline Morocojo.... & $\ldots \ldots . . .$. & 15 \\
\hline Morongo Basin. & $\ldots \ldots \ldots$ & 1,500 \\
\hline$\ldots \ldots \ldots$ & $\ldots \ldots \ldots \ldots$ & 2,065 \\
\hline Spr & Whee & 2,010 \\
\hline Mountain House & Wheeler.. & 5,641 \\
\hline Mud Spring, Amandor road. ...... . . & Wheeler & 5,973 \\
\hline Mud Springs ......................... & Wheeler . & 4,671 \\
\hline Murphy ...... . & U. S. C. \& G. S............ & 2,703 \\
\hline Murphỳ's Cabin, Lake Tonaiya ........ & Wheeler ..................... & 7,$9 ; 1$ \\
\hline hyy's Mining Village . ................. & $\ldots \ldots \ldots \ldots . . . . .$. & 195 \\
\hline Murphy's Ranch, Buffalo Salt Works...... & Wheeler .... & 3,845 \\
\hline Myers' Ferry . ................ & Wheeler. & 7,434 \\
\hline$\ldots \ldots \ldots$ & Wheeler & 3,759 \\
\hline$\ldots \ldots \ldots$ & Wh & 2,394 \\
\hline Napa & C. P. R. R & 18 \\
\hline $\mathbf{N}$ & C. P. R. R & \\
\hline Junction (Adalante) ............ & C. P. R. R. & \\
\hline h's Ranch ..................... & Wheeler... & 4,431 \\
\hline & Ton & 125 \\
\hline da City : & G. R. R........ & 2,531 \\
\hline bury Peak & 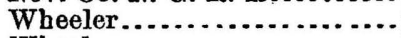 & 3,375 \\
\hline y Park. & Wheeler... & 830 \\
\hline 10..... & C. P. R. R. & 956 \\
\hline whall $\ldots . .$. & 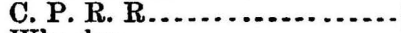 & 1,152 \\
\hline whall's Ranch ....... & Wheeler... & 974 \\
\hline w Pass .............. & P. R. R. Re & 3,164 \\
\hline York Tent . . . . . . . . . . & Wheeler... & 1,143 \\
\hline yara Creek, Sonora road... & 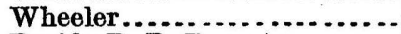 & 6,690 \\
\hline holas (near) ............... & Pacific R. R. Reports ....... & 289 \\
\hline ols Point ....................... & 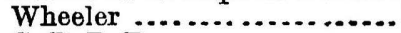 & 6,262 \\
\hline Junc. with San José Branch .... & C. P. R. R................... & 88 \\
\hline 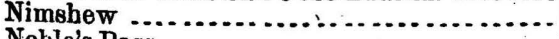 & Wheeler...$\ldots \ldots \ldots \ldots$ & 2,451 \\
\hline & & 5,963 \\
\hline
\end{tabular}




\begin{tabular}{|c|c|c|}
\hline Station. & Authority. & Elevation. \\
\hline & . & Feet. \\
\hline Noble's Pass .. & Williamson............ & 6,260 \\
\hline Noman's Spring & 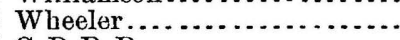 & 3,735 \\
\hline Nora .......... & C. P.R.R & 153 \\
\hline Nordhoff... & Wheeler. & 819 \\
\hline North Dome (above valley 3,633 ) . . . . . . . . & Wheeler. & 7,481 \\
\hline North End Peak................. & Wheeler. & 8,472 \\
\hline Northups (Excelsio & $\mathrm{S} \ldots \ldots \ldots \ldots$ & 4,519 \\
\hline$\ldots \ldots \ldots \ldots \ldots \ldots$ & .............. & 95 \\
\hline . & $\ldots \ldots \ldots \ldots \ldots$ & 7,110 \\
\hline 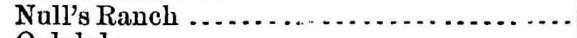 & $\ldots \ldots \ldots . . . . . .$. & 1,299 \\
\hline 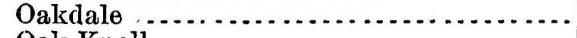 & $\ldots \ldots \ldots$ & 149 \\
\hline 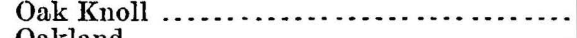 & C.P.R.R & 102 \\
\hline - & C. P.R.R. & 12 \\
\hline 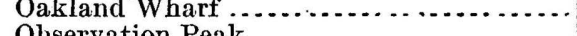 & C.P.R.R. & 14 \\
\hline 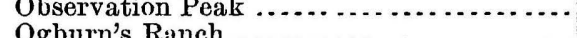 & $\ldots \ldots$ & 8,009 \\
\hline 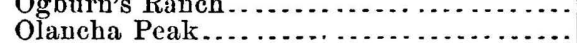 & $\begin{array}{l}\text { Wheeler ( } \mathrm{T} \\
\text { Wheeler... }\end{array}$ & $\begin{array}{r}2,270 \\
12,250\end{array}$ \\
\hline Old Bony Mountain . . . . . . . . . . . & Wheeler. & $\begin{array}{r}12,200 \\
1,892\end{array}$ \\
\hline shew Settlement ............. & Wheeler.. & $4,99 \cdot 2$ \\
\hline 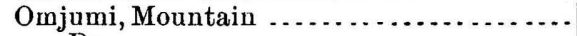 & Whitney . & 8,378 \\
\hline 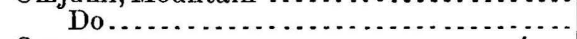 & Wheeler.. & 8,292 \\
\hline Orango & ( . P. R.R. & 134 \\
\hline Orot & Wheeler. & 188 \\
\hline Oso & Wheeler. . & 5,982 \\
\hline (n. & Whitney .. & 3,363 \\
\hline 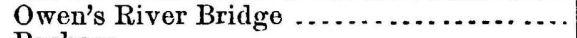 & 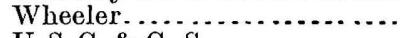 & $3,61^{R}$ \\
\hline 1eco $\ldots . . . .$. & U.S. C.\& G. S............... & 21 \\
\hline$\ldots \ldots \ldots \ldots \ldots \ldots$ & 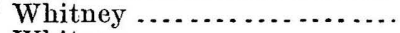 & 1,470 \\
\hline 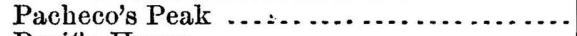 & Whitney .................. & 2,845 \\
\hline 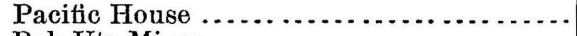 & Wheeler................... & 3,451 \\
\hline ( & Wheeler............................ & 6,607 \\
\hline 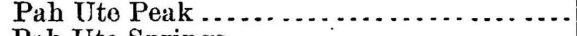 & $\ldots \ldots \ldots$ & $8,34 \%$ \\
\hline . & 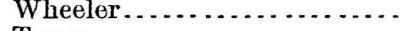 & 2,849 \\
\hline$\ldots \ldots . . .$. & To & 22 \\
\hline Palet & Wheoler. & 4,507 \\
\hline Palmer's Ranch ... & $\ldots \ldots$. & 2,346 \\
\hline 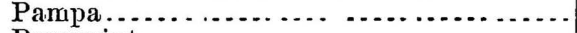 & ........... & 871 \\
\hline & Wheeler :- & 6,605 \\
\hline 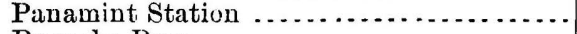 & Wheeler........ & 3,549 \\
\hline . & Whitney ......... & 2,500 \\
\hline 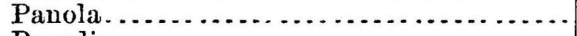 & $\ldots \ldots \ldots$ & 48 \\
\hline (n. & Toner..... & 125 \\
\hline$\cdots$ & C.P.R.R & 400 \\
\hline $\mathrm{Pa}$ & L. A. \& I. R. R & 178 \\
\hline Park & Wheeler...... & 4,136 \\
\hline ott's (formerly Pando'a) Ferry ........ & Wheeler. & 834 \\
\hline | Spring & Wheeler........ & 5,303 \\
\hline dler's Hill ...... & Wheeler......... & 6,831 \\
\hline Peña Blanca (Haigh & Wheeler................. & 1,807 \\
\hline 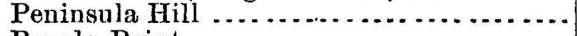 & U.S.C. \& G.S ... & 367 \\
\hline$\ldots \ldots \ldots \ldots . . .6 . . .6$ & U.S. C. $\&$ G.S ................. & 68 \\
\hline 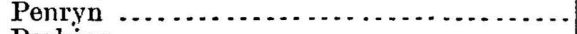 & er $\ldots \ldots \ldots \ldots \ldots$ & 624 \\
\hline (n............. & S. P. & 51 \\
\hline & U. & 111 \\
\hline Ranch & Wh & 6,999 \\
\hline Ranch ... & Wheeler. & 242 \\
\hline llips Station ... & Wheeler................... & 6,871 \\
\hline Pilot Knob. . . . . . . & Wheeler............................ & 5,525 \\
\hline Pilot Peak ........ & Whitney ..................... & 7,605 \\
\hline Pinos Mountain ... & Petermann .................... & 9,500 \\
\hline 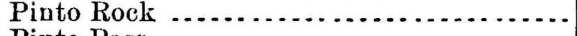 & Wheeler............................ & 3,903 \\
\hline 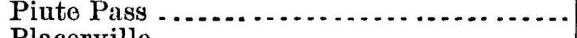 & K. P. R. R. surveys ............. & 2,579 \\
\hline Placerville & Toner $\ldots \ldots \ldots$ & 2,109 \\
\hline
\end{tabular}




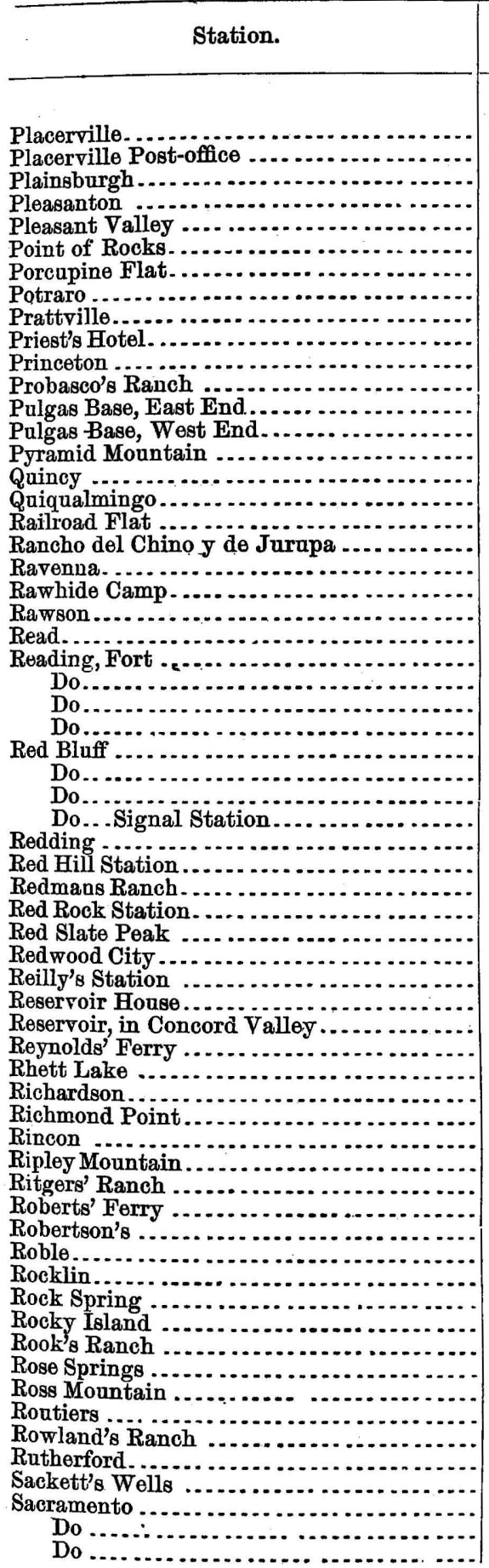

Authority. Eleva

Williamson

Wheeler

Toner $\begin{aligned} & \text { T. R. R . . } \\ & \text { C. }\end{aligned}$

Wheeler

Wheeler.

Wheeler.

Wheeler

Wheeler

Wheeler

Wheeler.

Wheeler.

U. S. C. \& G. S

U.S. C. \& G. S.

Wheeler

Wheeler

Toner...

Med. Dept. U. S. A

C. P.R. R

Wheeler

U. S. C. \& G. S ................

Pacific R. R. Reports.........

Pacific R. R. Reports..........

Pacific R. R. Reports ..........

Med. Dept. U. S.A.

Wheeler.

Williamson

C. P. R. R

U. S. Sig. Office ................

C. P. R. R . . . . . . . . . .

U. S. C. \& G. S ................

Wheeler.

Wheeler.

Whitney

Toner .

Wheeler.

Wheeler.

Wheeler.

Wheeler.

P. R. R. Reports

U. S. C. \& G.S
U.S. C. \& G.S

Toner.

Petermann

Wheeler.

Wheeler.

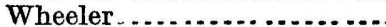

Toner ........................

C. P. R. R . . .

Pacific R. R. Reports.........

U. S. C. \& G. S................

P. R. R. Reports .............

Wheeler

U. S. C. \& G. S .....................

S. P. R. R ..................

Wheoler

C. P. R. R . . . . . . . . . . .

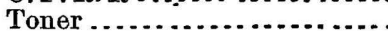

C. P. R. R .................

Smithsonian Inst .............

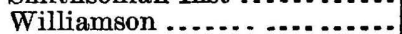

Feet.

1,965

1,893
209

353

2,405

2,542

7,749

1,028

4,394

2,558

2,104

973

19

129

10,127

3,381

1, 084

2,606

1,000

2,347

1, 556

228

474

596

675

674

518

307

370

308

324

556

188

1,181

2,394

13,400

10

1,477

1, 013

202

543

4,014

1,116

192

2,050

7,500

4,345

184

819

179

249

4, 898

157

4, 181

3,545

2,205

72

6,222

168

312

Bul. 5-4 


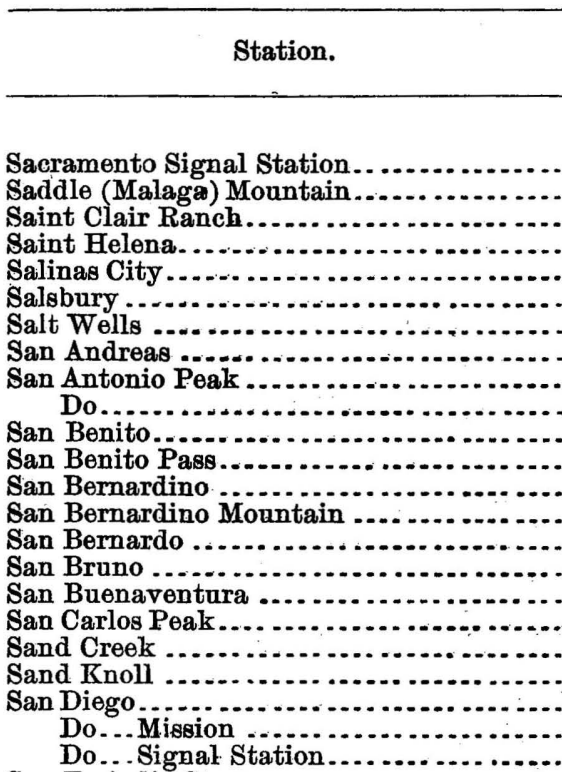

San Emigdio Store

San Fernando.

$$
\text { Do }
$$

San Fernando Pass

San Fernando Peak

San Fernando Tunnel, south mouth

San Felipe

Do

San Francisco, Signal Station

Do ......... Presidio

Do

San Francisquito Cañon

San Francisquito Pass

San Gabriel

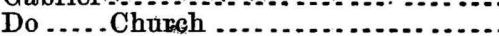

San Gabriel Mine

San Gabriel Peak

San Gabriel Range

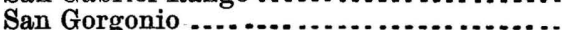

San Gorgonio Pass

$$
\text { Do.............. }
$$

San Isabel Rancho

San Jacinto Mountain

San Jose

$$
\text { Do.. }
$$

San Leandro

San Lorenzo

San Luis Obispo

San Luis Pass

San Luis Rey

San Mateo

San Miguel

San Pablo Point

San Pascual.

San Pedro.

San Pedro Point

San Pedro Hill

San Quentin, Point

Santa Ana

$$
\text { Do }
$$

Santa Ana Hotel

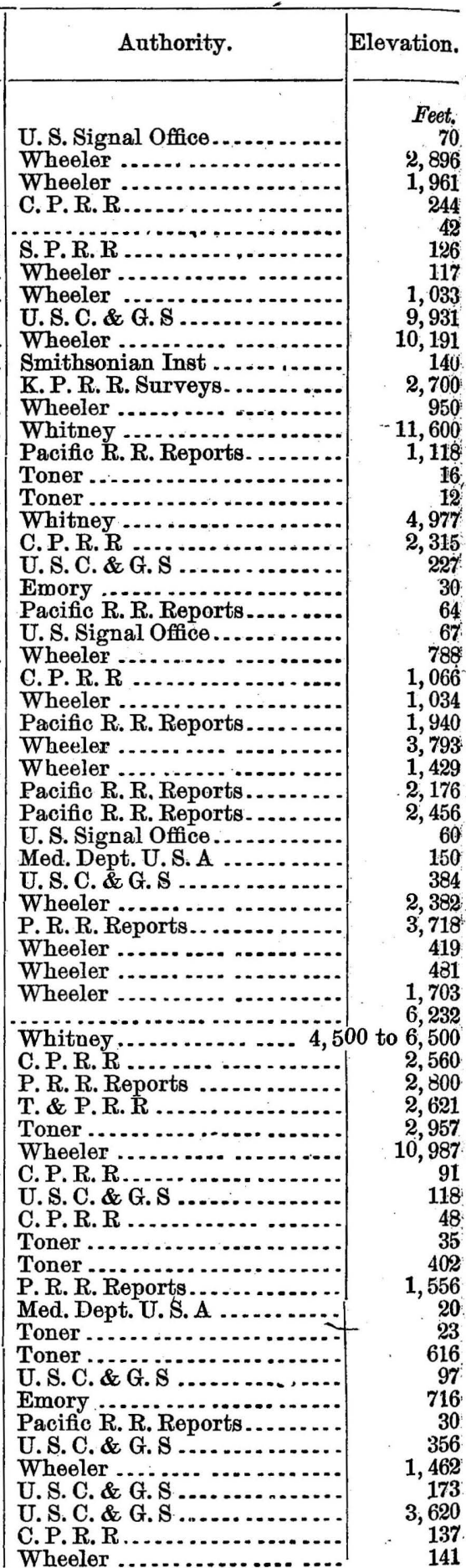




\begin{tabular}{|c|c|c|}
\hline Stat & Authority. & Elevation. \\
\hline & & Feet. \\
\hline 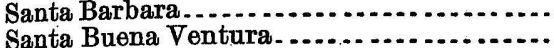 & Smithsonian Inst........ & $\begin{array}{r}20 \\
146\end{array}$ \\
\hline Santa Catalene........ & Toner... & 3,000 \\
\hline Santa Clara ........ & Smithson & 98 \\
\hline Santa Cruz Station & U. S. C. & 359 \\
\hline Santa Cruz Point. . & S.................. & 32 \\
\hline Santa Isabella ........ & & 3,050 \\
\hline Santa Isabella Ranch & R. Reports ......... & 2,957 \\
\hline Santa Monica ........ & $\mathbf{R} \ldots \ldots$ & 20 \\
\hline 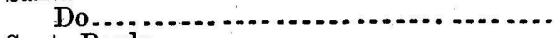 & . . . . . & 15 \\
\hline Santa $\mathrm{Pa}$ & Wheeler. & 384. \\
\hline Santa Rosa Valley . & Wheeler. & 175 . \\
\hline San Vincente....... & R............... & 167 \\
\hline$\ldots \ldots$ & Wheeler - & 5,553 \\
\hline$\ldots \ldots$ & $\log _{2}$ & 6,864 \\
\hline ool House ...... & $\ldots \ldots \ldots \ldots \ldots$ & 109 \\
\hline Schultz, Mount...... & Wheeler . . . & 2,275 \\
\hline - & Wheeler............................. & 2,715 \\
\hline 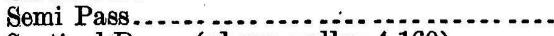 & 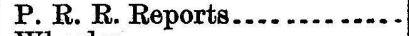 & 1,577 \\
\hline alley 4,160 ) . . . . . . . & 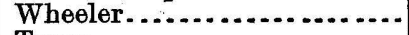 & 8,011 \\
\hline . & $\ldots \ldots . . . .$. & 229 \\
\hline Sevastapol Flat ..... & Whe & 2,210 \\
\hline ................. & C.P.R.R. & 1,126 \\
\hline Shafer's Station ....... & Wheeler. & 4,026 \\
\hline asta $\ldots \ldots \ldots . . . . .$. & ............. & 1,160 \\
\hline Shasta, Mount .......... & Whitney . & 14,442 \\
\hline 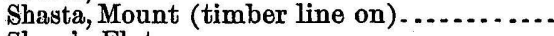 & (2) & 8,000 \\
\hline 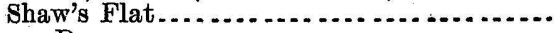 & .................... & 2,270 \\
\hline (n) & - & 2,036 \\
\hline Shaw's Ranch ... & $\ldots \ldots \ldots . . . . . .6$ & 6,311 \\
\hline Shear's Bridge.. & Wheeler. & 2,007 \\
\hline Head ........... & Wheeler... & 3,914 \\
\hline . & $\ldots \ldots \ldots \ldots \ldots$ & 4,094 \\
\hline . & & 113 \\
\hline . & S. P. R. R & 1,427 \\
\hline 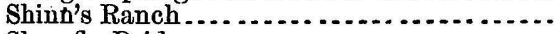 & 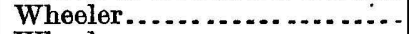 & 5,040 \\
\hline . & $\ldots \ldots \ldots$ & 3,071 \\
\hline n. & $\ldots \ldots$ & 5,067 \\
\hline$\theta y \ldots . . .$. & ler. . & 4,910 \\
\hline Sierravillo, Junc. of S. & Whe & 4,904 \\
\hline Do.....Post-office... & $\mathrm{Wl}$ & 4,880 \\
\hline Silliman, Mt............. & Wh & 11,623 \\
\hline (n) & 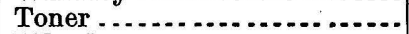 & 3,700 \\
\hline n. & $\ldots \ldots \ldots$ & $\mathbf{7}, 174$ \\
\hline . & $\ldots \ldots \ldots$ & 10,934 \\
\hline 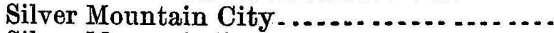 & $\ldots \ldots \ldots \ldots$ & 6,446 \\
\hline ntain Pass............... & $\ldots \ldots \ldots$ & 8,793 \\
\hline Ranch.$\ldots \ldots \ldots \ldots \ldots \ldots$ & & 674 \\
\hline$\ldots . . .$. & & 1,047 \\
\hline & W] & 4,163 \\
\hline g Post-office.. & W] & 252 \\
\hline r's Store ......... & Wheeler. & 4,925 \\
\hline 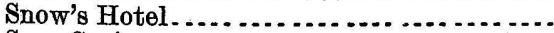 & Wheeler. & 5,217 \\
\hline Spring $\ldots \ldots \ldots$ & gler $\ldots \ldots \ldots \ldots$ & 706 \\
\hline Soda Lake ....... & Pacific R. R. Reports ......... & 1,002 \\
\hline$\ldots \ldots \ldots \ldots$ & Ier. . . . . . . . . . . . & 1,128 \\
\hline edad City ..... . . . . & . & 2,513 \\
\hline (n) & A. \& P. R. R. surveys ......... & \\
\hline tectes & ler & 5,908 \\
\hline ma Mountain.... & U.S. & 2,292 \\
\hline ra Mountain..... & Wheeler......... & 11,478 \\
\hline ora Pass ........... & P. R. R. Reports........... & 10,115 \\
\hline Sonora post-office $=\ldots \ldots \ldots$ & 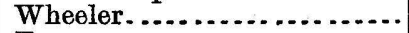 & . 1,816 \\
\hline Soto $\quad: \quad n_{1}$ & Toner ..... & 186 \\
\hline
\end{tabular}




\begin{tabular}{|c|c|c|}
\hline Station. & Authority. & Elevation. \\
\hline & & \\
\hline South Dome (lip) (a & Wheeler. & 8,804 \\
\hline South Fork Mountain .. & Wheeler. & 7,408 \\
\hline Spadra........................ & C. P.R.R & 705 \\
\hline Do.............. & Wheeler. - & 802 \\
\hline Spanish Ranch :- & Wheeler. . & 3,636 \\
\hline Sprague's Ranch. & Wheeler. . & 2,950 \\
\hline Springville...... & Wheeler.. & 48 \\
\hline Stanford Mov & Whitney ..... & 9,175 \\
\hline Starr King, Mt. (above valley & Wheeler.. & 9,022 \\
\hline State Line Peak .................. & Wheoler. & 8,405 \\
\hline Stevens Bar Ferry . & Wheeler: & 614 \\
\hline Stevens Mountain. & Wheeler. & 10,011 \\
\hline Stevens Ranch, Hor & Wheeler. . & 7,382 \\
\hline Stockton, Junc. with & C.P.R.R. & 23 \\
\hline Stockton's Cabin ... & Wheeler.. & 5,877 \\
\hline Stockton Mill & Wheeler...... & 4,639 \\
\hline Stokes Mou & Wheeler...... & $\overrightarrow{2}, 069$ \\
\hline Stonebreakers .... & Wheeler..... & 4,360 \\
\hline Stony Point ...... & - & 500 \\
\hline Storms............ & ............. & 2,424 \\
\hline berry & Wheeler. . & 5, 238 \\
\hline Strawberry Station (toll-house) ........... & Wheeler... & 5,695 \\
\hline Strawberry Valley . . . . . . . . . . . . . . . . . & Toner ..... & 3,567 \\
\hline 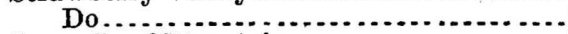 & Williamson.... & 5,707 \\
\hline ugar Loaf Mounts & Wheeler....... & 8,416 \\
\hline Sulphur Peak ...... & U.S. C. \& G. S... & 3,471 \\
\hline 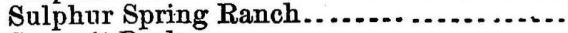 & Wheeler........ & 4,466 \\
\hline mit Peak ....... & Wheeler...... & 8,301 \\
\hline Summit Post-office, west of Beckwith's Pass & Wheeler..... & 4,875 \\
\hline Summit Station & Wheeler........ & 6,983 \\
\hline Summit Valley ..... & Toner ............. & 6,765 \\
\hline . & C.P.R.R..... & 415 \\
\hline Sunday Peak. & Wheeler.. & 8,335 \\
\hline Do........... & Wheeler.. & 11,089 \\
\hline unoe............... & $\ldots$ & 264 \\
\hline 'Surveyors' Wèlls .. & .......... & 3,567 \\
\hline Susanville & Wheeler....... & 4,195 \\
\hline Suspension Bridge, Mokelumne River ..... . & Wheel er........ & 2,092 \\
\hline 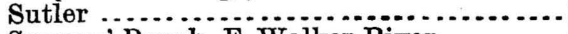 & Toner........... & 919 \\
\hline h, E. Walker River & Wheeler.. & 5,042 \\
\hline Countain ......... & Wheeler. & 11,778 \\
\hline A & $\cdots$ & 302 \\
\hline ore Grove... & Wheeler.. & 447 \\
\hline Tahoe City & Wheeler... & 6,252 \\
\hline Tahoe Lake. & R. R. Reports .. & 6,247 \\
\hline 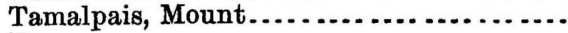 & Whitney ...................... & 2,597 \\
\hline c & & 6,209 \\
\hline Tan & Wh & 6,234 \\
\hline Tar & Wheeler. & 4,400 \\
\hline Tapo Ranch. & Wheeler....... & 1,373 \\
\hline Tas: & & 83 \\
\hline Taylor's Ranch.... & Whoeler.... & $\mathbf{1 , 0 4 7}$ \\
\hline Taylorville... & Wheeler.... & $\overrightarrow{3}, 479$ \\
\hline hapai, Moun & Wheeler (Theod.) .............. & 9,214 \\
\hline Tehachapai Pass. & 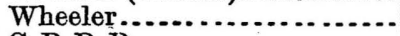 & 3,832 \\
\hline Tehama........... & R.R.... & 222 \\
\hline Tejon, Fort . & Med. Dept. U. S. A........... & 3,240 \\
\hline & 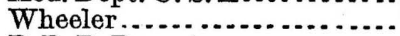 & 3,245 \\
\hline Tejon Pass... & P. R. R. Reports............. & 5,364 \\
\hline Tejon Ranch .... & 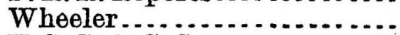 & 1,450 \\
\hline Telegraph Hill ..... & U. S. C. \& G. S $\ldots \ldots \ldots \ldots \ldots$ & 300 \\
\hline Telescope Mountain & Wheeler (Theod.).............. & 10,937 \\
\hline 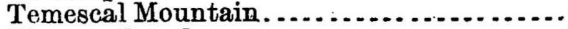 & Wheeler (Theod.) ............. & 5,730 \\
\hline Thomas Ranch.$\ldots \ldots \ldots \ldots \ldots \ldots \ldots \ldots$ & Wheeler....................... & 3,772 \\
\hline
\end{tabular}




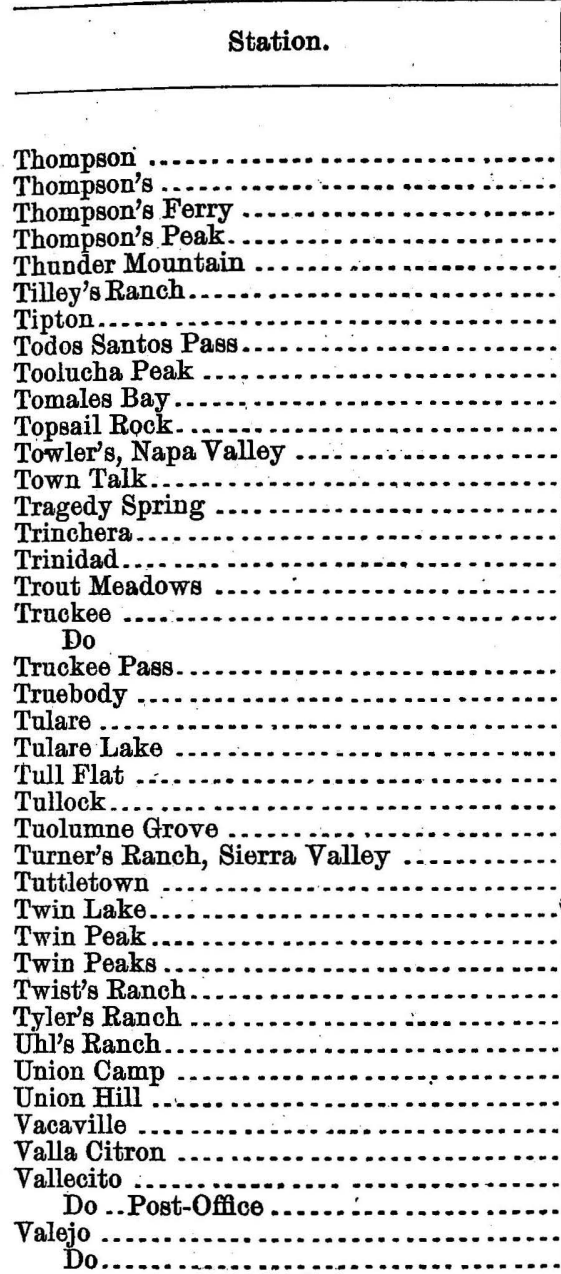

Vallejo (North)

Vallejo (South)

Vergennes Ranch

Vina.

Visalia

Visalia, Signal Station

Volcano

Wabler Lake House

Wades' Meadows

Wades' Peak

Wahguyhe Mountain

Walker's Pass

Do

Wallace's Ranch, Warner Lake

Walnut Grove.

Warner Lake .............

Warm Springs, Sonora Road

Warner's Pass

Warner's Ranch

Warren's Peak

Washington, Mount

Washington Quartz Mill

C. P. R. R

Wheeler

Wheeler.

Wheeler.

Wheeler.

Wheeler.

C. P. R. R

P.R. R. Reports

Wheeler.

U. S. C. \& G.S

U.S. C. \& G.S.

Nev. Co. N. G. R. $\mathrm{R}$

Wheeler.

Wheeler.

Wheeler

C.P.R. R

Wheeler

P. R. R. Reports

C. P. K. R

C. P.R. R

Pacific R. R. Reports

Wheeler.

Toner

Wheeler.

Wheeler.

Wheeler

Wheeler.

Wheeler.

Whitney

Wheeler

Wheeler.

Wheoler.

nian Inst

Nev. Co. N. G. R. R

Toner

Emory

Toner.

Wheeler.

U.S. C. \& G. $\mathbf{s}$

U.S. C. \&

C. P. R. R

年

C. P. R. R...................

Wheeler...............................

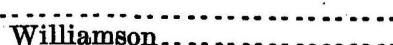

U. S. Signal Öfice.

Wheeler

Wheeler

Wheeler

Wheeler.

Wheeler (Theod.)

P. R. R. Reports.

Wheeler

Wheeler

C. P. R. R

C.P.R. R

Wheeler.

P. R. R. Reports.

P.R. R. Reports

Wheeler.

Wheeler

Wheeler
Elevation.

Feet.

2,114

188

7,752

9,121

2,609

267

637

7,022

673

81

369

2,774

7, 989

7,567

5,820

5,998

5,819

5,795

7,200

88

282

398

5,594

106

5, 794

4,904

1,321

5,106

8,824

8,925

1,121

4,802

2,662

54

2,706

175

1,539

1,643

1,748

87

371

26

13

211

384

348

2,075

6,808

4,567

7,153

8,527

5, 302

5, 322

4, 487

308

46

7, 385

3,870

3, 022

9,6 เे 8

10,802

1, 032 


\begin{tabular}{|c|c|c|}
\hline Station. & & Elevation.' \\
\hline & & Feet. \\
\hline Waucoba Peak & Wheeler. & 11,267 \\
\hline (2.2. & C.P.R.R. & 24 \\
\hline 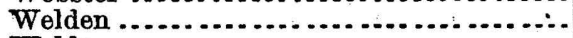 & Wheeler.. & 2,668 \\
\hline 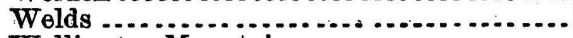 & Wheeler.. & 2,217 \\
\hline 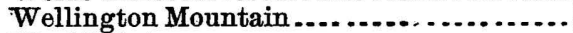 & Wheeler.. & 7,665 \\
\hline West Point. $\ldots \ldots \ldots \ldots \ldots \ldots$ & Wheeler.. & 2,749 \\
\hline West's Ranch . . . . . . . . . . . & Wheeler.. & 596 \\
\hline 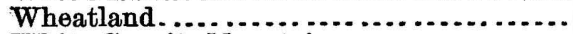 & ................... & 84 \\
\hline White Granite Mountain .................... & Wheeler.. & 7,045 \\
\hline 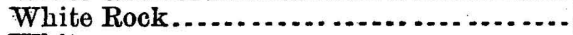 & S.P.R.R. & 495 \\
\hline Whitney ............... & Wheeler.. & 10,051 \\
\hline 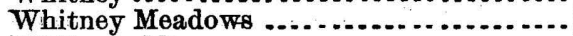 & Wheeler.. & 9,371 \\
\hline 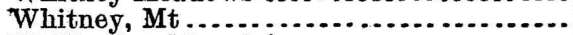 & Whitney. & 14,898 \\
\hline 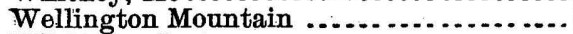 & Wheeler... & 7,665 \\
\hline 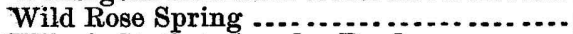 & Wheeler... & 4,683 \\
\hline Wiley's Station, Amador Road ............. & Wheeler.... & 5,027 \\
\hline Williamson River $\ldots \ldots \ldots \ldots \ldots$ & - & 4,387 \\
\hline Williamson's Lake ........................ & & \\
\hline 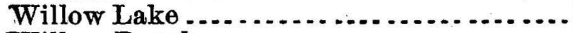 & . & $5,38 \%$ \\
\hline Willow Ranc & Wheeler. & 4,275 \\
\hline Willow Spring $\ldots \ldots \ldots \ldots \ldots \ldots$ & Wheeler.. & 420 \\
\hline Willow Spring (Head of Willow Creek) . . & Wheeler. . & 5,084 \\
\hline Willow Tree Spring & Wheeler... & 2,500 \\
\hline 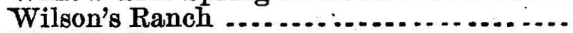 & Wheeler... & 1,115 \\
\hline Woodford's $\ldots \ldots \ldots \ldots \ldots \ldots$ & Wheeler.... & 5,676 \\
\hline 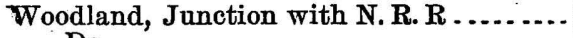 & C.P.R.R... & 63 \\
\hline Do $\ldots \ldots \ldots \ldots$ & U.S.C.\& G.S . & 58 \\
\hline ................... & ................... & 10,552 \\
\hline Workman's Hill ... . & Wheeler... & 1,363 \\
\hline Workman's Ranch .. & Wheeler... & 362 \\
\hline Wright Lake...... & P. R. R.Reports . . . . . . . & 4,470 \\
\hline Yallowbally ..... & Petermann .. & 8,000 \\
\hline Yankee Jim's ..... & & 3,185 \\
\hline Yerba Buena .... & U.S.C.\&.G. S ... & 345 \\
\hline Yosemite Valley & Whitney ........................ & 4,060 \\
\hline $\begin{array}{l}\text { Yosemite Valley (cliffs and domes about it } \\
\text { range from } 7,000 \text { to } 9,900 \text { feet above sea). }\end{array}$ & Williamson. & 3.935 \\
\hline 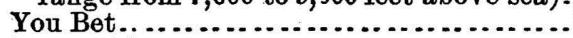 & 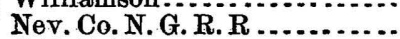 & 2,172 \\
\hline Yountville. & C.P.R.R................... & 97 \\
\hline Do.............. & Cal. P.R. R . . . . . . . . & 105 \\
\hline Yreka...$\ldots \ldots \ldots$ & 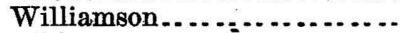 & 2,731 \\
\hline Yreka Gap $\ldots \ldots \ldots \ldots \ldots$ & 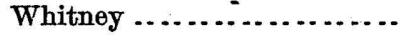 & 6,642 \\
\hline
\end{tabular}


COLORADO.

\begin{tabular}{|c|c|c|}
\hline Station. & Authority. & Elevation. \\
\hline & & \\
\hline Abeyta Pass... & Hayden & 9,300 \\
\hline Do .......... & D.\& R. G. & 9,399 \\
\hline cequia ... & D. \& R. G. & 5,508 \\
\hline a Spring ...... & King ........... & $\begin{array}{r}9,279 \\
14,054\end{array}$ \\
\hline olus, Mount. & & $\begin{array}{l}14,054 \\
14,211\end{array}$ \\
\hline $\begin{array}{l}\text { Do .......... } \\
\text { ate }\end{array}$ & $\begin{array}{l}\text { Wheeler... } \\
\text { K.P. R. R. }\end{array}$ & $\begin{array}{r}14,211 \\
5,458\end{array}$ \\
\hline ency Knob & Wheeler... & 12,274 \\
\hline ron........ & R. V. \& B. \& & 4,656 \\
\hline mosa, S & D. \& R. G. R. & $\begin{array}{l}7,524 \\
8,981\end{array}$ \\
\hline Jun & $\begin{array}{l}\text { Wheeler. } \\
\text { Wheeler. }\end{array}$ & $\begin{array}{l}8,981 \\
8,905\end{array}$ \\
\hline & R. G.R & 11,148 \\
\hline .. & $\mathrm{Wh}$ & 4,136 \\
\hline & D. \& R.R. & $y, 428$ \\
\hline & & 8,513 \\
\hline -.. & $\overline{\mathrm{Wl}}$ & 10,254 \\
\hline tar Peak & Wh & 13,254 \\
\hline & D. & 8,118 \\
\hline anch & Wh & 4,249 \\
\hline City.... & G.I & 6,532 \\
\hline (n) & Wh & $\begin{array}{r}6,622 \\
10,661\end{array}$ \\
\hline$\cdots$ & KII & $\begin{array}{r}10,661 \\
6,433\end{array}$ \\
\hline $\mathbf{P e}$ & R. I & 8,050 \\
\hline tero, & Whee & 13,853 \\
\hline & & 14, \\
\hline to & & 7,866 \\
\hline & D. & 5,917 \\
\hline & & 6.137 \\
\hline & $\tilde{\mathbf{K}}$. & 4,006 \\
\hline Arap: & & 13,520 \\
\hline $\mathrm{D}$ & & 11, \\
\hline Arbole & . G. R. R & 5,991 \\
\hline Pags & & 13,286 \\
\hline & & 5 \\
\hline Bridge. & lex. & 7,741 \\
\hline , Mount. & & 13,647 \\
\hline s Pass.... & & 11,445 \\
\hline a...... & K. P. R. R & \\
\hline & $\begin{array}{l}\text { C. C. R. R. } \\
\text { D. P.R.R. }\end{array}$ & $\begin{array}{l}5,322 \\
6,301\end{array}$ \\
\hline Mount, Timb & en $\ldots . . . .$. & $\begin{aligned} 0,301 \\
11,325\end{aligned}$ \\
\hline (.............. & R. G. R. H & 6,743 \\
\hline$\ldots$ & eler. & \\
\hline $\mathrm{D}$ & & 6,530 \\
\hline Mine .... & & 11,956 \\
\hline $110 . . . . .$. & & 9,753 \\
\hline & \& S.U.R.R ... & 9,676 \\
\hline tain............. & en $\ldots . . . . . . . .$. & 11,493 \\
\hline Do..........Timber line on..... & Hay & 11,100 \\
\hline Baldy $\ldots$ & D. \& R. G. R. R. & 7,592 \\
\hline $\begin{array}{l}\text { Baldy Peak } . . . . . . . \\
\text { Banded Peak. }\end{array}$ & Hayden ........... & 14,176 \\
\hline $\begin{array}{l}\text { Banded Peak. ........ } \\
\text { Do.... }\end{array}$ & ler $\ldots . . . . .$. & 12,824 \\
\hline (n) & en $R \cdot R$ & $\begin{array}{r}12,860 \\
6,207\end{array}$ \\
\hline & & 11,906 \\
\hline
\end{tabular}




\begin{tabular}{|c|c|c|}
\hline Sta & Authority. & Elevation. \\
\hline & & \\
\hline Basalt Peak & Wheeler. & 11,565 \\
\hline Bear Creek ....... & G. \& S. P. R. I & 5,547 \\
\hline $\begin{array}{l}\text { Bear Creek Pass. } \\
\text { Do }\end{array}$ & $\begin{array}{l}\text { Hayden :........ } \\
\text { Wheeler....... }\end{array}$ & $\begin{array}{l}12,600 \\
11,606\end{array}$ \\
\hline Bear Cre & Parry ..... & 7,198 \\
\hline Beaver $\mathrm{I}$ & C. C. R. R & 6,391 \\
\hline Beaver Cre & D. \& R. G. & 4,976 \\
\hline Belden .......... & D. \& R. G. R & $\begin{array}{r}8,346 \\
19\end{array}$ \\
\hline $\begin{array}{r}\text { Belleview Peak } \\
\text { Do........... }\end{array}$ & $\begin{array}{l}\text { Wheeler ... } \\
\text { Hayden .... }\end{array}$ & $\begin{array}{l}12,673 \\
12,350\end{array}$ \\
\hline Bennett & K. P. R. $\mathrm{R}$ & $\begin{array}{r}12,300 \\
5,496\end{array}$ \\
\hline ent $\mathrm{Ca}$ & Wheeler & 4,696 \\
\hline ent & D.\& R. G. & 6,941 \\
\hline erg & Hayden ... & 9,555 \\
\hline arg & Hayden .... & 9,773 \\
\hline & Hayden .... & 7,643 \\
\hline $\begin{array}{l}\text { Bergen's Ranch } \\
\text { Berthoud Pass.. }\end{array}$ & $\begin{array}{l}\text { Parry } . . . . . .4 \\
\text { Parry }\end{array}$ & $\begin{array}{r}7,752 \\
11,349\end{array}$ \\
\hline & U. P.R.R. Surv & 11,350 \\
\hline esse & D. \& R.G.R.R. & 4,751 \\
\hline$g$ & C.C.R.R......, & 6,823 \\
\hline Big . & Wh & 7,478 \\
\hline Bird's & D. \& & 10,161 \\
\hline Bisn & Wh & 7,736 \\
\hline & & 12,237 \\
\hline Blackb & D. 8 & 7,357 \\
\hline Blac. & C. C. R. R & 8,031 \\
\hline & & 7,543 \\
\hline Black & & 12,514 \\
\hline . & A. & 3,573 \\
\hline & B. O. C. R & 4,705 \\
\hline Blaine & Wheeler (Theod) & 14,249 \\
\hline & & 13,905 \\
\hline Blane & & 14,269 \\
\hline & & 14,464 \\
\hline Blod & & \\
\hline .. & \& S.F.R.R & 4,458 \\
\hline & D. \& R. G. R.R & 6,811 \\
\hline oul & & \\
\hline & & \\
\hline Bould & & 11,670 \\
\hline & V. & \\
\hline Boul & & \\
\hline Bou & $\mathrm{Ha}$ & 12,840 \\
\hline & K. & \\
\hline n... & & \\
\hline & G.R.R . & 9,893 \\
\hline & $\mathrm{Ha}$ & 11,274 \\
\hline & & 9,674 \\
\hline Pass.. & & 11,503 \\
\hline ....... & R.G.R.R. & 4,727 \\
\hline & D. & 4,979 \\
\hline Bristo & & 12,800 \\
\hline & ler (Theod) ... & 12,637 \\
\hline ... & $n$ & 9,468 \\
\hline .... & G. L. \& S. J & 9,151 \\
\hline$\cdots$ & R. V. \& B. \& C. R. R... & $\begin{array}{r}4,235 \\
10,8-7\end{array}$ \\
\hline (n) & 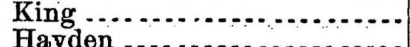 & $\begin{array}{l}10,817 \\
14.296\end{array}$ \\
\hline zin Mountain .... & D. \& R. G. R. R. & $\begin{array}{r}14,290 \\
7,948\end{array}$ \\
\hline Do.....S. P. Switch & D. \& R. G.R.R. R & 7,921 \\
\hline Do......S. P. Switch & 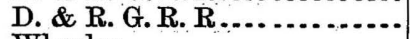 & 7,927 \\
\hline & Wheeler..................... & 13,755 \\
\hline & Hayden .... & 13,541 \\
\hline
\end{tabular}




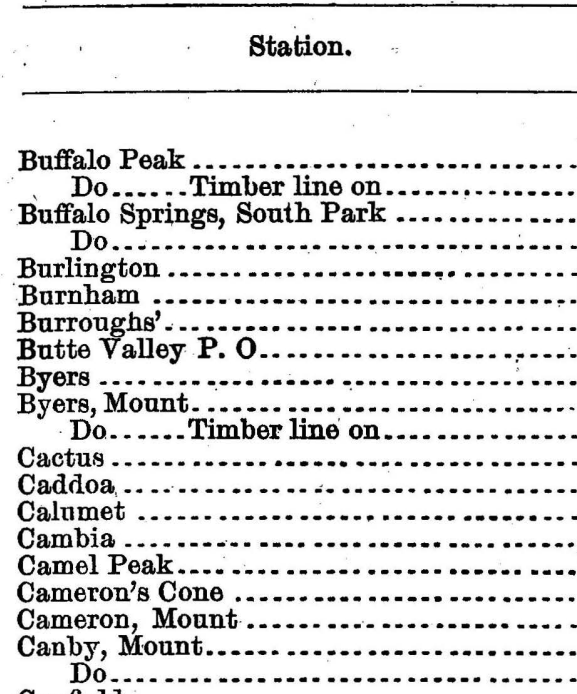

\section{Canfield}

Cañon City

$$
\begin{aligned}
& \text { Do.... (Old Depot) } \\
& \text { Do....... (New Depot)..... } \\
& \text { Do..... (Leadville Junc.) } \\
& \text { Do.... (Grape Creek).... }
\end{aligned}
$$

\begin{tabular}{|c|c|}
\hline Authority. & Elevation. \\
\hline & Feet. \\
\hline Hayden ... & $\begin{array}{l}10,020 \\
12,041\end{array}$ \\
\hline Wheeker... & 8,952 \\
\hline Whitney : & $\begin{array}{l}8,901 \\
4,976\end{array}$ \\
\hline D. \& R. G. & 5,220 \\
\hline Wheeler....... & 10,878 \\
\hline $\begin{array}{l}\text { Wheeler. } \\
\text { K. P. R. R.... }\end{array}$ & $\begin{array}{l}5,894 \\
5,203\end{array}$ \\
\hline Hayden ... & 12,778 \\
\hline Hayden $\ldots \ldots$ & 11,400 \\
\hline $\begin{array}{l}\text { D. \& R. G. R. R } \\
\text { A. T. \& S. F. R }\end{array}$ & $\begin{array}{l}4,859 \\
3,756\end{array}$ \\
\hline D. \& R.G.R. R & 8,849 \\
\hline G. \& S. P. R. R. & 5,944 \\
\hline Hayden... & 11,460 \\
\hline Hayden. & 13,274 \\
\hline Wheeler....... & 13,356 \\
\hline D. \& B. V.R. R & 5,048 \\
\hline $\begin{array}{l}\text { Wheeler. } \\
\text { D. \& R. G. R. R }\end{array}$ & $\begin{array}{l}5,396 \\
5,320\end{array}$ \\
\hline D. \& R. G. R. & 5,322 \\
\hline D. \& R. G. R.R & 5,313 \\
\hline D. \& R. G. R.R. & 5,357 \\
\hline Hayden & 13,997 \\
\hline $\begin{array}{l}\text { D. \& R. G. R. R } \\
\text { D. \& R. G. R. R. }\end{array}$ & $\begin{array}{l}6,151 \\
6,402\end{array}$ \\
\hline Wheeler........ & 12,078 \\
\hline Hajden... & 9,905 \\
\hline D. \& R. G. R. R & 4,929 \\
\hline $\begin{array}{l}\text { A., T. \& S. F. R } \\
\text { D. P. R. R ..... }\end{array}$ & $\begin{array}{l}3,517 \\
5,696\end{array}$ \\
\hline R. V. \& B. \& C. & 4,357 \\
\hline $\begin{array}{l}\text { Wheeler...... } \\
\text { D.\& R. G. R. R }\end{array}$ & $\begin{array}{l}9,989 \\
7,763\end{array}$ \\
\hline D. \& R. G.R. R & 4,982 \\
\hline Hayden ........... & 14,115 \\
\hline D. \& R. G. R. R & 6,198 \\
\hline 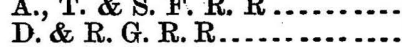 & $\begin{array}{l}4,234 \\
7,330\end{array}$ \\
\hline D. \& R. G.R.R. & 6,728 \\
\hline $\begin{array}{l}\text { K. P. } \\
\text { C. } \\
\text { R. R. }\end{array}$ & $\begin{array}{l}5,712 \\
8,484\end{array}$ \\
\hline Parry & 8,300 \\
\hline Wheoler............ & 7,727 \\
\hline D. \& R. G. R. R... & 7,942 \\
\hline Wheeler...................... & 12,248 \\
\hline D. \& R. G. R. R........ & 5,193 \\
\hline 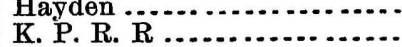 & $\begin{array}{l}9,940 \\
4,277\end{array}$ \\
\hline Hayden .................... & 11,500 \\
\hline A., T. \& S. F. R. R ....... & 4,530 \\
\hline Wheeler & 6,076 \\
\hline 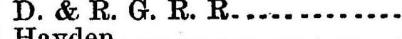 & $\begin{array}{r}6,095 \\
11,833\end{array}$ \\
\hline 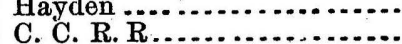 & $\begin{array}{r}11,833 \\
5,909\end{array}$ \\
\hline 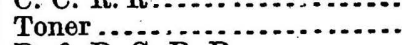 & $\begin{array}{l}0,909 \\
7,186\end{array}$ \\
\hline D. \& R. G. R. R... & 6,874 \\
\hline Hayden................ & 12,600 \\
\hline $\begin{array}{l}\text { King } \\
\text { Hayden }\end{array}$ & $\begin{array}{r}13,167 \\
9,500\end{array}$ \\
\hline
\end{tabular}

Capitol Mountain

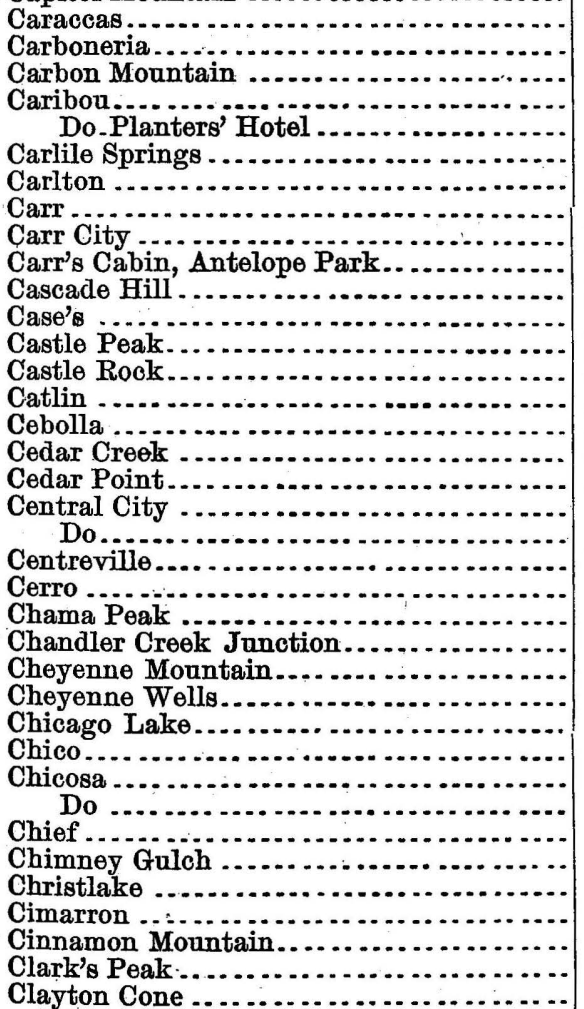




\begin{tabular}{|c|c|c|}
\hline & Authority. & Elevation. \\
\hline & & \\
\hline $\begin{array}{l}\text { Clelland ...... } \\
\text { Cleora ...... }\end{array}$ & $\begin{array}{l}\text { A., T. \& S. F. } \\
\text { D.\& R. G. R. }\end{array}$ & \\
\hline Clond City .. & D.\& R.G.R. R & 8,940 \\
\hline reek... & D. \& R. G. & 5,338 \\
\hline nction. & D. \& R. G & 6,186 \\
\hline intain & 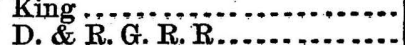 & $\begin{array}{l}8,430 \\
7,821\end{array}$ \\
\hline $\begin{array}{l}\text { Coch } \\
\text { Corh }\end{array}$ & Wheeler,... & 11,673 \\
\hline topa or I & Whoeler. & 9,088 \\
\hline topa Pass & Wheeler & $\begin{array}{l}10,032 \\
10,032\end{array}$ \\
\hline Do....... & $\begin{array}{l}\text { P. R. R. I } \\
\text { Hayden. }\end{array}$ & $\begin{array}{l}10,032 \\
10,000\end{array}$ \\
\hline Coffintop.... & Hayden . & 8,003 \\
\hline vens.... & D. \& R. G. & 5,932 \\
\hline $\begin{array}{l}\text { Colfa } \\
\text { Colin }\end{array}$ & $\begin{array}{l}\text { Wheeler. } \\
\text { D. \& R. G. R. R. }\end{array}$ & $\begin{array}{l}8,599 \\
6,712\end{array}$ \\
\hline Collins, Fort .... & Toner :........ & 4,815 \\
\hline Colorado City ... & D. \& R. G. R. & 6,092 \\
\hline $0 \ldots \ldots$ & Par & 6,344 \\
\hline Do Dolorado Sprir & Hay & 6.049 \\
\hline $\begin{array}{l}\text { Jolorado Sprir } \\
\text { Do......... }\end{array}$ & $\begin{array}{l}\text { Wheeler... } \\
\text { D. \& R. G. }\end{array}$ & $\begin{array}{l}6,010 \\
5,970\end{array}$ \\
\hline & D. \& R. G. I & 5,967 \\
\hline Do....... f & R. G & 5,960 \\
\hline & $\mathrm{U}$ : & \\
\hline Comanche Peak. & Kin & 11,929 \\
\hline$\cdots \ldots . .$. & $\mathrm{Ha}$ & 7,880 \\
\hline & & \\
\hline Peak _. & Hayden ... & 13,183 \\
\hline Springs. & Hayden ... & $\begin{array}{l}9,848 \\
5,985\end{array}$ \\
\hline Coro & R. V.\& B.\& C & $\begin{array}{l}0,900 \\
4,547\end{array}$ \\
\hline eak... & den ........ & 11,333 \\
\hline ........... & W. & \\
\hline Pass .... & Palmer.... & 9,520 \\
\hline i................... & D. \& R. G. R. I & 6,364 \\
\hline awood..... & C. C. R.R. R & 7,178 \\
\hline ark ..... & $\begin{array}{l}\text { R. G. R. R } \\
\text { R. G. R. R }\end{array}$ & $\begin{array}{r}9,731 \\
10,097\end{array}$ \\
\hline Peak... & & 10,255 \\
\hline atte (M & & 12,052 \\
\hline & D. & \\
\hline Butte (I & D. & 8,853 \\
\hline Crest & & \\
\hline & & \\
\hline , timber line & & 12,107 \\
\hline & ...... & \\
\hline Creek . & G. R. R ... & \\
\hline$a k \theta_{\ldots} .$. & D. & \\
\hline tain...... & $\begin{array}{l}\mathrm{Ha} \\
\mathrm{Wh}\end{array}$ & $\begin{array}{r}10,623 \\
8,529\end{array}$ \\
\hline 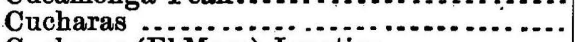 & D.\& R.G.R.R .... & 5,921 \\
\hline action. & & \\
\hline ......... & & 994 \\
\hline$F \theta$ & & 12,341 \\
\hline Church .. & W1 & 8,010 \\
\hline (............. & Ha & 14,069 \\
\hline & G.R, R ...... & 9,993 \\
\hline ngham Pass & & 12,090 \\
\hline ningham Pass, Timber line on ......... & Hayc & 11,500 \\
\hline se & R. G.R.R R.... & 4,493 \\
\hline Creek Pass & R. G. R. R ...... & $\begin{array}{l}7,052 \\
9,654\end{array}$ \\
\hline ; Creek Pass & $\begin{array}{l}\text { Wheeler...... } \\
\text { Hayden ....... }\end{array}$ & $\begin{array}{r}\mathbf{y}, 004 \\
13,193\end{array}$ \\
\hline
\end{tabular}




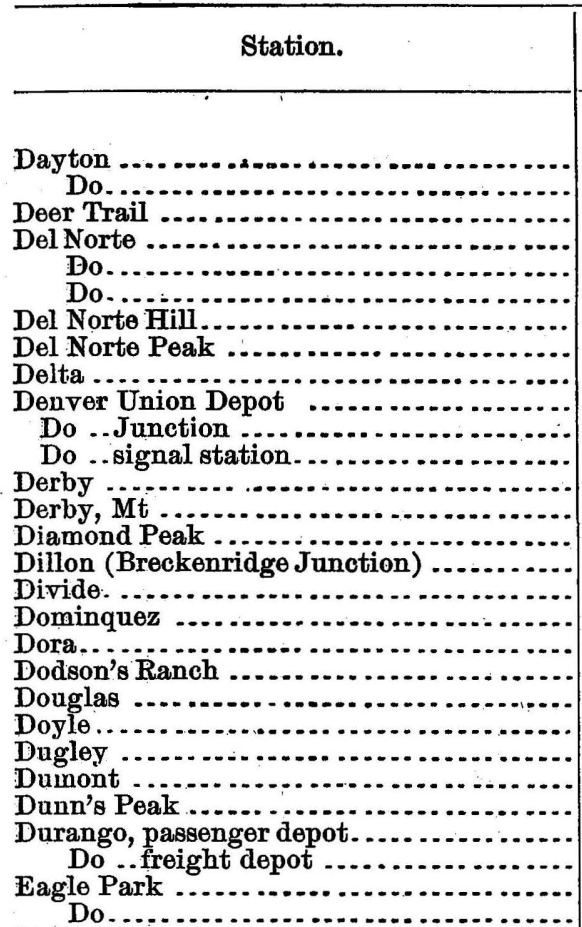

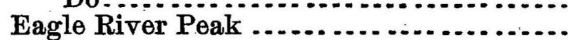

Earl

East Mountain

East River Pass

Eckley

Edgerton

Eighteen-mile Mountain

Eiler's

Eiler's Junction

Eiler's Smelter Junction

Elbert, Mt

Do...Timber line on

Elk Mountain

EIk Park

El Lat6

El Moro

El Moro Mines . .

Empire

Do.

Engineer Mountain

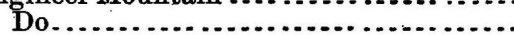

Énglemann, Mt., 'Timber line on

Erie

Escalante

Ethel Peak

Evans

Do.

Do

Evaus, Mount

Do...... Timber line on ...................

Do....... Timber line on................

Excelsior

Fairplay

Do.

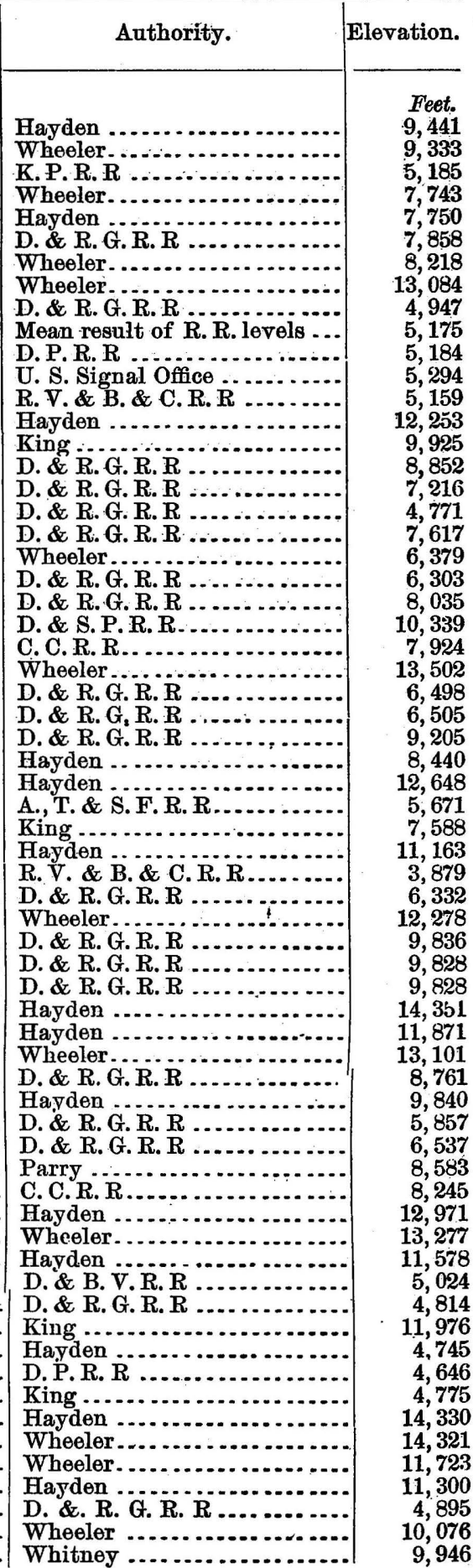




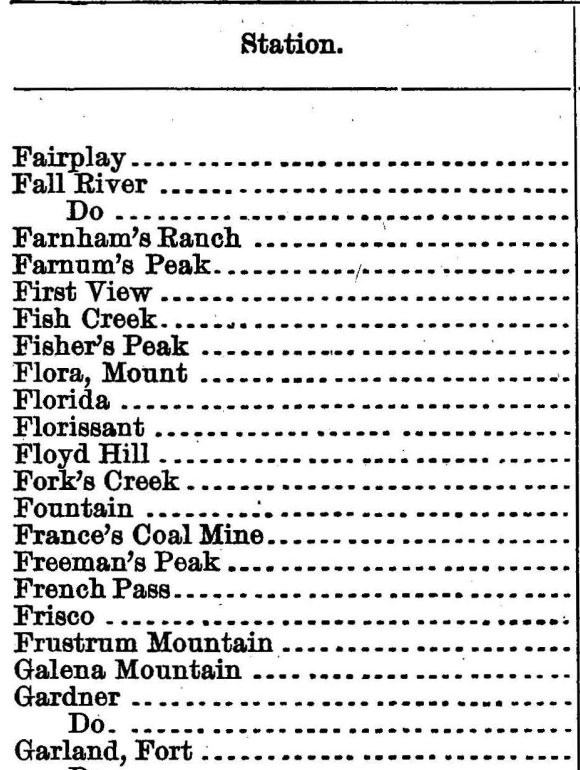

Garland, Fort

Do

Do

Do .........flagstaff

Garlick's Ranch

Georgetown

Do

Do ...... Astronomical Station

Do....... Barton House

Georgia Pass.

Do

Do

Gibson Peak

Girardodt

Glacier, Mount

Glacier Peak.

Glade

Godfrey

Golden

Do

Golden Peak

Gold Hill

$$
\text { Do... }
$$

Goodnight

Gore's Pass

Gothic Mountain

Granada

Grand Junction

Grand Lake

Grand Mesa

Granite

$$
\text { Do }
$$

Grant.

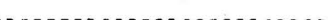

Do.................................. Do.......

Gray's Peak.

Do Podk

Do ...... Timber line on ..............

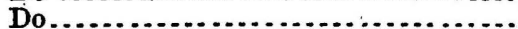

Gray's Peak, divide between north and south
D. \& S. P. R. R................

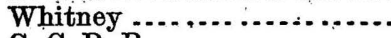

C. C. R R

Hayden

Hayden

K. P. R. R.

Hayden

Parry

D. \& R. G. R. $\mathrm{R}$.

Wheeler

C. C. R. R

C. C. R. R. $\mathrm{R}$. $\mathrm{R}$. $\mathrm{R}$

Hayden

Hayden

Wheeler.

D. \& R, G, R, R

Wheeler

Hayden

Wheeler

Hayden

Wheeler

D. \& R, G, R, $\mathbf{R}$

Hayden,$R_{1} R$.............

Wheeler, $\mathbf{R}, \mathbf{R}$, levels

Parry $\mathbf{C}, \mathbf{R}, \mathbf{R}$.

Parry ...

Wheoler

Whitney

Wheeler

Parry

Wheeler

D. \& R. G. R. R

Wheeler

\section{Hayden}

\section{Wheeler}

D. \& R. G. R. R

K. P. R. R

Whitney

Hayden

\section{Hayde \\ Parry}

D. \& R. G. R. $\mathrm{R}$

Hayden

Hayden

A. T. \& S. F. R.

D. \& R. G. R. R

Hayden

Hayden

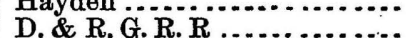

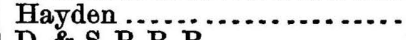

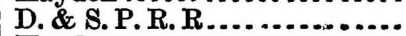

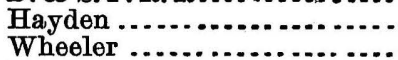

Hayden

Whitney

Hayden

Wheeler

Wheeler
Elevation.

Feet.

9,941

7,708

7,665

9,548

1.1, 400

4,577

6,889

9,460

12,878

6,695

8,184

7,201

6, 878

5,503

6,693

11,600

12,044

9,064

13,893

13,290

6,956

7,000

7,849

7,914

7,996

7,937

7,105

8,474

8,452

8,587

8,594

11,770

11,487

11, 811

13,729

6,358

14,243

12,654

13,360

6,515

5,603

5,684

5,690

9,650

8,463

8,636

4, 708

9,570

12,570

3, 436

4,561

8,153

10,000

8,923

8, 883

8,491

8,587

8,497

14,341

14, 319

11,100

14,380

13, 929 


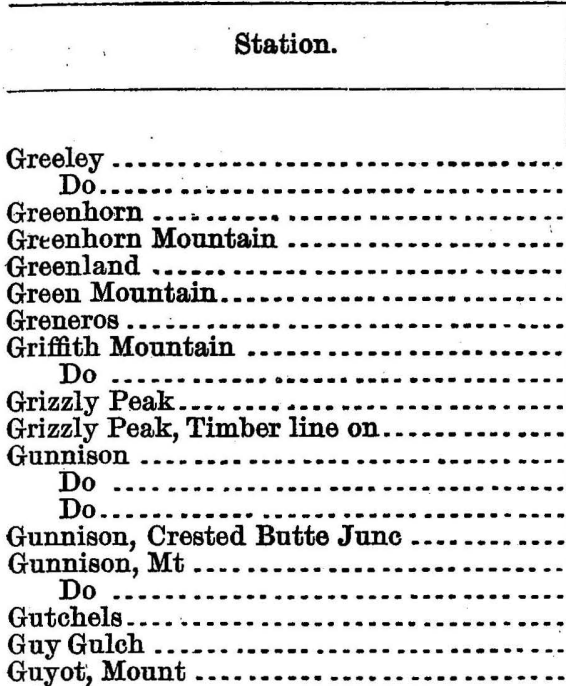

Do

Guyot, Mount, Timber line on

Hague's Peak

Hall's Ranch, San Luis Valley

Hall's Smelting Works

Hamilton

$$
\text { Do }
$$

Hamilton Pass

Do ....... Timber line on

Hamilton's Ranch

Hancock Pass

Handie's Peak

$$
\text { Do }
$$

Hanz Peak

Hardscrabble Mines

Harp

Hartzell's Ranch

Harvard, Mount

Do

Do.

Do ...... . Timber line on

Hayden Pass

Haydens.

Haydensville

Hay Station, Deer Range

Hecla

Helmet Peak

Henderson's Island

Henderson's Ranch.

Hepburn's Park

Hereford

Hermano Peak

Hermosa

$$
\text { Do }
$$

Hesperis Mountain.

Heyde

Hilton

Hoehne's

Holley's

Holy Cross Mountain

Homer.

Homestake Peak

Hoosier Pass

\begin{tabular}{|l|l|}
\hline Authority. & Elevation. \\
\hline
\end{tabular}

D. P. R. R

D.\& R.G.R. R

Hayden

D. \& R. G. R. R

Hayden $\ldots$.....

Wheeler...........................

Hayden ........................

Hayden

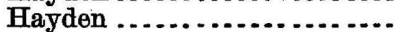

Hayden

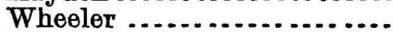

D. \& R.G. R. $\mathbf{R}$

D. \& R. G. R. R

Hayden

Wheeler.

D. \& R. G. R. R

C. C. R. $\mathbf{R}$

Hayden

Hayden

King

Wheeler

Wheeler

Hayden

Whitney

Ruffner

Wheeler

Wheeler

Hayden

Wheeler

King.

D. \& R. G. R. R

Wheeler

Hayden

Whitney

Wheeler

Hayden

Wheeler

D. \& R. G. R. R

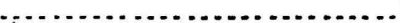

Wheeler

D. \& R. G. R. R

Hayden

D. P. R. R . . .

Whitney $\ldots$

D. \& R. G. R. R

Hayden

Wheeler....................

D. \& R. G. R. R

\section{Hayden}

R. V. \& B. \& C. R. R

A., T. \& S. F. R. R

A., T. \& S. F. R. R

A., T. \& S. F. R. R

Hayden

D. \& $R$.

D. \& R. G. R. R ............

Wheeler.
Feet.

4,642

4,779

5,076

12,230

6,899

7,993

5,782

11,589

11,273

13,956

11, 758

7,743

7,419

7,658

7,660

12,688

12,242

9,965

6,212

13,565

13,223

11,811

13, 832

7,839

9,916

9,743

9,875

12,370

10,840

7.,227

12,263

13,997

14,149

10, 906

8,800

7,323

8,828

14, 375

14, 452

14, 151

12,117

10,780

9,136

6,382

9,613

7,349

12,042

5,028

8,257

8,141

4,460

9,014

6,618

6, 623

13,135

4,220

3,877

5,704

3, 377

14, 176

8,935

13,687

11,627 


Station.

Hoosier Pass

$$
\text { Do }
$$

Horsefly, Mount.

Horseshoe Mountain

Hot Springs.

Howard's

Howardville

$$
\text { Do }
$$

Hudson .

Huerfan

Hughes, Astronomical Station

\section{Hngo}

Hukili.

Hunchback Mountain

Hunt's Mountain

Hunt's Peak

$$
\text { Do }
$$

Hunt's Peak, foot of.

Hurricane Peak

Husted's

Idaho Springs

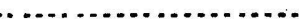

Ignacio

Indian Creek Pass

Iron Mine

Iron Springs

Jack's Cabin

James' Peak

Do Timber line on

Jamestown

Jefferson.

Do $\ldots \ldots \ldots \ldots \ldots$

Do

Johnson ...............................

Johnson's Ford, Umcompahgre River

Juniata.

Junction Honse

Junction Peak

Kahnah

Keèldar.

Kelley

Kelso Cabin

Kendall, Mount

Do

Kenny's Ranch, on Dead Man's Creek.

Kenosha Cones.

Kenosha House

Kenosha Summit.

Kezax

Kit Carson

Kit Carson's Peal

Kokomo

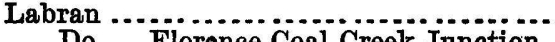

Do.... Florence Coal Creek Junction

La Jara.

La Junte

Do.

Lake

Lake City

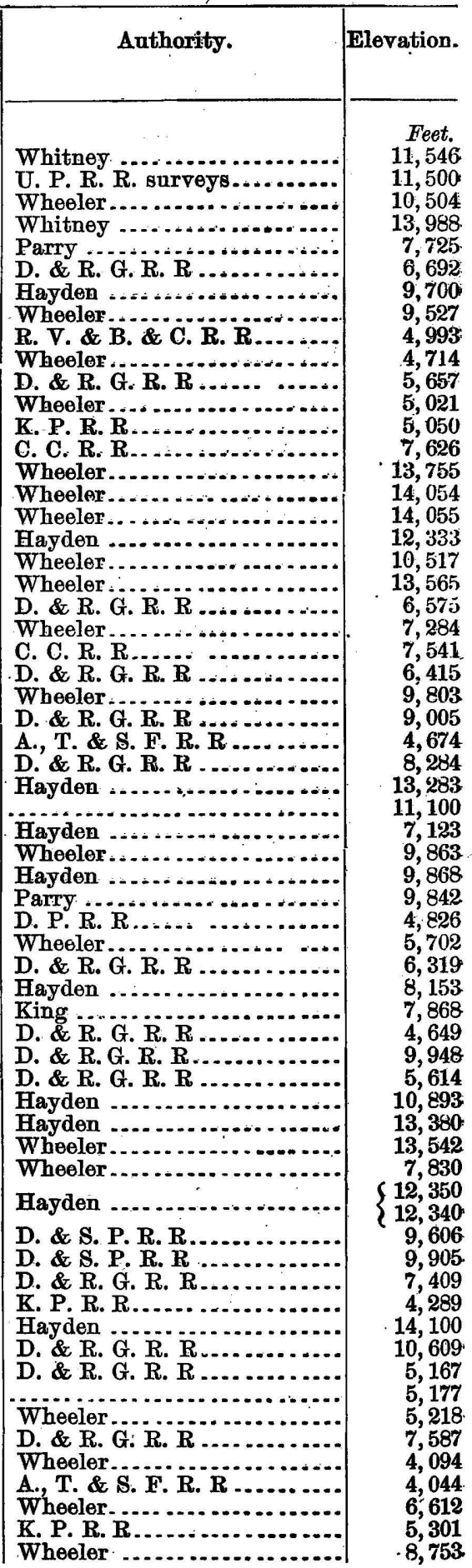




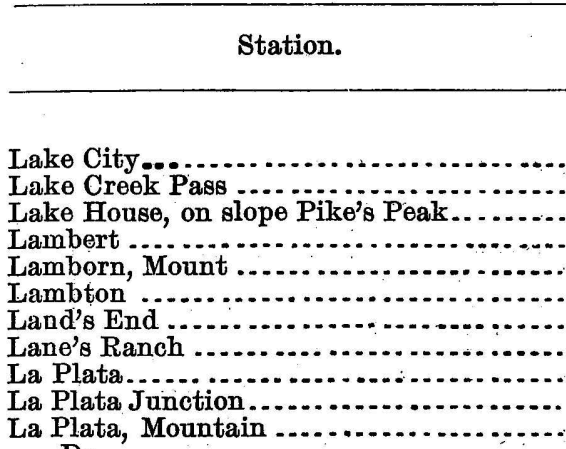

Do

Do.

Timber line on

Laporte

Lariat ...................................

Larkspur

Las Animas (west) Do.

Latham

La Veta

La Veta Peak

Lawson...

Leadville

Lennox

Leonard's Ranch, Arkansas River

Leon Peak Do

Lillie's Mountain

$$
\text { Do. }
$$

Timber line on

Lime Kiln

Do

Lime Stone

Lime or White Earth Creek Pass

Lincoln, Mount

Do.

Do........ Timber line on

Lindstrom's Mill

Little Butte

Little Giant

Littleton

L'ockwood's Ranch

Loma, Camp

Lone Cone.

Long's Cañon

Long's Pass

Long's Peak

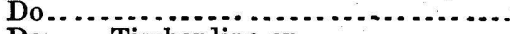

.... Timber line on

Los Piños ..........................

Do...Agency

Do

Lost Knife Pass

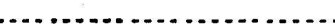

Lost Park Mountain.

Lumber Spur.

Lyon, Fort.

Machissett ................................

Macomb's Peak

Madge

Magnolia

Malta.

Do... Lizzie Smelter Junction

Manitou

Do...Adobe Smelter Junction

\begin{tabular}{|l|l} 
Authority. & Elevation. \\
\hline
\end{tabular}

Hayden

Feet.

Wheeler

Wheeler

Wheèler.

Hayden

D. \& R. G.R.R

Hayden

Wheeler.

D. \& R. G. R. R

D. \& R. G. R. R

Wheeler

\section{Hayden}

Hayden

King

D. \& R. G. R. R

D. \& R. G. R. R

W., T. \& S. F. R. R

D. \& R. G. R. R

Wheeler.

C. C. R. $\mathrm{R}$

D. \& R. G. R. R

D.\& R. G. R. R

Wheeler

Hayden

Wheeler

Hayden ..........

Hayden

D.\& R.G.R.R

D. \& R. G.R.R

D.\& R.G.R.R

Wheeler.

Wheeler

Hayden

Hayden

Parry

D. \& R.G.R.R

D. \& R. G. R. R

D.\& R. G. R. R

Wheeler

Ruffner.

Hayden

Palmer .

Hayden

King

Hayden

D. \& R. G. R. $R$

Hayden

-Wheeler

Palmer.

Hayden

D.\& R. G. R. R

D.P.R. $R$

Wheeler.

Wheeler

D. \& R. G. R.R

K. P. R. R

D. \& R. G. R. R

D.\& R. G.R.R

D. \& R. G. R. R

D. \& R. G. R. R
8,550

12,226

10,108

6,561

11, 337

6, 537

10,634

6,380

9,950

9,950

13,316

14,311

12,080

5,065

7,643

6,649

3,886 .

3,854

4,600 .

7,002

11,654

8, 111

10,178

4, 705

8,335

10,954

11,218

11,433

11,100

5, 552

6,219

4, 862

11,314

14, 375

14,297

12,051

8,738

5,346

10,046

5,350

4,997

8,069

12,761

8,402

7,660 .

14,271

14,050

11,100

9,615

9,290

9,065

8,400

11,800

9,074

4, 896

3,910

5,520

13,154

6,716

- 5,336

9,558

9,608

9,661

6,302 


\begin{tabular}{|c|c|c|}
\hline & Authority. & Elevation. \\
\hline & & Feet. \\
\hline Manitou ........ & $\begin{array}{l}\text { Hayden .... } \\
\text { Hayden .... }\end{array}$ & \\
\hline & Wheeler.... & \\
\hline Carlestor & Wheeler. & 10,874 \\
\hline [armot ] & Hayc & $\begin{array}{l}11,600 \\
14,003\end{array}$ \\
\hline $\begin{array}{l}\text { Maroon Mo } \\
\text { Marshall... }\end{array}$ & $\begin{array}{l}\text { Hayden ..... } \\
\text { G. B.\& C.R }\end{array}$ & $\begin{array}{r}14,003 \\
5,529\end{array}$ \\
\hline Tarshalleg & Wheeler.... & 10,852 \\
\hline farshes. & D. \& R. G.R. R & 6,325 \\
\hline a.. & Toner ..... & $\begin{array}{l}9,324 \\
6,960\end{array}$ \\
\hline Mass & Hayden. & 14,298 \\
\hline Do. & Hay & 11,607 \\
\hline [ays & D. & 8,298 \\
\hline McClellar & Hay & 13,423 \\
\hline & $\mathrm{Wh}$ & 13,842 \\
\hline [cClu & Wheeler........ & 5,318 \\
\hline McLa & Wheeler & $\begin{array}{l}9,672 \\
4,796\end{array}$ \\
\hline $\begin{array}{l}\text { Mead } \\
\text { Mear }\end{array}$ & D.\&R.G.R.R & $\begin{array}{l}4,790 \\
8,412\end{array}$ \\
\hline & D. \&.R.G.R. & 8,417 \\
\hline Iear' & Whe & 13,008 \\
\hline$\therefore$ & Wheeler.... & 13,394 \\
\hline$\ldots$ & Hayden ..... & $\begin{array}{r}7,360 \\
0-8,500\end{array}$ \\
\hline & Wheeler....... & 13,308 \\
\hline & $\ldots$ & \\
\hline & & \\
\hline$\cdots$ & D. \& R. G. R. R & 7,380 \\
\hline & $R \cdot G \cdot \ddot{R}$ & $\begin{array}{l}7,826 \\
6,009\end{array}$ \\
\hline$\cdots \ldots$ & $\begin{array}{l}\text { R. G. R. } \mathbf{R} \\
\text { R. G. R. R }\end{array}$ & $\begin{array}{l}0,009 \\
9,319\end{array}$ \\
\hline & Wh & 11,474 \\
\hline & Ha & 11,098 \\
\hline & R. R..... & 4,841 \\
\hline & & 9,073 \\
\hline & G. R. & 9,900 \\
\hline & ......... & 11,270 \\
\hline onte & & 10,295 \\
\hline & ...... & 9,652 \\
\hline ontg & ..... & 10,783 \\
\hline & $\therefore .$. & 11,181 \\
\hline. & D. \& R. G. R. & 5,771 \\
\hline & D. \& R. G. R. R & 6,953 \\
\hline Trading Post & Med. Dept., U. & $\begin{array}{l}4,500 \\
6.229\end{array}$ \\
\hline 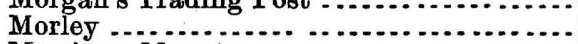 & A., T. \& S. F. R. R & 6,746 \\
\hline Mount.. & Hayden .......................... & \\
\hline …......... & …........... & 7,950 \\
\hline Pass...... & $\mathrm{r} \ldots$ & 9,787 \\
\hline & urveys...... & 9,577 \\
\hline & $\cdots \ldots \ldots, \ldots$ & 9,713 \\
\hline$\ldots . . .$. & & \\
\hline Pass ... & r........ & 13,308 \\
\hline Jreek Pass & & 8,772 \\
\hline $0 \theta \ldots$ & R. G. R. R. & 8,732 \\
\hline & D. \& R. G. R.R............. & 4,701 \\
\hline & & 5,224 \\
\hline & D. \& R. G. R. R & $\begin{array}{l}7,673 \\
6,566\end{array}$ \\
\hline & (G) & \\
\hline & & 8,263 \\
\hline & R. G. R. R & 8,118 \\
\hline & & 4,354 \\
\hline
\end{tabular}




\begin{tabular}{|c|c|c|}
\hline Station. & & Elevation. \\
\hline & & \\
\hline Nevada City. & A. $T \& \&$ & $\begin{array}{l}\text { Feet. } \\
8,800\end{array}$ \\
\hline New Mexico Line. & A., T. \& S. & 7,556 \\
\hline New Orleans Crossi & D. \& R. G & 5,239 \\
\hline New Yorl & Whitney & 7,170 \\
\hline North Mam ...... & Hayden .. & 11,973 \\
\hline North Park . . . . . . . & & $0-9,000$ \\
\hline Oak Creek ........ & D. \& R. C & 5,331 \\
\hline Oak Creek Junctio & D. \& R. & 5,187 \\
\hline Ohio Peak . . ........ & Hayden. & 11,972 \\
\hline Ojo .......... & D. \& R. G & $\gamma, 167$ \\
\hline Ormus, Mount... & Hayden. & 12,185 \\
\hline Osborn's Lake . ... & Toner .. & 8,821 \\
\hline Osier .............. & D. \& R. & 9,615 \\
\hline Oso, Mount. ...... & Hayden ... & 13,640 \\
\hline Otis ....... & R. V. \& B & 4,392 \\
\hline . & Hayden .. & 7,640 \\
\hline 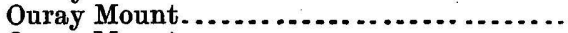 & Hayden & 14,043 \\
\hline Owen, Mount. . . . . . . . . . . . . . . . . . . . & Hayden. & 12,969 \\
\hline Oxford $\ldots . . . . . .$. & A., T. \& & 4,322 \\
\hline Pagoda Peak. & Hayden. & 11,251 \\
\hline Pagosa Peak .. & Wheeler. & 12,676 \\
\hline 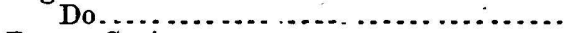 & Hayden. & 12,674 \\
\hline 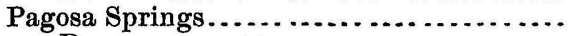 & Hayden .. & 7,095 \\
\hline Do............. & Wheeler. . & 7,108 \\
\hline Park Cone ....... & Hayrlen .. & 12,021 \\
\hline$\ldots \ldots \ldots \ldots \ldots$ & R. ............ & 5,715 \\
\hline 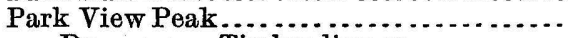 & $\ldots \ldots . . . .$. & 12,433 \\
\hline Do......... Timber line on .......... & $\ldots \ldots \ldots$ & 11,100 \\
\hline$\ldots \ldots \ldots \ldots \ldots \ldots \ldots$ & $\ldots . . . . .$. & 7,928 \\
\hline Parrott City... & Hayden & 8,633 \\
\hline Parry's Peak ...... & Parry ... & 13,133 \\
\hline Pass Mountain ... & $\ldots . .$. & 11,200 \\
\hline$\ldots \ldots \ldots \ldots \ldots \ldots$ & $\ldots \ldots \ldots$ & 13,004 \\
\hline$\ldots \ldots \ldots \ldots$ & den . & 5,650 \\
\hline 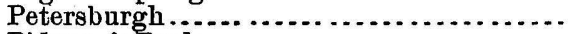 & D. \& R. G & 5,302 \\
\hline - & $\ldots \ldots \ldots \ldots$ & 13,928 \\
\hline & $\ldots \ldots \ldots$ & \\
\hline Pike's Peak .............................. & (............ & 14,147 \\
\hline$\ldots \ldots \ldots \ldots \ldots$ & $\mathrm{Pa}$ & 14,216 \\
\hline Signal Station ............. & $\theta \ldots . . . . . .$. & 14,134 \\
\hline , Do .... Timber line on .......... & $\mathrm{Ha}$ & 11,720 \\
\hline Pine Creek . . . . & $\mathrm{D}$ & 8,738 \\
\hline .................. & $\ldots \ldots \ldots$ & 5,016 \\
\hline$\ldots \ldots \ldots \ldots \ldots$ & $\ldots \ldots \ldots$ & 13,176 \\
\hline . & ........... & 239 \\
\hline$\ldots \ldots \ldots \ldots \ldots \ldots \ldots$ & $\ldots \ldots \ldots$ & 10,322 \\
\hline Do ......... & $\ldots . .$. & 487 \\
\hline Pisgah Peak, Littl & $\ldots \ldots$ & 10,027 \\
\hline Placer & $\ldots \ldots$ & \\
\hline Placer Iron Mine.. & $\ldots \ldots$ & \\
\hline Placer Iron Mine Ju & $\ldots . .$. & \\
\hline Platte Mount & len. & 9,343 \\
\hline 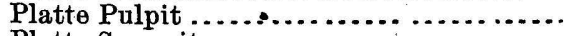 & lon $\ldots \ldots \ldots \ldots . . . . . . . .$. & 8,000 \\
\hline atte Summit $\ldots \ldots \ldots \ldots \ldots \ldots \ldots \ldots \ldots$ & R. V. B. \& C. R. R.......... & 094 \\
\hline$\ldots \ldots \ldots \ldots$ & D. P. R. R .. & 312 \\
\hline Platte Junction .... & D. \& R. G. R. R. & \\
\hline nt Valley .. & $\ldots \ldots \ldots . . .$. & 271 \\
\hline le Creek Mountain................ & $\therefore \ldots \ldots . . . .$. & 13,400 \\
\hline Poncho,$\ldots \ldots \ldots \ldots \ldots \ldots \ldots \ldots$ & D. \& R. G. R. R & \\
\hline Poncho, Maysville Junction . . . .... ... & $\ldots \ldots \ldots . . . .$. & 188 \\
\hline ncho Pass $\ldots \ldots \ldots \ldots \ldots \ldots \ldots \ldots \ldots \ldots$ & gler .... & 945 \\
\hline & & \\
\hline
\end{tabular}

Bull. 5-5 


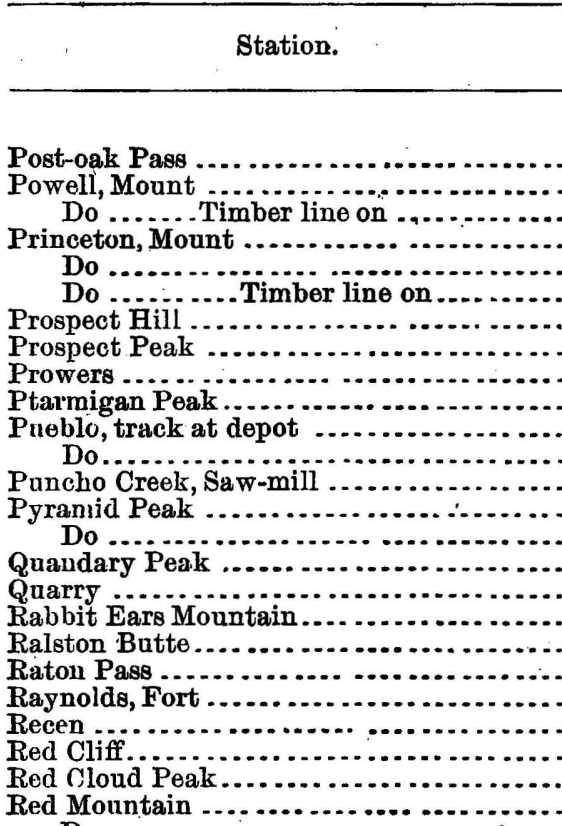

Do

Do........ Timber line.

Red Peak .

Reed's Ranch

Rhyolite Peak

Rio Grande Pyramid

Rito Alto

Rito Alto, Mount

Do

Do - Timber line on

River Bend

Riverside Do

Roan ........................

Robinson's

Robinson

Rock Cliff Post-Office

Rock Creek

Rockvale

Rockwood

Rocky Butte

Rocky Ford

Rollinsville

Rosalie, Mount Do

Rosita

Rosita (sun dial)

Roubideau's Pass.

Round Hill

Ronnd Mountain P. $\mathrm{O}$

Round Peak.

Rowter, Mount

Ryau's

Sagnache

$$
\text { Do }
$$

Saint John's

Saint Mary's

\begin{tabular}{|c|c|}
\hline & Elevation. \\
\hline & \\
\hline Palmer...: & 7,475 \\
\hline Hayden .. & 13,398 \\
\hline Hayden. & 11,600 \\
\hline Hajden . & 14,196 \\
\hline Whoeler & 14,041 \\
\hline Hayden. & 11,500 \\
\hline Hayden . & 8,893 \\
\hline Wheeler & 9,909 \\
\hline A., T. \& S & 3,668 \\
\hline Hayden. & 13,200 \\
\hline Wheeler. & 4,669 \\
\hline A., T. \& S & 4,639 \\
\hline Wheeler. & 8,259 \\
\hline Hayden. & 11,611 \\
\hline Hayden. & 13,885 \\
\hline Hayden ... & 14,269 \\
\hline D. \& R. G & 6,212 \\
\hline Hayden. & 10,719 \\
\hline Hayden. & 10,593 \\
\hline Wheeler & 7,893 \\
\hline Wheeler .. & 4,300 \\
\hline D. \& R. G. & 10,589 \\
\hline D. \& R. G. & 8,649 \\
\hline Wheeler & 14,092 \\
\hline Wheeler & 12,611 \\
\hline Whoeler & 13 , \\
\hline Wheeler & 11,746 \\
\hline Hayden. & 12,382 \\
\hline Wheeler & 7,972 \\
\hline Hay & 10,400 \\
\hline HaJ & $13, \pi 73$ \\
\hline ........ & 8,169 \\
\hline$\ldots \ldots$ & 12,989 \\
\hline ...... & $12,8 \mathrm{t} 3$ \\
\hline Wh & 11,817 \\
\hline Kans. Pac. R. R & 4 \\
\hline Wheeler & \\
\hline D. \& R. G & \\
\hline . \& R. G & 4,50 \\
\hline Hayden & to 8,50 \\
\hline $\begin{array}{l}\text { D. \& R. G } \\
\text { A. T. \& S }\end{array}$ & 10,84 \\
\hline$\ldots$ & \\
\hline 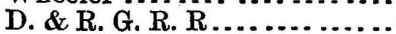 & 8,28 \\
\hline & \\
\hline 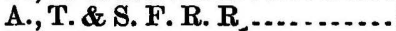 & \\
\hline 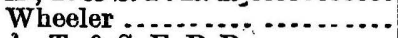 & \\
\hline$R \ldots \ldots$ & \\
\hline - & \\
\hline & \\
\hline & 14, \\
\hline & 8,932 \\
\hline & 8,736 \\
\hline R. R. Repor & 9,772 \\
\hline R. G.R.R . . . . . & 8,668 \\
\hline 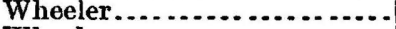 & \\
\hline eler.. & \\
\hline & 13 , \\
\hline & \\
\hline $\mathbf{r} \ldots \ldots$ & 7, \\
\hline & \\
\hline & \\
\hline & \\
\hline
\end{tabular}




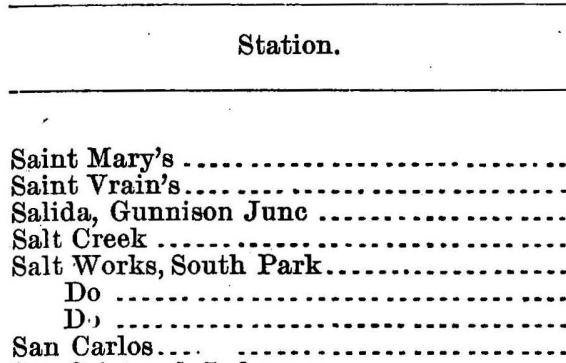

San Cristoval, Lake

San Francisco Pass

Sangre de Christo

Sangre de Christo Pass

1:0

San Isabel $\ldots \ldots \ldots$

San Juan City

San Luis de Culebra.

San Luis Lake Do

San Luis Peak

San Luis Valley.

San Miguel Lake

Santa Clara.

Sapinero

Sargents

Scraggs Butte

Sedalia

Sedgwick, Fort

Serape.

Shale ...............

Sheep Mountain

Shingled Mountain

Shirley

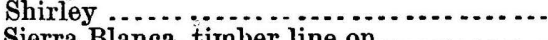

Sigual Butte

Silesia Butte

Silla

Silverheels, Mount
Do

Silver Plume

Silverton

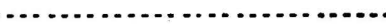

Simpson Mount

Sizer's Ran

Sknll Creek Pass

Slate Mountai

Slate Ranch

Smith Hill

Smith's Peak.

Sneffels, Mount

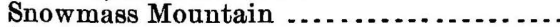

Snyder's Ranch ........................

Soda Springs, Animas Park

$$
\text { Do }
$$

Soledad

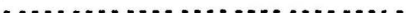

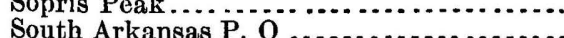

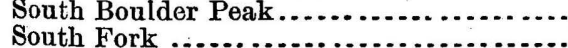

South Mam

South Park

South Platte

\begin{tabular}{|l|l} 
Authority. & Elevation. \\
\hline
\end{tabular}

Hayden .....................

Parry

D.\& R. G. R. $\mathbf{R}$

D. \& R. G.R.R

D. \& S.P.R.R

Whitney

Hayden

D. \& R. G. R. R

Hayden

Palmer \& $\mathrm{R}$ G.

(n)

Wheeler

U. P. R. R. Surveys.

Hayden

Wheeler

Wheeler

Wheeler

Hayden

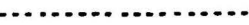

Hayden

Hayden

Feet.

6,067

5,256

7, 028

5,442

8,894

9,008

8,749

4,912

9,000

8,560

9,042

9,578

9,186

9,454

7,537

8,901

7, 596

7,535

7, 592

14,100

D.\& R G R R

D. \& R. G. R. R ............ 7, 2223

D. \& R. G. R. R ............ 8,456

Hayden .................... 8,600

D. \& R. G. R. R........... 5,815

Smithsonian Inst ........... $\quad 3,600$

D. \& R. G. R. R ............ $\quad 6,210$

D. \& R. G. R. R ........... 4,575

Hayden .................. 14, 239

Hayden ........................ 12,589

Hayden .................... 12,072

D. \& R. G. R. R ............ 8,654

Hayden .................... 10,410

Hayden .................... 8,800

Wheeler ................... 13,699

D. \& R. G. R. R ............ $\quad 6,650$

Hayden ...................... 13,897

Whitney ................... 13,832

Hayden ...................... $\quad 11,549$

G. L.\& S. J. R. R .......... 9,074

Hayden ..................... $\quad 9,400$

D. \& R. G. R. R ............ 9,202

Wheeler ................... 14,055

Wheeler .................. 4,040

Hayden .................... $\quad \mathbf{7 , 7 9 7}$

Hayden .................... 12,891

Wheeler.................... $\quad 9,257$

Col. Cent. R. R............. 7,626

Hayden ..................... 13,093

Hayden ..................... 14,158

Hayden ...................... 13,970

Wheeler ................. 8,127

D. \& R. G. R. R ........... 6, 628

Hayden ................... $\quad 6,853$

D. \& R. G. R. R ............ 6,355

Hayden .................. 12,823

Wheeler ................. 7,383

Hayden .................... 8,533

D. \& R. G. R. R ............ $\quad 8,166$

Hayden ......................... 10,897

Hayden ................ 8,000-10,000

G. \& S. P. R. R.......... 5,049 


\begin{tabular}{|c|c|c|}
\hline & Authority. & Elevation. \\
\hline & & \\
\hline $\begin{array}{l}\text { South Platte Brid } \\
\text { South Pueblo }\end{array}$ & Wheeler & 7,978 \\
\hline 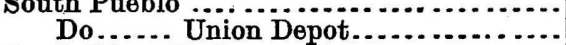 & $\begin{array}{l}\text { D. \& R. G. } \\
\text { A. T. \& S. }\end{array}$ & $\begin{array}{l}4,647 \\
4,639\end{array}$ \\
\hline South River Peak ................ & Hayden ... & 13,160 \\
\hline Spanish Peaks, I & Hayden . & 12,720 \\
\hline Do....... I & Hayden . & 13,620 \\
\hline 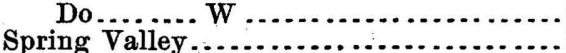 & Wheeler & $\begin{array}{r}13,718 \\
6,647\end{array}$ \\
\hline Squaw............ & Hayden .. & 11,733 \\
\hline Starl & A.T. \& S. F. R. R & 6,331 \\
\hline Star Ranch & Wheeler ......... & 7,327 \\
\hline Stern's Store ... & $\begin{array}{l}\text { Wheeler } \\
\text { Col Cent. }\end{array}$ & $\begin{array}{l}9,06 \varepsilon \\
8,126\end{array}$ \\
\hline $\begin{array}{l}\text { Stevens, } \ldots . . . . \\
\text { Stevens' Mine.. }\end{array}$ & Hayden ........... & $\begin{array}{r}8,126 \\
11,943\end{array}$ \\
\hline Stewart's Peak. & Hayden ........... & 14,032 \\
\hline & Wheeler.... & 14,032 \\
\hline tormy Pea & Hayden ... & 9,200 \\
\hline Suga & Hayden ... & 8,933 \\
\hline Sultan Mountain. & Hayden ... & 13,366 \\
\hline ummit Mines, & $\begin{array}{l}\text { Whe } \\
\text { Whe }\end{array}$ & $\begin{array}{l}13,501 \\
11,089\end{array}$ \\
\hline Sumn & Haj & 13,323 \\
\hline Table Mour & Hayden. & 6,650 \\
\hline Do.... & Hayc & $(i, 167$ \\
\hline ank $\mathrm{P}$ & & 8,929 \\
\hline [arryall . & Whit & 10,078 \\
\hline & Hay & 10,254 \\
\hline & & 9,932 \\
\hline Tarryall $\mathrm{Pa}$ & Hay & 12,176 \\
\hline Tarr & Haj & 12,466 \\
\hline Tem & A.T.\& S. H & 4,407 \\
\hline Tennes & Wheeler.. & 10,702 \\
\hline & & \\
\hline Teocal & Fa) & 13,113 \\
\hline Terrible Mine & Hay & 9,243 \\
\hline & & 14,198 \\
\hline reek .... & D. & 6,196 \\
\hline That & R. R & 5,399 \\
\hline Mount & Wh & 11,496 \\
\hline Tho & D. & 4,987 \\
\hline Park ... & Hay & 7,530 \\
\hline Block... & Wh & 12,190 \\
\hline ttte.... & $\mathrm{Ha}$ & 9,500 \\
\hline & R. G. R. R . & 8,197 \\
\hline e, Poncho Creek Road & ler .... & 8,216 \\
\hline Torrey's Peak ... & $\mathrm{Ha}$ & 14,336 \\
\hline & Wh & 14,375 \\
\hline y Mountain .......... & Hai & 13,200 \\
\hline Tri & & \\
\hline Tring & R. R. surveys. & 7,079 \\
\hline Trinch & Hay & 13,546 \\
\hline & ler $\ldots . . . . . . . . . .$. & 13,681 \\
\hline Crinidad ......... & Toner $\ldots \ldots \ldots \ldots$ & 5,820 \\
\hline ronomical monume & A.T. \& S. F. R. R ... & 5,965 \\
\hline 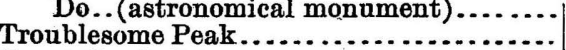 & en & $\begin{array}{r}5,990 \\
11,500\end{array}$ \\
\hline Tront Creek Pass ........ & D. \& S. P. R. R. & 9,386 \\
\hline & n $\ldots \ldots \ldots \ldots$ & 9,346 \\
\hline ........... & er $\ldots \ldots \ldots \ldots \ldots \ldots$ & 9,612 \\
\hline Tront Lake .... & Wheeler..................... & 9,700 . \\
\hline umichi Dome... & Hayden...$\ldots \ldots \ldots \ldots \ldots$ & 11,384 \\
\hline Twin $\mathrm{L}$ & $\begin{array}{l}\text { Wheeler....... } \\
\text { D. \& R. G. R. R. }\end{array}$ & $\begin{array}{l}8,568 \\
9.005\end{array}$ \\
\hline
\end{tabular}




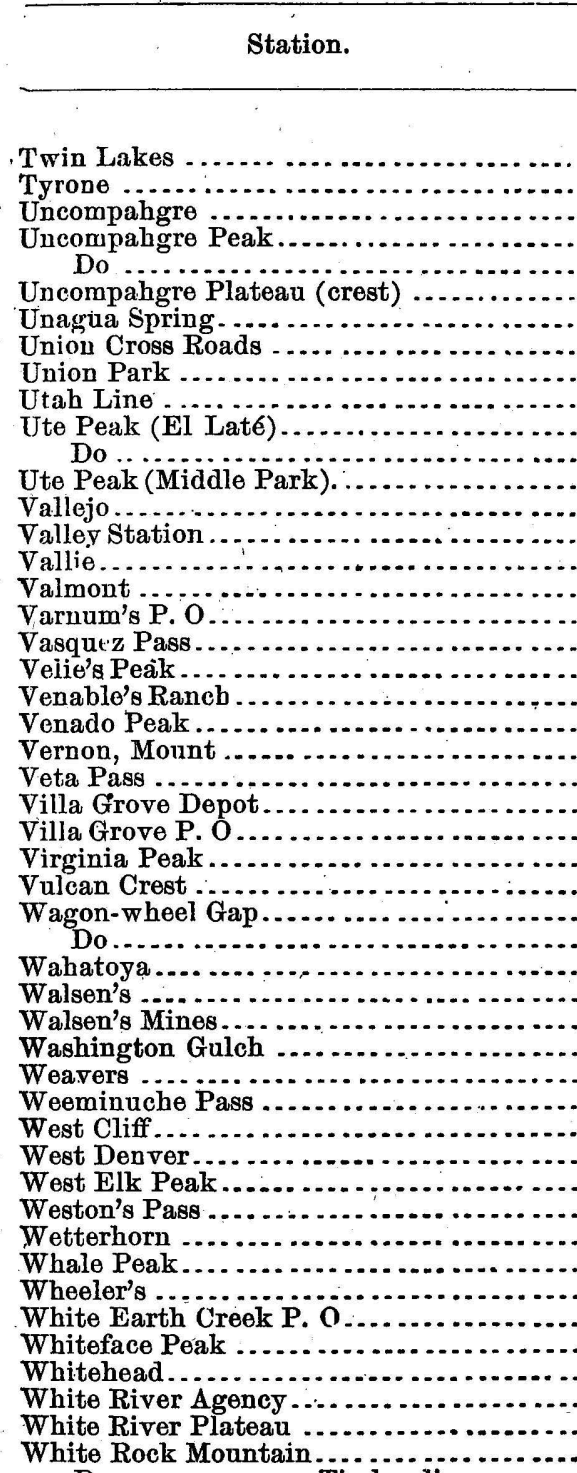
Do

White Ranche, Huerfano Park

White Water

Widefield

Wigwam

Wilcox Ranch, Antelope Park

Wild Flax Pass

Wild Horse . .

Williams, Mount

Willow Creek Pass

Wilson, Mount

$$
\text { Do. }
$$

Wilson's

Wood Valley

\begin{tabular}{|c|c|}
\hline Authority. & Elevatic \\
\hline 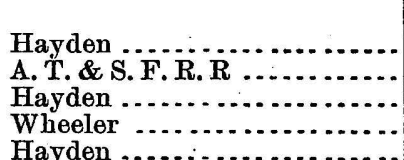 & $\begin{array}{r}F e \\
9, \\
5 \\
6 \\
14 \\
14\end{array}$ \\
\hline
\end{tabular}

Hayden ..................... 14, 235

Hayden ...................9,000-10,000

Hayden .................... 8, 141

Wheeler ..................... 9,655

D. \& R. G. R. R......... 4, 725

Wheeler ................... 10,152

Hayden .................... $\quad 9,884$

Hayden $\ldots \ldots \ldots \ldots \ldots \ldots . . . . . .11,968$

D.\& R. G.R. R ............

(.......................... 4,500

D. \& R.G.R.R . . .

D. \& B. V.R.R ............ $\quad 5,172$

Wheeler................... 7,990

Parry..................... 11,500

Wheeler.................... 7,628

Hayden ............................ 12,800

Parry ........................ $\quad 6,479$

Wheeler ..................... $\quad 9,494$

D. \& R. G. R. R .......... 7,951

Wheeler.................... 8,127

Hayden . . . . . . . . . . . . . . . . . 10,600

Wheeler...................... 13,971

Wheeler ................... 8,372

D. \& R. G. R. R. ................ 8,427

D. \& R. G. R. R. . . $6 . \ldots \ldots \ldots$

D. \& R. G. R. R............ 6,167

D. \& R. G. R. R. .......... 6,200

Wheeler.................... 10,132

D. \& R. G. R. R . . .

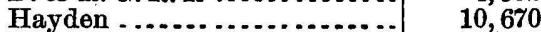

D. \& R. G. R. R .

D. \& R. G. R. R ........... 5,179

Hayden ................... 12,920

Wheeler............................ 12,109

Wheeler..................... 14,069

Hayden...................... 13,104

D. \& R. G. R. R .......... 9,759

Wheeler.................... 8,163

Hayden ...................... 11,493

King ........................... 10,817

Hayden .................... 6,491

Hayden .................. 11,000-12, 000

Hayden ...................... 13, 357

Hayden ..................... 11,919

Wheeler..................... $\quad \mathbf{7}, 469$

D. \& R. G. R. R ......... 4,635

D. \& R. G. R. R .......... $\quad 5,697$

D. \& R. G. R. R ......... 5,211

Wheeler.................. $\quad \mathbf{9}, 000$

Palmer ...................... $\quad 8,900$

Kans. Pac. R. R ............. 4, 438

Hayden ..................... 11, 413

Hayden ...................... $\quad 9,683$

Hayden ..................... $\quad 14,280$

Wheeler..................... 14,309

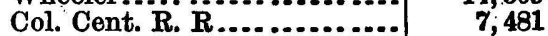

$\mathbf{5}, \mathbf{5 3 3}$ 


\begin{tabular}{|c|c|c|}
\hline Station. & Authority. & Elevation. \\
\hline 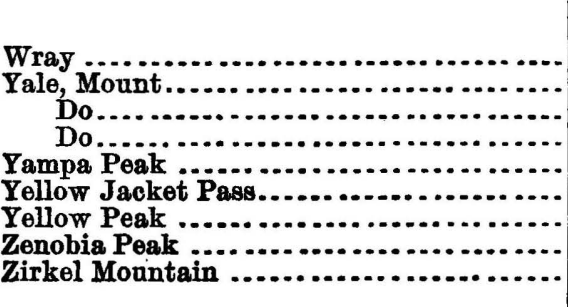 & 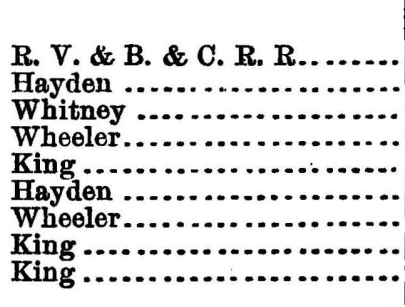 & $\begin{array}{r}\text { Feet. } \\
3,519 \\
14,187 \\
14,263 \\
14,121 \\
8,022 \\
7,493 \\
13,618 \\
9,297 \\
12,126\end{array}$ \\
\hline
\end{tabular}


CONNECTICUT.

\begin{tabular}{|c|c|c|}
\hline Statio & Authority. & Elevation. \\
\hline & & Feet. \\
\hline $\begin{array}{l}\text { Avon ..... } \\
\text { Berlin }\end{array}$ & N. H. \& Northampton $R$. & 242 \\
\hline Berlin, junction with & N. Y., N. H. \& Ftfd R. & $\begin{array}{l}63 \\
64\end{array}$ \\
\hline Bridgeport, bridge ne & U. S.C. \& G. S .......... & \\
\hline Do..... station, $\mathrm{J}$ & N. Y. \& N. H. R. R. . & \\
\hline Brookfield & Housatonic R. R.... & 338 \\
\hline & Housatonic R. R... & \\
\hline Carmel Mou & U. S. C. \& G. S......... & 739 \\
\hline Cheshire... & N. H. \& Northampton R. & 166 \\
\hline & N. H. \& Northampt & 227 \\
\hline East Rock .. & U. S. C. \& G. S... & 362 \\
\hline Danbury, $\mathrm{D}$. & Bost. \& N. Y. R. R. & 397 \\
\hline Farm & N. H. \& Northampto & 204 \\
\hline Gra & N. H. \& Northamp & 204 \\
\hline Great Rock or Rab & U. S. C. \& G. S..... & 376 \\
\hline Hartford ............ & N. Y., N. H. \& Htfd R. R & 39 \\
\hline Hawleyville & Housatonic $R$. R........ & 306 \\
\hline Hick & U. S. C. \& G. S... & 651 \\
\hline Jos & U. S. C. \& G. S... & 608 \\
\hline Lake & Conn. Western R. R.... & 670 \\
\hline Merid & N. Y., N. H. & 131 \\
\hline Midi & N. Y., N. H. \& Htfd R. R & 23 \\
\hline Mou & N. H. \& Northampt & 114 \\
\hline Mystic... & N. Y., Prov. \& Bc & 7 \\
\hline New Brita & N. Y., N. H. \& Ht & 179 \\
\hline New Har & Conn. Weste & 389 \\
\hline New Haven, Chapel street Bridge. & U. S. C. \& G. S... & 6 \\
\hline Do........ State & U. S. C. \& G. S...... & \\
\hline .New Road......... & ................... & \\
\hline .. large granite post in gre & U. S. C. \& G. S................... & 30 \\
\hline Do... & N. Y., N.H. \& Htfd.R.R & \\
\hline Do.. & U. S. C. \& G. S.... & 38 \\
\hline eline Junction & N. Y., N. H. \& Hftd. & 20 \\
\hline Do....... Signal & U. S. Sigual Office .. & 106 \\
\hline New London .... & N. Y., N. H. \& Htf.R. R & 9 \\
\hline Do ....... Sig & U. S. Signal Office...... & 47 \\
\hline New Milford ... & Housate & \\
\hline New Roc & N. Y., N. H. \& Htfd. R. R.. & 82 \\
\hline Noank .. & N. Y., Prov. \& Boston R. R .. & 14 \\
\hline Norfolk ........................................... & Conn. Western R. R ....... & 1,220 \\
\hline Norwalk Station, Junction with Danbury & & \\
\hline $\begin{array}{l}R . R \\
\text { Norwich, Main st }\end{array}$ & $\begin{array}{l}\text { N. Y. \& N. H. R. R ......... } \\
\text { New London Northern R. R. }\end{array}$ & $\begin{array}{r}25 \\
7\end{array}$ \\
\hline & $\begin{array}{l}\text { New London Northern K. K.. } \\
\text { Htfd., Prov. \& Fishkill R. R. }\end{array}$ & 162 \\
\hline & N. H. \& Northampton R. R .. & 191 \\
\hline & N. Y., Prov. \& Boston R. R.. & 8 \\
\hline Saybrook, junction with Connecticut Val & & \\
\hline Simsbury & N. H. \& Northampton R. R... & $\begin{array}{r}24 \\
167\end{array}$ \\
\hline Southington & N. H. \& Northampton R. R.. & 152 \\
\hline
\end{tabular}


[BUI.L. 5.

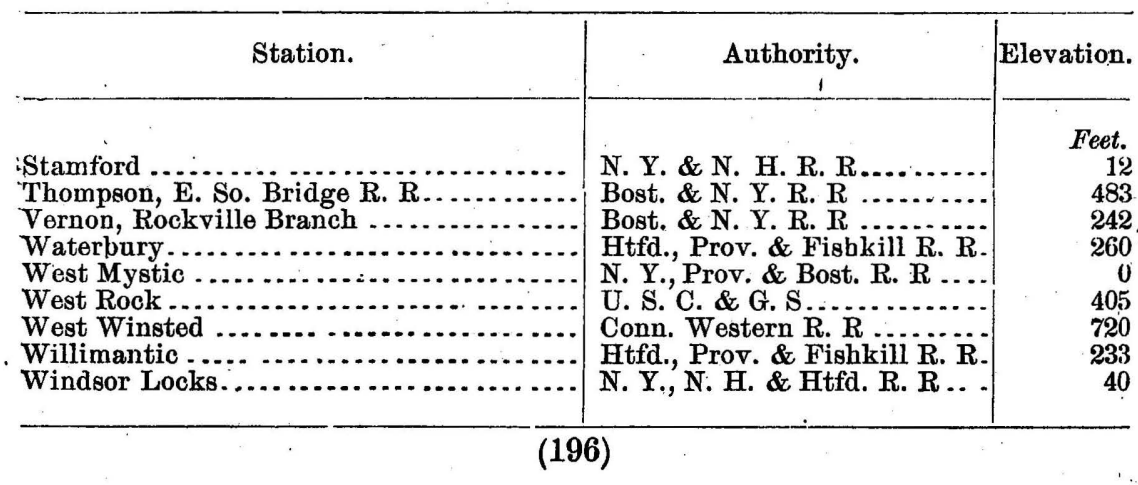


DAKOTA.

\begin{tabular}{|c|c|c|}
\hline Station. & & Elevation. \\
\hline & & \\
\hline Abraham . & & \\
\hline Alcester - & Dak. Cent. & 1,355 \\
\hline Altamont & C. N. W. R & 834 \\
\hline Apple Creek Valley. & N. P. R.R & 711 \\
\hline Aurora ............... & C. \& N. & 630 \\
\hline Austin .. & & 221 \\
\hline Bel & N.P.R.I & 2,577 \\
\hline tt, Fort, Signal & Sig & 440 \\
\hline ford ............... & $\ldots . .$. & 505 \\
\hline ld, Fort ......... & & 373 \\
\hline oux River, water $2 d$ crossing. & C. M. \& S & 479 \\
\hline k $\because \cdots$ & N.P. R. R & 1,668 \\
\hline Do... Signal Statio & U. S. Signa & 1,677 \\
\hline Do...Mo. Ri & $\cdots$ & 1,616 \\
\hline (n) & Fargo \& & 965 \\
\hline & & \\
\hline & C. \& N & 60 \\
\hline $\mathbf{B u}$ & Smithso & 017 \\
\hline Do.. & & 1,876 \\
\hline 年 & & \\
\hline 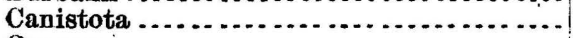 & $\tilde{\mathrm{Da}}$ & $\mathbf{1}$, \\
\hline$\ldots . . .$. & Dal & 1,525 \\
\hline$\ldots \ldots$ & S. C. \& D. & 1,291 \\
\hline (2) & Dak. Cent. & 1,440 \\
\hline Casse & N.P.R.R & 930 \\
\hline Ca & C. \&.N.W & $\mathbf{1}, \mathbf{3 1 1}$ \\
\hline & Dak. Cent. & 233 \\
\hline ............ & .......... & 785 \\
\hline Clark, Fort ............ & ......... & 327 \\
\hline land & $\ldots \ldots$ & 840 \\
\hline Miss & .......... & 000 \\
\hline des Prairies. & & 800 \\
\hline Depot........ & $R \ldots \ldots \ldots$ & 909 \\
\hline wer $\ldots \ldots$ & Jenı & 7,600 \\
\hline Depot & Fargo \& ís. W & 921 \\
\hline ction. & S. C. \& D.R. & 1,130 \\
\hline n... & $\ldots \ldots \ldots$ & 30 \\
\hline D & ............... & 1 \\
\hline$\cdots$ & .............. & 2 , \\
\hline ake. & $\ldots \ldots . . .$. & 167 \\
\hline & $\ldots \ldots \ldots$ & \\
\hline .. & N.P.R.R . & \\
\hline .. & P.R.R. & \\
\hline .. & C. M. \& St. P. R. & \\
\hline & & 142 \\
\hline on Lake (station) ....... & N.P.R.R. & \\
\hline on Lake, water ... & N.P.R. R. & 1, \\
\hline & Dak. Cent. R. R . . . . . . . & \\
\hline airview. & C. \& N. W.R.R . . . . . . . & 342 \\
\hline Do... & S. C. \& D.R.R.. & 1,231 \\
\hline & N.P.R.R...... & 903 \\
\hline & & \\
\hline & 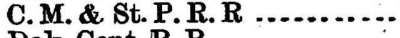 & \\
\hline (1) & Dak. Cent. R. R & 1,296 \\
\hline Y.................... & 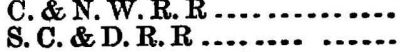 & $\begin{array}{l}1,484 \\
1,178\end{array}$ \\
\hline
\end{tabular}




\begin{tabular}{|c|c|c|}
\hline Station. & Authority. & Elevation. \\
\hline & & \\
\hline Gladstone.... & N.P. R.R & 2,346 \\
\hline lenullin .... & N.P. R.R & 2,070 \\
\hline $\begin{array}{l}\text { Good win..... } \\
\text { Greenwood . }\end{array}$ & $\begin{array}{l}\text { C.\& N.W. R.R R ... } \\
\text { Smithsonian Inst }\end{array}$ & $\begin{array}{l}1,996 \\
1,900\end{array}$ \\
\hline Harney's Peak & Ludlow ............ & 9,700 \\
\hline Hartford Siding & St.P.\& S.C.R. & \\
\hline Hawarden ..... & Dak. Cent. R. R. & 1,182 \\
\hline Henry ........ & Dak. Cent. R. R. & 1,810 \\
\hline Herman ..... & C. M. \& St. P. R. & 1,654 \\
\hline Herman, Lake. & C. M. \& St. & 1,646 \\
\hline Horace Depot. & Fargo \& S. & 917 \\
\hline Hurley ........ & Dak. Cent. I & 1,272 \\
\hline Huron ........ & C. \& N.W.I & 1,285 \\
\hline Junction & C. \& N.W. 1 & 1,312 \\
\hline Iroq & C. \& N.W. 1 & 1,401 \\
\hline Jamestown & N.P. R. R ... & 1,395 \\
\hline Jefferson ........ & S. C. \& D.R.R.. & 1,130 \\
\hline eska, Lake & C. \& N.W.R.R. & 1,650 \\
\hline Kampeska, Lake, n & $\begin{array}{l}\text { C.\&N. W.R.R . } \\
\text { P.R.R.Reports. }\end{array}$ & $\begin{array}{l}1,714 \\
2,431\end{array}$ \\
\hline $\begin{array}{l}\text { Kamskusahkee La] } \\
\text { Kranzbury ....... }\end{array}$ & $\begin{array}{l}\text { P.R. R. Reports. } \\
\text { C. \& N. W.R.R. }\end{array}$ & $\begin{array}{l}2,431 \\
1,982\end{array}$ \\
\hline Missouri. & N. P.R.R... & 2,255 \\
\hline Missouri $\mathrm{Bu}$ & Jenney $\ldots . . . .$. & 5,500 \\
\hline $\begin{array}{l}\text { McCook ............. } \\
\text { McKenzie....... }\end{array}$ & S.C.\& D.R.R.. & $\begin{array}{l}1,123 \\
1,696\end{array}$ \\
\hline Madison Lake.. & C.M.\& St.P.R.R & 1,576 \\
\hline Man & N.P.R.R R...... & 1,644 \\
\hline $\operatorname{ton} . .$. & R.R... & 903 \\
\hline$\ldots$ & S. C. \& D.R.R. & 1,167 \\
\hline Junction & C. M. \& St.P.R.R & \\
\hline $\begin{array}{l}\text { Siding... } \\
\text { falo....... }\end{array}$ & $\begin{array}{l}\text { St.P.\& S. C.R.R. } \\
\text { N.P.R.R.... }\end{array}$ & $\begin{array}{l}1,471 \\
1,206\end{array}$ \\
\hline $\begin{array}{l}\text { New Buffalo....... } \\
\text { New Madison .... }\end{array}$ & 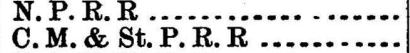 & 1,646 \\
\hline Noreland & C.\&N.W.R.R.... & 1,846 \\
\hline wood Settlement & Nicollet .......... & 1,341 \\
\hline ............ & $R \ldots \ldots$ & \\
\hline $\begin{array}{l}\text { Parker ............ Signal Stg } \\
\text { Pembina, Sil }\end{array}$ & int. R. R... & $\begin{array}{r}1,340 \\
791\end{array}$ \\
\hline $\begin{array}{l}\text { Pembina, Signal Sta } \\
\text { Pierre ............... }\end{array}$ & $\begin{array}{l}\text { U. S. Signal Office. } \\
\text { C. \& N.W. R. R... }\end{array}$ & 1,438 \\
\hline Fort ...... & Med. Dep't, U. S. A & 1,456 \\
\hline Lake... & V.R.R... & 1,696 \\
\hline & & 1,2 \\
\hline Fort .... & Med. Dep't, U. S. A. & 1,438 \\
\hline son...... & N.P.R.R .......... & 2,464 \\
\hline Sale & S.C.R.R. & \\
\hline ........... & N.P. & \\
\hline 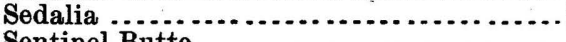 & N.P.R.R. & \\
\hline 1 Butte .... & N. R.R. R.. & \\
\hline e River... & $\begin{array}{l}\text { N.P.R.R } \\
\text { N.P.R.R. }\end{array}$ & $\begin{array}{l}1,200 \\
1,960\end{array}$ \\
\hline 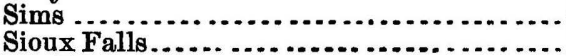 & $\begin{array}{l}\text { N.P.R.R } \\
\text { S.C.\& D. R. R..... }\end{array}$ & $\begin{array}{l}1,960 \\
1,411\end{array}$ \\
\hline 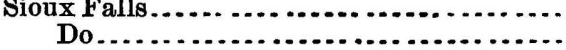 & C. M. \& St.P.R. R & $\begin{array}{l}1,411 \\
1,638\end{array}$ \\
\hline & St.P.\& S. C.R. R... & \\
\hline Falls Junctio & C.M. \& St.P. R. R . . . . & 1,496 \\
\hline Heart ........ & N.P.R.R R $\mathbb{R}_{\mathrm{N}}^{R} \ldots \ldots \ldots \ldots$ & 2,470 \\
\hline & N.P.R. K ........ & $\begin{array}{l}1,47 \\
2,12\end{array}$ \\
\hline & N.P.R.R & \\
\hline Stevenson, Fort, Signal Station.... & U. S. Signal Office....... & 1,734 \\
\hline $\begin{array}{l}\text { Sully, Fort } \\
\text { Sully }\end{array}$ & U.S. Signal Office................. & $\begin{array}{l}1,688 \\
2,647\end{array}$ \\
\hline & N.P.R.R . & $\begin{array}{l}2,046 \\
1,760\end{array}$ \\
\hline
\end{tabular}




\begin{tabular}{|c|c|c|}
\hline Station. & Authority. & Elevation. \\
\hline 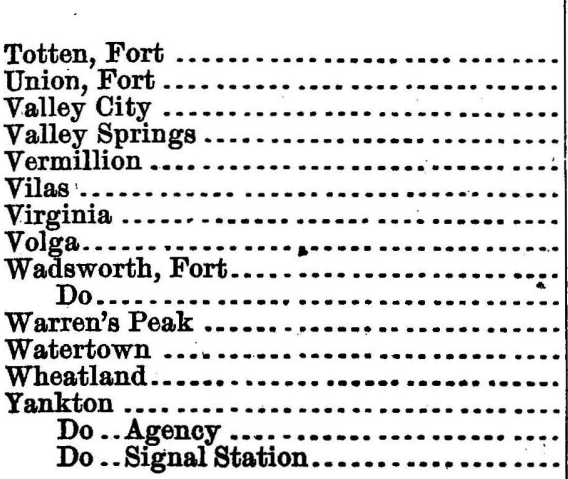 & 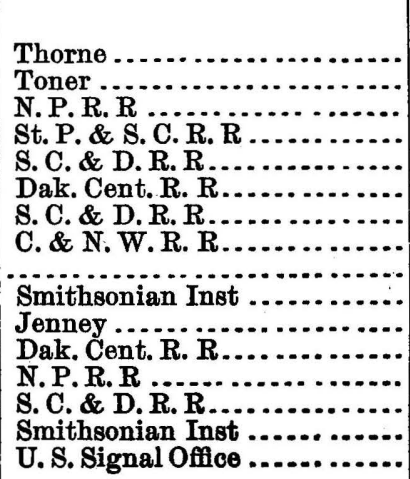 & $\begin{array}{l}\text { Feet. } \\
1,480 \\
1,970 \\
1,218 \\
1,392 \\
1,161 \\
1,480 \\
1,221 \\
1,636 \\
1,896 \\
1,650 \\
6,900 \\
1,735 \\
985 \\
1,196 \\
1,900 \\
1,228\end{array}$ \\
\hline
\end{tabular}




\section{DELAW ARE.}

\begin{tabular}{|c|c|c|}
\hline Station. & Authority. & Elevation. \\
\hline & & Ireet. \\
\hline Bellevue ........ & P. W.\& B. I & 14 \\
\hline Bridgeville ...... & D. R.R.... & 55 \\
\hline Canterbury ...... & D. R. R . . - & 69 \\
\hline Centre & W.\& R.R. R & 263 \\
\hline 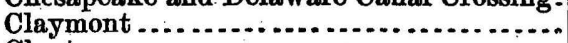 & P.W.\& B.R. R & 30 \\
\hline Clayton.............. & D. R.R R... & 45 \\
\hline Delaware Breakwater & U. S. Signal Offic & 20 \\
\hline Delaware City ....... & Pa. \& Del. R. R . & 16 \\
\hline Delmar $\ldots \ldots \ldots$ & E. S. R. R $\ldots$ & 32 \\
\hline $\begin{array}{l}\text { Dover } \\
\text { Dupont's ........... }\end{array}$ & $\begin{array}{l}\text { D.R. R. } \\
\text { W. \& R.R. R }\end{array}$ & $\begin{array}{r}39 \\
282\end{array}$ \\
\hline Georgetown ..... & J. \& B.R.R . & 52 \\
\hline Harrington ...... & D. R. R & 63 \\
\hline Holly Obak ..... & P. W. \& B.R.R & 10 \\
\hline Lewes .......... & J. \& B. R. R. . . . . $\ldots \ldots \ldots$ & 9 \\
\hline $\begin{array}{l}\text { Middletown ....... } \\
\text { Milford ........... }\end{array}$ & D. R. R $\ldots$ & $\begin{array}{l}66 \\
15\end{array}$ \\
\hline Newark ........... & P.W.\&B. R. R.. & 106 \\
\hline Do.. crossing o & Pa. \& Del. R. $\mathbf{R}$ & 118 \\
\hline $\begin{array}{l}\text { Newport } \ldots \ldots \ldots \\
\text { Oak Grove }{ }^{2} \ldots \ldots\end{array}$ & P. W. \& B. R. R $\ldots \ldots \ldots$ & 21 \\
\hline Rodney, junction with New Castle and & & \\
\hline 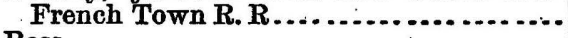 & D. R. R & \\
\hline Ross $\ldots \ldots \ldots \ldots$ & D. R. R ........... & 42 \\
\hline Stannton ... & P. W.\& B.R.R. & 17 \\
\hline 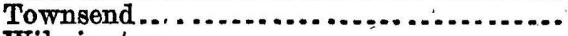 & 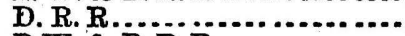 & $\overline{71}$ \\
\hline Wilmington & P.W.\& B. R. R .............. & \\
\hline
\end{tabular}

(200) 


\section{DISTRICT OF COLUMBIA.}

\begin{tabular}{|c|c|c|}
\hline Station. & Authority. & Elevation. \\
\hline Canitol dome & $\mathrm{IUC} \& \mathrm{~S}$ & Feet. \\
\hline Do..east front, ground in front of cen- & & 379.00 \\
\hline 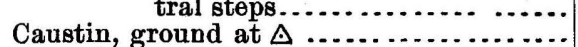 & $\begin{array}{l}\text { U.S.C.\& G. S. } \\
\text { U.S.C.\& G. S. }\end{array}$ & $\begin{array}{r}89.00 \\
386.00\end{array}$ \\
\hline $\begin{array}{l}\text { Coast Survey Office, top of roof at flagstaff. } \\
\text { Do }\end{array}$ & U.S.C.\& G. S. & 137.00 \\
\hline 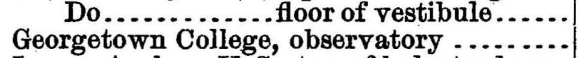 & 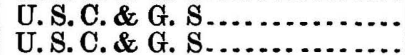 & $\begin{array}{r}78.00 \\
209.00\end{array}$ \\
\hline Insane Asylum, U.S:, top of balustrade on & U.S.C.\& G S & 255.00 \\
\hline 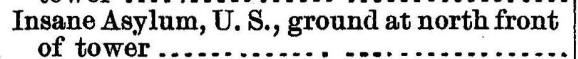 & U.S. C. \& G. & 173.00 \\
\hline $\begin{array}{c}\text { Naval Observatory, U. S., top of small } \\
\text { dome }\end{array}$ & U. S. C. \& G. S & 150.00 \\
\hline $\begin{array}{l}\text { Navy-yard, bridge, curbstone east end, B. } \\
\text { M. No.2 }\end{array}$ & U.S.C.\& G. S. & 8.00 \\
\hline $\begin{array}{l}\text { Navy-yard, tide gauge } \\
\text { Do } \ldots . . . \text { B. M }\end{array}$ & $\begin{array}{l}\text { U.S. C. \& G.S. } \\
\text { U.S.C. \& G. S.. }\end{array}$ & $\begin{array}{l}0.27 \\
5.00\end{array}$ \\
\hline Do .... platform foot of flagstaff ....... & U.S.C.\& G. S. & 39.00 \\
\hline $\begin{array}{l}\text { Smithsonian Institution, top of balustrade } \\
\text { on highest tower ........................................... }\end{array}$ & U.S. C.\& G. S.. & 175.00 \\
\hline 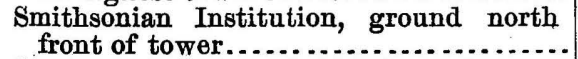 & U.S. C. \& G. S. & 34.00 \\
\hline $\begin{array}{l}\text { Soldiers' Home, top of old balustrade } \ldots . . . . \\
\text { Do } \ldots . . . . . \text {. foot of tower, south side... }\end{array}$ & 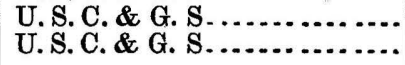 & $\begin{array}{l}410.00 \\
330.00\end{array}$ \\
\hline
\end{tabular}


FLORIDA.

\begin{tabular}{|c|c|c|}
\hline Station. & Authority. & Elevation. \\
\hline & & Feet. \\
\hline $\begin{array}{l}\text { Anthony Place } . . . . . . . . . \\
\text { Archer }\end{array}$ & A. G. \& W.I. T. R. R & $\begin{array}{r}78 \\
80\end{array}$ \\
\hline $\begin{array}{l}\text { Archer } \\
\text { Arredonda }\end{array}$ & $\begin{array}{l}\text { A. G. \& W. I. T. R. R...... } \\
\text { A. G. \& W. I. T. R. R....... }\end{array}$ & $\begin{array}{l}80 \\
70\end{array}$ \\
\hline 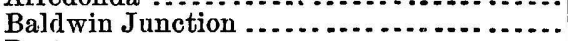 & A. G. \& W.I.T.R.R. & 47 \\
\hline Barton ........... & A. G. \& W. I. T. R. R. & \\
\hline 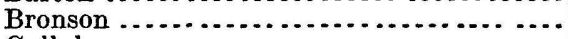 & A. G. \& W. I.T. R. R. & 27 \\
\hline Callahan ... & A. G. \& W. I.T. R. R. & 30 \\
\hline Cedar Key. & A. G. \&. W. I.T. R. R. & 10 \\
\hline Do .... Signal Station... & U. S. Signal Office.... & 22 \\
\hline Dutton ................... & A. G. \& W. I.T. R. R. & 45 \\
\hline Fernandina & A. G. \& W. I. T. R. R. & 10 \\
\hline Gainesville. & A. G. \& W. I. T. R. R. & 128 \\
\hline Hawthorne $\ldots \ldots \ldots \ldots \ldots \ldots \ldots \ldots$ & A. G. \& W. I. T. R. R. & 150 \\
\hline Highland, Trail Ridge, summit of R. R.... & A. G. \& W.I. T. R. R. - & 210 \\
\hline Jacksonville, Signal Station ............... & U. S. Signal Office.... & 43 \\
\hline 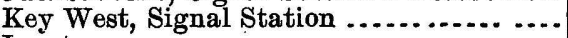 & U. S. Signal Office.... & 27 \\
\hline 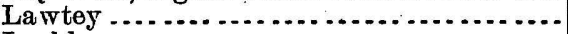 & A. G. \& W. I. T. R. R. & 140 \\
\hline Lochloosa . . . . . . . . . . . . . . . . . . . . . . . . & A. G. \& W. I. T. R. R. & $\mathbf{5 3}$ \\
\hline 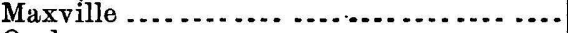 & A. G. \& W.I.T. R. R & 57 \\
\hline Ocala ........ & A. G. \& W. I. T. R. R & 100 \\
\hline Orange Lake.................. & A. G. \& W.I.T. R. R & 53 \\
\hline Pensacola, Signal Station ................ & U. S. Signal Office... & 30 \\
\hline Punta Rassa, Signal Station ............... & U. S. Signal Office.... & 13 \\
\hline 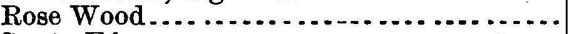 & A. G. \& W. I. T. R. R........ & 12 \\
\hline Santa F6́........ & A. G. \& W. I.T. R. R........ & 150 \\
\hline Silver Spring . . . . . . & A. G. \& W.I.T. R. R....... & 32 \\
\hline$\ldots \ldots \ldots \ldots \ldots \ldots \ldots$ & A. G. \& W.I.T. R. R...... & 150 \\
\hline$\ldots \ldots \ldots \ldots \ldots \ldots$ & A. G. \& W. I. T. R. R.......... & 140 \\
\hline Waldo $\ldots \ldots \ldots \ldots \ldots \ldots$ & A. G. \& W.I. T. R. R........ & 150 \\
\hline
\end{tabular}


GEORGIA.

\begin{tabular}{|c|c|c|}
\hline Station. & Authority. & Elevation. \\
\hline & & Feet. \\
\hline Academy Mountain $\ldots \ldots \ldots \ldots$ & U.S. C. \& G.S & 1,136 \\
\hline 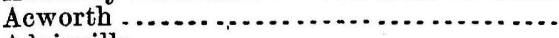 & W. \& A.R.R & 926 \\
\hline dairville............ & W. \& A: R. R & 710 \\
\hline lappeha Station .. & B. \& A.R.R. & 267 \\
\hline Albany ........... & S. W.R.R ... & 232 \\
\hline Do $\ldots \ldots$ & B. \& A.R.R & 168 \\
\hline 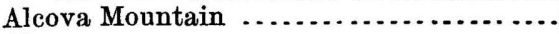 & U.S.C.\& G & 1,090 \\
\hline Alcory & Ga. R. R.... & 721 \\
\hline Do..level low water in Alcovy River. & Ga. R. R... & 654 \\
\hline 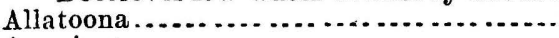 & W. \& A.R.R & 878 \\
\hline ericus $\ldots \ldots \ldots \ldots \ldots \ldots$ & S. W. R. R ... & 362 \\
\hline 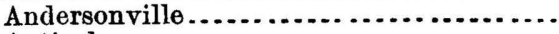 & S. W.R.R.: & 396 \\
\hline 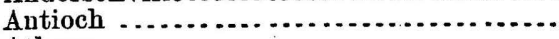 & Ga. R. R ... & 778 \\
\hline$\ldots \ldots \ldots \ldots$ & Ga. R. R. & 694 \\
\hline 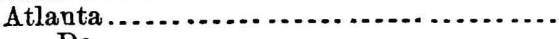 & Ga. R. R ... & 1,050 \\
\hline Do ..... & M. \& W. R. R & 1,050 \\
\hline Do ... & W. \& A. R. R & 1,050 \\
\hline Do & A. \& R. A. L. ] & 1,050 \\
\hline Do... signal station...$\ldots \ldots \ldots \ldots$ & U. S. Signal O & 1,131 \\
\hline Augusta $\ldots \ldots \ldots$ & Ga. R.R $R$ & 134 \\
\hline on $\ldots . . . \ldots \ldots . . . . . .$. & U. S. Signal of & 183 \\
\hline Baruesville ......... & M. \& W. R. R. & 875 \\
\hline . & Ga. R. R .... & 647 \\
\hline on $\ldots \ldots \ldots \ldots \ldots \ldots$ & W. \& A. R. R & 845 \\
\hline . & M. \& B. R. R . & 210 \\
\hline 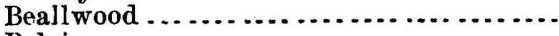 & C. \& R. R. R.. & 387 \\
\hline 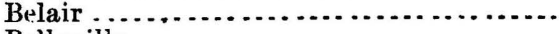 & Ga. R. R .. & 309 \\
\hline$\ldots \ldots \ldots \ldots$ & A. \& R. R. R. & 1,342 \\
\hline 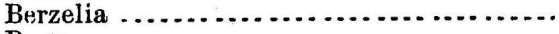 & Ga. R. R .... & 502 \\
\hline$\ldots \ldots \ldots$ & W. \& A. R. R. & 750 \\
\hline Shanty ........ & W. \& A. R. R. & 1,100 \\
\hline . & U. S. C. \& G. S & 4,463 \\
\hline . & W. \& A. R. R. & 850 \\
\hline … & - $\ldots \ldots \ldots \ldots$ & 934 \\
\hline …, & $\ldots \ldots \ldots . . . .$. & 796 \\
\hline 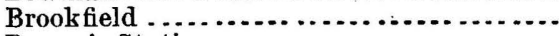 & B. \& A. R. R & 303 \\
\hline . & S. W. R. R .. & 371 \\
\hline 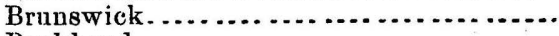 & M. \& B. R. R. & 14 \\
\hline$\ldots \ldots \ldots \ldots$ & Ga. R. R .... & 620 \\
\hline$\ldots \ldots \ldots \ldots \ldots$ & A. \& R. A. L. $\mathrm{H}$ & 1,207 \\
\hline$\ldots \ldots \ldots \ldots \ldots \ldots \ldots$ & $\ldots \ldots \ldots \ldots$ & 265 \\
\hline 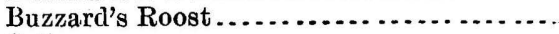 & M. \& B. R. R.. & 240 \\
\hline . & W. \& A. R. R.. & 657 \\
\hline . & Ga. R. R ..... & 592 \\
\hline 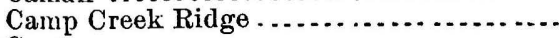 & $\ldots \ldots$ & 308 \\
\hline Canton...$\ldots \ldots \ldots \ldots \ldots$ & M. \& N. G. R. R .......... & 894 \\
\hline How ah River...... & M. \& N. G. R. R .......... & 859 \\
\hline pitol Tower Mountain, Atlanta ..... & 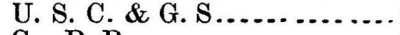 & 1,163 \\
\hline . . . . . . . & Ga. R. R .... & 507 \\
\hline vill & W. \& A. R. R. & 760 \\
\hline 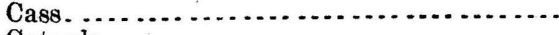 & W. \& A. R. R . & 765 \\
\hline (n) & C. \& R. R. R $R \ldots \ldots$ & 692 \\
\hline . & S. R. \& D. R. R & 672 \\
\hline & \& $B \cdot R \cdot R$ & 850 \\
\hline $\mathrm{C}$ & Ga. R. R . & 1,012 \\
\hline le C. H. & U. S. C. \& & 1,478 \\
\hline (1) & & 1,616 \\
\hline
\end{tabular}




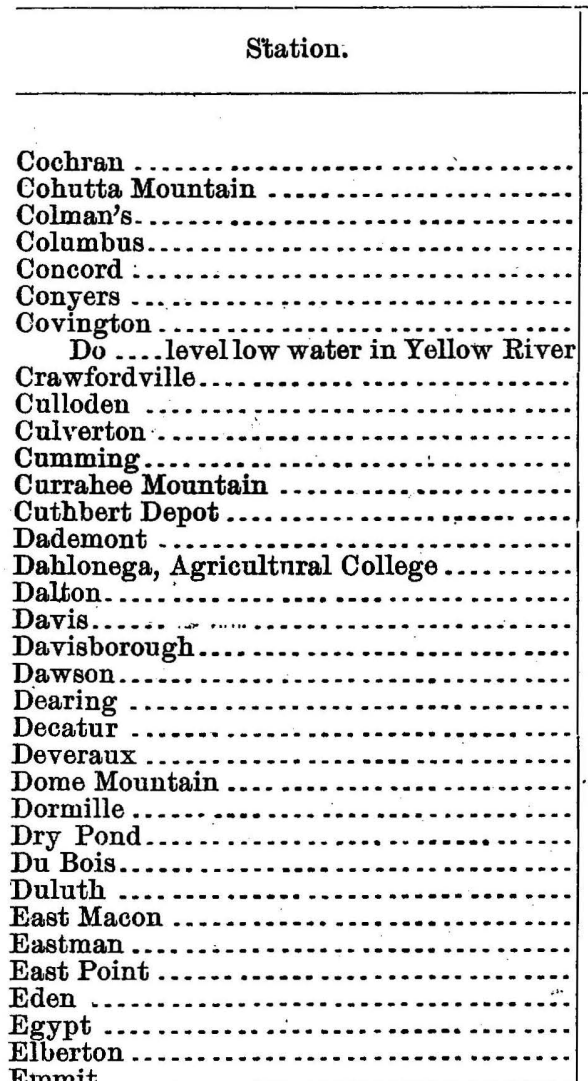

Enota Mountain

Fairburn

Fair Grounds

Fellowship Church.....................

Fodder's Bald Mountain

Forrest

Forsyth .....................................

Fort Gaines Depot

Do ...... Bridge

Fortsons

Fort Valley

Fosterville.

Gainesville.

Gillsville

Gilmore.

Goodwin

Gordon

Goss Store

Graham.

Grantville

Grassy Mountain

Graysville.

Greensboro Depot

Do

Griffin .

Griswold

Haddocks

Halcyondale

M. \& B. R. R..

U. S. C. \& G. S.

S. W. R. R.

C. \& R. R. R.

R. R. surveys

Ga. R. R

Ga. R. R

Ga. R. R

Ga. R. R

$R$. R. surveys

Ga. R. R

U. S. C. \& G. S

U. S. C. \& G. S

S. W. R. R .

Ala. G. S. R. R

U. S. C. \& G. S

S. R. \& D. R. R

B. \& A. R. R

C. R. R .

n..............

S. W. R. R

Ga. R. R

Ga. R. R

U. S. C. \& G. S

A. \& R. A. L. R. R

A. \& R. A. L. R. R

M. \& B. R. R

A. \& R. A. L. R. R

C. R. $\mathbf{R}$

M. \& B. R. R .

A. \& W. P. R. R

C. R. $\mathbf{R}$

C. R. R

Elberton R. R

C. R. R.

U. S. C. \& G. S.

A. \& W. P. R. R

W. \& A. R. R

Elberton R. R:

Guyot

Ga. R. R

M. \& W. R. R

S. W. R. R.

S. W. R. R.

C. \& R.R. R

S. W. R. R.

M. \& W. R. R

A. \& R. A. L. R. R

N. E. R. R

W. \& A. R. R

A. \& R. A. L. R. R

Cent. R. R.

Elberton R. R

M. \& B. R. R.

A. \& W.P.R. R

U. S. C. \& G. S

W. \& A. R. R.

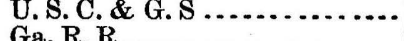

M. \& W. R. $\mathbf{R}$

Cent. R. R.

Cent. R. R

Ga. R. R.
Elevation.

Feet.

341

4,155

393

262

804

894

748

608

603

696

558

1,316

1,740

448

832

1,519

757

207

291

354

478

1,033

569

4, 042

1,098

$1,03$.

394

1,107

297

362

1,043

34

126

706

210

4,798

1, 034

979

857

4,821

511

735

166

192

522

530

960

1, 227

1, 052

903

975

343

727

250

869

3,290

706

611

612

975

464

77

487

110 


\begin{tabular}{|c|c|c|}
\hline Station. & Authority. & Elevation. \\
\hline & & Feet. \\
\hline 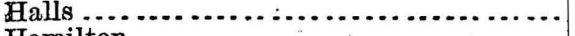 & W. \& A. R. & 771 \\
\hline 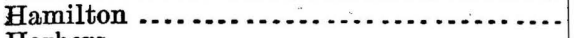 & C. \& R.R.I & 786 \\
\hline 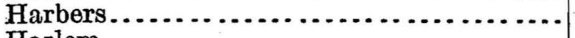 & S. R. \& D.I & 648 \\
\hline$\ldots \ldots \ldots \ldots \ldots \ldots \ldots$ & Ga. R. R. & 562 \\
\hline ................... & N.E.R.R & 954 \\
\hline fille* $\ldots . .$. & M. \& B. R. R & 235 \\
\hline$\ldots \ldots \ldots \ldots \ldots \ldots$ & B. R. R & 261 \\
\hline$\ldots \ldots \ldots \ldots \ldots \ldots$ & Cent: R. R. & 174 \\
\hline 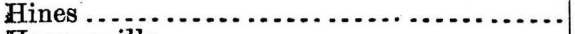 & C. \& R. R. R & 592 \\
\hline 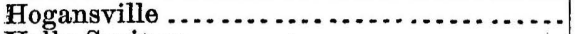 & A. \& W.P.R. I & 731 \\
\hline 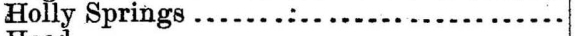 & M. \& N. G.R & 1,114 \\
\hline 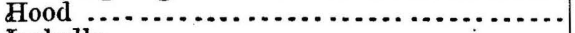 & C. \& R. R. F & 912 \\
\hline$\ldots \ldots \ldots \ldots \ldots \ldots$ & B. \& A.R. I & 340 \\
\hline Jes & M. \& B. R. I & 100 \\
\hline boro.... & M. \& W. R. & 905 \\
\hline Kenesaw ...... & U.S. C. \& G & 1,809 \\
\hline Kingsboro $\ldots \ldots \ldots \ldots \ldots \ldots \ldots \ldots$ & C. \& R.R. & 612 \\
\hline 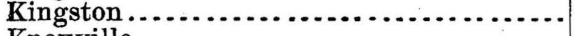 & W.\& A. R. & 710 \\
\hline lle........................... & R.R. & 640 \\
\hline 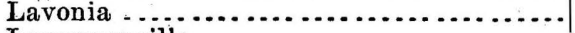 & Elber & 865 \\
\hline lceville.............................. & L. B. R. R & 1,082 \\
\hline La Grange . . . . . . . . . . . . . . . . . . . . . . . . & A. \& W. P & 742 \\
\hline 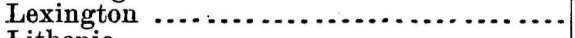 & Ga. R: R. & 770 \\
\hline 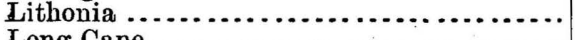 & Ga. R. R. & 937 \\
\hline$\ldots \ldots$ & A. \& W.P. & 624 \\
\hline$\ldots \ldots$ & N. E. R. & 1,334 \\
\hline City ..... & B. R. & 150 \\
\hline els $\ldots \ldots \ldots$ & W. \& A. R & 663 \\
\hline$\ldots \ldots \ldots$ & t. R. R & 245 \\
\hline s.............. & . \& A. R. & 965 \\
\hline rson Barracks. .................. & $\cdots$ & 1,078 \\
\hline 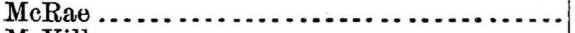 & 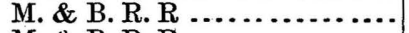 & 148 \\
\hline 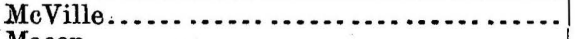 & $\cdots \ldots \ldots$ & 148 \\
\hline$\ldots \ldots \ldots \ldots \ldots$ & $\ldots \ldots$ & 334 \\
\hline - $\ldots \ldots \ldots \ldots$ & $\mathrm{G}$ & 681 \\
\hline Ma & M. \&.N & 1,133 \\
\hline ulsville..... & D. W. R. R & 493 \\
\hline …................ & $\ldots \ldots . . . . .$. & 917 \\
\hline 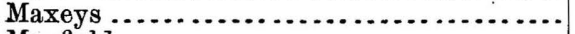 & $\because \ldots \ldots \ldots$ & 742 \\
\hline . & & 402 \\
\hline 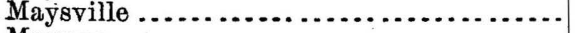 & N.E. R. R & 1,001 \\
\hline$\ldots$ & $\ldots .$. & 559 \\
\hline .............. & - & 310 \\
\hline$\ldots \ldots \ldots \ldots$ & $\ldots$. & 158 \\
\hline$\ldots \ldots$ & B. & 130 \\
\hline$\ldots \ldots$ & & 863 \\
\hline (n) & & 5,039 \\
\hline 然 & & 244 \\
\hline$\ldots, \ldots, \ldots, \ldots$, & L. R. R . . . . . . . . . & 1,587 \\
\hline reek Bald Mountain ... . . . . . . . . . . . . & $\ldots \ldots \ldots \ldots \ldots$ & 4,705 \\
\hline ................ & & 537 \\
\hline Holliand....... & A. & 1,182 \\
\hline & R. R :.. & 959 \\
\hline ............... & N.1 & 89 \\
\hline Bridge & & \\
\hline y Valley.... & M. \& N. G. R. R ... & 949 \\
\hline & & 1,078 \\
\hline od. & Ga. R. R .............. & 602 \\
\hline onee $\ldots$ & Ga. R.R... & 447 \\
\hline Do.level low water in Oconee River ... & Ga. R. R ... & 412 \\
\hline 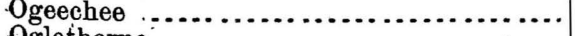 & C. R. R $\ldots \ldots \ldots \ldots \ldots \ldots$ & 106 \\
\hline$\ldots$ & S. W. R. R. & 301 \\
\hline & & \\
\hline
\end{tabular}




\begin{tabular}{|c|c|c|}
\hline & Authority. ' & Elevation. \\
\hline & & Feet. \\
\hline $\begin{array}{l}\text { Palmetto........ } \\
\text { Paramore Hill. }\end{array}$ & A. \& W.P. R. & \\
\hline & 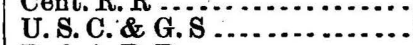 & 1,068 \\
\hline earson. & B. \& A.R.R & 175 \\
\hline end & M. \& & \\
\hline ine Log & U.S. C.\& G.S & 2,340 \\
\hline ine Mou & C. \& R.R.R & 1,052 \\
\hline 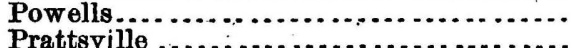 & A.\&W.P.R.R & 987 \\
\hline $\begin{array}{l}\text { Prantsville ..... } \\
\text { Pirors......... }\end{array}$ & S.R.\& D.R. & $\begin{array}{l}625 \\
819\end{array}$ \\
\hline & A.\&W.P. & 939 \\
\hline Rabunor, Mud & U. S. C. \& & 4,717 \\
\hline & A.\&W.P. & 1,025 \\
\hline eid & M. \& & 281 \\
\hline Resaca. & W.\&A.R. & 654 \\
\hline & & 619 \\
\hline idg & U.S. & 1,070 \\
\hline$\ldots \ldots$ & W.\& A.R. R & 785 \\
\hline aws & Ala. & 797 \\
\hline side .. & B. \& & 255 \\
\hline Rive & S. R.\& D. & 633 \\
\hline & & 542 \\
\hline ogers.. & W.\&.A.R. & 734 \\
\hline & S. R. \& D.R. & 627 \\
\hline and Ready & & 1,004 \\
\hline Roystonis............. & Elbes & 909 \\
\hline Ruffe & W. & $.1,080$ \\
\hline ge $\ldots . .$. . & & 725 \\
\hline and Hill ......... & M. & 58 \\
\hline$\ldots \ldots \ldots$ & & 159 \\
\hline Savannah, Depot & R.... & 32 \\
\hline Do..... Signa & U. S. Signal Offic & 87 \\
\hline wdust .......... & Ga. R.R.......... & 536 \\
\hline$\because \cdots$ & U.S. C. \& G. S . & 1,967 \\
\hline gh . & $\mathbf{R} \ldots$ & $\begin{array}{l}148 \\
.362\end{array}$ \\
\hline Seba & R... & 190 \\
\hline Cut $\ldots$ & R. R. & 1,166 \\
\hline ull Mount & & 5,04 \\
\hline . & S. 1 & 684 \\
\hline & & 2,076 \\
\hline ille ..... & S. W & 33 \\
\hline (irele & W. \& $A_{1} R . R$. & 1,069 \\
\hline rcle & $\begin{array}{ll}\text { Ga. R. R } \\
\text { Ga. R. R }\end{array}$ & 875 \\
\hline Mountain... & R. R & $\begin{array}{r}1,040 \\
567\end{array}$ \\
\hline & R. R .... & 23 \\
\hline .......... & A. R.R. & 84 \\
\hline ter & . R.R... & \\
\hline & $\&$ G. S & 1,686 \\
\hline$\cdots$ & \& G.S. & 1,037 \\
\hline x Spring & A.\& R.A.L.R. F & 1,252 \\
\hline$\cdots$ & $\begin{array}{l}\text { Ala.G.S.R.R. } \\
\text { B.\& A.R.R... }\end{array}$ & $\begin{array}{l}907 \\
340\end{array}$ \\
\hline $\operatorname{lrr}$ & R.R... & $\begin{array}{l}340 \\
192\end{array}$ \\
\hline & & 1,027 \\
\hline Sweat Mountain . ............. & U.S.C.\& G.S. & 1,694 \\
\hline Tallulah, S. E. Summit. $\ldots \ldots \ldots \ldots \ldots$ & 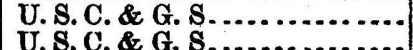 & 2,849 \\
\hline 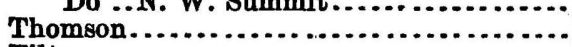 & 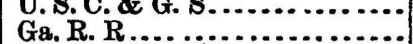 & $3, \frac{177}{517}$ \\
\hline & A. R.R. R & , 665 \\
\hline & on R.R......... & 1,040 \\
\hline $\begin{array}{l}\text { onah Mc } \\
\text { ooni... }\end{array}$ & $\begin{array}{l}\text { U.S.C.\& G. S. } \\
\text { M.\& N:G.R. R...... }\end{array}$ & $\begin{array}{l}3,168 \\
1,035\end{array}$ \\
\hline
\end{tabular}




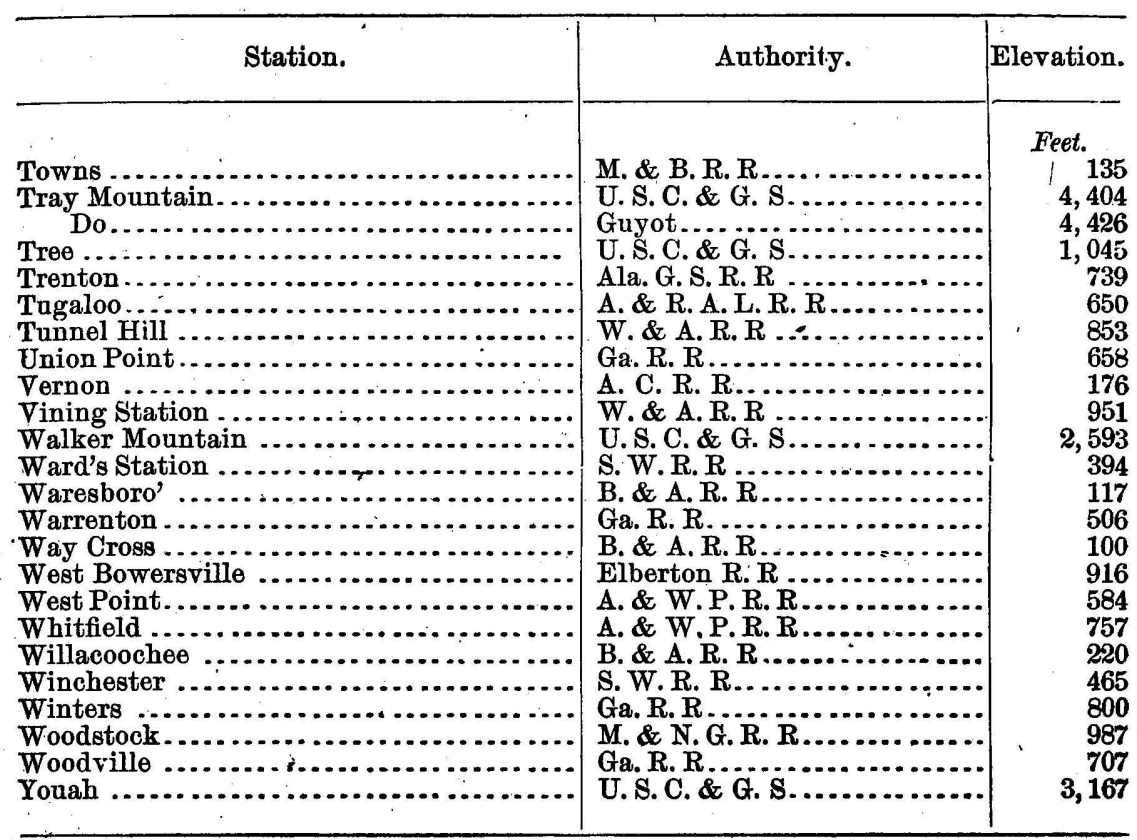


IDAHO.

\begin{tabular}{|c|c|c|}
\hline Station. & Authority. & Elevation. \\
\hline & & \\
\hline Algoma .... & N.P.R.R .. & 2,214 \\
\hline merican Falls...... & Wheeler.... & $4,45 \%$ \\
\hline nderson's ............. & Wheeler.. & $\begin{array}{l}4,492 \\
7,282\end{array}$ \\
\hline $\begin{array}{l}\text { ntelope Peak } \ldots \ldots \\
\text { riomo } \ldots . . . . . . . .\end{array}$ & $\begin{array}{l}\text { Wheeler.... } \\
\text { U.\& N.R.R }\end{array}$ & $\begin{array}{l}7,282 \\
4,659\end{array}$ \\
\hline dger Peak .......... & Wheeler.... & 6,389 \\
\hline ock Peak .... & Wheeler.. & 8,359 \\
\hline Creek ...... & U. \& N. R. & 4,500 \\
\hline $\operatorname{ar} \mathrm{La}$ & Wheeler. & 5,960 \\
\hline iver Bridge. & Hayden . & 5,744 \\
\hline ñon Stage & Pre. R. R. & 5,925 \\
\hline Beer Springs ....... & Hayden.. & 5,529 \\
\hline & Land & 5,850 \\
\hline Bennington & Hayd & 5,708 \\
\hline Do... & Whe & 6,132 \\
\hline Big But & Whee & 7,659 \\
\hline & U.\& N.R. & 4,515 \\
\hline Fork, $\mathrm{B}_{\mathrm{I}}$ & $\mathrm{Ha}$ & 4,456 \\
\hline alk. ... & & 7,490 \\
\hline Stago St: & Whe & 4,447 \\
\hline Iounta & Wh & 9,386 \\
\hline Black Rock Stage S & Whe & 4,589 \\
\hline Bloomington...... & Wheeler.. & 6,076 \\
\hline & Hay & 5,985 \\
\hline Bloomi & & 9,354 \\
\hline Signal s & U. S. Signa & 2,768 \\
\hline ( & $\ldots \ldots \ldots$ & \\
\hline$\ldots \ldots$ & Wheeler. & 10,451 \\
\hline k Ranch & Hayden . & 4,722 \\
\hline tain. & & 9,854 \\
\hline Station. & Ha & 4,666 \\
\hline $\mathrm{ck} \ldots \ldots \ldots$ & Wheeler. & 9,610 \\
\hline & & 7,587 \\
\hline & N.P.R.R & 2,450 \\
\hline Cit & King ..... & 6,700 \\
\hline & & 4,853 \\
\hline Clift & Whe & 4,926 \\
\hline & Hay & 4,893 \\
\hline Coeur d'Al6ne Lake ..... & Mullan... & $2,150_{4}$ \\
\hline 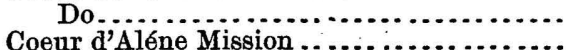 & Pacific R. & 2,240 \\
\hline Crater Buttes, mouth Henry's Fork .. & $\begin{array}{ll}\text { Pacih } \\
\text { Hayd }\end{array}$ & E. 5,638 \\
\hline & Haydeu - & W. 5,425 \\
\hline & & \\
\hline s ... & Haýden.. & 4,816 \\
\hline D & Whe & 3,601 \\
\hline iation .. & Hay & 5,689 \\
\hline $\mathbf{E}$ & Mull & 7,500 \\
\hline$k \ldots \ldots \ldots \ldots$ & U. $\& \mathrm{~N} . R . R$ & 4,723 \\
\hline & U. S. Signal Office...... & \\
\hline le Mountain .. & Hayden ................. & 9,332 \\
\hline Elkhorn Mail Station .............. & Wheeler...................... & 4,998 \\
\hline 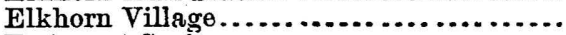 & Wheeler..................... & 4,950 \\
\hline Emigrant Springs.$\ldots \ldots \ldots \ldots \ldots \ldots \ldots \ldots \ldots$ & Wheeler...................... & 5,271 \\
\hline 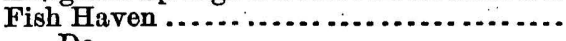 & Wheeler...................... & 5,963 \\
\hline Do $\ldots . . .$. . & Hayden .......... & $\begin{array}{l}5,932 \\
8,000\end{array}$ \\
\hline
\end{tabular}




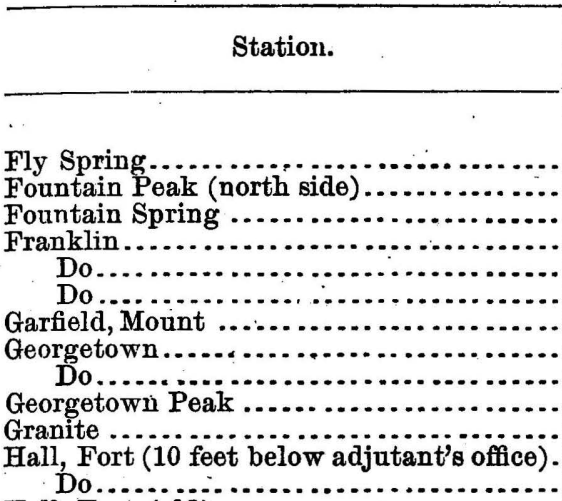

Hall, Fort (old)

Hall

Farkness's Toll Gate

Henry's Lake

Higham's Ranch

Hiteling's Ranch

Junction Station

Kalispelm, Lake

Keenam City -..

Keeney's Stage Station

Lane's Butte.

Lane's Ranch, Marsh Lake

Liberty

Lone Cone

McCrae's Ränch

Malade City

Do.

Malade Divide

Malade Spring

Market Lake.

Do

Marsh Cone

Meade Peak

Montpelier

Montpelier (co-operative store)

Mormon Salt Works

Morristown

Do

Monnd Spring Station ....

Mule Spring, head of Gentile Valley

Newton

Nine Mile...

Oneid

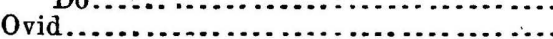

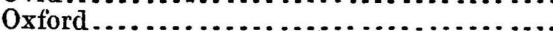

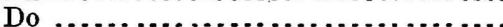

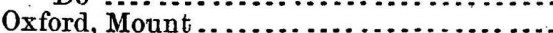

Packer's Bridge .........................

Paris

Paris Peak

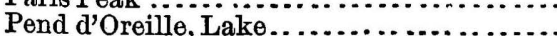
Do

Pillar Butte

.....................

Pleasant Valley Station

\begin{tabular}{|r|r}
\hline Authority. & Elevation. \\
\hline Feet.
\end{tabular}

Wheeler.

5,912

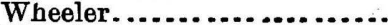

8,900

Wheeler

U. \& N. R. R

Wheeler

Hayden

Hayden

Wheeler

Hayden

Hayden

N.P. R. R

Wheeler

Hayden

De Lacy

Wheeler

Hayden

Hayden

Hayden

Toner

Wheeler

Hayden

Wheeler

Wheeler

Hayden

Wheeler

Wheeler

Hayden

Wheeler

Hayden

Wheeler.

TT. \& N. R. R

Hayden

Hiayden

Wheeler.

Hayden

Wheeler

Wheeler.

Wheeler

Hayden

Wheeler

Hayden

U. \& N. R. R

Hayden

Hayden

Hayden

Wheeler.

U. \& N. R. R

Hayden

Wheeler

Wheeler.

Hayden

Wheeler

Hayden

Petermann

N. P.R. R

Wheeler.

Wheeler.

Hayden
5, 235

4,513

4,585

4,516

9,704

6,019

5,800

8,466

2,290

4,752

4,754

4,500

4,996

4,802

6,443

4,601

5,205

6,329

2,093

6,641

4,933

7,823

4, 323

6,060

6,105

9,246

4,331

4,700

4,663

5,690

5,150

4, 792

4, 795

7, 663

10,541

5,793

5,936

6,598

5,716

5,700

4, 429

5,245

4, 500

4,756

9,413

5,700

6,341

6,300

6,022

4, 774

4, 862

4,866

9,386

4,500

6,018

9,522

2, 093

2,059

5,301

9,695

6, 086 


\begin{tabular}{|c|c|c|}
\hline & & Elevation. \\
\hline & & Feet. \\
\hline $\begin{array}{r}\text { Pocatello ........... } \\
\text { Do.... Stage Sta }\end{array}$ & $\begin{array}{l}\text { U. \& N. R. R.. } \\
\text { Hayden ....... }\end{array}$ & $\begin{array}{l}4,495 \\
4,512\end{array}$ \\
\hline Do..... Stage Sta & Whyoeler.. & $\begin{array}{l}4,620 \\
4,620\end{array}$ \\
\hline $\begin{array}{l}\text { Portage......... } \\
\text { Port Nenf... }\end{array}$ & $\begin{array}{l}\text { Wheeler... } \\
\text { U. \& N. R. }\end{array}$ & $\begin{array}{l}4,700 \\
4,514\end{array}$ \\
\hline Prescott ...... & $\begin{array}{l}\text { U. } \\
\text { N.P.R.R.. }\end{array}$ & $\begin{array}{l}4,514 \\
2,210\end{array}$ \\
\hline reuss, Mount & Hayden .. & 9,979 \\
\hline $\begin{array}{l}\text { Putnam, Mount } \\
\text { Do............. }\end{array}$ & $\begin{array}{l}\text { Wheeler.. } \\
\text { Hayden .. }\end{array}$ & $\begin{array}{l}8,905 \\
8,933\end{array}$ \\
\hline Raft River Rang & King ..... & 7,000 \\
\hline Raft R & eler... & $\begin{array}{l}5,041 \\
4,792\end{array}$ \\
\hline $\begin{array}{l}\cdots \\
\cdots\end{array}$ & $\begin{array}{l}\text { Hayden }(r \varepsilon \\
\text { Hayden } . .\end{array}$ & $\begin{array}{l}4,792 \\
4,792\end{array}$ \\
\hline rry, s & Wheeler.. & 4,192 \\
\hline $\mathrm{ch}$. & Wheeler. & 4,723 \\
\hline 's & Wheeler. & 5,268 \\
\hline Richmo & Wheeler. & 4,656 \\
\hline Ross Fork Ind & Hayden .. & 4,394 \\
\hline Doss Fork Stat & U. \& N. $\mathrm{R}$ & 4,561 \\
\hline rles. & Hayden... & 5,932 \\
\hline rge.... & Hayden ... & 5,771 \\
\hline $\begin{array}{l}\text { Salmon City .. } \\
\text { Samaria }\end{array}$ & $\begin{array}{l}\text { De Lacy.. } \\
\text { Wheeler.. }\end{array}$ & $\begin{array}{l}4,030 \\
4,561\end{array}$ \\
\hline & Hayden... & 4,800 \\
\hline landpo & N.P. P. R. R. & 2,100 \\
\hline Peak & $\begin{array}{l}\text { U. \& } \\
\text { Hayd }\end{array}$ & $\begin{array}{r}4,723 \\
10,013\end{array}$ \\
\hline Peak. & Wheeler.. & 9,207 \\
\hline ake.. & Whee & 4,310 \\
\hline Mount.. & Wheeler $\ldots . . .$. & 9,572 \\
\hline Shoshone .... & U.\& N.R.R... & 4,587 \\
\hline ntain. & Hayden. & $\begin{array}{r}-4,500 \\
9,269\end{array}$ \\
\hline Soda Peak ....... & Hayden ... & 9,683 \\
\hline Soda Springs...... & Hayden ... & 5,779 \\
\hline Do. & Wheeler..... & 5,778 \\
\hline $\begin{array}{l}\text { Sohon Pass....... } \\
\text { Stahn's Ranch... }\end{array}$ & $\begin{array}{l}\text { Mullan ....... } \\
\text { Wheeler..... }\end{array}$ & $\begin{array}{l}5,100 \\
4,403\end{array}$ \\
\hline & Wheeler....... & 6,057 \\
\hline Mount............... & Petermann & 7,400 \\
\hline $\begin{array}{l}\text { venson's Junction, Lemhi Valley .. } \\
\text { ner's Station ........................ }\end{array}$ & De Lacy...... & $\begin{array}{l}6,084 \\
4,621\end{array}$ \\
\hline 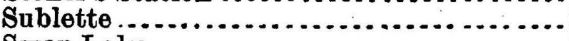 & Wheeler....... & 5,155 \\
\hline (n) & U.\& N. R. R.... & 4,794 \\
\hline nch ... & Wheeler.......... & 4,509 \\
\hline idge... & $\begin{array}{l}\text { Hayden ...... } \\
\text { Wheeler }\end{array}$ & $\begin{array}{l}4,627 \\
5,548\end{array}$ \\
\hline oponce Ranch & er...................... & 5,072 \\
\hline West Malade, Mount........................ & Hayden ....................... & 9,220 \\
\hline 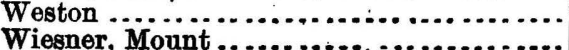 & 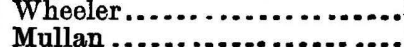 & $\begin{array}{l}5,011 \\
6,000\end{array}$ \\
\hline & & \\
\hline
\end{tabular}




\section{ILLINOIS.}

\begin{tabular}{|c|c|c|}
\hline Station. & Authority. & Elevation. \\
\hline & & Feet. \\
\hline Addieville ....... & St. L. \& S. E. I & 467 \\
\hline Allendale & C. \& V. R. R. . & 464 \\
\hline Altamont (crs'ng) St. L., V., T. & O.\&.M.R.R & 616 \\
\hline Alton...$\ldots \ldots \ldots$ & C. \& A. R. R. . & 470 \\
\hline Amboy ........... & Ill. Cent. R. R & 733 \\
\hline America ....... & C. \& V.R.R... & 344 \\
\hline Antioch & I., D.\& S. R.R . & 661 \\
\hline Antioch (geodetic station) & U. S. Lake Surve & 861 \\
\hline Arcola ................ & Ill. Cent. R. R... & 674 \\
\hline Ashby.......... & St. L.\& S. E.R. & 554 \\
\hline Ash Grove (geodetic statio & U. S. Lake Surv & 666 \\
\hline Ashkum ............ & Ill. Cent. R. R. & 600 \\
\hline Ashland ....... & O.\& M.R.R. & 625 \\
\hline Ashley........ & Ill. Cent. R: R. & 549 \\
\hline Atlanta ............. & C. \& A. R. R.. & 744 \\
\hline Atwater (or Zanesville)... & J.S.E. R. R ... & 652 \\
\hline Atwood ............. & I., D.\& S. R. R & 669 \\
\hline $\operatorname{arn} . . . . . . .$. & C. \& A.R.R... & 666 \\
\hline Aurora......... & C., B. \& Q. R.R. & 649 \\
\hline & St. L. \& S. E. R. & 536 \\
\hline Baland ( $3 \frac{1}{2}$ miles east of Omaha) .. & O.\& M.R.R .. & 458 \\
\hline Bardstown, (crossing R., R. I. \& St. & O.\& M. R. R & 435 \\
\hline Barnett...................... & J.S. E.R.R R .. & 672 \\
\hline Barnhill (valley of Watson's Creek) & O.\&M.R.R. & 385 \\
\hline Barry $\ldots \ldots \ldots \ldots \ldots$ & H. \& N. R. R. & 670 \\
\hline & O. \& M.R.R. & 386 \\
\hline & R.R. & 869 \\
\hline Beardstown (surface of Illinois & Mich. \& Ill. Can & 423 \\
\hline$p$ & \& S. E. R. & 538 \\
\hline & $0 . \&$ & 610 \\
\hline ir (geodetic station). & U. S. Lake Surve & 588 \\
\hline le $\ldots \ldots \ldots . . . .$. & B. \& & 479 \\
\hline & B. \& E. R. R. & 485 \\
\hline (geodetic station).... & U. S. Lake Sur & 595 \\
\hline & P., D. \& E. R. R & 665 \\
\hline tesnake...:.... & C. \& St. L.R.R. & 405 \\
\hline 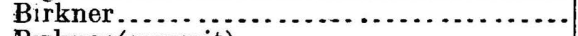 & St. L. \& S. E. R. R & 499 \\
\hline & & 568 \\
\hline (surface ground).. & St. L. \& S. E. R. R & 583 \\
\hline neville.............. & T., P.\&W. R. R . & 730 \\
\hline & & 688 \\
\hline ield (summit).......... & C. \& V.R.R. & 405 \\
\hline (surface ground) & R.R.. & 430 \\
\hline on ........ & & 823 \\
\hline Blu & St. L., V. \& T. H. R. R . & 505 \\
\hline & C. \& A. R. R........... & 603 \\
\hline & O.\&M.R.R...... & 581 \\
\hline & T., P.\&W.R. R .. & 491 \\
\hline $\mathrm{n} \ldots . . . .$. & C. \& A.R. R...... & 694 \\
\hline well ...... & C. \& A. R. R.... & \\
\hline Bud & Q. R.R... & 768 \\
\hline anction & P.K.\& P.R. R... & 455 \\
\hline & W. R.R = & 638 \\
\hline & Q.R.R . & 664 \\
\hline ; Geodetic Station..... & U. S. Lake Survey & 787 \\
\hline & C. \& St. L. R. R . & 425 \\
\hline Cairo $\ldots \ldots \ldots \ldots \ldots \ldots \ldots \ldots \ldots \ldots$ & Ill. Cent. R. R... & 322 \\
\hline Do.Ohio River, high water ....... & C. \& V.R.R ...... & 320 \\
\hline
\end{tabular}




\begin{tabular}{|c|c|c|}
\hline Stat & Authority. & Elevation. \\
\hline $\begin{array}{l}\text { Cairo Ohio River, low water................. } \\
\text { Do....railroad track on Commercial }\end{array}$ & & $\begin{array}{l}\text { Feet. } \\
272\end{array}$ \\
\hline Do..... Signal Station ....... & U. S. Signal Of & 312 \\
\hline aledonia.................... & C. \& V.R.R.. & \\
\hline 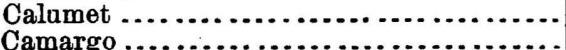 & Ill. Cent. R. R. & 591 \\
\hline Camargo ............... & $\begin{array}{l}\text { I.D.\& S.R.R } \\
\text { P.\& R. I. R.R }\end{array}$ & 638 \\
\hline Point (T. W. \& & C. B.\&.Q.R.R & \\
\hline$\ldots \ldots$ & T.P.\& W.R.R & 656 . \\
\hline le & Ill. Cent. R. R.. & 394 \\
\hline Carlyle, station ledge un & A. R. R... & \\
\hline $\begin{array}{l}\text { face of C. H. B. M. on } \\
\text { Carlple }\end{array}$ & U.S.C.\& G.S . & 47 \\
\hline arlyle $\ldots \ldots \ldots \ldots \ldots$ & O. \& M. R.R... & 450 \\
\hline (n...... & St. L. \& S. E. R. R & 401 \\
\hline …...... & St. L., V.\&'T. H. R. R. & $\begin{array}{l}680 \\
649\end{array}$ \\
\hline teodetic Station & U. S. Lake Survey....... & 631 \\
\hline$\cdots \cdot \cdot$ & St. L., V. \& T. H & 432 \\
\hline 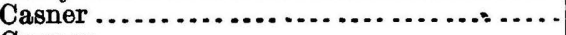 & I. D. \& S. R. R & 604 \\
\hline ............ & C. \& A. R. R... & 703 \\
\hline e............... & t. L. R. 1 & \\
\hline$\ldots \ldots \ldots \ldots$ & $\mathrm{R}$. & 494 \\
\hline gn $\ldots . . . \ldots \ldots . . . . .$. & Ill. ( & $73 \%$ \\
\hline .... Signal Station. & U. S. Signal Office . & 768 \\
\hline $\operatorname{th} \ldots \ldots \ldots$ & A. R. R ......... & 642 \\
\hline & $\begin{array}{l}\text { W.R.R... } \\
\text { W.R.R... }\end{array}$ & $\begin{array}{l}732 \\
724\end{array}$ \\
\hline oint........... & I. D. \& S. R. R.... & 649 \\
\hline , city base.......... & U. S. Lake Survey.. & 580 \\
\hline Do...... \& N. W. R. R. Station. & City Engineer ...... & \\
\hline I. \& P.R. R. Station............... & City Engineer & 593 \\
\hline 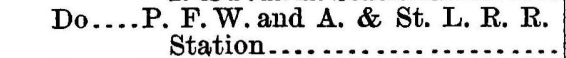 & g- & \\
\hline Do..... mll. Cen & Gilly sugineor .. & \\
\hline $\begin{array}{l}\text { Do.....Signal Station. } \\
\text { hrismau ................. }\end{array}$ & U. S. & 661 \\
\hline 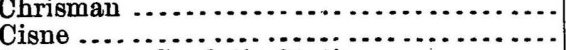 & I. D. & 644 \\
\hline it, Geodetic Statio & $\begin{array}{l}\text { U. \& } \\
\text { U. S. }\end{array}$ & 460 \\
\hline , Geouode nuavio & Ili. & 646 \\
\hline godetic S & U. & 662 \\
\hline lint & & 727 \\
\hline & P. D. & 666 \\
\hline Collin & St. L. & \\
\hline ank road. & H. R. R. & 417 \\
\hline atio & & 542 \\
\hline eodetic Sta & $\mathrm{U}$. & 791 \\
\hline (n) & St. \& \& $R . R$ & 674 \\
\hline netion ........... & & $\begin{array}{l}641 \\
363: \\
-\end{array}$ \\
\hline & & $\begin{array}{l}363 \\
340\end{array}$ \\
\hline & St. L & 508 \\
\hline & C. $\&$ & 604 \\
\hline $\operatorname{anv}$ & P. R. R. & 608 \\
\hline unction ......... & & \\
\hline ............... & Ill. & 666 \\
\hline (5) & St. I & \\
\hline Geodetic Station. & $\underset{T}{\mathrm{U}} \cdot \mathrm{S}$ & 544 \\
\hline & Iil. $\mathrm{C}$ & 718 \\
\hline & $\mathrm{BR}$ & \\
\hline
\end{tabular}




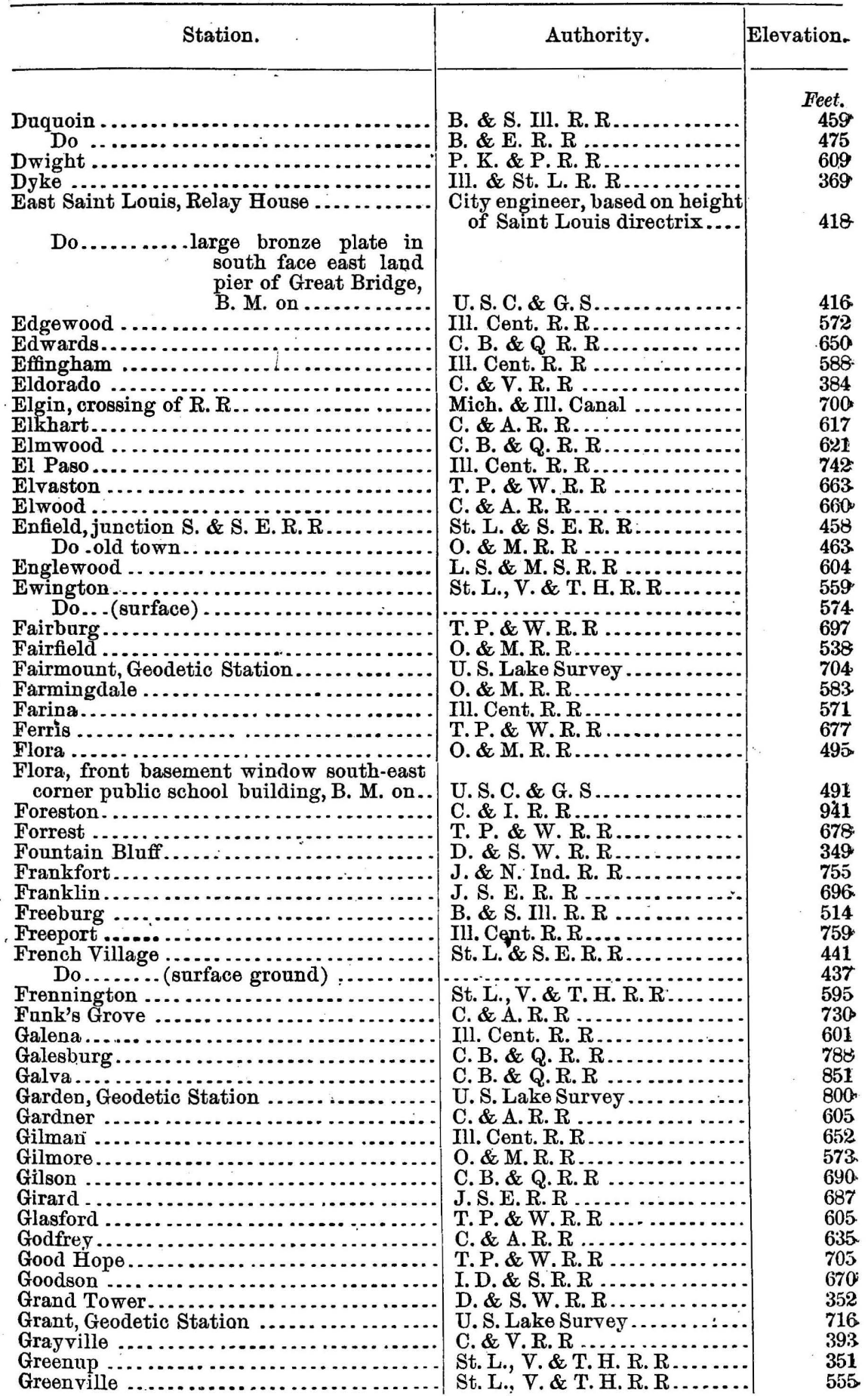




\begin{tabular}{|c|c|c|}
\hline Station & Authority. & Elevation. \\
\hline & & Feet \\
\hline Gridley ......... & T. P.\& W. R. & 758 \\
\hline $\begin{array}{l}\text { Griggsville } \\
\text { Groveland Park . }\end{array}$ & $\begin{array}{l}\text { H. \& N.R.R. } \\
\text { Ill. \& St. L. R. R }\end{array}$ & $\begin{array}{l}685 \\
523\end{array}$ \\
\hline Guerpert's Pass .. & C. \& St. L.R.R. & 472 \\
\hline Hadley ..... & H. \& N.R.R.. & \\
\hline Hammond. & I. D.\& S.R.R & 672 \\
\hline $\begin{array}{l}\text { Hampton .. } \\
\text { Harmony .. }\end{array}$ & $\begin{array}{l}\text { P.D.\& E. R. R } \\
\text { III.\& S. L. R. R }\end{array}$ & $\begin{array}{l}665 \\
563\end{array}$ \\
\hline $\begin{array}{l}\text { Harmony ........ } \\
\text { Harrisburg .... }\end{array}$ & $\begin{array}{l}\text { III. \& S. L.R.R } \\
\text { C. \&V.R.R... }\end{array}$ & $\begin{array}{l}503 \\
367\end{array}$ \\
\hline Havana........ & P.P.\& J.R.R. & 472 \\
\hline Haw & St. L. \& S. E. R & 395 \\
\hline Henn & P. K. \& P.R.R. & 394 \\
\hline $\begin{array}{l}\text { Hervey City } \ldots . . \\
\text { Highland ........ }\end{array}$ & $\begin{array}{l}\text { P.D.\&E.R.R. } \\
\text { St.L. V.\& T. F }\end{array}$ & $\begin{array}{l}707 \\
527\end{array}$ \\
\hline Do... (surfa & St. L., V. \& T. & $\begin{array}{l}527 \\
543\end{array}$ \\
\hline inck & C. \&I. R.R. & 746 \\
\hline Holl? & M.R.R & \\
\hline Holli & W.R.I & 451 \\
\hline & C. \& E. Ill. R. R & 718 \\
\hline $\begin{array}{l}\text { d's Point } \ldots . . . . ~ \\
\text { Crossing Q. A. \& }\end{array}$ & St. L., V.\& T. H. R & $\begin{array}{l}612 \\
468\end{array}$ \\
\hline ing Q. A. \& & $\begin{array}{l}\text { H.\& N.R.R... } \\
\text { I.D.\&S.R.R... }\end{array}$ & $\begin{array}{l}468 \\
649\end{array}$ \\
\hline Hur & ake Snrve & 551 \\
\hline Indi & $0 . \&$ & 393 \\
\hline Indian Lake, water & St. L., V.\& I & 402 \\
\hline Do...... railroad sta & St. L., V.\& T. H. & 416 \\
\hline Iowa. & T.P.\& W. R, F & 546 \\
\hline ville & . R. R.. & 619 \\
\hline Mes Mines & C. & 541 \\
\hline Des Moines & & 543 \\
\hline Jones & & 631 \\
\hline Kank & & 626 \\
\hline $\mathrm{D}$ & & 642 \\
\hline Kans: & & 839 \\
\hline & C. $\mathrm{E}$ & 543 \\
\hline Junction. & Q. R. & $56 \%$ \\
\hline & & 596 \\
\hline & & 478 \\
\hline (B. \& O.R. R. shops) & & 588 \\
\hline 8....................... & Q. R.R & 777 \\
\hline & W. R. . . . . . & \\
\hline & St. L., V.\& T. H.R.R . & 466 \\
\hline & O.\&M.R.R........ & 618 \\
\hline & & 702 \\
\hline $\mathrm{rg}$. & & 519 \\
\hline La S & & 510 \\
\hline face of & nal & 439 \\
\hline enceville & D.\& S.W.R.R... & 424 \\
\hline & Ill. \& St. L. R. R ........ & 539 \\
\hline ill of bas & & \\
\hline & $\cdots \cdots+\ldots$ & $\begin{array}{l}460 \\
441\end{array}$ \\
\hline & R.R & $\begin{array}{l}441 \\
959\end{array}$ \\
\hline$\ldots$ & T.P.\&W.R.R... & 499 \\
\hline & C. \& A & 751 \\
\hline & & 613 \\
\hline Geld ...... & J.S. & 464 \\
\hline & Ill. Cent. R. R & 777 \\
\hline Creek ... & I. D. \& S. R.R.. & \\
\hline ille.......... & $\begin{array}{l}\text { P. K. \& P.R. R } \\
\text { O.\& M.R.R. }\end{array}$ & $\begin{array}{l}668 \\
480\end{array}$ \\
\hline & 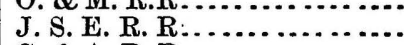 & 712 \\
\hline & C. \& A.R. R....... & 625 \\
\hline Ludlow & Ill. Cent. R. R ................. & 767 \\
\hline
\end{tabular}




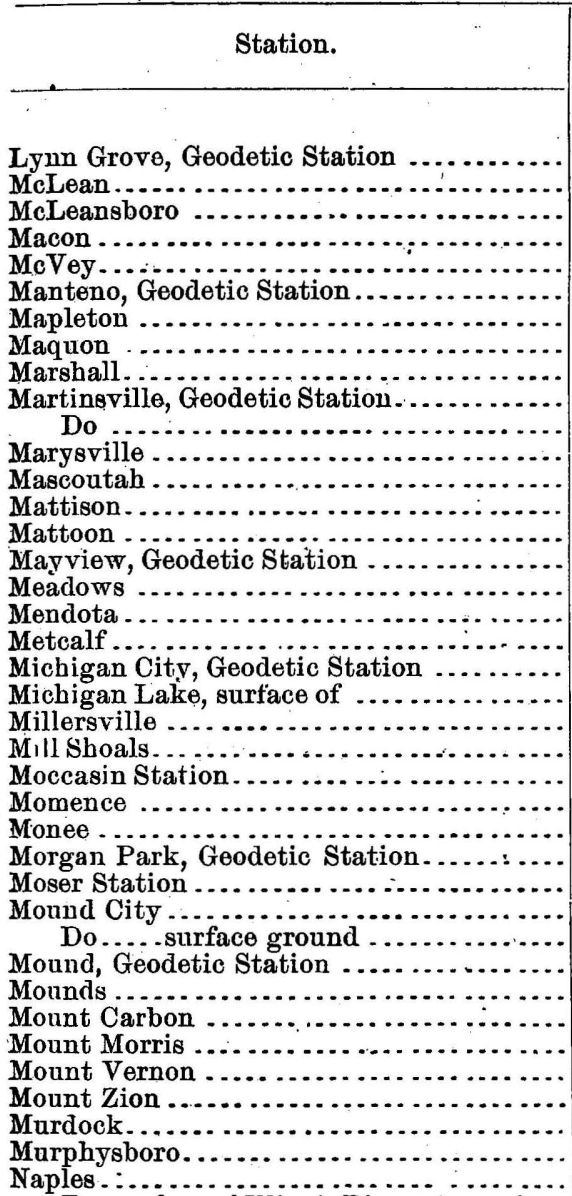

Do.surface of Illinois River at crossing of railroad.

U. S. Lake Survey

C. \& A. R. R

St. L. \& S. E. R. R

Ill. Cent. $R$. $R$

J.S. E. R. R

U.S. Lake Survey

T. P. \& W. R. R

C., B. \& Q. R. R

D. \& S W R R

U. S. Lake Survey .................... $\quad 678$

St. L., V. \& T. H. R. R ......

H. \& N. R. R............

St. L. \& S. E. R. R .........

Ill. Cent. R. R............... 699

Ill. Cent. R. R............... 733

U. S. Lake Survey .................

T. P. \& W. R. R.......... $\quad 764$

Ill. Cent. R. R............ 749

I. D. \& S. R. R............. 618

U.S. Lake Survey............. $\quad \mathbf{7 3 0}$

P., Ft. W. \& C. R. R ........ $\quad 582$

O. \& M. R. R................. $\quad 642$

O. \& M. R. R.............. 344

O. \& M. R. R............... 606

P. K. \& P. R. R ........... 625

Ili. Cent. R. R.............

U. S. Lake Survey ............... $\quad 666$

St. L. \& S. E. R. R.........

C. \& V. R. R ............ , 323

318

U. S. Lakè Survey ............. . . 522

Ill. Cent. R. R ............. $\quad 346$

D. \& S. W. R. R ........... 372

C. \& 1. R. R.............. $\quad 906$

St. L. \& S. E. R. R.......... 506

P. D. \& E. R. R............ 694

I. D. \& S. R. R............. 639

C. \& St. L. R. R ...........

H. \& N. R. R ........... 438

Mich. \& Ill. Canal. ........... 418

St. L. \& S. E. R. R...............

P. D. \& E. R. R............. 657

Nashville .............................

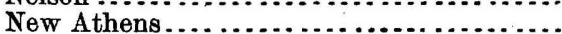

New Boston

Newman .

New Memphis

New Philadelphia

Now Salem.

Do... crossing C. \& V. R. $R$

Oak Hill: .........

Oakland, Geodetic Station.

Oakwood

Oblong, Geodetic Station

Ocoya

Odell

Odin Station, coping-stone east ond of a long, arched culvert, B. M. on ............ Odin

O'Fallon

Okawville

Olney, base of one of the columns of north face of C. H., B. M. on

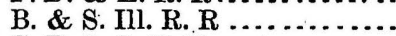

C. B. \& Q. R. R .............

I. D. \& S. R. R . . . . . . . .

St. L. \& S. E. R. R..........

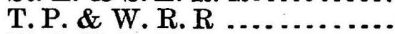

H. \& N. R. R . . . . . . . . . . .

C. \& V.R. R.

O. \& M. R. R ...............

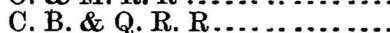

U. S. Lake Survey . ............

Iil. Cent. R. R . .....................

U. S. Lake Survey ...........

C. \& A. R. R.

C. \& A. R. R..................

U. S. C. \& G. S .............

Ill. Cent. R. R . . . . . . .

O. \& M. R. R ...............

St. L. \& S. E. R. R.

404

573

641

411

664

778

.420

424

597

693

585

U. S. C.\& G.S. 


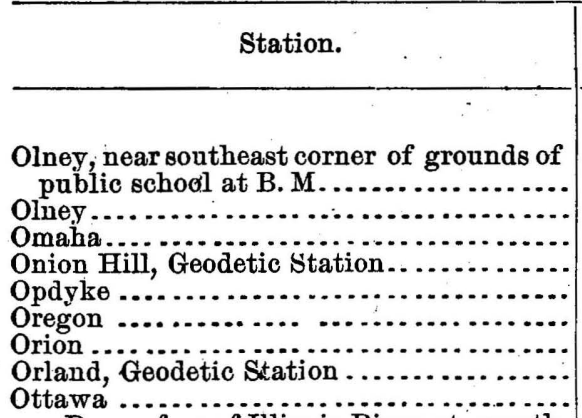

Do.surface of Illinois River at mouth Fox River.....................

Owaneco...............................

Palermo, Geodetic Station.

Pana

Paris

Parkersburg, Geodetic Station.

Paxton, Geodetic Station

Pekin.

Peoria

Do.Adams Street Depot

Do.surface of Illinois River.

Peru, surface of Illinois River

Philadelphia

Philins Ferry

Pilot Grove, Geodetic Station

Pisgah.

Plateau

Pleasant Plains

Pleasantville.

Pocahontas

Polo

Pontiac .

Princeville.

Prospect .

Queen's Lake, railroad track

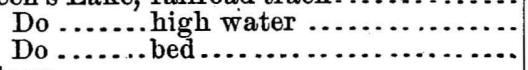

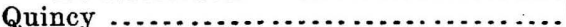

Rantoul, Geodetic Station

Redbud.

Richland.....

Ridge Farm

Ridgway

Rinard .

Roaches..............................

Robinson

Rochelle

Rochester

Rock Island

$$
\text { Do }
$$

Rushville

Sacramento, Bear Creek Valley

Saint Anne, I. C. \& L. R. R ...

Do .... Geodetic Station

(n)...........

Saint Jacobs : . ........................

Salem ................................................. of C. H., B. M. on ...............

Saline City, railroad track .................

Sanburn railroad track.

Sandoval.

\begin{tabular}{|r|r}
\hline Authority. & Elevation. \\
\hline & Ftet.
\end{tabular}

U. S. C. \& G. S .............. 482

O. \& M. R. R .............. 480

O.\& M. R. R ............... 369

U. S. Lake Survey ............ 569

St. L. \& S. E. R. R......... 509

C. \& I. R. R .............. 704

P. \& R. I, R. R............. 751

U. S. Lake Survey ........... $\quad 768$

C. B. \& Q. R. R.............. 486

Mich. \& Ill. Canal............ 448

O.\& M. R. R ............... 616

U. S. Lake Survey ........... $\quad 742$

IIl. Cent. R. R .............. . $\quad 676$

D. \& S. W. R. R ............. 705

U. S. Lake Survey ............ . . $\quad 569$

U. S. Lake Survey ...........

P. P. \& J. R. R ............ $\quad 475$

T. P. \& W. R. R ............. 463

C. R. I. \& P. R. R............. 458

Mich.\& IIl. Canal ............ 433

Mich. \& IIl. Canal........... $\quad 439$

O.\& M. R.R .............. : 616

H. \& N. R. R .............. 439

U. S. Lake Survey ........... $\quad 780$

B. \& S. Ill. R. R ............ 444

J. S.E. R. R.............. 687

St. L., V. \& T. H. R. R ....... 568

O.\& M. R. R ............... 606

St. L., V. \& T. H. R. R ....... $\quad 585$

St. L., V. \& T. H. R. R ...... 498

Ill. Cent. R. R .............. 849

C. \& A. R. R ................ 668

P. \& R. I. R. R ............ $\quad 719$

J.S. E. R. R................ 685

St. L. \& S. E. R. R ........... 408

St. L. \& S. E. R. R........... 401

St. L. \& S. E. R. R........... $\quad 379$

C. B. \& Q. R. R R........... 48 \&

U. S. Lake Survey ........... 821

C. \& St. L. R. R ........... 457

O. \& M. R. R ............. 605

D. \& S. W. R. R ........... 685

O. \& M. R. R .............. $\quad 379$

O.\& M.R.R ............... 457

St. L. \& S. E. R. R .......... 498

D.\& S.W.R.R............ 508

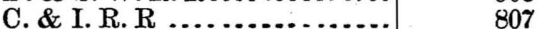

O.\& M. R. R ............... 569

C., R. I. \& P. R. R.......... 584

R.R.I. \& St. L. R. R ..........

C. B. \& Q. R. R ........... 676

O.\& M. R. R ................ 413

C. \& E. Ill. R. R............ 659

U. S. Lake Survey ............. 676

C. \& V. R. R.............. 458

St. L., V. \& T. H. R. R ...... 605

O.\& M. R. R .............. 538

U. S. C. \& G. S .............. 546

C. \& V.R.R R

C. \& V.R. R .............. $\quad 609$

Ill. Cent. R. R............... 494 


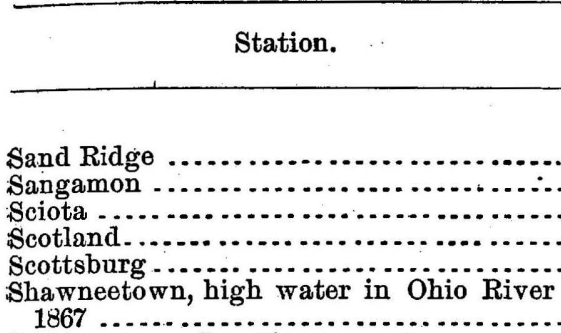

shawneetown Junction.

Sheldon.

sherman

Shipman

shirley

Smithfield ....

South Chicago

$$
\text { Do }
$$

South Chicago Avenue

Sparta.

Spencer.

Spring Creek, Geodetic Station

Springertown

Springfield, O. \& M. \& Illinois Central

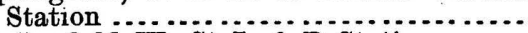
Springfield, W., St. L. \& P. Station...... S. Grand Avenue

springfield, C. A. \&' St. L. depot Do ........................................

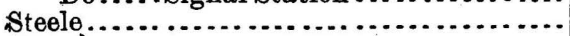

Streatior

Streator............................

Sullivan, W., St. L. \& P. Crossing

Sumner...

Swanwicli

Tenpin

Teutopolis

Thackery

Thorn Thicket

Tilden

Tilton Junction

Tolono.

Toulon

Towanda

Trenton

Troy

Trumbull

Tuscola

Ullin

Union

Unity Do..Crossing, ill Cent. $\mathrm{R}$.

Venedy

Virden.

Virğinia

(n........................... Do.Geodetic Station

Washington.

Waterloo

Watseka

Do.. Geodetic Station

Waveriy

West Chicago...........................

Westfield, Geodetic Station

White's Hill.

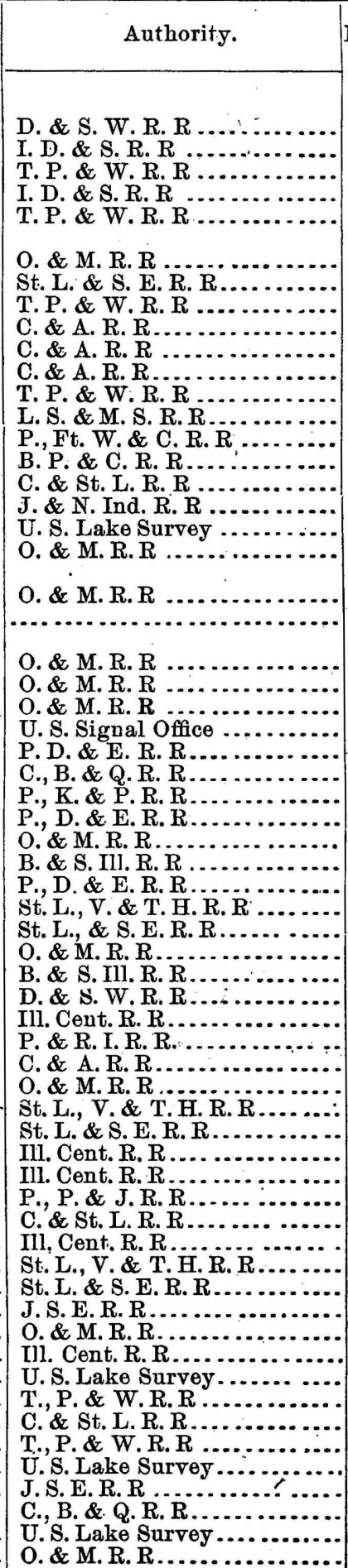

Elevation.

Feet.

351

$63 \%$

748

629

677

363

486

708

609

662

770

645

591

589

587

549

712

666

382

589

594

592

582

545

644

667

620

600

698

457

572

682

605

505

383

523

673

729

723

810

500

539

460

657

338

505

332

500

500

412

691

608

1,005

730

745

664

627

670

691

591

773 
[BULL. 5.

\begin{tabular}{|c|c|c|}
\hline Station. & Authority. & Elevation. \\
\hline 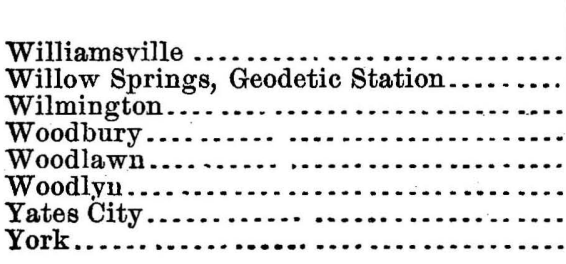 & 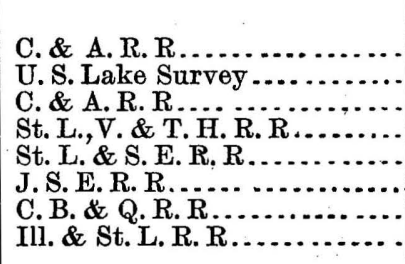 & $\begin{array}{r}\text { Feet. } \\
627 \\
729 \\
561 \\
559 \\
495 \\
701 \\
673 \\
524\end{array}$ \\
\hline
\end{tabular}

(218) 


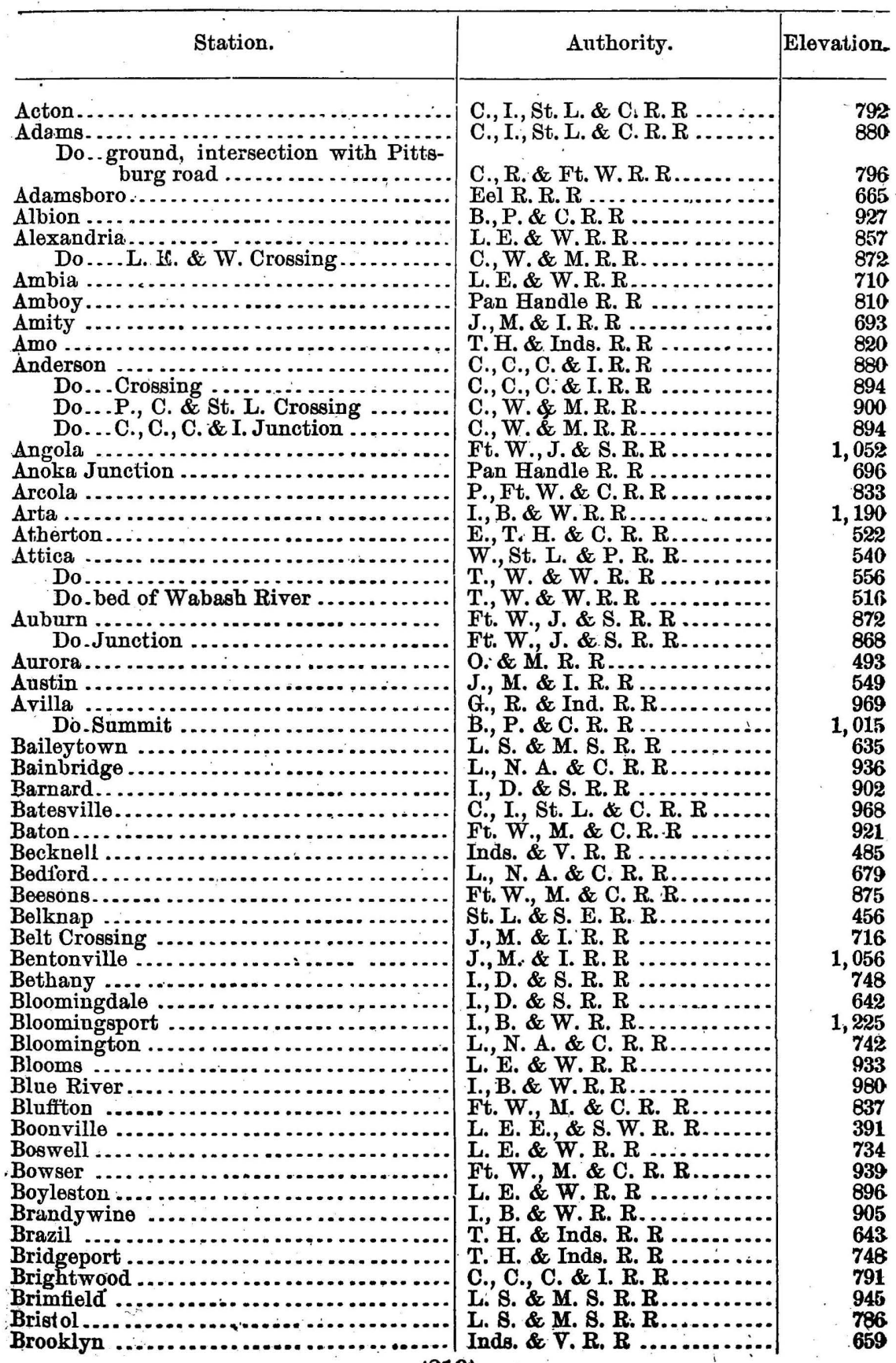




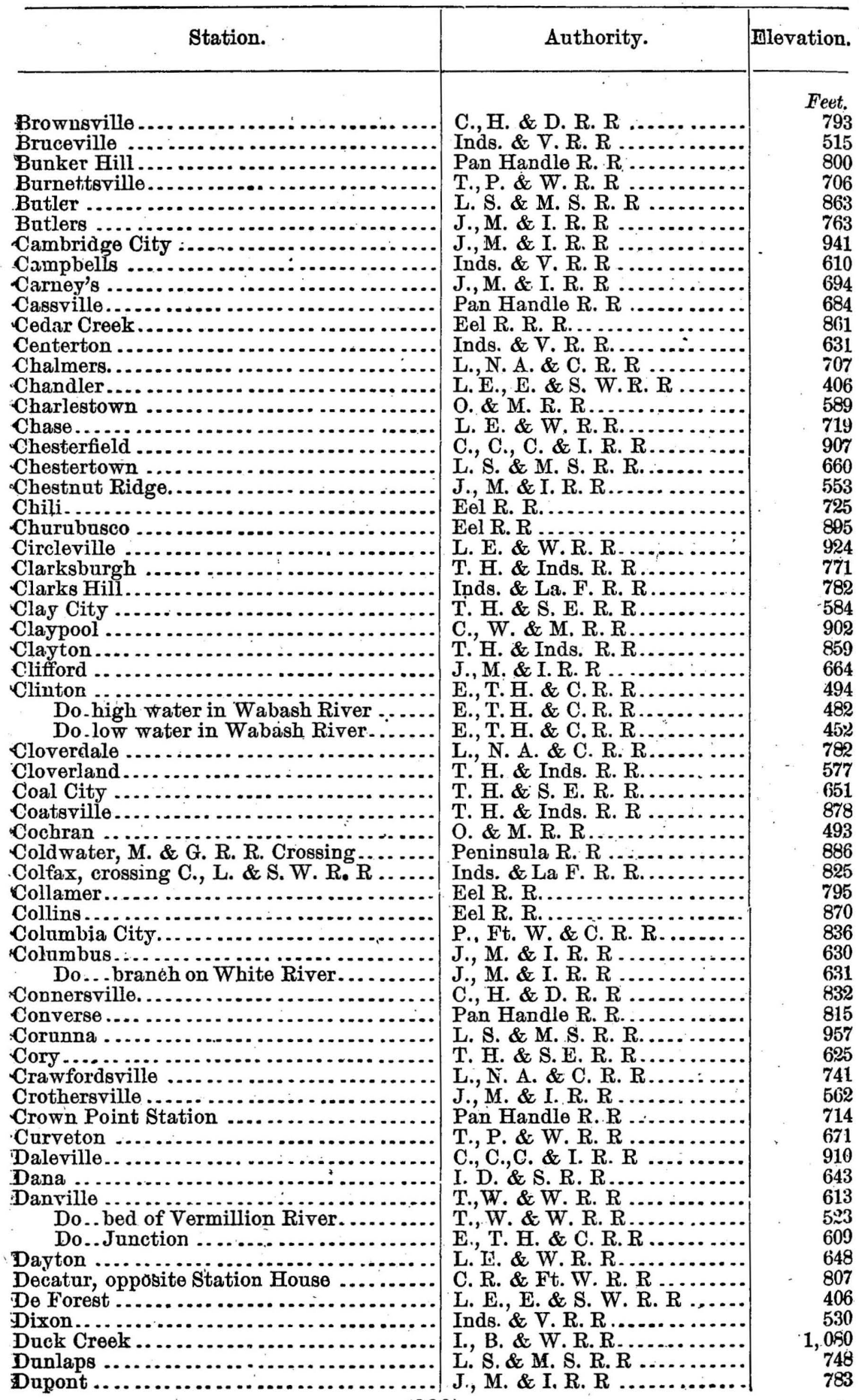




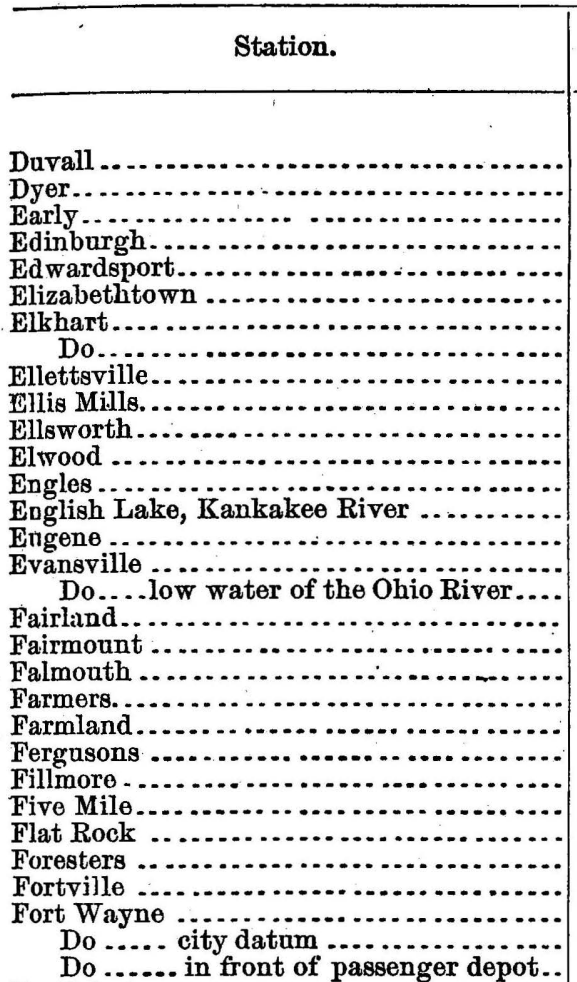

Frankfort.

Franklin .............................

Do.. Martinsville $R . R$. crossing

Freedom.

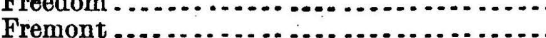

Do... Geodetic Station

French

Friendswood

Furnessville

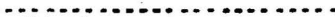

Gallaudet

Garvin City

(1)

Gessie

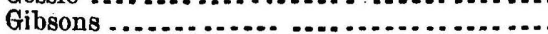

Gillman

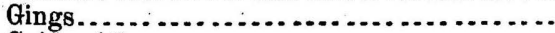

Goldsmith

Goodland

Goshen

Gosport.

Gravel Pit

Greencastle Do.

Junction

Green Fork

Greensburgh

Greenville Creek

Greenwood

Guilford

Gaion

Gundrum
I., B. \& W. R. R

J. \& M. Ind. R. R.

E., T. H. \& C. R. R J., M. \& I. R. R

Inds. \& V. R. R J., M. \& I. R. R. L. S. \& M. S. R. R C., W. \& M. R. R. L., N. A. \& C. R. R Inds. \& V. R. R. E., T. H. \& C. R. R L., E. \& W. R. R T. H. \& Inds. R. R P., Ft. W. \& C. R. R E., T. H. \& C. R. R L. E., E. \& S. W. R W. \& E. Canal C., I., St. L. \& C. $\mathrm{R}, \mathrm{R}$ C., W. \& M. R. R........... J., M. \& I. R. R Inds. \&. V. R. R. C., C., C. \& I. R. R Ft. W., M. \& C. R. R T. H. \& Inds. R. R J., M. \& I. R. R. J., M. \& I. R. R. L., S. \& M, S. R. R C., C., C. \& I. R. R G. $R$ \& Ind $R$. City Engineer P., Ft. W. \& C. R. R L. E. \& W. R. R J., M. \& I. R. R J., M. \& I. R. R Inds. \& V. R. R Ft. W., J. \& S. R. R U. S. Lake Survey I., D. \& S. R. R Inds. \& V. R. R M. C. R. R

U. S. Lake Survey

C., I., St. L. \& C. R. R B., P. \& C. R. R L. E., E. \& S. W. R. R Pan Handle R. $R$ E., T. H. \& C. R. R M.C. R. R L. E. \& W. R. R J., M. \& I. R. R L. E. \& W. R. R T., P. \& W. R. R L. S. \& M. S. R. R Inds. \& V. R. R . C., C., C. \& I. R R T. H. \& Inds. R. R. L., N. A. \& C. R. R I., B. \& W. R. R C $\mathrm{I}$ St \& $\mathrm{C}$. $\mathrm{R}$ I., B. \& W. R. R ................ Ft. W., M. \& C. R. R ........ C., I., St. L. \& C. R. R...... I., D. \&S. R. R

Pan Handle R. R
Elevation.

Feet.

885

635

497

674

460

646

755

741

682

558

488

858

677

663

507

378

326.

774

893

1, 048

528

1,037

806 .

844

465

695

852

857

752

768

775

841

732

736

538

1,055

1, 142

500

238

669

958

852

892

378

$76 \%$

616

600

852

1,000

903

718

789

595

879

834

773

1,120

942

1, 165

858

508

630

710

Bull. 5-7 


\begin{tabular}{|c|c|c|}
\hline Sta & Authority. & Elevation. \\
\hline & & \\
\hline Hamlet ...... & P., Ft. W. \& C. R. & 698 \\
\hline $\begin{array}{l}\text { Hamricks ....... } \\
\text { Harmans...... }\end{array}$ & T. H. \& Inds. R. R & 703 \\
\hline $\begin{array}{l}\text { Harmans....... } \\
\text { Harmony ...... }\end{array}$ & 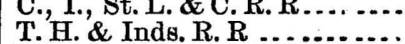 & $\begin{array}{l}747 \\
67 \%\end{array}$ \\
\hline Harrodsburgh. & L., N. A.\& C.R.R & 506 \\
\hline Harristown .... & L., N.A.\& D.R.I & 872 \\
\hline Harrisville... & C., C., C.\& I.R. & 1,101 \\
\hline $\begin{array}{l}\text { Harford City ... } \\
\text { Hart's Crossing. }\end{array}$ & Ft. W., M. \& C.R.R. & $\begin{array}{l}895 \\
457\end{array}$ \\
\hline Hastings ... & Inds. \& V.R. R... & 607 \\
\hline [ebron ..... & Pan Handle R. R & 714 \\
\hline Lenryville. & J., M.\& I. R.R . & 479 \\
\hline lillsburgh...... & L.E.\& W.R.R. & 910 \\
\hline illsclale.......... & I., D., \& S.R.R R $\ldots$ & $\begin{array}{l}452 \\
623\end{array}$ \\
\hline obbs ..... & L.E.\& W.R.R... & $\begin{array}{l}623 \\
867\end{array}$ \\
\hline ulmesville... & L.S.\& M.S.R.R & 800 \\
\hline omer ......... & J., M. \& I.R.R... & 913 \\
\hline bbells........ & T.H.\& S.E.R.R .. & 517 \\
\hline $\begin{array}{l}\text { untertown } . . . \\
\text { untington }\end{array}$ & $\begin{array}{l}\text { G.R. \& Inds. R. R.. } \\
\text { T. W.\& W.R.R .. }\end{array}$ & 827 \\
\hline untington $\cdot$ & W.\& E. Canal... & $\begin{array}{l}734 \\
741\end{array}$ \\
\hline ynds. & Inds. \& V.R.R. & 600 \\
\hline Idaville..... & T.P.\&W.R.R . & 712 \\
\hline Indianapolis, city & City Engine & 676 \\
\hline Do..... Belt & J.M. \& I. I & 722 \\
\hline ......Sig & U.S. Signa & 753 \\
\hline Do. & C., C., C.\& I. R. R & 709 \\
\hline Jamesboro .. & C., W.\& M. R. R. & $87 \%$ \\
\hline Jeffer & L.E.\& W.R.R. & 823 \\
\hline Do...Geod & U. S. Lake & 970 \\
\hline Do...Junct & J., M.\& I. R.R.. & 450 \\
\hline Jeffersonville & O.\&M.R.R... & 455 \\
\hline Jone & Pan Handle R. R & 846 \\
\hline Jon & J., M.\& I.R.R.. & 594 \\
\hline Kanka & P.R.\& P. F & 622 \\
\hline ... Brid & B.P.\& C. & 700 \\
\hline$\ldots .$. & L.E.\&W. & 920 \\
\hline ille. & L.S.. M.S. & 974 \\
\hline & I., B. \&W. & 1,057 \\
\hline $\operatorname{tion} . . . .$. & T., P.\& W. R. R - & 681 \\
\hline .......... & Ft. W., M. & 871 \\
\hline$\ldots \ldots \ldots$ & Peninsul & 742 \\
\hline tore... & W, R. R.... & 378 \\
\hline$\ldots \ldots \ldots$ & Pan Handle R. R ....... & 688 \\
\hline La Crosse ........ & Pan Handle R. R . & 675 \\
\hline $\begin{array}{l}\text { La Fayette... } \\
\text { La Grange... }\end{array}$ & G., R. \& Ind. R. R . & $\begin{array}{l}596 \\
915\end{array}$ \\
\hline & T., W. \& W. R. R. & 698 \\
\hline ake & M.C.R.R................... & 617 \\
\hline 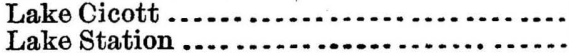 & $\begin{array}{l}\text { T. P. \& W. R.R..... } \\
\text { J. \& N. Ind.R.R .... }\end{array}$ & $\begin{array}{l}703 \\
613\end{array}$ \\
\hline $\begin{array}{l}\text { Lake Station . } \\
\text { Laketon ..... }\end{array}$ & Eel R.R.R............. & 762 \\
\hline dons.... & J., M. \& I. R. R..... & 539 \\
\hline (n)....................... & 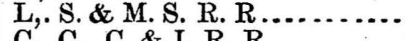 & 811 \\
\hline 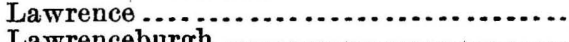 & C., C., C.\& I. R. R .............. & 872 \\
\hline coburgh .... & O. \& M. R. R. & 479 \\
\hline Do of the court-house & C. \& G. S .... & \\
\hline therwood............................ & I., D. \& S. R.R. . .... & 572 \\
\hline Leba & 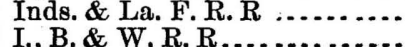 & $\begin{array}{r}925 \\
135\end{array}$ \\
\hline & J., M. \& I. R. R & 713 \\
\hline
\end{tabular}




\begin{tabular}{|c|c|c|}
\hline Station. & Authority. & Elevation. \\
\hline & & Fect. \\
\hline $\begin{array}{l}\text { Lexington }{ }_{1} \\
\text { Liberty }\end{array}$ & O. \& M.R.R... & 626 \\
\hline 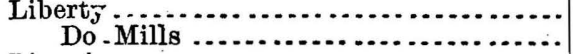 & $\begin{array}{l}\text { C., H. \& D. R. R. } \\
\text { Eel R. R. R..... }\end{array}$ & $\begin{array}{l}979 \\
773\end{array}$ \\
\hline Ligonier .............. & L.S. \& M. S.R.R & 886 \\
\hline Lima ....... & G. R. \& Ind. R. R & 882 \\
\hline Lisbon..... & G. R. \& Ind. R. ] & 1,022 \\
\hline Logansport & W., St. L. \& P.I & 606 \\
\hline London .... & C., I., St. L. \& C & 775 \\
\hline Loogootee - & O.\& M.R.R... & $5: 32$ \\
\hline Losantville. & I., B. \& W. R. R. & 1,140 \\
\hline Lowell ....... & J., M. \& I. R. R.. & 636 \\
\hline Lowrys ....... & L. E. \& W. R. R. & 874 \\
\hline 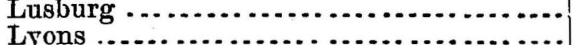 & C. W.\& M.R.R & 880 \\
\hline 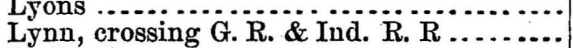 & 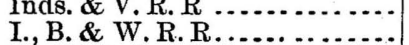 & $\begin{array}{r}509 \\
1,174\end{array}$ \\
\hline 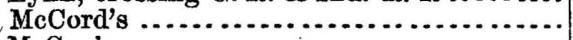 & C., C., C. \& I. R. & 854 \\
\hline McCoy's ........... & C., I., St. L. \& C & 1,027 \\
\hline McMillan's....... & J., M. \& I. R. R. & 1,010 \\
\hline 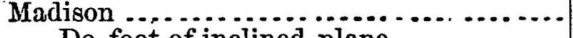 & J., M. \& I. R. R . & 451 \\
\hline Do.foot of inclined plane.......... & M. \& I. R. R . & 472 \\
\hline 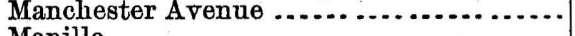 & , C. \& l. R. & 721 \\
\hline Manilla .............. & M. \& I.R.R & 896 \\
\hline Maplewood ....... & D. \& S. R. R. & 842 \\
\hline (n. & s. \& V.R.R & $48 \%$ \\
\hline 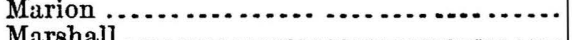 & Hande $\kappa$. & 811 \\
\hline 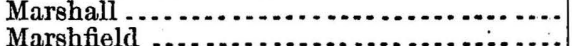 & T., W.\&W.R.R & $\begin{array}{l}700 \\
721\end{array}$ \\
\hline $\begin{array}{l}\text { rshfield } \ldots . . . . . \\
\text { Do............. }\end{array}$ & J., M. \& I. R.R & $\begin{array}{l}721 \\
543\end{array}$ \\
\hline $\begin{array}{l}\text { Do } \\
\text { artinsville }\end{array}$ & Inds. \& V.R. R & $\begin{array}{l}543 \\
598\end{array}$ \\
\hline Matthews & Inds. \& V. R. R & 691 \\
\hline Maxwells $\ldots \ldots \ldots \ldots \ldots \ldots \ldots \ldots \ldots \ldots \ldots$ & I., B. \& W.R. R & 920 \\
\hline May & Inds. \& V.R. R & 695 \\
\hline Memphis... & M. \& I. R. R & 490 \\
\hline Messick .... & 3. \& W. R. R & 1,090 \\
\hline 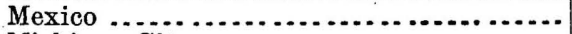 & R.R. R... & 700 \\
\hline Michigan City $\quad . . \ldots \ldots \ldots \ldots \ldots \ldots$ & C. R.R . & 603 \\
\hline 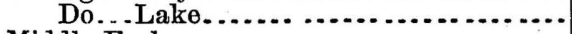 & Lake Sum & 582 \\
\hline Middle Fork $\ldots \ldots \ldots \ldots \ldots \ldots$ & $\ldots .$. & 813 \\
\hline . & Handle R. $\mathrm{H}$ & 816 \\
\hline (n) & \& M. R.R... . & 985 \\
\hline (1) & W. \& M.R.] & 850 \\
\hline tion $\ldots$ & P. \& C.R. I & 841 \\
\hline Millers.... & \&. \&. S.R & 625 \\
\hline odetic Station . . . . . . . . . . . . . . . . & .......... & 710 \\
\hline Millersburgh $\ldots \ldots \ldots \ldots$ & 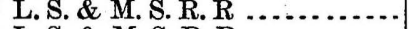 & 886 \\
\hline 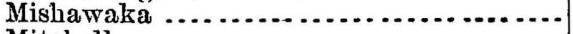 & $\ldots \ldots$ & 722 \\
\hline Mitchell $\ldots \ldots \ldots \ldots \ldots \ldots \ldots \ldots \ldots$ & O.\& M. R. R... & 676 \\
\hline $\begin{array}{l}\text { Do... B. M. ou window-sill W. corner of } \\
\text { S. iV face, M. N. Moore's store....... }\end{array}$ & $\ldots . .$. & 68 \\
\hline Mitchellville...$\ldots \ldots \ldots \ldots \ldots \ldots$ & \&. S.R.R & 872 \\
\hline Geodetic Station................. & & 1,027 \\
\hline Mon & S. R. R. & 759 \\
\hline Mon & I., & 494 \\
\hline$\ldots \ldots \ldots . . . . . .6$ & T.,P.\& W. R. R & $67 \cdot 2$ \\
\hline (n. & L. E. \& W. R. R................ & 672 \\
\hline . & Ft. W., M. \& C. R. R ......... & 867 \\
\hline 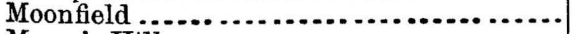 & I., D. \& S. R. R........... & 705 \\
\hline s Hill ..... & O. \& M. R. R... & 916 \\
\hline Mooresville ..... & Eel R.R.R . . & 877 \\
\hline Do............ & Inds. \& V. R. R. & 685 \\
\hline orris ............. & C., I., St. L. \& C. & 982 \\
\hline$\cdots \ldots$ & $\begin{array}{l}\text { C., H.\& D.R.R. } \\
\text { I.,B.\& W. R.R. }\end{array}$ & $\begin{array}{l}842 \\
8 \% 0\end{array}$ \\
\hline nt & & 407 \\
\hline
\end{tabular}




\begin{tabular}{|c|c|c|}
\hline Station. & Authority. & Elevatior. \\
\hline & & Feet. \\
\hline Mulberry ....... & L. E. \& W.R.R R & $\begin{array}{l}7 € 4 \\
948\end{array}$ \\
\hline Luncie ..... & Inds. \& V.R.R......... & $\begin{array}{l}940 \\
569\end{array}$ \\
\hline New Allony ... & J.M. \& I.R.R R . . . & 438 \\
\hline $\begin{aligned} \text { Do......low } \\
\text { Newbys........ }\end{aligned}$ & $\begin{array}{l}\text { L.,N.A.\&C.R.R.... } \\
\text { L.E.\&W.R.R...... }\end{array}$ & $\begin{array}{l}362 \\
861\end{array}$ \\
\hline New Carli & L.S.\& M.S.R. R .... & 772 \\
\hline New Castl & I. B. \& W. R. R .. & 1,075 \\
\hline New Era. & Ft. W., J. \& S. R. & 859 \\
\hline New Hav & T. W. \& W.R.R. & 753 \\
\hline New Jud & P. K. \& P. R. R ... & 681 \\
\hline New Lisbc & Ft. W., M.\& C.R.R & 1,098 \\
\hline ew Paris & 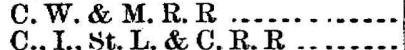 & 828 \\
\hline $\begin{array}{l}\text { New Point } \\
\text { Newport... }\end{array}$ & $\begin{array}{l}\text { C., I., St. L.\& \& C.R.R } \\
\text { E., T. H. \&. C.R.R.. }\end{array}$ & $\begin{array}{l}981 \\
494\end{array}$ \\
\hline New Provid & L., N.A.\& C.R.R. & 551 \\
\hline ixon ....... & I. B. \& W. R. R... & 1,015 \\
\hline North GI & Pan-Handle R. R. & 817 \\
\hline & Pan-Handl & 702 \\
\hline orth $\mathrm{Ma}$ & J., M. \& I. R. R.. & 878 \\
\hline Do. & J., M. \& I. R. R. & 876 \\
\hline & J., M. \& I.R.R... & 880 \\
\hline orth Mane & Eel R.R. R ........ & 775 \\
\hline orth & I., D.\& S.R. R . & 888 \\
\hline North Vern & J., M. \& I. R. R. & 727 \\
\hline Do .... & J., M. \& I. R. R ... & 727 \\
\hline uma... & E., T. H.\& C.R. R & 52:2 \\
\hline akla & C., C., C. \& I. R. R & 846 \\
\hline akle & I., D. \& S. R. R . . & 898 \\
\hline & Pan-Handle R. R & 763 \\
\hline Ope & E., Т. H. 8 & 510 \\
\hline Orle & L., N. A. \& C. R. R & 633 \\
\hline & L. S. \& M. S.R.R & 737 \\
\hline Osgr & O. \& M.R.R.... & 950 \\
\hline Ose & Ft. W., M. \& C. R. & 831 \\
\hline $\mathrm{Ot}$ & L. S. \& M & 765 \\
\hline en ........ & L. E. \& W. R. R. & 685 \\
\hline & L. E. & 703 \\
\hline de .... & C., H. \& D. R. R & $8: 31$ \\
\hline$\ldots$ & Inds. \& V. R. R. & 577 \\
\hline Parl & C., C., C. \& I. R. & 1,023 \\
\hline Pex & C., C., C.\& I. R. R & 847 \\
\hline odetic & U. S. Lake Survey & 891 \\
\hline Perr & E., T. H. \& C. R. R & 562 \\
\hline sville....... & C. \& E. IIl. R. R ... & 468 \\
\hline eru.... & W., St. L. \& P. R.R. & 655 \\
\hline & W. \& E. Canal ........ & 657 \\
\hline iereeville .... & O.\&M.R.R...... & 1,010 \\
\hline & L. S. \& M. S.R.R & 699 \\
\hline$\theta . . . . .$. & T. H. \& Inds. R. R . & 742 \\
\hline Lake.. & Ft. W., J. \& S. R. R. & 975 \\
\hline Plym & P., K. \& P.R. R .... & 769 \\
\hline Pljmou & P., Ft. W. \& C. R. $\mathbb{R}$ & 781 \\
\hline Yellow River & P., Ft. W.\& C.R.R......... & 760 \\
\hline ir Grove..... & C., I., St. L. \& C. R. R ....... & 849 \\
\hline Porter & M. C. R. R . . . . & 647 \\
\hline Portland, low water Salamonie R... & C., R. \& Ft. W. R. R ............ & 904 \\
\hline 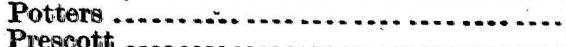 & Eel R. R. R $\ldots \ldots \ldots$ & 881 \\
\hline $\begin{array}{l}\text { Prescott .............. } \\
\text { Princeton .......... }\end{array}$ & C., I., St. L.\& C. R. R...... & 792 \\
\hline Prison Station... & J., M. \& I. R. R............. & $\begin{array}{l}485 \\
462\end{array}$ \\
\hline Putnamville .... & L., N. A. \& C. R. R . & 687 \\
\hline (n...................... & L., N.A.\& C. R. R .. & 749 \\
\hline & & \\
\hline
\end{tabular}




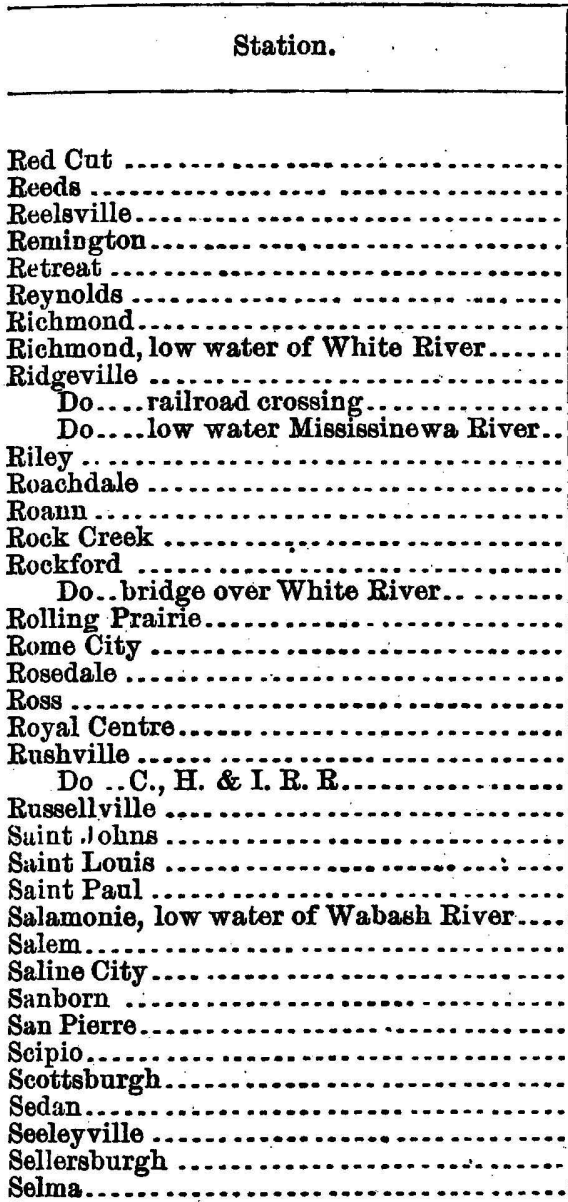

\section{Seymoro}

Do.Ind., O. \& M. R. R. crossing Shelbyville Do........... It. L. \& C.R. R. crossing Shoals, east front Wabash River........... Silver Lake

Sinedley

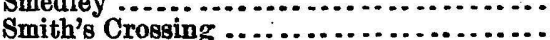

Smithville

Sinyth ........

Do ....... S. \& M. S, R. R. erossing....
Do ..... Saint Joseph's River ........... Southport.

South Wiveland

South Whitley

Spades

Sparta Lake

Speeds

Spencer

Spring Hill

Springport

Star City
Stanton

Authority. Elev

L., N. A. \& C. R. R

L. E. \& W. R. R $\ldots \ldots \ldots$

T. H. \& Inds. R. R .............

T., P. \& W..R. R...........

J., M. \& I. R. R .................

T., P. \& W. R. R ............

C., R. \& Ft. W. R. R

C., R. \& Ft. W. R. R ........

Pan-Handle R. R ...

C., R. \& Ft. W. R $\mathbf{R}$........

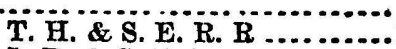

I.,D. \& S. R. $R$

Eel R. R. R .

J., M. \& I R. R .............

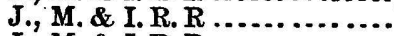

J., M.\& I. R. R

L.S.\& M. S. R. R .............

G. R. \& Ind. R. R

Pan-Haudle R. R ............

J. \& N. Ind. R. R..............

Pan-Haudle R. R ............

J., M. \& I. R. R .

J., M. \& I. R. R .............

I., D.\& S. R. R .............

J., M. \& I. R. R .............

J., M.\& I. R. R .............

C., I., St. L. \& C.R. R .......

W. \& E. Canal ..

L., N. A. \& C. R. R ...........

T.H. \& S. E. R. R..........

lud. \& V.R. R ..............

P., K.\& P.R. R

J., M. \& I. R. R

J., M. \& 1. R. R

L. S. \& M. S. R. $\mathrm{R}$

T. H. \& Inds. R. R

J., M. \& I. R. R

C., C., C. \& I. R. R...............

J., M. \& I. R. R .............

J., M. \& I. R. R .................

J., M. \& I. R. R ............

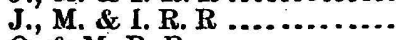

C. \& M. R. R $R$. R. R .

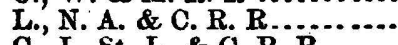

C., I., st. L. \& C. R. R .......

L., N. A. \& C. R. R .......

L., E. E. \& S. W. R. R .......

L. S. \& M. S. R. R.............

Mich. A. L. R. R ...........

Peniusula R. R ..............

Peninsula $R . R . . . . . . .$.

J., M. \& I. R. R .

I., D. \& S. R. R .................

Eel R. R. R . . . . . . . . .

C., I., St. L. \& C. R. R ......

B., P. \& C. R. R.

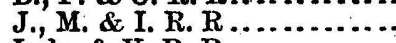

Inds. \& V. R. $R$

T. H. \& S. E. R. R .........

Ft. W., M. \& C. R. R........

Pan-Handlo R. $R$

T. H. \& Inds. R. $\mathbf{R}$
Elevation.

Feet.

832

936

732

540

692

969

885

994

993

964

569

839

750

665

$5 \times 5$

585

821

920

722

636

735

964

972

828

763

683

852

667

714

555

472

689

630

570

923

585

478

1,005

605

608

769

768

480

927

875

1,003

717

379

725

679

733

699

761

789

808

1; 013

938

465

557

508

1,018

706

643 


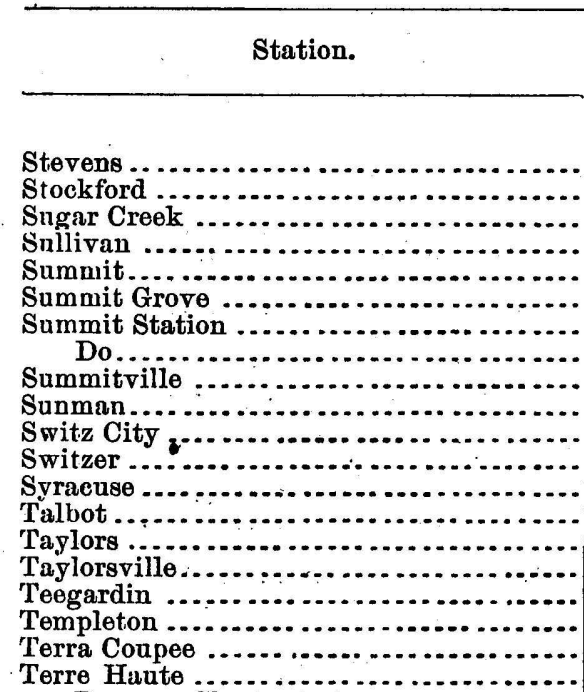

Doute

Do........ Chestnut street...............

Do....... public square

Do....... Rockville R. R. crossing .

Do....... Alton R. R. crossing .

Do......7th street R. R. crossing .

Do.......3d street R. R. crossing .

Thorntown .............................

Tippecanoe Crossing

Tipton

Tolleston

Turkey Lake, high water

Tyrone

Upton, R. R. transfer

Urhana

Valentine

Valley Mills

Valparaiso

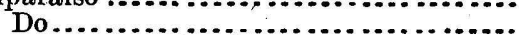

Van Buren, Geodetic Station

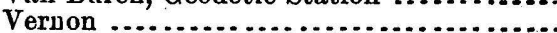

Do. Muscatatack Bridge ..................

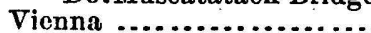

Vincennes

Vincennes

Do .... bench-mark on stone ledge northwest front court-house.

Vincennes, bench-mark on center of top of easternmost st one pier of United States Coast and Geodetic Survey astronomical observatory, court-house grounds.

Vistula.

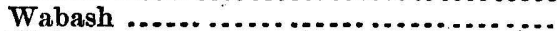

Wabash

Do.Court Honse Square

Do.W., St. L. \& P., over crossing

Walcott ......

Walcottsville ..........................

Waldron

Walesboro

Wallen

Walnut Grove

Wanatab

Authority: Ele

L., E., E. \& S. W. R. R...... St. L. \& S. E. R. R .......... I., B. \& W. R. R ............ E. \& T. H. R. R J., M. \& I. R. R ............ E., T. H. \& C. R. R ......... P., Ft. W. \& C. R. R ........ Ft. W., J. \& S. R. R C., W. \& M. R. R ... C., I., St. L. \& C. R. R Iuds. \& V. R. R Pan-Haudle R. R

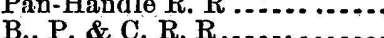
L., E. \& W. R. R............ Eel R. R. R.

J., M. \& I. R. R B., P. \& C. R. R.

$0 .+\ldots . . .$. L., E. \& W. R. R L. S. \& M. S. R. R. E., T. H. \& C. R. R ......... C. \& E. Ill. R: R C. \& E. Ill. $R \quad R$ T. H. \& Inds. R. $\mathrm{R}$ T. H. \& Inds. R. R T. H. \& Inds. R. R T. H. \& Inds. R. R Ind. \& La F. R. R Pan-Handle R. R L., E. \& W. R. R M. C. R. R

B., P. \& C. R. R I., D. \& S. R. R C., C., C. \& I. R. R St. L. \& S. E. R. R C., W. \& M. R. R G. $R$. \& $\mathrm{M}$, $R$, $R$. Inds. \& V. R. R.. P., Ft. W. \& C. R. R U. S. Lula R. R...............

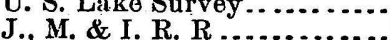
J., M. \& I. R. R J., M. \& I. R. R ............ E. \& T. H. R. R................ C. \& V. R. R.................. U. S. C. \& G. S................... U. S. C. $\&$ G. S............. Feet. 379 380 855 538 618 520 851

1,015

526

836

870

710

864

656

794

675

760

492

492

445

498

492

492

496

494

813

671

868

607

868

872

1,108

369

815

95.

759

738

801

950

686

669

566

463

417

435

431

L. S. \& M. S. R. R

809

W., St. L. \& P. R. R ........

T. W. \& W. R. R......... 740

W. \& E. Canal .............. $\quad 730$

C. W. \& M. R. R ........... 742

T. P.\& W. R. R.......... $\quad 715$

G. R. \& Ind. R. R ........ $\quad 938$

C. I., St. L. \& C. R. R........ 819

J., M. \& I. R. R ...........

G. R. \& Ind. R. R ......... $\quad 839$

E., T. H. \& C. R. R.......

P., Ft. W. \& C. R. R......... 


\begin{tabular}{|c|c|c|}
\hline tion. & Authority. & Elevation. \\
\hline 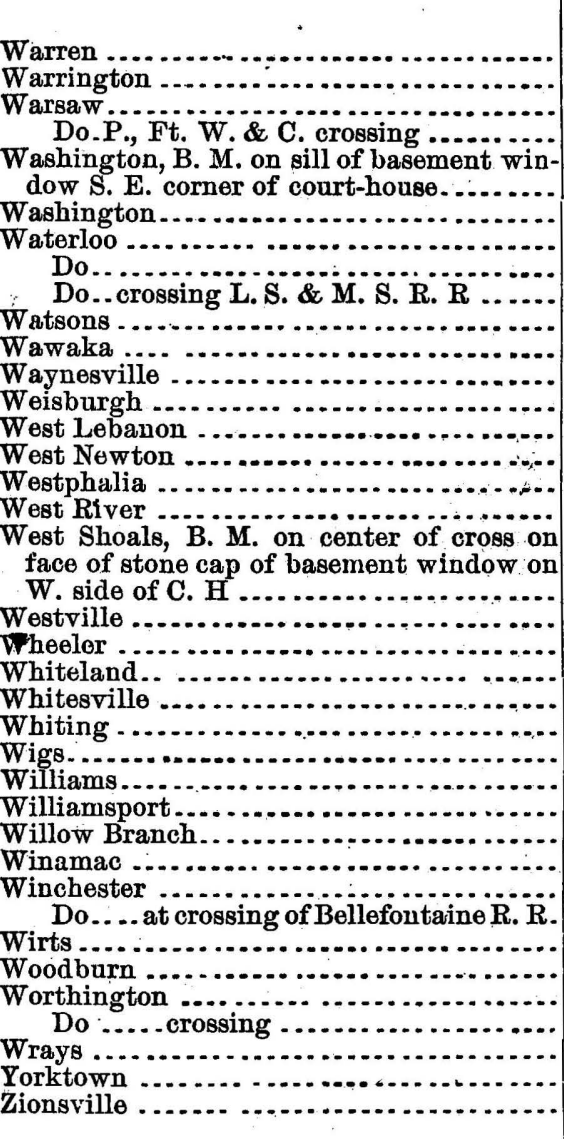 & 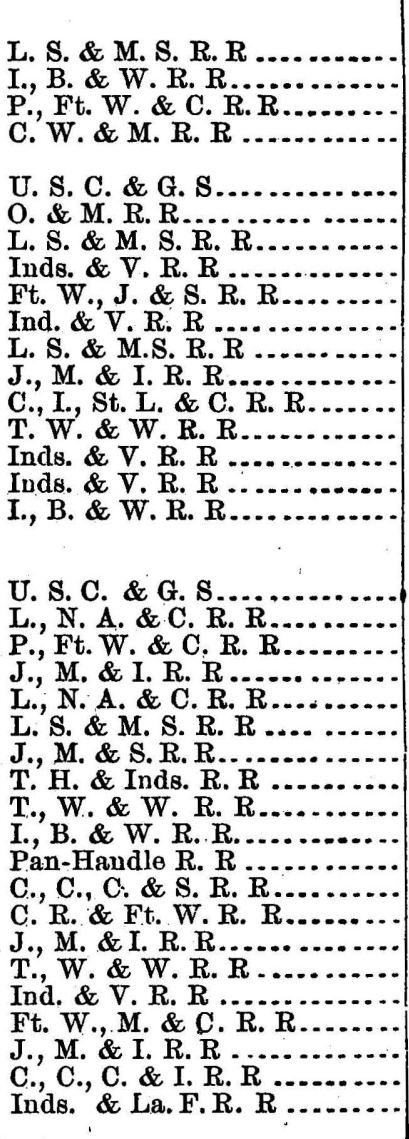 & $\begin{array}{r}\text { Feet. } \\
\mathbf{7 3 1} \\
\mathbf{1 , 0 2 0} \\
824 \\
839 \\
\\
510 \\
484 \\
897 \\
723 \\
914 \\
521 \\
896 \\
607 \\
\mathbf{9 2 9} \\
\mathbf{7 2 0} \\
\mathbf{7 7 9} \\
\mathbf{4 5 6} \\
\mathbf{1 , 1 1 0}\end{array}$ \\
\hline
\end{tabular}




\section{INDIAN TERRITORY.}

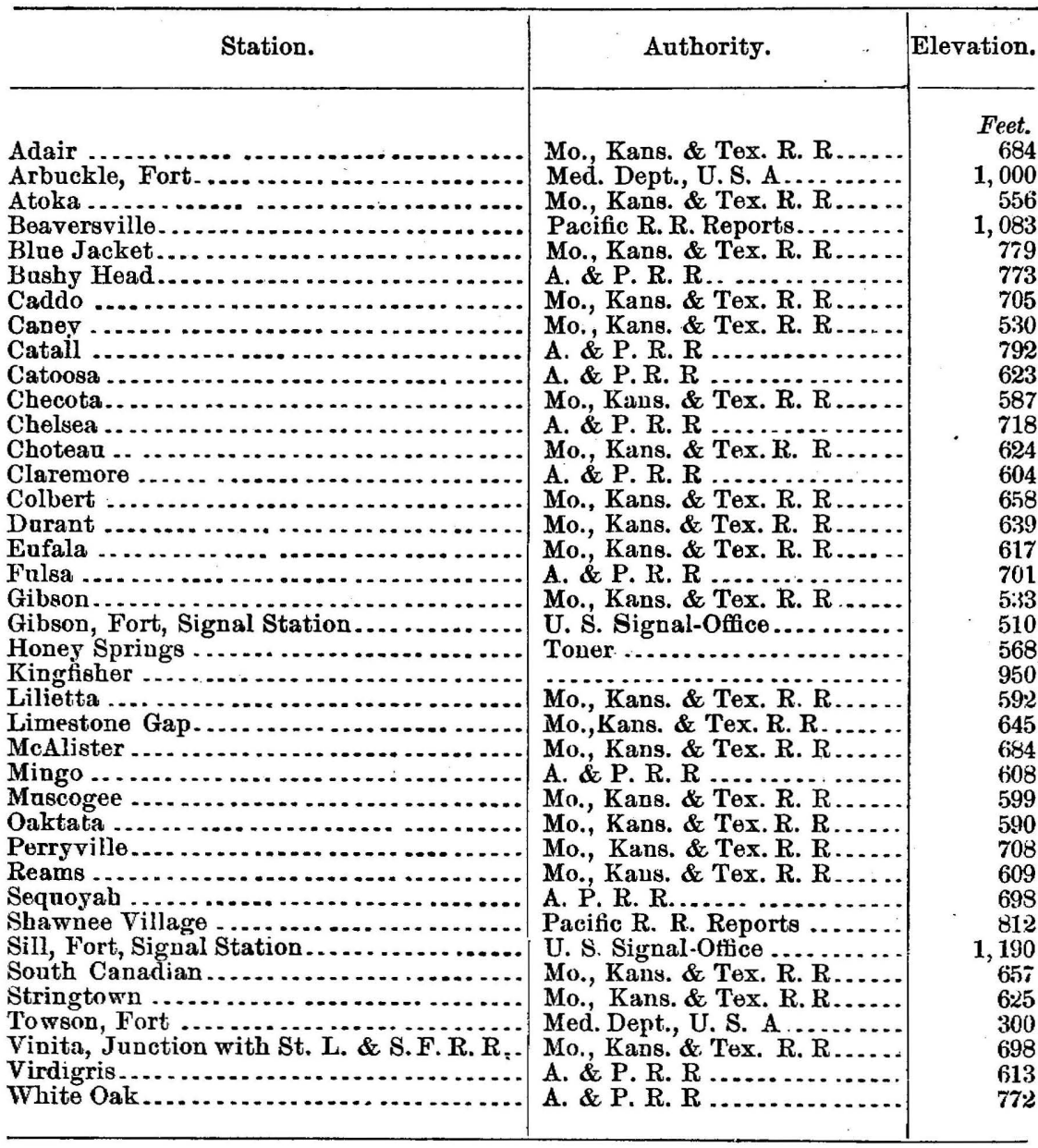




\section{IOWA.}

\begin{tabular}{|c|c|c|}
\hline & Authority. & Elevation. \\
\hline & & Feet. \\
\hline $\begin{array}{l}\text { Abbott........... } \\
\text { Ackley }\end{array}$ & $\begin{array}{l}\text { Cent. Iowa R. R. } \\
\text { Cent. Iowa R. R. }\end{array}$ & $\begin{array}{l}1,176 \\
1,177\end{array}$ \\
\hline dams ......... & B., C. R. \& N.R.R & 608 \\
\hline lel .... & D., M. \& N. W. R & 901 \\
\hline $\begin{array}{l}\text { Aurency } \\
\text { Albia ... }\end{array}$ & $\begin{array}{l}\text { B. \& M. R. R. R. } \\
\text { B. \& M. R. R. R. }\end{array}$ & $\begin{array}{l}801 \\
945\end{array}$ \\
\hline lbion.. & Cent. Iowa $\mathbf{R} . \mathbf{R}$ & 968 \\
\hline iden...... & Toner $\ldots \ldots \ldots$ & 1,165 \\
\hline Algona ... & Smithsonian Inst & 1,500 \\
\hline Ita.... & Ill. Cent. R. R... & 1,519 \\
\hline $\begin{array}{l}\text { Ames }-. . \\
\text { Areadia. }\end{array}$ & $\begin{array}{l}\text { C. \& N. W.R. R } \\
\text { C. \&N. W. R. R }\end{array}$ & $\begin{array}{r}936 \\
1,439\end{array}$ \\
\hline Ashawa. & D. M. \& Ft. D. R & $\begin{array}{r}1,405 \\
905\end{array}$ \\
\hline Atkinson, $\mathrm{F}$ & Med. Dept., U. S. & 700 \\
\hline Atlee ..... & Ft. M. \& N. W. I & 827 \\
\hline s City & B., C. R. \& N. R & $\begin{array}{r}900 \\
172\end{array}$ \\
\hline Barn & Toner $\ldots . . .$. & 1,173 \\
\hline Bartle & K. C., St. J. \& & 949 \\
\hline Batavia & B. \& M. R. R.R & 640 \\
\hline Bayfield & B., C. R. \& N.R. & 674 \\
\hline eas & C., R. I. \& P. R. & 1,256 \\
\hline Beaver. & C. \& N. W. R.R R & 1,041 \\
\hline Belkna & W., St. L. \& P. R & 857 \\
\hline$\cdots$ & C. \& N.W.R.R & 840 \\
\hline$\cdots$ & S. C. \& D. R. R & 1,257 \\
\hline$\ldots .$. & Ft. M. \& N. W. & 813 \\
\hline ve... & & 1,216 \\
\hline Bertrai & C. \& N. W & 733 \\
\hline ............ & Toner ..... & 590 \\
\hline Blair & Ill. Cent. R. I & 1,237 \\
\hline Blairstown ........ & C. \& N. W. R. R & 855 \\
\hline$\ldots \ldots \ldots$ & S. C. \& P. R. & 1,056 \\
\hline Bloomfield $: . . . .$. & W., St. L. \& P. & 855 \\
\hline 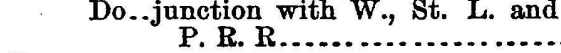 & S. K. K & \\
\hline 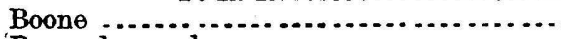 & N. W. R. R & 1,155 \\
\hline & Toner ............ & 1,160 \\
\hline airie .- & & 800 \\
\hline Brainard. & B., C. R. \& & 909 \\
\hline & M. \& St. L. R. R & 1,230 \\
\hline Brook & I. \& P.R.R & 836 \\
\hline Barlington, H.W. Miss. River, 1851 & B. \& M. R. R.R & 523 \\
\hline Do ... H. W. Miss. River, $1870 \ldots$ & B. \& M. R. & \\
\hline Do ..L. W. Miss. River, $1870 \ldots$ & B. \& M. R. R & 505 \\
\hline Do crossing Main street .... & C., B. \& Q. R. R. & 526 \\
\hline Do...bridge........... & C., B. \& Q. R. R................. & 537 \\
\hline Calamus $\ldots \ldots \ldots \ldots \ldots \ldots \ldots$ & 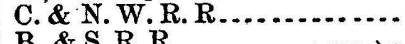 & 721 \\
\hline $\begin{array}{l}\text { Caldwell ....... } \\
\text { California Ju }\end{array}$ & $\begin{array}{l}\text { B. \& S.R.R. R. } \\
\text { S. C. \& P.R. R }\end{array}$ & $\begin{array}{r}887 \\
1,024\end{array}$ \\
\hline $\begin{array}{l}\text { California Juncti } \\
\text { Callender ....... }\end{array}$ & D. M. \& Ft. D. R. R........... & 1,167 \\
\hline liope $\ldots \ldots . . . .$. & S., C. \& D. R. R... & 1,200 \\
\hline & C., M. \& St. P. R. R ........... & 1,269 \\
\hline Can & B. \& S.R.R.... R & 776 \\
\hline roll........ & C. \& N. W. R. R & 1,240 \\
\hline cade....... & & $\begin{array}{r}770 \\
1,226\end{array}$ \\
\hline alia & C., M. \& St.P. R. R.... & $\begin{array}{l}1,220 \\
1,257\end{array}$ \\
\hline Cedar Creek.... & Toner $\ldots \ldots \ldots$ & 616 \\
\hline
\end{tabular}




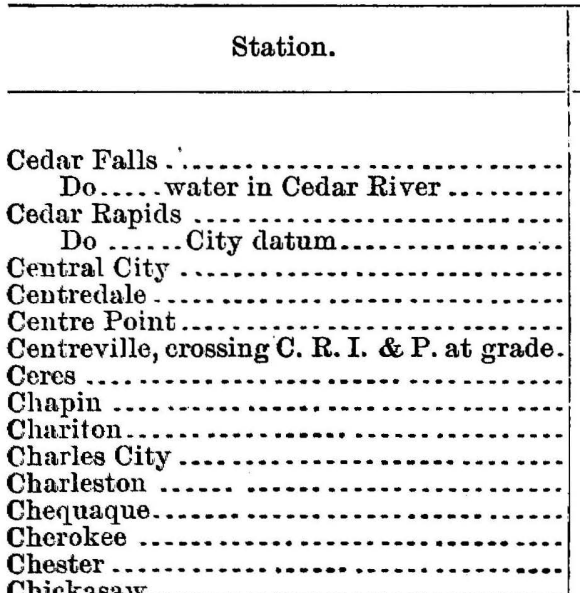

Chickasaw

Chillicothe.

Cincinnati

Clarence

Clarenda

Clarenda Junction

Clarksville

Clear Lake.

Do.... depot

Clermont

Clinton (station grounds)

Do _H. W. Miss. River

Cloriuda

Coalfield

Colburg

Colfax

Colo

Columbus Junction ....

Cone

Conover .

Coon River

Corinth

Corning

Corydon

Council Bluffs U. P. transfer depot

Do....... U. P. bridge, abutments

Do....... Bed Mo. $\mathrm{R}$

$100 \ldots . . .$. H. W. Mo. R

Do....... L. W. Mo. R

Crawfordsvillo

Crescent

Creseo .

Cromwell ....

Dallas Centre

Danville

Davenport

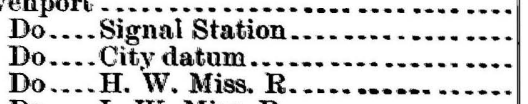

Do ..... H. W. Miss. R.

Do.... I. W. Miss. $\mathbf{R}$

Davis Creek

Deain.

Decerah

Delassus

Delavan

Delaware

Do.. crossing D. \& I. R. R

B., C. R. \& N. R. R ........

C., F. \& M. R. R

C. \& N. W. R. R..............

City engineer.

Toner

B., C. R. \& N. R. R........

B., C. R. \& N. R.R

Mo., Iowa \& Neb. R. R

Toner

Cent. Iowa R. R

B. \& M. R. R.

C. M. \& St. P. R. R

K. \& N. W. R. R

B. \& M. R. R. R

Ill. Cent. R. R . .

C., M. \& St. P. R. R

B. \& M. R. R. R.

B. \& S. R. R.

C. \& N. W. R. R

B. \& M. R. R. R.

B. \& M. R. R. R

B., C. R. \& N. R. R

C., M. \& St. P. R. R

B., C. R. \& N. R. R

C. \& N. W. R. R

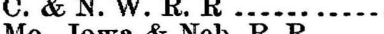

Mo., Iowa \& Neb. R. R

Cent. Iowa R. R

B. \& M. R. R. R

Toner.

C. \& N. W. R. R

B., C. R. \& N. R. R

B., C. R. \& N. R. R

C. M. St. P. R. R

M. \& St. L. R. R

B. \& M. R. R

Mo., Iowa \& Neb. R. R.

K. C. St. J. \& C. B. R. R ...

K. C. St. J. \& C. B. R. R ....

K. C. St. J. \& C. B. R. R ...

K. C. St. J. \& C. B. R. R ...

K. C. St. J. \& C. B R. R. ....

B. \& M. R. R. R.

C., M. \& St. P. R. R.

B. \&. M. R. R. R

D., M. \& Ft. D. R. R ..........

B. \&. M. R. R. R.................

C., R. I. \& P. R. R.

U. S. Signal Office ..............

City engineer.

City engineer

City engineer.

Toner

Mo., Iowa \& $\mathrm{Neb}, \ddot{R}$.

C., M. \& St. P. R. R

Toner

Toner

Ill. Cent. R. $\mathbf{R}$.

D. \& St. P. R. R
Elevation.

Feet.

844

859

744

719

986

715

809

1,013

1,246

1,030

1,012

676

605

1,211

1,244

1,148

645

1,037

841

1,069

1,097

914

984

1,237

856

609

587

1, 032

725

1,008

753

1,059

585

608

1,247

1,017

$1,1 \in 0$

1,127

1,092

989

1,038

953

986

967

692

1,209

1,312

1,220

1, 085

715

578

615

534

556

539

649

825

900

1, 068

6336

1,084

938 


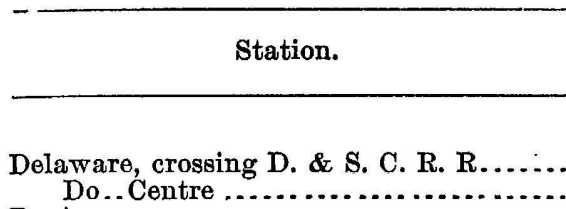

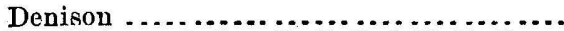

Des Moines, station C., R. I. \& P. R. R... Do..... station C., \& N. W. R. R.... Do..... station D., M. \&, N. W. R. R . Do.... . station K. \& D. M. R. R

Do..... L. W. in Des Moines R.

Des Moines, Signal Station

De Soto

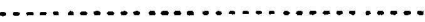

Dewitt, crossing D. \& St. P. $\ddot{\text { R. }}$ R

Dexter........

Dillon

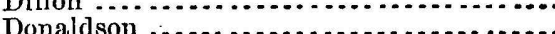

Donnellson

Doon

Downey

Dry Creek

Dnbuque

Do. City datum

Do. Signal Station

Duncombe

Durant

Dye

Dyersville.

Dysart.

East Nebraska City, Junction

$$
\text { Do............ Depot }
$$

East Orange

East Plattsmouth.

Eastport

Do. rail K. C., St. J. \& C., B. R. R... Do.. water in Mo. River March 12, 1871

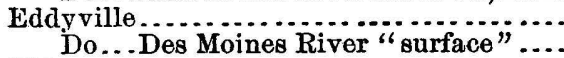

Eldora .... . . . . . . . . . . . . . . . . . . . . . .

Elm Creek.................

Elmira

Ely

Essex

Exline.

Fairbanks

Fairfax

Fairfield

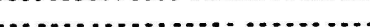

Farley ....................

Farragut

Fayette Village.

Flopd

Forest Citj

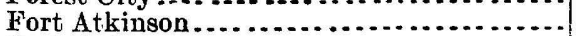

Fort Dodgo

Do

Do.

Fort Madison

Do..............

Franklin

$$
\text { Do.. }
$$

Frederic

Fredericksburgh

Authority. Elovation.

D. \& St. P. R. R C. \& N. W. R. $\ddot{R}$

City engineer

City engineer
City engineer..

City engineer..

City engineer

U. S. Signal Office

C., R. I. \& P. R. R

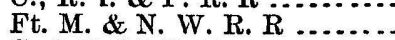

C. \& N. W. R. R..

C., R. I. \& P. R. R

Cent. Iowa R. R.

B. \& S. R. R
K. \& N. W. R. R

St. P. \& S. C. R. R.

C., R. I. \& P. R. R.

Toner ..

Ill. Cent. R. R

Ill. Cent. R. R

U. S. Signal Office

Iil. Cent. R. R.

C., R. I. \& P. R. R

Ft. M. \& N. W. R. R.

Ill. Cent. R. R.....

B., C.R. \& N. R. R............

C., R. I. \& P. R. R.

K. C., St. J. \& C. B. R. R

K. C., St. J. \& C. B. R. R..... St. P. \& S. C. R. R

Mo., Iowa \& Neb. R. R

Mo., Iowa \& Neb. R. R
Mo., Iowa \& Neb. R. R

Cent. Iowa R. R

Cent. Iowa R. R

Cent Iova $R$. $R$

B., C. R. \& N. R. R.

Toner $\ldots \ldots \ldots \ldots$

B., C. R.\& N. R. R...........

B. \& M. R. R. R ...............

B. \& S. R. R

Toner.

C. \& N. W. R. R

B. \& M. R.R.R

Ill Cent. R. R

B. \& S. R. R.

B. \& M R R R

Smithsonian Inst

I. C., C., F. \& M. R. R

M. \& St. L. R. R...

C., M. \& St. P. R. R.

D.M. \& Ft. D. R. R.

Ill. Cent $\mathrm{R}$. M. \& St. L. R. R.

C. B. \& Q. R. R.

Ft. M. \& N. W. R. IR

Ft. M. \& N.W.R. R.

B. \& S.R.R.

Cent. Iowa R. R

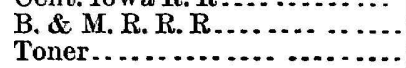

Feet.

1,083

1,106

1,192

800

798

807

799

776

849

886

813

699

1, 146

985

707

681

1,282

68:3

1, 603

614

5

665

1, 118

712

814

940

958

1,106

926

928

1, 302

$9: 24$

926

929

$91 \%$

672

698

1,153

833

1, 094

741

731

996

1,016

1,083

784

767

1,111

571

963

1,000

1,107

$1,2 \div 0$

1,023

1,015

1,032

1, 120

516

632

644

702

1, 193

735

765 


\begin{tabular}{|c|c|c|}
\hline Station. & Authority. & Elevation. \\
\hline & & Feet. \\
\hline ulton... & C., M. \& St. P.R. I & $\begin{array}{r}758 \\
1,227\end{array}$ \\
\hline arrison & B.; C.R.\&N.R.R. & 849 \\
\hline Geneva. & Cent. Iowa R. R.. & 1,181 \\
\hline & Cent. Iowa R. R. & 1,030 \\
\hline $\ln$ & Cent. Iowa & 1,035 \\
\hline Giver & Cent. Iowa R. R & 703 \\
\hline & B. \& M. R. R. R & 745 \\
\hline Glenwood & B. \& M. R. R. R.. & $\begin{array}{r}979 \\
154\end{array}$ \\
\hline & $\begin{array}{l}\text { D. M. \& Ft. D. R. } \\
\text { B., C. R. \& N. R. I }\end{array}$ & $\begin{array}{r}1,154 \\
816\end{array}$ \\
\hline rand & C. \& N. W. R. R. & 1,055 \\
\hline Gran & C. \& N. W & 736 \\
\hline & B., C. R. \& & 943 \\
\hline Grin & C., R. I. \& P. R. R & 1,011 \\
\hline Grove & C., R. I. \& P. R. F & 1,271 \\
\hline & $\begin{array}{l}\text { B., C. R. \& N. R. R } \\
\text { C., R. I. \& P. R. R }\end{array}$ & $\begin{array}{r}966 \\
1,269\end{array}$ \\
\hline Gntten & $\begin{array}{l}\text { C., K. } 1 . \& \\
\text { Toner.... }\end{array}$ & $\begin{array}{r}1,269 \\
690\end{array}$ \\
\hline Hamburg $\ldots . . . . .$. & K. C., St. J & 912 \\
\hline Do... Semaphor & K. C., St. . & 902 \\
\hline Hampt & dent. Iowa R. R.. & 1,240 \\
\hline Greek. & Toner $\ldots \ldots \ldots$ & 1,026 \\
\hline Harmon & B., \& M. R. R. R . & 1,040 \\
\hline Grove... & & 900 \\
\hline side tra & $\begin{array}{l}\text { K. C., St. J. \& C. B. I } \\
\text { Toner }\end{array}$ & 955 \\
\hline Hazar & 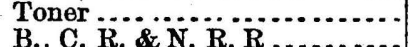 & 1.405 \\
\hline $\mathrm{Ha}$ & B., C. R. \& N. R. R & 988 \\
\hline & B., \& M. R. R. R . & 1,078 \\
\hline Hes & Snithsonian Inst & 720 \\
\hline Grove & Iowa & 810 \\
\hline & B., C. R. \& N. R. R & 985 \\
\hline Hom & C., R. I. \& P. R.R. & 866 \\
\hline Ho & St. P. \& S. C. R. R & 1,338 \\
\hline um & Ft. D. \& F & 1,082 \\
\hline & S. N. & 1,089 \\
\hline Indepen & IIll. $\mathrm{c}$ & 921 \\
\hline Do & B., C. R. \& & 1,111 \\
\hline Iowa C & B., C. R. \& & 644 \\
\hline Do. & C., R. I. \& P. R. R... & 671 \\
\hline letion ... & B., C. R. \& N. R. R..... & 618 \\
\hline & Iil. Cent. R. R . & $\begin{array}{l}892 \\
990\end{array}$ \\
\hline & Tor & 845 \\
\hline & B., C. R. \& N. R & 651 \\
\hline ........ & C., R. I. \& P. R. R... & 839 \\
\hline & D. M. \&. N. W. R.R.. & 968 \\
\hline Kensett . & Cent. Iowa R. R.... & 1,306 \\
\hline Keokuk, & C. B. \& Q. R. R. & 501 \\
\hline y da & City Eng & 480 \\
\hline & City & 5 ins \\
\hline & U.S. & 618 \\
\hline Kesw & B., C.R.\& N. R.R... & 862 \\
\hline $38 \ldots \ldots$ & B., C. R. \& N. R. R.. & 757 \\
\hline Kossuth ..... & B., C. R. \& N. R. R. & \\
\hline $\begin{array}{l}\text { Lacey } \ldots . . . . . . \\
\text { Lake Mills ... }\end{array}$ & $\begin{array}{l}\text { Cent. Iowa R. R } \\
\text { M. \& St. L. R. R }\end{array}$ & $\begin{array}{r}850 \\
1.264\end{array}$ \\
\hline La Porte . . . . . . . & B., C. R. \& N. R. R... & 1, 802 \\
\hline & C. \& N. W.R. R ....... & $\therefore 953$ \\
\hline Le Mars. & St. P. \& S. C. R. R .... & 1,221 \\
\hline branch, B. \& M & 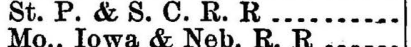 & 1,197 \\
\hline orancen, B. \& & C. M. \& St. P. R. R & $\begin{array}{l}1,020 \\
1,298\end{array}$ \\
\hline
\end{tabular}




\begin{tabular}{|c|c|c|}
\hline Stati & Authority. & Elevation. \\
\hline & & \\
\hline $\begin{array}{l}\text { Lime Springs } \\
\text { Linden ......... }\end{array}$ & $\begin{array}{l}\text { C. M. \&. St. P. R. } \\
\text { D. M. \& N. W. R. }\end{array}$ & 1,258 \\
\hline & B., C. R. \& N. R. & 1,156 \\
\hline & C. \& N. W. R.R & 888 \\
\hline & Cent. Iowa R. R. & 1,078 \\
\hline Do. & $\begin{array}{l}\text { M. \& St. L. R.R... } \\
\text { Ft. D. \& Ft. R. R. R }\end{array}$ & $\begin{array}{l}1,135 \\
1,128\end{array}$ \\
\hline gan. & Toner $\ldots \ldots \ldots \ldots$ & 928 \\
\hline & $\begin{array}{l}\text { C. \& N. W. R.R... } \\
\text { B. C.R.\& N.R.R }\end{array}$ & $\begin{array}{r}733 \\
718\end{array}$ \\
\hline velanc & Mo., Iowa \& Neb. & $\begin{array}{r}718 \\
962\end{array}$ \\
\hline & B., \& M. R.R.R & 1,205 \\
\hline & C., M. \& St. P. I & 1,132 \\
\hline$\cdots$ & Toner .... & 857 \\
\hline $\begin{array}{l}\cdots . . \\
\cdots \ldots .\end{array}$ & $\begin{array}{l}\text { Smithsoni } \\
\text { C., M. \& : }\end{array}$ & $\begin{array}{l}630 \\
616\end{array}$ \\
\hline $\begin{array}{l}\text { r, L. W } \\
\text { …H. W }\end{array}$ & C., M. \& \& & $\begin{array}{l}616 \\
634\end{array}$ \\
\hline cPaul & K. C., St. J.\& C. & 940 \\
\hline.. & ill Cent $\mathrm{R} R$ & $\begin{array}{l}600 \\
450\end{array}$ \\
\hline 10n & $\begin{array}{l}\text { III. Cent. R. R.... } \\
\text { B., C. R. \& N. R. }\end{array}$ & $\begin{array}{r}950 \\
1,189\end{array}$ \\
\hline & Cent. Iow & 1,291 \\
\hline Mans & IIl. Cen & 1,245 \\
\hline ock - . & B., C. R. \& N. R. R .... & 992 \\
\hline ng Iowa Central $R$ R. & $\begin{array}{l}\text { Ill. Cent. R. R............. } \\
\text { C. \& N. W. R. }\end{array}$ & $\begin{array}{r}1,469 \\
898\end{array}$ \\
\hline Ford....... & Toner.... & $1,-148$ \\
\hline & C., & 1,130 \\
\hline & B., & 1,096 \\
\hline & C. & 912 \\
\hline & B. & 756 \\
\hline & B. & 853 \\
\hline M & R.R... & 1,412 \\
\hline & B. & 725 \\
\hline & B. & 806 \\
\hline Min & D. M. \& Ft. I & 1,062 \\
\hline ri Valley, junction with S. C. \& P. & & \\
\hline & C. \& $\mathrm{N}$ & 1,022 \\
\hline ville... & $\begin{array}{l}\text { C. F.\& M. } \\
\text { C., R. I.\& }\end{array}$ & $\begin{array}{r}1,202 \\
966\end{array}$ \\
\hline & S.C.\& P.R.R.... & 1,029 \\
\hline Moing & C. \& N.W.R.R.... & \\
\hline Do .....L. W. Des Moines Rive & & 870 \\
\hline 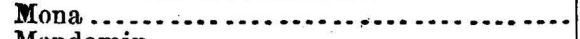 & Iil. ( & 1,203 \\
\hline & & 1,038 \\
\hline$\cdots$ & C., M. \& & 1,221 \\
\hline & B., & 94 \\
\hline lo (near) & & 800 \\
\hline - & & 86 \\
\hline g Sun..... & N.R.R. & 835 \\
\hline $30 \mathrm{n} . . . . . . .$. & & 937 \\
\hline & B., C. R. \& N.R.R & 753 \\
\hline (n.............. & C., R. I. \& P. R.R R... & 652 \\
\hline junction with W., St. L. \& P.R.R. & $\begin{array}{l}\text { W., St. L.\& } \\
\text { B.\&S.R.R : }\end{array}$ & $\begin{array}{l}994 \\
994\end{array}$ \\
\hline Mount Auburn & B.,C.R.\& N.R.R ... & 853 \\
\hline & $\&$ Neb. R. R & 1,236 \\
\hline ant . & B. \& M.R.R & \\
\hline & & 649 \\
\hline Union .... & B. \& N.R.R.... & 712 \\
\hline unt Vernon .. & C.\&N.W.R. R........... & 1,183 \\
\hline & & \\
\hline
\end{tabular}




\begin{tabular}{|c|c|c|}
\hline Station. & Authority. & Elevation. \\
\hline & & \\
\hline Anscatine & B., C. R.\& N. R. R & \\
\hline Nashua ... & C., F.\&M.R.R. & 975 \\
\hline Nevada ... & C.\& N.W.R.R & 1,017 \\
\hline New Bosto & K.\&N.W.R.R. & 674 \\
\hline Newlourgh & Cent. lowa R. R. & 1,036 \\
\hline Now Hau & C., M. \& St. P. R. R & \\
\hline New Jeffe & C.\&N.W.R.R... & 1,071 \\
\hline New Shar & Cent. Iowa R. R. & 877 \\
\hline ENon. & C., R. I. \& P. R. R. & 958 \\
\hline Nichols. & B., C.R.\& N.R.R & 628 \\
\hline & Toner ............ & 1,151 \\
\hline .. & B. \& M.R.R.R . & 1,080 \\
\hline Nora Ju & B., C. R.\& N.R. & 1,052 \\
\hline & M. \& St. L. R. R... & 1,279 \\
\hline North & C., M.\& St.P.R. H & \\
\hline North $U_{I}$ & Toner ............. & 1,250 \\
\hline & B. C., R.\& N.R.R & 1,210 \\
\hline Norw & C.\& N.W.R.R.. & 809 \\
\hline Oakl & B. \& M.R.R.R & 1,193 \\
\hline & B., C. R.\& N.R.R. & 790 \\
\hline Oelwein & B., C. R.\& N.R. R & 1,039 \\
\hline Ogd & C. \& N.W.R.R. & 1,109 \\
\hline & S. C. \& P.R.R. & 1,064 \\
\hline Ortonvil & D. M.\&N.W.R & 1,055 \\
\hline & I. C., C. F. \& M. & 1,178 \\
\hline & B.\& M. R. & 1,123 \\
\hline Oskaloos & Cent. Iowa & 850 \\
\hline Oss & C., $\mathrm{M}$ & 1,281 \\
\hline & B. \& & 630 \\
\hline Oxfo & C., R.I. & $7: 20$ \\
\hline Pacific J & K. C. & 960 \\
\hline Do.. & K. C., St. J & 961 \\
\hline Palo... & B., C.R.\& & 741 \\
\hline & D.M.\&N. & 1,074 \\
\hline burgh . & Tor & 953 \\
\hline Pat & $\mathrm{D}$. & 1,116 \\
\hline & & 747 \\
\hline & K. & 933 \\
\hline & D. & 977 \\
\hline & Ft. & $\varepsilon 31$ \\
\hline & C. F. \& M. & 926 \\
\hline Plain & Smi & 950 \\
\hline Junction. & R. \& N.R.R. & 1,114 \\
\hline & Tor & 1,244 \\
\hline & S. C. & 1,162 \\
\hline $\mathrm{Po}$ & C.M. \& St. P. R. R. & 1,207 \\
\hline & .............. & 800 \\
\hline eek. & & 699 \\
\hline $\mathrm{Pul}$ & S. R. R .... & 840 \\
\hline & N. W. R. R & 898 \\
\hline & Smithsoni & 888 \\
\hline a...... & B., C.R. \& N. R. R .... & 1,096 \\
\hline & D. M. \& N.W.R. R ... & 968 \\
\hline & & 1,033 \\
\hline k.... & B., C.R.\& N.R. R ......... & 916 \\
\hline & & 1,335 \\
\hline Rip & D. M. \& Ft. D. R. R..... & $1,0 \times 0$ \\
\hline & B., C.R.\& N. R. R .... & 631 \\
\hline onx.... & S.C. \& P. R. R ........ & 1,053 \\
\hline$\ldots . . .$. & B. \& M.R.R.R.. & 931 \\
\hline Falls. & B., C. R. \& N.R. R .......... & 1,094 \\
\hline ........ & B., C.R.\& N.R. R . . & 1,011 \\
\hline $18 \ldots$ & St. P. \& S. C. R. R & 1,344 \\
\hline & Cent. Iowa R. R......... & 1,219 \\
\hline
\end{tabular}




\begin{tabular}{|c|c|c|}
\hline Sta & Authority. & Elevation. \\
\hline olfe .... & Smithsonian Inst & Feet. \\
\hline $0 s \cos \ldots$ & B. \& N. R. R.... & $\begin{array}{r}1,000 \\
755\end{array}$ \\
\hline ossville & sonian Inst & \\
\hline owley & B., C.R.\& N.R. & \\
\hline issell & B. \& M. R. R .. & 1,017 \\
\hline & Toner $\ldots \ldots \ldots$ & 900 \\
\hline $\begin{array}{l}\text { St. Ausg } \\
\text { St. Gilm }\end{array}$ & C. F. \& M.R.R & $\begin{array}{l}1,179 \\
1,442\end{array}$ \\
\hline t. Gilma & Smithsonizn Inst. & $\begin{array}{l}1,442 \\
1,200\end{array}$ \\
\hline lem.. & 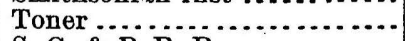 & 694 \\
\hline$\cdots$ & S. C. \& P. R. R... & $\begin{array}{r}1,097 \\
799\end{array}$ \\
\hline$\ldots$. & $\begin{array}{l}\text { Toner } \ldots \ldots \ldots \\
\text { D.\& } \mathrm{St}, \mathrm{P}, \mathrm{R} .\end{array}$ & $\begin{array}{l}792 \\
938\end{array}$ \\
\hline$\cdots$. & S. C. \& P.R.R... & 1,008 \\
\hline ar: & Cent. Iowa R. R. & 810 \\
\hline Sedan, crossin & Mo., Iowa \& Neb. $\mathrm{R}$ & 827 \\
\hline ney .......... & St. P. \& S. C. R. R & 1,221 \\
\hline .... & Cent. Iowa R. R. & 1,163 \\
\hline aeld & St. P. \& S. C. R. I & 1,406 \\
\hline hellshingeh & B., C. R. \& N. R. R & 911 \\
\hline hellsburgh. & B., C. R. \& N. R. R... & $\begin{array}{l}764 \\
979\end{array}$ \\
\hline ........ & B. \& M. R. R.R R & $\begin{array}{r}979 \\
1.509\end{array}$ \\
\hline (.... & $\begin{array}{l}\text { St.P. \& S.C.R.R.... } \\
\text { St.P.\& C.R.R. }\end{array}$ & $\begin{array}{l}1,509 \\
1,122\end{array}$ \\
\hline$\ldots$ & $\begin{array}{l}\text { St. P.\& C. R. R ....... } \\
\text { S. C.\& D.R.R }\end{array}$ & $\begin{array}{l}1,122 \\
1,185\end{array}$ \\
\hline ey - & $\begin{array}{l}\text { S.C.\& D.R } \\
\text { S.C.\& P.R. }\end{array}$ & $\begin{array}{l}1,185 \\
1,089\end{array}$ \\
\hline$\ddot{. .}$ & 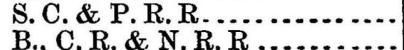 & $\begin{array}{r}1,089 \\
784\end{array}$ \\
\hline Euglisl & $\begin{array}{l}\text { R. \& N.R.R. } \\
\text { R \& N.R.R. }\end{array}$ & $\begin{array}{l}784 \\
830\end{array}$ \\
\hline & B., C. R \&N.R.R. & 830 \\
\hline & $\begin{array}{l}\text { B., C. R. \& N. R. R .... } \\
\text { D. \& St. P.R.R. }\end{array}$ & $\begin{array}{r}747 \\
1,236\end{array}$ \\
\hline$\ldots$ & D. \& St. P. R. R ....... & $\begin{array}{r}1,236 \\
915\end{array}$ \\
\hline & ......... & 915 \\
\hline $\begin{array}{l}\text { Stat } \\
\text { Star }\end{array}$ & Tone & 1,004 \\
\hline Star & $\mathrm{N} \cdot \mathrm{W} \cdot \mathrm{R} \cdot \mathrm{R}$ & 863 \\
\hline -. & N. W. R. R . & 1,086 \\
\hline & R. R.. & 1,061 \\
\hline & R. R & 1,218 \\
\hline & R.R... & 1,084 \\
\hline $\mathrm{u}$ & K. \& N. W. R. R .. & 666 \\
\hline & N. W. R. R....... & 832 \\
\hline & \& Ft. D. R. R. & 1,159 \\
\hline & Ton & 1,002 \\
\hline & B. & 868 \\
\hline & & 770 \\
\hline Tr: & $\&$ C. B. R. R .... & \\
\hline & R. R ...... & 906 \\
\hline Ty & B. & 819 \\
\hline n.......... & & 1,013 \\
\hline צ' & & 1,058 \\
\hline r's Grove & asonian Inst $\ldots . .$. & 1,500 \\
\hline $\begin{array}{l}\text { Victor } \ldots . . . \ldots \\
\text { Victoria } . . . . . .\end{array}$ & , I. \& P. R. R . . . . & $\begin{array}{l}806 \\
788\end{array}$ \\
\hline $\begin{array}{l}\text { Victoria } . . . . . . . . . \\
\text { Viele ................. }\end{array}$ & S.R. R......... & 543 \\
\hline Vinton................. & R.\& N.R. R & 800 \\
\hline & Smithsonian Inst & 607 \\
\hline $\begin{array}{l}\text { Walcott ..... } \\
\text { Walker }\end{array}$ & C., R. I. \& P. R. R & 733 \\
\hline Walker ........... & B., C. R. \& N.R R $\ldots \ldots \ldots \ldots$ & $\begin{array}{r}880 \\
1,040\end{array}$ \\
\hline $\begin{array}{l}\text { Walnut ........... } \\
\text { Wapello ........ }\end{array}$ & 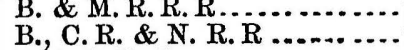 & \\
\hline & B.\& S. R. R & 709 \\
\hline Washburn ........... & B., C. R \& N. R. R....... & 817 \\
\hline $\operatorname{ton} \ldots \ldots \ldots \ldots$ & B. \& N. R, R $\ldots \ldots \ldots$ & 738 \\
\hline $\mathrm{k}(\ldots \ldots \ldots \ldots \ldots \ldots$ & $\begin{array}{l}\text { Ill. Cent. R. R. } \\
\text { Toner } \ldots \ldots \ldots \ldots\end{array}$ & $\begin{array}{l}862 \\
988\end{array}$ \\
\hline $\begin{array}{l}\text { Waubeck } \\
\text { Wauke } \ldots \ldots \ldots\end{array}$ & D., M. \& Ft. D. R. R......... & 1,049 \\
\hline
\end{tabular}


[BUaL. 5.

\begin{tabular}{|c|c|c|}
\hline Station. & Anthority. & Elevation. \\
\hline & & Feet. \\
\hline 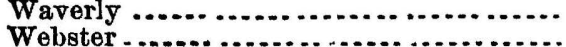 & $\begin{array}{l}\text { I., C., C., F. \& M. R. R } \\
\text { Iil. Cent. R. R. }\end{array}$ & $\begin{array}{r}942 \\
1,054\end{array}$ \\
\hline Wellman ....... & B., C., R.\& N.R.R. & $\begin{array}{r}1,004 \\
688\end{array}$ \\
\hline Wesley ........... & C., M.\& St. P.R. R. & 1,254 \\
\hline West Branch..... & B. C. R.\& N.R.R.. & 708 \\
\hline $\begin{array}{l}\text { Westfield .......... } \\
\text { West Grove...... }\end{array}$ & $\begin{array}{l}\text { S. C. \& D. R. R.... } \\
\text { W., St. L. \& P.R.R. }\end{array}$ & $\begin{array}{r}1,148 \\
946\end{array}$ \\
\hline West Liberty ...... & C., R. I. \& P. R. R.. & 666 \\
\hline West Nishnabotany & 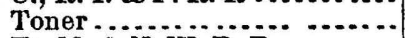 & 938 \\
\hline West Point ........ & Ft. M. \& N.W.R.R & 866 \\
\hline West Union... & B., C. R. \& N.R.R. & 1,105 \\
\hline What Cheer... & B., (.R.\& N.R.R & 741 \\
\hline Wheatland. & C. \& N. W.R. R ... & 695 \\
\hline Whitfield .. & B. \& M. R. R ... & 677 \\
\hline Whiting ........ & S. C. \& P.R.R. & 1,074 \\
\hline Willets ........... & B. \& S.R.R...... & 604 \\
\hline Wilton....... & C. R. I. \& P.R.R. & 672 \\
\hline Winfield ... & 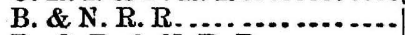 & 690 \\
\hline Winslow $\ldots . . . \ldots \ldots \ldots \ldots$ & B., C.R. \& N.R. R ............ & $8 \pi 4$ \\
\hline Winthrop......... & Ill. Cent. R. R..................... & 1,053 \\
\hline Yarmouth................... & B. \& N. R. R . . . . . . . . & 696 \\
\hline
\end{tabular}




\section{KANSAS.}

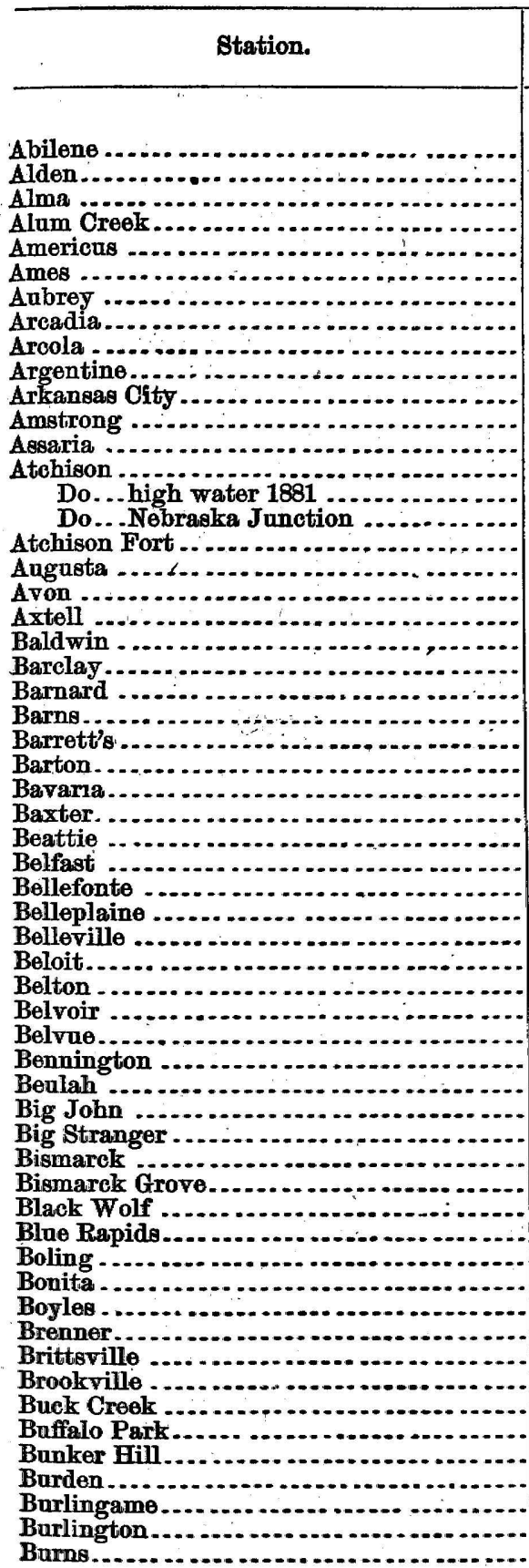

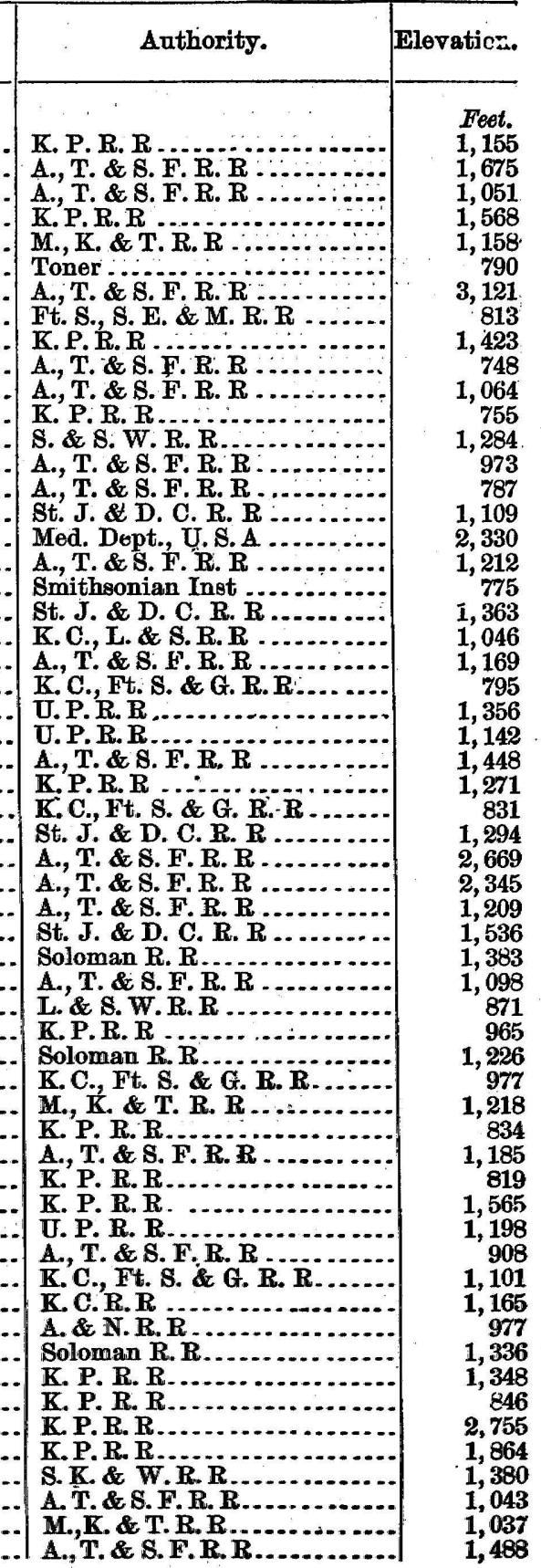




\begin{tabular}{|c|c|c|}
\hline Stati & Authority. & Elevation. \\
\hline & & \\
\hline Caldwell... & A., T.\& S.F.R.R. & 1,102 \\
\hline mbridge. & S. K. \& W.R.R... & 1,248 \\
\hline nton..... & A., T.\&S.F.R.R. & $\begin{array}{l}1,582 \\
952\end{array}$ \\
\hline rbon ...... & A., T.\&S.F.R. R. & ${ }^{\circ} 1,072$ \\
\hline $\mathrm{n} \mathbf{t}$ & L.\& S.W.R.R... & \\
\hline$\theta . .$. & A., T. \& S. F.R.R. & 3,178 \\
\hline rlyle.... & K.C., L. \& S.R. R. & 984 \\
\hline rthage & A., T.\& S.F.R.R. & $\begin{array}{l}5,009 \\
1,237\end{array}$ \\
\hline $\begin{array}{c}\text { Cedar Grove ..... } \\
\text { Do...Junc }\end{array}$ & $\begin{array}{l}\text { A., T. \& S.F.R.R. } \\
\text { A., T. \& S.F.R.R. }\end{array}$ & $\begin{array}{r}1,237 \\
778\end{array}$ \\
\hline Centralia........ & 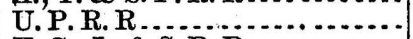 & 1,270 \\
\hline $\begin{array}{l}\text { Chanute } \\
\text { Do. }\end{array}$ & K. C., L.\&S.R.R. & $\begin{array}{l}910 \\
914\end{array}$ \\
\hline hase.. & $\begin{array}{l}\text { M., K.\& } \& . \text { R. R.. } \\
\text { A., T.\& S. F. R. }\end{array}$ & $\begin{array}{r}914 \\
1,708\end{array}$ \\
\hline & K. C., Ft. S. \& G. F & 933 \\
\hline$\cdots$ & K. C., L. \& S. R. R & 836 \\
\hline & M., K. \& T. R. R. & $\begin{array}{r}832 \\
2.106\end{array}$ \\
\hline & A., T. \& S. F. R. & $\begin{array}{r}2,106 \\
764\end{array}$ \\
\hline & A., T. \& S. F. R. R & 1,306 \\
\hline & A., T. \& S. F. R.R & 2,116 \\
\hline & St. J. \& D. C. R. I & 1,116 \\
\hline & Ft. S., S. E. \& M. & 890 \\
\hline & J. C., \& Ft. K. R. & 1,201 \\
\hline & K. P. R. R........... & 3,046 \\
\hline & J. C., \& Ft. K. R. I & 1,278 \\
\hline & A., T.\& S. F. R.R. & 1,018 \\
\hline & Toner $\ldots . . . . .$. & 762 \\
\hline & $\& \mathrm{Ft}$ & 1,299 \\
\hline & an R. R... & 1,197 \\
\hline & $\mathrm{E}: \& \mathrm{M}$ & 832 \\
\hline & K. C., L. \& & 728 \\
\hline & K. P.R. R........ & 2.586 \\
\hline e bet & K. C., L.\& S. R. R & 1,121 \\
\hline & K. C., Ft. S. \& G. R & 905 \\
\hline & J. C. \& Ft. K. R. R. & 1,366 \\
\hline & A., T. \& S. F.R.R. & 1,527 \\
\hline & A., T. \& $\mathrm{S}$ & 3,339 \\
\hline & U.'P. R. R........ & 1,369 \\
\hline & M., K. \& T. R. R. & 1,238 \\
\hline & S. K. \& W & 783 \\
\hline & A., T. \& S. & 1,220 \\
\hline & F. R. R & 981 \\
\hline & R. R & 2,933 \\
\hline & Sol & 1.301 \\
\hline & F.R.R & 1,271 \\
\hline & F. R. R & 790 \\
\hline & K.'P. R. & 1,135 \\
\hline ity $\ldots \ldots \ldots$ & A., T. \& & 2,475 \\
\hline .. Signal Station. & U.'S.Sign & 2,512 \\
\hline & K. P. R. R ........ & 1,730 \\
\hline & A., T. \& S. F. R. R & \\
\hline -..... & A., T. \& S. F. R. R .... & 1,895 \\
\hline & A., T.\& S. F. R. R ..... & 1,188 \\
\hline$\ldots$ & K. P. R. R . . . & 3,434 \\
\hline .... & K. C., L. \& S. R. R .... & 960 \\
\hline $\begin{array}{l}\mathrm{E} 2 \\
\mathrm{E} d\end{array}$ & $\begin{array}{l}\text { K. C.R.R R } \\
\text { K. C. L. \& S.R. R. }\end{array}$ & $\begin{array}{l}909 \\
962\end{array}$ \\
\hline ville ... & K. P. R. R ......... & $\begin{array}{l}902 \\
783\end{array}$ \\
\hline Effingham ... & U. P. R. R ........ & 1,144 \\
\hline Eldorado .... & A., T. \& S. F. R. I & 1,282 \\
\hline $\begin{array}{l}\text { Elk } \\
\text { Ellinor }\end{array}$ & $\begin{array}{l}\text { K. C. R. R } \\
\text { A., T. \& S. F. R. R } \ldots \ldots \ldots\end{array}$ & $\begin{array}{r}979 \\
1,154\end{array}$ \\
\hline
\end{tabular}




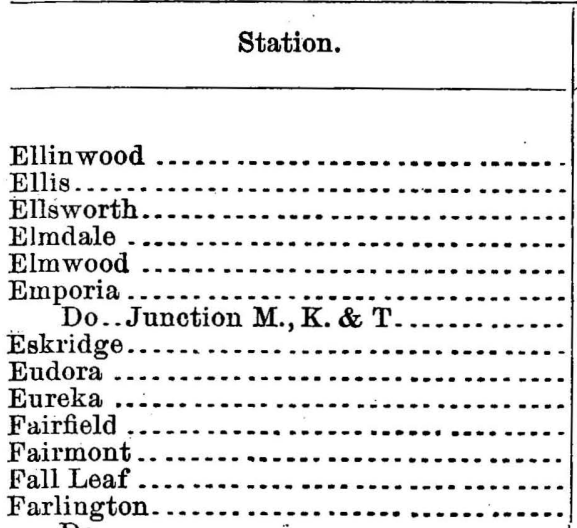

Do
Florence.......

Fontana

Frankfort

Fullerton

Fulton...

Galesburg

Galoa .......

Garden City

Gardner

Garfield

Garnett

Girard

Glasco.

Glendale

Godfrey

Gorham .

Grainfield.

Grantville

Grasshopper Falls

Great Bend

Greenleaf .

Green Ridge.

Grenola

Grinnell

Half Mound

Halstead Junction, $\mathrm{H}$. C. R. R

Hamlin

Hanover

Harker, Fort

Hartford

Hartland

Haweville.

Haves.

Hazleton

Hepler

Hevels

Hiattsville

Hiawatha

Highland

Hillsboro'

Hillsdale

Hoge

Hollenburgh

Holliday

Holton

Homer

Horners

Horton

\begin{tabular}{|c|c|}
\hline Authority. & Elevation. \\
\hline & \\
\hline A., T. \& S. F. R. R & 680 \\
\hline K. P. R. R . & 2,117 \\
\hline K. P. R. R . & 1,538 \\
\hline A., T. \& S. F. & 1,193 \\
\hline $\begin{array}{l}\text { Toner } \\
\text { A., T. \& S. F. R. R }\end{array}$ & $\begin{array}{r}775 \\
1,132\end{array}$ \\
\hline A., T. \& S. F. R. R & 1,132 \\
\hline A., T. \& S. I & 1,403 \\
\hline A., T. \& S. & 811 \\
\hline A., T. \& S. Y & 1,073 \\
\hline A., T. \& S. I & $1,060^{\circ}$ \\
\hline K. P. R. R - & 955 \\
\hline K. P. R. R. & $809^{\prime \prime}$ \\
\hline K. C., Ft. S. \& & 988 \\
\hline $\begin{array}{l}\text { A. \& N. R. } \\
\text { A. T. \& S. I }\end{array}$ & 914 \\
\hline K. C., Ft S. \& G. I & 1, $260 \%$ \\
\hline U. $\mathbf{P}$ R. R & 1,155 \\
\hline A., T. \& S. F & 1,091 \\
\hline K. C., Ft. S. \& G. & 813 \\
\hline M., K. \& T. R. R & 979 \\
\hline A., T. \& S. F. R. R & 1,537 \\
\hline A., T. \& S. F. R. R & 2,827 \\
\hline Smithsonian Inst ... & 800 \\
\hline A., T. \& S. F. R. R & 2,0006 \\
\hline K. C., L. \& & 1,056 \\
\hline K. C., Ft. S & 990 \\
\hline Solomon R. & 1,321 \\
\hline A., T. \& S. & 849 \\
\hline K. C., Ft. S. & 962 \\
\hline K. P. R. R . & 3,318 \\
\hline K. P. R. R & 1,912 \\
\hline K. P. R. R & 2,811 \\
\hline K. P. R. R & $8 \pi 7$ \\
\hline K. C. R. R & 917 \\
\hline A., T. \& S. & 1,841 \\
\hline U. P. R. R & 1,462 \\
\hline Tor & 1,013 \\
\hline$\& W$ & 1,112 \\
\hline$\ldots \ldots$ & 2,904 \\
\hline$\ldots \ldots \ldots$ & 979 \\
\hline A., T. \& S. F. R. R & 1,386 \\
\hline St. J. \& D. C. R. R & 983 \\
\hline St. J. \& D. C. R. R . . . . & 1,228 \\
\hline K. P. R. R . . . . & 1,582 \\
\hline M., K. \& T.R. R & 1,087 \\
\hline A., T. \& S. F. R. R & 3,047 \\
\hline A., T. \& S. F. R. R & 1,104 \\
\hline K. P. R. R. & 1,991 \\
\hline K. P. R. R ..... & 1,114 \\
\hline M., K. \& T. R. R. & 1,002 \\
\hline M., K. \& C. R. R . & 914 \\
\hline M., K. \& T. R. R. & $1,003$. \\
\hline St. J. \& D. C. R. R & 1,094 \\
\hline A. \& N.R. R . . & 893 \\
\hline A., T. \& S. F. R. R & 1,424 \\
\hline K. C., Ft. S. \& G. & 903 \\
\hline K. P. R. R...... & 854 \\
\hline St. J. \& D. C. R. R $\ldots \ldots$ & 1,259 \\
\hline A., T. \& S. & 75.3 \\
\hline K. C. R. R. & 1,026 \\
\hline$\therefore$ n. $\mathrm{n}$. & 1,874 \\
\hline A T \& S F R & $\begin{array}{l}1,314 \\
1,186\end{array}$ \\
\hline
\end{tabular}




\begin{tabular}{|c|c|c|}
\hline Station. & Authority. & Elevation. \\
\hline & & \\
\hline Howard.. & A., T.\& S. F. R. & 1,006 \\
\hline EHowell. & A., T. \& S. F. R. & 2,535 \\
\hline Hugo ..... & Toner $\mathrm{L} \& \mathrm{~L}$. $\mathrm{R}$ & $\begin{array}{l}4.937 \\
.952\end{array}$ \\
\hline $\begin{array}{l}\text { Humboldt...... } \\
\text { Hunds Station }\end{array}$ & 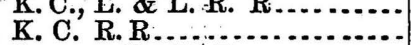 & 835 \\
\hline Hunnewell ... & Sumner Co. R. $\mathbf{R}$ & 1,102 \\
\hline Hutchinson . & A., T. \& S. F. R.R & 1,524 \\
\hline Independence & K. C., L. \& S.R.R & 794 \\
\hline Irving ... & U. P. R. R .......... & 1,152 \\
\hline Junction Cit & K. P. R.R.... & 1,082 \\
\hline Kansas Falls & 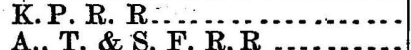 & $\begin{array}{l}1,106 \\
1,491\end{array}$ \\
\hline Kent....... & A., T. \& S. F.R.R. & 2,162 \\
\hline Kingsville.. & K.'P.R.R... & 920 \\
\hline Labette............ & M., K. \& T.R.R... & $\begin{array}{l}864 \\
825\end{array}$ \\
\hline $\begin{array}{l}\text { La Cygne } \\
\text { Ladore... }\end{array}$ & M., K. \& T. C.R.. & $\begin{array}{l}825 \\
909\end{array}$ \\
\hline Lakeview & A., T. \& S. F. R.R & 828 \\
\hline Gakin... & A., T. \& S. F. R.R & 2,989 \\
\hline Larkins ......... & K. C. R. R & 944 \\
\hline Larned.......... & A., T. \& S. F. R.R.. & $\begin{array}{l}1,993 \\
1,932\end{array}$ \\
\hline 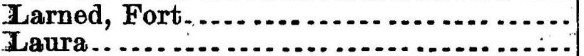 & A. T. \& S. F. R. R & $\begin{array}{l}1,932 \\
1,638\end{array}$ \\
\hline 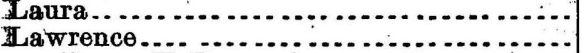 & A., T. \& S. F. R. R & $\begin{array}{r}1,038 \\
849\end{array}$ \\
\hline Do..... K. I & A., T. \& S. F: R.R & 822 \\
\hline Meavenworth . & K. P. R. R........... & 765 \\
\hline Do........Ju & A., T. \& S.F.R.R. & 764 \\
\hline & Med. Dept., U. S. A & \\
\hline Do........Sig & U. S. Signal Office : & 842 \\
\hline Uecompton ...... & A. T. \& S. F. R.R & 844 \\
\hline & Smithsonian Inst - & 825 \\
\hline Kehigh.... & A.; T. \& S. F. R. & 1,520 \\
\hline Le Loup & K. C., L. \& S. R. R & 949 \\
\hline Lenape.... & K. P.R. R.... & 781 \\
\hline Lenexa ....... & K. C., Ft. S. \& G. R. R.. & 1,050 \\
\hline Le Roy ............ & M., K. \& T. R. R $\mathbb{R}_{\mathrm{R}}$ & 994 \\
\hline Liberty ............. & K. C., L. \& S. R.R & $\begin{array}{r}744 \\
1332\end{array}$ \\
\hline $\begin{array}{l}\text { Lindsborg ........ } \\
\text { Linwood...... }\end{array}$ & $\begin{array}{l}\text { S. \& S. W. R.R ... } \\
\text { K. P.R. R. }\end{array}$ & $\begin{array}{r}1,332 \\
789\end{array}$ \\
\hline & $\begin{array}{l}\text { K. P. R. R. } \\
\text { A., T. \& S. F.R.R R }\end{array}$ & $\begin{array}{r}789 \\
1,572\end{array}$ \\
\hline $\begin{array}{l}\text { Little River. } \\
\text { Longton ..... }\end{array}$ & S.K. \& W.R.R... & $\begin{array}{r}1,572 \\
919\end{array}$ \\
\hline Loriy & K. P. R. R. . & 789 \\
\hline Louisville.... & St. J. \& D. C. R. R & , 1,006 \\
\hline $\begin{array}{l}\text { Lowell ...... } \\
\text { Lyons..... }\end{array}$ & $\begin{array}{l}\text { S. C. \& J. R. R } \\
\text { A., T. \& S. F. R. R }\end{array}$ & $\begin{array}{r}823 \\
1,691\end{array}$ \\
\hline McCune.... & M., K. \& C. R. R .... & 924 \\
\hline McIntosh ... & A., T. \& S. F. R. R. & 1,125 \\
\hline McLouth ........... & A., T. \& S. F. R. R & 1,157 \\
\hline McPherson ........ & A., T. \& S. F. R. R & 1,488 \\
\hline Madison ............. & $\begin{array}{l}\text { A., T. \& S. F. R. R } \\
\text { A., T. \& S. F. R. R.. }\end{array}$ & $\begin{array}{l}1,068 \\
1,000\end{array}$ \\
\hline Manville ............ & St. J. \& D. C. R. R & 972 \\
\hline ( & A. T. \& S. F. R. R .......... & 1,299 \\
\hline Marmaton...... & M., K. \& T. R. R............. & 917 \\
\hline Marysville ..... & St. J. \& D. C. R. R $\ldots \ldots \ldots$ & 1,156 \\
\hline $\begin{array}{l}\text { Mathewson .... } \\
\text { Medina ........ }\end{array}$ & K. P. R.R................. & $\begin{array}{l}853 \\
853\end{array}$ \\
\hline Medway ........ & A., T. \& S. F. R. R.... & 3,284 \\
\hline (n) & Ft. S., S. E. \& M. R. R.... & 842 \\
\hline Menoken........ & K. P. R. R $\mathrm{R}_{\mathrm{T}} \ldots$ & 902 \\
\hline ation & $\begin{array}{l}\text { A., T. \& S. F. R. } \\
\text { A., T. \& S. F. R. }\end{array}$ & $\begin{array}{l}964 \\
945\end{array}$ \\
\hline Do..crossing L, S.\& S.T.R.R ........ & A., T. \& S. F. R. R ............. & \\
\hline
\end{tabular}




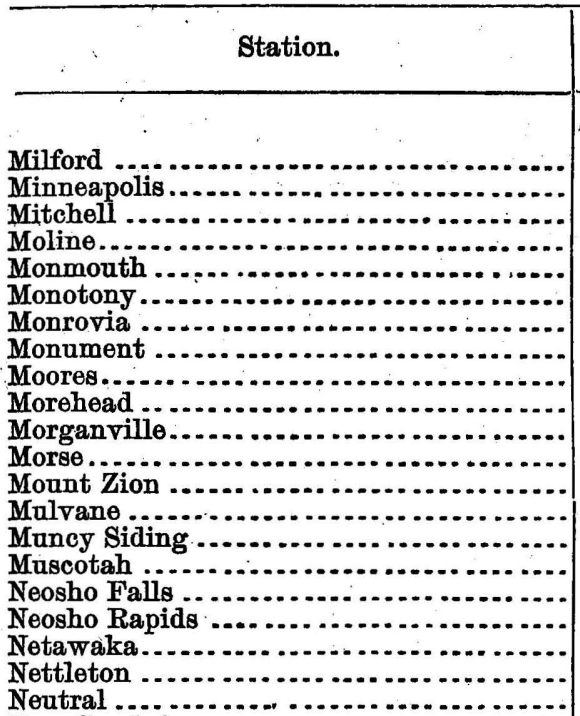

New Cambria

Newman ....

New Salem.

Newton

Nichols

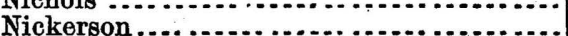

North Topeka, K. P crossing

North Robinson

Nortb Wichita

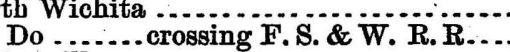
Nortonville

Norway

Norwood

Ochiltreo.

Offerlo

Ogallah

Ogdensburgh

Olathe

Osage

Osage Mission

Osawkee

Oskaloosa.

Oswego

Ottawa, Marias des Cygnes River

Oxford.

Paola.

Pardee

Parker......

Parkersville

Parnell

Parsong Janction

Pauline

Pavilion

Pawneo

Pawnee Rock

Paw Paw

Peabody

Peace

Perry

Perryville

Perth

Petersbuxgh

Peterton
J. C. \& Ft. K. R. R Solomon $\mathbf{R}$. $\mathbf{R}$ A., T. \& S. F. R. R. S. K. \& W. R. R. M. K. \& C. R. R. K. P. R. R

U. P. R. R

K. P. R. $\mathbf{R}$

K. P. R. R

K. C. L. \& L R R

J. C. \& F. K. R. R

A., T. \& S.F.R. R

K.P.R. R

A., T. \& S. F. R. R

K. P. R. R.

U. P. R. R

M., K. \& T. R. R

M., K. \& T. R. R

St. J. \& D. C. R. $\mathbf{R}$

A., T. \& S. F. R. R

K. C., Ft. S. \& G. R. R

K. P.R. R

K. P. R. R

S. K. \& W. R. R

A., T. \& S. F. R. R

A., T. \& S. F. R. R

A., T. \& S. F. R. R

A., T. \& S. F. R. R

St. J. \& D. C. R: R

A., T. \& S. F. R. R

A., T. \& S. F. R. R

A., T. \& S. F. R. R

St. J. \& D. C. R. R

K. C., L. \& S. R. R

K. C., Ft. S. \& G. R. R.

A., T. \& S..F. R. R

K. P. R. R.

K. P. R. R.

A., T. \& S. F: R. $\mathbf{R}$

A., T. \& S. F. R. R

M., K. \& T. R.R

A.; T. \& S. F. R. R

A., T. \& S. F. R. R

M., K. \& T. R. R .

K. C., L. \& S. R. R

S. K. \& W. R. R.

K. C., Ft. S. \& G. R. R.

Toner

\section{Toner}

M., K. \& T. R. R

A., T. \& S. F. R. R

M., K. \& T. R. R ..

A., T. \& S. F. R. R

A., T. \& S. F. R. R...

A., T. \& S. F. R. R

A., T. \& S. F. R. R

A., T. \& S. F. R. R

Toner

Ft. S., S. E. \& M. R. R

K. P R R . . . . R .

A., T. \& S. F. R. R .........

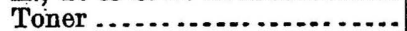

A., T. \& S. F. R. R........
Elevation.

Feet.

1,112

1,257

1,731

1,050

881

3, 774:

1, 054

3, 18k

915.

900 .

1, 238:

1,085 .

1,654

1, 085 .

767

973

980 .

1,076

1,140

2, 112:

862.

1, 189:

861

1,242

1, 438 :

1,001

1,592:

872.

949

1,294

1,302

1,158

1,041

938

1,078

2,261 .

2,367

1, 060

1,030

1,075 .

890

876.

989

895

896.

1,160

854

1,155 .

$92 \mathrm{t}$

1,337

1,039

902:

1, 067:

1, 096.

988

1,939

1,110

1, 349n

1,613

799

852

1, 201

2,224

1,065 


\begin{tabular}{|c|c|c|}
\hline & - Author & Elevation. \\
\hline & & \\
\hline hilips.. & A., T. \& S. F & \\
\hline & A., T: \& S & 2,750 \\
\hline Pleasan & A., T. \& S. F.R.R & $\begin{array}{l}945 \\
849\end{array}$ \\
\hline Pleasanto & K. C., Ft. S: \& G.R. R & $\begin{array}{l}849 \\
860\end{array}$ \\
\hline & K. C.R. R & 1,086 \\
\hline Plym & A., T. \& S. F & 1,135 \\
\hline & $\begin{array}{l}\text { K. C.; L. \& S. R } \\
\text { K. C., Ft, S. \& C }\end{array}$ & $\begin{array}{l}1,052 \\
887\end{array}$ \\
\hline Prin & K. C., L. \& S. R & $\begin{array}{l}887 \\
966\end{array}$ \\
\hline & A., T. \& $\mathrm{s}$ & 1,721 \\
\hline & A., T: \& & 1,097 \\
\hline Rea & A., T. \& S & 1,073 \\
\hline & St., J. \& D & 1,046 \\
\hline & K.P.R.R & 835 \\
\hline Ric & L. \& S. W & 901 \\
\hline & K. C., L. \& & 1,017 \\
\hline & A., T. \&, S. F. R.R. & 2,514 \\
\hline Rid & Toner ..... & 903 \\
\hline & K. P.R.R R & 1,07 \\
\hline $\mathrm{Cre}$ & A., T. \& S. F. R & 1,057 \\
\hline Ron & S. C. R. R.... & 1,216 \\
\hline Ros & K. P. R. R & 933 \\
\hline Rus & $\begin{array}{l}\text { K. P. R. R } \\
\text { St.J. \& D }\end{array}$ & $\begin{array}{r}1,832 \\
892\end{array}$ \\
\hline & St. J. \& D & 1,308 \\
\hline & A., T. \& & $\mathbf{1}, 140$ \\
\hline & 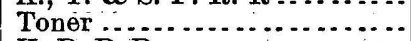 & \\
\hline rg & K. P. R. R.. & 1,000 \\
\hline rye & K. P. R. R $\cdot$. & 955 \\
\hline$\therefore$ & K. P. R. $\stackrel{R}{R} \therefore$ & $\begin{array}{l}1,558 \\
1,225\end{array}$ \\
\hline Sal & $\begin{array}{l}\text { K. P.R. R } \\
\text { K. P. R. R ..... }\end{array}$ & $\begin{array}{l}1,225 \\
1,146\end{array}$ \\
\hline & & 3,425 \\
\hline Sco & M., K. \&T. & 802 \\
\hline & K. C. R. R & 1,025 \\
\hline $\begin{array}{l}\text { Ser } \\
\text { Sed }\end{array}$ & $\begin{array}{l}\text { A., T. \& S. F. R. R. } \\
\text { A.T. \& S.F. R. R. }\end{array}$ & $\begin{array}{l}1,099 \\
1,369\end{array}$ \\
\hline $\begin{array}{l}\text { Sed } \\
\text { Sod }\end{array}$ & $\begin{array}{l}\text { A., T. \& S. F. R. R. } \\
\text { A., T. \& S. F. R. R. }\end{array}$ & $\begin{array}{l}1,369 \\
\mathbf{1}, 368\end{array}$ \\
\hline & A., T. \& & 1,16 \\
\hline & St. J. \& I & 1,15 \\
\hline & $\& \mathrm{D}$ & 90 \\
\hline & A., T. \& S. F. R.R. & 1,09 \\
\hline & A., T.\& S. F. R. R. & 2,87 \\
\hline & K.P.R.R. & 3,10 \\
\hline & $\&$ & $\begin{aligned} 2,925 \\
893\end{aligned}$ \\
\hline & S. C. \& J. & $\begin{array}{l}89.3 \\
817\end{array}$ \\
\hline & A., T. \& S. F. R. R .. & 2,78 \\
\hline & …… & 75 \\
\hline Lake & $\ddot{K} \ddot{R}$ & 1,779 \\
\hline $\begin{array}{l}\text { Sil } \\
\text { Ski }\end{array}$ & $M,, K$ K T R R & 1,226 \\
\hline Solon & Solomon $\mathbf{R} . \mathbf{R}$ & $1,17$. \\
\hline & D....... & 1,172 \\
\hline & R.R $R \ldots$ & 1,179 \\
\hline$\cdots$ & & $\begin{array}{l}1,124 \\
993\end{array}$ \\
\hline ound & $\begin{array}{l}\text { M., K. \& T. R. R } \\
\text { A., T. \& S. F. R. R }\end{array}$ & 2,449 \\
\hline & A., T. \& S. F. R. R & 8,59 \\
\hline$\therefore \cos 2>0$ & A., T. \& S. F. H. R & 1,032 \\
\hline$\cdots$ & $\begin{array}{l}\text { K. C, Ft. S. \& G. R. R } \\
\text { A., T. \& S. F. R. R...... }\end{array}$ & $\begin{array}{r}1,028 \\
-\quad 973\end{array}$ \\
\hline & & 1,27 \\
\hline
\end{tabular}




\begin{tabular}{|c|c|c|}
\hline Station. & Anthority. & Elevation. \\
\hline & & \\
\hline Sterling $: . . .$. & \& S. $\mathrm{H}$ & 1,635 \\
\hline Stilson ........ & K. C., Ft. S. & 909 \\
\hline $\begin{array}{l}\text { Strawn } \\
\text { Strong City. }\end{array}$ & M., K. \& T. R. R & 1,047 \\
\hline $\begin{array}{l}\text { Strong City ...... } \\
\text { Summit "Șanta F' }\end{array}$ & $\begin{array}{l}\text { A., T. \& S. F. R. R. } \\
\text { K. C., L. \& S. R. R }\end{array}$ & 1,172 \\
\hline Syracuse & A., T. \& S. F & 3,218 \\
\hline ecumseh .... & A., T. \& S. I & 860 \\
\hline erra Cotta ....... & K. P. R. R. & 1,470 \\
\hline Thayer ............... & K. C., L. \& & 1,445 \\
\hline iblow:....... & K. P. R. R. & 793 \\
\hline Tonganoxie.. & K.P. R. R.. & 851 \\
\hline Topeka ...................... & A., T. \& S. F & 884 \\
\hline Troy ............................ & St. J. \& D. C. R. R . . . . . & 1,091 \\
\hline Do...Junction ........ & A. \& W. R. R & 1,150 \\
\hline $\begin{array}{l}\text { Turner. } \\
\text { Udall } \ldots \ldots \ldots \ldots \ldots \ldots \ldots\end{array}$ & A., T. \& S. I & 762 \\
\hline $\begin{array}{l}\text { Udall } . . . . . . . \\
\text { Urbana ....... }\end{array}$ & A., T. \& S. & 1,272 \\
\hline $\begin{array}{l}\text { Urbana ....... } \\
\text { Valléy Center }\end{array}$ & $\begin{array}{l}\text { M., K.\& } \mathrm{K} .1 \\
\text { A., T. \& S. }\end{array}$ & 931 \\
\hline Valley Falls Junction, Kansas Cent. R. R.. & $\begin{array}{l}\text { A., T. \& S. } \\
\text { A., T. \& S. }\end{array}$ & $\begin{array}{r}1,339 \\
907\end{array}$ \\
\hline 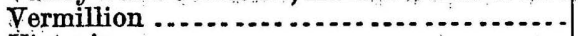 & U.P.R.R. & 1,198 \\
\hline ria .......... & K. P. R. R & 1,928 \\
\hline and ...... & K. C., L. \& & 881 \\
\hline Wabaunsee ............... & A., T. \& S. F & 1,011 \\
\hline Wakarusa ............. & A., T. \& S. F & 946 \\
\hline Wa Keeney .... & K. P. R.R... & 2,456 \\
\hline Wakefield....... & J. C. \& Ft. I & 1,152 \\
\hline $\begin{array}{l}\text { Walker ........ } \\
\text { Wallace....... }\end{array}$ & K. P. R. R. & 1,944 \\
\hline Wallace... & K. P. R. R .. & 3,301 \\
\hline $\begin{array}{l}\text { Walnut } \ldots \ldots . . . . . . \\
\text { Walnut Ridge......... }\end{array}$ & M., K. \& T. & $\begin{array}{r}931 \\
199\end{array}$ \\
\hline 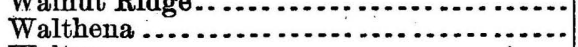 & $\begin{array}{l}\text { A., T. \& S. H } \\
\text { St. J. \& D. C }\end{array}$ & 1,199 \\
\hline Valton...$\ldots \ldots \ldots \ldots \ldots$ & (1) & 1,527 \\
\hline go ........... & K. P. R. R.. & 1,000 \\
\hline Washington.... & St. J. \& D. C & 1,316 \\
\hline .................... & U. P.R. R... & 1,183 \\
\hline Weir City............ & M., K. \& C. R. & 921 \\
\hline Welda ...... & K. C., L. \& S. R. R & 1,098 \\
\hline Wellington & A., T. \& S. F & 1,219 \\
\hline Wellsville.. & K. C., L. \& & 1,041 \\
\hline . & U. P. R. R.- & 1,153 \\
\hline $\begin{array}{l}\text { White City.... } \\
\text { White Cloud. }\end{array}$ & K. \& T. R & 1,476 \\
\hline d $=\ldots$ & \& N. R. R & 858 \\
\hline $\begin{array}{l}\text { Whiting . } \\
\text { Wichita.. }\end{array}$ & R. R . & 1,126 \\
\hline $\begin{array}{l}\text { Wichita.... } \\
\text { Wilder.... }\end{array}$ & $\begin{array}{l}\text { R. } R \\
\text { R. } R\end{array}$ & 1,291 \\
\hline $\begin{array}{l}\text { Wildiamstown } \\
\text { Will }\end{array}$ & R. $\mathbf{R}$ & 770 \\
\hline Wilson............ & (........... & 851 \\
\hline ester & $\begin{array}{l}\text { K. P. } \mathbf{R} \\
\text { K. C. } \mathrm{R}\end{array}$ & 1,684 \\
\hline old ......... & $\begin{array}{l}\text { K. C. R } \\
\text { A. T. T. }\end{array}$ & 1,164 \\
\hline Wolf River Crossi & $\begin{array}{l}\text { A., T. \& S. F. R } \\
\text { St. J. \& D. C. R }\end{array}$ & 1,112 \\
\hline ndotte....... & $\begin{array}{l}\text { St. J. \& D. C. R } \\
\text { Smithsonian In }\end{array}$ & $\begin{array}{l}819 \\
707\end{array}$ \\
\hline Zarah, Fort ... & & 1,870 \\
\hline
\end{tabular}




\section{KENTUCKY.}

\begin{tabular}{|c|c|c|}
\hline on. & Authority. & Elevation. \\
\hline & & Feet. \\
\hline Aden. & O.R. R. I & 626 \\
\hline 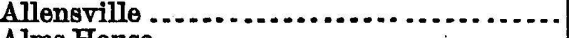 & T. S. \& N.A & 552 \\
\hline 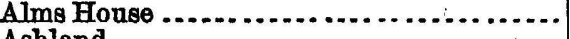 & L., N.S. \& N.A & 425 \\
\hline Ashland . & C. \& O.R.R ... & 544 \\
\hline Athens....... & C. \& O. R. R & 1,006 \\
\hline Anburn..... & L., N. S. \& & 603 \\
\hline Bacon Creek & L., N. S. \& & 619 \\
\hline akers & $\operatorname{lucah}_{\mathrm{C}}$ \& & $\begin{array}{l}477 \\
837\end{array}$ \\
\hline arastow & $\begin{array}{l}\text { C. \& L. R } \\
\text { N. S. \& N }\end{array}$ & 837 \\
\hline & & $\begin{array}{l}635 \\
415\end{array}$ \\
\hline 3eaver & ucah \& & $49 \%$ \\
\hline Belmon & $\mathbf{R} \ldots \ldots$ & 429 \\
\hline$\ldots \ldots \ldots$ & R. R . . . . & 730 \\
\hline$\ldots . . . . . . .$. & R............. & 733 \\
\hline ........... & R........ & 512 \\
\hline Bishops .... & C. S. R. R & 887 \\
\hline . & R. $\mathbf{R}$ & 968 \\
\hline 's. & N.S. S. & 423 \\
\hline oston ..... & L. & 429 \\
\hline Bowling Green .. & . S. 8 & 466 \\
\hline Bracht $\ldots \ldots \ldots$ & C. S. R. R. & 934 \\
\hline , & R. R. & $\mathbf{1 , 3 1 8}$ \\
\hline 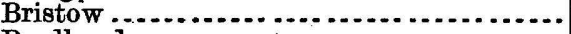 & $\mathbf{R} \ldots \ldots$ & 515 \\
\hline ............ & $\mathbf{R} \ldots \ldots$ & 901 \\
\hline (- & $\mathbf{R} \ldots \ldots$ & 488 \\
\hline$\ldots$ & $\mathbf{R} \ldots . . .$. & 1,012 \\
\hline - $\ldots \ldots \ldots$ & C & 902 \\
\hline & & 1,034 \\
\hline 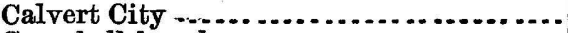 & $\ldots$ & 494 \\
\hline$\ldots \ldots$ & $\ldots$. & 904 \\
\hline & ....... & 621 \\
\hline 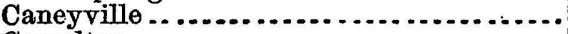 & ........... & 450 \\
\hline 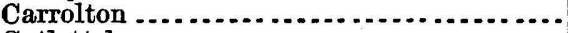 & ... & 472 \\
\hline . & $\ldots . .$. & 544 \\
\hline 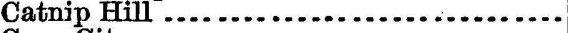 & & 990 \\
\hline .......... & $\mathbf{R}$ & 611 \\
\hline$\ldots$ & R............. & 586 \\
\hline$\ldots \ldots \ldots$ & $\ldots . . .$. & 400 \\
\hline n' & & 688 \\
\hline . & $\mathbf{R} \ldots \ldots . . .$. & 675 \\
\hline (1) & & 851 \\
\hline - & & 671 \\
\hline ngton Junotion ...... & & 435 \\
\hline$\ldots \ldots \ldots$ & ...... & 321 \\
\hline (2) & & 1,023 \\
\hline & & 423 \\
\hline abia ......... & , R. $\mathbf{R} \ldots \ldots$ & 313 \\
\hline M & $\ldots . . . . . . . . . . . . .$. & 309 \\
\hline s Ferry. & $R \ldots \ldots \ldots$ & 954 \\
\hline - & & 968 \\
\hline$\ldots$ & R ....... & 917 \\
\hline & & 923 \\
\hline & & 1,260 \\
\hline illo .......... & & 970 \\
\hline 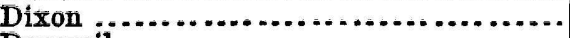 & S. R. R & 936 \\
\hline$\ldots \ldots \ldots \ldots \ldots \ldots \ldots$ & C. & 897 \\
\hline & & 964 \\
\hline
\end{tabular}




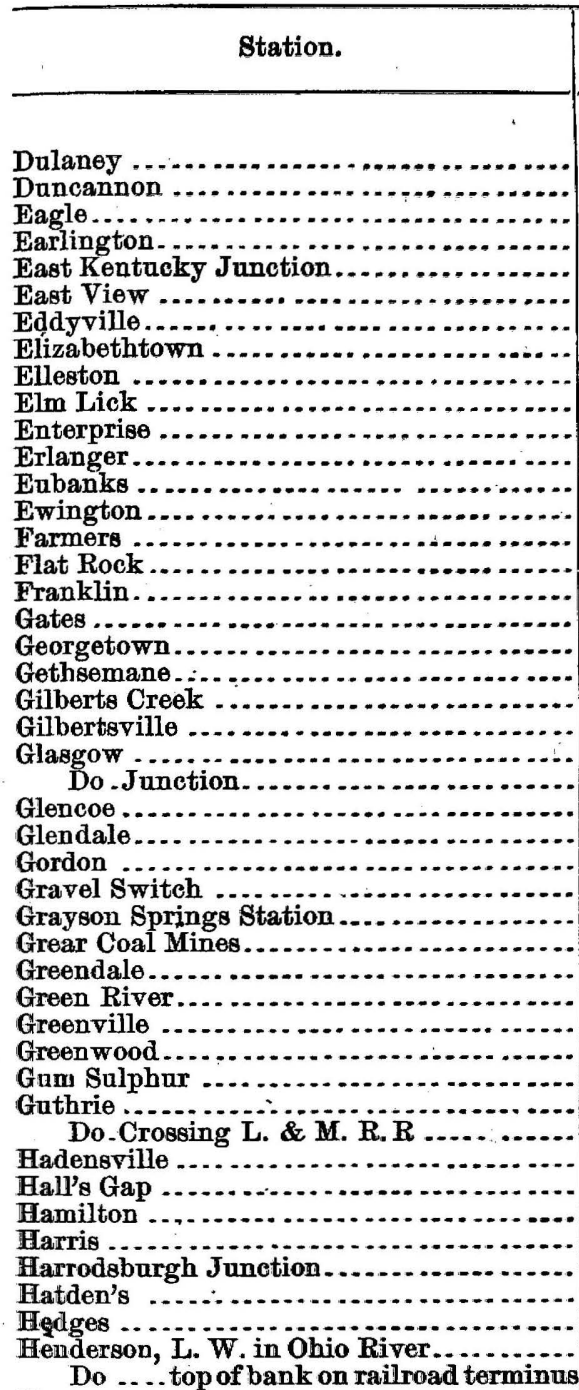

\section{Herat}

Hickman .

High Bridge

Hinton

Hopkinsville

Horee Branch

Do.....

Hubers

Hyattsville

Independence

Jessamine

Jordan

Junction

Junction City

Kelly's Station

Kenton Heights

King's Mountain

\section{Paducah \& E. R. R...........}

L., N. S. \& N. A. R. R .......

L., C. \& L. R. R

E., H. \& N. R. R ...........

C. \& O.R.R.

Paducah \& E. R. R

Paducah \& E. R. R.

L., N. S. \& N. A. R. R .

L., C. \& L. R. R.

Paducah \& E. R. R.

C. \& O. R. R

C. $\mathbf{S}$. R. $\mathbf{R}$

C. $\mathbf{S}$. R. $\mathbf{R}$

C. \& O. R. $\mathbf{R}$

C. \& O. R. R

C. S. R. $R$

L., N. S. \& N. A. R. R

C. \& O. R. R

C. B. R. R.

L., N. S. \& N. A. R. R

L., N. B. \& N. A. R. R

Paducah \& E. R. R.

L., N. S. \& N. A. R. R

L., N. S. \& N. A. R. R

L., C. \& L.R. R.

L., N. S. \& N. A. R. R

Paducah \& E. R. R..

L., N. S. \& N. A. R. R

Paducah \& E. R. R.

C.S. R. R

C. S. R. $\mathbf{R}$

C. S. R. $\mathbb{R}$

Padueah \& E. R. R

C.S. R. R

L., N. S. \& N. A. R. R

L., N. S. \& N. A. R. R

E., H. \& N. R. R
L., N. S. \& N. A. R. R

L., N. S. \& N. A. R. R.

Paducah \& E. R. R..

L., N. S. \& N. A. R. R

C. S. R. R

L., N. S. \& N. A. R. R

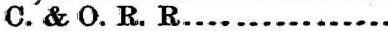

E., H. \& N. R. R ...........

E., H. \&. N. R. R..........

E., L. \& B. S. R. R .........

N. \& N. R. R ..................

C. S. R. R .

C. S. R. R . . . . . . . . . . .

E., H. \& N. R. R...........

Paducah \& E. R. R

Paducah \& E. R. R

L., N. S. \& N. A. R. R........

L., N. S. \& N.A.R.R.

L., N. S. \& N. A. R. R.........

L., C. \& L. R. R

C. S. R. $\mathbf{R}$................

M. \& O. R. R . . . . .

E., L. \& B. S. R. R............

C. S. R. R

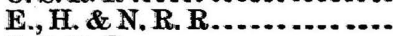

C. S. R. R

C. S. R. R
Elevation.

Feet.

684

987

$47: 3$

370

613

812

487

681

593

507

831

915

1,187

992

668

1,309

689

819

883

456

828

482

778

621

550

638

480

894

709

1, 325

957

927

537

1,207

…25

545

532

991

493

1,007

915

821

976

335

402

619

301

777

958

550

527

782

601

456

1,002
760

760
901

404

618

997

705

845

1,183 


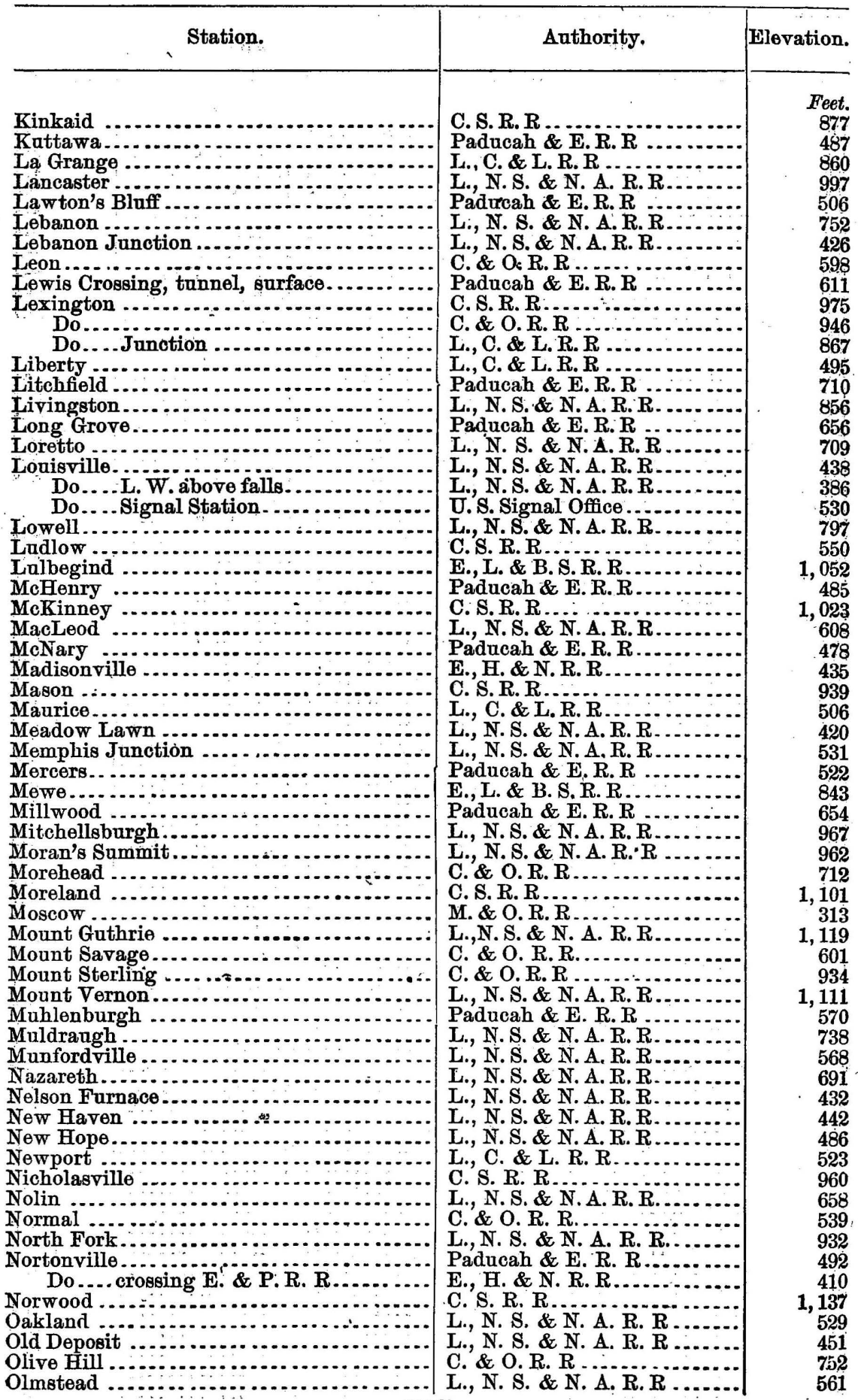




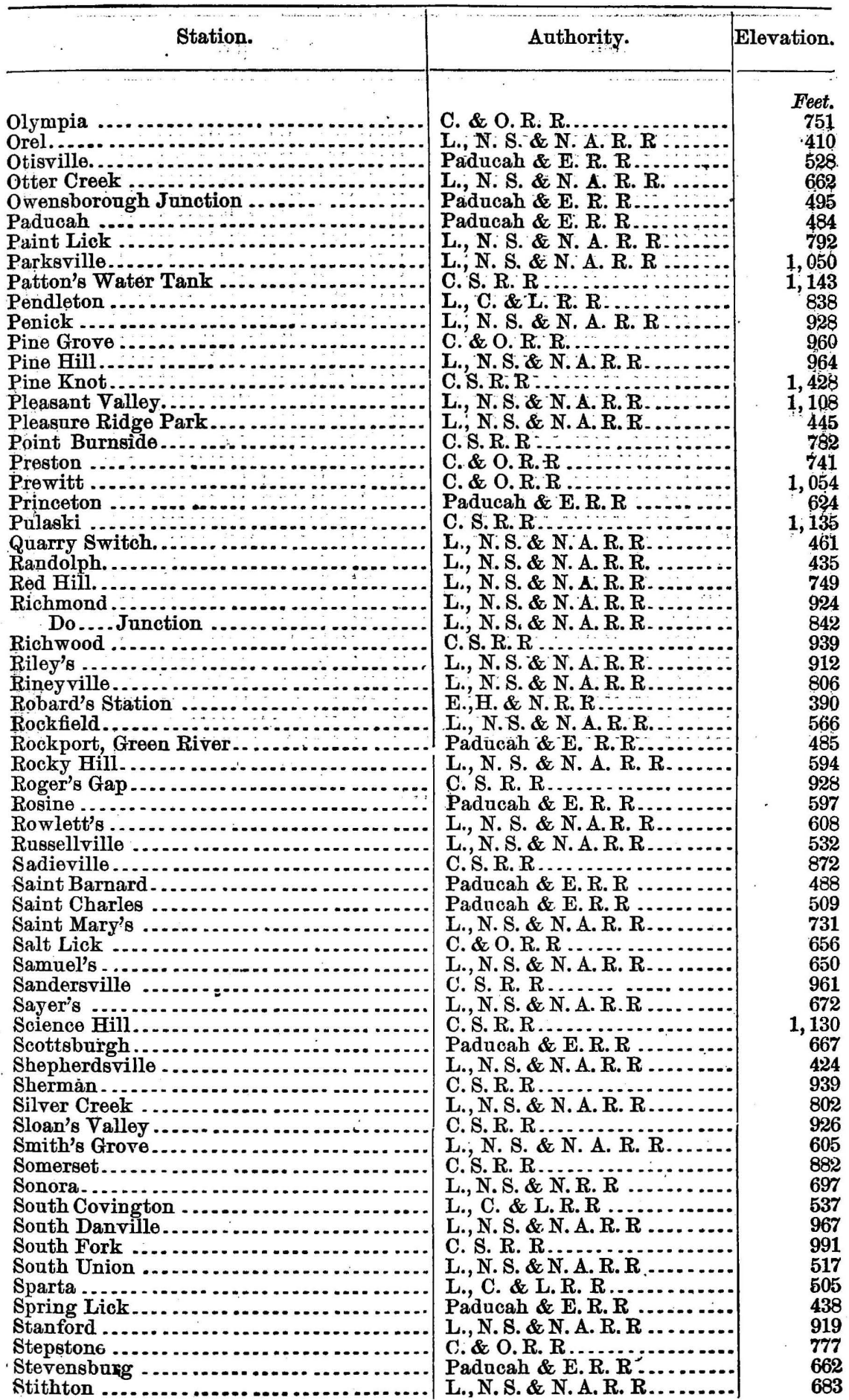




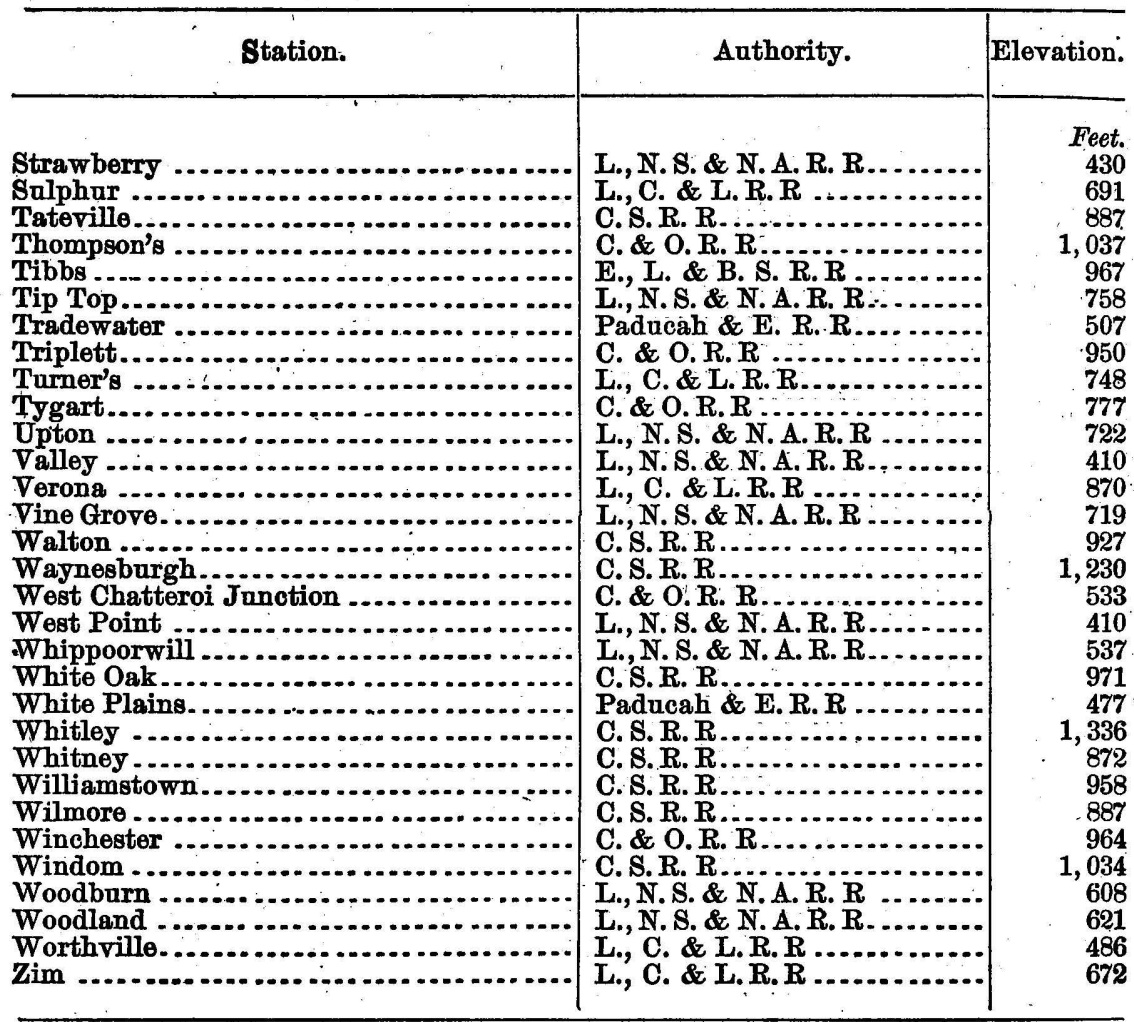




\section{LOUISIANA.}

\begin{tabular}{|c|c|c|}
\hline Station. & Authority. & Flevation. \\
\hline & 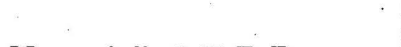 & Eeet. \\
\hline Algiers .......... & Morgan's L. \& T. R. R........ & 11 \\
\hline Baldwin ..................... & Morgan's L. \& T. R. R........ & 16 \\
\hline Bayou Sali. ...... & Morgan's L. \& T. R. R & 10 \\
\hline Berwick .... & Morgan's L. \& T. R. R. & 6 \\
\hline Boouf...... & Morgan's L. \& T. R. R. & 2 \\
\hline Boutte. .......... & Morgan's L. \& T.R. R & 6 \\
\hline Broussardville. & Morgan's L. \& T. R. R & 40 \\
\hline Carencro.......... & Morgan's L. \& T. R. R & 45 \\
\hline Chatahoula..... & Morgan's L. \& T.R. R....... & 6 \\
\hline Chef Menteur .... & 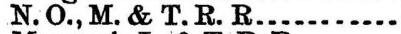 & 9 \\
\hline Des Allemands .. & Morgan's L. \& T. R. R & 4 \\
\hline Franklin......... & Morgan's L. \& T. R. R & 13 \\
\hline Grand Cotieau .. & Morgan's L. \& T. R. R & 51 \\
\hline Jeannerette .... & Morgan's L. \& T. R. R & 22 \\
\hline Lake Charles & Toner ............ & 22 \\
\hline Morgan City . & Morgan's L. \& T. R. ] & 6 \\
\hline New Iberia.. & Morgan's L. \& T. R. F & 22 \\
\hline New Orleans & Jackson \& Gt. N. R. R & 4 \\
\hline Do...... & Pontchartrain R. R.. & 15 \\
\hline Do..... & N. O., M. \& T. R. R. & 16 \\
\hline Do...... Sigr & U. S. Signal Office..... & 52 \\
\hline Opelousas ........ & Morgan's L. \& T. R. R........ & 59 \\
\hline Pattersonville & Morgan's L. \& T. R. R........ & 9 \\
\hline Port Eads, South Pass, Signal Station..... & U. S. Signal Office $\ldots \ldots \ldots \ldots$ & 7 \\
\hline Raceland ..... & Morgan's L. \& T. R. R . . . . & 6 \\
\hline $\begin{array}{l}\text { Rigolets } \\
\text { Shreveport..... }\end{array}$ & 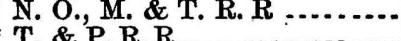 & \\
\hline $\begin{array}{l}\text { Ireveport... } \\
\text { Do...Sign }\end{array}$ & $\begin{array}{l}\text { T. \& P. R. R } \\
\text { U. S. Signal Office }\end{array}$ & $\begin{array}{l}198 \\
227\end{array}$ \\
\hline Slidell ........ & N. O. \& N.E.R.R & 8 \\
\hline Sorrell ....... & Morgan's L. \& T. R. R & 20 \\
\hline Surel ........ & Morgan's L. \& T. R. R. & 20 \\
\hline Terre Bonne & Morgan's L. \& T. R.R. & 16 \\
\hline Tigerville.... & Morgan's L. \& T. R. R & 4 \\
\hline Vermillionvill & Morgan's L. \& T. R. R & 42 \\
\hline Washington : & Morgan's L. \& T. R. R & 42 \\
\hline 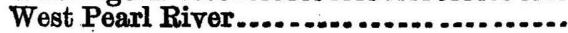 & N. O. \& N. E: R. R ... & 30 \\
\hline
\end{tabular}


MAINE.

\begin{tabular}{|c|c|c|}
\hline Sta & Authority. & Elevation. \\
\hline & & Feet. \\
\hline $\begin{array}{l}\text { Abraham, Mount. } \\
\text { Agamenticus, Mou }\end{array}$ & Geol. Survey of Maine & 3,388 \\
\hline $\begin{array}{l}\text { Agamenticus, Mo } \\
\text { Alleguash, Lake.. }\end{array}$ & Water-pówer of Máine. & $\begin{array}{l}673 \\
950\end{array}$ \\
\hline Attean, Lake ... & Water-power of Maine. & 1,094 \\
\hline Äuburn ....... & Me. Cent. R. R....... & \\
\hline Angusta ...... & Mé. Ceñt. R. R.... & 48 \\
\hline Bancroft .... & European \& N. A.R.R. & 333. \\
\hline Bangor ........... & Mé. Cent. R. R & 13. \\
\hline Do..Thomas Hi & U.S. C. \& G. S... & 242 \\
\hline Do... Exchange st & European \& N. A. R. R & 11 \\
\hline $\begin{array}{l}\text { Do_..Signal Stati } \\
\text { Barker's Mountain... }\end{array}$ & Toner signal Utlce......... & 2,20 \\
\hline Basin Mills.... & European \& N.A.R. R & $2,0.56$ \\
\hline Baskaliegan, & Water-power of Maine. & 400 \\
\hline Belfast, City I & Me. Cent. R. R....... & 29 \\
\hline Bethel .... & Grand Trunk R. R . & 646 \\
\hline Bowdoinhan & Me. Cent. R. R........ & 10 \\
\hline Brewer .... & Bucksport \& Bangor $R$ & 34 \\
\hline Brewer Village & Bucksport \& Bangor $\mathbf{R}$. & 24 \\
\hline Brooks ..... & Me. Cent. R. R...... & 376 \\
\hline Brov & Port. \& Ogden. R. R. & 396 \\
\hline Brunswick... & Me. Cent. R. R....... & 64 \\
\hline Pond. & Graud Trunk R. R.. & 706 \\
\hline & and Bangor & 5 \\
\hline Buck & Bucksport and Bangor $\mathrm{I}$ & 99 \\
\hline Bur & Me. Cent. & 157. \\
\hline & of Maine. & 930 \\
\hline Chambe & of Maine. & 926 \\
\hline Che & f Mäine. & 900 \\
\hline ook, Lake. & er of Maine. & 382 \\
\hline ook, Lake, G & Water-power of Maine. & 449 \\
\hline Chi & jower of Mai & 914 \\
\hline & Me. Cent. $R$. $R$....... & 133 \\
\hline $\mathrm{Cos}$ & in \& N. A. R. R & 111 \\
\hline and $J$ & Me. & 59 \\
\hline Cumberland. & Me. Cent. R. R & 56 \\
\hline & Grand Trunk R. R & \\
\hline Curti & Me. Ce & 331 \\
\hline & N.A.R.R & 379 \\
\hline Junc .. & d Trunk R. R .... & 200 \\
\hline Lake......... & & 88 \\
\hline win Station.. & Port. \& O & 312 \\
\hline & R. R. & 881 \\
\hline & Me. Cen & 327 \\
\hline rt, Signal Station. & 1 Office. & 61 \\
\hline & European \& N. A. R. R. & 400 \\
\hline re Road ............. & Grand Trunk R. R .... & 278 \\
\hline & pean \& N. A.R. R. & 190 \\
\hline uth . & Grand Trnnk R. R .... & 49 \\
\hline River Lake....... & Water-power of Maine. & 660 \\
\hline & an \& N. A. R. R & 435 \\
\hline & Me. Cent. R. R............ & 127 \\
\hline rgh Station........ & Port. \& Ogden. R. R... & 420 \\
\hline 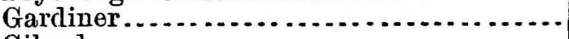 & Me. Cent. R. R ............. & \\
\hline Gilead ............ & Grand Trunk R. R..... & 711 \\
\hline$\cdots$ & Mé. Cent. R. R ... & $\begin{array}{c}106 \\
85\end{array}$ \\
\hline ...... & $\begin{array}{l}\text { European \& N. A. R. R. } \\
\text { Me. Cent. R. R }\end{array}$ & $\begin{array}{r}85 \\
297\end{array}$ \\
\hline Green......... & European \& N. A. R. R & 124 \\
\hline
\end{tabular}




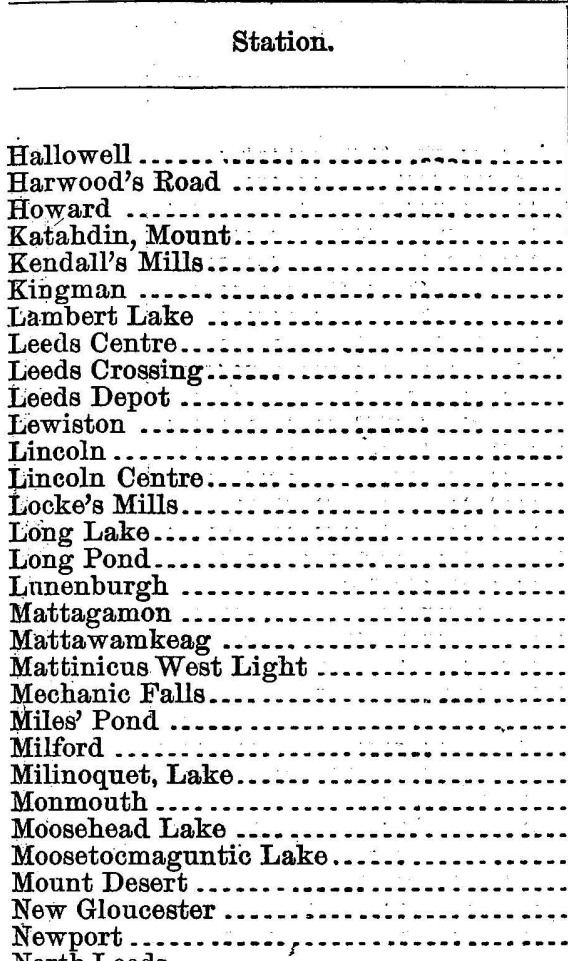

North Leeds........ . . . . . . . . . . . . . . . . . . . .

North Yarmouth .......................

Oaks Hill

Olamon . . . . . . . . .

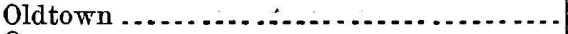

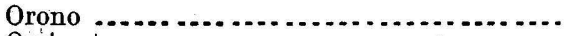

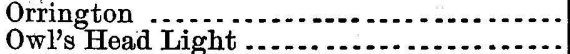

Oxford ................

Paris

Passadumkeag

Pawnal

Pigeon Hill

Pittsfien Hill ..............................

Pleasant, Mount

Pomgocwahem Lake

Portage Lake

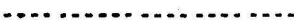

Do

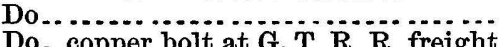

Do.. copper bolt at G. T. R. R. freight

Do . Brownhall Hill ..................

Do..R. R. wharf.

Do . . Signal Station.

Pownal .

Prince Regent's Redoubt

Ragged Mountain

Rangeley Lake.

Richardson, Lake .........................

Richmond

Richville Station (Standish)

Authority. $\mid \begin{array}{r}\text { Elevation } \\ \text { Feet. }\end{array}$

Me. Cent. R. R

54

Me. Cent. R. R ............. 17

U. S. C. \& G. S...........

Eastman ..................... $\quad 5,200$

Me. Cent. R. R ............. 122

European \& N. A. R. R...... $\quad 325$

European \& N. A. R. R...... . $\quad 417$

Me. Cent. R. R ............. 285

Me. Cent. R. R ............. 271

Me. Cent. R. R .............. 266

Me. Cent. R. R .............. 200

European \& N. A. R. R..... 205

European \& N. A. R. R...... 167

Grand Trunk R. R.......... $\quad 718$

Water-power of Maine....... 603

Water-power of Maine........ $\quad 1,094$

Port. \& Ogden. R. R .......... 850

Water-power of Maine........ $\quad 850$

European \& N. A. R. R...... 205

U. S. C. \& G. S............. $\quad 37$

Grand Trunk R. R............ , 298

Port. \& Ogden. R. R ....... 1,026

European \& N. A. R. R...... 114

Water-power of Maine........ $\quad 500$

Me. Cent. R. R .............. 266

Water-power of Maine....... $\quad 1,023$

Water-power of Maine........ $\quad 1,486$

U. S. C. \& G. S. ............ 1,527

Me. Cent. R. R ........... 113

Me. Cent. R. R . . . $20 \ldots \ldots$

Me. Cent. R. R ............ 280

Grand Trunk R. R......... 100

Me. Cent. R. R ............... 125

European \& N. A. R. R..... 121

European \& N. A. R. R..... 88

European \& N. A. R. R..... 71

Bucksport \& Bangor R. R ... 146

U. S. C. \& G. S..............

Grand Trunk R. R .........

Water-powér of Maine ....... $\quad 500$

Grand Trunk R. R...........

European \& iN. A. R. R.....

Grand Trunk R. R..........

U. S. C. \& G. S. ...........

Me. Cent. R. R .............. 210

Guyot..................... $\quad 2,021$

Water-power of Maine....... 914

Water-power of Maine.......

Me. Cent. R. R ............ 13

Grand Trunk R. R.........

Portland \& Roch R. R ....... 10

Port. \& Ogden. R. R......... 16

U. S. C. \& G. S............. 176

U. S. C. \& G. S............ 4

U. S. Signal Office ...........

Grand T'runk R. R.......... 143

U. S. C. \& G. S............ 197

U. S. C. \& G. S............. 1,301

Water-power of Maine ....... 1,511

Water-power of Maine ...... $\quad \mathbf{1 , 4 5 6}$

Me. Cent. R. R ............. 77

Port. \& Ogden, $\mathbf{R} . \mathbf{R}$........ 315 


\begin{tabular}{|c|c|c|}
\hline & Authority. & Flovation. \\
\hline & & \\
\hline Ripogenus, Lake .. & Water-power of & 878 \\
\hline $\begin{array}{l}\text { Schoodic Lake } \\
\text { Sebago, Lake ....... }\end{array}$ & $\begin{array}{l}\text { Water-power of Maine } \\
\text { Water-power of Maine }\end{array}$ & $\begin{array}{r}300 \\
-247\end{array}$ \\
\hline Do & Port. \& Ogden. R. R .. & 274 \\
\hline $\begin{array}{l}\text { Do.......... (wate } \\
\text { ebattis Mountain . }\end{array}$ & $\begin{array}{l}\text { Port. \& Ogden. R. R } \\
\text { U. S. C. \& G. S...... }\end{array}$ & 801 \\
\hline bec Lake ........ & Water-power of Main & 375 \\
\hline uth Gardiner... & ent. $\mathbf{R} \cdot \mathbf{R}$ & 25 \\
\hline outh Orrington & $\begin{array}{l}\text { Bucksport \& Bangor } \\
\text { Grand Trunk R R }\end{array}$ & $\begin{array}{r}34 \\
389\end{array}$ \\
\hline $\begin{array}{l}\text { South } \\
\text { South }\end{array}$ & $\begin{array}{l}\text { Grand Trunk R. K } \\
\text { European \& N. A. R. }\end{array}$ & $\begin{array}{l}0.99 \\
194\end{array}$ \\
\hline nd-Cross I & Water-power of Main & 587 \\
\hline & Wa & 580 \\
\hline ins (n: & Por & 299 \\
\hline Station & Port. \& Ogden. R & 305 \\
\hline 1510 & Me. C & 305 \\
\hline bor L & U. $\mathrm{s}$ & \\
\hline Tho & Me. C & 267 \\
\hline Tom & Eu & 375 \\
\hline Tops & Me. & \\
\hline g Lake. & $\overline{\mathbf{W}} \mathbf{a}$ & 1,256 \\
\hline Unit & & 223 \\
\hline orough & Ear & 394 \\
\hline Vea: & Eư & 110 \\
\hline Wal & Me. & 180 \\
\hline le ....... & Me. & 117 \\
\hline We & . R. I & 68 \\
\hline ldwin Station. & Po & 364 \\
\hline hel ........... & Gra & \\
\hline Westbrook ................................ & Me. Cent. R. R.. & 101 \\
\hline Do.... Station, orossing Port. \& Roch. & & \\
\hline & & \\
\hline almouth $\ldots . . . . \ldots \ldots \ldots$ & & 43 \\
\hline is ...... & Gr: & 483 \\
\hline ad Light ................ & & \\
\hline ck Station (Gorham). & & 213 \\
\hline & A. R. I & 467 \\
\hline Station (south) .... & . R. R .. & 131 \\
\hline Win & N. A. R. R. & 201 \\
\hline Wir & Me. & 220 \\
\hline Woodford's ..... & $\mathbf{R} \ldots \ldots \ldots$ & 61 \\
\hline Wood Lake ..... & of Maine. & 1,094 \\
\hline Wytopitlock ................................ & an \& N. A. R. R. & 343 \\
\hline 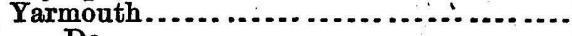 & Me. Cent. & 88 \\
\hline Do & Grand Tru & \\
\hline & & \\
\hline
\end{tabular}




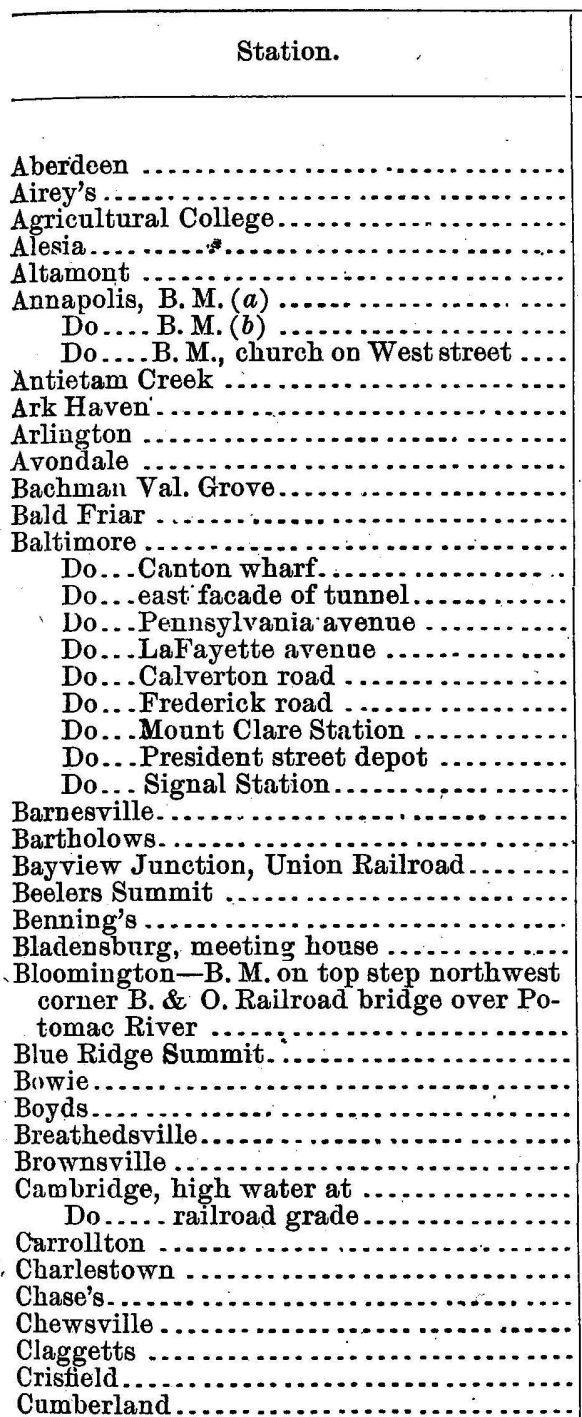

Do.................................

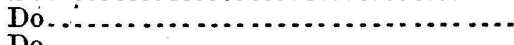

Do............ on coping stone of feedlock west terminus of Chesapeake and Ohio Canal

Do..... Basin Chesapeake and Obio Canal ......................

Dickerson's. . . . . . . . . . . .

Double Pike Creek . ... . . . . . . . . . . . .

Eakil's Mill
P. W. \& B. R. R ........... Feet.

D. \& D. R. R ............. 74

U. S. C. \& G. S........................

B. \& H. R. R........... 702

B. \& O.R. R ............. 2,620

U. S. C. \& G. S $\ldots \ldots \ldots \ldots \ldots \ldots . \ldots$

U. S. C. \& G.S $\ldots \ldots \ldots \ldots \ldots \ldots$
U. S. C. \& G.S $\ldots \ldots \ldots \ldots \ldots \ldots$

W.Md. R.R .................

C. \& P. D. R. R........... 80

W. Md. R. R .............. 420

W. Md. R. R ............. $\quad 540$

B. \& H. R. R ............. 741

C. \& P. D. R. R ........... 78

B. \& O.R. R ............. 24

W.MA. R. R .............. 20

B. \& P. R. R ............. 67

B. \& P. R.R .......... 131

B. \& P. R. R . . . . 164

B. \& P.R. R ............ 133

B. \& P.R. R .............. 154

B. \& P. R. R ............... 68

P. W. \& B. R. R ........... 9

U. S. Signal Office............ $\quad 45$

B. \& O.R. R .............. $\quad 500$

B. \& O. R. R ............... 463

P. W. \& B. R. R ........... $\quad 36$

B. \& O. R. R .......... 678

B. \& P. R. R .............

U. S. C. \& G. S .......... 51

U. S. C. \& G. S

... 1,008

B. \& P. R. R ............... $\quad \begin{array}{r}1,373 \\ 149\end{array}$

B. \& O.R. R .............. 418

B. \& O.R. R .............. 472

B. \& O. R. R ............ 535

D. \& D. R. R ............ 57

D. \& D.R.R ............. 62

W. Md. R. R .............. $\quad 550$

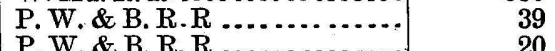

P. W. \& B. R. R $\ldots \ldots \ldots$
W. Md. R. R ..............

B. \& O.R.R R........... 619

E. S. R. R................ . 3

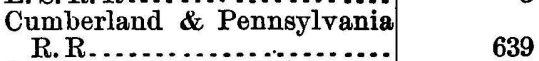

Cumberland Turnpike ........ 635

Penna. R. R................. 642

B. \& O.R.R R............ 639

U. S. C. \& G. S .............. 624

C. \& O. Canal ................ 609

C. \& O. Canal ...............

W. Md.R.R .............. 300

B. \& O.R. R .............. 412

Bull. $5 \square 9$ 


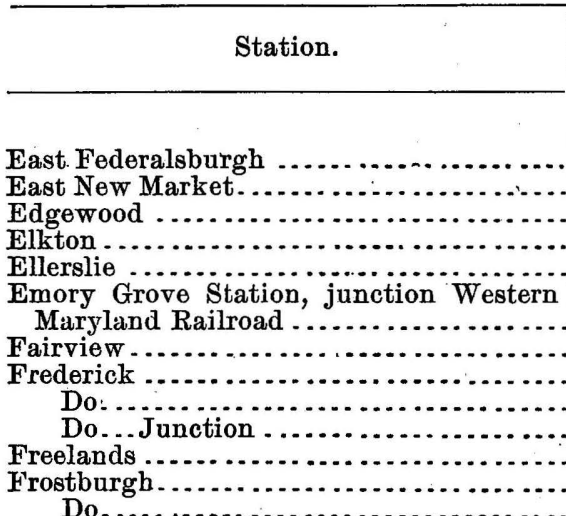

Gaithersburgh

\section{Galt's}

Georgetown

Germantown

Glendale

Glen Falls .

Glen Morris

Glyndon

Graceham

Green Mount

Greenwood .

Grimes

Gunpowder Bridge

Hagerstown ...

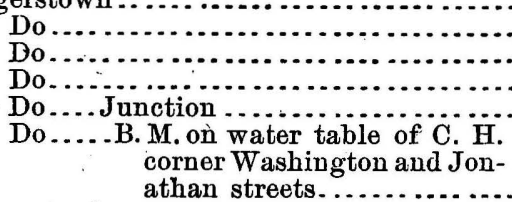

Hampstead . ........................... 53

Hancock, B. M. on coping stone of lock 53, aqueduct .

Do...B. M. on coping stone middle north side Chesapeake and Ohio Canal aqueduct ..........

Harmans.............................

Harmony Grove

Havre de Grace ........................

Ilowardsville

Hurlock's

Junction

Keedysville .

Knowles

Ladiesburgh

Lanham's

Linkwood

Linsted

Linwood

Lonaconing

Loys

Magnolia

Marriott

Martinsburg, junction with B.\& O.R.R

Maryland Heights

Mechanicstown

\begin{tabular}{|l|} 
Authority. \\
Elevation.
\end{tabular}

D. \& D.R.R

D. \& D. R. R..

P. W. \& B. R. R

P. W.\& B.R. R

B. \& O. R. R.

B. \& H. R. R

B.\& H.R.R

Pa.R.R.

W. Md. R. R

B. \& O.R. R

N. C. R.R.

Cumberland Turnpike .......

Cumberland \& Penusylvania

R. R

B. \& O. R.

Pa.R.R

Pa.R.R R.

B.\& O.R.R

B. \& P. R. R

W.Md. R. R

W. Md. R. R

W. Md. R. $R$

W. Md. R. R

B. \& H.R.R

W. Md.R.R

Shenandoah Valley R. R ....

P. W. \& B. R. R ..............

Shenandoah Valley R. R

W. Md.R.R Cumberland Valley R. R

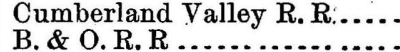

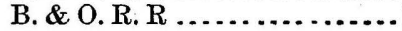

U.S. C. \& G.S

Feet.

71

30

28

729

652

676

280

415

264

596

1,890

1,920

516

486

290

422

150

375

580

600

400

820

425

382

978

566

520

572

527

249

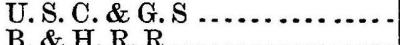

552

880

U. S. C.\& G.S

474

U. S. C. \& G. S

B. \& P. R. R . . . . . .

Pa. R. R

Pa. R. R

W. Md. R. R

D. \& D. R. R

W. Md. R. R

B. \& O. R. R

B. \& O. R. R

Pa. R. R

B. \& P. R. R

D. \& D. R. R

U. S. C. \& G. S. ...............

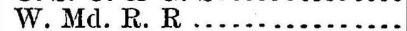

Cumberland \& Pennsylvania

R. R .

W. Md. R. $\mathrm{K}$

P. W \& B R R . . . . . . .

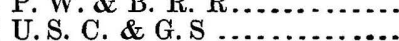

Cumberland Valley $\mathbf{R} . \mathbf{R}$.....

U. S. C. \& G. S.............

W. Md. R. R. 


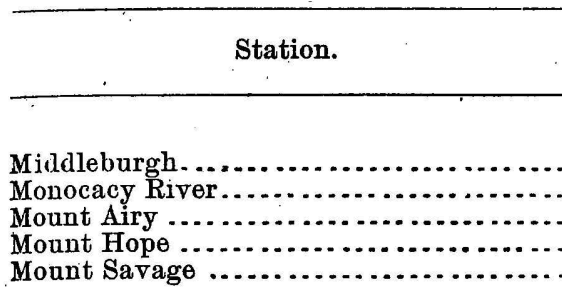

Do ....... Junction

Do....... Station

Mount Vernon

New Midway

New Windsor

North East.

Oalkland

Oakland Do.. (A.\& E. R. R)

Owing's Mills

Parktou

Patapsco

Patuxen

Pen Mar

Perrymansville

Perry ville, Port Deposit Br. $\mathrm{R} . \mathrm{R}$

Piedmont

Pikesville

Point of Rocks, junction with main line $B$. \& O.R.R

Port Deposit . . . . . . . . . . . . . . .

Potomac Bridge

Princess Anne.

Relay Station

Rohrersville

Ritter's

Rockville

Rocky Ridge.

Sabillasville

Saint James

Salisbury

Seabrook

Sharpsburgh

Silver Spring

Snaithsburgh

Soper .......

Stabler

Stimmer's Run

Stoney Run

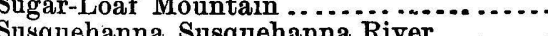

Sykesville

Tannery

Taylor.

Terra Cotta ...............................

Timber Grove

Timonium

Tuscarawa

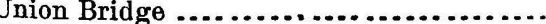

Washingten Junction

Webb

Westminster

Authority. Elev

W. Md. R. R

W. Md. R. R .............. 280

B. \& U. R. R .............. 813

W. Md. R. R .............. 440

Cumberland \& Pennsylvonia $\mathbf{R} \mathbf{R}$

B. \& O. R. $\mathbf{R} \ldots \ldots \ldots$

1,206

687

703

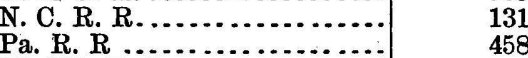

Pa. R. R . . . . . .

P. W. \& B. R. R.......... 43

B. \& O. R. R............ $\quad 2,371$

W. Md. R. R ............. 380

U. S. C. \& G. S.............. 164

B. \& P. R. R ............ 160

W. Md. R. R ............. 480

N. C. R. R ............... 420

W. Md. R. R .............. $\quad 360$

U. S. C. \& G. S........... 84

B. \& P. R. R .

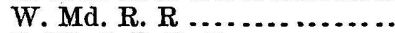

P. W. \& B. R. R

85
1,200
46

P. W. \& B.R. R............ 21

Cumberland \& Pennsylvania

R. R ................ $\quad 928$

W. Md. R. R ............. 435

B. \& O. R. R

C. \& P. D. R. R ...............

Cumberly.nd Valley R. R....

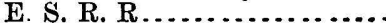

B. \& O. R. R..............

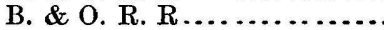

Pa. R. R ...

B. \& O. R. R

W. Md. R. R

W. Mr. R. R

Shenandoah Valley $R . R$

E. S. R. R.

B. \& P. R. R

B. \& P. R. R

Shenandoah Valley R. R

B. \& O. R. R

W. Md. R. R

U. S. C. \& G. S

B. \& P. R. R

U. S. C. \& G. S

P. W. \& B. R. R

B. \& P. R. R

U. S. C. \& G. S.

P. W. \& B. R. R

B. \& O. R. R

Pa. R. R ...

W. Md. R. R .

U. S. C. \& G. S

B. \& O.R.R.

W. Md. R. R

B. \& O. R. R

W. Md. R. R

B. \& O. R. R . . . . . . . . .

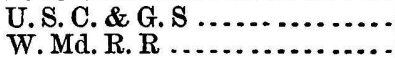

229

9
376

14

66

591

309

421

370

1,120

467

26

159

166

445

336

750

469

167

571

24. 50 .

61

1,281

16

410

493

.610

95

168

550

381

224

350

229

236

700 


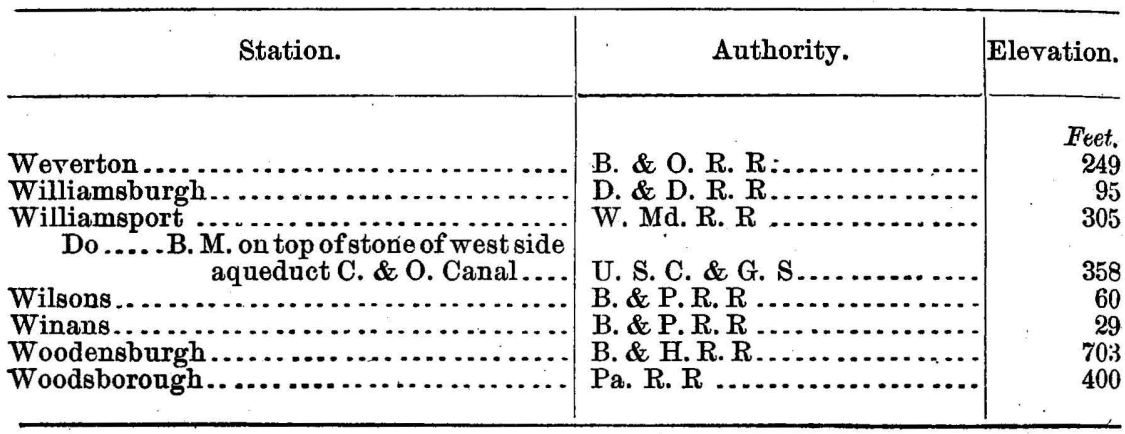




\begin{tabular}{|c|c|c|}
\hline Station. & Authority. & Elevation. \\
\hline 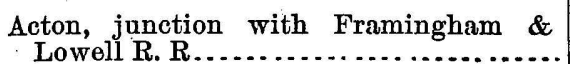 & $N A \& B, R$ & Feet. \\
\hline Allston & Boston \& Albany R. R & 24 \\
\hline Amherst . . . . . . . . . . . & N. L. N.R.R...... & 258 \\
\hline Do.... & Mass. Cent, R. R . & 235 \\
\hline Ashburnham ......... & Vt. \& Mass. R. R. & 1,106 \\
\hline Do...... Summit . & Cheshire R. R .... & 1,084 \\
\hline Ashland $\ldots \ldots \ldots \ldots \ldots \ldots$ & Boston \& Albany $\mathrm{R}$ & 184 \\
\hline Athol, junction with Vt. \& Mass. R. R.... - & Spring., Athol \& North & 546 \\
\hline Attleborough $\ldots \ldots \ldots \ldots \ldots \ldots$ & Bost: \& Prov. R. R. & 129 \\
\hline Auburndalo ....................... & Boston \& Albany $R$ & 63 \\
\hline Ayer Junction ............... & Worcester \& Nashv & 230 \\
\hline Bald Peak $\ldots \ldots \ldots \ldots \ldots \ldots \ldots \ldots$ & Guyot.................... & 2,624 \\
\hline Baldwinsville $\ldots \ldots \ldots \ldots$ & Boston \& Aibany $\mathrm{K}$ & 901 \\
\hline $\begin{array}{l}\text { Do.:- } \\
\text { Bandvill }\end{array}$ & Fitchburg R. R.... & 891 \\
\hline $\begin{array}{l}\text { Bandville } \\
\text { Hardwell. }\end{array}$ & Mass. Cent. R. R ...... & 405 \\
\hline . & N. H. \& Northampton & 238 \\
\hline $\begin{array}{l}\text { Barnstade... } \\
\text { Barre Plains }\end{array}$ & Boston \& Albany R. R & $\begin{array}{r}57 \\
588\end{array}$ \\
\hline Barrett's Junction, crossing $\mathrm{N}$ & Spring., Atbol \& Northern R.R & 329 \\
\hline (n. & Bost. \& Albany R. R ..... & 1,207 \\
\hline Belchert & Mass. Cent. R. R... & 460 \\
\hline Belmon & Mass. Cent. R. R. & 73 \\
\hline Bernardstown.. & Conn. River R. R .. & 359 \\
\hline Billerica & Boston \& Albany R. R & 110 \\
\hline Blackston & Prov. \&. Worces. R. R . & 197 \\
\hline 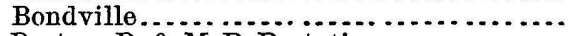 & Spring., Athol \& Northern R.R & 350 \\
\hline $\begin{array}{l}\text { Boston, B. \& M. R. R. station } \ldots . . . . . \\
\text { Do.sidewalk in front of B. \& M. R. R. }\end{array}$ & Boston \& Maine R. R ........ & 14 \\
\hline station...$\ldots \ldots \ldots \ldots \ldots \ldots$ & Engil & 8 \\
\hline Cent. R. R. station & Mass. Cent. R. R. & 10 \\
\hline Do... Fitchburg R. R. station . & Fitchburg R: R . & 11 \\
\hline Do.sidewalk in front of Fitchburg $R$. & & \\
\hline R. station $\ldots \ldots \ldots$ & City Engineer.. & 6 \\
\hline 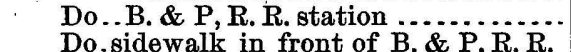 & Bost. \& Prov. R. R & 6 \\
\hline & City Engineer.... & \\
\hline Do...B. \& A. R. R. station ............... & Boston \& Albany R & 10 \\
\hline Do.. sidewalk in front of B.\& A. R. R. & & \\
\hline & City Enginee & 7 \\
\hline L. R. R. station $\ldots \ldots \ldots \ldots \ldots$ & Boston \& Lowell R. I & 12 \\
\hline ralk in front of B. \& L. R. R. & & \\
\hline & City Engir & \\
\hline Do.s & U.S. Signal Office & 142 \\
\hline Boylston. & Bost. \& Prov. IR. R .... & 31 \\
\hline Braggs & Bostou \& Albany R. R. & 243 \\
\hline Brightor & Boston \& Albany R. R. & 24 \\
\hline Brookfield & Boston \& Albany R. R. & 606 \\
\hline Brookline... & Boston \& Albany R. R. & 15 \\
\hline Do....Junction & Boston \& Albany R. R. & 9 \\
\hline Brooks' Farm .... & well R. R. & 30 \\
\hline Jun & Bost. \& Prov. R. R.. & 101 \\
\hline Char & $\mathrm{A} \& \mathrm{All}$ & 888 \\
\hline & Boston \& Albany R. R. & 595 \\
\hline Chico & n. River R. R... & 79 \\
\hline don Hills. & Bost. \& Prov. R. R...... & 50 \\
\hline Clinto & Worcester \& Nashua R. R ... & 309 \\
\hline Coldbrook .. & Boston \& Albany R. R....... & 672 \\
\hline College Hill:. & Boston \& Lowell R. R...... & 31 \\
\hline Concord .... & Fitchburg R. R............. & 135 \\
\hline Cordaville ......... & Boston \& Albany R. R...... & 248 \\
\hline 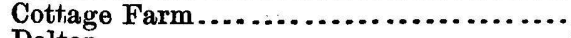 & Boston \& Albany R. R. & \\
\hline & Boston \& Albany R. R. & \\
\hline
\end{tabular}




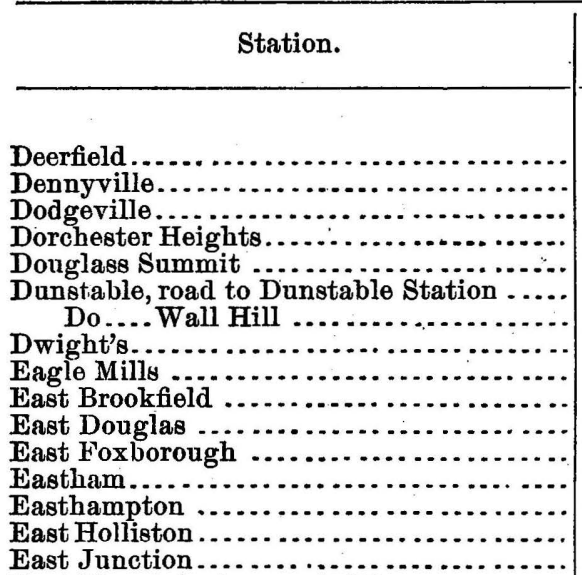

East Mountain (copper bolt)

Do.......... (south peak)

East Woburn ...........................

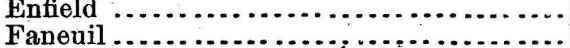

Farnum's .............................

Fitchburgh

Florence.

Forest Hills.

Foxborough

Framingham Center

Franklin.

Wremont or N. Wareham.

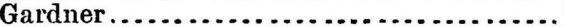

Gilbertville .

Grafton

Grantville

Greenfield

Green Lodge

Greenwich.

Greenwich Village

Greenwich Village

Greylock

$$
\text { Do. }
$$

Groton

Do.Cow Pond Brook

Groton Junction

$$
\text { Do. }
$$

Hadley

Harrard

Hatfield

Haverhill

Haydensville

Heath

Hinsdale.

Holden

Holliston

Holyok

Holyoke, Mount

Hoosac, Mount

Do....... east summit over tunnel

Hoosac Tunnel, east portal.

Do........ central shaft

Do........ west portal .

Hudson .... :

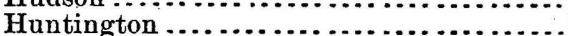

Hyannis

Hyde Park.

Indian Orchard
Conn. River R. R

Mass. Cent. R. R ............

Bost. \& Prov. R. R...........

U. S. C. \& G. S

Boston \& N. Y. R. R

N., A. \& B. R. R

N., A. \& B. R. R

Mass. Cent. R. R

Boston \& Albany $\mathrm{R}, \mathrm{R}$

Boston \& N. Y. R: R

Bost. \& Prov. R. R

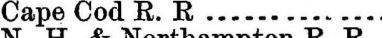

N. H. \& Northampton R. R..

Boston \& Albany R. R

Bost. \& Prov. R. R

Appalachian Club

Appalachian Club

Boston, Lowell \& Nashua R. R

Boston \& Albany R. R.

Prov. \& Worces. R. R ......

Fitchburg R. R

N. H. \&. Northampton R. R.

Bost. \& Prov. R. R..........

Framingham \& Mansfield R. R

Bost.,Clinton \& Fitchburg R.R

Boston \& N. Y. R. R.

Cape Cod R. R

Fitchburg $R$ R

Boston \& Albany R. R

Bostou \& Albany R. R.

Boston \& Albany R. R

V., M., T. \& G. R. R Spring., Athol \& Northern Spring., Athol \& Northern R.R Appalachian Club

Worcester \& Nashua R. $\mathrm{R}$

N., A. \& B. R. R............ Worcester \& Nashua R. R.

Fitchburg R. R.............. Mass. Cent. R. R

Worcester \& Nashua $\mathrm{R}$. R... Conn. River R. R

Boston \& Maine R. R....... N. H. \& Northampton R. R. Bost. \& Prov. R. R.......... Boston \& Albany R. R. Mass. Cent. R. R Boston \& Albany R. R Conn. River R. R...........

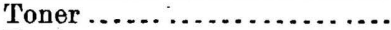
Toner V., M., T. \& G. R. R. R Troy \& Greenfield R. R Troy \& Greentield R. R Troy \& Greenfield R. R Mass, Cent, R. R Boston \& Albany R. R...... Cape Cod R. R ............. Bost. \& Prov. R. R ........... Boston \& Albany R. R.
Elevation.

Feet,

221

625

114

140

630

51

88

245

555

621

517

211

18

169

169

108

2, 276

1,917

33

415

24

284

430

273

36

284

188

292

60

1,009

546

368

140

181

54

455

484

3,535

3,505

303

82

230

234

122

288

148

33

432

25

1, 431

758

191

94

1,120

2,510

2,269

759

819

759

221

373

25

51

241 


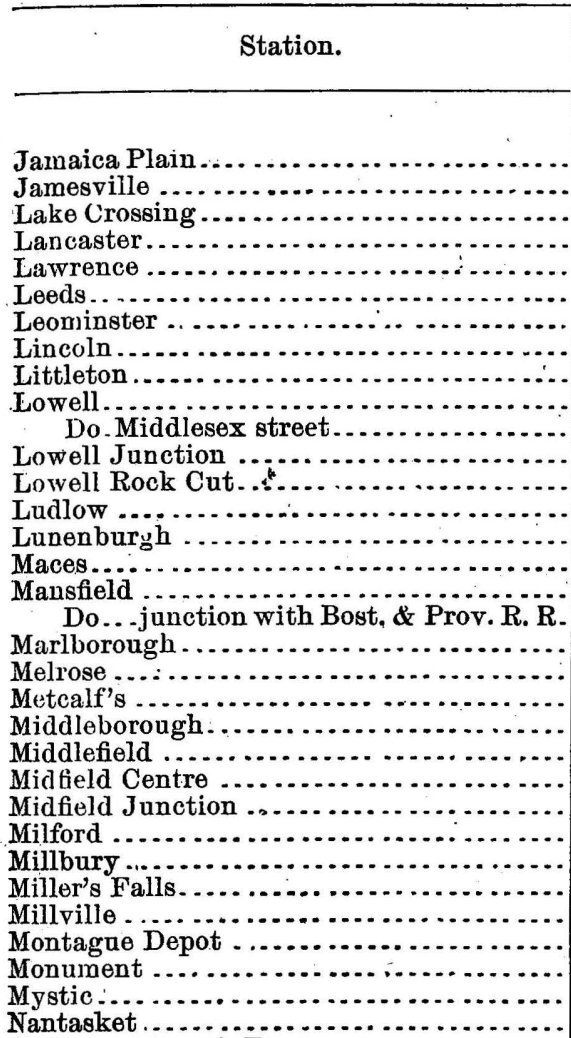

Do... Tower's Tavern .

Nantucket Cliff, Nantucket.

Natick

Newburyport ..............................

New Salem.

Newton

Newtonville

North Adams

Northampton

North Ashburnham

North Billerica.

Northborough

Northbridge

North Dana.

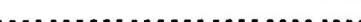

North Eastham .........................

North Hatfield .........................

North Wilbraham .......................

North Wrentham......................

Oakdale ..............................

old Furnace .............................. Do

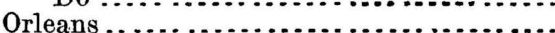

Palmer ...............................

Pepperill ..............................

Pittsfield .............................

Pratt's Junction..........................

Quinsigamond Lake

Readville ...............................

Rice's Crossing.

Richmond
Bost. \& Prov. R. R.

Boston \& Albany R. R

Boston \& Albany R. R......

Worcester \& Nashua R. R...

Manch. \& Lawrence R. R

N.H. \& Northampton R. R.

Fitchburg R. R..............

Fitchburg R. R............

Fitchburg R. R

Bost:, Lowell \& Nashua R. R.

Boston \& Lowell R. R .......

Lowell \& Andover R. R......

Boston \& Lowell R. R ........ Spring. $\curlyvee$ Athol \& North'n R. R. Fitchburg R. R.............. Lowell \& Andover R. R...... Bost. \& Prov. R. R ........... Framingham \& Mansfi'd R. R. Bost., Clinton \& Fitch. R. R. Boston \& Maine R. R.

Boston \& Albany R. R........ Cape Cod R.R.

Boston \& Albany R. R........ Framingham \& Mansfi'd R. R. Framingham \& Mansfi'd R. R. Boston \& Albany R, R. Boston \& Albany R. R........ N. L. N. R. R ............... Prov. \& Worces. R. R ........ V., M., T. \& G. R. R ......... Cape Cod R.R.............. Boston \& Lowell R. R .

U.S.C.\& G.S ................. U.S. C. \& G.S .................. U.S. C. \& G. S .................... Boston \& Albany R. R.:.:.

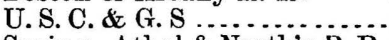
Spring., Athol \& North'n R. R. Boston \& Albany R. R....... Boston \& AlbanyR. R . . . . . . . Troy \& Greenfield R. R ...... Conn. River R. R.

Cheshire R. R .

B. \& I R. R . Bost., Clinton \& Fitch. R. R. Prov. \& Worces. R. R........ Spring., Athol \& North'n R. R. Cape Cod R. R

Conn. River R. R ................ Boston \& Albany R. R...... Boston \& N. Y. R. R .......... Worcester \& Nashua R. R .. Mass. Cent. R. R ............. Boston \& Albany R. R....... Cape Cod R. R ................. Boston \& Albany R. R...... Worcester \& Nashua R. R... Bos' on \& Albany R. R. Boston, Clinton \& Fitchburg R. R.

Toner

Bost. \& Prov. R. R ........... Boston \& Albany R. R.

Boston \& Albany R. R.
Elevation.

Feet. 33

564

168

259

65

356

373

205

228

99

103

108

239

375

151

169

172

378

62

242

96

918

171

136

244

393

292

208

129

20
128

11

58

170

124

522

46

57

686

125

1,066

120

296

269

462

. 54

172

264

222

382

561

581

44

336

205

1,013

429

361

61

106

1,047 


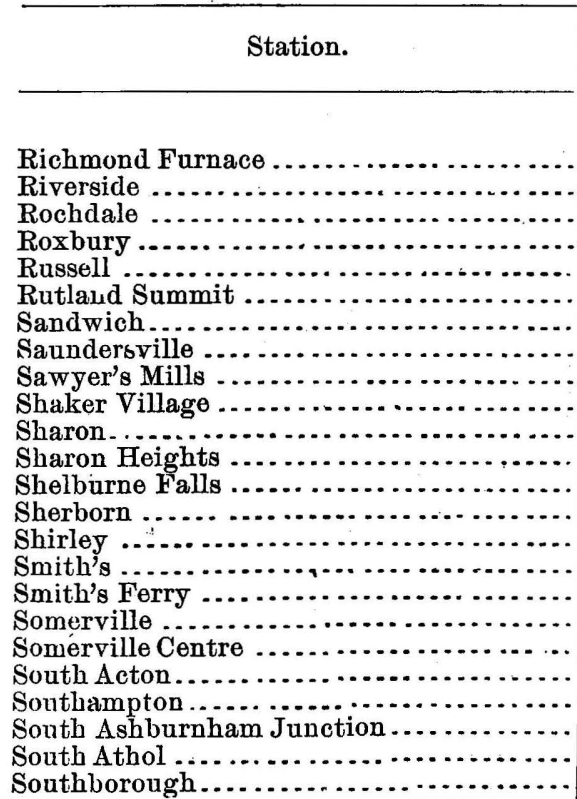

South Dedham

South Deerfield............................

Soutb Framingham

South Lancaster.

South Lawrence

South Spencer.

Southville

South Walpole

South Wellfleet

Southwick ..................................

South Worcester.

Springfield

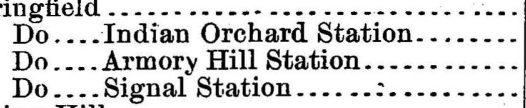

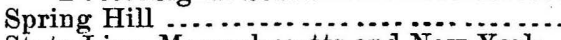

State Line, Massachusetts and New York.

Sterling Junction

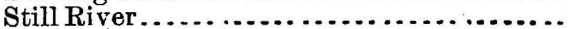

Stone Hill, Williamstown ..................

Stony Brook..........................

Sudbury ..............................

Summit Station ......................

Sunderland

Sunset Rock ............................

Sutton ...............................

Tatham .............................

Templeton ...........................

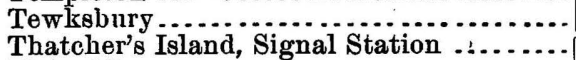

Thorndike .............................

Tom, Mount ...........................

Turner's Falls ........................

Uxbridge

Wachusett Mountain Walpole Junction, B., H. \& E. R. R.........

Waltham

Ware

Wareham

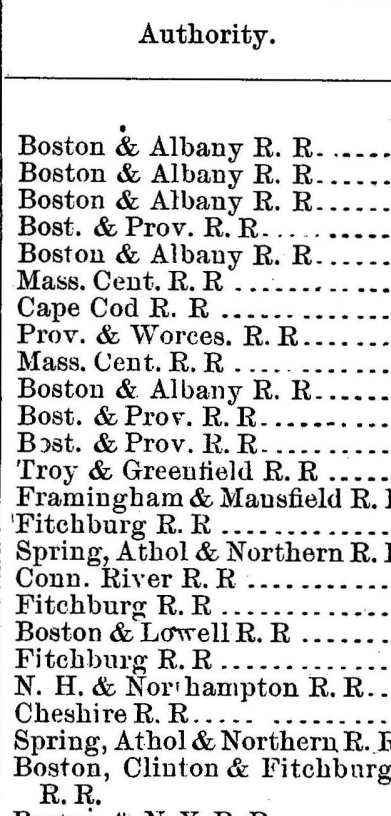

Elevation.

Bostun \& N.Y.R.R.........

Conn. River R. R.............

Boston \& Albany R. R ........

Worces. \& Nashua R. R .......

Boston \& Maine R. R

Boston \& Albany R. R ........

Boston \& Albany R. R .......

Framingham \& Mansfield R.R.

Cape Cod R. R.

N. H. \& Northampton R. R....

Prov. \& Worces. R. R

Boston \& Albany R. R .......

Boston \& Albany R. R.......

N.Y. \& N. E. R. R ..........

U. S. Signal Office............

Cape Cod R. R..............

Boston \& Albany R. R........

Worcester \& Nashua R. R....

Worcester \& Nashua R. R ...

Appalachian Club

Fitchburg R. R .

Mass Cem R R ......

Mass. Cent. R. R ..............

Worces. \& Nashua R. R ......

Mass. Cent. R. R ..............

Appalachian Club............

Pruv. \& Worces. R. R........

Boston \& Albany R. R........

Boston \& Albany R. R.........

Lowell \& Andover R. R......

U. S. Signal Office .

Boston \& Albany R. R ........

Conn. River R. R ..............

N.H. \& Northampton R. R ...

Prov. \& Worces. R. R........

Guyot .............................

Fitchburg R. R ..............

Boston \& Albany R. R ........

Cape Cod R. R...............

Feft.

64
721

20

273

1,043

13

311

301

1,114

220

260

4:30

171

282

424

122

8
34

199

195

1,014

561

307

141

207

163

272

49

704

263

227

14

242

463

70

175

204

120

. 24

914

436

243

1,086

91

127

619

120

1, 992

331

129

964

124

48

345

123

170

231

2, 018

157

45

489 


\begin{tabular}{|c|c|c|}
\hline Station. & Authority. & Elevation. \\
\hline Wayren & & Feet. \\
\hline 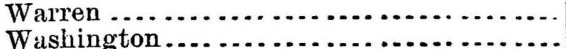 & Boston \& Albany R. R & 593 \\
\hline 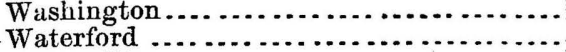 & Boston \& Albany R. R & 1,437 \\
\hline 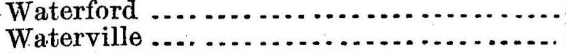 & Prov. \& Worces. R. R .. & 188 \\
\hline 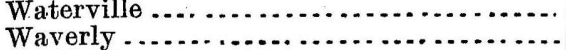 & Boston \& Albany R. R & 918 \\
\hline $\begin{array}{l}\text { Waverly } \\
\text { Wayland } \ldots \ldots \ldots\end{array}$ & 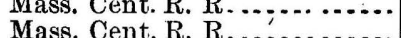 & 132 \\
\hline $\begin{array}{l}\text { Wayland } 1 . \ldots \\
\text { Wellesley }\end{array}$ & 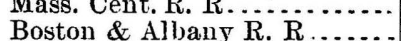 & 113 \\
\hline Wellfleet & Cape Cod R.R........ & $\begin{array}{r}140 \\
14\end{array}$ \\
\hline West Acton ...... & Fitchburg R. R... & $2 * 1$ \\
\hline West Barnstable. & Cape Cod R. R ... & 37 \\
\hline West Berlin ....... & Mass. Cent. R. R ... & 295 \\
\hline Westborough .... & Boston \& Albany R. R. & 300 \\
\hline West Boylston .... & Worcester \& Nashua R. R & 442 \\
\hline 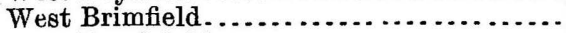 & Boston \& Albany R. R.... & 391 \\
\hline 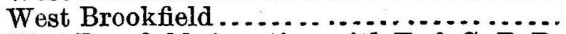 & Boston \& Albany R. R & 604 \\
\hline West Deerfield, junction with T. \& G. R. R. & Mass. Cent. R. R .... & 267 \\
\hline 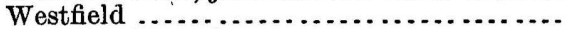 & Boston \& Albany R. R & 147 \\
\hline 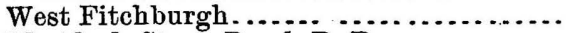 & Fitchburg R. R..... & 539 \\
\hline Westford, Stony Brook R. R ......... & N., A. \& B. R. R.... & 102 \\
\hline 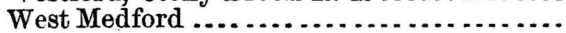 & Boston \& Lowell R. R & 21 \\
\hline Westminster .... & Fitchburg R. R...... & 704 \\
\hline West Newton ... & Boston \& Albany R. R. . & 55 \\
\hline Weston ......... & Mass. Cent. R. R ....... & 65 \\
\hline 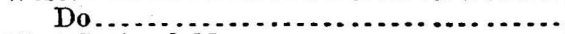 & Fitchburg R. R ...... & 95 \\
\hline West Springfield. & Boston \& Albany R. $\mathrm{B}$ & 100 \\
\hline West Walpole........ & Boston \& N. Y. R. R . & 211 \\
\hline .................... & Spring, Athol \& Northern R. R & 387 \\
\hline . & Boston \& Albany R. R... & 514 \\
\hline Whately .......... & Conn. River R. R ....... & 186 \\
\hline Whitins.......... & Prov. \& Worces. R. R. & 268. \\
\hline Whitney's... & Boston \& Albany R. R .... & 166 \\
\hline Williamsburgh $\ldots \ldots \ldots \ldots \ldots \ldots \ldots \ldots \ldots$ & N. H. \& Northampton R. R .. & 492 \\
\hline Williams College, old observatory ........ & Appalachiain Club........... & 708 \\
\hline Williamstown & Troy \& Greenfield R. R. & 580 \\
\hline Williamsville ...... & Boston \& Albany R. R .... & 833 \\
\hline 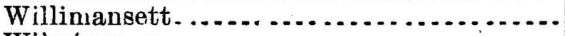 & Conn. River R. R ........... & 77 \\
\hline Wilmington.... & Boston \& Lowell R. R & 97 \\
\hline Wilmington Junction . ................. & Boston \& Maine R. R . & 88 \\
\hline 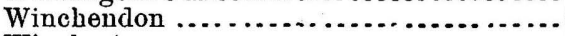 & Boston \& Albany R. R ... & 993 \\
\hline Winchester ....... & Bost., Lowell \& Nashua R. R. & 27 \\
\hline Wood's Holl, Signal Station ............ & Signal Office................ & 35 \\
\hline Worcester, Union Station ............. & Boston \& Albany R. R. & 473 \\
\hline Do.....Lincoln Square Station ...... & Boston \& Albany R. R, & 475 \\
\hline Do..... South Worcester Junction ..... & Boston \& Albany R. R...... & 477 \\
\hline Yarmouth $\ldots \ldots \ldots \ldots \ldots \ldots \ldots$ & Cape $\operatorname{Cod}$ R. R .............. & 40 \\
\hline
\end{tabular}


MICHIGAN.

\begin{tabular}{|c|c|c|}
\hline Stat & Authority. & Elevation. \\
\hline & & Feet. \\
\hline $\begin{array}{l}\text { Abronia........ } \\
\text { Ada } . . . . . . .\end{array}$ & $\begin{array}{l}\text { L. S. \& M. S. R. R } \ldots . . \\
\text { D., G. H. \& M. R. R... }\end{array}$ & $\begin{array}{l}714 \\
666\end{array}$ \\
\hline Adrian............. & L., S. \& M. S. R. R..... & 806 \\
\hline 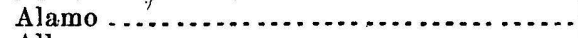 & K. \& S. H. R. R & 765 \\
\hline llba........... & G. R. \& Ind. R. R & 1,177 \\
\hline Albion......... & Mich. Cent. R. R & 943 \\
\hline Allegan ... & L. S. \& M. S. R. & 708 \\
\hline Allen's ................ & L. S. \& M. S. R. I & 1,064 \\
\hline $\begin{array}{l}\text { lpena, Signal Station } \\
\text { madore.............. }\end{array}$ & $\begin{array}{l}\text { U. S. Signal Office } \\
\text { P. H. \& N. W. R. }\end{array}$ & $\begin{array}{l}609 \\
756\end{array}$ \\
\hline Inderson ... & P. H. \& N. W. R. & $\begin{array}{l}7: 6 \\
742\end{array}$ \\
\hline or. & Mich. Cent. R. R. & 771 \\
\hline & L.S. \& M.S.R.R & 772 \\
\hline Arland ....... & G.R.V.R.R..... & 9.31 \\
\hline Ashton..... & G. R. \& Ind. R. R & 1,152 \\
\hline a $\ldots \ldots \ldots$ & C. \& G.T.R.R .. & 898 \\
\hline Augusta $\ldots$ & Mich. Cent. R. R. & 789 \\
\hline $\begin{array}{l}\text { Austin Lake....... } \\
\text { Avery's .......... }\end{array}$ & G.R. \& \& Ind. R. R & $\begin{array}{l}862 \\
655\end{array}$ \\
\hline $\begin{array}{l}\text { Avery's ........ } \\
\text { Bagley ...... }\end{array}$ & $\begin{array}{l}\text { Mich. Cent. R. R . } \\
\text { J., L. \& S. R. R . }\end{array}$ & $\begin{array}{r}655 \\
1,285\end{array}$ \\
\hline om, Geodetic Station & U.' S. Lake Survey & $\begin{array}{r}1,200 \\
822\end{array}$ \\
\hline 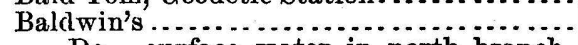 & Ft. W., J. \& S. R & 1,011 \\
\hline Do... surfa & & \\
\hline Balmer's ..... & $\begin{array}{l}\text { Ft. W., J. \& S. R. } \\
\text { P.H.\& N.W.R.R }\end{array}$ & $\begin{array}{r}1,002 \\
744\end{array}$ \\
\hline Bancrof & C.\& G. T.R.R... & $\begin{array}{l}744 \\
856\end{array}$ \\
\hline & M., H. \& O.R.R.. & 936 \\
\hline & Ft. W., J.\& S. R. R & 1,067 \\
\hline Lake... & Mich. A. L. R.R. & 768 \\
\hline & \& $M$ & 946 \\
\hline Batt & Mic & 819 \\
\hline Bay & D. & 592 \\
\hline action & & 604 \\
\hline$\cdots .$. & $\begin{array}{l}\text { G. R. } \\
\text { J. L }\end{array}$ & $\begin{array}{r}616 \\
1954\end{array}$ \\
\hline $\begin{array}{l}\text { Beav } \\
\text { Bedfc }\end{array}$ & J., I & $\begin{array}{r}1,254 \\
809\end{array}$ \\
\hline 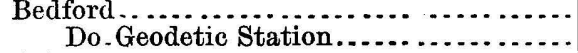 & $\begin{array}{l}\text { Mich. } \\
\text { U. S.I }\end{array}$ & $\begin{array}{l}809 \\
679\end{array}$ \\
\hline th $\ldots \ldots \ldots$ & G. I & 661 \\
\hline & C. & 777 \\
\hline & & 900 \\
\hline ont ... & K. \& & 700 \\
\hline & D., $G$ & 687 \\
\hline , Geodetic Station & U.'S & $9 \varsigma 9$ \\
\hline Big R & G. I & 916 \\
\hline D & D., I & 1,001 \\
\hline & D.', & 779 \\
\hline & D., & 930 \\
\hline Bliss & L., S.\& M. S. R. I & 684 \\
\hline & & 687. \\
\hline ............... & K. \& S.H.R.R. & 731 \\
\hline & G. R. & 1,416 \\
\hline Mine ....... & M., H. \& C & 1,562 \\
\hline & G.R.V.R.] & 734 \\
\hline & & 712 \\
\hline ... & G. R & 757 \\
\hline 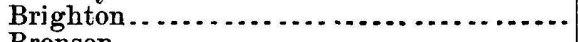 & D., L. \& N.R.R.... & 929 \\
\hline $\begin{array}{r}\text { ronson } \\
\text { Do.Geodetic Station }\end{array}$ & $\begin{array}{l}\text { L.S. \& M. S. R. R ... } \\
\text { U.S. Lake Survey .. }\end{array}$ & $\begin{array}{l}927 \\
998\end{array}$ \\
\hline & & \\
\hline
\end{tabular}




\begin{tabular}{|c|c|c|}
\hline Station. & Authority. & Eleration. \\
\hline & & Feet. \\
\hline 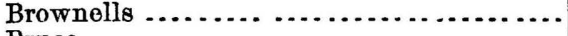 & K. \& S. H. R. R... & 938 \\
\hline 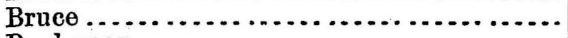 & M., H.\& O.R. R & 1,258 \\
\hline$\ldots \ldots \ldots \ldots . . .6$. & Mich. Cent. R. R. & 733 \\
\hline Bunday, Geodetic S & U. S. Lake Survey & 1,284 \\
\hline Burlington ........... & Mich. A. L. R. R.. & 947 \\
\hline Geodetic Station... & U. S. Lake Survey & 817 \\
\hline Burr Oak ... & L. S. \& M. S. R. R. & 896 \\
\hline Byers ............... & G. R. \& Ind. R. R & 902 \\
\hline 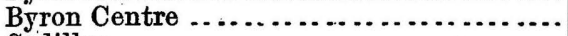 & L. S. \& M. S. R. R & 740 \\
\hline$\ldots \ldots \ldots \ldots \ldots \ldots \ldots$ & G. R. \& Ind. R. R & 1,292 \\
\hline ................... & G.R.V:R.R .... & 799 \\
\hline an. $\ldots \ldots \ldots \ldots \ldots$ & M. R. R. R .... & 1,228 \\
\hline Calvin, Geodetic Station ........ & U. S. Lake Surve & 1,011 \\
\hline$\because \ldots \ldots \ldots$ & C. \& G. T. R. R & 817 \\
\hline lisle, Geodetic St & U. S. Lake Survey & 914 \\
\hline Carp .................. & M., H. \& O.R. R & 1,400 \\
\hline Carpenters.......... & D. \& B. C.R.R.. & 801 \\
\hline Carp River Furnace & M., H. \& O.R. R & 625 \\
\hline$\ldots \ldots \ldots \ldots \ldots \ldots$ & C., D. \& C. G.T.J & 768 \\
\hline$\ldots \ldots \ldots \ldots \ldots \ldots \ldots \ldots$ & Mich. A. L. R. R. & 881 \\
\hline$\ldots \ldots \ldots \ldots \ldots \ldots \ldots$ & G. R. \& Ind. R. R & 846 \\
\hline$\ldots \ldots \ldots \ldots \ldots \ldots \ldots$ & Mich. A. L. R. R. & 813 \\
\hline ................. & Mich. Cent. R. R. & 892 \\
\hline Chadwick....... & D., L. \& N. R. R . & 856 \\
\hline Champion.......... & M., H. \& O. R. R & 1,597 \\
\hline$\ldots \ldots \ldots \ldots \ldots \ldots \ldots$ & M., H. \& O. R. R ... & 1,715 \\
\hline Charlesworth ..... & L., S. \& M. S. R. R ......... & 916 \\
\hline Charlotte........ & G. R. V.R.R................ & 906 \\
\hline$\ldots \ldots \ldots \ldots \ldots \ldots$ & Mich. Cent. R. R. & 913 \\
\hline . & J., L. \& S. R. R .. & 1,203 \\
\hline …............... & J., L. \& S. R. R ............. & 632 \\
\hline$\ldots \ldots \ldots \ldots \ldots$ & G.R.V.R.R . . . . & 883 \\
\hline$\ldots \ldots \ldots \ldots \ldots \ldots$ & D., L. \&. N.R.R & 874 \\
\hline Child's Mill ... & G. R. \& Ind. R. R & 680 \\
\hline Chowlay ..... & D., M. \& M. R. R. & 617 \\
\hline$\ldots \ldots \ldots \ldots \ldots \ldots \ldots$ & Mich. A. L. R. R. & 966 \\
\hline$\ldots \ldots \ldots \ldots \ldots \ldots$ & M., H. \& O. R. R . & 1,544 \\
\hline$\ldots \ldots \ldots \ldots \ldots \ldots$ & D., G. H. \& M. R. R. . & 1,008 \\
\hline$\ldots \ldots \ldots \ldots \ldots \ldots$ & L. S. \& M. S. R. R .. & 905 \\
\hline$\ldots \ldots \ldots \ldots \ldots \ldots$ & M., H. \& O. R. R. . & 1,427 \\
\hline$\ldots \ldots \ldots \ldots \ldots$ & M., H. \& O.R. R. & 1,661 \\
\hline$\ldots \ldots \ldots \ldots \ldots \ldots$ & L. S. \& M. S. R. R & 832 \\
\hline , & J., L. \& S.R.R. & 767 \\
\hline - & D., L. \& N. R. R & 867 \\
\hline$\ldots \ldots \ldots \ldots \ldots$ & L., S. \& M. S. R. & 983 \\
\hline . & D. I. \& N.R.R. & 777 \\
\hline$\ldots \ldots$ & Mich. A. L. R. R. & 838 \\
\hline$\ldots \ldots \ldots \ldots \ldots \ldots$ & K. \& S. H. R. R. & 682 \\
\hline a Mine .............. & M. H. \& O.R.R. & 1,510 \\
\hline iaville.... & D.\& B. C. R. R. & 777 \\
\hline$\ldots \ldots$ & D., L. \& N. R. R. & 91 \\
\hline$\ldots \ldots \ldots \ldots \ldots \ldots$ & Mich. Cent. R. R. & 78 \\
\hline 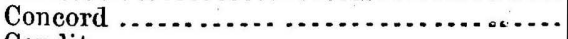 & Mich. A. L.R.R.... & 987 \\
\hline & $\mathrm{S}, \mathrm{R}, \mathrm{R}$ & 952 \\
\hline & G. R. \& Ind. R. R. & 912 \\
\hline 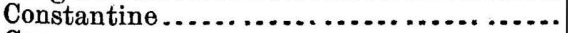 & L. S. \& M. S. R. R......... & 803 \\
\hline Cooper... & G. R. \& Ind. R. R......... & 772 \\
\hline Do.............. & L. S. \& M. S. R. R.......... & 749 \\
\hline Coopersville ...... & D., G. H. \& M. R. R ........ & 646 \\
\hline$\ldots \ldots \ldots \ldots \ldots \ldots$ & D., L. \& N. R. R ........... & 897 \\
\hline Coreys..... & Mich. A. L. R. R............ & 871 \\
\hline$\ldots \ldots \ldots \ldots \ldots$ & D., G. H. \& M. R. R .......... & 776 \\
\hline $\begin{array}{l}\text { Crapo } \ldots \ldots \\
\text { Crofton } . \ldots \ldots \\
\end{array}$ & 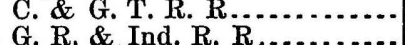 & 777 \\
\hline
\end{tabular}




\begin{tabular}{|c|c|c|}
\hline Sta & Authority. & Elevation. \\
\hline & & Feet. \\
\hline Crosswell ........ & P. H. \& N. W. R. & 730 \\
\hline $\begin{array}{l}\text { Daileys } \\
\text { Dalliba Mine.... }\end{array}$ & $\begin{array}{l}\text { Mich. A. L. R. R. } \\
\text { M. H. \& O. R. R. }\end{array}$ & $\begin{array}{r}871 \\
1,726\end{array}$ \\
\hline Dana ................. & D., L. \& N. R. R & 897 \\
\hline Danby............. & D., L. \& N. R. R & 782 \\
\hline Davisburgh ... & D., G. H. \& M. R. & 959 \\
\hline Davison............ & C. \&. G. T. R. R. & 791 \\
\hline Dayton ............ & Mich. Cent. R. R. & 718 \\
\hline Dearborn .... & Mich. Cent. R. R. & 614 \\
\hline (3........... & Mich. Cen & 781 \\
\hline Deerfield............ & L. S. \& M. S. R. R & 670 \\
\hline Delta ..... & D., L. \& N. R. R & 867 \\
\hline Dennis & D., G. H. \& M. & 640 \\
\hline Denton & Mich. Cent. R. R & 703 \\
\hline Detroit & Wich. Cen & 581 \\
\hline Do & U. S. Sign & 661 \\
\hline Devereus & L. S. \& M. S. R. & 990 \\
\hline Dex & Mich. Cent. R. I & 858 \\
\hline$\cdots \cdots$ & D., L. \& N. R. R & 897 \\
\hline le Crossin & L. S. \& M. S. R. & 860 \\
\hline Divide, Geodetic St & U. S. Lake Surv & 1,029 \\
\hline Dorr ............... & L. S. \& M. S. R. & 696 \\
\hline Dow & Mich. Cent. R. $\mathrm{R}$ & 760 \\
\hline lains. & D., G. H. & 967 \\
\hline Dundee, Geodetic S & U. S. Lake Surv & -681 \\
\hline Durand ............... & D., G. H. \& M. R. & 801 \\
\hline $\begin{array}{l}\text { Do ..Crossing D. G. } \\
\text { (grade)............... }\end{array}$ & C. \& G. T.R. R ... & \\
\hline Durton .............................. & D., M. \& M. R. R & 752 \\
\hline Eagle ... & D., L. \& N. R. R & 851 \\
\hline Eagle $M_{1}$ & M., H. \& O.R. R . & 1,280 \\
\hline Eagl & L. S. \& M. S. R. F & 601 \\
\hline East & J., L. \& S. R. R. & 588 \\
\hline Eaton Rapids .... & G.R.V.R.R... & 876 \\
\hline Do & L., $\mathrm{S}$ & 864 \\
\hline Edgetor & G. R. \& I & 755 \\
\hline & D., L. \& & 946 \\
\hline line. & M., H. \& O. R. R & 1,544 \\
\hline Ell & C. \& G. T. R. R . & 859 \\
\hline ........ & G. R. \& Ind. R. R & 1,234 \\
\hline & $\&$ N.R.R. & 638 \\
\hline Emn & C. \& G. T. R. R. & 779 \\
\hline & M., H. \& O. R. R . & 1,571 \\
\hline Signal S & U. S. SigI & 612 \\
\hline & M. H. \& C & 1,447 \\
\hline Gendetic & U.S & 799 \\
\hline & & 909 \\
\hline & D., L. \& N. R & 848 \\
\hline & D., G. H. \& I & \\
\hline River, Geodetic Statio & U. S. Lak & 856 \\
\hline & G. R. & 682 \\
\hline & & 631 \\
\hline ling........ & D., L.\& N.R.R & 934 \\
\hline & C. \& G.T. R. R. & 715 \\
\hline Flowerfield....... & L.S.\& M. S.R.R & 864 \\
\hline Forrest.. ......... & J., L. \& S. R. R.. & 1,226 \\
\hline & D., G. H. \& M. R. R & 748 \\
\hline Fowlerville ........ & & 902 \\
\hline Francisco .......... & Mich. Cent. R. R $\ldots \ldots \ldots$ & 1,016 \\
\hline Frazer .............. & C., D. \& C. G. T. J. R. R .... & $\begin{array}{r}604 \\
1019\end{array}$ \\
\hline $\begin{array}{l}\text { Fyfe Lake ...... } \\
\text { Gaines }\end{array}$ & $\begin{array}{l}\text { G. R. \& Ind. R. R } \\
\text { D. G. H. \& M. R. R }\end{array}$ & $\begin{array}{r}1,019 \\
859\end{array}$ \\
\hline $\begin{array}{l}\text { Gaines } \ldots \ldots \\
\text { Galesburgh }\end{array}$ & $\begin{array}{l}\text { D., G. H. \& M. R. } \\
\text { Mich. Cent. R. R .. }\end{array}$ & $\begin{array}{l}859 \\
788\end{array}$ \\
\hline Galion & Mich. Cent. R. R ............ & $6=2$ \\
\hline
\end{tabular}




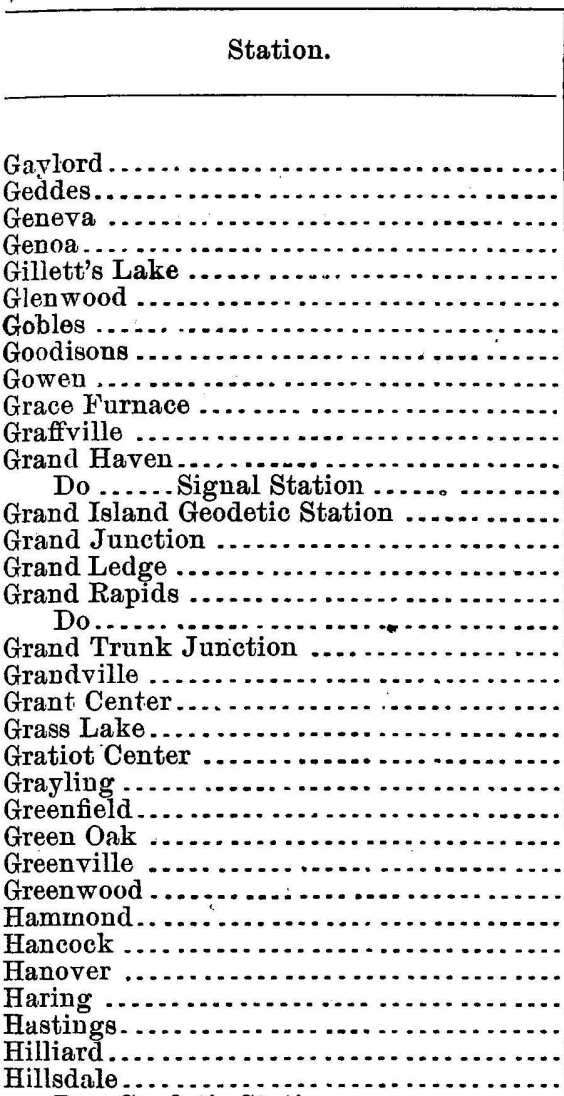

Do.. Geodetic Station.

Hobert

Holly

Homer Air Line Crossing

Horse Shoe Lake, low water ...............

Houghton, Mount

Howard City

Hudson

Humboldt ..................................

Humboldt Mine

Hunter's Creek.

Huron Mountain, Geodetic Station.

Huron, Lake

Ida

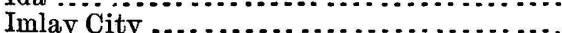

Inaple

Ingersoll

Ionia

(..................................

Ishpeming .............................

Ives' Hill, Geodetic Station

Jackson

Jackson, Mich. C. R. R., crossing on Grand

River

Jackson Junction

Jackson Miue

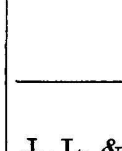

J., L. \& S. R. R

Mich. Cent. R. R

K. \& S. H. R. R

D., L. \& N. R. R

Mich. Cent. R.

K. \&. S. H. R. R

D. \& B. C. R. R

D., L. \& N. R. R

M., H. \& O. R. R

D., L. \& N. R. R.

D., G. H. \& M. R. R

U. S. Signal Office

U. S. Lake Survey

C. \& M. L. S. R. R

D., L. \& N. R. R.

L., S. \& M. S. R. R

D., G. H. \& M. R. R

L., S. \& M. S. R. R ..........

L., S. \& M. S. R. R

P. H. \& N. W. R. R..............

Mich. Cent. R. R ............

P. H. \& N. W. R. R..........

J., L. \&. S. R. R

D., L. \& N. R. R

D., L. \& N. R. R

D., L. \& N. R. R

J., L. \& S. R. R ............

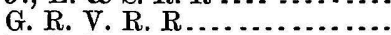

M. R. R. R ..............

Ft. W., J. \& S. R. R..........

G. R. \& Ind. R. R

G.R.V.R. R.......

L.S. \& M. S. R R

L., S. \& M. S. R. R

U. S. Lake Survey

G. R. \& Ind. R. R

D. G. H. \& $\mathrm{I}$ R. R

L., S. \& M. S. R. R ...........

L., S. \& M. S. R. R

Ft. W., J.\& S. R. R

Foster \& Whitney

G. R. \& Ind. R. R .

L., S. \& M. S. R. R...........

M., H. \& O. R.R R............

M.. H. \& O. R. R ...........

D.\& B. C. R. R

U. S. Lake Survey

U. S. Lake Survey .

L., S. \& M. S. R. R ...........

C. \& G.T.R.R...

G.R.\& Ind.R.R............

D., L.\& N.R.R

D., L.\& N. R. R............

G. R. V. R. R.

M., H. \& O. R. R

U. S. Lake Survey

U. S. Lake Survey

L., S. \& M. S. R. R.

Mich. Cent. R. R

Ft. W., J. \& S. R. R

Mich. Cent. R. R.

M., H. \& O. R. R

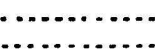

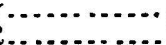

(n........

Elevation.

Feet.

1,349

743

695

978

932

751

803

842

848

632

969

594

620

990

678

860

605

639

586

624

745

986

$61 \%$

1,135

636

893

819

824

754

607

1,114

1,322

791

719

1,095

1,239

1, 307

938

972

703

1,054

1, 511

872

945

1,535

1,557

917

1,532

582

632

430

887

861

659

1,443

1,062

1,632

928

927

931 


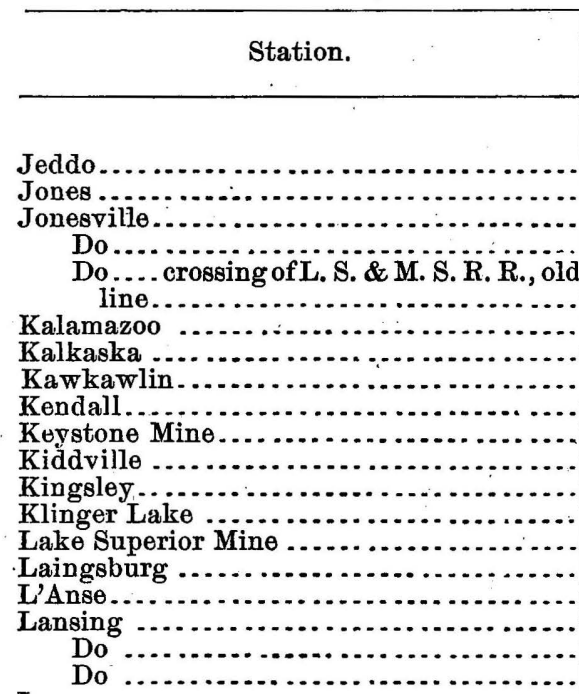

Lapeer

Lawton.............................

Leelsville

Lenawee Junction ......................

Leoni

Leroy.

Litchfield

Livonia

Lockwood

Lowell.

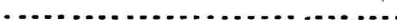

Lions

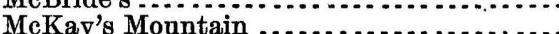

McOmber Mine

Macon

Magnetic Mine

Mancelona

Manchester

Manganese Mine

Mangers

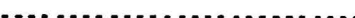

Manton .................................

Maple Valley.

Marengo

Marquette ............................

Do ..... Signal Station .

Marshall

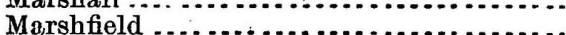

Martin ...............................

Mason ...................................

Mattawan...........................

Mecosta ................................

Melrose ...............................

Mendon

Meridian

Do

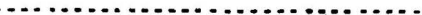

Metamora

Michigamme ............................

Michigamme Mine

Michigan Furnace

\begin{tabular}{|c|c|}
\hline Authority. & Elevation \\
\hline 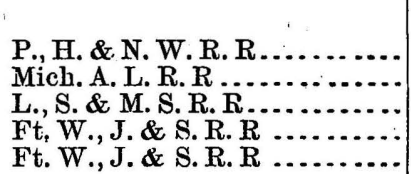 & $\begin{array}{r}\text { Feet. } \\
737 \\
916 \\
1,097 \\
1,056 \\
1,077\end{array}$ \\
\hline
\end{tabular}

L. S. \& M. S. R. R

G. R. \& Ind. R. R.

J., L. \& S.R.R

K. \& S. H. R. R

M., H. \& O.R.R

D., L. \& N. R. R.

P.H.\& N. W.R. R

L. S. \& M. S. R. R

M., H. \& O.R. R

J., L. \& S. R. R

M., H. \& O. R. R

J., L. \& S. R. R

L. S. \& M. S. R. R ............

C.\& G.T.R.R

C. \& G.T.R. R ...............

Mich. Cent. R. R ............

G. R. \& Ind. R. R

L. S. \& M. S. R. R

Mich. Cent. R. R

G. R. \& Ind. R. R

D., G. H. \& M. R. R

L. S. \& M. S. R. R

D., L. \& N. R. R.

G. R. \& Ind. R. R.

D., G. H. \& M. R. R..........

M., H. \& O. R. R

D., L. \& N. R. R

D. L \& $\mathrm{R}$ R.

Foster \& Whitney

D., L. \& N. R. R

M., H. \& O. R. R

A. \& D.R. R .

M., H. \& O.R. R

G. R. \& Ind. R. R.

L. S. \& M. S. R. R

M., H. \& O. R. R

D. \& B.C.R.R

G. R. \& Ind. R. R

D., L. \& N. R. R

Mich. Cent. R. R

M., H. \& O.R.R

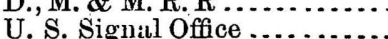

Mich. Cent. R. R

D., L.\& N.R.R

G. \& Ind R.

J L \& S.R.R

Mich. Cent. R. R ............

D., L.\& N.R.R.

G. R. \& Ind. R. R ...........

G. R. \& Ind. R. R

C. \& G.T.R.R.

D., L. \& $\mathrm{N}$ R. R...................

U. S. Lake Survey ...........

D. \& B. C. R. R

M., H. \& O.R. R

M., H. \& O. R. R

M., H, \& O. R. R
Feet.

916

1,097

1,077

777

1,022

597

792

$1,66 i$

802

736

890

1,447

806

608

852

827

840

830

778

1, 050

714

980

1,232

874

1,007

669

882

641

1,497

734

942

1,627

647

1,458

870

1,563

1, 119

907

1,503

597

1,142

925

921

649

622

673

898

1, 011

827

883

860

962

677

842

850

885

1,124

1,055

1,584

1,552

1,537 


\begin{tabular}{|c|c|c|}
\hline Station. & Authority. & Elevation. \\
\hline Michigan, Lake $\ldots \ldots \ldots \ldots \ldots \ldots \ldots \ldots$ & U. S. Lake Sur & Feet. \\
\hline Middleville & G. R. V.R.R... & 717 \\
\hline ................. & D., L.\& N.R.R. & 938 \\
\hline . & G. R. \& Ind, R. R & 625 \\
\hline . & D. \& B. C. R. R . & 757 \\
\hline Milton, Geodetic Station ................... & U. S. Lake Surve. & 896 \\
\hline Milwaukee Junction . . . . . . . . . . . . . . . . . . . & Grand Trunk R. i & 625 \\
\hline 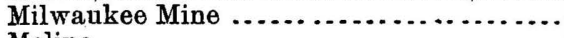 & M., H. \& O. R. R & 1,562 \\
\hline . & G. R. \& Ind. R. R & 785 \\
\hline Monistique, Geodetic Station . ........... & U. S. Lake Survey & 997 \\
\hline Monroe, Geodetic Station $\ldots \ldots \ldots \ldots \ldots \ldots$ & U. S. Lake Surve & 626 \\
\hline Monroe Junction $\ldots \ldots \ldots \ldots \ldots \ldots \ldots \ldots \ldots \ldots \ldots \ldots$ & L. S. \& M. S. R. R & 579 \\
\hline 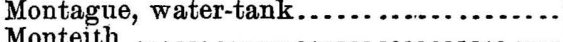 & C. \& M. L. S. R. & 587 \\
\hline 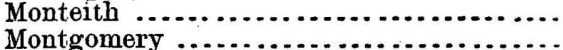 & G. R. \& lnd. R. I & 828 \\
\hline 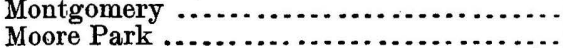 & $\begin{array}{l}\text { Ft. W., J.\&. S. R } \\
\text { L., S. \& M. S. R. }\end{array}$ & $\begin{array}{l}1,035 \\
846\end{array}$ \\
\hline $\begin{array}{l}\text { Voore Fark ....... } \\
\text { Morley ............ }\end{array}$ & 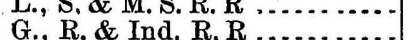 & $\begin{array}{l}842 \\
887\end{array}$ \\
\hline 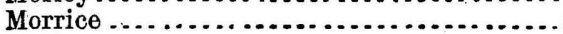 & C. \& G. T.R.R. & $\begin{array}{l}887 \\
890\end{array}$ \\
\hline 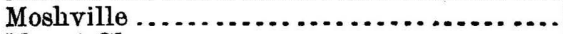 & Ft. W., J. \& S. R & 1,015 \\
\hline Mount Clemens...$\ldots \ldots \ldots$ & C. D.\& C. G. T. J & 602 \\
\hline Mud Lake, Geodetic Station ........... & U. S. Lake Surve & 1,005 \\
\hline 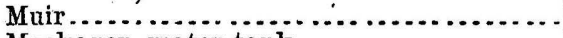 & D., G. H. \& M. R. R & 657 \\
\hline 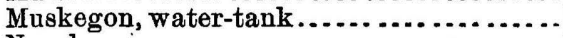 & C. \& M.L.S. R. R & 594 \\
\hline 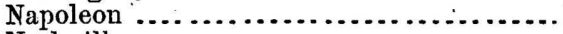 & L. S. \& M. S. R. R & 964 \\
\hline 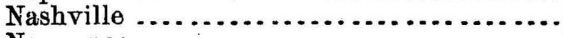 & G. R. V.R.R R... & 807 \\
\hline Negaunee $\ldots . . . . . . . . . . . . . . . . . . . . .$. & M., H. \& O.R.R. & 1,379 \\
\hline New Buffalo.......................... & Mich. Cent. R. R. & 602 \\
\hline 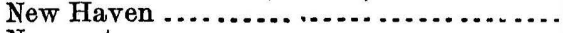 & C., D. \& C. G.T. J. R. R ..... & 617 \\
\hline 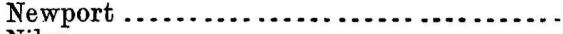 & L. S. \& M. S. R. R. . . . . . . . . & 584 \\
\hline 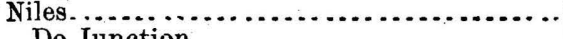 & Mich. Cent. R. R . & 681 \\
\hline$\ldots \ldots \ldots \ldots \ldots$ & Mich. A. L. R. R .. & 697 \\
\hline 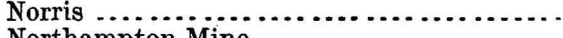 & D. \& B. C. R. R . & 631 \\
\hline (n) & $\cdots \cdots \cdots$ & 1,675 \\
\hline $\begin{array}{l}\text { Concord .... } \\
\text { Lansing .... }\end{array}$ & $\cdots \cdots \cdots$ & 968 \\
\hline 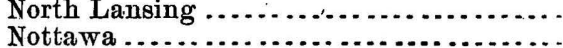 & $\begin{array}{l}\text { U., L. \& N. R. R... } \\
\text { G. R. \& Ind. R. R }\end{array}$ & $\begin{array}{l}85 \% \\
85{ }^{\circ}\end{array}$ \\
\hline $\mathbf{n} \mathbf{a} \ldots \ldots$ & L. S. \& M. S. R. R & $\begin{array}{l}85 \% \\
942\end{array}$ \\
\hline ca............. & C. \& M. L.S.R.R & $\begin{array}{l}942 \\
631\end{array}$ \\
\hline 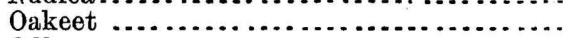 & C. \& G.T.R.R ... & 874 \\
\hline 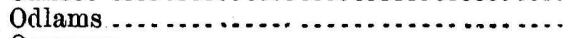 & P. H. \& N.W.R. & 730 \\
\hline 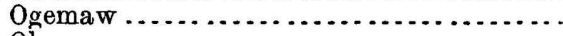 & J., L.\& S. R. R. & 1,088 \\
\hline Okemos .... & D., L. \& N.R. R. & 874 \\
\hline .............. & G. R. V.R.R R ... & 895 \\
\hline Ontonagon Junction ............... & M., H. \& O. R. R & 1,434 \\
\hline$\ldots \ldots \ldots$ & D. \& B. C. R.R . & 995 \\
\hline Orono.......... & G., R. \& Ind. R. & 1,072 \\
\hline Osseo ..... & $\ldots \ldots \ldots$ & 1,126 \\
\hline Ostemo .... & Mich. Cent. R. R & 962 \\
\hline to $\ldots \ldots \ldots$ & L. S. \& M. S. R. R & 710 \\
\hline 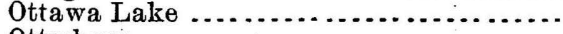 & L. S. \& M. S. R. R & $68: 3$ \\
\hline$\ldots$ & C. \& G. T.R. R. & 774 \\
\hline Otter Lake. & D. \& B. C.R.R. R & $\begin{array}{l}869 \\
735\end{array}$ \\
\hline Ovid.. & D., G., H. \& M. 1 & 735 \\
\hline $\begin{array}{ll}0 \text { Owos } \\
0 \times f a 1\end{array}$ & N., G. H. \& M. I & 1058 \\
\hline cific Fur & D. \& B. C.R.R & 1,058 \\
\hline ucific Furnace $\quad \ldots \ldots \ldots \ldots \ldots \ldots$ & $\begin{array}{l}\text { M., H. \& U. K. R . } \\
\text { D., L. \& N. R.R. }\end{array}$ & $\begin{array}{l}62 \% \\
868\end{array}$ \\
\hline $\begin{array}{l}\text { Palmer .... } \\
\text { Palmyra... }\end{array}$ & L. S. \& M. S. R. R . & 707 \\
\hline & \& Ind R. R .......... & 927 \\
\hline & t. R. R $\ldots \ldots \ldots \ldots$ & 986 \\
\hline lee ... & G. F & 713 \\
\hline ter, tu & & 595 \\
\hline & & $87 \%$ \\
\hline & & 892 \\
\hline
\end{tabular}




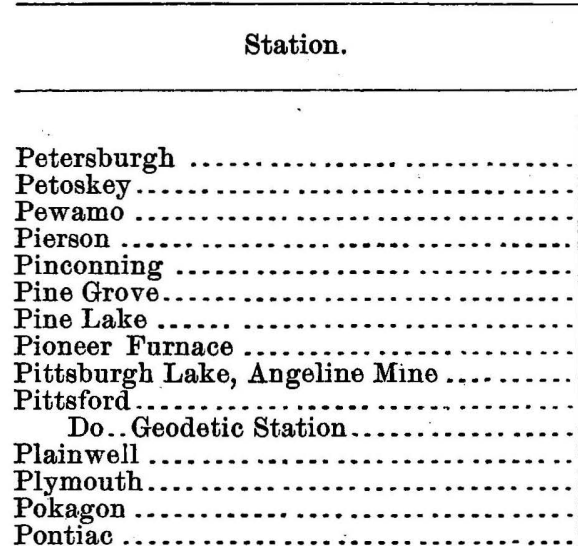

Do. Stanley Station crossing, O. \& Pt. A. R. R. Porcupine Mountain, Geodetic Station

Portage

Portage Lake

Porter, Geodetic Station.

Port Huron, Signal Station

Portland

Pulaski

Quimby

Quincy.

Do.Geodetic Station

Rack's Mills

Raisin Do.Geodetic Station

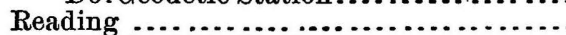
Do.Geodetic Station

Redford.

Reed City

Reese ........

Remick

Republic .

Republic Mine

Riga

Rives' Junction

Rochester

Rockford

Rockwood

Rodney

Rolling Mill Mine

Rosconmon

Ross

Royal Oak

Saganin

Saginaw

Saginaw City

Saginaw Mine

Saint Charles

Saint Helens

Do...... (L. W: of Maumee River) Do...... Bridge

Salem

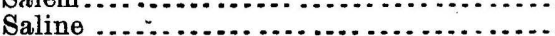

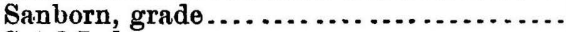

Sand Lake

Sand River

Saranac

Schooleraft

.

L. S. \& M. S. R. R

G. R. \& Ind. R. R.

G.

J., L. \& S. R. R.............

K. \& S.H.R.R

C. \& G. T. R. R.

M., H. \& O. R. R

M., H. \& O. R. R

L. S. \& M. S. R. R

U. S. Lake Survey

L. S. \& M. S. R. R

D., L. \& N. R. R

Mich. Cent. R. R

D., G., H. \& M. R. R

C. \& G. T. R. R

U. S. Lake Survey

L. S. \& M. S. R. R

G. R. \& Ind. R. R

U. S. Lake Survey

U. S. Signal Office

D., L. \& N. R. R.

Mich. A. L. R.R.

L. S. \& M. S. R.

U. S. Lake Survey.

C. D. \&C. G. T. R. R

U. S. Lake Survey

Ft. W., J. \& S. R. R

U. S. Lake Survey

D. L. \& N. R. R

D. \& B. C. R. R.

D. L. \& N. R. R

M. H. \& O. R. R

M. H. \& O. R. R.

L. S. \& M. S. R. R.

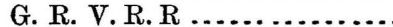

D. \& B. C. R. R..................

G. R. \& Ind. R. R

L. S. \& M. S. R. R

D. L.\& N. R. R .

M. H. \& O. R. R.

J., L \& S. B. R

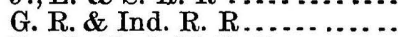

D., G. H. \& M. R. R .......

J., L. \& S. R.' R .............

M., H. \& O. R. R............

J., L. \& S. R. R.

M. H. \& O. R. R..........

J., L. \& S. R. R.

J., L. \& S. R. R . . . . . . . . . .

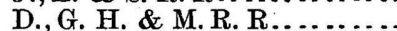

Ft. W., J. \& S. R. R ........

C. \& M. L. S. R. R ...........

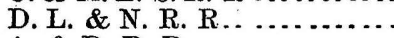

A. \& D. R. R ... . . .

C. \& G. T. R. R ..........

G. R. \& Ind. R. R...............

D. M. \& M. R. R ..........

D., G. H. \& M. R. R........

L. S. \& M. S. R. R ..........
Elevation.

Feet.

670

658

744

906

599

777

859

1,382

1,442

1,109

1,149

744

747

733

934

897

2,023

860

834

950

633

730

1,043

794

1,027

1, 061

759

862

851

1,200

1,209

631

1, 027

629

934

1,510

1,536

692

904

747

689

582

1,062

1,402

1,128

$7 \pi 7$

663

616

1,441

591

1,552

591

1, 158

767

996

596

953

889

710

912

627

643

884 


Station.

Shaftsburg .

Shelby Depot

Shelbyville.

Shelter Bay, Geodetic Station

Shenango Mine

Shepardsville.

Sheridan

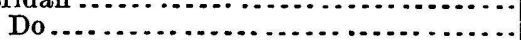

Sherman, Geodetic Station.

Sherwood

Shiloh

Simons

Snyder

South Boardman

South Haven .

South Lyon

Spinnings ...

Spring Arbor

Spring Lake

Springport .

Spurr Mountain

Standard Mine

Standish

Stanton

Stanton Junction

Stanwood

State Road

Stimpson's

Sterling

Steward Mino

Sturgeon .............................

Sturgeon River, Geodetic Station .........

Sturgis

Suffield

Superior, Lake

Swartz Creek

Taylor

Taylor Mine.

Tecumseh

Tekonsha

Terry's

Thomas.

Three Lakes

Three Oaks

Three Rivers.

Travis .

Trenton

Tribola, Geodetic Station

Trowbridge.

Trufant's

Tustin

Union City

Upper Big Rapids

Utica .

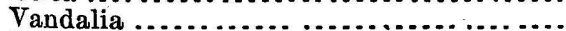

Vassar.

Vermontville

Vernon

Vicksburg .

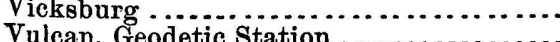

Wagar's

Authority. Elevation

Mich. Cent, R, R

$\quad 845$

C. \& M. L. S. R. R ......... 808

G. R. \& Ind. R. R........... 832

U. S. Lake Survey........... $\quad 1,054$

M. H. \& O.R. R............ 1,421

D., G. H. \& M. R. R........ 749

D., L. \& N. R. R ...........

856

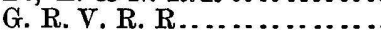

U. S. Lake Survey ...........

Mich. A. L. R. R.

D., L. \& N. R. R

..............

Mich. A. L. R. R ............

G. R. \& Ind. R. R

K. \& S. H. R. R

D. L. N.R. R

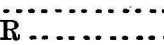

D. \&. B. C. R. R

Mich. A. L. R.R

D., G. H. \& M. R. R

L. S. \& M. S. R. R.

M. H. \& O. R. R

M. H. \& O. R. R

M. H. \& O. R. R.

J., L. \& S. R, R

D., L. \& N. R. R

D., L. \& N. R. R

G. R. \& Ind. R. R

J., L. \& S. R. R

A. \& D. R. R .

M. H. \& O. R. R

M. H. \& O. R. R

M. H. \& O. R. R

U. S. Lake Survey

L. S. \& M. S. R. R

C. \& G. T. R. R .

U. S. Lake Surrey
C. \& G. T. R. R ..

M., H. \& O. R. R

M., H. \& O. R. R
L. S. \& M. S. R. R

Mich. A. L. R. R

J., L. \& S. R. R

D. \& B. C. R. R

M., H. \& O. R. R

Mich. Cent. R. R

L. S. \& M. S. R. R

G. R. \& Ind. R. R

L. S. \& M. S: R. R

U. S. Lake Survey

C. \& G. T. R. R

D., L. \& N. R. R

G. R. \& Ind. R. R

Mich. A. L. R. R

G. R. \& Ind. R. R

D. \& B. C. R. R

Mich. A. L. R. R.

D. \& B. C.R.R.

G. R. V. R. R.

D., G. H. \& M. R. R

806

1,038

$87 \%$

848

1,219

1,005

583

807

933

621

994

596

986

1,607

1,602

1,538

627

904

821

954

602

865

1,567

1,602

1,643

947

934

783

602

782

1,225

1,516

807

937

589

1,093

1,616

669

805

742

584

1,237

851

884

1,212

900

927

650

878

643

817

770

G. R. \& Ind. R. R

U. S. Lake Survey

D., L. \& N. R. R 


\begin{tabular}{|c|c|c|}
\hline & Authority. & Elevation. \\
\hline 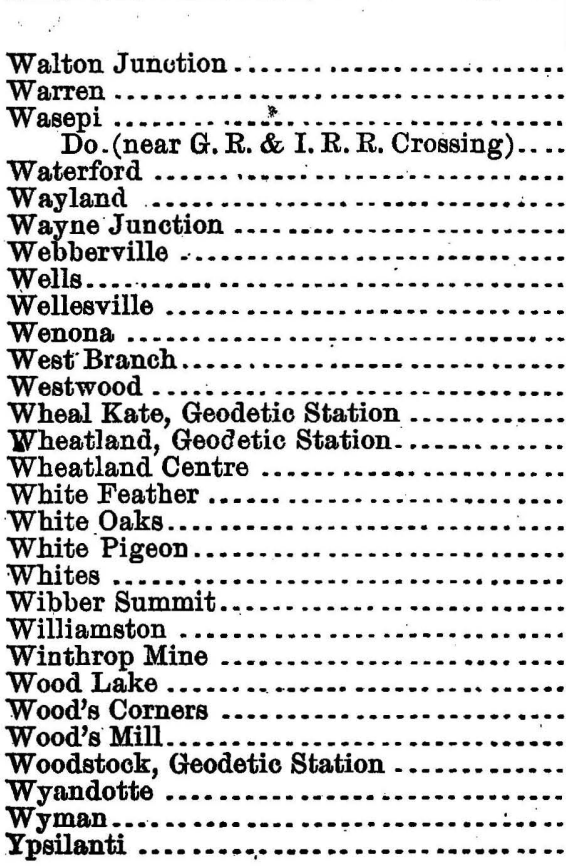 & 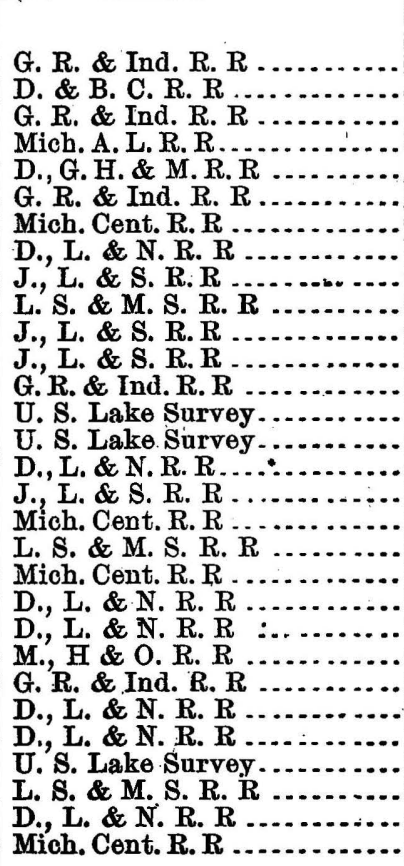 & $\begin{array}{r}\text { Feet. } \\
1,047 \\
641 \\
842 \\
835 \\
988 \\
747 \\
662 \\
892 \\
774 \\
690 \\
589 \\
957 \\
1,089 \\
1,508 \\
1,248 \\
1,004 \\
604 \\
842 \\
824 \\
900 \\
781 \\
891 \\
1,456 \\
891 \\
851 \\
926 \\
1,191 \\
580 \\
960 \\
714\end{array}$ \\
\hline
\end{tabular}




\begin{tabular}{|c|c|c|}
\hline Station. & Authority. & Elevation. \\
\hline & 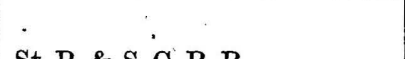 & Feet. \\
\hline drian..... & St.P. \& S. C.R.R. & 1,538 \\
\hline iken....... & N.P.R.R...... & $\begin{array}{r}950 \\
1,204\end{array}$ \\
\hline irlie ....... & (., M. \& St.P.R. & 1,629 \\
\hline itkin, water & U.S. Engineer Corp & 1,190 \\
\hline albany ...... & St.P.\&P.R. R .... & 1,187 \\
\hline llbert Lea. & C., M. \& St.P.R.R & 1,221 \\
\hline lden ....... & C., M.\& St.P.R. R & 1,261 \\
\hline drich .... & U.P.R.R ...... & 1,328 \\
\hline Alexandria. & st. P.\& P.R.R. & 1,382 \\
\hline Alma, water. & U. S. Engineer Corp & 657 \\
\hline Amiret ...... & C. \& N.W.R.R... & 1,283 \\
\hline noka, water & U. S. Engineer Corps & 827 \\
\hline Anoká......... & St. P. \& P.R.R ....... & \\
\hline Appleton ... & C., M. \& St. P.R. R & 1,007 \\
\hline Armstrong . & C., M. \& St. P.R. R & 1,270 \\
\hline Ash Creek & St. P. \& S.C.R.R... & 1,396 \\
\hline abur & C., M. \& St. P.R. R & 861 \\
\hline & N.P.R. R & 1,310 \\
\hline $\mathrm{An}$ & C., M.\& St.P.R.R & 1,253 \\
\hline usti & C., M. \& St. P.R.R & 1,197 \\
\hline unct & C., M. \& St. P. R. R & 1,194 \\
\hline erill...... & St.P.\& P.R.R .... & 918 \\
\hline Avoc: & St. P.\& S. C. R.R & 1,533 \\
\hline$\ldots$ & St. P.\& P.R.R .. & 1,116 \\
\hline Balaton.... & C. \& N.W.R.R... & 1,528 \\
\hline ake. & C.\& N.W.R.R... & 930 \\
\hline & Toner ............... & 1,600 \\
\hline r Bay & Snithsonian Inst & 1,270 \\
\hline & St. P.\& P. & \\
\hline lain, $\mathrm{v}$ & U. S. Engineer Corps. & . 695 \\
\hline Plain & St.P.\& S. C. R. R.... & 725 \\
\hline & St. $]$ & 1,134 \\
\hline & St.P.\& P.R.R & 1,084 \\
\hline Belt & St.P.\& P.R.R & 903 \\
\hline & St.P.\& P.R.R.. & 1,042 \\
\hline & St.P.\& S. & 1,631 \\
\hline $\operatorname{ake} \ldots$ & St. P.\&P. & 930 \\
\hline Big Sto & U. S. Engin & 978 \\
\hline & M. \& St.P.R. R. & 963 \\
\hline & Nicollet....... & \\
\hline am Lake.. & St.P. \& S. & 1,420 \\
\hline and & C., M.\& St.P.R.R & 1,089 \\
\hline & St. P.\& S. C.R.R... & 728 \\
\hline Prairie & C., M. \& St. P. R. R .. & 1,286 \\
\hline $\mathrm{Bl}$ & St.P.\& S. C & 738 \\
\hline Bl & St. P. \& S. C. R. R.... & 1,425 \\
\hline & Toner ..... & 1,238 \\
\hline , water & U. S. Engineer Corps. & 1,152 \\
\hline & 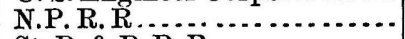 & 1,207 \\
\hline Breckenridge .... & St.P.\& P.R.R R . & 957 \\
\hline Do......, Sign & U. S. Signal Office... & 968 \\
\hline Brentwood........ & St.P. \& S. C. R. R................. & 749 \\
\hline Brgwnsdale...... & C., M. \& St. P. R. R .. & 1,271 \\
\hline vnton ........ & C., M. \& St. P. R. R . & 1,024 \\
\hline station & Toner U. & $\begin{array}{l}600 \\
744\end{array}$ \\
\hline $\begin{array}{l}\text { Durmington, Geo } \\
\text { Burtor ......... }\end{array}$ & C., M. \& St. P. R.R .. & 947 \\
\hline
\end{tabular}




\begin{tabular}{|c|c|c|}
\hline & Authority. & Elevation. \\
\hline & & \\
\hline Butterfield & St., P.\& S. C. R. R & 1,184 \\
\hline & C. \& N. W.R.R. & 1,250 \\
\hline mpe & C. \& N.W.R.R. & $\begin{array}{r}977 \\
1,243\end{array}$ \\
\hline & C., M. \& St. I & 815 \\
\hline $\begin{array}{l}\text { Do } \\
\text { Cass I }\end{array}$ & $\begin{array}{l}\text { M. \& St. L. } 1 \\
\text { U. S. Engine }\end{array}$ & $\begin{array}{r}719 \\
1,300\end{array}$ \\
\hline at] & C. M. \& St. & $\begin{array}{l}1,300 \\
935\end{array}$ \\
\hline ntrevi. & L., S.\& M. R. R. R & 929 \\
\hline Chask & C., M. \& St. & 728 \\
\hline Chatfi & $\begin{array}{l}\text { C., M. \& St. I } \\
\text { C. \& N. W. }\end{array}$ & $\begin{array}{l}730 \\
976\end{array}$ \\
\hline & C. \& N.W. W & 1,275 \\
\hline & C. \& N. W. R. R & 1,122 \\
\hline & St. P. \& P. R. R. & 1,219 \\
\hline$\ldots$ & $\begin{array}{l}\text { C. \& N. W. R. R } \\
\text { St. P. \& P. R. R. }\end{array}$ & $\begin{array}{r}1,280 \\
993\end{array}$ \\
\hline & U. S. Engine & 936 \\
\hline & C., M. \& St. P. R. & 1,107 \\
\hline & St. P. \& P. R. R. & 1,022 \\
\hline & C. \& N. W. R. R & 936 \\
\hline Do .- brid & C. \& N. W. R. R . & 811 \\
\hline rookston & & \\
\hline Crow & St. P.\& P & \\
\hline & U. S. Engi & 1,146 \\
\hline Lake.... & St. P. \& S. & 994 \\
\hline$\cdots$ & C., M. \& St. P. R. & 982 \\
\hline$\cdots$ & St. P. \& P. R. R.... & 1,127 \\
\hline uff. & $\begin{array}{l}\text { Pacific R. R. Reports } \\
\text { C., M. \& St. P. R. R. }\end{array}$ & $\begin{array}{l}994 \\
710\end{array}$ \\
\hline laff. & C., M. \& St. P. R. F & 1,446 \\
\hline & St. P. \& P. R. R.. & 1,056 \\
\hline$\ldots$ & St. P. \& P & 923 \\
\hline 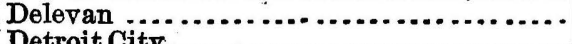 & C., M. \& St. P. R. R & \\
\hline ity....... & N. P. R. I & $\begin{array}{l}1,364 \\
1,412\end{array}$ \\
\hline ntre..... & $\begin{array}{l}\text { C., M. \& St. P. R. } \\
\text { C. \& N. W. R. R. }\end{array}$ & $\begin{array}{l}1,412 \\
1,288\end{array}$ \\
\hline ........ & St. P.\& P. R. R . & 968 \\
\hline (........... & C. \& N. W. R. R .. & 1,310 \\
\hline us $\ldots \ldots \ldots$ & $\begin{array}{l}\text { St., \& P. R. R } \\
\text { C. \& N. W. R. R }\end{array}$ & $\begin{array}{l}1,121 \\
1,138\end{array}$ \\
\hline & St. P. \& S. C. R, R. & 1,516 \\
\hline ch......... & R. R. . & 676 \\
\hline$\cdots \ldots$ & N. & 607 \\
\hline $\begin{array}{l}\text { 0..Lake Sup } \\
\text { o..Signal St }\end{array}$ & $\begin{array}{l}\text { N. P. R. . } \\
\text { U. S. Sig. }\end{array}$ & \\
\hline 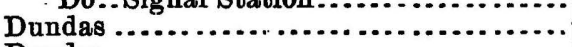 & C., M. \& St. P. R. R.... & \\
\hline & St. P. \& S. C. R. R. & 1,443 \\
\hline Lake ....... & $\mathrm{K}$ & 1,012 \\
\hline Henderson, $w$ & neer Corps .... & \\
\hline $\begin{array}{l}\text { East Henderson ... } \\
\text { East Minneapolis.. }\end{array}$ & St. P.\& S. C. R. R........ & 734 \\
\hline East Minneapolis.. & M. \& St. L. R. R & $\begin{array}{r}830 \\
1,569\end{array}$ \\
\hline & C., M. \& St. P. R. R .... & 1,046 \\
\hline & C., M. \& St. P. R: R .... & \\
\hline$\cdots \cdots$ & St. P. \& P. R. R $\ldots \ldots \ldots$ & 1,011 \\
\hline $\begin{array}{l}\text { East Saint Peter } \\
\text { East Sawteeth, Ge }\end{array}$ & $\begin{array}{l}\text { St. P. \& S. C. R. R ....... } \\
\text { U. S. Lake Surver ..... }\end{array}$ & \\
\hline Eckelson, Lake & orts................ & 1,454 \\
\hline Prairie ....... & L.R.R $\ldots \ldots \ldots$ & \\
\hline & & \\
\hline
\end{tabular}




\begin{tabular}{|c|c|c|}
\hline Station. & Autho & Elevation. \\
\hline & & \\
\hline Elk Lake & P.R.R. Reports. & \\
\hline Elk River........ & St. P.\& P.R. R . & 903 \\
\hline Elk River, water.. & Engineer C & 850 \\
\hline Ellea.............. & Toner .............. & 1,600 \\
\hline Empire & C., M. \& St. P. R & 889 \\
\hline Evansville ........ & St. P. \& P. R. R. & 1,345 \\
\hline Eyota ................ & C. \& N.W.R.R.. & 1,237 \\
\hline$\ldots \ldots \ldots . . .$. & C., M. \& St. P. R. R & 943 \\
\hline int ......... & C., M. \& St. P. R. R & 1,176 \\
\hline$\ddot{H} \ddot{B} \ddot{R}^{\prime}$ & C., M. \& St. P. R. R & 1,002 \\
\hline $\begin{array}{l}\text { Farmington, } \mathrm{c} \\
\text { Do }\end{array}$ & C., M. \& St. P. R & 901 \\
\hline Do........ crossing Iowa and Minnesota & C., M. \& St. P. R & 904 \\
\hline division & U., di. Ce No. 1. & 904 \\
\hline iter ..... & U. S. Enginee & 699 \\
\hline , Big Sio & St. P. \& S. C & 1,501 \\
\hline ac ......... & N.P.R.R...... & 608 \\
\hline ake...... & L. S. \&. M. R. R. R & 906 \\
\hline 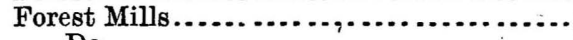 & C. \& N. W. R. R... & 1,023 \\
\hline ........... & Minn. Mid. R. R.... & 970 \\
\hline$\ldots \ldots . .$. & C., M. \& St. P. R. & 1,302 \\
\hline ity ...... & N.P.R. R......... & 1,411 \\
\hline Frontenac ......... & C., M. \& St. P. R & 721 \\
\hline$\ldots \ldots \ldots \ldots$ & C., M. \& St. P. R & 1,508 \\
\hline ...... & St. P. \& P. R. R. & 709 \\
\hline •...... & Minn. Mid. R. R. & 821 \\
\hline (n...... & C. M. \& St. P. R. R & 1,007 \\
\hline & B. C. R. \& R. R. R. & 1, 223 \\
\hline . & R. R....... & 925 \\
\hline der........ & C.,M. \& St. P. R. F & 974 \\
\hline$\ldots . .$. & L., S. \& M. R. R. R & 888 \\
\hline & St. P. \& P. R. R ... & 1,017 \\
\hline low...... & C., M. \& St. P. R. R & 1,338 \\
\hline$\ldots \ldots \ldots$ & C. \& N. IV.R.R... & 1,173 \\
\hline Falls........ & C., M. \& St. P. R. R & 941 \\
\hline ......... & St.P. \& S. C. R. R . & 714 \\
\hline & Mid. R. R. & 805 \\
\hline$\ldots .$. & \& P.R.R ... & 1,150 \\
\hline$\ldots .$. & M. \& St. L. R. R.. & 1,240 \\
\hline .......... & \& St. P. R & 709 \\
\hline L. W.... & U.' & 664 \\
\hline Junction & \&St. P. R. R & 709 \\
\hline Hatfield & I. \& St. P. R. R & 1,662 \\
\hline Ha & C., M. \& St. P. R. R. & 1, \\
\hline $\mathrm{H}$ & 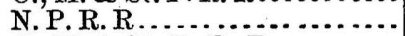 & 152 \\
\hline$\ldots . . .$. & C., M. \& St. P. R. R......... & \\
\hline $\mathrm{He}$ & C., M. \& St. P. R. R ....... & 1,081 \\
\hline$\ldots \ldots$ & M. \& St. L. R. R... & \\
\hline ........ & $\ldots \ldots \ldots$ & \\
\hline Lake....... & $\ldots \ldots \ldots$ & 1,284 \\
\hline & R.R. & 1,063 \\
\hline 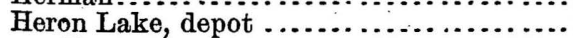 & \& S. C.R.R & 1,417 \\
\hline & C. R. R & 1,403 \\
\hline (Brewster).... & \& S. C. R. R. & \\
\hline ley .......... & L., S. \& M.R.R.R. & 1,025 \\
\hline $\mathrm{H}$ & N.P.R.R......... & 1,386 \\
\hline .... & C., M. \& St. P. R. R.......... & 649 \\
\hline er .... & C., M. \& St. P. R. R . . . . . . & 663 \\
\hline kins ... & M. \& St. L. R. R............. & 919 \\
\hline Hospers........... & Toner $\ldots \ldots \ldots \ldots \ldots \ldots$ & 1,438 \\
\hline ................. & C., M. \& St. P. R. R. ......... & \\
\hline$\ldots \ldots \ldots \ldots \ldots$ & M. \& St. L. R. R................. & 247 \\
\hline & & \\
\hline
\end{tabular}




\begin{tabular}{|c|c|c|}
\hline & Authority. & Elevation. \\
\hline & & \\
\hline Iceland ..... & St.P.\& S.C.R.I & 998 \\
\hline da ........ & St. P. \& P.R.R & 1,402 \\
\hline $\begin{array}{l}\text { Iona .. } \\
\text { Iosco.. }\end{array}$ & $\begin{array}{l}\text { C., M. \& St. P. R. H } \\
\text { M. \& St. L. R. R.. }\end{array}$ & $\begin{array}{l}1,608 \\
1,146\end{array}$ \\
\hline Isinours.:- & C., M. \& St. P.R.R & 899 \\
\hline land Lal & N.P.R.R.............. & 1,307 \\
\hline asca..... & St. P.\& P.R.R $\ldots$ & 895 \\
\hline ville & C. \& N.W. R. R. & $\begin{array}{r}1,063 \\
792\end{array}$ \\
\hline ssie Lak & $\begin{array}{l}\text { Minn. Mid. R. R . } \\
\text { P. R. R. Reports . }\end{array}$ & $\begin{array}{r}792 \\
1,260\end{array}$ \\
\hline 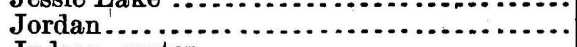 & M. \& St. L. R. R. & . 753 \\
\hline$\cdots$ & U. S. Engineer Co & $\begin{array}{r}759 \\
\end{array}$ \\
\hline n.......... & St. P. \& S. C. R. & 1,633 \\
\hline neja L & St. P.\& P.R.R. & $\begin{array}{l}1,092 \\
1,216\end{array}$ \\
\hline Kasot & C. \& N. W.R.R. & 794 \\
\hline & St. P.\& S. & 800 \\
\hline Do.M & C.\& N.W.F & 781 \\
\hline Do.Min & N.W & 723 \\
\hline Do.Min & N.W & 735 \\
\hline Kasson ..... & N.W. & 1,252 \\
\hline & $\mathbf{M i}$ & 759 \\
\hline n's L & C., & 948 \\
\hline & C., & 702 \\
\hline $\mathbf{K e}$ & L. & 1,114 \\
\hline & M. & 1,056 \\
\hline & N.I & 1,233 \\
\hline & St. P. \& & 1,104 \\
\hline & St. P. \& & 886 \\
\hline Parle & U. s & 940 \\
\hline scent.... & & 647 \\
\hline .....high- & & 648 \\
\hline Do......lime-k & & 650 \\
\hline ke Benton..... & & 1,759 \\
\hline Lake City.... & C., M. \& & 705 \\
\hline & & 658 \\
\hline gtal. & St. 1 & 987 \\
\hline & C., M. \& St. P. & 1,463 \\
\hline & N.P.R.R... & 1,335 \\
\hline rerse, we & & 970 \\
\hline & & 1,144 \\
\hline & & 660 \\
\hline & & 841 \\
\hline & & 813 \\
\hline & St. & 1,063 \\
\hline & St. & 1,224 \\
\hline & & 850 \\
\hline ake ....... & ps..... & 1,292 \\
\hline & & 1,073 \\
\hline Geodetic Station. & & 1,157 \\
\hline - & & \\
\hline & U.S. En & \\
\hline ... high water in Minnesota River... & & 735 \\
\hline & . R. R.. & 1,211 \\
\hline & C. R. R & 1,042 \\
\hline .... & St. P & 1,125 \\
\hline 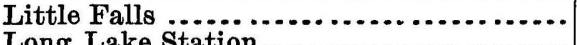 & St. P. \& P. R. R. & 1,118 \\
\hline 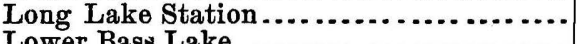 & St. P. \& P. R. R. & $\begin{array}{l}949 \\
893\end{array}$ \\
\hline r Bass Lake. & St. P., S. \& T. F. R. R.... & $\begin{array}{r}893 \\
1,451\end{array}$ \\
\hline nne......... & 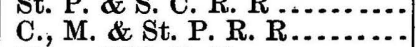 & $\begin{array}{l}1,401 \\
1,199\end{array}$ \\
\hline acken.. & Minn. Mid. R. R... & 732 \\
\hline adison $\ldots . . . . .$. & $\begin{array}{l}\text { C., M. \& St. P. R. R } \\
\text { M. \& St. L. R. R R } \ldots\end{array}$ & $\begin{array}{l}1,250 \\
1,258\end{array}$ \\
\hline & & \\
\hline
\end{tabular}




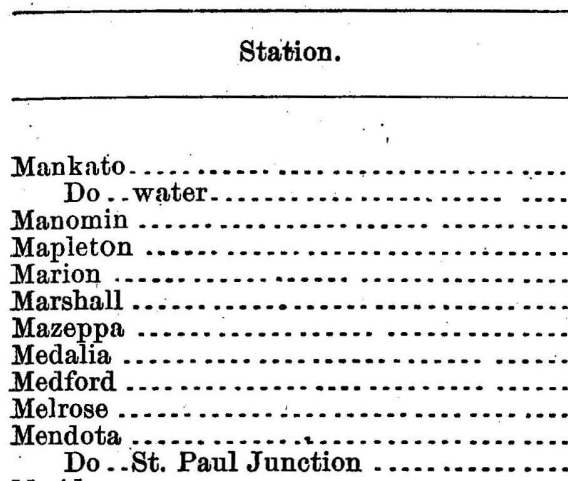

Meriden

Merriam Junction, St. P.\& S. C. R. R.

Midland Junction

Milan

Millville

Minneapolis

Do .... Miss. Riv

Minneiska L. W

Minneopa

Do.. bridge, 68 feet above water

\section{Minneota}

Minnesota City.

Minnesota Falls

$$
\text { Do.......... (foot of) }
$$

Minnesota Lake.

Minnetonka Lake

Money Creek

Montgomery.

Monticello, water.

Montievideo

Moorhead, Signal Station

Do... East bank Red River.

Moose Lake, depot.

Morris

Motley

Mound Prairie.

Mountain Lake.

Mulford's Siding

Muskoda

Newport Do... L. W.

Nö Prague.

New Richland.

New UIm

Do .. high water in Minnesota River .. Nicollet

Nicols.

Nininger, water

Norman

North Branch

Northfield

Oakdalo

Oakes

Oak Lake

Oakland

Odessa.

-

Olivia.

Olmstead

Oronoco

Ortonville.

Authority. Elevat

C., M. \& st. P. R. R ........

U. S. Engineer Corps ......... St. P. \& P. R. R... C., M. \& St. P. R. R Toner.

C. \& N. W. R. R

Minn. Mid. R. R .

St. P. \& S. C. R. R

C., M. \& P. R. R.

St. P. \& P. R. R.

St. P. \& S. C. R. R

C., M. \& St. P. R. R

C. \& N. W. R. R

M. \& St. L. R. R

Minn. Mid. R. R

C., M. \& St. P. R. R

Minn. Mid. R. R.

M. \& St. L. R. R.

U. S. Engineer Corps

U. S. Engineer Corps

St. P. \& S. C. R. R

St. P. \& S. C. R. R

C. \& N. W. R. R.

C., M. \& St. P. R. R

C., M. \& St. P. R. R.

U. S. Engineer Corps

Do

St. P. \& P. R. R.

C., M. \& St. P. R. R

M. \&.St. L. R. R

U. S. Engineer Corps.

C., M. \& St. P. R. R

U.S. Signal Office..

N. P. R. R . . .

St. P. \& P.R. R.

N.P.R.R.

C., M. \& St. P. R. R

St. P. \& S. C. R. R.

M. \& St. L. R. R.

N.P.R.R

C., M. \& St. P. R. R.

U.S. Engineer Corps

M. \& St. L. R. R . . . . . . . . . .

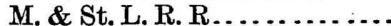

C. \& N. W. R. R

C. \& N. W. R. R..............

C. \& N. W. R. R.

St. P.\& S. C. R. R.

U. S. Engineer Corps,

N. P. R R

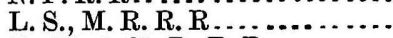

C., M. \& St. P. R. R........

St. P., S. \& T. F. R. R.

St. P.\& P. R. R..............

N.P.R.R.

C., M. \& St. P. R. R

C., M. \& St. P. R. R

C., M. \& St. P. R. R.

C., M. \& St. P. R. R

C. \& N. W. R. R

C. \& N. W.R. R.

C., M. \& St. P. R. R
Feet.

$7 \% 0$

751

842

1,031

1,200

1,174

935

1,021

1,098

1,189

718

759

1,149

753

695

995

787

825

794

644

871

863

1,179

677

1,041

869

1,038

922

692

1,063

890

927

923

906

1,054

1,122

1,222

660

1,300

1,060

1,085

751

672

973

1,178

837

808

980

706

665

1,287

885

915

879

1,228

1,369

1,265

963

1,410

1,082

1,054

1,041

990 


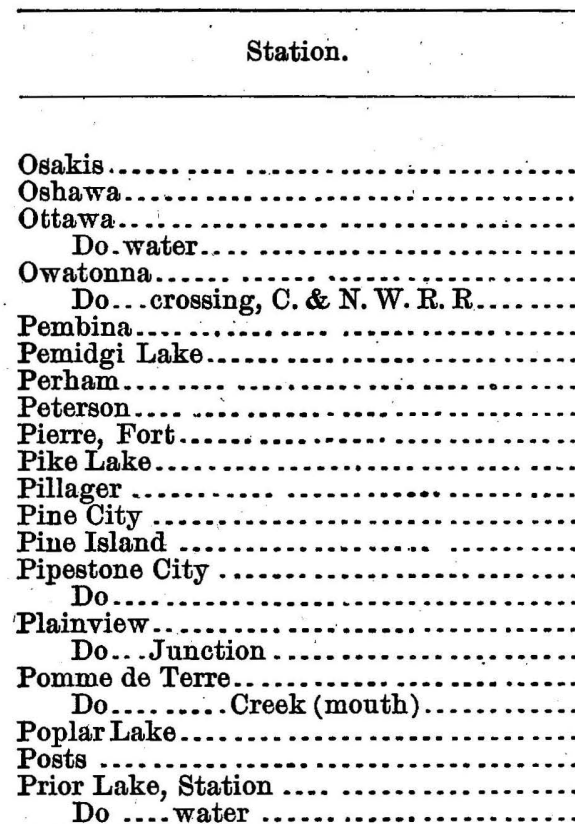

Qui Parle, Lake

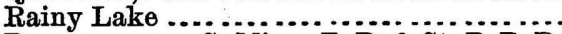

Ramsey, cross. S. Minn.R.R.\& St. P.R.R. Randall

Rapidan

Read's Landing

Do........L. W

Red Wing.......

Redwood Falls

Reinville Trading Post

Renville

Richmond.

Ridgeley, Fort, water.

Ripley, Fort

Rochester

Rolette

Rosemount

Rush City

Rushford.

Rushmore

Sacred Heart

St. Anthony's Falls

Do........ Junction

Do........ . . low water, MississippiRiver.

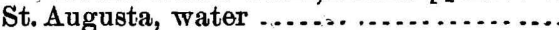

St. Charles

St. Cloud, east shore Mississippi River

Do ... west shore Mississippi River.

Do... low water, Mississippi River.

St. Cloud Mill, Mississippi River ...........

Do ........ low water, Mississippi River

St. Croix Lake, draw-bridge . ............

Do......... low water.................

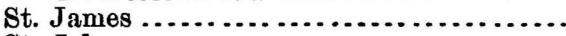

St. Johns ...............................

St. Joseph

St. Oloff.
St. P. \& P. R. R

C. \& N. W. R. R

St. P \& S.C. R R .

U. S. Engineer Corps

C. \& N. W. R. R . .

C., M. \& St. P. R. R.

Toner.

Toner.

N. P. R. R

C., M. \& St. P. R. R

Pacific R. R. Reports

Pacific R. R. Reports

N. P. R. R

L., S. \& M. R. R. R

C. \& N. W. R. R.

St. P. \& S. C. R. R.

C., M. \& St. P. R. R

C. \& N. W. R. R

C. \& N. W.R.R

St. P. \& P. R. R

U. S. Engineer Corps

P. R. R. Reports

C. \& N. W. R. R

C., M. \& St.P.R. R

C., M. \& St. P. R. R

Petermann

Hind .

C., M. \& St. P. R. R

St. P. \& P.R. R

C., M. \& St. P. R. R

C., M. \& St. P. R. R.

U.S. Engineer Corps

C., M. \& St. P.R. R

U. S. Engineer Corps

C. \& N.W.R.R.

C., M. \& St. P. R. R

C., M. \& St. P. R. R

U. S. Engineer Corps

St. P. \& P. R. R.

C. \& N.W. R R

St. P. \& P. R. R

C., M. \& St. P. R. R ........

L., S. \&.M. R. R. R

C., M. \& St. P. R. R..........

St. P. \& S. C. R. R.

C., M. \& St. P.R. R

Smithsonian Inst

St. $P$ \& $P$ R $R$

St. P. \& P. R. R

U. S. Engineer Corps

C. \& N. W. R. R .

St. P. \& P. R. R

St. P. \& P. R. R

St. P. \& P. R. R

U. S. Engineer Corps

St. P. \& P. R. R .

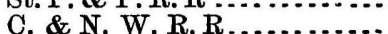

St. $\mathrm{P}$ \& $\mathrm{S} . \mathrm{C}, \mathrm{R}, \mathrm{R}$

St. P. \& P. R. R

St. P. \& P. R. R

St. P. \& P. R. R
Elevation.

Feet.

1,328

982

790

$7 \% 2$

1,144

1,144

900

1,456

1,368

756

828

1,134

1,197

946

998

1,715

1,693

1,167

1,275

1,066

948

1,562

835

949

909

946

$1,100^{\circ}$

1,215

1,045

979

667

$683:$

$68 \%$

659

1,028

946

1,064

674

793

1, 162

991

894

959

911

722

1,665

1,061

820

838

800

946

1,139

1,020

1, 034

962

960

953

691

671

1,073

1,116

1,074

1,335 


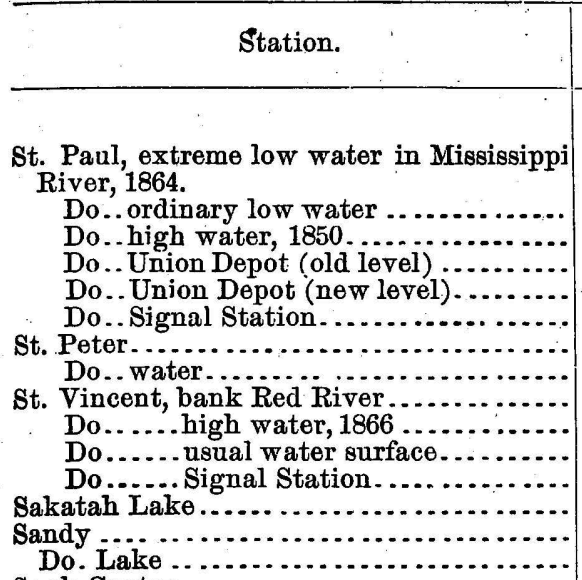

Sauk Center

Sauk Rapids ..........................

Shakopee, crossing of St. P. \& S. C. R. R. Do.... low water, Minnesota River .... Do.... high water, Minnesota River ...

Sherburne ....

Shetek Lake . ........................

Sicottes........................................

Sleepy Eye. Do .... Lake Station

Smith Lake.............................

Snelling, Fort.

South Bend .....

Spirit Lake

Split Rock Ge.........................

, Geodetic Station

Springfield . ............................

spring Valley

Stanley

Stewart...

Do... low water in Saint croix Lake.

Stillwater Junction....................

Stockton ......................................

Sueur Lake .............................

Sumter .............................

Superior, Lake

Swede Grove.

Takara Iake:...

Theilman .

Tigr

Do .... water.......................

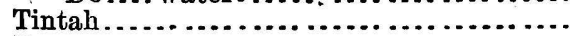

Tracey ...............................

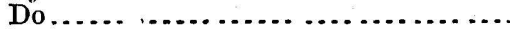

Traverse Lake, water ... .................

Tumuli

Twin Lakes............................

Twin Rivers, water.....................

Tyler

Upper Bass Lake

Utica

Victoria

Viola

Wabash

Do... water

\begin{tabular}{|l|l}
\hline Authority. & Elevation. \\
\hline Feet.
\end{tabular}

U. S. Engineer Corps .

Feet.

Mean result from R. R. levels.

U. S. Engineer Corps ..........

Mean result from $R$. R. levels

Mean result from R. R. levels.

U. S. Signal Office

C. \& N.W. R.R..................

U. S. Engineer Corps

St.P.\& P.R.R

St. P. \& P. R. R ............

St.P. \& P. R. R............

U. S. Signal Office ............

Toner. \& N. W. R. R

(n)...........

Toner \&. P. R. R

St. P.\& P. R. R

C., M. \& St. P. R. R

C., M. \& St. P. R. R

C., M. \& St. P. R. R

C., M. \& St. P. R. R

Petermann

C. \& N. W. R. R

C. \& N. W. R. R

S., P.\& P. R. R ..............

Med. Dep., U. S. A.

St. P. \& S. C. R. R...

Toner U. Lake Survey.

C. \& N. W. R. R..

C., M. \& St.P. R. R.

St. P. \& P. R. R....

St. P., S. \& T. F. R. R

St. P., S. \& T. F. R. R

C. \& N. W. R. R

C. \& N. W. R. R

Petermann . M S S. P. R. R

U. S. Engineer Corps

St. P.\& P.R. R.

Minn. Mid. R. R

N. P. R. R

R. R. reports

C., M. \& St. P. R. R

St. P. \& P. R. R .

C. \& N. W. R. R
Minn. Mid. R. R

U. S. Engineer Corps

St. P. \& P. R. R.

M. \& St I R R

St. P.\& P. R. R.

C. \& N. W. R. R...........

St. P., S. \& T. F. R.R

C. \& N.W.R.R................

St.P.\& P. R. R.............

C. \& N.W.R.R

C., M. \& St. P. R. R .........

U. S. Engineer Corps..........
683.

685 .

702

688

702 .

811

812

729

792

787.

758.

804.

932

1,089

1,450

1,232

1,008

756

692

719

1,273

1,518

1,282

1,034

$1,020^{\circ}$

1,049 .

820

808

755

1,310

765

1, 025 .

1,266 .

902

1,064

680

660

876

753 .

1, 011

1,035

602

1. 186 .

1,168

743.

1, 038

979

979

991

1,403 .

756.

950

$1,196$.

1,255

1,117

1,750

$920 ె$

1,170

1,366

1,129

712

656 


\begin{tabular}{|c|c|c|}
\hline Station. & Authority. & Elevation. \\
\hline & & Feet. \\
\hline Wacouta, low water & U. S. Engineer Corps. & 659 \\
\hline $\begin{array}{l}\text { Wadena } \text {........ } \\
\text { Walnut Grove... }\end{array}$ & $\begin{array}{l}\text { N.P.R.R. } \\
\text { C. \& N. W. R. }\end{array}$ & $\begin{array}{l}1,351 \\
1,223\end{array}$ \\
\hline Waseca ........ & C. \& N.W.R.R... & $\begin{array}{l}1,2253 \\
1,153\end{array}$ \\
\hline Watab $\ldots \ldots \ldots$ & St.P.\& P. R.R.. & 1,057 \\
\hline $\begin{array}{l}\text { Do_Mississippi } \\
\text { Waterville }\end{array}$ & U. S. Engineer Co & 1,001 \\
\hline $\begin{array}{l}\text { Waterville .......... } \\
\text { Watson ............. }\end{array}$ & M. \& St. L. R. R & 1,008 \\
\hline $\begin{array}{l}\text { Watson } . . . . . \\
\text { Waverly .... }\end{array}$ & $\begin{array}{l}\text { C., M. \& St. P.R. R } \\
\text { St. P. \& P. R. R. }\end{array}$ & $\begin{array}{l}1,029 \\
1,007\end{array}$ \\
\hline Wayzota ...... & St.P.\& P.R. R....... & 931 \\
\hline Weaver... & C., M.\& St.P.R. & 674 \\
\hline Wells .......... & C., M. \& St.P.R. R. & 1,153 \\
\hline $\begin{array}{l}\text { Westeott } \\
\text { West Saint Clot }\end{array}$ & C., M. \& St. P. R. R .. & $\begin{array}{r}882 \\
1,025\end{array}$ \\
\hline $\begin{array}{l}\text { West Saint Cloud, } \\
\text { West Sawteeth, Ge }\end{array}$ & U. S. Lake Survey.... & $\begin{array}{l}1,025 \\
1,532\end{array}$ \\
\hline West Union....... & St.P.\& P.R. R.. & 1,323 \\
\hline Whalan..... & C., M. \& St. P. R. R & 786 \\
\hline White Bear ... & M. \& St. L.R.R ... & 919 \\
\hline White Bear Lake & L. S. \& M. R. R. R... & 928 \\
\hline White Earth ...... & Toner & 1,670 \\
\hline $\begin{array}{l}\text { Wilder } \\
\text { Wild Rice }\end{array}$ & 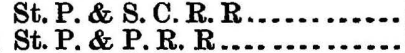 & $\begin{array}{r}1,448 \\
903\end{array}$ \\
\hline 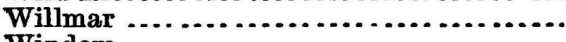 & St. P.\& P. R. R & 1,124 \\
\hline Windom ............... & St.P.\& S.C.R.R... & 1,353 \\
\hline $\begin{array}{l}\text { Winnebago City .... } \\
\text { Winnibigoshish Lal }\end{array}$ & C., M.\& St.P.R.R . & 1,096 \\
\hline $\begin{array}{l}\text { Winnibigoshish I } \\
\text { Winona......... }\end{array}$ & U. S. Engineer Corps... & $\begin{array}{r}1,290 \\
66^{\circ}\end{array}$ \\
\hline $\begin{array}{r}\text { Wrnona } \\
\text { Do.low water } \ldots \ldots \ldots\end{array}$ & U. S. Engineer Corps.... & 634 \\
\hline 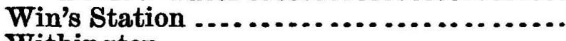 & St.P., S.\& T.F.R.R .... & 916 \\
\hline $\begin{array}{l}\text { Withington } \\
\text { Woods, La] }\end{array}$ & N.P.R.R R................ & 1,272 \\
\hline $\begin{array}{l}\text { Woods, Lake o } \\
\text { Woodstock.... }\end{array}$ & $\begin{array}{l}\text { Hinds } \& \text { St. P. S. } . \text { R. R. } \\
\text { St }\end{array}$ & $\begin{array}{r}978 \\
1,822\end{array}$ \\
\hline Worthington............. & St.P.\& S.C.R.R.. & 1,582 \\
\hline $\begin{array}{l}\text { Wykoff } \ldots \ldots \ldots \ldots \ldots \ldots \\
\text { Wyoming }\end{array}$ & C., M. \& St.P. R. R ..... & 1,310 \\
\hline $\begin{array}{l}\text { Wyoming ......... } \\
\text { Young America }\end{array}$ & L.S.\& M.R.R.R.......... & \\
\hline & Minn. Mid. R. R........ & 856 \\
\hline & Minn. Mid. R. R......... & \\
\hline
\end{tabular}




\section{MISSISSIPPI.}

\begin{tabular}{|c|c|c|}
\hline Station. & Authority. & Elevation. \\
\hline & & Feet. \\
\hline$\ldots \ldots \ldots \ldots$ & M. \& N. W.R. & 85 \\
\hline$\ldots \ldots \ldots \ldots \ldots . . . \ldots \ldots$ & M. \& O.R.R. . & 244 \\
\hline$\ldots \ldots \ldots \ldots \ldots$ & M.\&O.R.R. & 379 \\
\hline 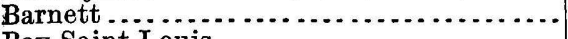 & N. O.\& N.E. & 306 \\
\hline 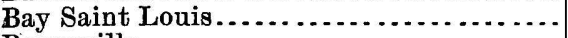 & N. O., M. \& T. & 24 \\
\hline Booneville $\ldots \ldots \ldots \ldots \ldots \ldots \ldots \ldots$ & M. \& O.R.R. & 511 \\
\hline ............................ & M. \& O.R.R.. & 513 \\
\hline 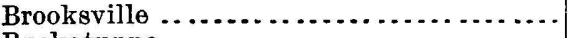 & M. \& O.R.R. & 275 \\
\hline 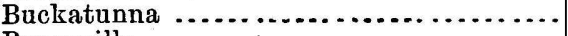 & M. \& O.R. R. & 150 \\
\hline 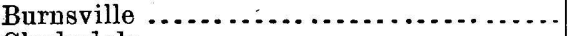 & Memphis \& C. R. R & 463 \\
\hline Clarksdale ....... & M. \& N.W.R.R... & 87 \\
\hline Corinne.......... & N. O. \& N. E. R.R. & 276 \\
\hline$\ldots \ldots \ldots \ldots \ldots \ldots \ldots$ & Memphis \& C & 434 \\
\hline 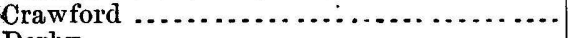 & M. \& O.R.R. & 316 \\
\hline Derby ........... & N. O.\& N.E. 1 & 168 \\
\hline (n) & M. \& O. R. R. & 210 \\
\hline Eastabutchie $\ldots . . . \ldots \ldots \ldots \ldots$ & N. O.\& N. E. R & 169 \\
\hline East Pearl River...$\ldots \ldots \ldots \ldots \ldots \ldots \ldots \ldots$ & N. O. \& N. E.R.R. & 48 \\
\hline (n) & M. \& O. R. R...... & 306 \\
\hline$\ldots \ldots \ldots \ldots \ldots$ & N. O.\& N. E. I & 239 \\
\hline rise ....... & M. \& O.R.R. & 248 \\
\hline Err & \& N. E. R. R. & 260 \\
\hline Glenda & bis \& C. R. R & 495 \\
\hline Do.......... & M. \& N:W.R.R .. & 936 \\
\hline 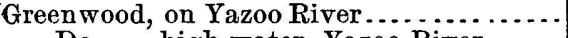 & V.\& N.R.R.... & 154 \\
\hline Do..... high water, Yazoo River..... & V.\& N.R.R... & 158 \\
\hline Grenada, Miss. Cent. R. R . ........... & N. R.R... & $\ddot{213}$ \\
\hline 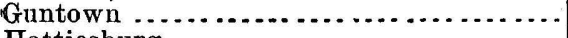 & . R.R..... & 386 \\
\hline 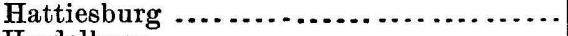 & N. O. \& N. E. R.R. & 144 \\
\hline berg $\quad \ldots \ldots \ldots \ldots$ & N.O.\&.N. E. R.R. & 315 \\
\hline 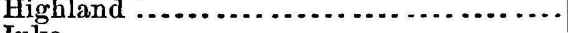 & N.O.\& N. E. R.R. & 174 \\
\hline 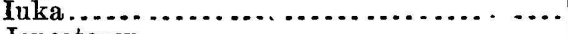 & is \& C. R. R & 455 \\
\hline$\ldots, \ldots \ldots$ & T. W.R.R... & 86 \\
\hline 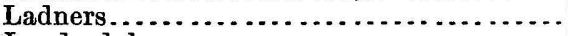 & N. E. R. & 235 \\
\hline dale ...... & . R. R... & 213 \\
\hline$\ldots \ldots \ldots \ldots \ldots \ldots .6$. & N. O.\& N. E.R.R. & 239 \\
\hline Lockhart $\ldots . . .$. & M. \& O.R. R... & 360 \\
\hline Do... Summit & & 426 \\
\hline Lyon ........... & M. \& N. W. R. R & 87 \\
\hline . & M. \& O. R. R.... & 185 \\
\hline . & M. \& N.W.R.R. & 93 \\
\hline 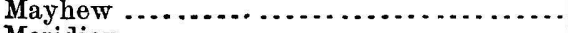 & M. \& O.R.R... & 213 \\
\hline$\ldots \ldots \ldots \ldots$ & M.\& O.R.R...... & 336 \\
\hline pi City .. & N. O., M. \& T. R. R & 10 \\
\hline$\ldots . . . .$. & N. O. \& N. E.R.R. & 69 \\
\hline . & M. \& O.R. R..... & 304 \\
\hline$\ldots \ldots \ldots \ldots \ldots \ldots$ & M. \& O.R.R.... & 183 \\
\hline an Springs ... & N.O., M. \& T. R. R ......... & 28 \\
\hline$\ldots \ldots \ldots \ldots \ldots \ldots \ldots$ & N. O.\& N.E.R.R.............. & 219 \\
\hline 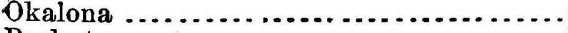 & M. \& O. R. R.... & 311 \\
\hline & N. O. \& N. E. R. & 282 \\
\hline hristian... & N. O. M. \& T. R. R .... & 10 \\
\hline & N. O. \& N. E. R. R..... & 271 \\
\hline Ridge.. & V. \& N. R. R............ & 516 \\
\hline & N. O. \& N. E. R. R...... & 313 \\
\hline & M. \& O. R. R............... & 311 \\
\hline & N. O. \& N. E. R. R......... & 360 \\
\hline & & 231 \\
\hline
\end{tabular}




\begin{tabular}{|c|c|c|}
\hline Station. & Authority. & Elevation. \\
\hline & & \\
\hline ienzi .......... & M. \& O. R. R... & 441 \\
\hline ussells........ & Ala. G. S. R. R & 412 \\
\hline tillo $\ldots . . . . . . . .$. & $\begin{array}{l}\text { M. \& O. R. R... } \\
\text { M. \& O. R. R... }\end{array}$ & $\begin{array}{l}318 \\
193\end{array}$ \\
\hline a. & M. \& O.R. R... & 249 \\
\hline 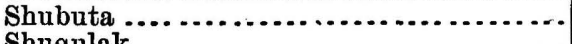 & M. \& O. R. R.. & 197 \\
\hline heo.. & M. \& O. R. R... & 221 \\
\hline Sulo & 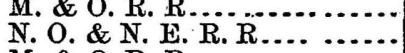 & 245 \\
\hline $\begin{array}{l}\text { Tibbee. } \\
\text { Toomsu. }\end{array}$ & M. \& O.R. R R & 207 \\
\hline $\begin{array}{l}\text { Toomsu } \\
\text { Tupelo }\end{array}$ & $\begin{array}{l}\text { Ala. G. S. R. R. } \\
\text { M. \& O. R. R.. }\end{array}$ & $\begin{array}{l}295 \\
280\end{array}$ \\
\hline & N. O. \& N. E.R.R & 223 \\
\hline Verona ............... & . R. R... & $30 \pi$ \\
\hline $\begin{array}{l}\text { Vicksburg \& Merid } \\
\text { Vicksburg, Signal S }\end{array}$ & N. O.\& N. E.R. & 308 \\
\hline $\begin{array}{l}\text { Vicksb } \\
\text { Vossbu }\end{array}$ & N. O.\&N. E. R. R.... & $\begin{array}{ll}244 \\
424\end{array}$ \\
\hline Waha & M. \& O. R. R......... & 187 \\
\hline ................ & N. O. \& N. E. R. R... & 349 \\
\hline Waynesboro' $\ldots . . . \ldots \ldots \ldots \ldots \ldots \ldots$ & M. \& O.R.R............ & 191 \\
\hline 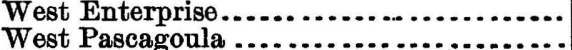 & N. O. \& N. E. R. R........... & $\begin{array}{r}256 \\
8\end{array}$ \\
\hline $\begin{array}{l}\text { West Pascagoula.. } \\
\text { West Point ........ }\end{array}$ & M. \&O.R.R... & 243 \\
\hline & M. \& O. R. R......... & 165 \\
\hline Yazoo Pass .. & M. \& N. W. R. R.... & \\
\hline
\end{tabular}




\begin{tabular}{|c|c|c|}
\hline & Authority. & Elevation. \\
\hline & & Feet. \\
\hline $\begin{array}{l}\text { Alexandria, H. W. Mississippi } \\
\text { Do .....L. W. Mississippi }\end{array}$ & $\begin{array}{l}\text { Mo., Iowa \& Neb. R. } R \\
\text { Mo., Iowa \& Neb. R.R. }\end{array}$ & 487 \\
\hline Allenville & St. L., I. Mt. \& S. R & $\begin{array}{l}400 \\
353\end{array}$ \\
\hline $\mathrm{ma}, \mathrm{L} . \mathrm{W}$. & U. S. Eng'r. Corps . & 651 \\
\hline mazonia ....... & K. C., St. J. \& C. E & 833 \\
\hline anapolis & St. L., I. Mt. \& S. & 635 \\
\hline pleton .......... & Mo., Kans. \& Tex. R. R & 868 \\
\hline to & $\begin{array}{l}\text { Mo., lowa \& Neb. } \\
\text { St. L. \& S. F. R. R }\end{array}$ & $\begin{array}{l}655 \\
708\end{array}$ \\
\hline & H. \& St. J. R. R.. & 739 \\
\hline a Hills & St. L., I. Mt. \& S. B & 335 \\
\hline & Toner $\ldots . . . . .$. & 482 \\
\hline $\operatorname{ta} . . .$. & W., St. L. \& P. R. 1 & 906 \\
\hline & $\begin{array}{l}\text { Smithsonian Inst } \\
\text { St. L. \& S. F. R. }\end{array}$ & $\begin{array}{r}780 \\
1,357\end{array}$ \\
\hline & Mo., Kans. \& Te & $\begin{array}{r}1,357 \\
748\end{array}$ \\
\hline $\operatorname{tra}(-2>$ & W., St. L. \& P. & 647 \\
\hline$\ldots$ & St. L., I. Mt. \& S. I & 417 \\
\hline$\stackrel{B}{B}$ & H. \& St. J. R. R . . & 637 \\
\hline$\dddot{\ldots}$ & K. C., St. J. \& C. B. R. I & 943 \\
\hline tatio & 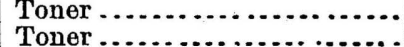 & 512 \\
\hline & Mo., Kans. \& Tex & $\begin{array}{l}506 \\
765\end{array}$ \\
\hline & H. \& St. J. R. R. & 589 \\
\hline B & St. L., I. Mt. \& S. I & 313 \\
\hline & Tor & 470 \\
\hline & & 515 \\
\hline & Tor & 317 \\
\hline & & 531 \\
\hline Bever & K. C., St. I. & 769 \\
\hline Do.crossin & K. C., St. J. \& C. B.R. R & 769 \\
\hline Bevi & H. \& St. J. R. R.. & 780 \\
\hline & K. C., St. J. \& C. B. R. & 861 \\
\hline ld ... & C., G. \& S.L. R. R .... & 317 \\
\hline..... & St. L. \& S. F.R. R . & 1,360 \\
\hline at... & St. L., I. Mt. \& & 321 \\
\hline & St. L., I. Mt. & 1,024 \\
\hline H... & St. L., I. Mt. \& S. R. R .. & 592 \\
\hline & K. C., St. J.\& C. B. R. R & .930 \\
\hline & Ton & 1,000 \\
\hline tills. & Ton & 542 \\
\hline ek. & & 949 \\
\hline & Cans. \& Tex. R. R...... & 607 \\
\hline $\mathrm{B}$ & Tone & 763 \\
\hline & St. L. \& S. F. F & 941 \\
\hline Green ... & L. \& M. R. R. H & 881 \\
\hline$\ldots . .$. & B. \& S. R.R... & 879 \\
\hline idge & St. J. R. R. & 926 \\
\hline & & 516 \\
\hline Broc & H. \& St. J. R. R... & 757 \\
\hline$\ldots . . .6$ & St. L. \& S. F. R. R .... & 1,289 \\
\hline Broo & L. R., V. \& Ark. R. R.. & 285 \\
\hline & $\begin{array}{l}\text { B. \& S. R.R } \dddot{R} \\
\text { W St. L \& R. R. }\end{array}$ & $\begin{array}{l}760 \\
644\end{array}$ \\
\hline & & 1,359 \\
\hline & St. L. \& S. F. R. R. & 897 \\
\hline Bucklin ............................... & H. \& St. J. R. R...... & 786 \\
\hline
\end{tabular}




\begin{tabular}{|c|c|c|}
\hline Stat & Authority. & Elevation. \\
\hline & & Feet. \\
\hline $\begin{array}{l}\text { unkernill } \\
\text { urton } \therefore . .\end{array}$ & Mo.. Kans. \& Tex. $R$ & $\begin{aligned} 1,434 \\
672:\end{aligned}$ \\
\hline ushberg. & St. L., I. Mt. \& S. R. & 411 \\
\hline adet..... & Toner ............... & 805 \\
\hline thoka.... & Mo., Iowa. \& Neb. R & 697 \\
\hline airo ....... & W., St. L. \& P. R. R & 860 \\
\hline Calhoun ... & Mo., Kans. \& Tex. R & 774 \\
\hline alifornia ... & Toner ....... & 864 \\
\hline Callao .......... & H. \& St. J.R.R... & 812 \\
\hline Camden, Missou & W., S. L.\& P.R.R. & 724 \\
\hline Cameron Junctio & H. \& St.J.R.R... & 1,026 \\
\hline Do........... & H \& \& St.J & \\
\hline Camp Branch & Mo., Kans. & $\begin{array}{r}1,028 \\
903\end{array}$ \\
\hline ane Ridge & L. R. V. \& A & 288 \\
\hline Caney Cre & St. L., I. Mt. \& S.R. & 338 \\
\hline Girardeau & C. G. \& S.L.R.R... & 338 \\
\hline Carbon ... & H.\& St.J.R.R.... & 867 \\
\hline Cen & 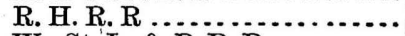 & 772 \\
\hline Carrollton ... & W., St.L.\& P.R. R. & 667 \\
\hline Carthage ...... & St. L. \& S.F.R.R... & 1,269 \\
\hline Cass: & Smithsoni & 3,000 \\
\hline eek. & W., St. L. \& P.R. R. & 854 \\
\hline Cedar City, 2d l & L. \& M.R.R.R .... & 550 \\
\hline Cent & W., St. L. \& & 879 \\
\hline Cent & Toner.... & 856 \\
\hline Centreview ... & Toner .... & 875 \\
\hline Cha & Ton & 531 \\
\hline Cha & St. L., I. Mt. \& S. R. R. & 326 \\
\hline Chillicothe.... & H. \& St.J.R.R...... & 764 \\
\hline Do.....H. \& & W., St. L. \& P.R.R & 746 \\
\hline Chittenham ...... & Toner ... & 458 \\
\hline$\ldots \ldots \ldots$ & Mo., Kan & 751 \\
\hline & H. \& St & 824 \\
\hline ity .... & Mo., Iowa \& Neb. & 694 \\
\hline & W., St. L. & 722 \\
\hline & Mo., Kans. \& & \\
\hline ey........ & St. L. \& S. F. I & 497 \\
\hline & L. R. V.\& & 289 \\
\hline ption .... & W., St. L. \& P. R. R 、 & 1,018 \\
\hline $\operatorname{cse} . . . .$. & W., St.L. \& P.R.R. & 1,075 \\
\hline Con & St. L. \& S.F.R.R... & $\overrightarrow{1}, 408$ \\
\hline & 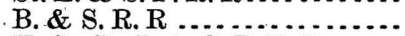 & 789 \\
\hline & K. C., St. J.\& C. B.R.R. & \\
\hline Ronde... & Ton & 732 \\
\hline & K. C., St. J. \& & 871 \\
\hline r............... & St. L. \& S. F. & 1,132 \\
\hline ked Creek Valley & St. L., S. \& L. R. R. R & 881 \\
\hline ................. & St. L.\& S.F.R.R... & 1,010 \\
\hline & & , 637 \\
\hline ne, high water... & W., St. L. \& & 438 \\
\hline Darley ......................... & 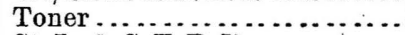 & 1,627 \\
\hline & St. L. \& S. F. R.R.. & \\
\hline Branch... & & 1,205 \\
\hline Deerfield....... & Mo., Kans. \& Tex. R. R. & 791 \\
\hline Lassus..... & St. L., I., Mt. \& S. R. R . & 889 \\
\hline & St. L., I., Mt. \& S. R. R & 547 \\
\hline De & St. L., I., Mt. \& S. R. R & 497 \\
\hline ....... & W., St.L.\& P.R.R & 644 \\
\hline ter........ & St. L., I..Mt. \& S.R. R & \\
\hline 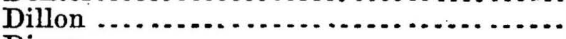 & St.L.\& S.F.R.R... & 1,193 \\
\hline ( $\ldots \ldots \ldots$ & St. L. \& S. F.R.R.. & 1,215 \\
\hline ester & St. L. \& S. F. R. & \\
\hline
\end{tabular}




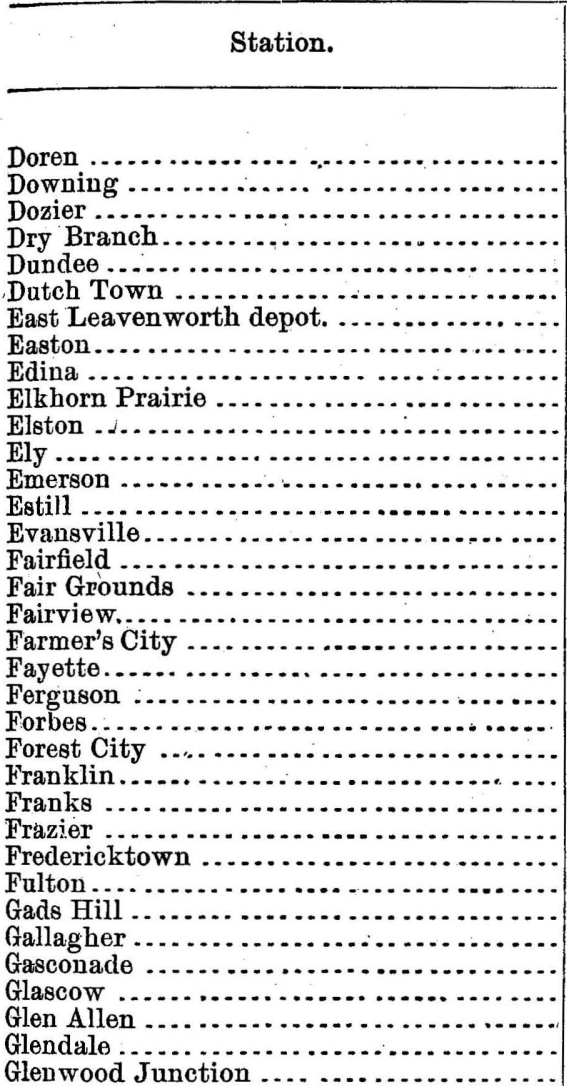

Do ............ cross St. L., K. C.\& N. R. R. at grade.

Gower

Granby City

Grand Prairie

Granger

Gray's Ridge

Greenfield

Greenfield's Landing

Green Prairie

Green Ridge

Green Top

Greenwood.

Hall

Halliday

Halls

Hamilton

Hancock

Hannibal, crossing H. \& St. J. R. R

Do... high water Mississippi River, 1851

Do... low water Mississippi River, 1851.

Do... depot.

Do... junction with H. \& St. J., B. \& Mo. R. \& T. W. \& W. R. R

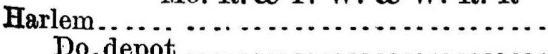

Do.depot $\ldots \ldots \ldots \ldots \ldots$
Do.semaphore $\ldots \ldots \ldots \ldots \ldots \ldots$

\begin{tabular}{|l|l} 
Authority. & Elevation. \\
\hline Feet.
\end{tabular}

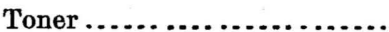

Feet.

Mo., Iowa \& Neb. R. R

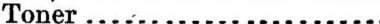

869

St. L. \& S. F. R. R

Smithsonian Inst

K. C., S.J. \& C. B. R. R

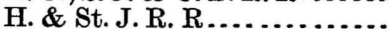

B. \& M, R, R, R

.

St. L. \& S. F. R. R

Toner

.............

H. \& St. J. R. R...

W., St. L. \& P. R. R

Mo., Kans. \& Tex. R. R

Mo., Kans. \& Tex. R. R

$\mathrm{H} \& \mathrm{C} \mathrm{M} \mathrm{R}$

Toner

Toner

Mo., Kans. \& Tex. R. R .

W., St. L. \& P. R. R

K. C., St. J. \& C. B. R. R

K. C., St. J. \& C. B. R. R

Mo., Kans. \& Tex. R. R

St. L. \& S. F. R. R

W., St. L. \& P. R. R...

St. L., I. Mt. \& S. R. R

L. \& M. R. R. R............

St. L., I. Mt. \& S. R. R .

Toner

St. L., I. Mt, \& S. R

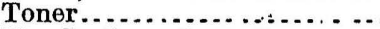

W., St. L. \& P. R. R.......

Mo., Iowa \& Neb. R. R .....

W., St. L. \& P. R. R ........

St. L. \& S. F. R. R..............

St. L. \& S. F. R. R

Mo., Iowa \& Neb. R. R

St. L., I. Mt. \& S. R. R

Smithsonian Inst.

Toner.

St. L. \& S. F. R. R

Mo., Kans. \& Tex. R. R

W., St. L. \& P. R. R. .

Toner.

Toner

K. C. St. J. \& C. B. R. R...

Mo., Kans. \& 'Tex. R. R.

H. \& St. J. R. R

H. \& St. J, R. R

St. L. \& S. F. R. R

H. \& N. R. R.

H. \& St. J. R. R . . . . . . .

H. \&. St. J. R. R ...........

H. \& St. J. R. R.............

Mo., Kans. \& Tex. R. R

455

835

536

329

764

912

738

1,409

715

731

866 .

662

731

977

518.

977

885.

657

505

848

859

591

935.

957

721

843

843.

785

483

630

458

568

998

990

935

$1,0 \% 7$

1,363

759.

300

1,800 .

320

1,374

903

992

907

531

803

772

804

987

1,109

470

469.

447

470

469

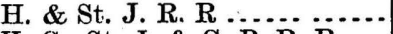

735

K. C., St. J. \& C. B. R. R.... 746 .

K. C., St. J. \& C. B. R. R.... 749 


\begin{tabular}{|c|c|c|}
\hline Stat & Authority. & Elevation. \\
\hline & & \\
\hline Harris .... & Mo., Kans. \& Tex & 853 \\
\hline $\begin{array}{l}\text { Hassard ........ } \\
\text { Hematite }\end{array}$ & Mo., Kans. \& Te & \\
\hline Hendrickson... & St. L., I. Mt. \& S & $\begin{array}{l}476 \\
373\end{array}$ \\
\hline Lermann. & Smithsonian Inst & 598 \\
\hline Hermitag & Toner ............ & 1,037 \\
\hline Do... & St. L. \& S. F. R. R & 1,045 \\
\hline Hibernia & L. \& M. R. R. R..... & 860 \\
\hline Higginsville, Lex. & $\begin{array}{l}\text { Mo., Kans. \& Tex. R. } \\
\text { L. \& M. R. R. R.... }\end{array}$ & $\begin{array}{l}877 \\
647\end{array}$ \\
\hline $\operatorname{logan} . . .$. & St. L., I. Mt. \& S. & 892 \\
\hline Holt ................ & H. \& St. J. R. R.. & 861 \\
\hline Hopewell Summit. & St. L., I. Mt. \& S. I & 985 \\
\hline Topkins........... & K. C., St. J. \& C. B. R. & 1,048 \\
\hline Howard...... & R. H. R. R $\ldots \ldots \ldots$ & 901 \\
\hline nd ..... & H.\& St.J.R. R. & $\begin{array}{l}983 \\
755\end{array}$ \\
\hline un & W., St. \& P.R.R. & 795 \\
\hline 100 & L.\& M.R.R.R. & 995 \\
\hline on $\mathrm{C}$ & St. L. \& S. F. R.R . & 1,036 \\
\hline on & St. L., I. Mt. \& S. & 796 \\
\hline in Sta & St. L., I, M & 1,077 \\
\hline h ........ & St.L.\& S.F.R.R & 1,093 \\
\hline Iront & St. L., I. Mt. \& S. & \\
\hline e....... & W., St. L. \& P.R. R & 861 \\
\hline 3arracks. & $\begin{array}{l}\text { W., St. L. \& P.R.R. R } \\
\text { St, L., I. Mt. \& S.R.I }\end{array}$ & $\begin{array}{l}793 \\
418\end{array}$ \\
\hline Jeron & St. L.\& S.F.R. R... & 693 \\
\hline thes & S.C.\& J.R.R .. & 1,018 \\
\hline Kansas City, Unio & Mea & 748 \\
\hline Do.......Grand & U.T. & 783 \\
\hline ... high wate & H. \& St. J. R. R ... & 752 \\
\hline Do....... Missouri River, & W., St. L. \& P.R.R.... & 746 \\
\hline Do........ high & & \\
\hline $\begin{array}{l}\text { bridge. ......... } \\
\text {.......track on bridge } . .\end{array}$ & K. C., St. J. \& C. I & 753 \\
\hline erack ou orruge ... & H. \& St. J. R. R... & 768 \\
\hline ille bottom & W., St. L. \& P.R. & 635 \\
\hline Kid & H. \& St. J. R. R & 1,017 \\
\hline & St. L., I. Mt & 415 \\
\hline irie .... & St. L. \& S. & 1,442 \\
\hline & Tone & 924 \\
\hline Kịksville $\ldots . . . \ldots \ldots$ & L.\& P.I & 975 \\
\hline Do..junction Saint Louis, Kan & & \\
\hline & B. \& M. R. I & $\begin{array}{l}975 \\
636\end{array}$ \\
\hline & St. L., I. Mt & 926 \\
\hline r ... & Ton & 822 \\
\hline ......... & & 1,084 \\
\hline & B.\& M.R.R.R . & 768 \\
\hline & Ton & 486 \\
\hline & B. \& M.R.R.R . & 741 \\
\hline le................... & B. \& S.R. R ...... & 786 \\
\hline Do...crossing B.\& S. W., grade at & H. \& St. J. R. R........ & 787 \\
\hline 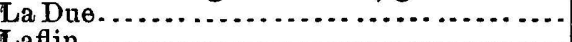 & Mo., Kans. \& Tex. R. R. & 745 \\
\hline & $\begin{array}{l}\text { St. L., I. Mt. \& S. R. R ... } \\
\text { H. \& St. J. R. R ........ }\end{array}$ & 287 \\
\hline & K. C., St. J. \& C. B. R. R... & 826 \\
\hline Do ....... crossing $\mathrm{H} . \&$ St. J. & K. C., St. J. \& B. R. R...... & 829 \\
\hline ceville $\ldots \ldots \ldots \ldots \ldots \ldots$ & C. G. \& S. L. R. R. . . . . . & 351 \\
\hline & Toner ...... & $\begin{array}{l}878 \\
972\end{array}$ \\
\hline & & 1,050 \\
\hline & W.. St. L. \& P. R. R ......... & \\
\hline
\end{tabular}




\begin{tabular}{|c|c|c|}
\hline Station. & Authorit & Elevation. \\
\hline & & Feet. \\
\hline Lathrop ............... & W., St. ] & 948 \\
\hline Leasburg ................... & St. L. & 1,030 \\
\hline 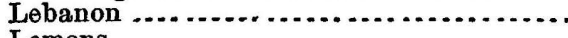 & & 1,269 \\
\hline Lemons $\ldots \ldots \ldots$ & B. \& S. R. R & 1,078 \\
\hline Lentner . . . . . . . . . . & H. \& St. J. R. & 790 \\
\hline Lewis ...................... & Mo., Kans. \& Te & 785 \\
\hline Lewis Prairie..... & L. R. V. \& Ark. & 311 \\
\hline Liberty $\ldots \ldots \ldots \ldots$ & H. \& St. J. R. R & 846 \\
\hline Liberty Landing ... & W., St. L. \& P. & 735 \\
\hline Lingo . . . . . . & H. \& S. J. R. R & 809 \\
\hline 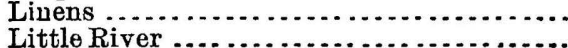 & B. \& S. R. R . & 425 \\
\hline $\begin{array}{l}\text { Little River ........... } \\
\text { Logan's .............. }\end{array}$ & L. R. V. \& Arl & 290 \\
\hline $\begin{array}{l}\text { Logan's .......... } \\
\text { Louisiana ....... }\end{array}$ & St. L. \& S. F. & 1,370 \\
\hline Do . . top of bluff & $\begin{array}{l}\text { L. \& M. K. R. K } \\
\text { L. \& M. R. R. R }\end{array}$ & $\begin{array}{l}460 \\
650\end{array}$ \\
\hline ove Lake........... & W., St. L. \& P. I & 896 \\
\hline Juray ........... & & 737 \\
\hline 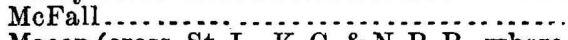 & & 980 \\
\hline $\begin{array}{l}\text { Macon (cross. St. L., K. C. \& N. R. R., where } \\
\text { latter is } 22.5 \text { feet higher. . . . . . . . . . }\end{array}$ & & \\
\hline $\begin{array}{l}\text { latter is } 22.5 \text { feet highe } \\
\text { Macon, depot............ }\end{array}$ & H. $\&$ & 864 \\
\hline Macon, depot........... & H. \& & 867 \\
\hline McQuirk's......... & Toner. & 585 \\
\hline 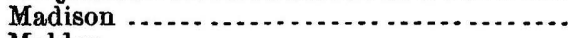 & Mo., Kans. \& Tex. R. R...... & 772 \\
\hline Malden ............ & L. R. V. \& Ark. R. R....... & 297 \\
\hline Malden Creek.... & W., St. L. \& P. R. & 854 \\
\hline Marble Hill . ...... & Toner ........ & 411 \\
\hline Marionsville ........... & St. L. \& S. F. R & 1,338 \\
\hline Marquand ........ & St. L., I. Mt. \& S. R. & 570 \\
\hline Marshall ............... & L. \& M. R. R. R... & 578 \\
\hline Marshfield $\ldots \ldots \ldots \ldots \ldots \ldots \ldots$ & St. L. \& S. F. R. & 1,483 \\
\hline Marysville ........ & K. C., St. J. \& C & 1,037 \\
\hline 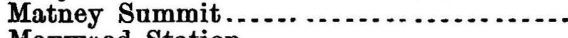 & W., St. L. \& P. & 1,027 \\
\hline Maywood Station ..... & B. \& M. R. R. & 524 \\
\hline Meadville $\ldots \ldots \ldots$ & H. \& St. J.R. R & 729 \\
\hline Memphis, county-seat $\ldots$ & Mo., Iowa \& Ne & $\begin{array}{l}749 \\
787\end{array}$ \\
\hline $\begin{aligned} \text { Do } & \text { Donda }\end{aligned}$ & Mo., lowa de & $\begin{array}{l}787 \\
885\end{array}$ \\
\hline Meramac & Tomer....... & 435 \\
\hline Mercenae & Touer ......... & 419 \\
\hline Mexico & W., St. L. \& P. R. R & 798 \\
\hline Middlebrook $\ldots \ldots \ldots \ldots$ & Toner ........ & 1,144 \\
\hline Milan .............. & B. \& S. R. R. & 840 \\
\hline Millard .......... & W., S. L. \& P. R. R. & 970 \\
\hline Miller's Landing. & Toner ........... & 508 \\
\hline Mill Spring ...... & St. L., I. Mt. \& S. & 443 \\
\hline Mineral Point.... & St. I., I. Mt. \& S. I & 863 \\
\hline Mingo ............. & C. G. \& S. L. R. R. & 338 \\
\hline Miugo Swamp.... & C. G. \& S. L. R. R. & 326 \\
\hline Missonri City.......... & W., St. L. \& P. R. & 722 \\
\hline Moberly ........... & St. L. \& P. R. R.... & 867 \\
\hline Do..junc. with St. L., K. C. \& N. R. R & Mo., & 865 \\
\hline 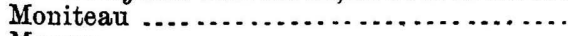 & Toner .. & 904 \\
\hline Monroe ............ & Mo., Kans. \& Tex. F & 728 \\
\hline Montgomery City & W., St. L. \& P. R. R & 831 \\
\hline Mnntrose........ & Mo., Kans. \& Tex & 824 \\
\hline Moody ............ & 1H. \& St.J.R.R & 473 \\
\hline Mooresville ...... & H. \& St. J. R. R & 921 \\
\hline$\ldots \ldots \ldots \ldots \ldots \ldots$ & Toner ............ & 522 \\
\hline (n. & St. L., I. Mt. \& S. R & 345 \\
\hline (n) & St. L. \& S. F. R. R. & 500 \\
\hline . & H. \& St. J. R. R. & 734 \\
\hline Neelysville .............. & & \\
\hline
\end{tabular}

Bull. 5-11 


\begin{tabular}{|c|c|c|}
\hline Station. & Authority. & Elevation. \\
\hline & & \\
\hline $\begin{array}{l}\text { Neosho } \\
\text { Nettleton. }\end{array}$ & St. L. \& S. F. R. R & 1,021 \\
\hline Nevada... & Mo., Kans. \& Tex. R. R. & $\begin{array}{l}928 \\
870\end{array}$ \\
\hline Now Bourbo & Toner.... & 641 \\
\hline New Cambri & H. \& St.J.R.R. & 821 \\
\hline New Madric & L. R. V.\& Ark. R. R & 299 \\
\hline Newport ... & Toner ............... & 482 \\
\hline New Springfie & Toner ................. & 1,532 \\
\hline Niangua .... & St. L. \& S. F. R. R. & 1,428 \\
\hline Nibbard . & W., st. L. \& P. R. R & 1,051 \\
\hline Nishnabotna, & K. C., St. J. \& C. B. F & 879 \\
\hline Nodaway, si & K. C., St. J. \& C. B. R. F & $\begin{array}{l}830 \\
693\end{array}$ \\
\hline North Lexing & $\begin{array}{l}\text { W., St. L. \& P. R. R... } \\
\text { W., St. L. \& P. R. R... }\end{array}$ & $\begin{array}{l}693 \\
741\end{array}$ \\
\hline $\begin{array}{l}\text { North Mo. Ji } \\
\text { North River. }\end{array}$ & H. \& St. J. R. R. & $\begin{array}{ll}741 \\
479\end{array}$ \\
\hline Nosborne. & W., St. L. \& P.R. & 707 \\
\hline Oakland . & Toner .............. & 611 \\
\hline Ogden & Toner ..... & 500 \\
\hline $\mathrm{Oh}$ & Toner ...... & 505 \\
\hline Oregor & Toner ........ & 1,100 \\
\hline Osage & Toner ............ & 541 \\
\hline & H. \& St. J. R. R & 1,036 \\
\hline ille... & Toner & 695 \\
\hline Pacific City & St. L. \& S. F. R. R & 457 \\
\hline myra Jun & H. \& St. J.R. R ........ & 649 \\
\hline Paris ........ & Mo., Kans. \& Tex. R. R. & 651 \\
\hline ( & K. C., St. J. \& C. B.R. R. & 753 \\
\hline & W., St. L. \& P. R: R. & 772 \\
\hline Pendleton (sum & W., St. L. \& P. R. R & 915 \\
\hline Pevel & St. L., I. Mt. \& S. R. & 441 \\
\hline $\mathbf{P h}$ & K. C., St. J. \& C. B. R & 895 \\
\hline Pjeker & K. C., St. J. \& C. B. R. & 1,023 \\
\hline Piedr & St. L., I. Mt. \& S. R. & 503 \\
\hline City .. & St. L. \& S.F.R. R. & 1,176 \\
\hline Pilot Grove .. & Mo., Kans. \& Tex. I & 832 \\
\hline Pla & W., St. L. \& P.R. R & 819 \\
\hline Pla & W., St. L. \& P.R. I & 948 \\
\hline Pleasan & Mo., Kans. \& Tex. & 775 \\
\hline Plymouth ........ & St. L. \& S. F.R. R & 1,326 \\
\hline $\begin{array}{l}\text { Pollock ....... } \\
\text { Poplar Bluff. }\end{array}$ & B. \& S.R.R R.... & 943 \\
\hline $\begin{array}{l}\text { Poplar Bluff.... } \\
\text { Do .... bank Black River. }\end{array}$ & St. L., I. Mt. \& S. R. & $\begin{array}{l}343 \\
326\end{array}$ \\
\hline $\begin{array}{r}\text { Do } \\
\text { Prairie City } . . .\end{array}$ & & $\begin{array}{ll}326 \\
771\end{array}$ \\
\hline & Mo., Kans. \& Tex & 695 \\
\hline Purdin........ & B. \& S.R.R ...... & 887 \\
\hline City ... & W., St. L. \& P. R. R & 1,004 \\
\hline Re & & 99 \\
\hline & Toner .................... & 372 \\
\hline $\mathrm{d}+\ldots \ldots \ldots$ & Mo., Kans. \& Tex. R. R & 733 \\
\hline ind $\ldots . . .$. & Smithsonian Inst ........ & 800 \\
\hline ill .... & R. H. R. R .......... & 784 \\
\hline & St. L. \& S.F.R. R .. & 1,143 \\
\hline & W., St. L. \& P.R. R. & 809 \\
\hline d and Lexingto & W., St. L. \& P. R. R & 699 \\
\hline & St.L.\& S. F.R.R... & 1,066 \\
\hline Robertson.......... & 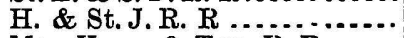 & 870 \\
\hline Rockville ........ & Mo., Kans. \& Tex. R. R. & 771 \\
\hline & St. L. \& S. F. R. R..... & 1,085 \\
\hline Ruseberry ....... & W., St. L. \& P. R. R ... & 977 \\
\hline $\begin{array}{l}\text { Rosedale.......... } \\
\text { Rosehill }\end{array}$ & K. C., Ft. S. \& G. R. R . & 795 \\
\hline dale......... & K. C., St. J. \& C. B. R. I & $\begin{array}{l}923 \\
920\end{array}$ \\
\hline Grove...... & H. \& St. J. R. R........ & 836 \\
\hline
\end{tabular}




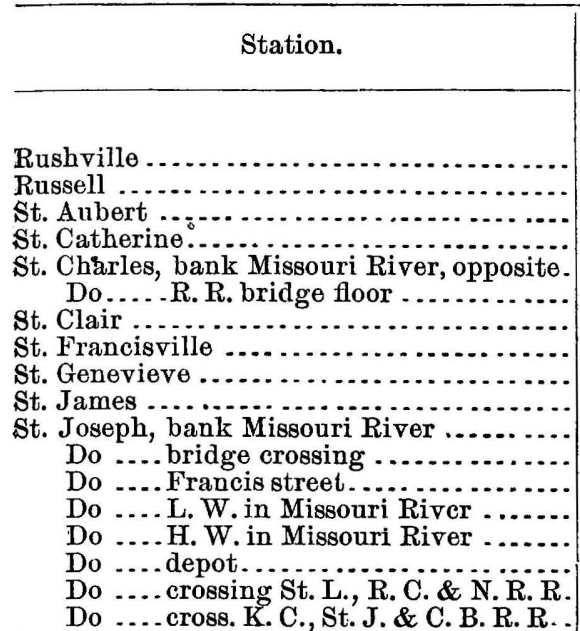

St. Louis, old station.

Do .. city directrix

Do

Do ... Union Depot.

Do .. Fourth street . . . . . . . . . . . . . . . .

Do ... H. W. 1844 ...

Do ... L. W. Miss. R.

Do ... bed Mississippi River.

Do .. bronze plate on W. land pier of great bridge...................

Do .. Signal Station . . . . . . . . . . . . . .

St. Paul .............................

Salem

Salina City

Salisbury Junction

Satan

Savannah, new station

Do.... old station

Saxton.

Schell City . . . . . .

Schockley's Gat.

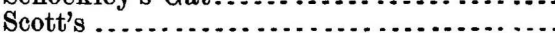

Selalia, junction M. P. \& St. L. \& L. R. R.

Seneca...

Shawnee...........................

Shelbina

Sikeston

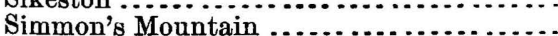

Sleeper

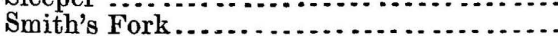

Smithton

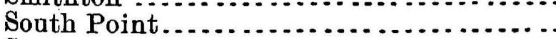

Sprague

Spring Creek

Springfield

Stafford

Stanberry 0 -

Stanton.

Stewartsville

Stockton ................

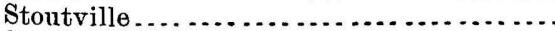

Stoutsville ...........................

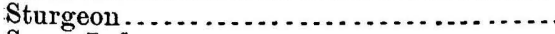

Sugar Lake .............................

Sullivan...

Sulphur Springs.

\begin{tabular}{|c|} 
Authority. \\
\hline
\end{tabular}

K. C., St. J. \& C. B. R. R Feet.

Mo., Kans. \& Tex. R. R ..... $\quad 760$

Toner ....................... 527

H. \& St. J. R. R........... 797

W., St. L. \& P. R. R ........ 444

W., St. L. \& P. R. R ........ 504

St. L. \& S. F. R. R ........... $\quad 759$

C. G. \& S. L. R. R .......... 333

Toner .................... $\quad \mathbf{3 7 2}$

St. L. \& S. F. R. R ........ 1,117

W., St. L. \& P. R. R ........ 823

K. C., St. J. \& C. B. R. R .... 826

K. C., St. J. \& C. B. R. R .... 824

St. J. \& D. C. R. R ......... 792

St. J. \& D. C. R. R......... 812

H. \& St. J. R. R............ $\quad 825$

H. \& St. J. R. R............ 827

H. \& St. J. R. R........... 839

St. L., I. Mt. \& S. R. R ........ 418

Levels of Miss. River Com.... $\quad 413$

U. S. C. \& G. S ..... . . . . . . . ... 416

City Engineer ............... 431

St. L., V. \& T. H. R. R ...... $\quad 470$

City Engineer ............... 428

City Engineer . .............. 379

St. L., V. \& T. H. R. R ...... 363

U. S. C. \& G. S .

U. S. Signal Office.

Toner $\ldots$ L \& L. R. R. R

R

L. \& M. R. R. R.

W., St. L. \& P. R. R .......

K. C., St. J. \& C. B. R. R

461

568

428

1,182

650

721

K. C. St. J. \& C. B. R. R K. C., St. J. \& C. B. R. R

709

1,100

1,023

H. \& St. J. R. R ...........

Mo., Kans. \& Tex. R. R

881

St. L \& S. F. R R R R .

Toner $\ldots \ldots \ldots \ldots \ldots \ldots$

754

1,367

622

907

St. L. \& S. F. R. R .......... 851

K. C., Ft. S. \& G. R. R

H. \& St. J. R. R........... 779

St. L., I. Mt. \& S. R. R...... 330

St. L., S. \& L. R. R........... $\quad 1,250$

St. L. \& S. F. R. R.......... $\quad 1,209$

W., St. L. \& P.R. R ......... 879

Toner .......................

Tower .....................

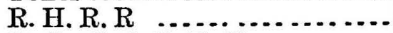

St. L., S. \& L. R. R

St. L. \& S. F. R. R

St. L. \& S. F. R. R

W., St. L. \& P. R. R

St. L \& S. F. R R . R ...

H. \& St. J. R. R ..............

Smithsonian Inst .

St. L. \& S. F. R. R.

Mo., Kans. \& Tex. R. R

W., St. L. \& P. R. R...

K., C. St. J. \& C. B. R. R

St. L. \& S. F, R. R

St. L., I. Mt. \& S. R. R.......

888

510

917

1,123

1,352

1,479

876

867

958

800

1.166

605

847

794

967

411 


\begin{tabular}{|c|c|c|}
\hline Station. & Authority. & Elevation. \\
\hline & & \\
\hline Sutton ..... & Toner........ & \\
\hline Sylvania... & Toner......... & $\begin{array}{r}339 \\
\end{array}$ \\
\hline 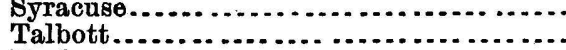 & Mo. Kans \& Tex.R.R. & $\begin{array}{r}944 \\
. \quad 620\end{array}$ \\
\hline Taplors................ & St.L.\& S.F.R.R...... & - 1,004 \\
\hline Taylorville. & 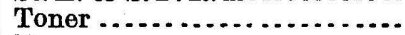 & 470 \\
\hline Tipton............... & Toner................. & 944 \\
\hline Tip-top Snmmit... & St. L., I. Mt. \& S. R. R & 1,200 \\
\hline Tolona........... & B. \& M.R.R. R..... & 697 \\
\hline Tower Grove........ & Smithsonion Inst.. & 500 \\
\hline Turnback Summit.... & St. L. \& S. F.R. R. & 1,383 \\
\hline Turney............... & H. \& St.J.R. R... & $-1,048$ \\
\hline Tuscumbia................ & Smithsonian Inst. & 600 \\
\hline Union ................ & Smithsonian Inst... & 616 \\
\hline Unionville..... & B.\& S.R. R....... & 1,068 \\
\hline Utica....... & H. \& St. J.R.R ... & 739 \\
\hline Verona...... & St.L.\& S.F.R.R..... & 1,262 \\
\hline Waldron... & K. C., St.J.\& C. B. R. R & 757 \\
\hline Walker's....... & Mo., Kans. \& Tex. R. R. & 856 \\
\hline Warrenton.... & W., St. L. \& P. R. R... & 853 \\
\hline Washington.... & Toner .............. & 487 \\
\hline Watson ......... & K. C., St. J.\& C. B. R. R & 904 \\
\hline Wayland............. & Mo., Iowa \& Neb. R. R.. & 531 \\
\hline Webster.............. & & 525 \\
\hline Wellsburgh.. & Toner $\ldots \ldots \ldots$ & 778 \\
\hline Weston......... & K. C., St. J. \& C. B. R. & 773 \\
\hline West Plattsburgh, summit. & W., St.L. \& P.R. R. & 1,020 \\
\hline West Quincy......... & H. \& St. J. R. R.... & 483 \\
\hline Do....... high-water in Missouri Rive & H. \& St.J.R.R. & 477 \\
\hline Do.......junction with H. \& S.J.R.R & B. \& M. R. R. R.. & 489 \\
\hline Wheeling & H.\&.St. J. R. R...... & 740 \\
\hline Williamsville... & St. L., I. Mt. \& S. R. R. & 401 \\
\hline Windsor....... & Mo., Kans. \& Tex. R. R. & 875 \\
\hline Windthrop ............. & H. \& St. J. R. R .......... & 801 \\
\hline Winthrop, junction .... & K. C., St. J. \& C. B. R. R & 789 \\
\hline Do.... depot & K. C., St. J. \& C. B. R. R & 789 \\
\hline Do..... top of rail C. \& A. bridge & K. C., St. J. \& C. B. R. R & 798 \\
\hline Do.... crossing R. I. \& P.R. R.. & K. C., St. J. \& C. B. R. R & .788 \\
\hline 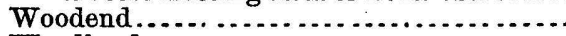 & St. L. \& S. F. R. R... & 1,113 \\
\hline Woodland. & H. \& St. J. R. R....... & 679 \\
\hline Yeatman....... & Toner.................... & \\
\hline
\end{tabular}


MONTANA.

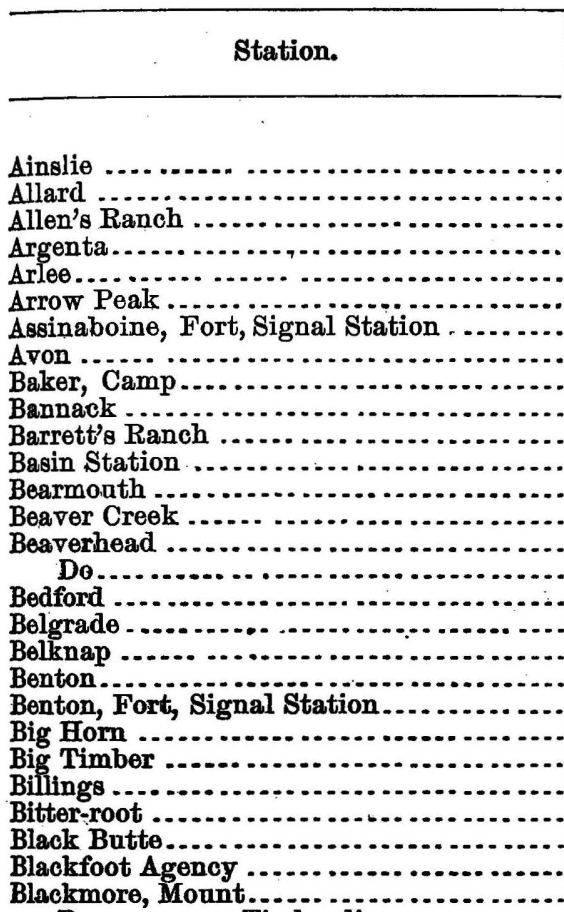
Do

Bonita

Boteler's Ranch

Do

Boulder City (opposite)

Bouldertown

Boundary Pass

Bozeman

Do

Brewer's Springs

Bridger's Pass.

Do....Peak

Do.... do

Butler

Cabinet

Cadotte's Pass

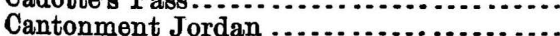

Carroll

Castle Rock

Clancy

Clark's Fork

Clasoil

Coal Spur.

Cone Butte.

Conical Peak.

Cook's Ranch

Coriacan Defile Pass

Cowan, Mount

Crazy Peak

\section{Timber line on}

Authority.

N.P. R. R

Hayden

De Lacy.

N. P.R. R

N.P.Transcontinental Survey.

U. S. Signal Office............

N.P. R. R

Ludlow

De Lacy.

De Lacy.

De Lacy

N.P.R. R

N.P. R. R

Hayden

De Lacy

Pre. R. R. levels

N. P. R. $\mathbf{R}$

N. P. R. R.

N. P.Transcontinental Survey:

U. S. Signal Office

N. P. R. R

N.P.R.R $R$

N.P.R.R.

N. P. Transcontinental Survey.

Hayden.

Hayden .......................

N.P.R.R.

Hayden

Pre. R. R. levels

De Lacy.......................

P. R. R. Reports..................

Hayden .

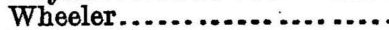

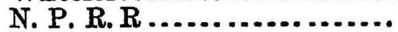

Ludlow

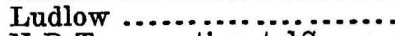

N.P. Transcontinental Survey.

Hayden

N.P.R.R

N.P.R.R

Pacific R. R. Reports

Ludiow

N.P.Transcontinental Survey.

Pre. R. R. levels

N.P.R.R.

N.P. R. R.

N. P. R. R . . . . . . . . . . . .

N.P.Transcontinental Survey

N.P. Transcontinental Survey.

De Lacy

N.P.R.R.

Hayden ......................

N. P.Transcontinental Survey.
Elevation.

Feet.

2,272

2,299

4,396

6,337

3,057

7,420

2,650

4,675

4,538

5,896

5,665

5,437

3,787

2,639

4,464

4,888

3,845

4,435

2,405

2,600

2,674

2,688

4, 070

3,115

2,480

5,540

3,169

10,134

9,550

3,564

4,825

4,873

4,904

5,000

6,030

4,900

4,839

4,752

4,957

6,147

9,106

9,002

4, 725

12,187

6,044

2,990

2,247

4,947

4,262

2,086

4, 123

4,735

5,820

10,737

4,995

3, 946

10, 351

11,178 


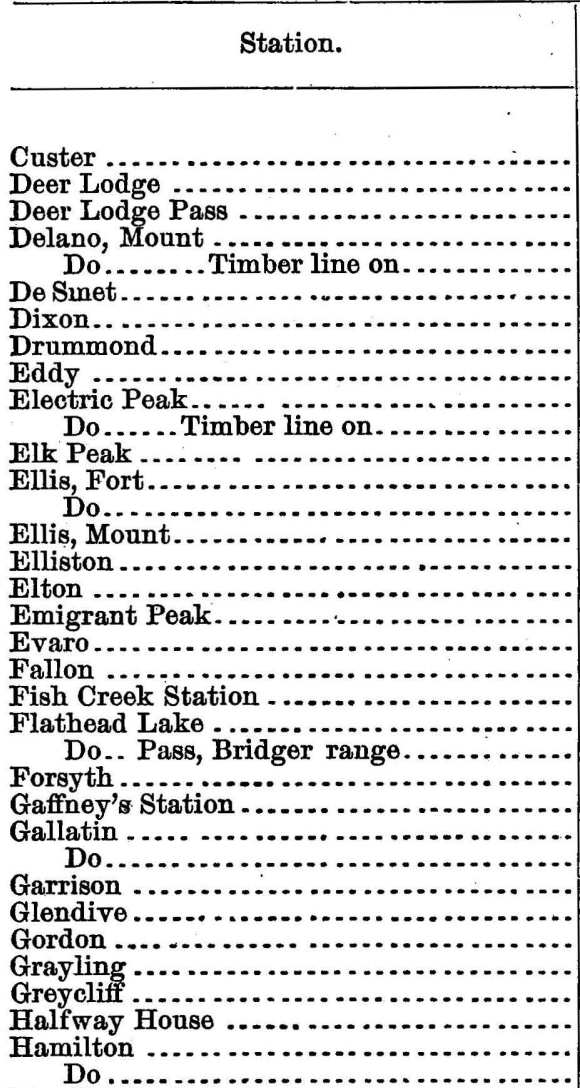

Hamilton's, Jeff. Davis Gulch.

Hathaway

Helena.

Do

Do B. M. in

Do.corner stone in basement of old school house.

Do.Signal Station

Hellgate .

Heron.

Highwood Peak

Hilgard, Mount

Hodges

Hope

Hopley's Hole

Horse Plain .

Horton

Huntley

Jefferson Bridge

Jefferson City

Jefferson City

Jocko

Judith Gap

Judith Peaiz

Keogh, Fort

Last Spike

Laurel.

\begin{tabular}{|l|l|}
\hline Authority. & Eleva \\
\hline
\end{tabular}

N.P.R.R

Stewart

N.P.R. R. Surveys

Hayden

Hayden

N.P.R. R

N.P.R.R

N.P. R. R

N.P.R. R

Hayden

Hayden ......................

N.P.Transcontinental Survey

Hayden

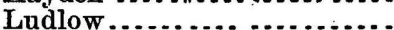

N.P.Transcontinental Survey. N.P.R. R

N.P.R.R

Hayden

N.P.R. R

N.P.R.R

Pacific R. R. Reports............

Hayden

N.P.R.R

De Lacy.

N. P. R. R

Hayden

N.P.R.R

N.P.R. R

N. P. R. R

U. \& N.R.R

N. P. R. R

Pre. R. R. levels

Hayden

N. P. R. R

De Lacy.

N.P. R. R

Hayden

N.P.R. R

De Lacy

Pre. R. R. levels.

Pre. R. R. levels

U. S. Signal Office

Toner

N. P. R. R

Hayden

N.P. R.R

N.P. R. R.

Ludlow

N.P.R. R

N.P.R. R

N.P.R. R

N.P.R. R

De Lacy.

De Lacy

Pre. R. R. level

N. P. R. R

N.P.Transcontinental Survej.

N.P.Transcontinental Survey.

N.P. R. R

N. P. R. R

N. P. R. R
Elevation.

Feet.

2,725

4,768

5,808

10,200

8,784

3,213

2,320

3,943

2,415

11, 155

9,442

8,606

4,935

4,747

8,419

5,036

4,280

11,034

3,946

2, 206

4,134

2,800

6,769

2,512

4,838

4,030

4, 132

4, 295

2,067

4,905

5,442

3,845

4,157

4,342

4,240

6,536

2,426

4, 266

3,930

4,296

4, 061

4,149

4,262

3,318

2,261

7,604

11,000

2,535

2,108

4,894

2,463

2,390

2,559

3,012 .

4. 672

4,776 .

4,575

2,507

4,650

6,386 .

2,365

4,253

3,253 


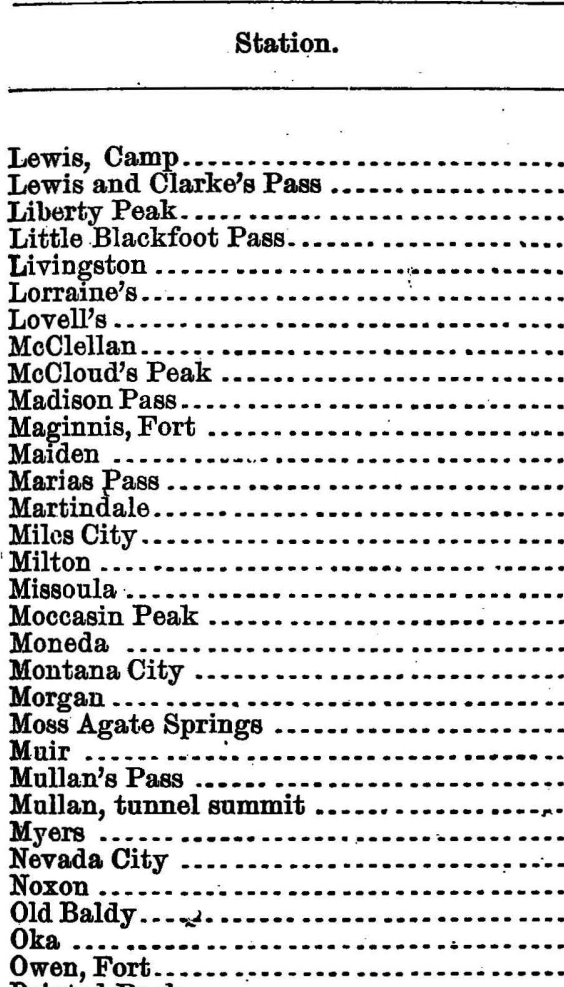

Painted Rock

Park City

Parson's Bridge, Jefferson River

Perma

Philbrook

Placer

Pómaine's

Pompey's Pillar

Porcupine Butte

Reynolds' Pass

Riverside

Salisbury Station on the bench

Sanders

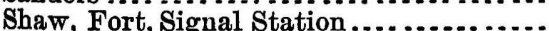

Sheridan

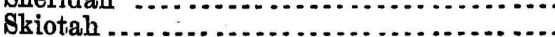

South Nez Percé Pass

Sphinx Mountain

Springdale

Square Butte

Sterretts

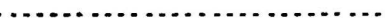

Taghee Pass

Ten Mile Station.

Terry

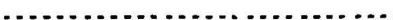

Three Buttes, highest.

Townsend.

Trout Creek

Twin Peaks

U-Bet.

Union Pass

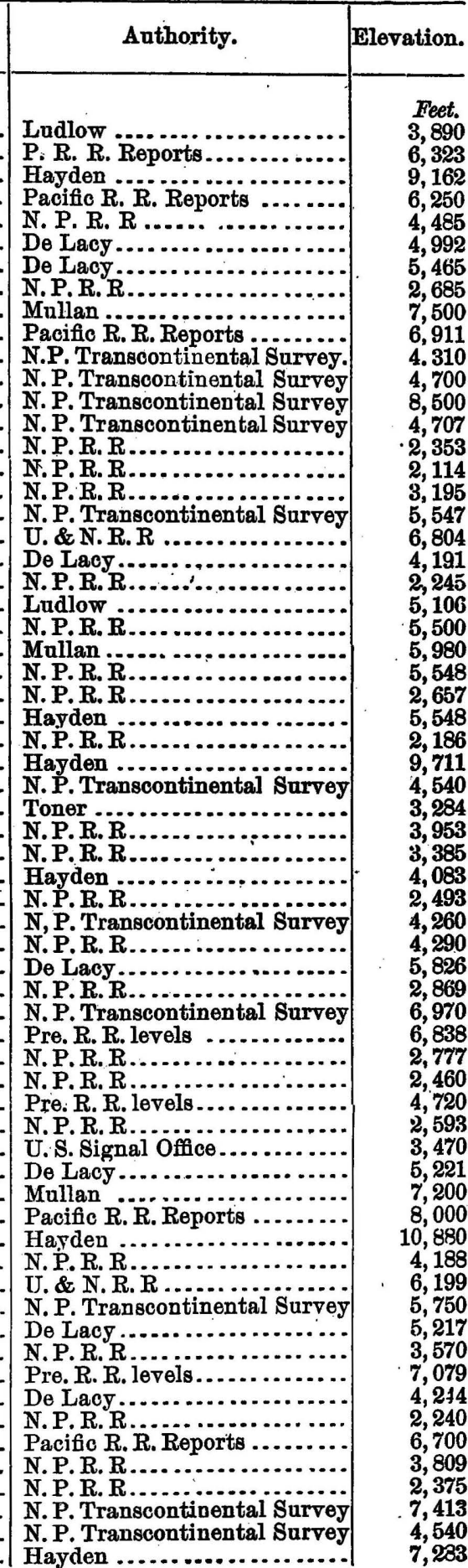




\begin{tabular}{|c|c|c|}
\hline Station. & Authority. & Elevation. \\
\hline & & \\
\hline U. N. Junction. & N.P.R.R. & 4,3 \\
\hline Utica....$\ldots \ldots$ & N.P.Transcontinental Survey & 4,420 \\
\hline Virginia City .... & Hayden ........................ & 5,824 \\
\hline 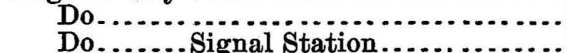 & 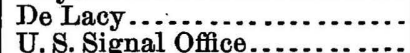 & $\begin{array}{l}5,778 \\
5,480\end{array}$ \\
\hline Ward's Peak & Hayden ............................... & $\begin{array}{r}0,400 \\
10,371\end{array}$ \\
\hline Do....... timber line on ............ & Hayden............... & 9,156 \\
\hline Warm Springs Hotel ......... & Pre.R.R. levels .. & 4296 \\
\hline Stage Statio & Pre. R. R. levels .. & 5,269 \\
\hline Wedge Monntain $\ldots . . . . . . .$. & Hayden .............. & 10,342 \\
\hline Whitehall Stage Station..... & Pre.R.R. levels ... & 4,493 \\
\hline 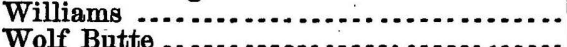 & U.\&N.R.R _................ & $\begin{array}{l}6,603 \\
4,760\end{array}$ \\
\hline & N.P.Transcontinental Survey & 4,760 \\
\hline
\end{tabular}


NEBRASKA.

\begin{tabular}{|c|c|c|}
\hline Stat & Authority. & Elevation. \\
\hline & & \\
\hline dams .... & A. \& N.R.R. & 1,250 \\
\hline lbion .... & O.N.\& B.H.R.I & \\
\hline & U.P.R.R............. & 1,922 \\
\hline lexandria & $\begin{array}{l}\text { St.J.\&. D.C.R. } \\
\text { U.P.R.R...... }\end{array}$ & $\begin{array}{l}1,308 \\
3,063\end{array}$ \\
\hline Alma ........... & R.V.\& B.\& & 1,944 \\
\hline Amboy ...... & B. \& M.R.R. & 1,693 \\
\hline e. & U.P.R.R... & 4,712 \\
\hline rapaho. & R. V.\& B. \& & 2,177 \\
\hline clington & B. \& M. R. R. & 1,226 \\
\hline shland... & B. \& M. R. I & 1,101 \\
\hline aburn .. & B. \& M. R. R. & 1,052 \\
\hline arora... & B. \& M. R.R. R & 1,803 \\
\hline $\mathrm{yr} \ldots \ldots$ & B. \& M. R. R. R.. & 1,847 \\
\hline Barton.......... & $\begin{array}{l}\text { Toner } \\
\text { S. C. \& P.R. R }\end{array}$ & $\begin{array}{l}3,421 \\
1,602\end{array}$ \\
\hline Beatrice ....... & B. \& M.R.R.R & 1,261 \\
\hline Belle Cree & S. C. \& P.R. R. & 1,175 \\
\hline elle Wood & A. \& N.R.R. & 1,451 \\
\hline videre. & St. J. \& D. C & 1,501 \\
\hline Benklema & R. V. \& B. \& C. I & 2,975 \\
\hline & B. \& M. R.R.R. & 1,256 \\
\hline Benton: & U. P. R. R ..... & 1,404 \\
\hline Berk & B. \& M. R.R.R. & 1,428 \\
\hline g Spring. & U.P.R.R........ & 3,371 \\
\hline Blair ....... & S. C. \& P. R. & 1,100 \\
\hline igto & B. \& $C$ & 1,848 \\
\hline iil. & B. & 1,978 \\
\hline Bradshaw & B. \& M.R.R. R ............... & 1,725 \\
\hline & U.P.R.R R $\ldots \ldots$ & 2,657 \\
\hline $\mathrm{Br}$ & R. V.R.R. & 1,687 \\
\hline .... & Ton & 4,200 \\
\hline & .......... & 894 \\
\hline & R.V.\& B.\& C. & 1,377 \\
\hline $\mathrm{Bu}$ & R. V.\& B.\& C.I & 1,367 \\
\hline$B$ & 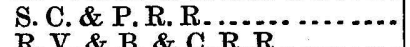 & 1,691 \\
\hline idge. & $\mathbf{R} \ldots \ldots$ & $\begin{array}{l}2,262 \\
1,275\end{array}$ \\
\hline $\begin{array}{l}\mathrm{Ca} \\
\mathrm{Ca}\end{array}$ & (n)................ & $\begin{array}{l}1,270 \\
1,327\end{array}$ \\
\hline $\mathrm{Ca}$ & St.J. \& D. C. R. R & 1,554 \\
\hline & R. V.\& B.\& C.R.R & 1,998 \\
\hline & M. R.R.R... & 1,028 \\
\hline & B. \& M.R.R.R & 1,708 \\
\hline 's...... & U.P.R. R & 1,77 \\
\hline s.... & & $\begin{array}{l}5,702 \\
1,435\end{array}$ \\
\hline s....... & R. V.\& B.\& C.R.R............. & 1,621 \\
\hline Fort.. & & \\
\hline & U.P.R.R K.... & \\
\hline Creek ... & O.\& R.V.R.R .. & 1,185 \\
\hline $10 . . .$. & Toner .............. & 1,518 \\
\hline cus.. & U.P.R.R ....... & 1,453 \\
\hline Cowle & B. \& M.R.R. R & 1,801 \\
\hline . & U.P.R.R $\ldots \ldots \ldots \ldots \ldots \ldots$ & 2,501 \\
\hline & B. \& M.R.R.F & 1,368 \\
\hline bertson... & S. C. \& P. R. R & 1,285 \\
\hline bertson.... & R.V.\& B.\& C. & $\begin{array}{l}2,57 \% \\
1,090\end{array}$ \\
\hline & & \\
\hline
\end{tabular}




\begin{tabular}{|c|c|c|}
\hline Station. & Authority. & Elevation. \\
\hline & & Feet. \\
\hline $\begin{array}{l}\text { Davenport ..... } \\
\text { David City ... }\end{array}$ & $\begin{array}{l}\text { St. J. \& D. C. R. R } \\
\text { A. \& N. R. R..... }\end{array}$ & \\
\hline Dawson........ & A. \& N.R.R.... & \\
\hline De Soto.. & Toner .......... & 1,100 \\
\hline Denton ... & B.\& M.R.R.R & 1,247 \\
\hline Dewitt ... & B. \& M. R. R.R & 1,299 \\
\hline Dexter... & Toner .......... & 3,000 \\
\hline Diller...... & R. V.\& B. \& C. I & \\
\hline Dorchester & B. \& M. R. R. R. & 1,501 \\
\hline Dunbar... & B. \& M. R. R. R & 1,051 \\
\hline Duncan.... & U. P. R. R ...... & \\
\hline Eden ... & St. J. \& D. C. R & 1,729 \\
\hline El Dorado & Toner ................. & 1,693 \\
\hline Elk Creek. & A. \& N. R. R ... & 1,072 \\
\hline Elk Horn. & U.P.R.R... & 1,165 \\
\hline Elm Creek & U.P. R. R...... & 2,273 \\
\hline Emerald ... & A. \& N. R. R.. & 1,206 \\
\hline Emerson. & Toner ............ & 1,300 \\
\hline Endicott.. & R. V. \& B. \& C. R & 1,291 \\
\hline Exeter..... & B. \& M. R. R. R. . & 1,622 \\
\hline Fairbu & St. J. \& D. C. R. & 1,316 \\
\hline Fairfield .... & St. J. \& D. C. R. R & 1,780 \\
\hline Fairmont .... & B. \& M. R. R. R... & 1,656 \\
\hline Falls City. & A. \& N. R. R ... & 904 \\
\hline Firth .... & A. \& N. R. R .... & 1,319 \\
\hline Fontenel & Smithsonian Inst. & 1,000 \\
\hline Frankl & R. V. B. \& C. R. R & 1,820 \\
\hline Fremont & U. P. R. R ...... & 1,203 \\
\hline Friendville... & B. \& M. R. R. R.. & 1,573 \\
\hline Gau & U.P.R.R........ & 2,752 \\
\hline Garrison ..... & A. \& N.R. R.... & 1,602 \\
\hline Genoa ....... & O. N. \& B. H. R.R & 1,584 \\
\hline rgetown.. & St. J. \& D. C. R. R & 1,844 \\
\hline mantown & B. \& M.R.R.R.. & 1,399 \\
\hline Gibl & U. P.R. R........ & 2,067 \\
\hline Gib & A. N N.R.... & 860 \\
\hline$\ldots .$. & U. P.R. R ........ & 998 \\
\hline Gle & hsonian Inst. & 1,300 \\
\hline & B. \& M. R. R. R.. & 1,699 \\
\hline Island & U. \& P. R. R.... & 1,871 \\
\hline ort. & Warren....... & 8,325 \\
\hline ood... & B. \& M. R. R. R.... & 1,141 \\
\hline ock. & R. V. \& B. \& C. R. R. & 1,650 \\
\hline & B. \& M. R. R.R R.... & 1,770 \\
\hline $\mathrm{Ha}$ & R. V.\& B.\& C.R.R. & 1,673 \\
\hline Har & R. V.\& B.\& C. R.R. & 1,512 \\
\hline & M.R.R.R ...... & 1,812 \\
\hline s........ & B. \& M.R.R. R .......... & 1,947 \\
\hline $\mathbf{r} \ldots \ldots$. & R.V.\& B.\& C.R.R.... & 3,265 \\
\hline Hickman ... & A. \& N.R.R. ......... & 1,247 \\
\hline & \& P. 1 & 1,237 \\
\hline & R. V.\& B.\& C. R.R ... & 1,460 \\
\hline oldt.... & A. \& N. R.R....... & 985 \\
\hline breys .. & O. N. \& B. H. & 1,650 \\
\hline ........ & St. J. \& D. C. R. R... & 2,003 \\
\hline & R. V. \& B. \& C. R. R & 1,729 \\
\hline & R. V. \& B. \& C. R. R & 2,380 \\
\hline & B. \& M.R.R.R . . . . & 1,868 \\
\hline Ionia ......... & Smithsonian Inst . & 3,000 \\
\hline & St. J.\& D. C. R. R & 2,079 \\
\hline & B. \& M. R. R. R . & 1,230 \\
\hline & B. \& M. R. R. R . & 2,054 \\
\hline 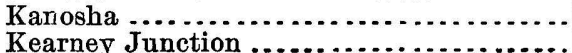 & Smithsonian Inst ..... & 1,050 \\
\hline Kearney Junction & U. P. R.R......... & 2,157 \\
\hline
\end{tabular}




\begin{tabular}{|c|c|c|}
\hline Station. & Autho & Elevation. \\
\hline$\pi$ & & \\
\hline , & U. P. R.R. & 2,088 \\
\hline $\begin{array}{l}\text { Kennard ...... } \\
\text { Ketcham ..... }\end{array}$ & S. C. \& P. R & 1,157 \\
\hline $\begin{array}{l}\text { Ketcham ........ } \\
\text { Liberty ........ }\end{array}$ & $\begin{array}{l}\text { Toner } \\
\text { R. } \mathrm{V} \& \mathrm{~B} \& \mathrm{C}\end{array}$ & 1,272 \\
\hline Lincoln ......... & B. \& M. R. R. R & 1,155 \\
\hline Lockwood. & Toner $\ldots \ldots \ldots \ldots$ & 1,800 \\
\hline $\begin{array}{l}\text { Pole. } \\
\text { Creek. }\end{array}$ & U. P. R. R.... & 3,833 . \\
\hline Louisville....... & O. N. \& B. H. I & 1,500 \\
\hline Houisvine....... & $\begin{array}{l}\text { B. \& M. K. R. K } \\
\text { B. \& M. R. R. R }\end{array}$ & $\begin{array}{l}1,040 \\
2,076\end{array}$ \\
\hline (............. & R., V., B \& C. R & 2,511 \\
\hline McPherso & & 2,695 \\
\hline - & O. N. \& B. H & 1,585 \\
\hline $\operatorname{lm} \ldots . .$. & B. \& M. R. R & 1,291 \\
\hline $\begin{array}{l}\text { Marquette .... } \\
\text { Maxwell ...... }\end{array}$ & $\begin{array}{l}\text { B. \& M. R. } \\
\text { U. P. R. R. }\end{array}$ & 1,825 \\
\hline Mead ...... & O.\&R. V.R.R & $\begin{array}{l}2,718 \\
1,218\end{array}$ \\
\hline (2) & R., V., B. \& C. I & 2,880 \\
\hline ilford ............ & A. \& N. R. R.. & 1,414 \\
\hline Millard ........... & U. P. R. R .... & 1,078 \\
\hline 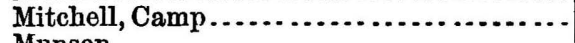 & $\cdots$ & 3,891 \\
\hline - & O. N. \& B. & 1,595 \\
\hline $\begin{array}{l}\text { Napaneo .......... } \\
\text { Nebraska City ... }\end{array}$ & $\begin{array}{l}\text { R., V., B. \& C. F } \\
\text { B. \& M. R. R. R }\end{array}$ & $\begin{array}{r}1,878 \\
941\end{array}$ \\
\hline Nemaha City....... & B. \& M. R. R. R & 885 \\
\hline Newark.......... & M. R. R. I & 2,126 \\
\hline ................. & Toner ......... & \\
\hline 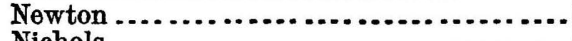 & B. \& M. R. R. F & 1,151 \\
\hline $\begin{array}{l}\text { Nichols ............. } \\
\text { Nickerson }\end{array}$ & an. & 2,920 \\
\hline $\begin{array}{l}\text { Nickerson ........ } \\
\text { Norfolk ........... }\end{array}$ & S. C. \& P. R. R & 1,211 \\
\hline $\begin{array}{l}\text { Norfolk } \ldots \ldots \ldots \\
\text { North Bend ........... }\end{array}$ & S. C. \& P. R. R & 1,532 \\
\hline North Platte ........... & $\begin{array}{l}\text { U. P. R. R } \\
\text { U. P. R. R... }\end{array}$ & $\begin{array}{l}1,279 \\
2,808\end{array}$ \\
\hline Station .................... & U. S. Signal Off & 2,841 \\
\hline Nuadilla.......... & B. \& M. R. R. R & 1,086 \\
\hline Nur & Smithsonian Ins & 1,266 \\
\hline Oak Dale & S. C. \& P R. R. & 1,722 \\
\hline Odell ..... & R. V. \& B. \& C. & 281 \\
\hline O'Fallon's ......... & U. P. R. R ........ & 2,892 \\
\hline Ogallala & U. P. R. R. . . . & 3,216 \\
\hline Omaha, high water Missouri River........ & result from R. R. levels. & 967 \\
\hline Do.low water Missouri River .......... & Mean result from $R$. R. levels. & 986 \\
\hline $\begin{array}{l}\text { Do.passenger der } \\
\text { Do.Signal Statio }\end{array}$ & Mean result from R. R. levels. & 1,039 \\
\hline $\begin{array}{l}\text { Do.Signal statio } \\
\text { Oreopolis } . . . . . . . . . .\end{array}$ & 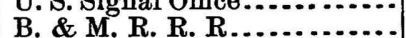 & 1,113 \\
\hline Osceola ............. & R. V.R. R & 1,642 \\
\hline erton .......... & U. P.R. R... & 2,326 \\
\hline rd $\ldots \ldots . . . .$. & R. V. \& B. \& C & 2,079 \\
\hline ra & M. R. R. R.. & 1,151 \\
\hline . & U. P. R. R & 1,005 \\
\hline ............ & R. V. \& B. \& C. R. R & 3,099 \\
\hline $\mathrm{Pa}$ & R. V. \& B. \& C. R. R..... & 1,180 \\
\hline $\mathrm{Pe}$ & B. \& M. R. R. R.. & 903 \\
\hline ger .......... & S. C. \& P. R. R... & 1,423 \\
\hline neer Grove .. & Smithsonian Inst .. & 1,400 \\
\hline itte Center ... & O. M. \& B. \& H. R. R . . . . & 1,537 \\
\hline attsmouth ..... & B. \& M. R. R. R........... & 983 \\
\hline sant Dale.... & A. \& N. R. R..... & 1,311 \\
\hline$\ldots \ldots \ldots \ldots$ & U. P. R. R . . . . . . & 2,394 \\
\hline$\ldots \ldots \ldots \ldots \ldots$ & U. P. R. R & 4,386 \\
\hline ( & A. \& N. R. R... & 885 \\
\hline$\ldots . . . .$. & B. \& M. R. R. R. & 378 \\
\hline anphlion & R........ & 1,944 \\
\hline
\end{tabular}




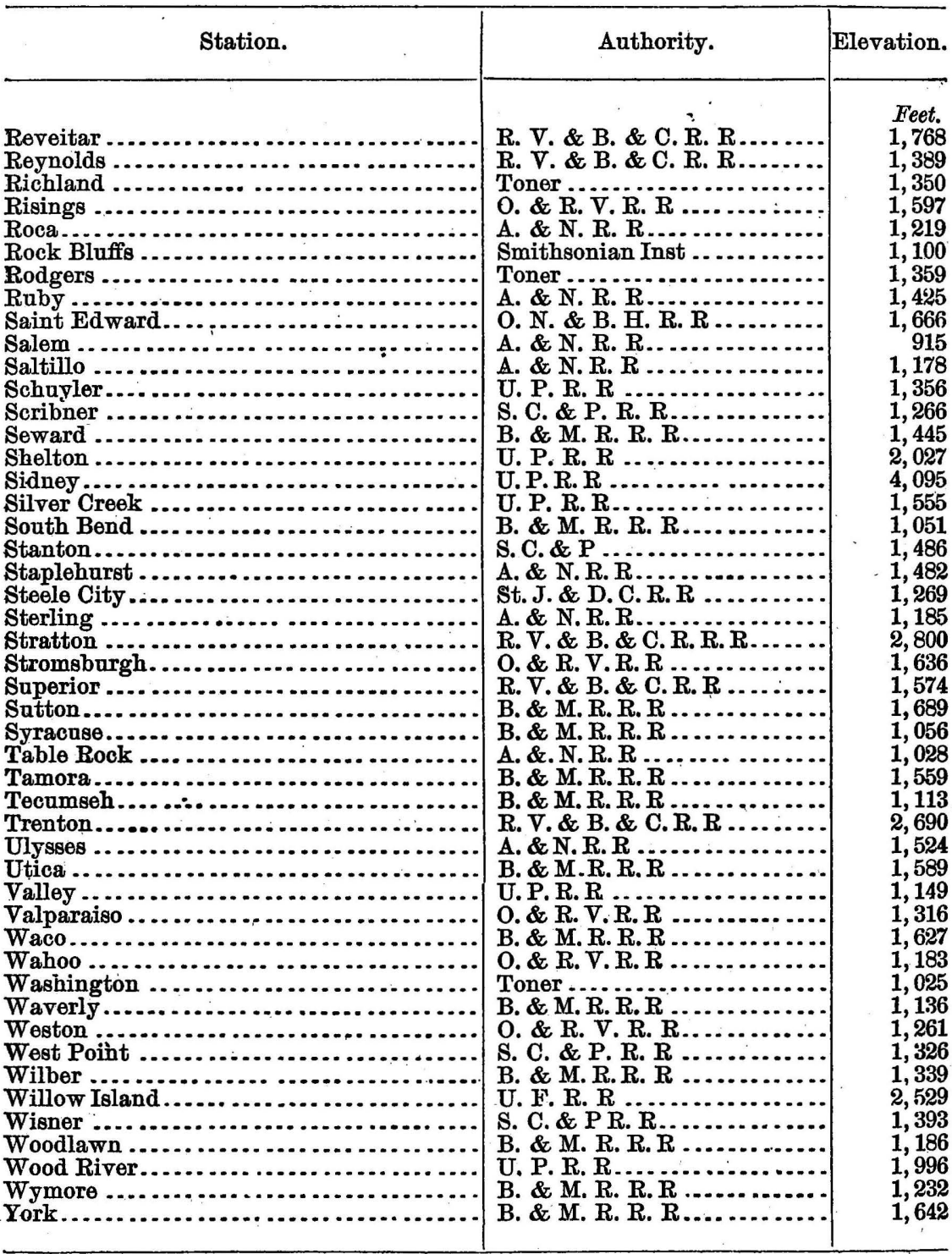




\section{NEVADA.}

\begin{tabular}{|c|c|c|}
\hline Station. & Authority. & Elevation. \\
\hline & & Feet. \\
\hline Agate Pass...... & Reports....... & 7,615 \\
\hline Ahle's Ranch... & $\therefore \ldots \ldots \ldots . . . . . . .$. & 5,990 \\
\hline Airy, Mount..... & ….............. & 6,787 \\
\hline Albion Peak ...... & . & 8,391 \\
\hline Allen's Springs... & . & 4,051 \\
\hline 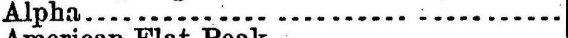 & . & 5,911 \\
\hline American Flat Pea & . & 6,659 \\
\hline $\begin{array}{l}\text { Anderson's Ranch } \\
\text { Antelope Spring. }\end{array}$ & Wheeler... & 4,041 \\
\hline $\begin{array}{r}\text { nterope spring. } \\
\text { Do............. }\end{array}$ & $\begin{array}{l}\text { Wheeler... } \\
\text { Wheeler... }\end{array}$ & $\begin{array}{l}7,201 \\
6,445\end{array}$ \\
\hline elope Valley.. & Simpson & s 5,633 \\
\hline & NTाір & \{ to 5,690 \\
\hline Ant & King ..... & 8,433 \\
\hline $\begin{array}{l}\text { Ant } \\
\text { Arg }\end{array}$ & Wheeler & 6,517 \\
\hline $\begin{array}{l}\text { Arge } \\
\text { Aur }\end{array}$ & C. P. R. R.. & 4,548 \\
\hline $\begin{array}{l}\text { Aurora. } \\
\text { Austin. }\end{array}$ & $\cdots$ & 7,441 \\
\hline Do. & (n) & 6,594 \\
\hline Bacon Mill Point & Wheeler. & 6,451 \\
\hline Baltimore Mine. & Wheeler & $\begin{array}{l}5,327 \\
5,729\end{array}$ \\
\hline Barkley Spring. & (................ & 5,729 \\
\hline Basalt Peak.... & Wheeler & $\begin{array}{l}5,906 \\
6,599\end{array}$ \\
\hline Battle Mountain & C.P.R.R & 4,511 \\
\hline . & Wheoler. & $\begin{array}{l}4,011 \\
8,092\end{array}$ \\
\hline Beowawe & C. P.R.R & \\
\hline Head... & Powell... & 7,570 \\
\hline Big Creek Pass... & King.... & 8,922 \\
\hline Bill Williams' Ran & & $\mathbf{3}, \mathbf{5 0 0}$ \\
\hline 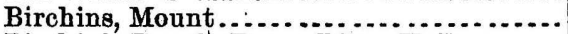 & Wheeler & 10,345 \\
\hline Birchin's Ranch, Reese River Valley ...... & Wheeler & 5,743 \\
\hline Bishop $\ldots \ldots \ldots \ldots \ldots \ldots \ldots \ldots \ldots$ & Toner... & 5,423 \\
\hline Black Rock Valley & Hayden.. & 4,900 \\
\hline Bonneville, Mount & 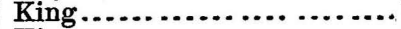 & 11,200 \\
\hline Bonpland, Mount. & King... & 11,321 \\
\hline Brown Knob Peak. & Wheeler. & 6,202 \\
\hline$\ldots \ldots \ldots \ldots \ldots$ & C. \& P.R. R... & 3,929 \\
\hline (n) & Wheeler............................. & 5,221 \\
\hline Carson River Valley... & ................. & 4,151 \\
\hline . & Simpson....... & 6,000 \\
\hline alo Peak.. & Kin & 8,387 \\
\hline alo Spring. & Wheeler. & 4,076 \\
\hline lion $\mathrm{Ci}$ & Wheeler. & 6,386 \\
\hline intain... & Wheoler. & 9,040 \\
\hline zer Hill Peak.... & Wheeler. & 11,405 \\
\hline Bunker's Peak.... & $\ldots \ldots \ldots \ldots$ & 6,780 \\
\hline ellsburgh.... & ............... & 4,278 \\
\hline terfield Spring................ & $\ldots \ldots \ldots \ldots$. & 5,324 \\
\hline$\ldots \ldots \ldots \ldots \ldots$ & $\ldots \ldots \ldots$ & 945 \\
\hline$\ldots \ldots \ldots . . . . .$. & $\ldots \ldots \ldots . . . . . .$. & 7,366 \\
\hline$\ldots \ldots \ldots . . . .$. & $\ldots \ldots \ldots$ & 5,628 \\
\hline Spring... & $\ldots \ldots \ldots$ & 2,050 \\
\hline$\ldots \ldots \ldots$ & $\ldots \ldots \ldots \ldots$ & $\begin{array}{l}8,035 \\
4,849\end{array}$ \\
\hline$\ldots \ldots \ldots$ & Wheeler $\ldots$ & \\
\hline $\begin{array}{ll}\text { Do } \\
\text { urlins }\end{array}$ & King K. K........ & \\
\hline le Point... & Powell ...... & 7,330 \\
\hline 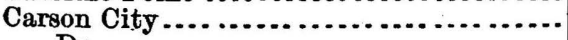 & C. P. R. R.. & 4,630 \\
\hline & Wheeler & \\
\hline
\end{tabular}




\begin{tabular}{|c|c|c|}
\hline Station. & Authority. & Elevation. \\
\hline & & Feet. \\
\hline Carson City, Friend & Wheeler. & 4,660 \\
\hline Carson Hot Springs & Wheeler: & 4,592 \\
\hline Carson Lake... & Wheeler. & 3,883 \\
\hline Do $\ldots . .$. & Pacific R. I & 3,840 \\
\hline Cave Valley. & Wheeler... & 6,464 \\
\hline Cedar Mounta & King .... & 6,500 \\
\hline Cedar Valley..... & Wheeler. & 7,073 \\
\hline Cerro Gordo Peak & Wheeler... & 9,432 \\
\hline Chalk Well ..... & Wheeler .. & 7,604 \\
\hline Chapman's Ranch.. & Wheeler. & 4,932 \\
\hline Charcoal Moun & Powell ... & 7,290 \\
\hline Charleston Pea & Peterman & 10,874 \\
\hline Chataya Peak. & King ..... & 7,766 \\
\hline Cherry Valley. & Wheeler & 7,473 \\
\hline Chinatowu.... & Simpson. & 4,360 \\
\hline Chollar Potosi Mining & Wheeler & 6,242 \\
\hline Churchill Butte ...... & Wheeler & 6,009 \\
\hline Churchill, Fort.. & Med. Dept. & 4,284 \\
\hline Do.......... & Williamson . & 4,319 \\
\hline Clark's . . . . . . & C. P. R. R... & 4,263 \\
\hline Clifton ............ & $\ldots \ldots \ldots$ & 6,339 \\
\hline Clover Valley se & Powell ... & 5,300 \\
\hline Do......... & Wheeler. & 4,902 \\
\hline Cluro ........ & Toner .... & 4,785 \\
\hline Cold Spring.. & Wheeler. & 6,173 \\
\hline Cold Springs. & Wheeler & 5,418 \\
\hline Cold Springs & Wheeler & 4,200 \\
\hline Como Peak. & Wheeler. & 9,017 \\
\hline Cortez Peak.. & King ...... & 8,363 \\
\hline Cory, Mount .. & Wheeler ... & 11,326 \\
\hline Cottage, Camp & Wheeler ... & 6,437 \\
\hline Cottonwood Sp & Wheeler ... & 3,450 \\
\hline les Peak .. & Wheeler .... & 9,980 \\
\hline Cox's Station & Wheeler ........... & 4,379 \\
\hline Coyote........ & & 2,440 \\
\hline te Spring & Wheeler .. & 3,674 \\
\hline Crescent ..... & Wheeler .. & 5,438 \\
\hline Crescent Mill. & Wheeler. & 6,100 \\
\hline Crosman Valley & Simpson & 4,814 \\
\hline Crossman's Spri & Wheeler ........... & 4,391 \\
\hline Crystal Spring & Wheeler ............ & 5,782 \\
\hline Cumberland . & 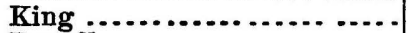 & 5,674 \\
\hline Curtis, Mount .. & $\ldots \ldots \ldots$ & 9,000 \\
\hline Custer Springs, & $\ldots \ldots \ldots \ldots$ & 5,843 \\
\hline Peak... & $\ldots \ldots \ldots$ & 9,23 \\
\hline Mine..... & Wheeler & 4,886 \\
\hline Davidson, Mount... & Wheeler & 941 \\
\hline Do........... & King .... & 7,827 \\
\hline Davies, Mount & Wheeler... & 11,756 \\
\hline Dayton ........ & Wheeler & 4,369 \\
\hline Horse Well .... & Wheeler & 4,117 \\
\hline Deep Hollow Station & Wheeler... & 5,244 \\
\hline Deeth............ & C. P. R. R & 5,340 \\
\hline Desatoya Peak. & Wheeler... & 9,921 \\
\hline Desert Wells.......... & (n) & 4,693 \\
\hline 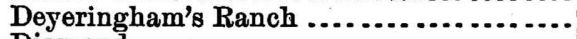 & Wheeler $\ldots \ldots \ldots \ldots \ldots$ & 6,497 \\
\hline ................. & Eureka \& P.R. R............ & 941 \\
\hline .................. & King $\ldots \ldots \ldots \ldots \ldots \ldots \ldots$ & \\
\hline g.................... & Wheeler. & \\
\hline ision.Peak... & Wheeler ...................... & \\
\hline Dodge Valley .... & Simpson ..................... & \\
\hline Duck Vallej. & Wheeler... & \\
\hline
\end{tabular}




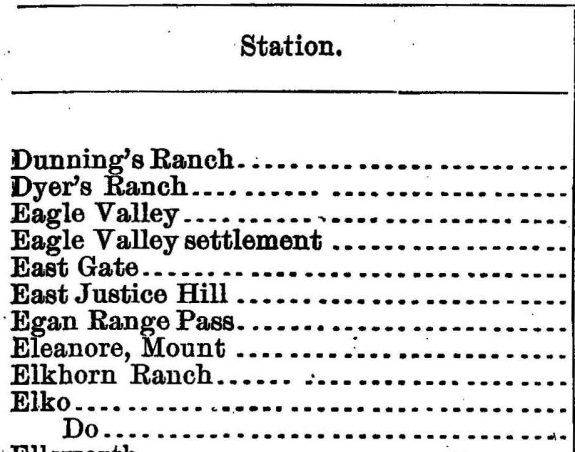

Ellsworth.

Emigrant Pass Peak

Emigrant Peak.

Emma, Mount

$$
\text { Do. }
$$

Empire City .

Erie Consolidatod Mine

Euclid Peak.

Eugene Peak.

Eureka

$$
\text { Do }
$$

Europa Mine.

Fairview Peak

$$
\text { Do ..... do }
$$

Fish Lake

$$
\text { Do. }
$$

Fossil Pass.

Franklin Lake

Eranklin Valley

Franktown

French's Peak

\section{Galena}

Gass Mountain

Gates' Ranch

Geneva Peak

$$
\text { Do. do. }
$$

Genoa

Do

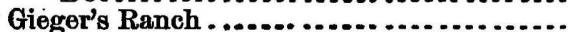

Gila Mina Post, near Succor Mine.

Gilson's Crest

Glenbrook

Glendalo.

Globe Peak

Gold Hill Mine.

Golgonda

Gosiute Lake

Gosiute Pass

Gosiute Peak

Grafton Peak

Grand Peak

Granite Peak

Grape Vine Spring

Grass Valley

Grono's Ranch

Grouse Valley

Gaide Valley.

\begin{tabular}{|c|c|}
\hline Authority. & Elevation. \\
\hline & Feet. \\
\hline Wheeler & 4,571 \\
\hline Wheeler & 6,037 \\
\hline Simpson & 4,587 \\
\hline Powell ... & 5,480 \\
\hline Wheeler & 5,291 \\
\hline Wheeler & 5,861 \\
\hline Wheeler ............... & 7,546 \\
\hline Powell.................... & 7,692 \\
\hline Wheeler $\ldots \ldots \ldots \ldots$ & 6,123 \\
\hline 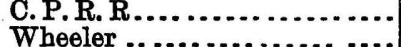 & $\begin{array}{l}5,063 \\
5,148\end{array}$ \\
\hline Wheoler ....................... & $\begin{array}{l}0,140 \\
6,871\end{array}$ \\
\hline Wheeler & 7,876 \\
\hline Wheeler & 8,509 \\
\hline King ... & 6,546 \\
\hline Wheeler & 6,439 \\
\hline Whoeler. & 4,593 \\
\hline Wheeler. & 5,268 \\
\hline King .... & 7,725 \\
\hline King .................. & 7,548 \\
\hline Wheeler $\ldots \ldots \ldots \ldots \ldots$ & 5,906 \\
\hline Eureka \& P.R. R . . . . . . . . & 6,371 \\
\hline Wheeler $\ldots \ldots \ldots \ldots \ldots$ & 5,978 \\
\hline $\begin{array}{l}\text { Whoeler } \ldots \ldots \\
\text { King }\end{array}$ & 8,412 \\
\hline Wheeler & 4,745 \\
\hline Wheeler & 6,866 \\
\hline King ..... & 8,028 \\
\hline R. Reports... & 6,200 \\
\hline Pacific R. R. Reports.... & 6,248 \\
\hline Wheoler ................ & 5,054 \\
\hline Wheeler ............. & 10,779 \\
\hline Wheeler ............. & 5,650 \\
\hline Petermann & 6,200 \\
\hline Wheeler ... & 4,154 \\
\hline King ....... & 10,994 \\
\hline Wheeler .................. & 11,077 \\
\hline Simpson. $\ldots \ldots \ldots \ldots \ldots \ldots$ & 4,824 \\
\hline Wheeler ......................... & 4,801 \\
\hline Wheeler .................. & 4,352 \\
\hline Wheeler ............. & 5,452 \\
\hline Powell ...................... & 11,000 \\
\hline (n. & 6,282 \\
\hline Wheeler & 4,453 \\
\hline King .... & 11,237 \\
\hline Wheeler & 6,374 \\
\hline 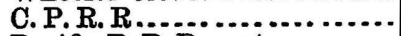 & 4,385 \\
\hline Pacific R. R. Reports....... & 5,952 \\
\hline U. P. R. R. surveys $\ldots \ldots \ldots$ & i, 065 \\
\hline King $\ldots \ldots \ldots \ldots \ldots \ldots$ & 10,491 \\
\hline Wheeler .................. & 10,964 \\
\hline King & 8,307 \\
\hline Wheeler ........ & 8,990 \\
\hline 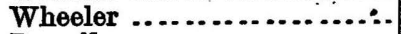 & 9,965 \\
\hline Powell .................... & 1,930 \\
\hline Hayden .. & 4,300 \\
\hline Wheoler ......... & 4,187 \\
\hline 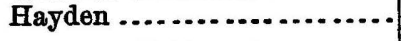 & 5,600 \\
\hline Pacific R. R. Reports .... & $\begin{array}{l}5,308 \\
6,139\end{array}$ \\
\hline Powell ... & 2,620 \\
\hline King ..... & 11,298 \\
\hline & 5,006 \\
\hline & \\
\hline
\end{tabular}

Hackberry Springs .

Hahn Peal

Half-way House.

Halleck 


\begin{tabular}{|c|c|c|}
\hline Sta & ity. & Elevation \\
\hline & & \\
\hline Halleck Camp .. & King ........ & 5,671 \\
\hline $\begin{array}{l}\text { Do. do.... } \\
\text { Do. do... }\end{array}$ & $\begin{array}{l}\text { Smithsonia } \\
\text { Wheeler }\end{array}$ & \\
\hline Hamilton ........ & Wheeler. & $\begin{array}{l}5,790 \\
7,600\end{array}$ \\
\hline Hastings Pass .. & U.P.R.R & \\
\hline Do.............. & Simpson. & 6,580 \\
\hline $\begin{array}{l}\text { Hawthorne .... } \\
\text { Hay Ranch .... }\end{array}$ & $\begin{array}{l}\text { Carson \& } \\
\text { Eureka \& }\end{array}$ & $\begin{array}{l}4,569 \\
5,171\end{array}$ \\
\hline Hiko............ & Wheeler.. & \\
\hline Hill and Gaines RaI & Wheeler & 3,944 \\
\hline Homer ................. & Wheeler. & 5,821 \\
\hline $\operatorname{ation...~}$ & Wheeler & $\begin{array}{l}4,816 \\
4,095\end{array}$ \\
\hline Hot Springs.... & C.P.R.R & \\
\hline & Wheeler & 4,731 \\
\hline $\begin{array}{l}\text { Hot Springs Peak } \\
\text { Hot Springs, Walk }\end{array}$ & Wheeler & $\begin{array}{l}7,692 \\
7,334\end{array}$ \\
\hline Lousta & Whoeler. & 4,149 \\
\hline Lum & C.P.R.R & 4,236 \\
\hline Humboldt River & & \\
\hline Huntington Valle & Wheeler.. & $\begin{array}{r}5,300 \\
\text { to } 6,300\end{array}$ \\
\hline ence ....... & C.P.R.R. & 6,007 \\
\hline 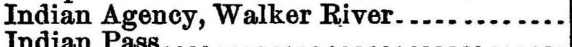 & Wheeler . & \\
\hline $8 \ldots . .$. & $\begin{array}{l}\text { King...... } \\
\text { Wheeler }\end{array}$ & 9,057 \\
\hline $\begin{array}{l}\text { Indian Spr } \\
\text { Inyo Peak }\end{array}$ & $\begin{array}{l}\text { Whe } \\
\text { King }\end{array}$ & $\begin{array}{r}3,402 \\
11,337\end{array}$ \\
\hline Ione $\ldots . . .$. & ler. & 6,844 \\
\hline Iron Point ........ & C.P.R.R. & 4,375 \\
\hline h .......... & Wheeler .. & \\
\hline ves Pass ............ & King........ & 6,672 \\
\hline Aills Ranch & $\begin{array}{l}\text { Wheeler. } \\
\text { Wheeler . }\end{array}$ & $\begin{array}{l}4,386 \\
5,736\end{array}$ \\
\hline Ranch.... & Powell ..... & 3,200 \\
\hline Iount ........ & King ....... & 6,187 \\
\hline & Ha & $\begin{array}{l}6,247 \\
4,850\end{array}$ \\
\hline ker Mir & Whe & 5,867 \\
\hline Kobeh Valley & Simpson.. & \\
\hline Iount. . & Wheeler & \\
\hline W6 & ic R. R. Repor & \\
\hline Las ] & Wheeler ............... & \\
\hline 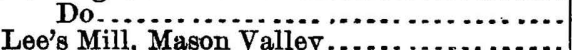 & $\begin{array}{l}\text { Whe } \\
\text { Whe }\end{array}$ & $\begin{array}{l}2,018 \\
4,350\end{array}$ \\
\hline Mawsull & Wh & 6,167 \\
\hline & $\mathrm{Wh}$ & \\
\hline & Whoeler ................. & \\
\hline Valley .. & Wheeler & 6,216 \\
\hline & $\mathrm{R}$ & $\begin{array}{l}6,195 \\
3,977\end{array}$ \\
\hline & Wh & 8,793 \\
\hline & & \\
\hline ass ... & R. Surveys ... & \\
\hline Mountain & (............... & 7,532 \\
\hline Cove... & Wh & \\
\hline Cam & U. S. A.... & 4,700 \\
\hline & Med. Dept., U. S. A ....... . & 6,600 \\
\hline 's, on Lake Tahoe. & Wheeler ................. & 6,232 \\
\hline sPass $\ldots \ldots$ & King $\ldots \ldots \ldots \ldots \ldots \ldots$ & 5,592 \\
\hline Aon's Ranch, Reese Ri & 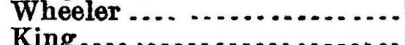 & $6,55 \%$ \\
\hline 1t. & or & $\begin{array}{l}8,100 \\
8,363\end{array}$ \\
\hline${ }_{1}$ & 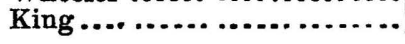 & 9,241 \\
\hline
\end{tabular}




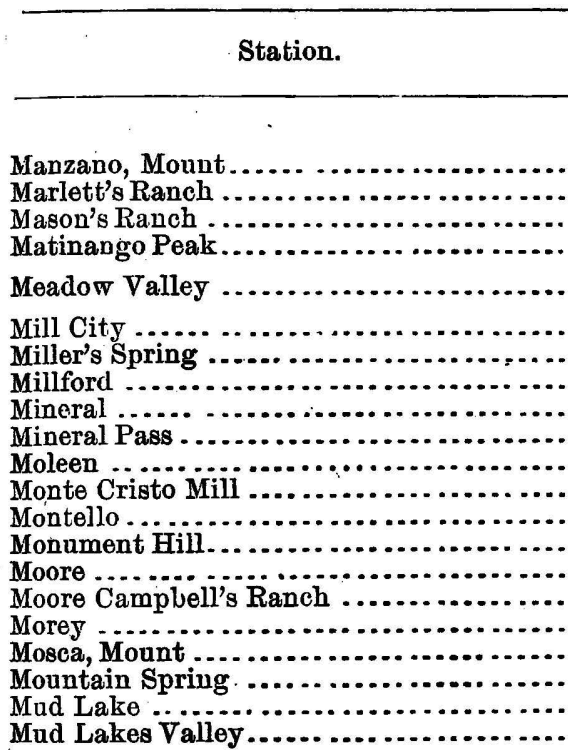

Do

Mud Meadows.........................

Mud Spring .

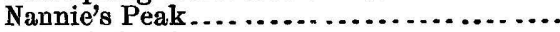

Nelson's Station ..........................

Nevada City .

Newton's Ranch, near Honey Lake.........

North Granite Peak

North Pass. .............................

Occidental Mills, Virginia City

Olcott, Monnt ............................

Old Kentuck Ranch, Sutro Tunnel Road..

O'Neill's Ranch ........................

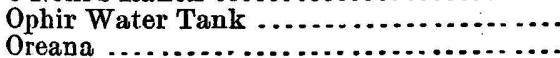

Ormsby Peak

Osha, Mount

Osino

Ory yee

Oxford Peak

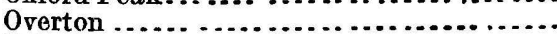

Pah-ghun-pah-ghun Spring

Pahranagut Lake

Pahrumpe Valley

Pah-Ute Peak

Palisade, junction with Eureka and Palisade R. R.

Panacca

Panacea Head ..............................

Paradise Peak.

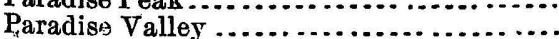

Park Peak

Patterson's Ranch

Peaviue Mount.

Peavine Peak.

Peavine Rauch.

Pedernal, Mount

Pelro.

Do

Bull. 5-16
Wheeler

Wheeler.

Wheeler

Wheeler.

Powell.

C. P. R, R

Powèll .

Wheeler

Eureka \& P. $\ddot{R} . \ddot{R}$

Wheeler.

C. P. R. $\mathbf{R}$.

Wheeler.

C. P. R. R

Whoeler.

C. P. R. R

Wheeler

Wheeler

Wheeler

Pacific R. R. Reports

King

Pacific R. R. Reports.

Wheeler

Wheeler

Wing .....

Toner...

Wheeler

Wheeler

C. P. R. R. Surveys...............

Wheeler

Wheeler

Wheeler

Wheeler

Wheeler

King ...

Wheeler

Wheoler

King.

Powell .

Wheeler

Simpson.

Wheeler

Authority.

Elevation.

Feet.

10,086

8,074

4,348

8,845

$\left\{\begin{array}{r}4,400 \\ \text { to }\end{array}\right.$

to 4,800

4,226

6,220

4,204

5,443

6,969

4,982

7, 596

5, 010

6,734

6,166

6,267

7,384

9, 723

5,501

4, 079

3,862

\{ 4,000

to 4,400

4,318

4,900

9,046

4,245

6,205

4,079

8,363

5,964

$5,4 \geq 0$

7,974

5,684

5,581

6,509

4,181

8, 388

10,023

5,100

5,392

7,556

1,360

2,282

3,400

$5 \quad 5,700$

to 6,000

8,618

C. P.R.R.

Powell .

Wheeler

Powell.

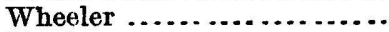

Hasden

Wheeler

Wheeler

King

Wheeler

Wheeler

Wheeier

C. P. R. $\mathrm{R}$

Wheeler
4, 821

4,718

7,450

8,662

4,500

8,683

5,213

6,217

8,275

4,952

7,580

5,204

5,180
4, 770 


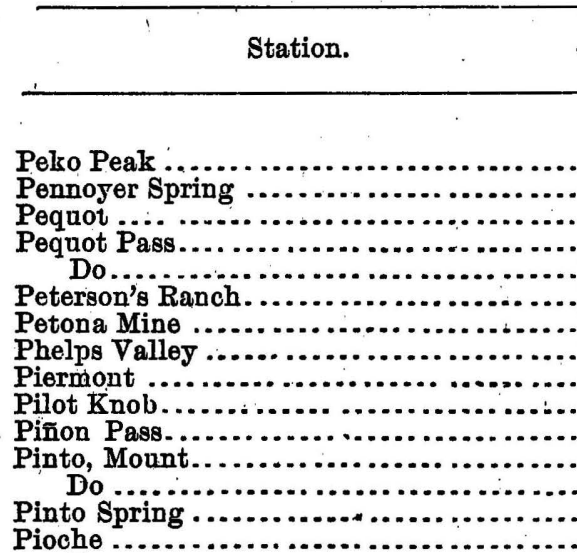

Do...............................

Do... Signal Station

Placer, Mount

Point of Rocks Peak

Proctor's Ranch, Truckee River Do

Pueblo Valley

Pyramid Lake

Pyramid Lake Indian Agency.

Quartz Mountain

Quinn's River Valley

Quinn Cañon

Quinn's River Valley.

Rabbit Hole Spring

Ragtown.

$$
\text { Do. }
$$

Rajlroad Peak

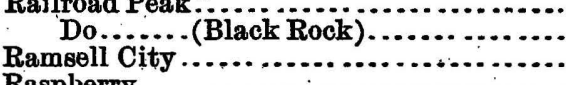

Raspberry

Rattlesnake Hill

Rattlesnake Spring

Raven's Nest Peak.

Raw Mountain

Red Dome Pass.

$$
\text { Do }
$$

Reese River Valley

\section{Reno.}

Do...Junction Virginia City and True-

Robert's Peak kee R. R

Rock Island Mine

Rose Creek

Rose Mountain

Rose Mountain

Rose Mountain

Rose Valley .

Rose Valley Settlement.

Ruby, Camp.

$$
\text { Do }
$$

Ruby Valley

Rye Patch.

Sacramento

Saint Clair

Saint Joseph

Saint Thomas.

Authority.

King

Wheeler....................

C. P. R. R.................

U. P. R. R. Surveys..........

C. P.R. R. Surveys...........

Wheeler.

Wheeler..........................

Simpson ....................

Wheeler

Wheeler

King ....

Wheeler.....................

King ........................

Wheeler

Wheeler.

Powell ....................

U. S. Signal Office.............

Wheeler

King

Wheeler.

Wheeler.

King ....

Wheeler.

Pacific. Rail................

Wheeler.

Wheeler.

Hayden

Wheeler

Haydon

King.

King ....

King .

Wheeler.

Wheeler.

C.P.R. R

Wheeler.

Wheeler.

King

Wheeler.

U. P.R. R. Surveys: ...........

C.P. R. R. Surveys.

Simpson.

Wheeler.

C. $P . R . R \ldots \ldots \ldots \ldots \ldots$

King $\ldots . . . \ldots \ldots \ldots \ldots \ldots$

Whoeler.

C. P. R. R

King

King ...

Wheeler

Wheeler:

Powell

Wheeler.

Medical Department, U. S. A

Simpson

C. P. R. R

Wheeler.

Powell

Powell
Elevation.

Feet.

6,620

6,652

6,184

6,143

6,180

6,137

6,639

6,150

5, 581

10,758

$6,5: 33$

8,618

8,715

5,683

5,942

6,100

6,220

8,965

9,610

11, 977

3,963

8,256

8,151

5,000

4,890 .

3,980

8,700

4,850

6,256

4,850

4,374

4,039

4,002

8,569

5,436

5,324

4, 327 .

6,616

6,038

8,393

8,404

4,720

4, 821

5,600

4, 484

4,497

10,132

$5 ; 678$

4,322

7,949

$6,44{ }^{\circ}$

10,820

5,401

5,450

6,153

5,922

6,000

4,257

$\mathbf{6}, 575$

3,989

1,490

1,180 


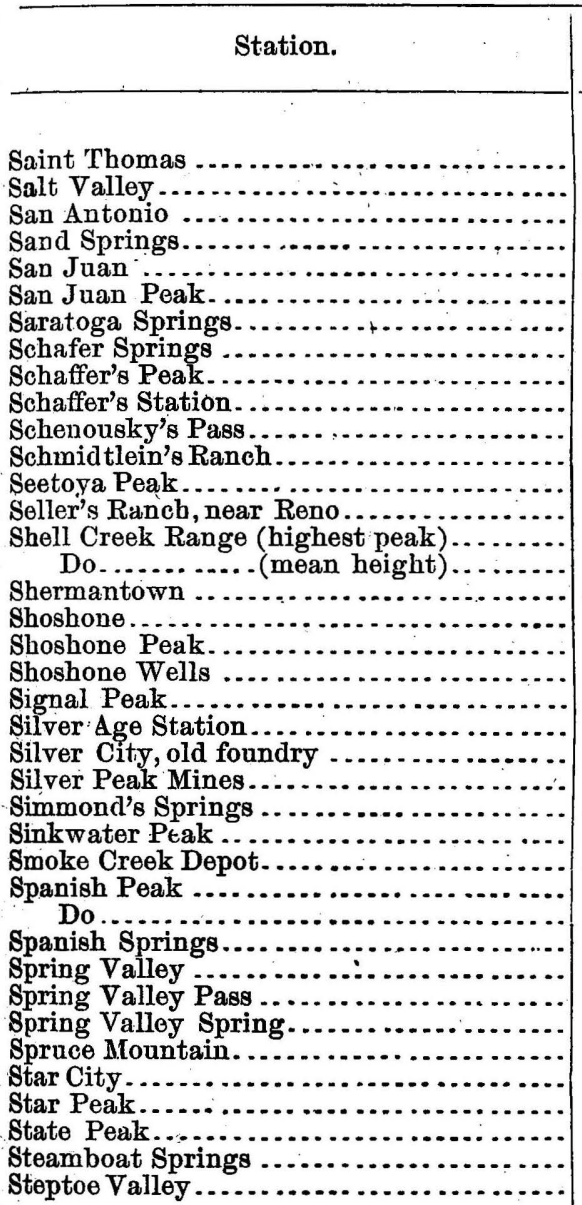

Do

Sterling Mill

Stone House

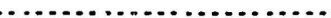

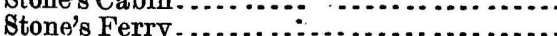
Do.

Stony Peak

Stamp's Ranch

Sue's Peak.

Sugar Loaf Mountain ....................

Sulphur Springs.....

Summit Spring Peak. .....................

Summit Springs

Superior G. \& S. mine......................

Susanville.

Sutro Sṕrings

Taroggua Peak

$$
\text { Do. }
$$

Taylor's Ranch

Tecolote, Mount

Tecoma

Telescope Peak

Tenabo Peak

Thousand Springs Valley

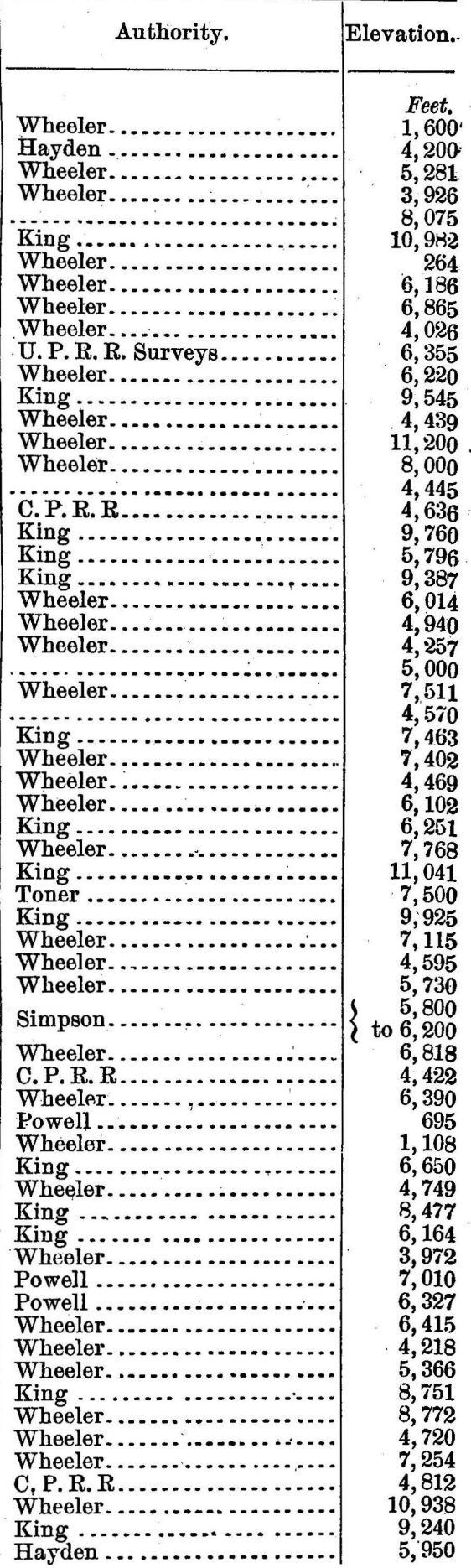




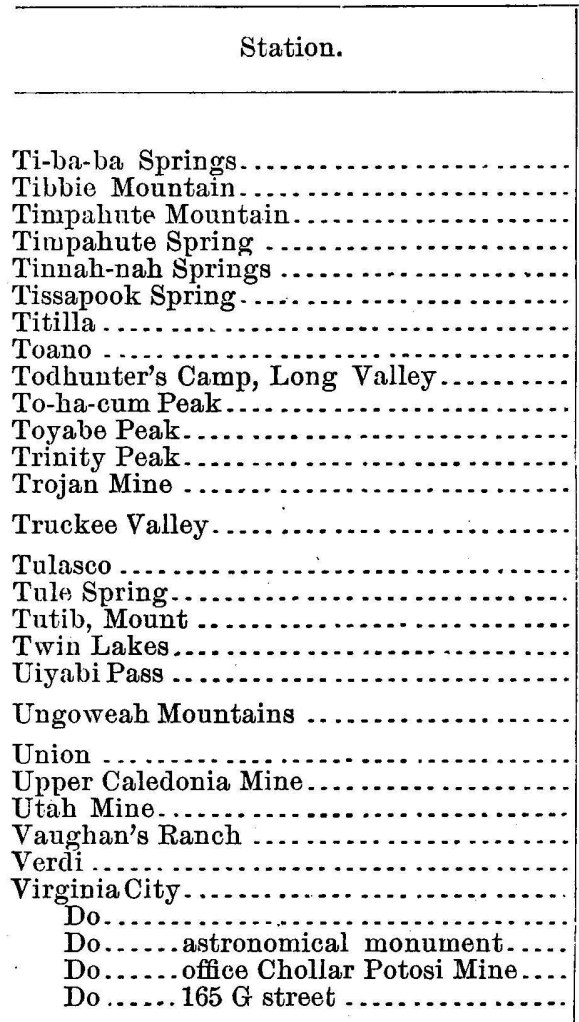

Virgin Valley (lower)

Vista

Vivian Mine.

Wadsworth

$$
\text { Do. }
$$

Wah-ya-bah Springs

Walker's Lake

Do

Walker's Ranch

Walker's River Basin

Walker's River Meadows

Wallace's Ranch.

Wall Spring.

Wanahonupe Valley

Washington

Washoe City.

Washoe Lake.

Washoe V.alley.

Water Cañon.

Wauguyhi Peak

Weahbah Range

Welche's Ranch

Welch's Station

Wells

West Belcher Mine

West Gate

West Point

Wheeler Peak

Do .... timber line on

White Bluff Spring

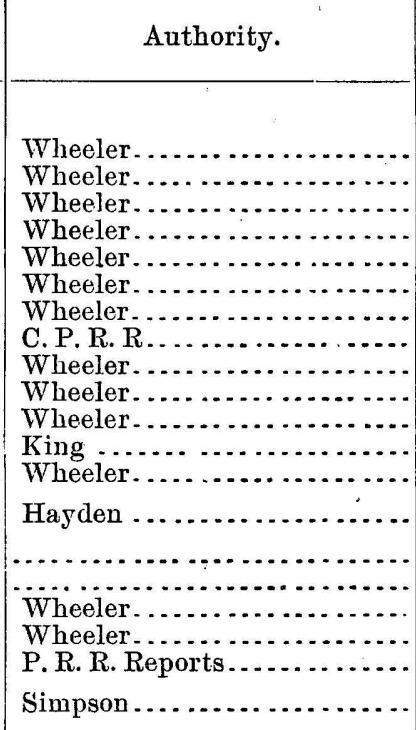

Toner

Wheeler

Wheeler.

Wheeler

C.P.R.R

C.P.R.R

Touer...

Wheeler

Wheeler

Wheeler

Powell

Toner .

Wheeler

C. P. R. R

Wheeler

Wheeler

Pacific R. R. Reports

Carson and Colorado R. R

Wheeler

Wheeler

Wheeler

Simpson

Wheeler

Wheeler

Wheeler

Hasden

.

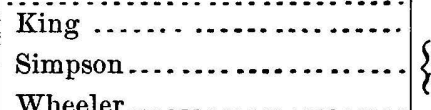

Wheeler

Wheeler

C. P. R. R

Wheeler

Wheeler

Wheeler

Wheeler

Wheeler
Elevation.

Feet.

6,248

$7,38: 3$

$\varepsilon, 600$

6,892

4, 080

5,832

7,060

5,973

5,779

8,174

10,144

7,500

6,086

\{ 4,000

to 5,100

5,418

2,366

7,062

7,843

6,233

7,500

to 8,000

6,160

6,987

5,996

5,316

4,895

6,205

6,200 .

6,339

6,242

6,118

700

to 2,000

4,400

5,415

4,077

4,095

5,188

3,840

4,147

5,146

4,100

6,500

5,787

3,912

5,543

6,992

5,021

5,046

4,000

2,140

8,528

7,000

to 8,000

5,771

5,236

5,629

6,098

4,504

1,755

13,036

11,500

5,020 


\begin{tabular}{|c|c|c|}
\hline Station. & Authority. & Elevation. \\
\hline 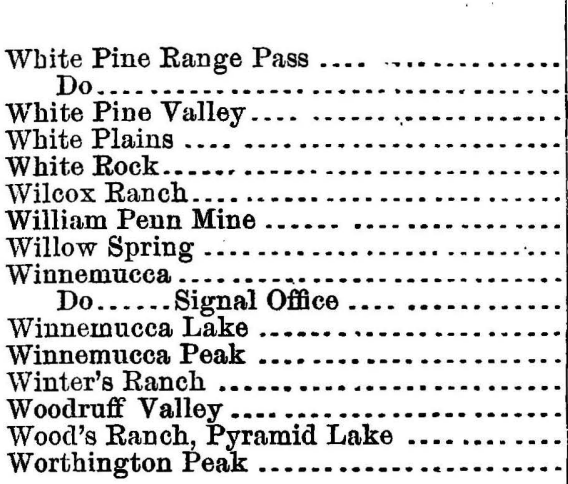 & 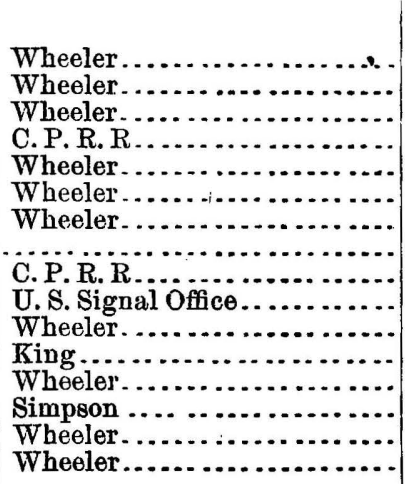 & $\begin{array}{l}\text { Page. } \\
5,860 \\
6.326 \\
6,000 \\
3,894 \\
4,818 \\
5,396 \\
5,236 \\
5,859 \\
4,332 \\
4,327 \\
3,825 \\
6,869 \\
5,145 \\
6,000 \\
3,861 \\
8,400\end{array}$ \\
\hline
\end{tabular}


NEW HAMPSHIRE.

\begin{tabular}{|c|c|c|}
\hline on. & Authority. & Elevation. \\
\hline & & \\
\hline Adam's Mountain & & 5,776 \\
\hline Do........... & $\begin{array}{l}\text { Appalachian Club. } \\
\text { Appalachian Club : }\end{array}$ & $\begin{array}{l}5,554 \\
5,384\end{array}$ \\
\hline & Appalachian Club : & 5,313 \\
\hline 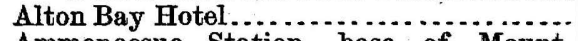 & Suncook Valley R. & \\
\hline $\begin{array}{c}\text { Ammonoosuc Station, base of Mount } \\
\text { Washington }\end{array}$ & Bost., Con. \& Mont. & 2,668 \\
\hline ndover $\ldots \ldots \ldots \ldots \ldots \ldots$ & Northern R. R ....... & 628 \\
\hline & Ashuelot R. R....... & 434 \\
\hline intain, Franconi & $\begin{array}{l}\text { Boston \& Maine R. I } \\
\text { Appalachian Club . }\end{array}$ & $\begin{array}{r}57 \\
2,310\end{array}$ \\
\hline 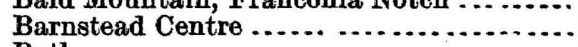 & $\begin{array}{l}\text { Apparacnias } \\
\text { Suncook Va }\end{array}$ & $\begin{aligned} 2,310 \\
527\end{aligned}$ \\
\hline & Bost., Con. & 521 \\
\hline Belknap Mo & Appalachi & 2,259 \\
\hline Berli & $\begin{array}{l}\text { Port. \& } 0 \\
\text { Grand Tru }\end{array}$ & $\begin{array}{r}996 \\
1,016\end{array}$ \\
\hline $\begin{array}{l}\text { Berlin Falls ... } \\
\text { Bethlehem ... }\end{array}$ & Bost. Con. \& Mont. R. & $\begin{array}{l}1,016 \\
1,187\end{array}$ \\
\hline Do.... Sinclair Ho & Appalachian Club ... & 1,459 \\
\hline $\begin{array}{l}\text { Do..... Maplewood } \\
\text { Black Mountain, Sand }\end{array}$ & Appalachian Club & 1,489 \\
\hline & Appalachian Clu & 4,071 \\
\hline $\begin{array}{l}\text { Do............ Jer } \\
\text { Do.......... Sac }\end{array}$ & App & 3,587 \\
\hline Do....... & & $\begin{array}{l}3,050 \\
2,967\end{array}$ \\
\hline Do.............. & App & 2,545 \\
\hline Do... & Apr & 2,391 \\
\hline lue ] & & 4,370 \\
\hline Boot & Apl & 5,524 \\
\hline & & 274 \\
\hline ain, & Apl & 2,278 \\
\hline & Col & 679 \\
\hline Braj: & Ap & 1,637 \\
\hline Brick & & 3,850 \\
\hline Camp & & 687 \\
\hline & & 645 \\
\hline ton Hollow $\mathbf{P}$. & Apl & 707 \\
\hline- & month $\mathrm{R}_{\mathrm{R}}$ & $\begin{array}{l}956 \\
445\end{array}$ \\
\hline .... & d \& Portsmouth R. R... & $\begin{array}{r}445 \\
3,850\end{array}$ \\
\hline & (1) & $\begin{array}{l}3,850 \\
4,678\end{array}$ \\
\hline & & 3,480 \\
\hline & & 4,830 \\
\hline & I Co. R.R. & 328 \\
\hline & A Co. R.R. & 376 \\
\hline atain.. & Guy & 3,670 \\
\hline er . & ook Valley R. i & 373 \\
\hline & & 3,540 \\
\hline$\cdots$ & Con & $\begin{array}{l}543 \\
473\end{array}$ \\
\hline nt & d \& Clare & \\
\hline Mount. . & Guyot. & $\begin{array}{l}5,553 \\
4,311\end{array}$ \\
\hline 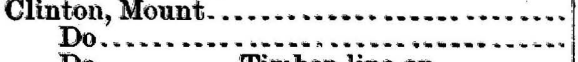 & 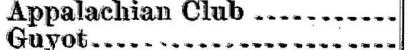 & $\begin{array}{l}4,311 \\
4,320\end{array}$ \\
\hline Do ........... Timber line o & Guyot & $\begin{array}{l}4,320 \\
4,250\end{array}$ \\
\hline & Concord \& Clarenont $\mathbf{R} . \mathbf{R}$.. & 252 \\
\hline iniver briag & & \\
\hline onnecticut Rive & Sullivan Co. R.R. & 351 \\
\hline
\end{tabular}




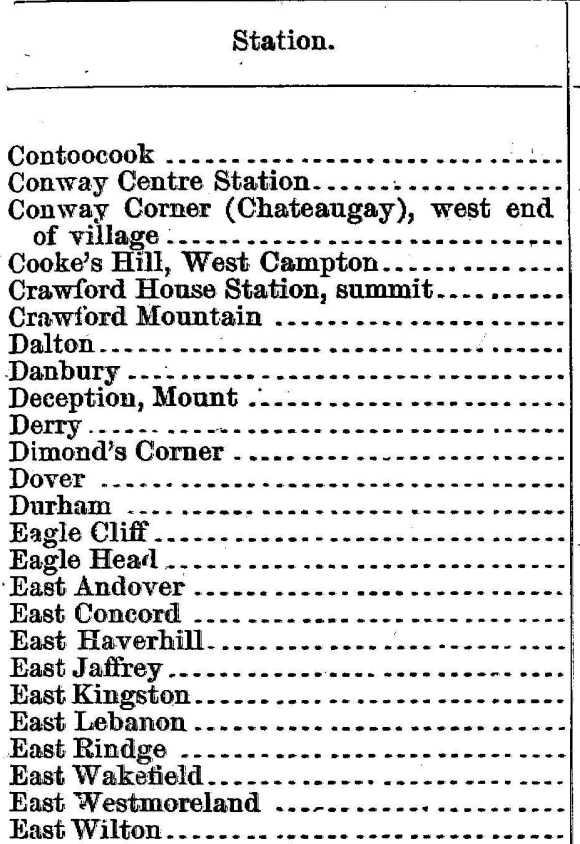

Echo Lake

Do

Echo Monntain .............................

Elkin's Fisher, Teeumseh Group ............

Elliot .

Enfield

Epping

Epsom

Do

Exeter

Do.M. M. Steele's residence............

Fabyan House . . . . . . . . . . . . . . . . . . . . .

Fisher Mountain, Teenmseh Group

Fitzwillian.

Fort Mountain, Epsom

Franconia, Franconia Honse

Do.... Lafayette House

Franklin...

Franklin, Mount

$$
\text { Do. }
$$

Gilford, Locke's Farm-house

Gilmanton Hill

Gilmanton, Iron Works Village

Glen Station

Goffstown Station

Gorham

Grafton

Grafton Centre

Great Falls.

Do .... (Conway) Junction

Greenfield

Green Mountain, Tecumseh Group

Green's Cliff.

Groveton

Groveton Junction

Gunstock Momintain

Hampstead \&tation

\begin{tabular}{|c|r}
\hline Authority. & Elevation. \\
\hline & \\
Contoocook Valley R. R....... & Feet. \\
Port. \& Ogden R. R.......... & 455 \\
\hline
\end{tabular}

P., G. F. \& C. R. R

Appalachian Club .............

Port. \& Ogden R. R............

Gayot.............

Bost., Con. \& Mont. R. R

Northern R. R

Guyot

Manch. \& Lawrence R. R

Concord \& Claremont R. $\mathbf{R}$.

Boston \& Maine R. R

Boston \& Maine R. R ..........

Guyot............................

Guyot.

Northern R. R

Geol. S'vy of $\mathbf{N}$...............

Bost., Con. \& Mont. R. R....

Monadnock \& Petersboro R.R

Boston \& Maine R. R.

Northern R. R

Monadnoek \& Petersboro $\$$. $\mathrm{R}$

P., G. F. \& C. R. R.

Cheshire R. R...................

Nashua, Wilton \& Petersborough R. R.

Guyot

Appalacian

Gupot

Appalachian Club

P., G. F. \& C. R. R . . .

Northern R. R.

Concord \& Portsmouth $\mathbf{R}$.

Geol. Survey of N. H

Suncook Valley R. R

Appalachian Club ..............

Boston \& Maine R. R ..........

Bost., Con. \& Mont. R. R...

Appalachian Club

Cheshire R. R ..............

Appalachian Club

Appalachian Club

Northern R. R...

Appalachian Club

Appalachian Club

(n.........

Gunot ..............

Port. \& Ogden R. $\mathbf{R}$

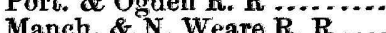

Grand Trunk R. R.........

Northern R. $\mathbf{R}$

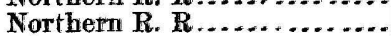

Boston \& Maine R. R

P., G. F. \& C. R. R.

Petersborough $\mathbf{R}$. R

Appalachian Clab

Grand Trunk R. R. $\mathbf{R}$

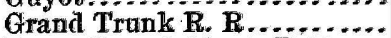

Bost., Con. \& Mont. R. $\mathbf{R}$

Appalacbian Clab ...........

Nashua \& Rochester R. R...
466

2,236

1,903

3,134

866

826

2,449

238

$4: 5$

72

3,446

4,216

661

246

773

1,032

130

766

1, $00 \mathrm{~g}$

678

709

328

1,926

1,929

3,170

2,621

21

768

154

342

362

781

58

1,571

3,470

1,063

1, 431

1,054

990

363

4,917

4,904

643

1,329

647

530

304

794

848

872

178

80

3,547

1, 329

884

901

2,400

258 


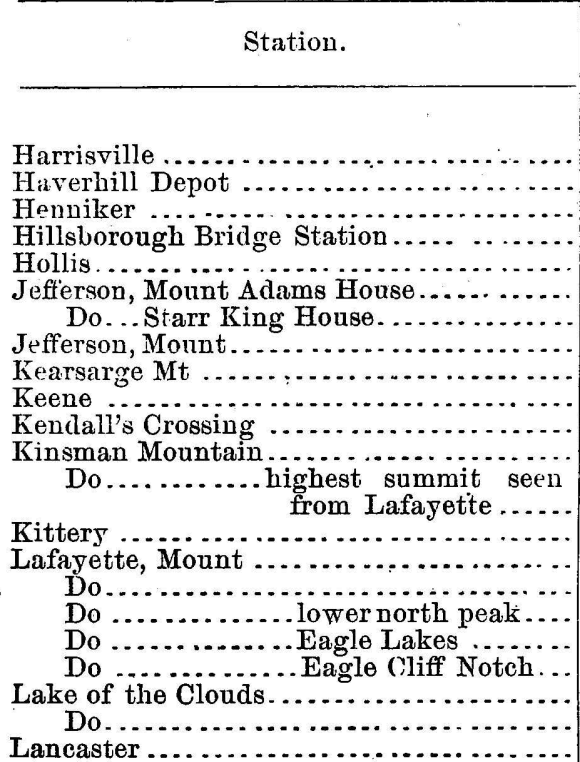

Lebanon.

Lincoln Mountain

Lisbon

Littlefield's Crossing .

Little Monroe Mountain Do.

\section{Littleton.}

Do .. Oak Hill House

Lyndeborough

Madbury

Madison, Mount.

Do.

Madison, N. W. end of 6-mile pond

Mad River Peak of 6-mile pond ........

Manchester

Marlborongh

Mast Yard

Meredith Village

Middle Fisher Mountain, Tecumseh Group

Milan.

Milton Station

Monadnock, Mount .

Monroe, Mount.

Moose hillock .

Morijah, Mount

Mornt Sunapee Station.

Nashua

Do. Main strect.

Do. Concord depot

Do Lake street. ury Summit, on rail ...................

Newmarket ......................................

New market Junction ...................

Newport . . . . . . . . . . . . . . .

Newton

Do.R. R. Summit

North Joscawen ..........................

North Charlestown .......................

North Conway Station.................

Do............. (village plains)....

Authority. Elevatic

Manch. \& N. Weare R. R ...

Conn. \& Pass. R. R........... Contoocook Valley R. R .... Contoocook Valley R. R Worcester \& Nashua R. R...

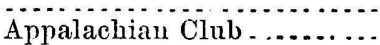
Gupot...

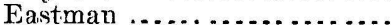

Ashnelot R. R ..

Sullivan Co. R. R.

Guyot........................

Appalachian Club P., G. F. \& C. R. R Appalachian Club

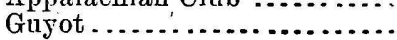
........................

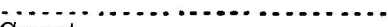
Guyot .

Appalachian Club Bost., Con. \& Mont. R. R Northern R. R Appalachian Club Bost., Con. \& Mont. R. R ... Concord \& Portsmouth R. R Guyot

Appalachian Clu b.

Bost., Con. \& Mont. R. R.

Appalachian Clnb ........... Petersborough R. R Boston \& Maine R. R Appalachian Club . Geol. S'v'y of N. H P., G., F.\& C. R. R Gurot . \& C. R. R. Manch. \& Lawrence R. R Cheshire R. R Concord \& Claremont R. R. Bost., Con. \& Mont. R. R

Appalachian Club

Grand Trunk R. R.

P., G., F. \& C. R. R

$\mathrm{R} . .$.

Guyot...................... Appalachian Club ........... Guyot.

Guyot .................................. Concord \& Claremont R. R . Boston, Lotell \& Nasbua R. R. Nashua, Acton \& Boston R. R. Worcester \& Nushna R. R... Nashua, Acton \& Boston R. R Concord \& Claremont R. R... Concord \& Claremont R. R.. Boston \& Maine R. R ....... Concord \& Portsmouth R. R . Concord \& Claremont R. R . Boston \& Maine R. R Boston \& Maine R. R Northern R. R .... Snllivan Co. R. R ............ P., G. F. \& C. R. R ......... Port. \& Ogden R. R............
Feet.

1,334

412

439

574

195

1,648

1,437

5,714

$2,7 \cdot 26$

466

$30: 3$

4,200

4,370

17

5,249

5,290

5,081

4,146

2,990

5,009

5,053

870

510

5,098

577

126

5,204

5,220

817

975

624

108

$.5,3: 36$

5,365

476

4, 397

181

789

375

542

2,790

1, 060

415

3,718

5,361

4,790

4,653

1,128

135

31

120

45

$1,1: 30$

1,181 .

40

52

802

126

142

290

417

516

521 


\begin{tabular}{|c|c|c|}
\hline Station. & Authority. & Elevation. \\
\hline North Lisoon. & Bost Con \& Mont & Feet. \\
\hline Nortb Stratford .... & Grand Trunk R. R . & 902 \\
\hline North Weare..... & Manch. \& N. Weare R. R. & 489 \\
\hline Osceola Mountain . & Appalachian Club...... & 4,417 \\
\hline Ossipee, $\frac{1}{4}$ mile from village $\ldots \ldots . . . . .$. & P., G. F. \& C. R. R & 642 \\
\hline Ossipee Mountain, East Knoll ............. & Appalachian Club. & 2,774 \\
\hline 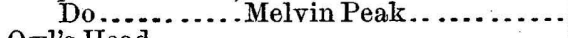 & Appalachian Club & 2,950 \\
\hline 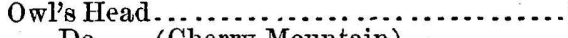 & 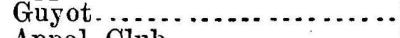 & 2,950 \\
\hline Do..... (Cherry Mountain) ............ & Appal. Club ....... & 3,302 \\
\hline Parker's Station $\ldots \ldots \ldots \ldots \ldots \ldots \ldots$ & Mauch. \& N. Weare & 319 \\
\hline$\ldots \ldots \ldots \ldots \ldots$ & 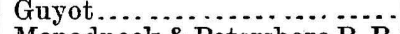 & 4,420 \\
\hline 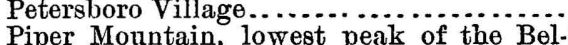 & Monadnock \& Petersboro R. R. & 744 \\
\hline 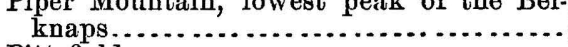 & Appalachian Club & 2,063 \\
\hline 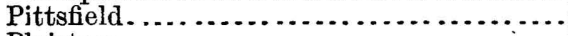 & Suncook Valley R. R & 493 \\
\hline Plaistow . . . & Boston \& Maine R. R & 92 \\
\hline Do..brickyard $\ldots \ldots \ldots \ldots \ldots \ldots \ldots$ & Boston \& Maine R. R & 86 \\
\hline 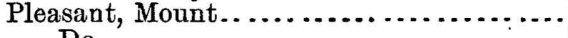 & Guyot................... & 4,764 \\
\hline Do....................... & Appalachian Club . & 4,771 \\
\hline Pleasant, Mount. . . . . . . . . . . . . . . . . . . & Club. & 1,896 \\
\hline$\ldots \ldots \ldots$ & Bost., Con. \& Mont. R. R .... & 473 \\
\hline$\cdots \ldots \ldots \ldots$ & Appalachian Club ....... & 1,747 \\
\hline Profile Monntain, true summit . . . . . . . . . . & alachian Club ... & 4,114 \\
\hline Prospect Mountain, Lancaster ............ & Appalachia & 2,062 \\
\hline$\cdots \ldots \ldots \ldots \ldots \ldots$, & ord \& Portsmouth R. & 198 \\
\hline$\ldots \ldots \ldots \ldots \ldots$ & Nashua \& Rochester R. R. & 226 \\
\hline Rollinsford $\ldots \ldots \ldots \ldots \ldots \ldots \ldots \ldots \ldots \ldots \ldots \ldots$ & Boston \& Maine R. R.... & 115 \\
\hline Rowe's Hill, West Campton ................ & Appalachian Club. & 1,235 \\
\hline & Bost., Con. \& Mont. & 520 \\
\hline sell's (or S. Greenfield) ................. & rsborongh R. R. & 820 \\
\hline 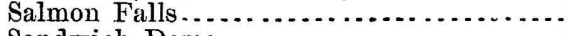 & Boston \& Maine R. R.... . & 107 \\
\hline$\ldots \ldots$ & 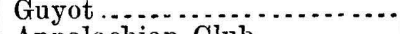 & 3,969 \\
\hline Sandwich Notch, Campton, Sandwich road. & Appalachian Club. & 1,754 \\
\hline Thornton & achian Club & 3,824 \\
\hline Scott's Mills, crossing B., C. \& M. R. R.... & Port. \& Ogden R. R. & 860 \\
\hline 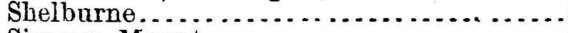 & Grand Trunk R. R... & 704 \\
\hline Sinapee, Mount . . . . . . . . . . . . . . . . . . . & nann .......... & 1,636 \\
\hline South Charlestown... & Co. R. R.. & 303 \\
\hline South Danbury....... & Northern R. R ... & 732 \\
\hline th Keene..... & shire R. R..... & 560 \\
\hline$\ldots \ldots . . . .$. & Bost., Con. \& Mont. & 867 \\
\hline 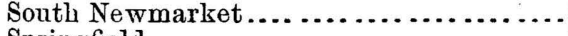 & Boston \& Maine R. R & 38 \\
\hline gfield $\ldots \ldots \ldots \ldots$ & an Co. R.R... & 374 \\
\hline & Trunk R. R. & 954 \\
\hline Star Lake, between Madison & in Club. & 4,890 \\
\hline$\ldots \ldots$ & n Club & 3,943 \\
\hline e Mountain, Tecumseh Group....... . & Appalachian Club. & 3,376 \\
\hline Stratford Hollow . ................. & Grand Trunk R. R...... & 863 \\
\hline Sugar Hill, Coast Survey Signal......... & & 1,781 \\
\hline Do.... Goodnow House .............. & Appalachian Club & \\
\hline Do..... post-office ......... & & \\
\hline Sunape & & 956 \\
\hline aset Hill, Campton Village. & achian Club & 1,035 \\
\hline$y \ldots \ldots \ldots \ldots \ldots \ldots$ & Asbuelot R. R.... & \\
\hline Table Mountain ........ & ot . . . . . n & \\
\hline Taylor's Hill, West Campton .............. & Appalachian Club. & \\
\hline seh Mountain, highest peak..... & (n) & \\
\hline & & $\begin{array}{l}3,818 \\
3,393\end{array}$ \\
\hline Tremont ........ & $\begin{array}{l}\text { Guyo } \\
\text { Ches }\end{array}$ & 1,002 \\
\hline Troy - & Guyot.. & 4,930 \\
\hline $\begin{array}{l}\text { Twin Mou } \\
\text { Twin Mou }\end{array}$ & Bost., Conl. \& Mont. R. R. & 1,375 \\
\hline lett Station.. & Port. \& Ogden R. R... & 660 \\
\hline Wakefiel & & \\
\hline
\end{tabular}




\begin{tabular}{|c|c|c|}
\hline Station. & Authority. & Elevatian \\
\hline Wallace Hill, Bethlehem. & Appalachian Clu & Feet. \\
\hline Walpole ................ & Cheshire R. R...... & 217 \\
\hline Warner ................. & Concord \& Claremon & 422 \\
\hline Warren.............. & Bost., Con. \& Mont. R. & \\
\hline Washington, Mount .............. & Guyot.:............... & \\
\hline Do.................... & Appalachian Club. & 6,285 \\
\hline Do... & Bost., Con. \& Mont. $R$ & 6,293 \\
\hline ...... Nelson's crag & Appalachian Club.. & 5,615 \\
\hline Do .............. Lion's Head . & Appalachian Club. & 5,016 \\
\hline Do.............. top south wall Hunt- & Appalachian Club. & \\
\hline Do .............. Signal Station.... & U. S. Signal Office.. & 6,286 \\
\hline Do $\ldots \ldots \ldots$. . Timber line on & Bost., Con. \& Mont. R. & 4,150 \\
\hline Waterville $\ldots . . . . . . . . .$. & Appalachian Club .... & 1,536 \\
\hline Weetamoo Mountain, C & Appalachian Club. & 2,546 \\
\hline Welch Mountain, lower peak ...... & Appalachian Club .... & 2,657 \\
\hline Do .......... highe & 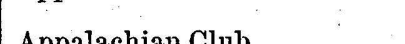 & \\
\hline West Andover.... & $\begin{array}{l}\text { Appalachiai } \\
\text { Northern R. }\end{array}$ & $\begin{array}{r}2,788 \\
677\end{array}$ \\
\hline West Campton, Blair's & App & 556 \\
\hline Do.........S.S & Ap & 571 \\
\hline Do.... & Apl & 623 \\
\hline ...Rising Sun Church & $\mathrm{Ap}$ & 1,103 \\
\hline (n......................... & A R.R... & 813 \\
\hline West C & Co. R. & 406 \\
\hline Wes & Cor & 354 \\
\hline West & Con & 392 \\
\hline Wes & Nort & 376 \\
\hline & runk R. R. & 996 \\
\hline and $\ldots \ldots \ldots \ldots \ldots$ & iire R. R..... & 512 \\
\hline Wes & \& C. R.R. & 428 \\
\hline ............. & lot R. R .... & 470 \\
\hline Whi & Guyot............ & 4,030 \\
\hline Whitefield $\ldots \ldots \ldots \ldots \ldots$ & Bost., Con. \& Mont. R. R .... & 931 \\
\hline Do... Dodge's Mountain View House & & \\
\hline & App & 1,279 \\
\hline Do .....Fiske's Cherry Mountain House & & 1,249 \\
\hline White Mountain House................... & Con. \& Mo & 1,556 \\
\hline$\ldots \ldots \ldots$ & & 4,350 \\
\hline ation ............... & \& Ogden R.R & 1,608 \\
\hline Will & Guyot........... & 4,300 \\
\hline Wilton & Petersborough R. R & 328 \\
\hline WinchendonVillage, crossing Cheshire Rail & & \\
\hline & Mone & 992 \\
\hline 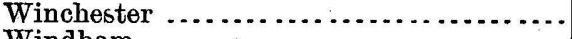 & & 448 \\
\hline 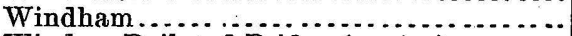 & MaI & 324 \\
\hline d Bridge (center).. & Co.R.R.... & 352 \\
\hline ogee Lake.... & s Valley R. R. & 500 \\
\hline Do. & P., C & 501 \\
\hline Do $\ldots \ldots \ldots \ldots \ldots \ldots \ldots$ March 26,1872 & P., G.F.\& C.R. R & 498 \\
\hline Wolfborough Junction, 50 miles from Ports & & \\
\hline & $\begin{array}{l}\text { P., G } \\
\text { App }\end{array}$ & $\begin{array}{l}574 \\
749\end{array}$ \\
\hline Woodsville & Bost., Cou. & 448 \\
\hline
\end{tabular}


NEW JERSEY.

\begin{tabular}{|c|c|c|}
\hline Station. & Authority. & Elevation. \\
\hline & & Feet. \\
\hline 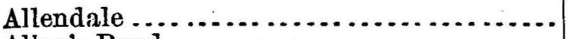 & N. Y., L. E. \& W. R. R & 270 \\
\hline Allen's Pond $\ldots . . . . . . . . . . . . . . . . . .$. & N. J. Geol. Survey .... & 897 \\
\hline Amboy, in passenger house on wharf..... & Camden \& A. R. R. & 12 \\
\hline Andover, depot..................... & Sussex R. R ...... & 636 \\
\hline A'sbury & N.J. C.R.R R.... & $4: 38$ \\
\hline Atlantic City, Signal Station . . . . . . . . . . . & U. S. Signal Office.. & 13 \\
\hline Augusta $\ldots \ldots \ldots$ & Sussex R. R $\ldots \ldots \ldots$ & 489 \\
\hline $\begin{array}{l}\text { Avondale } \ldots \ldots \ldots \ldots \ldots \\
\text { Barnesboro } \ldots . . . . . . . . .\end{array}$ & N. Y., L. E. \& W. R. R........ & 95 \\
\hline Basking Ridge ............ & P.\& D. R. R...... & $\begin{array}{r}64 \\
379\end{array}$ \\
\hline Beacon Hill ........... & U.S. C. \& G. S ....... & 373 \\
\hline Belleville ........ & N.Y., L. E. \& W. R. R & 26 \\
\hline 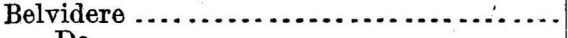 & Phil.\& T. R. R : & 271 \\
\hline Do $\ldots \ldots \ldots \ldots \ldots \ldots \ldots \ldots \ldots \ldots \ldots$ & Newton \& Belvidere R. R.... & 286 \\
\hline Bergen Junction ........ & N. Y., L. E. \& W. R. R.'...... & 4 \\
\hline Bergen Neck . . . . . . . . & U.S. C. \& G. S. ..... & 223 \\
\hline Berkeley Heights ...... . & P.\& D. R. R .......... & 230 \\
\hline Bernardsville ........ & P. \& D. R. R ........ & 372 \\
\hline field............ & Newark \& Bloomfiel & 127 \\
\hline$\cdots$ & N.J. C. R. R .. & 334 \\
\hline Blue Mountain, summit o & Geol. Su & 1,527 \\
\hline Boonton ................ & Morris \& Essex R. R & 413 \\
\hline Boonton Branch Junction ............ & Morris \& Essex R. R & 41 \\
\hline Boonton Crossing Junction . . . . . . . . . & Morris \& Essex R. R & 554 \\
\hline Boonton (Sheep's Hill, U. S. C. \& G. S. Sta- & & \\
\hline Bordentown, mean tide in Delaware River & Del. \& Raritan Canal ..... & $\begin{array}{r}943 \\
1\end{array}$ \\
\hline Brook $\ldots \ldots \ldots \ldots \ldots \ldots \ldots \ldots$ & N.J.C.R.R ............ & 36 \\
\hline chville Hotel .... & N.J.Geol. Sur & 582 \\
\hline Bray's Hill Summit..... & N.J.C.R.R.. & 365 \\
\hline Bridgeton ............. & W.J.R.R... & 51 \\
\hline Broa & Morris \& Es & 377 \\
\hline Bull's Island, head of. .............. & Phil. \& T. R. R ...... & 97 \\
\hline Do..... . low water in Delaware River. & Phil. \& 'T. R. R ... & 7,4 \\
\hline Camden, Court-Housé.............. & U. S. C. \& G. S ... & 30 \\
\hline Camden...$\ldots \ldots \ldots \ldots \ldots$ & W.J.R.R R . . . . & 6 \\
\hline May, Signal Station ............ & U. S. Signal Office... & 27 \\
\hline jerville $\ldots \ldots \ldots \ldots \ldots$ & Phil. \& T. R. R .... & 178 \\
\hline Centreville..... & N. Y., L. E. \& W. R. R. & 132 \\
\hline$\ldots \ldots \ldots \ldots$ & Morris \& Essex R. R. & 233 \\
\hline Chester & 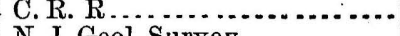 & 686 \\
\hline Do.U. S. C. \& G. S. Station ............ & N.J. Geol. & 790 \\
\hline hester Junction . . . . . . . . . . . . & is \& Esse & 691 \\
\hline Olayto & M. \& G. R. R & 123 \\
\hline Clementon, Jaggert's house . ............. . & U.S. C. \& G.S . ... & 76 \\
\hline 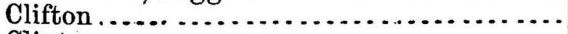 & N. Y., L. E. \& W. R. R........ & 56 \\
\hline ................ & N.J.C.R.R................. & 349 \\
\hline Convent ...... & is \& Essex R. R......... & 281 \\
\hline Cookstown ..... & U.S. C. \& G. S .................. & 99 \\
\hline Hill ....... & $-\ldots \ldots \ldots \ldots \ldots$ & 159 \\
\hline Cranberry Reservoir (water) $\ldots \ldots \ldots$ & $\ldots . . . .$. & 772 \\
\hline Do $\ldots \ldots \ldots$........ (summit) $\ldots \ldots \ldots$ & & 780 \\
\hline Crane Mountain, U. S. C. \&. G. S. Station & U. S. C. \& G. S & 665 \\
\hline & N. J. C.R.R R.... & $7 \%$ \\
\hline Crystal Lake.... & W. J. R. R................. & 2 \\
\hline Culver's Gap, 1st sunımit .... & N. J. Geol. Survey ............ & 896 \\
\hline Do......2d summit $\ldots . . . . .$. & N. J. Geol. Survey ............ & 931 \\
\hline Thanomatong & N. J. Geol. Survey ............. & \\
\hline
\end{tabular}




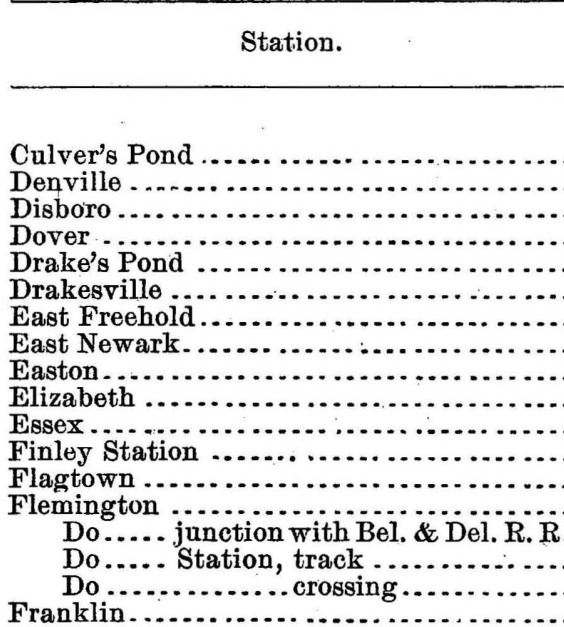

Franklin Furnace Pond

Franklinville

Freehold

Freehold Junction

Frenchtown

Glassborough

Gloucester

Do.... Buena Vista Hotel

Hackettsown

Haddonfield

Do

Hanan's Pond

Hardings

Hartford, Rodgers house

Hasteds.

Haw thorne

High Bridge .

High Knob

Do.....Pond

Hightstown

Hillsdale.

Hoboken

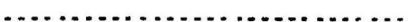

Holmdel Cross-Roads

Hopating Lake.

Hope

Hornerstown

Horton's

Howell P. O.

Ironia

Jamesburg

Do Junction

Jersey City

Johnsburg

Keyport, Morris house

Do:-: Broad street

Do... First street.

Kingsland

Kingston (Lock)

Do intersec $\mathrm{R} \ddot{H}$ \& $\mathrm{Old} \mathrm{C} \& \ddot{\mathrm{Br}}$

Lafayette, grade line in

Lake View

Lambertville.

Do. Do ...... lock

Lebanon
N. J. Geol. Survey
Morris \& Essex R.

U. S. C. \& G. S .

U. S. C. \& G. S...

N. J. Geol. Survey.

Morris \& Essex R. R

F. \& N. Y. R. R

N. Y., L. E. \& W. R. R

N. J.C.R. R

N. J. C. R. R . . .

N. Y., L. E. \& W. R. R.......

W.J.R. R .

N. J.C.R. R

F. R. R

F. R. R ...

N. J. C. R. R

N. Y., L. E. \& W. R. R

N. J. Geol. Survey

M. \& G.R. R

F. \& N. Y. R. R

F. \& N. Y. R. R

Phil. \& T. R. R

M. \& G. R. R

W. J. R. R

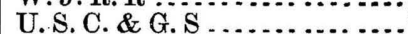

Mbrris \& Essex R. R ..

Camden \& Amboy' $R$. $R$.

U. S. C. \& G. S

W. J. R. R.

W. J. R. R

U. S. C. \& G. S

W. J. R. R

N. Y., L. E. \& W. R. R

N. J. C. R. R.

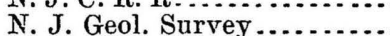

N. J. Geol. Survey .............

Camden \& Amboy R. R

F. \& N. Y. R. R.

Morris \& Essex $\mathrm{R} . \mathrm{R} \ldots$

N. Y., L. E. \& W. R. R........

U. S. C. \& G. S..................

Newton \& Belvidere R. $\mathrm{R}$

U. S. C. \& G. S

C. R. R

Newton \& Belvidere R. R

C. R R \& Belvidere R. R...

Camden \& Amboy R. R

Camden \& Amboy R. $R$

N. Y., L. E. \& W. R. R ........

New ton \& Belvidere R. R ....

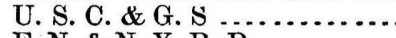

F. N. \& N. Y. R. R ..........

F. N. \& N. Y. R. R

Morris \& Essex R. R

Del. \& Raritan Canal

Camden \& Amboy R. R

Sussex R. R.

N. Y., L. E. \& W. R. R

Phil. \& T. R. R .............

Del \& Raritan Canal

Dél. \& Raritan Canal

N. J. C. R. R
Elevation.

Feet.

$8: 39$

523

276

575

581

797

173

32

160

29

127

107

137

182

76

195

104

81

531

103

168

33

128

149

16

11

569

72

74

87

140

48

90

50

335

1, 799

1,574

99

143

7

190

135

414

451

83

695

565

$70 \%$

73

92

5

571

16

32

16

24

57

60

566-571

93

75

59

69

298 


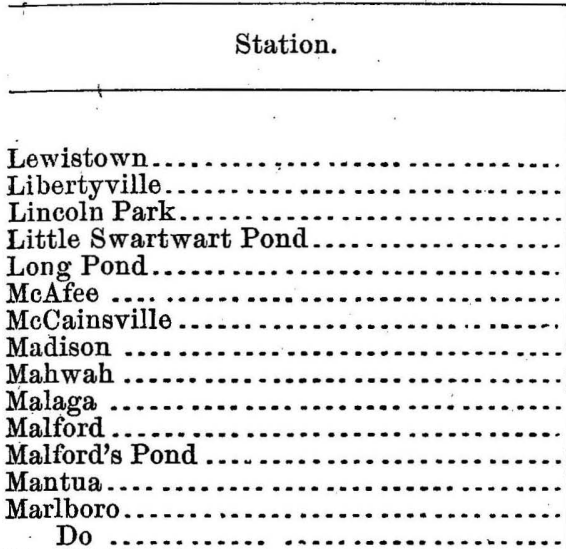

Martin's Creek

Mattawan

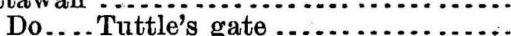

Do.... bench-mark at .

Milford

Millburn

Millington.

Millville

Morgan Station, bench-mark at

Monroe

Morgansville

Do ... . . Clarke's house

Do ........Frazer's house.

Morris Plains.

Morris Ponds

Morristown

Mount Airy

Mount Holly

Do... Garden street

Do....... Rhees House

Do ..... R, R. bridge

Mount Pisgah Hotel ...................

Mulford.

Navesink Highlands Light-house, benchmark at

Neshanic.

Newark.

Newfield

New Hampton (Junction)

$$
\text { Do....... Summit ... }
$$

New Market

Newton

Do. east end of depot.

North Branch

North Vineland

Orange.

Oxford

Palatine

Passaic .

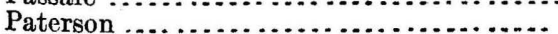

Do... Junction

Pemberton

Peru

Authority. $\mid$\begin{tabular}{c} 
Elevation. \\
\hline Fet.
\end{tabular}

U. S. C. \& G. S

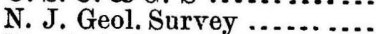

Morris \& Essex R. $\mathrm{R}$

N. J. Geol. Survey ................

N. J. Geol. Survey

W. V. R. R.

C. R. R

Morris \& Essex $\mathrm{R} . \mathrm{R}$

N. Y., L: E. \& W. R. R .....

M. \& G. R. R

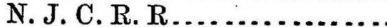

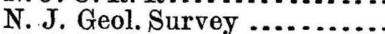

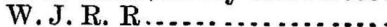

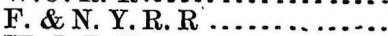

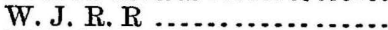

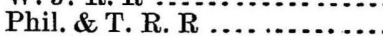

F. \& N. Y. R. R .............

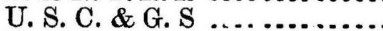

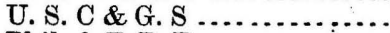

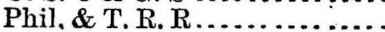

Morris \& Essex R. R ........

P. \& D. R. R

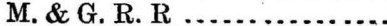

U. S. C. \& G.S

W. J.R.R

Newark \& Bloomfield $\mathrm{R}, \mathrm{R}$

F. \& N. Y. R. R..............

U.S.C. \& G.S

U. S. C. \& G.S

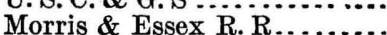

N. J. Geol. Survey

U.S. C. \& G. S

Morris \& $\mathrm{Essex} \mathrm{R}$

F. R. R. R

U.S. C. \& G. S

U.S. C. \& G. S

U. S. C. \& G. S

U.S.C. \& G.

N. J. G. S

N.J. R. R

U. S. C. \& G. S

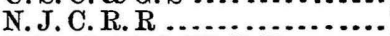

N. Y., L. E. \& W.R.R......

M. \& G. R. R

Warren R. R.

N.J.C.R. R

N. J. C. R. R

Newton \& Belvidere R. R .... $\quad 610$

Sussex R. R ............... 607

N. J.C. R. R .............. 93

M. \& G. R. R.............. 107

Morris \& Essex R. R ......... 187

Warren R. R............... 491

W. J.R.R ................ $\quad \mathbf{1 1 6}$

N. Y., L. E. \& W. R. R ...... 55

N. Y., L. E. \& W. R. R ......

N. Y., L. E. \& W. R. R ...... 92

U. S. C. \& G. S............. 42

U. S. C. \& G. S............ 35

Phil. \& T. R. R ........... $\quad 198$

W. J. R. R ................ 112

U. S. C. \& G. S............. 202

N. J. C. R. R ..............

85

176

862

419

13

310

76

36

156

234

58

56

150

36

6
134

239

116

172

20

328

187

41

15

850

77

94

14

508

13

1

.

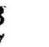

1

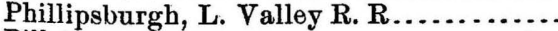

Pine Hill . . . . . . . . . . . . . .

Plainfield 


Station.

Port Jervis Depot

Port Morris

Port Murray

Princetown, on street-crossing in front of

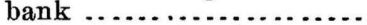

Do ..... on street-crossing in front of

library .....................

Princeton Junction......................

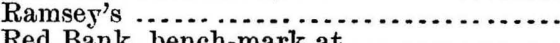

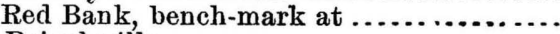

Reigelsville.

Ricefield .

Ringoes

Rockaway

Roseville Junction

Rossell's Freehold

Rutherford

Do... Park

Sand Pond

Sandy Hook, Signal Station

Sarepta

Scotch Plains

Seabright, bench-mark at . ..............

Somerville, bench-mark on court-house grounds ...................

Do ..... beuch-mark on base east pillar at court-house

South Orange .

South Vineland

Sparta (upper mill-dam)

Spotswood

Springfield

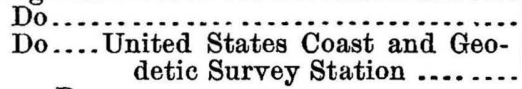

Spruce Run detic Survey Station

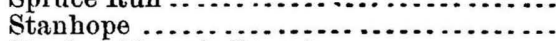

Stanley (Passaic River)

Stewartsville

Stirling .

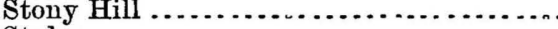

Styles

Succasuna

Sunfish Pond

Swartwout Hotel

Do ....Pond

Three Bridges

Trenton, Lock

Do..top of dam in Assapink Creek, above Green street bridge .....

Do . . Clinton street station ............

Do...Junction .

Tunis, Imlaystown

Union

Valley

Vernon

Vineland

Walkill

Washington

Washington's Crossing.

Waterloo

Authority. Elevatio

N. J. Geol. Survey

Morris \& Essex R. R.........

Morris \& Essex R. R..........

Phil. \& T. R. R ............

Camden \& Amboy R. R......

Camaden \& Amboy R. R.....

Camden \& Amboy R. R.....

N. Y., L. E. \& W. R. R .....

U. S. C. \& G. S....................

Phil. \& T. R. R .............

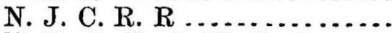

N. Y., L. E. \& W. R. R .....

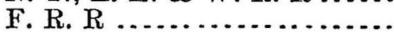

Morris \& Essex R. R ........

Newark \& Bloomfield R. R ..

U. S. C. \& G. S $\ldots \ldots \ldots$

Morris \& Essex R. R.

N. J. Geol. Survey

U. S. Signal Office............

Newton \& Belvidere R. R....

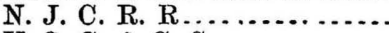

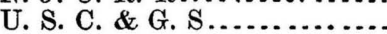

U. S. C. \& G. S .............

U. S. C. \& G. S. . ............

Morris \& Essex R. R

M. \& G. R. R . . . . . . . .

N. J. G. S

Camden \& Amboy R. R.....

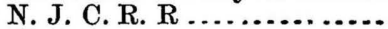

U. S. C. \& G. S

N. J. Geol. Survey ...........

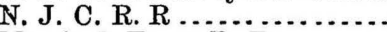

Morris \& Essex R. R

Morris \& Essex R. R.

Morris \& Essex R. R........

P. \& D. R. R .

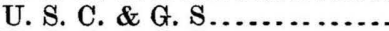

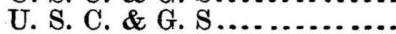

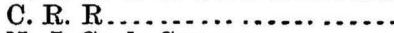

N. J. Geol. Survey .............

N. J. Geol. Survey ...........

N. J. Geol. Survey............

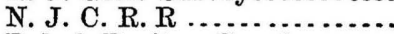

Del. \& Raritan Canal.........

Camden \& Amboy R. R......

Phil \& $\mathrm{T}$ R $\mathrm{R} . \ldots . . . .$.

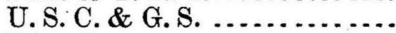

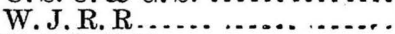

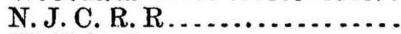

W. V.R. R.

M. \& G. R. R............

N. J. Geol. Survey............

Morris \& Essex R. R .........

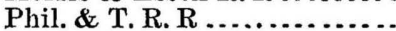

Morris \& Essex R. R.
Feet.

441

9\%2

588

86

221

207

83

345

38

166

109

137

248

557

144

166

50

37

1,500

28

364

156

9

82

91

141

92

696

29

312

523

495

471

873

239

377

226

235

34

703

504

529

478

114

50

26

33

63

228

143

398

392

110

383

507

67.

717 


\begin{tabular}{|c|c|c|}
\hline Station. & Authority. & Elevation, \\
\hline & & Feet. \\
\hline 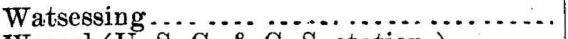 & W.\& B. R. R ................ & 134 \\
\hline Weasel (U. S. C. \& G. S. station.)........ & 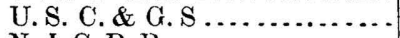 & 583 \\
\hline 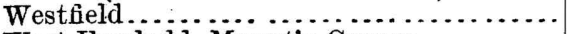 & N.J.C.R.R............... & 133 \\
\hline West Freehold, Mount's Coruer........... & U.S. C.\& G.S............... & 183 \\
\hline 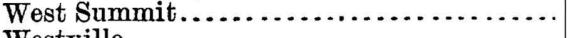 & 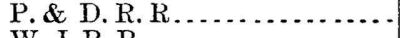 & 293 \\
\hline 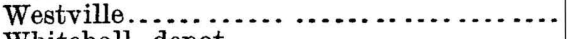 & 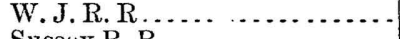 & 10 \\
\hline 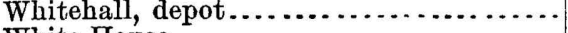 & Sussex R. R. . & 735 \\
\hline 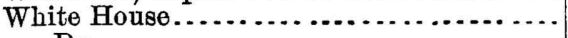 & N.J.C.R.R...... & 181 \\
\hline 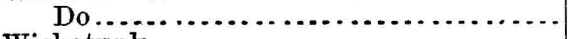 & U.S. C.\& G.S........... & 64 \\
\hline 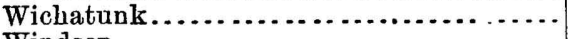 & F. \& N. Y.R. R............... & 141 \\
\hline 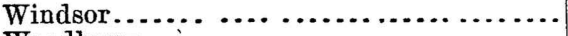 & Camden \& Amboy R. R...... & 85 \\
\hline 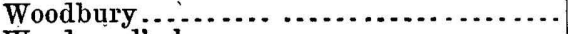 & 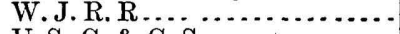 & 34 \\
\hline 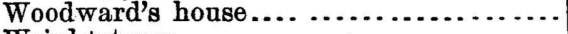 & U.S. C. \& G.S $\ldots \ldots \ldots \ldots \ldots$ & 188 \\
\hline Wrightstown $\ldots \ldots \ldots \ldots \ldots \ldots \ldots \ldots$ & U. S. C. \& G. S. . & 136 \\
\hline 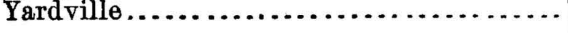 & Caụden \& Amboy R. R ...... & 53 \\
\hline
\end{tabular}


NEW MEXICO.

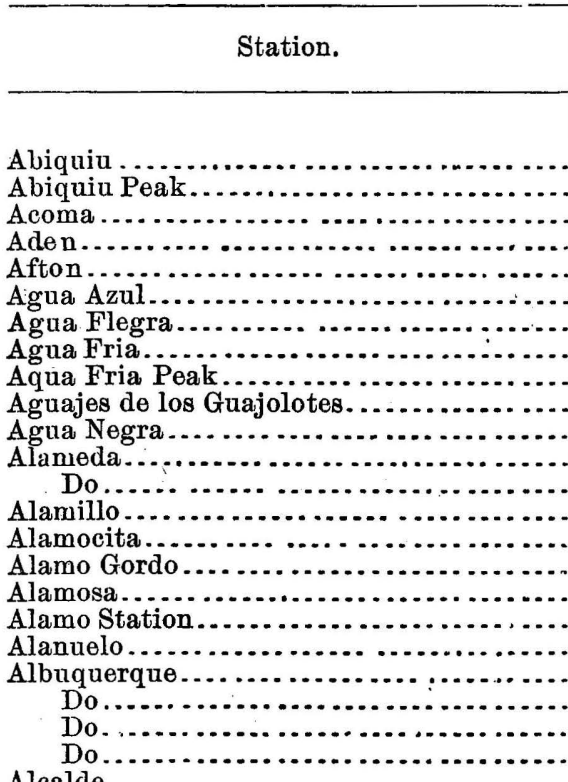

Alcalde

Aleman ...

Algodones. Do.

Amargo

Animas Peak

Annaya Spring.

Antelope Spring

Anthony

Anton Chico

Do

Apache

Apache Cienega

Apache Tejo.

Arch Spring

Do

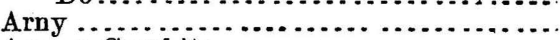

Arooyo Cuerbito.......................

Atlantic \& Pacific crossing

Atrisco

Azotea

Azul .........

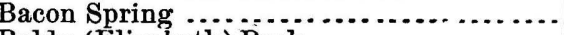

Baldy (Elizabeth) Peak

Baldy (Santa F6) Peak

Banded-Peak...

Barney Station.

Barranca

Bascom, Fort

Bayard, Fort.

$$
\text { Do.... }
$$

Belen

$$
\text { Do }
$$

\begin{tabular}{r|r} 
Authority. & Elevation. \\
Feet.
\end{tabular}

Wheeler

Wheeler

Wheeler

S. P.R. R

S. P. R. R.

Wheeler.

Wheeler

Wheeler.

Wheeler

Wheeler

Wheeler.

A., T. \& S. F. R. R

A., T. \& S. F. R. R

Wheeler.

Wheeler

Wheeler

Wheeler

Wheeler

Wheeler

A., T. \& S. F. R. R

Med. Dept., U. S. A

Pac. R. R. Reports

D. \& R. G. R. R

Wheeler.

Wheeler

A., 'T. \& S. F. R. R

D. \& R. G. R. R

Wheeler.

Wheeler

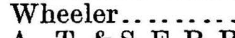

A., T. \& S. F. R. R

A., T. \& S. F. R. R

Pac. R. R. Reports

Wheeler.

Wheeler.

Wheeler.

Pac. R. R. Reports

Wheeler ............

A., T. \& S. F. R. R

A., T. \& S. F. R. R

Pac. R. R. Reports

D. \& R. G. R. R...

Wheeler

Wheeler

Wheeler

Wheeler

D. \& R. G.R. R

Toner.

Wheeler

Wheeler

Wheeler

Wheeler

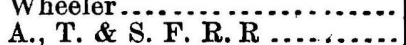

5,930

11,240

6,423

4,393

4,209

6,682

8,194

6,486

10,965

6,202

8,194

4,906

4,979

4,634

4,256

4,863

5,177

6,593

4, 693

4,919

4,933

5,032

5,026

5,687

4,594

5,104

5,087

6,987

6,106

4, 717

6,221

3,772

5,386

5,373

7, 324

5,291

5,478

6,350

6,485

4,512

4,848

4,874

5,033

7,701

6,670

7,189

12,491

12. 661

12,824

3,992

6,927

3,726

6,068

6,098

8,081

4,890

4,784 


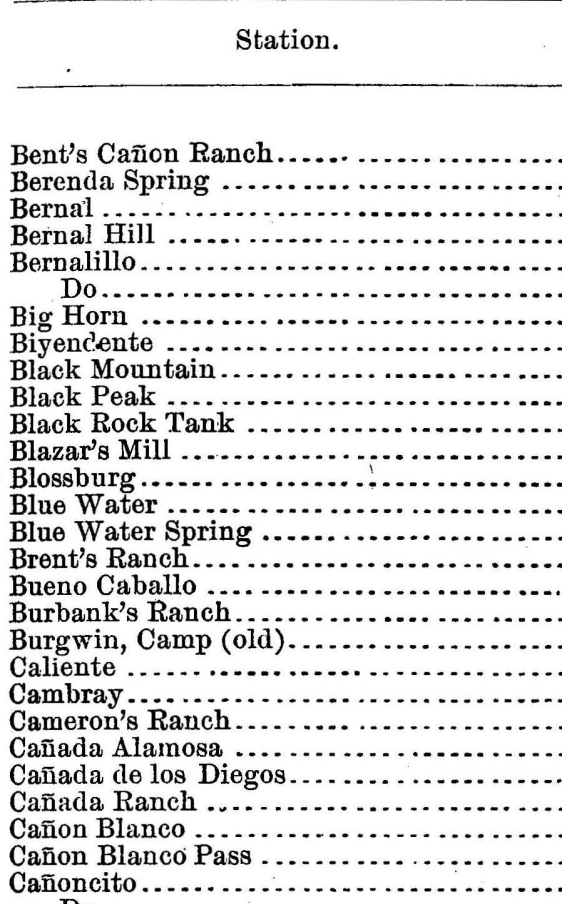
Do

Cañon del Agua

Cañon del Chaco

Cañon Pajareto

Capitan Pass

Capitan Peak

Carrizillo Spring Do......

Carrizo Spring

Casa Colorado

Caseta

Cerillos

Cerro Blanco .........................

Cerro Colorado.

Cerro de Culebra

Cerro Tecolote

Chama..

Chamisal

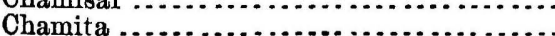

Chase, Camp

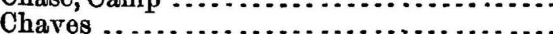

Chico Spring

Chili

Chittenden's Ranch

Chupadero Ranch

Ciboletta

Cibolo Hili

.

Cienega San Simon $\ldots \ldots \ldots . . . . . . . .$.

Cimarron

Cineguilla

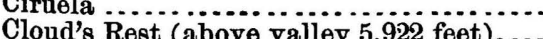

.

Toner

Wheeler

A., T. \& S. F. R. R

Wheeler.

Wheeler.

A., T. \& S. F. R. R

D.\& R. G. R. R

Toner.

Wheeler

Wheeler.

Wheeler.

Wheeler

A., T. \& S. F. R. R

A. \& P. R. R

Wheeler.

Wheeler.

Wheeler.

Wheeler

Wheeler

D.\& R. G. R. R

S.P. R. R

Wheeler

Wheeler

Toner .

Wheeler

Pac. R. R. Reports

K. P. R. R. Surveys

Wheeler............

Wheeler

Wheeler.

Wheeler

Wheeler.

Wheeler

Mexican Boundary Survey

Wheeler

Wheeler

Wheeler

Wheeler.

Wheeler

A. T. \& S. F. R. R

Wheeler.

Wheeler.

Wheeler

Wheeler

D. \& R. G. R. R

Wheeler.

D. \& S. P. R. R

Wheeler.

A. \& P. R.R

Wheeler

Wheeler.

Wheeler.

Wheeler.

Wheeler.

Wheeler.

Wheeler.

Wheeler.

Wheeler.

Wheeler

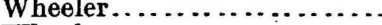

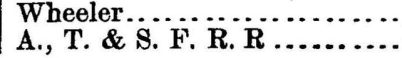

Elevation.

Feet.

4,820

6,056

7,029

5,084

5,031

9,000

5,406

8,909

8,910

4,180

6,449

6,840

6,609

6,778

4,021

6,948

6,882

7,277

7,302

4,226

$7,1: 33$

6,540

6,145

5,088

6,320

7,081

7,023

6,853

5,916

5,839

5,099

7,398

10,023

4,455

4,457

9,390

7, 32\%

4,679

6,101

5,667

14,269

5,654

6,992

7,254

7,841

7,527

5,619

5,374

6,969

6.882

5,647

5,004

5, 825

6,411

6,474

7, 179

3,855

6,385

6, 011

6,744

9,773

4,356

Bull. 5 


\begin{tabular}{|c|c|c|}
\hline & Autho & Elevation. \\
\hline & & \\
\hline olonas Ferry, & Wheeler. & - 7,443 \\
\hline lorado Moun & Wheeler. & 5,654 \\
\hline lorado Plaza, & Wheeler $\ldots . . .$. & 4,008 \\
\hline & D. \& R. G. R. R & \\
\hline $\begin{array}{l}\text { Comanche Cañon Pass } \\
\text { Cone Peak ............. }\end{array}$ & $\begin{array}{l}\text { Wheeler....... } \\
\text { Wheeler....... }\end{array}$ & $\begin{array}{r}8,284 \\
12,690\end{array}$ \\
\hline Conrad, Fort.... & Med. Dept. U. S. & $\begin{array}{r}1 \pi, 080 \\
4,576\end{array}$ \\
\hline & Wheeler.......... & 4,711 \\
\hline Cooke's Spring & Mexican Bo & 4,326 \\
\hline Cook, Mount ... & Wheeler... & 8,330 \\
\hline dge $\ldots . . . .$. & A. \& P. R. & 6,975 \\
\hline l's ........... & Wheeler.. & 6,800 \\
\hline Mines ........ & Emory ... & 6,167 \\
\hline Pass ............ & $\begin{array}{l}\text { eler. . } \\
\text { eler.. }\end{array}$ & $\begin{array}{r}5,091 \\
10,188\end{array}$ \\
\hline Costilla & Haj & 12,634 \\
\hline$\cdots$ & Whe & 12,615 \\
\hline ood Sp & Wh & 4,773 \\
\hline$\because \cdots \cdots \cdot$ & Pac.R.R. & 5,880 \\
\hline ring .... & Wheeler... & 5,001 \\
\hline ring $\ldots . . .$. & Whe & 7,202 \\
\hline te Water-holes. & Whe & 6,775 \\
\hline Craig, Fort ......... . & Wheeler. & 4,448 \\
\hline $\begin{array}{r}\text { Do................ } \\
\text { Crocker }\end{array}$ & Med. Dept & 4,576 \\
\hline $\begin{array}{l}\text { Crocker } \\
\text { Cross Spring...... }\end{array}$ & $\begin{array}{l}\text { A., T. \& S. } \\
\text { Wheeler.. }\end{array}$ & 4,707 \\
\hline Cubero & A. \& P.R. & $\begin{array}{l}6,260 \\
5,905\end{array}$ \\
\hline & Wh & 6,122 \\
\hline longa ..... & Wheele & 5,914 \\
\hline & & \\
\hline Negra...... & Whe & 4,56 \\
\hline$\ldots \ldots \ldots$ & Whe & 5,195 \\
\hline $\mathrm{C}$ & & 5,702 \\
\hline Peak ..... & Whe & 14,050 \\
\hline Cummings, Fort...... & Wh & 4,778 \\
\hline . old & & 4,840 \\
\hline 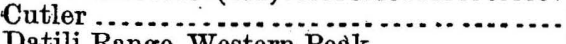 & A., T & 4,683 \\
\hline nge, Weste & Wheeler... & 9,440 \\
\hline nch........ & Wheeler. & 5,405 \\
\hline pring........ & Wheeler............. & 5,982 \\
\hline Defiar & P. R. R .... & 6,352 \\
\hline & $\ldots$ & 7,042 \\
\hline , junction wit & 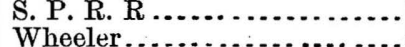 & \\
\hline Knoll......... & ........... & 7,617 \\
\hline ㅍ…........ & A., & 6,454 \\
\hline Iña.......... & S.F.R. & 3,899 \\
\hline & S.F.R. & \\
\hline Is Mill ..... . & Wh & 6,435 \\
\hline & S.F.R & 5,817 \\
\hline g Springs... & Whe & 5,623 \\
\hline & G. R. R. & 6,757 \\
\hline ley 3,818 feet) ..... & Wheeler.......................... & 7,669 \\
\hline . & Wheeler................... & 7,976 \\
\hline Peak .. & Wh & 12,491 \\
\hline town .. & & 8,465 \\
\hline ng .. & Wheeler.. & 7,415 \\
\hline & r & 7,414 \\
\hline & E. F. R.R. & 4,745 \\
\hline $\mathrm{E}$ & Mexican Boundary & 3,940 \\
\hline & A., T. \& S. F. R. R. & 5,123 \\
\hline El P & Wheeler. & 7,187 \\
\hline El Ri & Wheeler............... & 5,650 \\
\hline & A. \& P. R. R ... & 5,638 \\
\hline
\end{tabular}




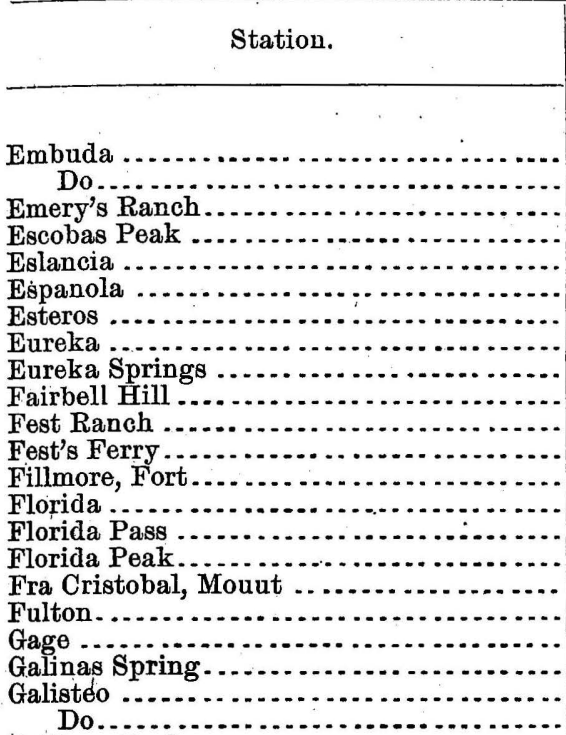

Gallinas Peak

Gallinas Ranch

Gallo Spring

Gallup

Garcia Peak

Georgetown

Geyser Spring

Glorieta

Grama

Gran Quivira

Grants

Guadalupita

Guy Fawkes

Hacheta Peak

Hanover Peak

Hatch

Hedionda Lake

Hendrick's Peak

High Peak.

Hillsborough.

Horse Springs.

Hot Springs

Do ..... Diamond Creek .................

Do..... (13 miles from Magruder's)

Hurricane Rock ........................ Indian Agency, near Blazer's Hill... . . . . . .

Indian Ranch

Inscription Rock

Isleta

Do.. (river bank)

Do... (top of bluffs)

Jackson Ranch

Jaruloso Spring

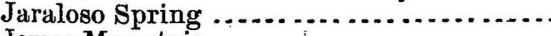

Jemes Mountain ............................

Jemes Peak

Jemes Pueblo

Juan de Dios

Juan Lujan Spring

Kettle Spring

Kingman ....

Authority.

Wheeler

D. \& R $\mathrm{G}$

Wheeler.

Wheeler.

Wheeler

D. \& R. G. R. R

Wheeler.

Wheeler

Wheeler

Wheeler

Wheeler.

Whieeler

Med. Dept., U. S. A.

A., T. \& S. F. R. R

P.R. R. Reports

Wheeler.

Wheeler

A., T. \& S. F. R.R

S. P. R. R

Wheeler

Wheeler.

Emory

Wheeler

Wheeler

Wheeler

A. \& P. R. R

Wheeler

Wheeler.

Wheeler

A., T. \& S. F. R. R

A., T. \& S. F. R. R

Wheeler.

A. \& P. R. R

Wheeler

Wheeler.

Wheeler

Wheeler

A., T. \& S. F. R. R

Wheeler

Wheeler

Wheeler

Wheeler

Wheeler

Wheeler

Wheeler

Wheeler

Wheeler.

Wheeler

Wheeler.

Pac. R. R. Reports

A., T. \& S. F. R. R

P. R. R. Reports.

P. R. R. Reports

Wheeler.

Wheeler

Wheeler

Wheeler.

Wheeler.

Wheeler

Wheeler

A. T. \& S. F. R. R

Whoeler. 


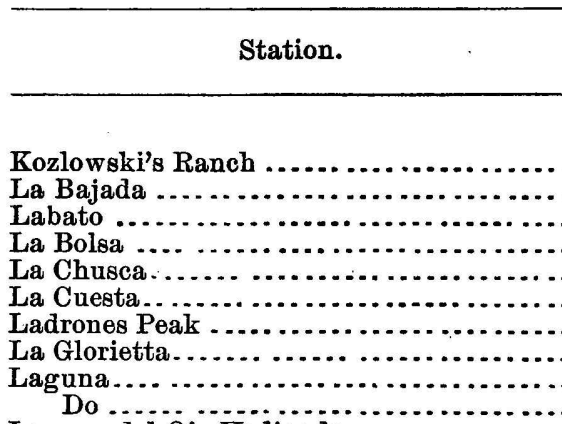

Laguna del Ojo Hediondo

Laguna Gallinas ...

Laguna Los Grieg

La Joya. Do...

Lake Peak

La Lacha Spring

La Laguna de Sal

La Mesilla

Do .. . Signal Station-

La Monica Springs

Lamy.

Lanark

Lansing

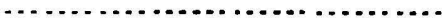

La Pilla................................

La Placita .............................

Largo Cañon .... ......................

Las Cruces. Do

Las Lunas

Las Lunitas

Las Playas

Las Tapiacitas.

Las Tenejas

Las Truchas Mountain

Las Vegas

$$
\text { Do ... . Hot Springs }
$$

La Tenaju. ......

Lava

$$
\text { Do. }
$$

La Veta ...........

Le Jarra

Lepai

Levy

Lisbon

Llano Spring

Loma ......

Lomitas Spring

Lone Mountain

Lordsburgh

Los Alamos

Los Brazos

Los Cerutos del Aquila

Los Chavez..

Los Cornudos

Los Machos

Los Ojos (Rio Chama)

Los Pinos

Authority. Elev

Wheeler

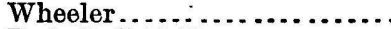

D.\& R. G. R.R ...............

Wheeler

Wheeler

Wheeler

Wheeler.

A. \& P. R. R

Wheeler

Wheeler

Whoeler

Wheeler

Wheeler

A., T.\& S. F. R. R

Wheeler

Wheeler

Wheeler

Wheeler

U. S. Signal Office

Wheeler

A. T. \& S. F. R. R

S. P.R. R.

A. T.\& S. F. R. R

Wheeler

Wheeler

Wheeler

A., T.\& S. F.R. R

A., T.\& S.F.R. R

A., T. \& S. F. R. R

Wheeler

Wheeler

Wheeler (Theod.)

Med. Dept. U.S. A

A., T. \& S. F. R. R

A., T.\& S. F.R. R

Wheeler

Wheeler

A., T. \& S.F.R. R

D. \& R. G. R. R.

Wheeler.

Wheeler.

S.P.R. R

A., T.\& S.F.R.R

S. P. R. R

Wheeler

Wheeler

Wheeler

Wheeler

S. P. R. R

Wheeler

Wheeler.

Wheeler

Wheeler

Wheeler

Wheeler

Wheeler.
Feet.

6, 405

5,515

8,281

5,829

6,703

5,792

9,214

7, 048

5,767

6,266

7,181

6,393

6,656

6,988

4,685

5,712

12,405

4,756

6,047

3,935

4,124

7,735

6,458

4,167

7, 053

5,850

5, 129

5,651

3, 797

3,871

4,831

4,806

4,190

8,810

4,749

13,150

6,418

6, 381

6,709

4,701

8,950

4,703

8,446

6,266

6, 401

6,942

4,505

$6, \approx 38$

4,280

7,452

5, 348

7,000

6, 393

5,731

5,986

4,247

6,789

7,321

7,943

4,775

4, 362

7,290

7,273

4,675 


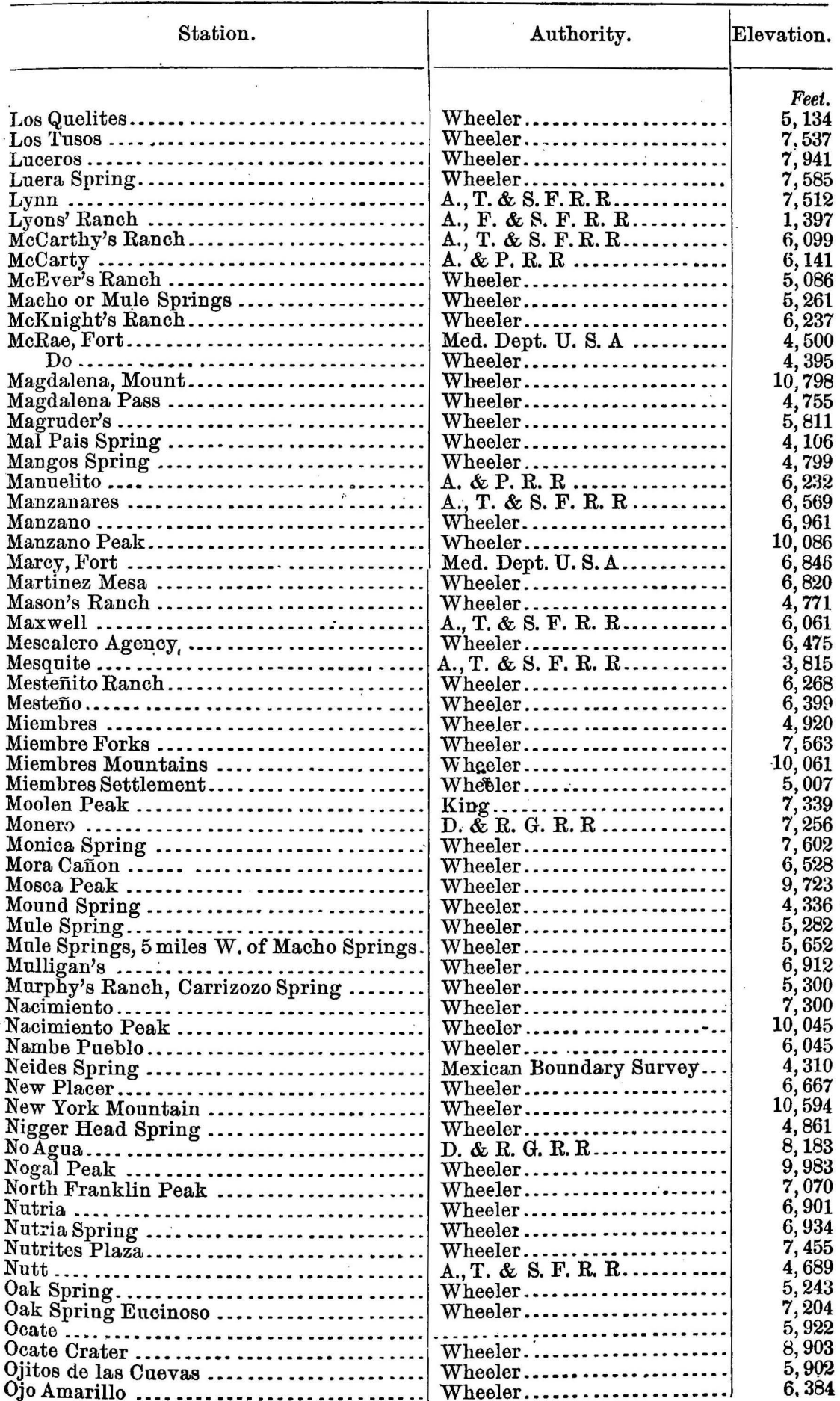




Station.

Ojo Caliente.

Ojo Chameleon

Ojo Datil

Ojo de Estancia

Ojo de Inez.

Ojo de Judio

Ojo de la Casa.

Ojo de la Culebra

Ojo del Alto Peak

Ojo de la Parida.

Ojo de la Quinea

Ojo de las Cañas

Ojo de la Tunisa.

Ojo del Cibolo.

Ojo del Indio.

Ojo de los Cazos

Ojo lel Oso

Ojo de los Valles

Ojo del Perro...

Ojo del Pescado

Ojo de Nuestra Signora

Ojo de Vaca.

Ojo Galle...

Ojo Milagro ...

Ojos Calientes.

Onava

Ord Peak

Organ Mountain

Organ Mountain Pass

Ortiz.

Oscura Water Holes

Osha Peak

Oteio ..................................

Pajarida

Palmillo

Palomas

Paraje

Paria Spring

Parida

Pastora

Patero

Patterson's Ranch

Payanda

Pecos Village

Pedernal

Pedernal Pass

Pedernal Peak

Pedernal Water Hole

Pelado Peak

Peloncillo Pass.

Peña Blanca

Peñasco.

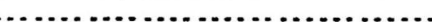

Peralta .................................

Pescado Spring.

Picacho Crossing.

Picacho de Sabinal

Picacho Peak

Picuris Pueblo

Pinos Altos.

Pinos Altos Peak

Pintado Pueblo

Placer Mountain

Plaza de Alcalde

Authority. $\mid \frac{\text { Elevation. }}{\text { Feet }}$

Wheeler

Feet.

Wheeler.

Wheeler

Wheeler.

Mexican Boundary Survey

Wheeler

Wheeler

Wheeler

Wheeler

Wheeler.

Wheeler

Wheeler

Wheeler

Wheeler

Wheeler

Wheeler

Wheeler

Wheeler

Mexican Boundary Survey

Pac. R. R. Reports

Wheeler

Wheeler

Mexican Boundary Survey

Wheeler.

Wheeler

Wheeler

A., T. \& S.F. R. R

Wheeler

Wheeler (Theod.)

P. R. R. Reports

A., T. \& S. F. R. R

Wheeler

Wheeler

A., T. \& S. F.R. R

D. \& R. G. R. $\ddot{R}$

Wheeler

Wheeler

Wheeler

Wheeler

Hayden

Wheeler

Wheeler

Toner

Emory .

Wheeler

Wheeler

Wheeler.

Wheeler

Wheeler

T. \& P. R. R. Surveys ....

Wheelér.

Wheeler

Wheeler

Wheeler

Wheeler

Wheeler

Wheeler

Wheeler

Wheeler

Wheeler

Wheeler

Wheeler

Wheeler
6,292

6,401

7,419

6,177

5,293

9,289

6,243

5,707

6,950

4,929

5,673

5,131

5,673

5,640

9,289

7,615

7,786

6,979

4,692

6,551

6,606

6,864

4, 989

7,943

5,173

5,594

6, 728

10,094

9,108

5,467

5, 819

5,461

10,023

6,377

4,833

8,236

4, 127

4,319

6, ع96

4,627

9, 332

6,123

4,590

4, 833

6,366

7,140

7,181

7,580

7,140

11, 260

4,446

5,170

7,452

4,661

6,546

3,784

4,676

4,825

7,108

6,845

8,128

6,506

8,827

5,756 


\begin{tabular}{|c|c|c|}
\hline Station. . & Authority. & Elevation. : \\
\hline$\therefore$ & & Feet. \\
\hline Plaza Mangos $\ldots \ldots \ldots \ldots \ldots \ldots \ldots$ & Wheeler. & $7.319=$ \\
\hline 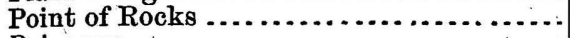 & Wheeler. & 4,268 \\
\hline Pojoaque ......... & Wheeler. & 5,750 \\
\hline Polvadero Peak... & Wheeler. & 7,324 \\
\hline Ponil Pass $\ldots \ldots \ldots \ldots \ldots \ldots \ldots \ldots \ldots \ldots \ldots \ldots \ldots$ & Wheeler. & 348 \\
\hline Pope. & A., T. \& & 4,557 \\
\hline (n) & Wheeler. & 6,055 \\
\hline Pueblo Colorado... & Wheeler. & $6,368:$ \\
\hline 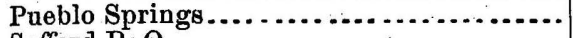 & Wheeler.. & 6,363 \\
\hline Safford P. $0 \ldots \ldots \ldots \ldots \ldots \ldots \ldots \ldots$ & Wheeler. & 2,712 \\
\hline (n. & Wheeler & 6,847 \\
\hline (n. & eler & 6,428 \\
\hline Puertocito Spring . & ler & 499 \\
\hline Punta del Agua............. & Wheeler. & $\begin{array}{l}6,599 \\
4,303\end{array}$ \\
\hline 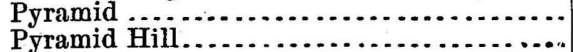 & $\begin{array}{l}\text { S. P. R. R } \\
\text { Wheeler. }\end{array}$ & $\begin{array}{l}4,303 \\
6,628\end{array}$ \\
\hline 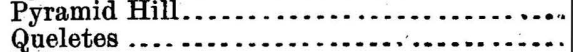 & Wheeler. & $\begin{array}{l}6,628 \\
5,193\end{array}$ \\
\hline 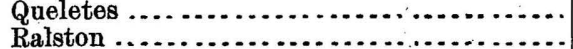 & Wheeler. & $\begin{array}{l}5,193 \\
4,488\end{array}$ \\
\hline 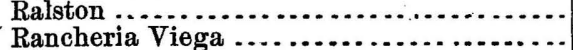 & Wheeler. & $\begin{array}{l}4,480 \\
7,300\end{array}$ \\
\hline $\begin{array}{l}\text { Rancheria Viega } . . . . . . . . . . . . \\
\text { Ranchos de Taos ............... }\end{array}$ & Wheeler. & 6,983 \\
\hline Randall. & A., T. \& S & 3,947 \\
\hline Raton $\ldots \ldots \ldots \ldots$ & A., T. \& S & 6,620 \\
\hline 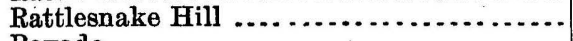 & Wheeler.. & 6,617 \\
\hline 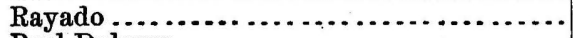 & & 6,946 \\
\hline Dolores ....................... & Wheeler. & 6,802 \\
\hline 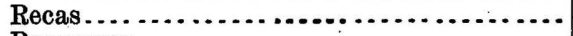 & A., T. \& S. & 7,049 \\
\hline Remances......$\ldots \ldots \ldots \ldots \ldots \ldots \ldots \ldots$ & Toner ....... & 6,185 \\
\hline Rincon $\ldots \ldots \ldots \ldots \ldots \ldots \ldots$ & A., T. \& S & 4,014 \\
\hline Rinconada $\ldots . . . \ldots \ldots \ldots \ldots \ldots \ldots$ & Wheeler... & 5,829 \\
\hline 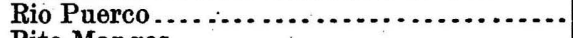 & A. \& P. R. & 5,026 \\
\hline 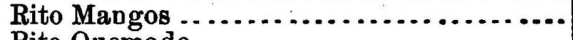 & Wheeler. & 7,319 \\
\hline 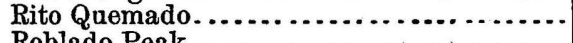 & Wheeler. & 6,827 \\
\hline $\begin{array}{l}\text { Roblado Peak } \\
\text { Rock Ranch }\end{array}$ & $\begin{array}{l}\text { Wheeler. } \\
\text { Wheeler. }\end{array}$ & $\begin{array}{l}5,575 \\
5,844\end{array}$ \\
\hline 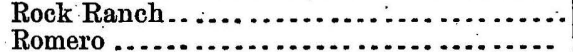 & $\begin{array}{l}\text { Wheeler.. } \\
\text { A., T. \& } \$\end{array}$ & $\begin{array}{l}5,844 \\
6,286\end{array}$ \\
\hline (n. & Wheeler. & \\
\hline . & S. P.R.R... & \\
\hline Rosario. & A., T. \& S. F. R. R.......... & 5,400 \\
\hline Sabinal & A., T. \& S. F. R. R. . . . . & 4,741 \\
\hline Do.......... & eeler................. & 5 \\
\hline Sabinal Agen & 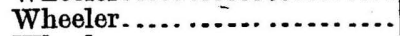 & 4,757 \\
\hline Salado & Wh & 6,321 \\
\hline$\ldots \ldots \ldots$ & Wheeler........ & 9,040 \\
\hline San Antonio. & A., T. \& S. F. R. R.......... & 4,517 \\
\hline Do. & R. R. Reports......... & 6,409 \\
\hline Do. & ler................... & 625 \\
\hline San Antonio Peak & ler................... & 10,912 \\
\hline$\ldots \ldots \ldots$ & $n \ldots \ldots . . . .$. & 10 , \\
\hline o Valley. & r. & \\
\hline San Aug & ............ & 302 \\
\hline$\ldots \ldots \ldots \ldots \ldots$ & $\ldots \ldots \ldots . . . . .$. & \\
\hline$\ldots \ldots \ldots \ldots \ldots \ldots$ & $\ldots \ldots \ldots \ldots$ & \\
\hline 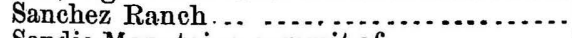 & $\ldots \ldots \ldots$ & \\
\hline ntains, summit of ....................... & Wheeler. & \\
\hline 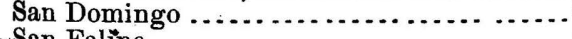 & & \\
\hline 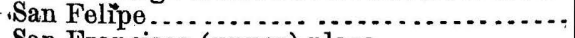 & Wheeler & \\
\hline San Francisco (upper) plaza ................... & Wheeler. & \\
\hline Do ........ (middle) plaza ........ & Wheeler. & \\
\hline . (lower) plaza .................... & Wheeler. & \\
\hline , on Rio Puerco....... & $\begin{array}{l}\text { Wheeler. } \\
\text { Wheeler. }\end{array}$ & \\
\hline$\ldots \ldots, \ldots, \ldots, \ldots$ & $\begin{array}{l}\text { Wheeler. } \\
\text { Wheeler. }\end{array}$ & \\
\hline an Ildefonso... & & 457 \\
\hline an & & 460 . \\
\hline
\end{tabular}




\begin{tabular}{|c|c|c|}
\hline Station. & Aut & Elevation. \\
\hline & & \\
\hline $\begin{array}{l}\text { an José....... } \\
\text { Do } \ldots . . . .\end{array}$ & $\begin{array}{l}\text { A. \& P. R. } \\
\text { Wheeler... }\end{array}$ & $\begin{array}{l}5,428 \\
5,648\end{array}$ \\
\hline an Juan & Wheeler... & 5,601 \\
\hline $\begin{array}{l}\text { Do...Pueblo } \\
\text { au Lorenzo .... }\end{array}$ & $\begin{array}{l}\text { Wheeler.. } \\
\text { Wheeler.. }\end{array}$ & $\begin{array}{l}5,554 \\
6,107\end{array}$ \\
\hline San Lorenzo Spring & Wheeler.. & 5,326 \\
\hline an Luis Rey.... & Wheeler. & 5,152 \\
\hline an Marcial.... & $\begin{array}{l}\text { A., T. } \text {. \& S. } \\
\text { Wheeler... }\end{array}$ & $\begin{array}{l}4,437 \\
4,385\end{array}$ \\
\hline an Marcos Sp & Wheeler. & 6,056 \\
\hline an Mat & Wheeler. & 7,323 \\
\hline $\begin{array}{l}\text { an Mateo Mountai } \\
\text { an Miguel }\end{array}$ & & $\begin{array}{r}10,209 \\
6,019\end{array}$ \\
\hline $\begin{array}{l}\text { San Miguel ......... } \\
\text { Sands................... }\end{array}$ & A., T. \& & $\begin{array}{l}0,019 \\
6,386\end{array}$ \\
\hline San Nicolas Spring & Wheeler.. & 4,218 \\
\hline$\ldots \ldots \ldots$ & Wheeler.. & 4,488 \\
\hline$\cdots$ & $\begin{array}{l}\text { Wheeler: } \\
\text { Wheeler: }\end{array}$ & $\begin{array}{l}6,509 \\
5,346\end{array}$ \\
\hline a...... & & \\
\hline a Moul & Wheeler ( & 11,50 \\
\hline $1 \mathrm{z} \ldots . .$. & & \\
\hline Santa Fé......... & Wheeler.. & 7,047 \\
\hline Signal $\mathrm{s}$ & $\begin{array}{l}\text { A., T. \& S } \\
\text { U.'S. Sign }\end{array}$ & $\begin{array}{l}6,937 \\
6,862\end{array}$ \\
\hline Santa FE Baldy P & wheeler. & 12,661 \\
\hline Santa & & 6,161 \\
\hline Santa I & $\cdots$ & 6,1 \\
\hline $\begin{array}{l}\text { Sant } \\
\text { Sant }\end{array}$ & $\begin{array}{l}\text { Mexi } \\
\text { Whee }\end{array}$ & $\begin{array}{l}6,106 \\
5,110\end{array}$ \\
\hline Sant & $\mathrm{W}$ & 7,41 \\
\hline Sapel & Wh & 6,876 \\
\hline & $\therefore \ldots \ldots \ldots . . .$. & \\
\hline nch..... & Wl & 6,69 \\
\hline ort ........ & F $\mathrm{R}, \ddot{\mathrm{R}}$ & $\begin{array}{l}3,90 \\
3,93\end{array}$ \\
\hline Sellers & A. & 4,49 \\
\hline Servil & & 6,7 \\
\hline & & \\
\hline ach, San & [............ & 4,3 \\
\hline ings ..... & & \\
\hline Camp (olc & $\mathbf{S} \mathbf{F} \mathrm{B}$ & 6,927 \\
\hline $\begin{array}{l}\text { Shoem } \\
\text { Silla. }\end{array}$ & $\mathbf{F}, \mathbf{R}$ & $\begin{array}{l}0,204 \\
6,677\end{array}$ \\
\hline Silver & F.R.R. & 5,771 \\
\hline Signal & 1 Office. & \\
\hline rings..... & & 7,6 \\
\hline Slocu & & \\
\hline 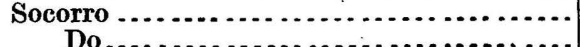 & S. F.R.R.......... & 4,5 \\
\hline Do................. & & $\begin{array}{l}4,659 \\
4,560\end{array}$ \\
\hline . Signal Sta & al Office....... & $\begin{array}{l}4,500 \\
4,565\end{array}$ \\
\hline Ford........ & & 4,5 \\
\hline & & \\
\hline Florida Peak & ..... & 7,261 \\
\hline Mountain & & 8,73 \\
\hline $\begin{array}{l}\text { andia Peak ..... } \\
\text { r .............. }\end{array}$ & S.F.R. R & $\begin{array}{l}0,004 \\
5,766\end{array}$ \\
\hline Fort (flagstaff) & eler............... & 6,151 \\
\hline Stiv & & \\
\hline Stone Ranch, on Canadian $\mathrm{F}$ & 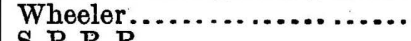 & \\
\hline $\begin{array}{l}\text { Strauss } \\
\text { Sublette }\end{array}$ & $\begin{array}{l}\text { S.P.R.R. } \\
\text { D.\&R.G. }\end{array}$ & $\begin{array}{l}4,085 \\
9,215\end{array}$ \\
\hline & $\begin{array}{l}\text { D. \& R. } \\
\text { A.T.\&. }\end{array}$ & $\begin{array}{l}9,210 \\
5,973\end{array}$ \\
\hline & & \\
\hline
\end{tabular}




\begin{tabular}{|c|c|c|}
\hline Station. & Authority. & Elevation. \\
\hline & & Feet. \\
\hline Sunday Peak .... & Wheeler & 6,030 \\
\hline 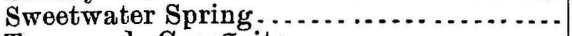 & Wheeler. & 6,343 \\
\hline 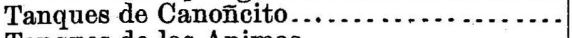 & Wheeler. & 5,083 \\
\hline 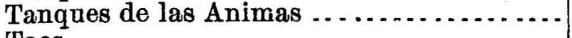 & Wheeler. & 6,404 \\
\hline 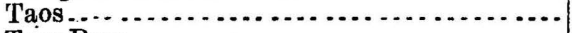 & Wheeler. & 6,983 \\
\hline 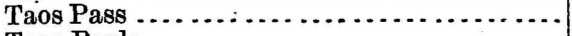 & Wheeler. & 9,282 \\
\hline 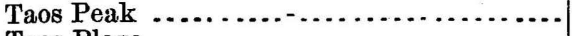 & Wheeler. & 13,145 \\
\hline 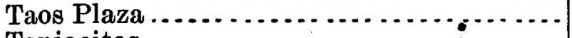 & Wheeler. & 6,949 \\
\hline . & Wheeler. & 8,870 \\
\hline Taylor, Mount $\ldots \ldots \ldots \ldots \ldots \ldots \ldots$ & Wheeler. & 11,391 \\
\hline Taylor's Ranch................... & Wheeler. & 7,226 \\
\hline Tecoloto $\ldots \ldots \ldots \ldots$ & Wheeler & 6,668 \\
\hline 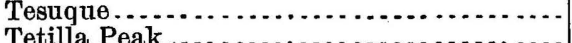 & $\cdots \ldots . .$. & 5,844 \\
\hline Tetilla Peak ............................ & gler.. & $\begin{array}{r}7,060 \\
11,975\end{array}$ \\
\hline 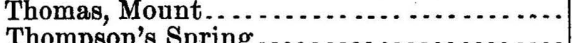 & Wheeler. & 11,275 \\
\hline Thompson's Spri & Wheeler... & 7, \\
\hline Thorn, Fort ......... & Med. Dept & $\begin{array}{l}4,500 \\
0,100\end{array}$ \\
\hline Thunder Peak & Wheeler... & 9,122 \\
\hline Tierra Amarilla...$\ldots \ldots \ldots \ldots \ldots \ldots$ & Wheeler & 7,466 \\
\hline$\ldots \ldots \ldots \ldots \ldots \ldots \ldots$ & Wheeler & 6,214 \\
\hline$\ldots \ldots \ldots \ldots \ldots \ldots \ldots \ldots \ldots$ & A., T. \& S & 6,363 \\
\hline 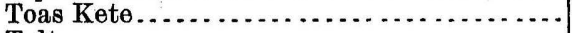 & Wheeler.... & 6,506 \\
\hline Toltec $\ldots \ldots \ldots \ldots \ldots \ldots$ & A. \& P. R. R & 9,443 \\
\hline Tomasceños Water Holes ................. . & Whieeler.. & 5,502 \\
\hline Tomè & Wheeler. & \\
\hline 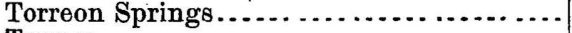 & Wheeler. & 5,980 \\
\hline 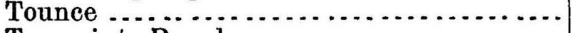 & ............. & 3,975 \\
\hline 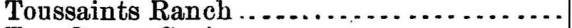 & $\ldots \ldots \ldots$. & 4,634 \\
\hline Tres Cerros Spring . . . . . . . . . . . . . . . . & $\ldots \ldots \ldots$ & 6,128 \\
\hline Tres Hermanos Peak .................. & Whe & 7,151 \\
\hline Tres Piedras.................... & D. \& R. G & 8,066 \\
\hline . . . . . & - & 5,725 \\
\hline$\ldots \ldots \ldots \ldots \ldots$ & Wheeler & 4,347 \\
\hline 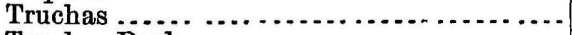 & Wheeler.. & 7,622 \\
\hline 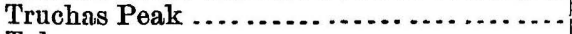 & Wheeler. & 13,150 \\
\hline Tularosa.$\ldots \ldots \ldots \ldots \ldots \ldots$ & Wheeler. & 4,344 \\
\hline 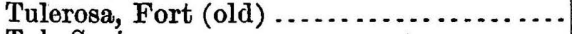 & $\ldots . . . . .$. & 6,740 \\
\hline 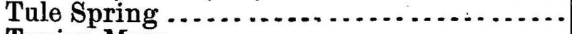 & ........... & 5,925 \\
\hline 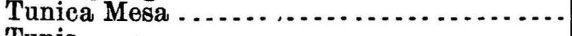 & Wheeler. & 5,510 \\
\hline Tunis & S.P.R.R. & 4,424 \\
\hline 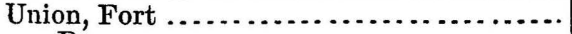 & Wheeler. & 6,711 \\
\hline & Med. Dept. U. & 6,670 \\
\hline United States Mountain . . . . . . . . . . . . . & Med. Dept. U. S. A........ & 10,734 \\
\hline Upham & A., T. \& S. F. R.R . . . . . . . & 4,537 \\
\hline Upper Abo Pass................... & Wheeler...................... & 431 \\
\hline Ute Peak ...... & Wheeler........ & 10,151 \\
\hline$\ldots \ldots \ldots \ldots \ldots \ldots \ldots \ldots \ldots$ & $\ldots . . . . .$. & \\
\hline - & A., T. \& S. F. R. R........... & 69 \\
\hline Van Brummer... & 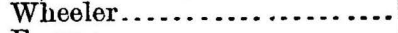 & 8,557 \\
\hline Vegas Village............................ & $\ldots \ldots \ldots$ & 6,418 \\
\hline 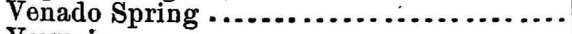 & $\ldots \ldots \ldots$ & 5,982 \\
\hline ....................... & $\ldots \ldots \ldots \ldots$ & \\
\hline 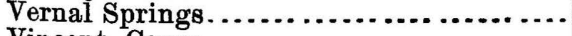 & ........... & 6,299 \\
\hline 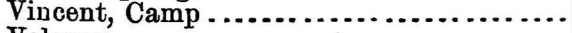 & Wheeler..- & 6,188 \\
\hline 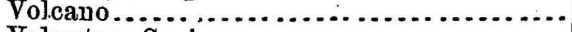 & D. \& R.G. I & 8,465 \\
\hline teer Spring.$\ldots \ldots \ldots \ldots \ldots \ldots \ldots$ & Wheeler............................. & 106 \\
\hline 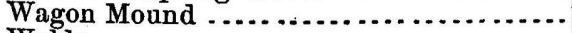 & A., T. \& S. F. R. R .......... & 6,176 \\
\hline 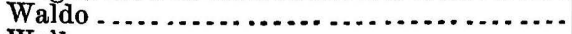 & A., T. \& S. F. R. R ............ & 604 \\
\hline 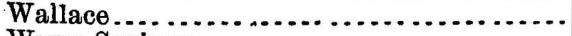 & A., T. \& S. F. R. R .......... & 246 \\
\hline 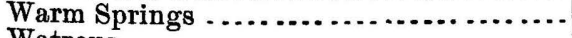 & Wheeler $\ldots \ldots \ldots \ldots$ & 5,008 \\
\hline Watrous $\ldots \ldots \ldots \ldots \ldots$ & 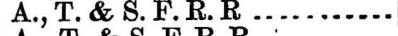 & 396 \\
\hline 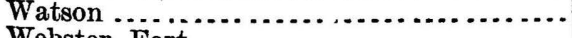 & A., T. \& S. F. R. R......... & 4,493 \\
\hline Webster, Fort West $_{\text {Gallinas }}$ & Med. Dept. U. S. A. ........... & $\begin{array}{l}6,350 \\
8,464\end{array}$ \\
\hline & & \\
\hline
\end{tabular}




\begin{tabular}{|c|c|c|}
\hline Station. & Authority. & Elevation. \\
\hline 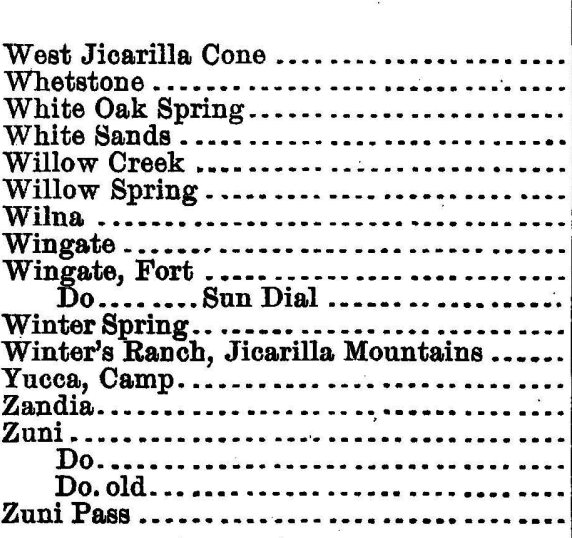 & 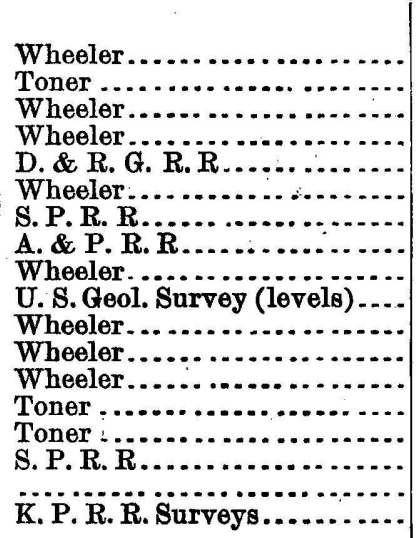 & $\begin{array}{r}\text { Feet. } \\
7,7277 \\
6,360 \\
6,618 \\
3,888 \\
7,720 \\
6,677 \\
4,559 \\
6,714 \\
7,038 \\
6,997 \\
7,182 \\
6,582 \\
4,374 \\
5,056 \\
6,355 \\
4,189 \\
7,414 \\
7,926\end{array}$ \\
\hline
\end{tabular}


NEW YORK.

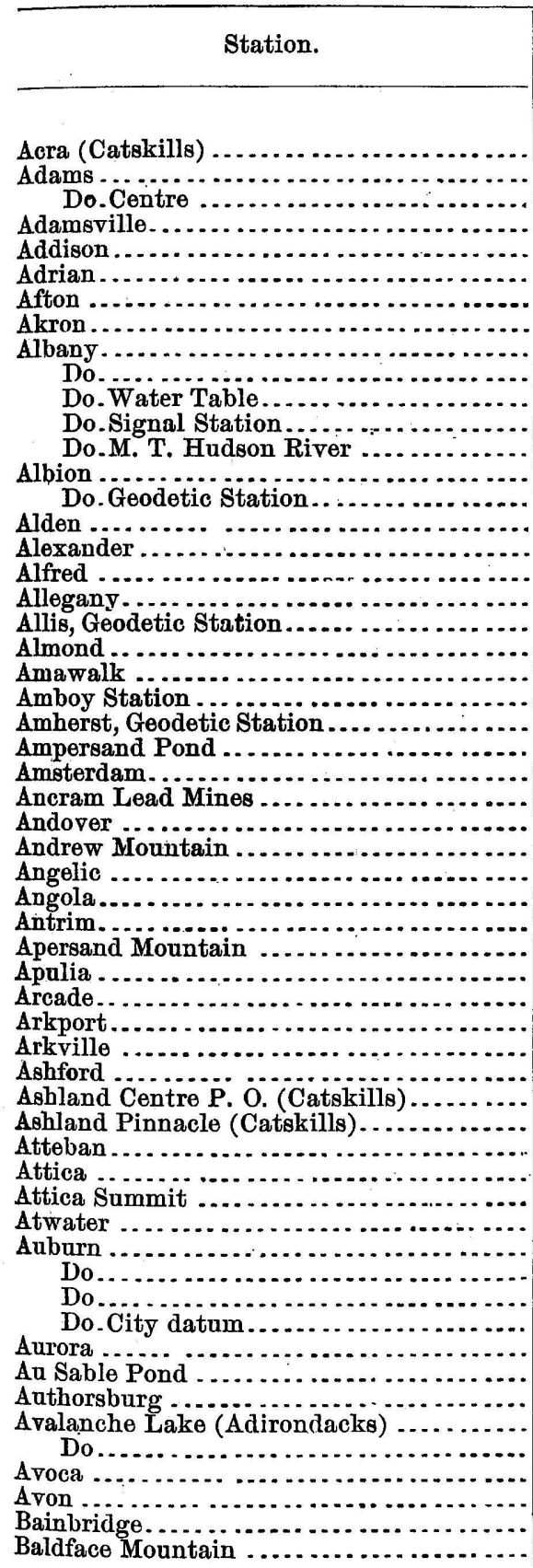

\begin{tabular}{|l|l}
\hline Authority. & Elevation. \\
\hline Feet
\end{tabular}

Appal. Club ................. $\quad 546$

R., W. \&, O. R. R............ 599

R., W. \& O.R. R........... 619

A. \& S. R. R ............... $\quad 212$

N. Y., L. E. \& W. R. R ....... 993

N. Y., L. E. \& W. R. R....... $\quad 1,112$

A. \& S. R. R ............. $\quad 979$

N. Y. C. \& H. R. R. R........ 765

N. Y. C. \& H. R. R. R........ $\quad 30$

A. \& S. R. R ............... 16

N. Y. C. \& H. R. R. R .......

U. S. Signal Office............ 75

U. S. C. \& G. S ............. 6

N. Y., C. \& H. R. R.......... 547

U. S. Lake Survey ............ 711

N. Y., L. E. \& W. R. R......... 864

N. Y., L. E. \& W. R. R....... 938

N. Y., L. E. \& W. R. R ....... $\quad 1,660$

N. Y., L. E. \& W. R. R ....... 1, 1,422

N. Y. State Survey ............ 503

N. Y., L. E. \& W. R. R...... $\quad 1,410$

N. Y. \& N. R. R ..............

N. Y. State Survey

U. S. Lake Survey

Geol. Survey of N. Y.

N. Y. C. \& H. R. R. R

P. H. \& B. R. R .

N. Y., L. E. \& W.R. R

Adirondack Survey

R. N. \& P. R. R

L. S. \& M. S. R. R.

C. C. \& A R, R

Adirondack Survey

S. \& B. R. R

B., N.Y. \& P. R. R

N. Y. L. E. \& W. R. R

U. \& D. R. R.

N. Y. \& N. R. R

Appal. Club.

Appal. Club

M., D. \& C. R. R

N. Y., L. E. \& W. R. R

N. Y., L. E. \& W. R. R

G., I. \& S. R. R ......

Southern Cent. R. R........

I., A. \& W. R. R..........

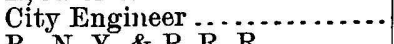

384

793

260

2,079

279

570

1,640

3,216

1,455

687

1,672

3,433

1,227

1,457

1, 199

1,342

139

1,435

3,420

451

998

1,086

394

674

666

639

673

B., N. Y. \& P. R. R........

N., D. \& C. R. R.

Adirondack Survey

Geol. Survey of N. Y.

N. Y., L. E. \& W. R. R

N. Y., L. E. \& W. R. R

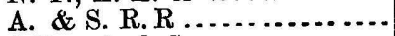

Adirondack Survey ...........

925

2,065

337

$2,846^{\circ}$

2,856

1,198

585

994

3,904 


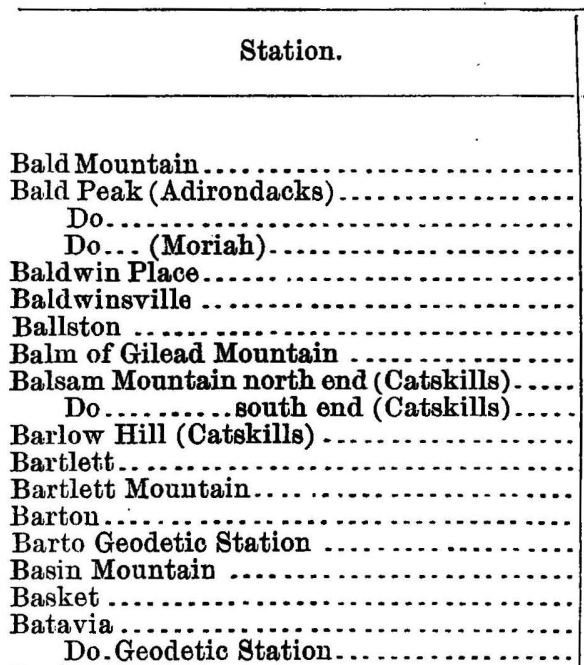

Bath.

Beach, Lake

Bear-pen Mountain, approx

Beaver Dam

Beaver Dams

Beaver Lake

Beaver Meadow Pond

Bedford

Beech Ridge Gap

Bee Line Mountain, approx

Belle Ayr Mountain

Bellona

Belvedere

BennetNotch

Bennett's Pond.

Bergen

Berkshire

Bessemers

Best Hill.

Bethel

Big Flats

Big Indian

Big Island

Big Westkill Mountain

Billings.

Binghams

Binghamton

Do.

Do ...

Do ........ Y. \& Erie Junction

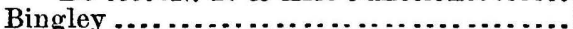

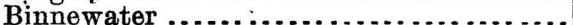

Birch Kill Notch .......................

Birds and Worms.

Black Dome

Black Head

Black River

Black Rock

Blanteville

Blodgett Mills

Bloods

Blosśburg .

Blue Mountain

Blue Mountain Lake

\begin{tabular}{|c|c|}
\hline Authority. & Elevation. \\
\hline 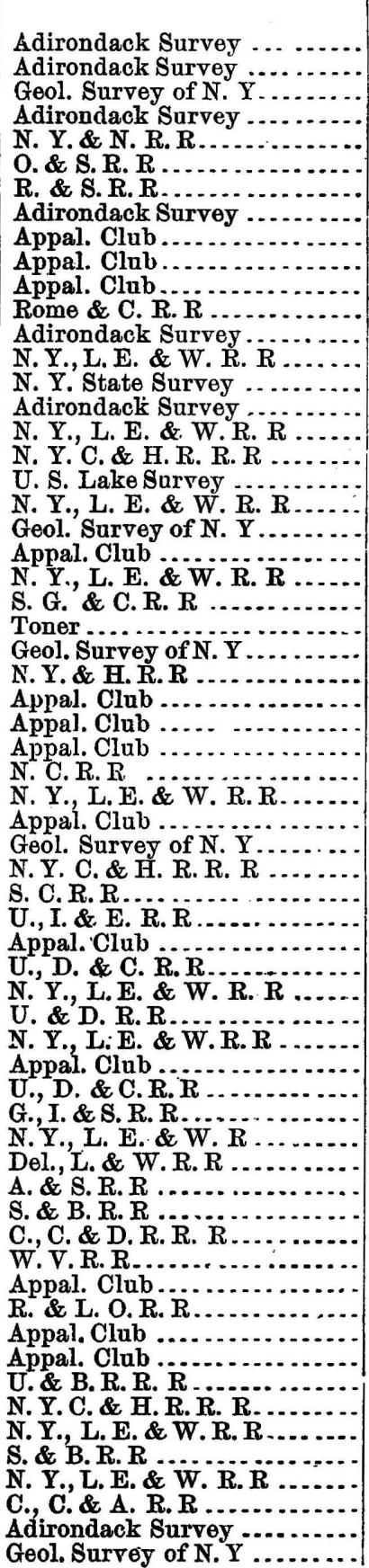 & 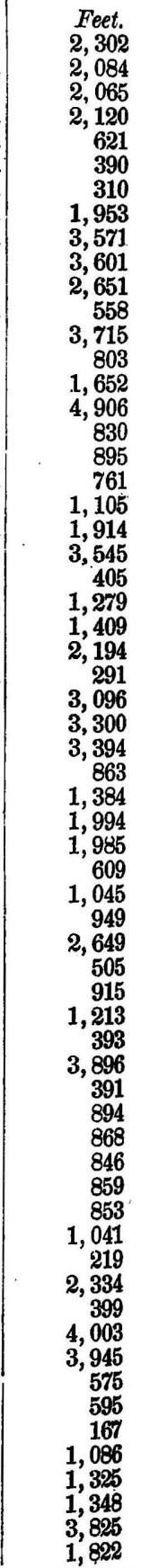 \\
\hline
\end{tabular}




\begin{tabular}{|c|c|c|}
\hline Station. & Authority. & Elevation. \\
\hline & & Feet. \\
\hline Bog Lake $\ldots \ldots \ldots$ & Geol. Survey o & 1,755 \\
\hline Boiceville $\ldots \ldots \ldots \ldots \ldots \ldots$ & U. \& D.R. R & 604 \\
\hline 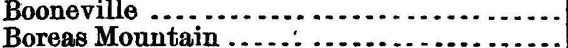 & U. \& B. R. R. F & 1,135 \\
\hline 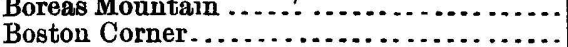 & $\begin{array}{l}\text { Geol. Survey of } \\
\text { P., H. \& B. R. }\end{array}$ & 3,726 \\
\hline Bouchon ........... & N.Y., L. E. \& V & 852 \\
\hline Breesport ... & U., I. \& E. R. & 1,097 \\
\hline$\ldots \ldots \ldots \ldots \ldots . . . \ldots \ldots$ & N.\& O. $\mathrm{R}$ & 384 \\
\hline 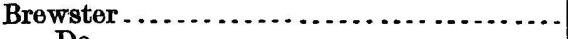 & \&. R. & 406 \\
\hline$\ldots \ldots \ldots . . . . . .$. & N. Y. \& H. R. & 414 \\
\hline Bridgewater & U. C. \& S. R. I & 1,188 \\
\hline Brier Hill .............. & U. \& B. R. R. & 276 \\
\hline Brinckerhoff $\ldots \ldots \ldots \ldots \ldots \ldots \ldots \ldots \ldots \ldots \ldots \ldots$ & U., D. C. R. R. & 223 \\
\hline $\begin{array}{l}\text { Brockport. } \\
\text { Do.. Geodetic Station }\end{array}$ & N. Y. C. \& H. F & 546 \\
\hline on $\ldots \ldots \ldots \ldots \ldots \ldots \ldots$ & $\begin{array}{l}\text { U. S. Lake Su } \\
\text { L., S. \& M. S. }\end{array}$ & $\begin{array}{l}731 \\
689\end{array}$ \\
\hline Brodhead's Bridge. & U. \& D. R. R & 504 \\
\hline 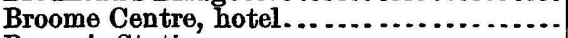 & 1. Club. & 1,973 \\
\hline Brown's Station .... & . R. R. & 530 \\
\hline Bucktooth ........ & A. \& G. W. R.R.. & 1,376 \\
\hline Buffalo $\ldots \ldots \ldots$. & N. Y., L. E. \& W. & 588 \\
\hline Do... Exchange street $\ldots \ldots \ldots \ldots$ & N. Y. C. \& H. R & 584 \\
\hline 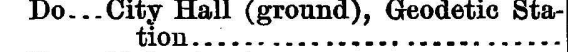 & & 609 \\
\hline 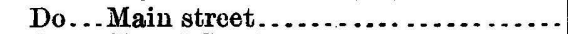 & L. E. \& & 630 \\
\hline Do... Signal Stati & Signal Offic & 664 \\
\hline Buffalo Plains, Geodetic Station ........... & ake Sur & 685 \\
\hline Bulger, Geodetic Station .................. & N. Y & 833 \\
\hline Bulwagga Mountain ....................... & Geol. & 1,260 \\
\hline Burns $\ldots \ldots \ldots \ldots \ldots \ldots \ldots \ldots \ldots \ldots$ & E. \& W. & 1,203 \\
\hline Burnt Mountain (Adirondacks) ........... & Adirondack Surv & 2,085 \\
\hline 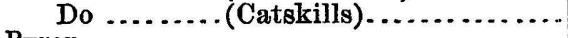 & Appa & 3,170 \\
\hline Byron $\ldots \ldots$ & C. \& H. & 695 \\
\hline Cade Mountain (Catskills)................. & 1. Clab .... & 2,390 \\
\hline Cadyville $\ldots \ldots \ldots \ldots \ldots \ldots \ldots$ & Chat. R.R ... & 732 \\
\hline 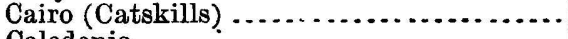 & Appal. Club .... & 346 \\
\hline 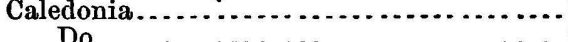 & N. Y. C. \& H. R. & 658 \\
\hline Callicoon $\ldots$ & 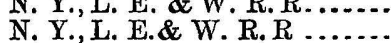 & $\begin{array}{l}667 \\
781\end{array}$ \\
\hline mden........... & R. W. \& O. R. R...... & 520 \\
\hline Camel's Hump..... & Adirondack Surv & 3,548 \\
\hline Cameron & N. Y., L. N. \& W. R. R. & 1,056 \\
\hline eron Mills ... & N. Y., L. E. \& W. R. R. & 1,029 \\
\hline ell ........ & N. Y., L. E. \& & 1,014 \\
\hline Campbell Hall ... & N.Y., L.E. \& V & 396 \\
\hline Camprille ....... & N. Y., L. E. \& W. & 830 \\
\hline . & Boston \& Albany R. R....... & 869 \\
\hline (n) & & 740 \\
\hline ga ...... & N. Y & 418 \\
\hline$\ldots \ldots$ & ., W.S. \& B. R. R ... & 426 \\
\hline Candor .. & $\mathbf{L}, \mathbf{R}$ & 822 \\
\hline Canisteo ......... & R. $\mathbf{R} \ldots$. & 1,134 \\
\hline$\ldots \ldots \ldots \ldots \ldots \ldots$ & R. W.\&. O.R & 253 \\
\hline Carmel ........... & \& N.R.R. & 519 \\
\hline$\ldots \ldots \ldots \ldots \ldots \ldots \ldots$ & $\ldots \ldots . . . .$. & 968 \\
\hline 1ter, Geodetic Station. . . . . . . . . . . & N. & 1,105 \\
\hline Carrollton ........ & ., L. E. \& W. & 1,399 \\
\hline Carthage ... & U. \& B. R. R. R .. & 740 \\
\hline 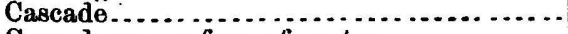 & S. C.R.R R & 724 \\
\hline Cassadaga, surface of water.......... & D., A. V.\& P. R. R . . . . & 1,309 \\
\hline $\begin{array}{l}\text { Cassville } \\
\text { Do Junction }\end{array}$ & $\begin{array}{l}\text { U. C. \& S. R. R.... } \\
\text { U. C. \& S. R. R...... }\end{array}$ & $\begin{array}{l}1,215 \\
1,179\end{array}$ \\
\hline Castile.......... & \& W. R.R... & 1,401 \\
\hline Do..summit . & & 1,431 \\
\hline
\end{tabular}




\begin{tabular}{|c|c|c|}
\hline Sta & Authority. & Elevatien. \\
\hline astleton. & U.S.C. \& G. S & Feel, \\
\hline Do...Boulo & 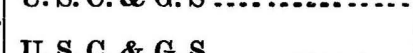 & \\
\hline astor Land & $\begin{array}{l}\text { U.S.C.\&G.S... } \\
\text { U.\&B.R.R.R .. }\end{array}$ & $\begin{array}{r}21 \\
745\end{array}$ \\
\hline & S. C. R. R ........... & 423 \\
\hline tskill Lakes & Appal. Club ....... & $\begin{array}{l}2,140 \\
2,225\end{array}$ \\
\hline $\begin{array}{l}\text { atskill Moun } \\
\text { attaraugus }\end{array}$ & $\begin{array}{l}\text { Appal. Club } \\
\text { N.Y., L. E. \& W. R. R }\end{array}$ & $\begin{array}{l}2,225 \\
1,411\end{array}$ \\
\hline upuga ......... & N.Y.C.\& H.R.R.R. & 388 \\
\hline Lake, $187 \varepsilon$ & G. I. \& S.R.R R & 383 \\
\hline 10via & C., C.\& D.R.R. R & 1,176 \\
\hline Do..... & S. \& C.V.R.R.:. & 1,191 \\
\hline Do .....Lak & Taner $\ldots . . . . . . .$. & 900 \\
\hline edar Lake . . & $\begin{array}{l}\text { Geol. Survey of N } \\
\text { Adirondack Surv }\end{array}$ & $\begin{array}{l}2,530 \\
2,493\end{array}$ \\
\hline edar River $\mathrm{S}$ & $\begin{array}{l}\text { Adrondack Surv } \\
\text { Adirondack . Sury }\end{array}$ & $\begin{array}{l}2,493 \\
1,670\end{array}$ \\
\hline & N. Y., L. E. \& W. & $\begin{array}{r}1,000 \\
476\end{array}$ \\
\hline & N.Y.C. \& H. R. R & 324 \\
\hline $110 .$. & A. \& S. R. R.... & 964 \\
\hline nds & Adirondac & 1,707 \\
\hline 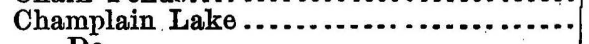 & Adir & 91 \\
\hline & R. R. Rep & 101 \\
\hline & N. Y. Stat & 1,280 \\
\hline Pond & $\begin{array}{l}\text { Adirondac } \\
\text { N. Y. C. \& }\end{array}$ & $\begin{array}{l}1,687 \\
255\end{array}$ \\
\hline Tum & N. Y. C. & 523 \\
\hline 1 & & 1,291 \\
\hline & on \& & 462 \\
\hline nter & Boston \& & 315 \\
\hline & R., W. \& & 294 \\
\hline & N.'Y., L. E. \& W. & 66. \\
\hline & N. Y., L. E. \& W. & 817 \\
\hline & U., I. \& E. R. R.. & 902 \\
\hline C & U., C. \& S. R. R. & 901 \\
\hline alley. & U. \& S. R. R ..... & 1,321 \\
\hline & N. Y., L. E & 459 \\
\hline Ridg $\theta$, & N. Y. Stat & 498 \\
\hline$=-$ & U., I. \& ] & 1,169 \\
\hline & N.Y. Y. \& & 417 \\
\hline Falls : & C., C. \& ] & 1,051 \\
\hline & N.Y. Y. & 570 \\
\hline & N. Y & 643 \\
\hline ls...... & & 528 \\
\hline & & 232 \\
\hline & & 2,006 \\
\hline 1... & Ac & 1,655 \\
\hline & & 418 \\
\hline & & 583 \\
\hline Corners.. & & 288 \\
\hline & R.R.R. R. & 637 \\
\hline & N., & 289 \\
\hline (atskills)... & & 2,372 \\
\hline ation. & $\begin{array}{l}\text { N. Y. C. } \\
\text { U.S. Lak }\end{array}$ & $\begin{array}{l}396 \\
642\end{array}$ \\
\hline Geodetic Station...... & $\begin{array}{l}\text { U. S. Lak } \\
\text { N. Y. Sta }\end{array}$ & $\begin{array}{l}642 \\
633\end{array}$ \\
\hline & B. C. \& P & 1,146 \\
\hline & Adirondacl & 1,936 \\
\hline & A.\& S. R. R............... & 903 \\
\hline & N. Y., L.E.\& W.R.R........ & 748 \\
\hline & 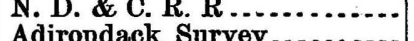 & \\
\hline $\begin{array}{r}\text { Colden, Lake .... } \\
\text { Do.............. }\end{array}$ & $\begin{array}{l}\text { Adirondack Survey } . . . \\
\text { Guyot........................... }\end{array}$ & $\begin{array}{l}2,748 \\
2,786\end{array}$ \\
\hline & Adirondack Survey ............ & 4,708 \\
\hline & & \\
\hline
\end{tabular}




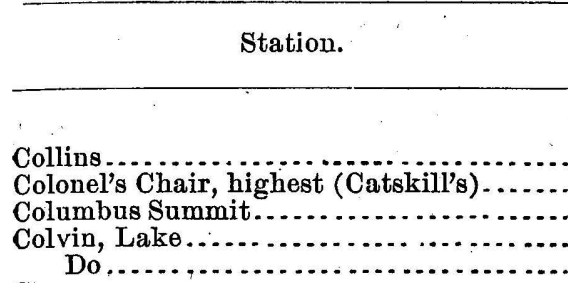

Conesus

Conewango Creek

Conklin

Conneaut, Geodetic Station.

Cooksburg (Catskill)

Coopers .

Corbettsville

Corfu ....................................

Coryell Mountain (Catskills)

Corning.

Cornwall

Cornwallville (Catskills)

Cortland Geodetic Station

Covert

Crafts

Craigville

Crain's Mills

Crain's Mountain

$$
\text { Do. }
$$

Cranberry Creek

Cranberry Lake

Crane's Village

Cranson, Geodetic Station

Crittenden

Crooked Lake.

Croton Falls

Croton Lake

Crystal Lake

Cuba

Cuba Summit

Canastota, Geodetic Station

Curtis

Cuyler

Cuylerville.

Dale

Dannemora

Dansville

Darien

Davison, Geodetic Station

Dayton

Deep Hollow Mountain (Catskills)

Deer Park Summit

Delong Mountain (Catskills)

Delphi .

Deposit

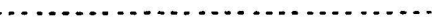

De Ruyter

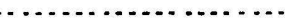

Devil's Ear Mountain

Dewiti.

$$
\text { Do.. Centre }
$$

Dickinson

Discovery Mountain Do

Dix's Peak Do

Dobb's Ferry

Dolbey's

Doubletop (Catskills)
Authority.

Elevation.

A. \& S. R. R

Appal. Club.

N. Y., K. \& S. R. R.

Geol. Survey of N. Y

Adirondack Survey:

N. Y., L. E. \& W. R. R

B. \& S. W. R. R..

Del., L. \& W. R. R

U. S. Lake Survey

Appal. Club

N.Y., L.E. \& W. R. R

Del., I. \& W. R.R...

N. Y., C. \& H. R. R. R

Appal. Club

N.Y., L. E. \& W.R.R

N. Y., L. E. \& W. R. R

Appal. Club ..

U., I. \& E. R. R .

N. Y. State Survey

G. I. \& S. R. R

N.Y. C. \& N. R. R

N. Y., L. E. \& W. R. R

C. C. \& De R:R.R

Adirondack Survey

Adirondack Survey

F. J. \& G. R. R

Toner

N. Y. C. \& H. R. R. R

N. Y. State Survey

N. Y. C. \& H: R. R. R

Geol. Survey of N. Y

N. Y. \& H. R. R

N. Y., B. \& M. R. R

Geol. Survey of N. Y.

N. Y., L. E. \& W. R. R

N. Y., L. E. \& W. R. R

N. Y. State Survey ..

N. Y., L. E. \& W. R. R

C. C. \& De R.R. R ..

N. Y., L. E. \& W.R.R

N. Y., L. E. \& W. R. R.

Chat. R. R

N. Y., L. E. \& W. R. R

N. Y., L. E. \& W. R. R

N. Y. State Survey ...

N. Y., L. E. \& W. R. R

Appal. Club.

N. Y., L. E. \& W. R. R

Appal. Club.

C. C. \& De R.R.R. .

N. Y., L. E. \& W. R. R

N. Y. State Survey

C. C. \& De R. R. R .

Adirondack Survey

N. Y. C. \& H. R. R. R.

$\mathrm{S} \& \mathrm{C}, \mathrm{V}, \mathrm{R}$

N.Y., L. E. \& W. R. R

Adirondack Survey

Adirondack Survey

Adirondack Survey

U. S. C. \& G. S

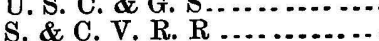

Appal. Club
Feet.

1,118

3,165

1,553

1,991

4,142

1,280

1,270

852

882

756

970

855

863

3,681

942

280

950

1,116

1,020

853

482

455

1,172

3,253

3,289

759

1,570

270

1,321

848

2,022

356

167

1,663

1, 542

1,698

588

997

1,225

528

1,190

1,356

691

1,024

631

1,346

3,500

901

2,540

1, 309

1, 009

832

1,276

3,904

417

410

954

1,583

1,376

4,916

5,200

12

408

3,875 


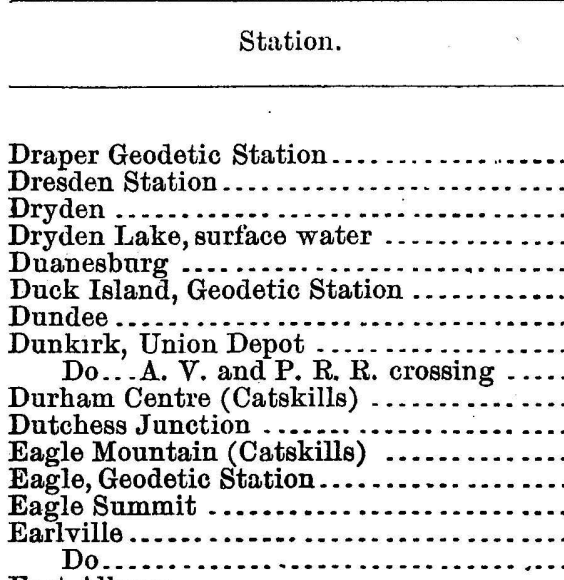

East Albany

East Bloomfield

East Buffalo .

East Chatham

East Clarion

East Creek

East Tarrytown

East Hill, Geodetic Station

East Homer

East Jewett (Catskills)

East Kill Mountain (Catskills)

East Pembroke

East River

East Sing Sing

East Waverly

Eaton, Geodetic Station

Eaton ...

Ebenezer

Eckford Lake

Eden

Edwards

Elk Lake

Elkland

Enlicottville.

Do .... . water surface, Great Valley Creek

Elma

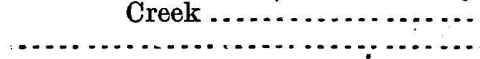

Elmira.........................

Emmons ............................

Do Mount

Do

Ephrata, Geodetic Station

Erie, Lake, water surface

Erieville.

Erin

Erwin Centre

Erwin's

Erwin's Mill

Esperance

Etna.

Evergreen Mountain, approx. (Catskills)

Fabius, Geodetic Station

Failing, Geodetic Station .................

Fairfield Academy, R. R. bench at labora-

tory, Geodetic Station .................

Fair Ground

Fairmount, Geodetic Station

Authority.

N:Y. State Survey ...........

S., G. \& C. R. R . ............

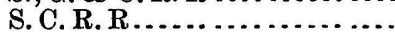

S. C. R. R

A. \& S. R. R

U. S. Lake Survey.

S., G. \& C. R. R .

L. S. \& M. S. R. R.

B. \& S. W. R. R

Appal. Club

N., D. \& C. R. R

N., D. \& C. R. R .

N. Y. State Survey

R. \& St. L. R. R...

U., C. \& S. V.R. R

S. \& C. V.R. R

N. Y. C. \& H. R. R. R

N. Y. C. \& H. R. R. R

N. Y., L. E. \& W. R. R

Boston \& Albany R. R

N. Y., R. \& P. R. R.

N. Y. C. \& H.R.R. R

N. Y. C. \& N: R. R

N. Y. State Survey

C., C. \& De R. R. R

Appal. Club ......

N.Y. C. \& H. R. R. R.

C., C. \& De R.R.R

N.Y.C. \& N.R.R

G., I. \& S. R. R.

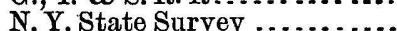

N. Y. \& O. R. R .

B., N. Y. \& P.R. R

Toner.

B. \& S.W.R.R.

Boston \& Albany R. R

Geol. Survey of N. Y

Corning $C$ \& $A$, R R

$R$ \& $S$. $R$

R. \& S. L. R. R

B. N. Y \& $\mathrm{P}$

N'Y Y E \& W R R

A. \& S. R. R . . . . . . . . . .

Geol. Survey of N. Y

Adirondack Survey

N. Y. State Survey .

U. S. Lake Survey

S. \& C. V.R. R .

U., I. \& E. R. R

Corning, C. \& A. R. R

N. Y., L. E. \& W. R. R

N. Y., L. E. \& W.R. R

A. \& S. R. R

U., I. \& E. R. R

Appal. Club

N. Y. State Survey

N. Y. State Survey

N. Y. State Survey

N. Y., L. E. \& W. R. R

N. Y. State Survey..........
Foat.

Elevation.

515

1,079

1,160

793

27

990

598

1,260

850

560

1,253

1,916

1,125

1,071

23

883

607

691

1,463

334

305

2,300

1,135

3,146

3,190

885

1,120

246

806

1,315

1,227

640

1, 791

810

939

2,053

1,142

1,560

1, 548

827

863

1,127

1, 000

3,596

1,029

573

1,577

1,249

976

983

1. 409

769

1,010

3,624

2,020

821

1, 281

540

736 


\begin{tabular}{|c|c|c|}
\hline Station. & Authority. & Elevation. \\
\hline & & \\
\hline Fairport ............. & $\begin{array}{l}\text { N. Y. C. \& H. R. R. } \\
\text { D., A. V.\& P.R.R }\end{array}$ & $\begin{array}{r}456 \\
1,258\end{array}$ \\
\hline 'alkirk, Geodetic Statio & U. S. Lake Survey . & 843 \\
\hline Fall Brook . . & Corning, $\mathrm{F} . \& \mathrm{~A}$. & 1,842 \\
\hline Farmer & L. S. \& M. S. R. R & $\begin{array}{l}860 \\
623\end{array}$ \\
\hline Fayetteville ..... & S.\& C.V.R. R . & 538 \\
\hline Mills $\ldots \ldots \ldots \ldots$ & U. \& B. R.R.R. & \\
\hline Fenner, Geodetic Station & N. Y. State Surve & 1,862 \\
\hline Fentonville $\ldots \ldots \ldots \ldots$ & D., A. V. \& P. R. & 1,243 \\
\hline $\begin{array}{l}\text { Fishkill.... } \\
\text { Flemingvilie }\end{array}$ & N., D. \& C. R. R & 213 \\
\hline 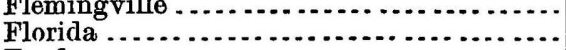 & N. Y., L. E.\&W & $\begin{array}{l}907 \\
404\end{array}$ \\
\hline & N. Y.C. \& H. R. & $\begin{array}{l}404 \\
299\end{array}$ \\
\hline Forestville........ & N. Y., L. E. \& W & 883 \\
\hline Lake............... & Toner & 1,704 \\
\hline ward ................. & R. \& S. R. R ... & 141 \\
\hline vix......... & $\begin{array}{l}\text { N.Y.C. \& H. R. } \\
\text { U.S.C.\&G.S }\end{array}$ & $\begin{array}{r}311 \\
9\end{array}$ \\
\hline ix .... & U. \& D.R.R ....... & $\begin{array}{r}9 \\
1,004\end{array}$ \\
\hline Frankfort. & N. Y. C. \& H. R. & 402 \\
\hline immit $\ldots . .$. & N. Y. \& O.R.R. & 1,764 \\
\hline ton (Catskills) . & Appal. Club $\ldots$ & 1,260 \\
\hline $\begin{array}{l}\text { Fra } \\
\text { Frec }\end{array}$ & $\begin{array}{l}\text { B., N. Y. \& P. R. } \\
\text { D., A. V. \& P. R. }\end{array}$ & $\begin{array}{r}1,593 \\
765\end{array}$ \\
\hline U., I. \& E.R. & 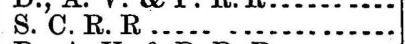 & 1,049 \\
\hline Frewsburg ................. & D., A. V.\& P. R. R & 1,261 \\
\hline ship $\ldots \ldots$ & N.'Y., L. E. \& W. & $\begin{array}{l}1,539 \\
387\end{array}$ \\
\hline ham Church (Cats) & $\begin{array}{l}\text { O. \& S. R. R .... } \\
\text { Appal. Club ..... }\end{array}$ & $\begin{array}{l}387 \\
714\end{array}$ \\
\hline lle $\ldots \ldots \ldots \ldots . . . . .$. & N. Y,, L. E. \& W. R. R & 1,407 \\
\hline Gainsville Summit .... & R. \& S. L. R. R ....... & 1,636 \\
\hline (3) & $\begin{array}{l}\text { W. V.R.R.R. R. } \\
\text { N. Y. L. F, \& W. R }\end{array}$ & $\begin{array}{r}311 \\
1983\end{array}$ \\
\hline $\begin{array}{c}\text { Garwoodd's } \\
\text { Gasport } \ldots \ldots\end{array}$ & N. Y.C. \& H. R. R. R. & $\begin{array}{r}1,283 \\
525\end{array}$ \\
\hline Do Geodetic Station ..... & U. S. Lake Survey..... & $\begin{array}{l}5 \% D \\
648\end{array}$ \\
\hline d's ................ & N.Y., L. E. \& & 784 \\
\hline - & N.Y., L. E.\& W. R & 1,526 \\
\hline Genesee Bridge & N. Y., L. E.\& W. R. R. & 548 \\
\hline $\begin{array}{l}\text { Geneseo Valley Junction } \\
\text { Genesseo ................. }\end{array}$ & $\begin{array}{l}\text { N.Y.C.\&.H.R.R.R... } \\
\text { N.Y.,L.E.\& W.R.R.. }\end{array}$ & $\begin{array}{l}543 \\
600\end{array}$ \\
\hline $\begin{array}{l}\text { Genesseo } \ldots \\
\text { Genera }\end{array}$ & N. Y.C.\& H.R.R.R... & $\begin{array}{l}600 \\
459\end{array}$ \\
\hline ake............ & Tone & 324 \\
\hline 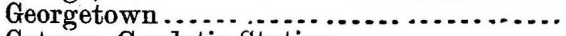 & S. \& C.V.R.R...... & 1,450 \\
\hline etic Station & N. Y. State Survey & 994 \\
\hline alley $\ldots . .$. & Adiro & 4,530 \\
\hline odetic St & N.Y. & 521 \\
\hline (atskills). & Appa & 1,036 \\
\hline tic Station & N.Y. S & 1,265 \\
\hline …..... & U. \&. I & 780 \\
\hline Lake & N. Y., & 50 \\
\hline & N., D.\& C.R.R. & 213 \\
\hline -... & Adir. R. R. & 712 \\
\hline 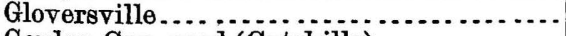 & F., J. & \\
\hline road (Catskills) ...... & $\operatorname{lub}_{10}$ & 2,504 \\
\hline (Catskills).... & Appal. Club \& $\mathrm{W}, \mathrm{R}$ & $\begin{array}{r}2,629 \\
431\end{array}$ \\
\hline$\ldots$. & $\begin{array}{l}\text { N. Y., L. E. \& W.R. R... } \\
\text { Adirondack Survey .... }\end{array}$ & 4,744 \\
\hline & 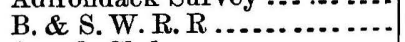 & 776 \\
\hline Mountain, Dry Brook ..... & Appal. Club .......... & 3,886 \\
\hline$\ldots \ldots \ldots \ldots \ldots$ & O.\& S.R.R.............. & $\begin{array}{r}390 \\
1574\end{array}$ \\
\hline & U.\& D. R. R..... & $\begin{array}{r}1,574 \\
420\end{array}$ \\
\hline $\begin{array}{l}\text { Grandview, Geode } \\
\text { Graves Mountain }\end{array}$ & $\begin{array}{l}\text { N.Y. State Survey } \\
\text { Adirondack Survey................... }\end{array}$ & 2,345 \\
\hline
\end{tabular}




\begin{tabular}{|c|c|c|}
\hline & & \\
\hline 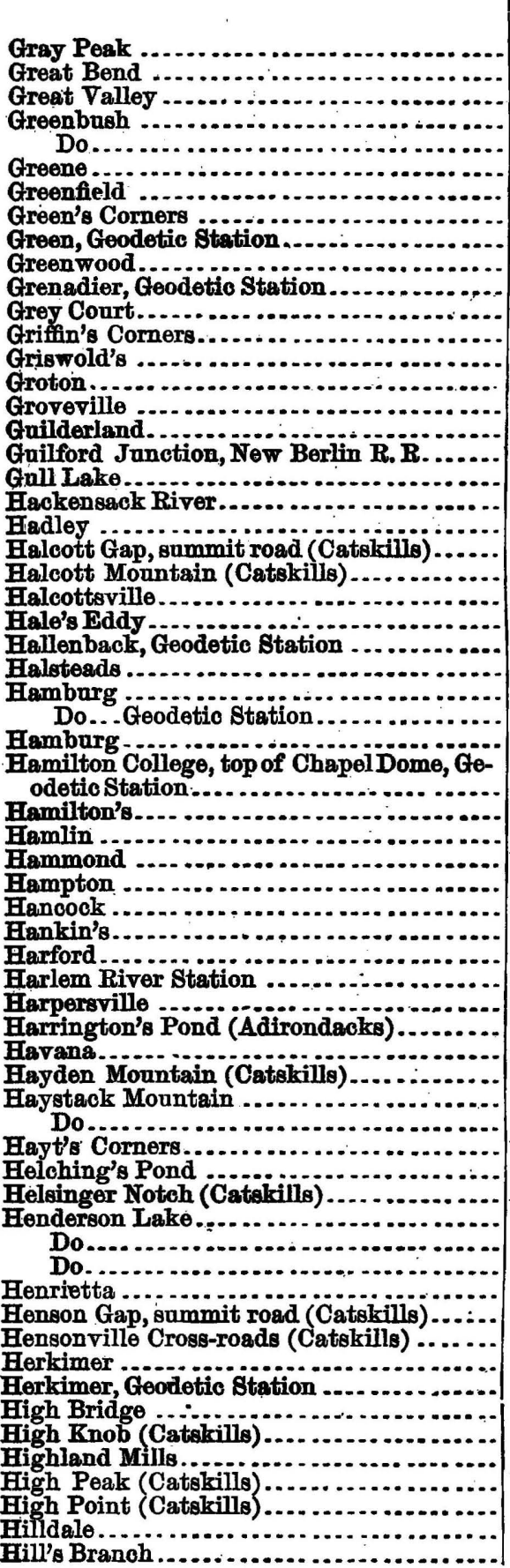 & 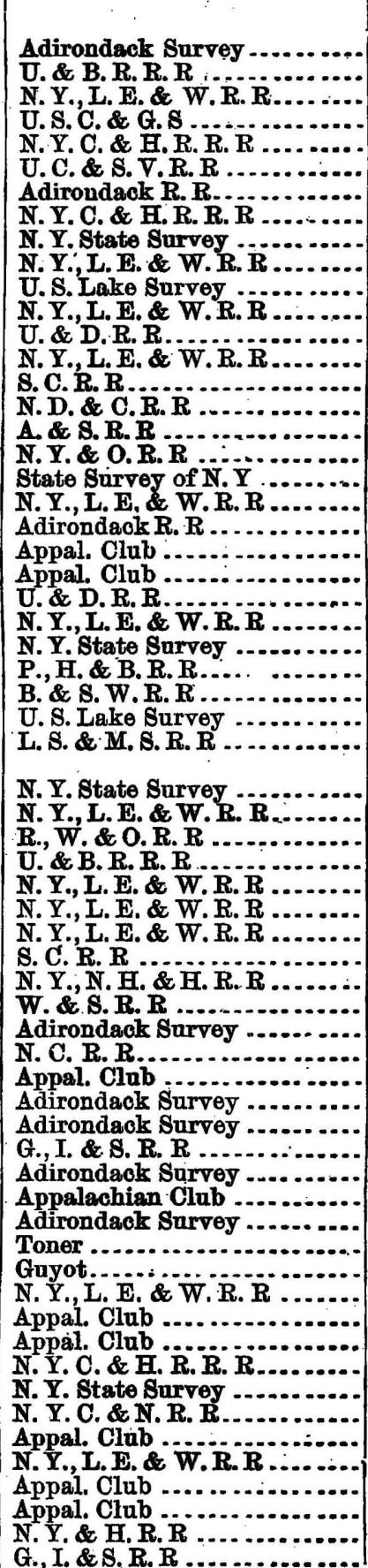 & 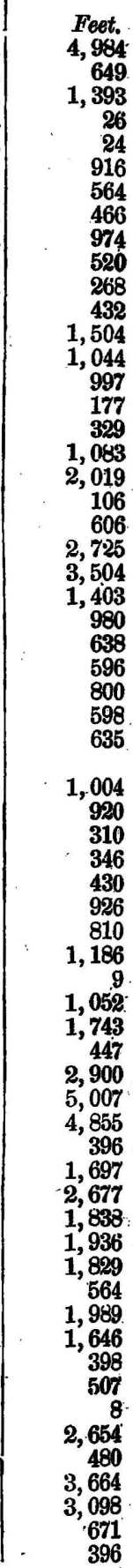 \\
\hline
\end{tabular}




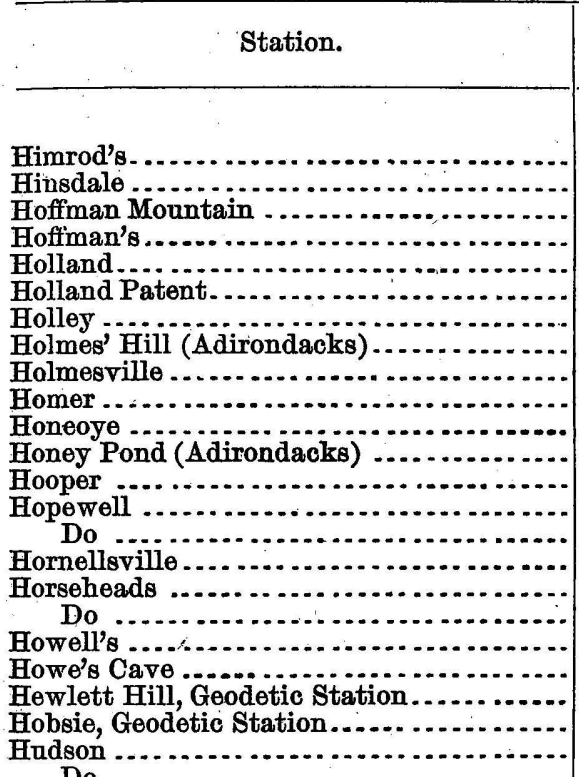

Do

Hunter Mountain (Catskills).

Hunter Village, Rusk's Catskills

Hunt's

Hurricane Mountain

Husteds

Hyndsyille.

Ilion.

Ilion, Geodetic Station

Indian Head, East peak (Catskills)

Do....... Middl $\theta$ peak (Catskills)

Do ....... West peak (Catskills).

Indian Lake (Adirondacks).

Indian Pass (Adirondacks).

Do..... (Catskills)

Iodus, Geodetic Station.

Iron Works (Adirondacks)

Irving

Ischua

Ithaca

Do

Jackson, Geodetic Station

Jamestown

Jamieson

Jessup's Landing

Johnston

Jordan

Kasvay Summit ..........................

Kelley's Corner

Kennedy

Kimball Mountain (Catskills)

Kinderhook

King's

King's Bridge

King's Ferry

Kingsley, Geodetic Station

Kingston

Kingston

Kirkland
N. C. R.R. . . Y. L. E. \& W. R

Adirondack Survey

N. Y., C. \& H. R. R. R

B., N. Y. \& P. R. R

U. \& B. R. R. R

N. Y., C. \& H. R. R. R

Adirondack Survey

R. W. \& O. R. R ..

S. \& B. R. R

N. Y., C. \& H. R. R. R

Adirondack Survey

N. Y., L. E. \& W. R. R

N. C. R. R

N., D. \& C. R.R

N.Y., L. E. \& W. R. R

N. C.R. R

U., I. \& E. R. R

N. Y., L. E. \& W. R. R

A. \& S. R. R.

Gardiner

Gardiner

U.S. C. \& G. S

N. Y. C. \& H. R. R. R

Appal. Club

Appal. Club

N.Y., L. E. \& W. R. R

Adirondack Survey

N., D. \& C. R. R.

A. \& S. R. R.

N. Y.C. \& H. R. R. R

N. Y. State Survoy

Appal. Club

Appal. Club

Appal. Club

Adirondack Survey

Adirondack Survey

Appal. Club

U. S. Lake Survey

Adirondack Survey

L. S. \& M. S. R. R

B., N. Y. \& P. R. R

G., I. \& S. R. R

C. \& S. R. R.

U., I. \& E. R. R .

N. Y. State Survey

A. \& G. W. R. R...

B., N. Y. \& P. R. R.

S. \& B. R. R .....

Adirondack R. R

N. Y. C. \& H. R. R. R

R. W. \& O.R.R.

U. \& D. R R

A. \& G. W. R. R.

Appal. Club.

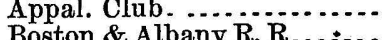

Adiron \& Albany R. R.......

N. Y. C. \& N. R.R ..........

G., I. \& S. R. R...............

N. Y. State Survey

W. V.R. R........................

U. \& D. R. R.....................

Rome \& C. R. R............
Elevation.

Feet.

799

1,501

3,728

266

1,176

$630^{\circ}$

537

2, 086 .

320

1,131

777

1,644

$839^{\prime}$

$850^{\prime}$

252:

1,161

865

899

699

782

1,136

1, 198

8

4,038

1,609

1,339

3,763

846.

1,112

400

549

3,380

3,510

3,581

1,669

2,902

2, 694

598

1,769

586

1,541

392

377

840

1,420

1, 321 .

894

591

606

659

406

639

1,378

1,264

3,960

318

588

8 


\begin{tabular}{|c|c|c|}
\hline & Authority. & Elevation. \\
\hline & & \\
\hline $\begin{array}{l}\text { Kirkville } \\
\text { Do...................... Geodic Stati }\end{array}$ & $\begin{array}{l}\text { N. Y. C. \& H. R. R. } \\
\text { N. Y. State Survey }\end{array}$ & $\begin{array}{l}423 \\
507\end{array}$ \\
\hline Kirkwood & N. Y., L. E. \& W. I & 876 \\
\hline Knowersvi & A. \& S. R. R & $\begin{array}{l}459 \\
385\end{array}$ \\
\hline & N., D. \& C. R. R... & $\begin{array}{l}385 \\
357\end{array}$ \\
\hline & G.,I. \& S. R. R... & 401 \\
\hline Lake Stati & W. V.R.R. R & 542 \\
\hline $\begin{array}{l}\text { Lake View. } \\
\text { Do...... }\end{array}$ & $\begin{array}{l}\text { L. S. \& M. S. R. R. } \\
\text { R. \& L. O. R. R... }\end{array}$ & $\begin{array}{l}709 \\
312\end{array}$ \\
\hline Lancaster. & N. Y., L. E. \& W. & 683 \\
\hline $\begin{array}{l}\text { Lanona } \\
\text { Lansing }\end{array}$ & $\begin{array}{l}\text { N. Y., L. E. \& W. } \\
\text { U. S. C. \& G. S... }\end{array}$ & $\begin{array}{r}1,157 \\
33\end{array}$ \\
\hline Laona. & D., A. V. \& P. R. & 810 \\
\hline & N.Y., L. E. \& W. & 572 \\
\hline Law & Corning, C. \& A. R. & 1,006 \\
\hline ill (Ca) & $\begin{array}{l}\text { S. \& C. V. R. R ... } \\
\text { Appal. Club....... }\end{array}$ & $\begin{array}{l}1,336 \\
2,649\end{array}$ \\
\hline Leol & N. Y., L. E. \& W. R & 872 \\
\hline Levant & A. \& G. W. R.R... & 1,267 \\
\hline Lewey Lake.. & Adirondack Survey & \\
\hline Do.......... Mour & $\begin{array}{l}\text { Geol. Surrey of N. } \\
\text { Adirondack Survey }\end{array}$ & $\begin{array}{l}1,738 \\
3,904\end{array}$ \\
\hline Lewiston ........ & N. Y. C. \& H. R. R. R. & $\begin{array}{r}3,904 \\
358\end{array}$ \\
\hline n Mov & Appal. Club $\ldots \ldots \ldots \ldots$ & 2,930 \\
\hline$\cdots$ & U. \& B. R. R. R . & 990 \\
\hline Hibe & N. Y., L. E. \& W. R & 1,293 \\
\hline $\lim \epsilon$ & $\begin{array}{l}\text { R. \& S. L. R. R... } \\
\text { N. Y., L. E. \& W. R }\end{array}$ & $\begin{array}{r}777 \\
1.416\end{array}$ \\
\hline & $\begin{array}{l}\text { N. Y., L. E. \& W. K. R } \\
\text { N. Y., L. E. \& W. R. R }\end{array}$ & $\begin{array}{l}1,416 \\
1,181\end{array}$ \\
\hline $\operatorname{Linc}$ & Corning, C. \& A. R. R & $\begin{array}{l}107 \\
977\end{array}$ \\
\hline & N. Y., L. E. \& W. R. & 390 \\
\hline alls & N. Y.C. \& H. R. R. & 376 \\
\hline on. & Y. Y. State $\delta 1$ & 797 \\
\hline 's Lak & Adirondack Su & 1,504 \\
\hline & N. Y., L. E. \& W. R. & 1,594 \\
\hline ville (Catskills & Appal. Club $\ldots \ldots \ldots$ & 1,100 \\
\hline & N. Y., L. E. \& W. R & 1,030 \\
\hline & S. C. R. R ......... & 799 \\
\hline & N. Y. & 600 \\
\hline Fili & $3 \ldots . . .$. & 628 \\
\hline tskills) & $\mathrm{R}$ & $\begin{array}{l}3,446 \\
3,670\end{array}$ \\
\hline atskills) .................. & $\begin{array}{l}\text { R. R } \\
\text { of } \mathrm{N} . \overline{\mathrm{Y}}\end{array}$ & $\begin{array}{l}3,670 \\
1,620\end{array}$ \\
\hline 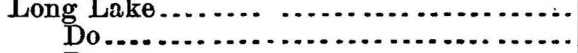 & $\begin{array}{l}\text { of N. Y. } \\
\text { of } \mathrm{N} . \mathrm{Y} \text {. }\end{array}$ & $\begin{array}{l}1,620 \\
1,575\end{array}$ \\
\hline & Adir & 1,584 \\
\hline ong & & 1,573 \\
\hline & & 1,925 \\
\hline Long Pond Mountain. . & ck Surve & 2,269 \\
\hline & & \\
\hline$r . . . . .$. & Adirondack Survey & 2,604 \\
\hline & N. Y., L.E.\& W. R. R & $\begin{array}{r}867 \\
1,115\end{array}$ \\
\hline$r_{\theta} \ldots \ldots . . .$. & $\begin{array}{l}\text { Geol. Survey of N. Y } \\
\text {. S De R. R. }\end{array}$ & 1,761 \\
\hline ac Lake . & of $N . Y$. & 1,527 \\
\hline & G., I. \& S. R. R........ & 396 \\
\hline Mountain .. & Chat. R. R... & 1,918 \\
\hline & N.Y. C.\& H.R.R.R.. & 407 \\
\hline (2) & U. \& B.R.R.R R & 845 \\
\hline 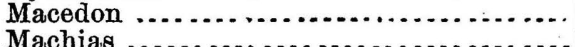 & N.Y.C. \& H. R & $\begin{array}{r}471 \\
1656\end{array}$ \\
\hline th B, N. Y.\&P.R. & $\begin{array}{l}\text { B., N. Y.\& P. R. R } \\
\text { R. \&. L. R. R ...... }\end{array}$ & $\begin{array}{l}1,656 \\
1,646\end{array}$ \\
\hline & & \\
\hline
\end{tabular}




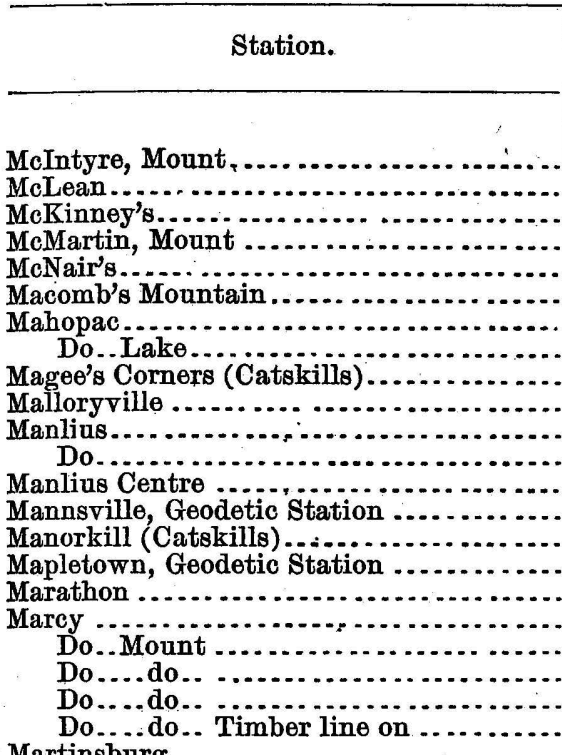

Martinsburg

Martville

Maryland .

Mason, Lake Do

Mattewan

Maxham, Mount Do.

Mayfield

Mayville

Medina

Memphis

Merritt's Corners

Merry, Geodetic Station

Mexico

Middleburg Railroad depot (Catskills) .... Middletown

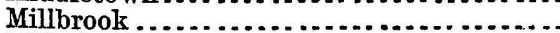

Miller's Corners

Millerton

Milo

Do. Geodetic Station

Minchell's

Mine Hill (Catskills)

Minetto

Mineville (Adirondacks).

Mink Mountain (Catskills)

Mitchell, Geodetic Station:

Monkey Hill (Mucky) (Catskills)

Monsey

Montgomery

Moon Lake

Moon?s

Moore's.

Moose Lake

$$
\text { Do }
$$

Moravia

Morgansville, Geodetic Station

Morian's

Morris Dock

Morrisonville

Morristown.

Authority. Elev

Geol. Survey of N. Y.

U. I. \& E. R. R . . . . . . . . .

G. I. \& S. R. R .

Geol. Survey of N. Y .........

N. Y., L. E. \& W. R. R ......

Adirondack Survey ..........

N. Y. C. \& N. R. R .............

N. Y., B. \& M. R. R.........

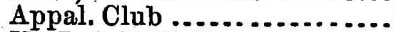

U., I. \& E. R. R

S. \& C. V. R. R............

U. S. Lake Survey .............

Appal. Club ....................

N. Y. State Survey

S. \& B. R. R

U. \& B. R. R. R . . . . $\ldots \ldots$

Adirondack Survey.

Guyot...............

...........

U. \& B. R. R. R.

S. C. R. R

A. \& S. R. R . . .

Geol. Survey of N. Y.

Adirondack Survey.

U., D. \& C. R. R .

Adirondack Survey

F., J. \& G. R. R.

B. C.\& P R R

N. Y. C. \& H. R. R. R

N. Y. C. \& H. R. R. R

N. Y. C. \& N. R. R .............

N. Y. State Survey..........

R.,W. \& Og. R. R...........

Appal. Club

N. Y., L. E. \& W.R.R

N., D. \& C. R. R .............

N. Y. C. \& H. R. R. R

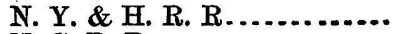

N. C. R. R ... . . . . .

N. Y. State Survey ...........

N., D. \& C. R. R...........

Appal. Club

O. \& S. R. R

Adirondack Survey Appal. Clab

N. Y. State Survey Appal. Club

N. Y., L. E. \& W. R. R N. Y., L. E. \& W. R. R Toner.

D., A. V. \& P. R. R

N., D. \& C. R. R

Geol. Survey of N. Y

Adirondack Survey

S. C. R. $\mathbf{R}$

U. S. Lake Survey

L. S. \& M. S. R. R.

N. Y. C. \& N. R. R

Chat. R. R

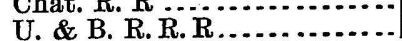

Feet.

5,183

1,090

396

5,000

576

4, 371

641

661

1,964

1,057

416.

742

435.

725

1, 520

1,214

1,026

587

5,403

5,379

5, 334

4, 851

760

367

1, 220

1,860

1,824

119

2, 511

2,474

759

1,300

545

410

346

1, 328

375

640

$56 \%$

566

896.

702

857

1,343

966

2,810

334

1,338

3,807

881

2,489

523

386

1, 772

1,303

439

2, 239

2,203

732

885

627 


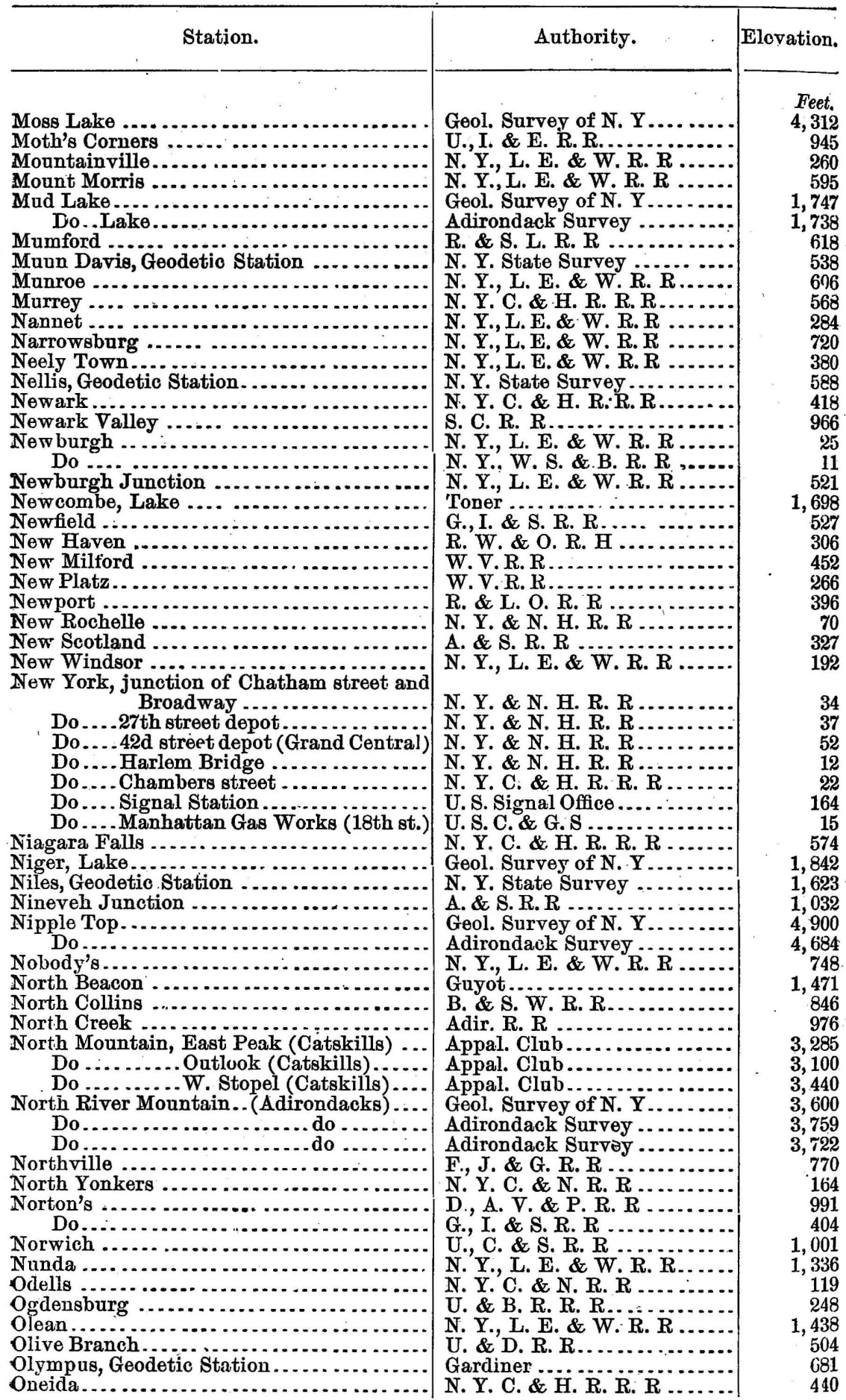




\begin{tabular}{|c|c|c|}
\hline Station. & Authority. & Elevation. \\
\hline & & Feet. \\
\hline 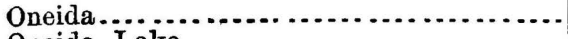 & N. Y. \& Oswego Micl. R. R... & 419 \\
\hline Oneida, Lake & Toner $\ldots \ldots$ & 360 \\
\hline Oneota & A. \& S. R. R .... & 1,087 \\
\hline - $\ldots \ldots \ldots \ldots \ldots \ldots \ldots$ & U. S. Lake Survey & 247 \\
\hline Ontario $\quad \ldots n_{1}$ & R., W. \& O. R & 415 \\
\hline Orangebnrg & N. Y., L. E. \& W. R. R & 67 \\
\hline Orange Farm & N. Y., L. E. \& I & 406 \\
\hline 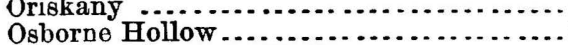 & $\begin{array}{l}\text { N. Y. C. \& H } \\
\text { A.\& S.R.R. }\end{array}$ & $\begin{array}{r}423 \\
115\end{array}$ \\
\hline 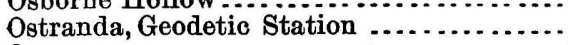 & $\begin{array}{l}\text { A. } \\
\text { N. Y. State Surv }\end{array}$ & 1,115 \\
\hline 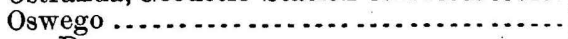 & O.\& S.R.R.... & $\begin{array}{r}1,2 / 4 \\
293\end{array}$ \\
\hline Do.... & R., W.\&. O.R.R & 280 \\
\hline Do.... & N.Y. \& O. R. I & 301 \\
\hline 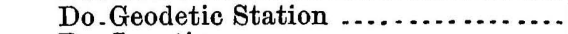 & U. S. Lake Su & 418 \\
\hline 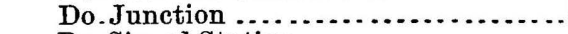 & S. C. R. R.. & 316 \\
\hline Do.Signal Station . . . . . . . . . . . . . . . & U. S. Signal Office....... & 304 \\
\hline 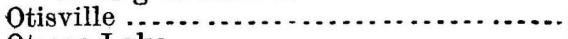 & N. Y., L. E. \& W. R. R & 870 \\
\hline Otsego Lake ...... & A.\& S.R.R. & 1,054 \\
\hline Otter Lake. . . . . . . . . . . & Toner ...... & 2,206 \\
\hline 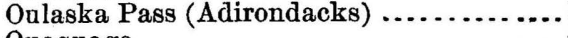 & Adirondack & 3,050 \\
\hline Ouaquaga. . . . . . . & A. \& S. R. R . . & 991 \\
\hline Oven Lake. . . & Geol. Survey of N. Y... & 2,025 \\
\hline Overlook Mountain (Catskills)............ & Appal. Club . ........... & 3,150 \\
\hline Ovid Centre. & G., I. \& S. R. R & 819 \\
\hline Owasco Lake, water-surface................ & S. C. R. R....... & 713 \\
\hline Owego & N. Y., L. E. \& V & $0_{0.05}^{822}$ \\
\hline Owl's Head & Adirondack Sur & 2,825 \\
\hline Orl's Head Mountain (Adirondacks)...... & Adirondack Su & 2,789 \\
\hline 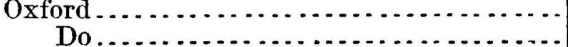 & $\begin{array}{l}\text { N. Y., L. E. \& W. } \\
\text { U., C. \& S. R. R. }\end{array}$ & $\begin{array}{l}540 \\
980\end{array}$ \\
\hline & N.'Y., L, E. \& W. R. R. & $\begin{array}{l}980 \\
945\end{array}$ \\
\hline ne Bridge & N. Y.C. \& H. R. R. R... & $\begin{array}{l}940 \\
304\end{array}$ \\
\hline $\begin{array}{l}\text { Palatine Bridge } \ldots . . . \\
\text { Palenville Overlook (Catskills) } \ldots \ldots \ldots \ldots\end{array}$ & 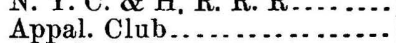 & 1,660 \\
\hline $\begin{array}{l}\text { Palenville Overlook (Catskills) } \ldots \ldots . . . . . \\
\text { Palenville Union Church (Catskills) } \ldots . . .\end{array}$ & 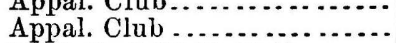 & $\begin{array}{r}1,000 \\
470\end{array}$ \\
\hline 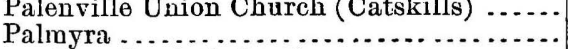 & N. Y. C. \& H. R. R. R & $43 \gamma$ \\
\hline $\begin{array}{l}\text { Palmyra } \\
\text { Do.. Geodetic Station. } . . .\end{array}$ & U. S. Lake Survey ..... & 669 \\
\hline Panama. ... & B., C. \& P. R. R.. & $\mathbf{1}, 545$ \\
\hline Pantherkill Mountain (Catskills) ......... & Appal. Club ........ & 3,828 \\
\hline 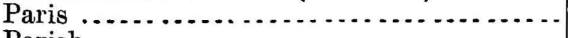 & U., C. \& S. V. R.R & 1,422 \\
\hline$\ldots \ldots \ldots \ldots \ldots$ & R., W. \& O. R. R. & 474 \\
\hline Parker Hill, Star Rock (Catskills) ........ & Appal. Club .... & 2,545 \\
\hline 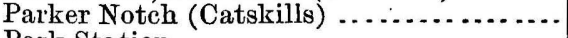 & Appal. Club ... & 2,415 \\
\hline Park Station............ & U., I. \& E. R. R... & 1,515 \\
\hline 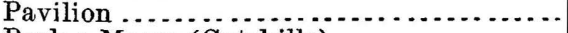 & R. \& S. L. R. R......... & 940 \\
\hline Peak-o-Moose (Catskills) ................. & Appal. Club............ & 3,875 \\
\hline$\ldots \ldots \ldots \ldots \ldots \ldots$ & R. \& S. L. R. R & 988 \\
\hline Peekskill ......... & U.S. C. \& G. S . & \\
\hline Pekin, Geodetic Station & U. S. Lake Survey. & 661 \\
\hline Peunyan......... & N. C. R. R....... & 756 \\
\hline Perrysburgh ... & N. Y., L. E. \& W. R. R ...... & 1,260 \\
\hline ........................ & C., C. \& De R. R. R ... & 1,041 \\
\hline (n) & N. Y., L. E. \& W. R. R....... & 1,350 \\
\hline 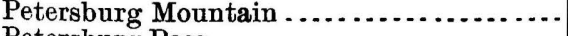 & 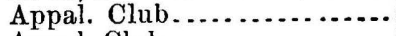 & 2,534 \\
\hline ................ & Appal. Club ..... & 2,075 \\
\hline 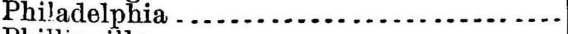 & U. \& B. R. R. R ........ & 485 \\
\hline ille....... . . & N. Y., L. E. \& W. R. R. & 1,390 \\
\hline nicia .......... & 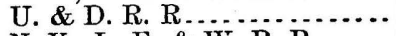 & 796 \\
\hline Piermont........... & N. Y., L. E. \& W. R. R ...... & \\
\hline Pike................ & R. \& S. L. R. R. . . . . . . . & 1,673 \\
\hline Pine Hill (Catskills) .. & Appal. Club $\ldots \ldots \ldots \ldots \ldots$ & 1,512 \\
\hline Pinehill.............. & N. Y., K. \& S. R. R........ & 1,889 \\
\hline Do $\ldots . . .$. & 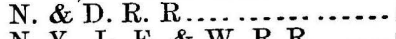 & 1,679 \\
\hline Pine Island & N. Y., L. E. \& W. R. R ...... & 406 \\
\hline Pine Island Mountain, approx. (Catskills) - & & 3,086 \\
\hline
\end{tabular}




\begin{tabular}{|c|c|c|}
\hline ion. & Authority. & Elevation. \\
\hline & & Feet. \\
\hline $\begin{array}{l}\text { Pine Plains .... } \\
\text { Pine Valley ... }\end{array}$ & $\begin{array}{l}\text { P., H. \& B. R. R. } \\
\text { N. C. R. R. }\end{array}$ & $\begin{array}{l}470 \\
865\end{array}$ \\
\hline Pinnacle Hill, Geodetic Station & U. S. Lake Survey & $\begin{array}{r}865 \\
, 752\end{array}$ \\
\hline isco, Lake & Toner ............... & 1,648 \\
\hline isgah (Catskills & Appal. Club . . & \\
\hline ittitsfield Summit & N. Y., K. \& S. R.R & 1,568 \\
\hline Plaaterkill Mounta & Appai. Club ...... & \\
\hline Placid Lake....... & & $\begin{array}{l}1,991 \\
1,950\end{array}$ \\
\hline Platt Creek Chu & $\begin{array}{l}\text { Adirondack Surve } \\
\text { Appal. Club ...... }\end{array}$ & 1,683 \\
\hline Plattsburgh..... & Chat. R. R.... & \\
\hline Pleasant Lake (A & Toner $\ldots \ldots \ldots$ & 1,706 \\
\hline & Adirondack Survey & 1,579 \\
\hline Pleasant Mountain & U. \& D. R. R........ & $\begin{array}{r}699 \\
199\end{array}$ \\
\hline Pleasant Valley ... & P., H. \& B. R. R & $\begin{aligned} 199 \\
2,17 \%\end{aligned}$ \\
\hline $\begin{array}{l}\text { Point of Rocks (Ca } \\
\text { Poland }\end{array}$ & & $\begin{array}{l}2,176 \\
1,269\end{array}$ \\
\hline $\begin{array}{l}\text { Poland } \ldots . . . . . . . . \\
\text { Poolville........ }\end{array}$ & $\begin{array}{l}\text { A. \& G. W. R.R. R. } \\
\text { U., C. \& S. B. R.R }\end{array}$ & $\begin{array}{l}1,269 \\
1,099\end{array}$ \\
\hline Portage ......... & N.'Y., L. E. \& W. I & 1,314 \\
\hline Port Bryan... & N. Y. C. \& H. R. I & 406 \\
\hline Port C & A. \& S. R. R..... & 1,041 \\
\hline Port Jervis.... & N. Y., L. E. \& W & 442 \\
\hline den . & U.\& B. R. R & \\
\hline Portv & B., N.Y.\&P.R.R & 1,44 \\
\hline Post Creek P. O & S., G. \& C. R. R . . & 1,187 \\
\hline Poughkeepsie .. & U., S. C. \& G. S . & \\
\hline Do......... & P., H. \& B.R.R R & 17 \\
\hline Do......... & N.Y. C. \& H. R. R & 13 \\
\hline Prattsville Hotel (C & Appal. Club ...... & 1,16 \\
\hline Preble & S. \& B. R. R. & 1,18 \\
\hline Preston Hollow & Appal. Club ...... & 87 \\
\hline Prospect $\ldots . . .$. & B., C. \& P. R. R & \\
\hline Do... (Center I & Appal. Club ........ & 2,59 \\
\hline Prospect, Geodetic & N. Y. State Survey & 1,38 \\
\hline $\mathbf{P}$ & U. \& B. R. R.R R & 1,01 \\
\hline Prote & B., N.Y. \& P. R. R & 1,38 \\
\hline nd (Ad & Adirondas & 2,19 \\
\hline Puls & R., W. \& Og. R. R & 377 \\
\hline Rafinesque I & U. S. C.\&G. S ..... & 1,18 \\
\hline Raft $\mathrm{H}$ & Adirond & 2,10 \\
\hline Mountain... & Adirond? & 4,16 \\
\hline .........by & Adirondac & 4,12 \\
\hline$\ldots \ldots$ & N. Y., L. E. \& W. R & 31 \\
\hline a............... & A. \& G. W. R.R... & 1,31 \\
\hline ville. & N. Y., L. E. \& W. F & 1,01 \\
\hline fill $\ldots \ldots \ldots \ldots \ldots$ & Adirondack Survey . & $\mathbf{1}, 98$ \\
\hline Geodetic Station & N. Y. St & 79 \\
\hline & N. Y., L. E. \& & \\
\hline P.O. (Catskills) & Appal. Club. & 1,27 \\
\hline Mount .......... & Ad & 4,68 \\
\hline .... & A. \& G. W. R. R. & 1,35 \\
\hline & U. \& B. R. R. R. & 1,18 \\
\hline & U. \& B. R.R.R. & 36 \\
\hline & S. C. R.R R.... & 1,09 \\
\hline & Geol. Sv & 1,54 \\
\hline Junction & R., W. \& O. R. R .. & 52 \\
\hline & R., W. \& O.R.R. & 52 \\
\hline I Cone (Catskills).. & & 3,20 \\
\hline ville $\ldots \ldots \ldots$ & A. \& S. R. R .. & 1,173 \\
\hline & N. Y. C. \& H. R & 828 \\
\hline & Adir & 2,141 \\
\hline & P., H. & 779 \\
\hline & L. S. \& M. S. R.R & 75 \\
\hline & L. S. \& M. S. R. R & \\
\hline
\end{tabular}




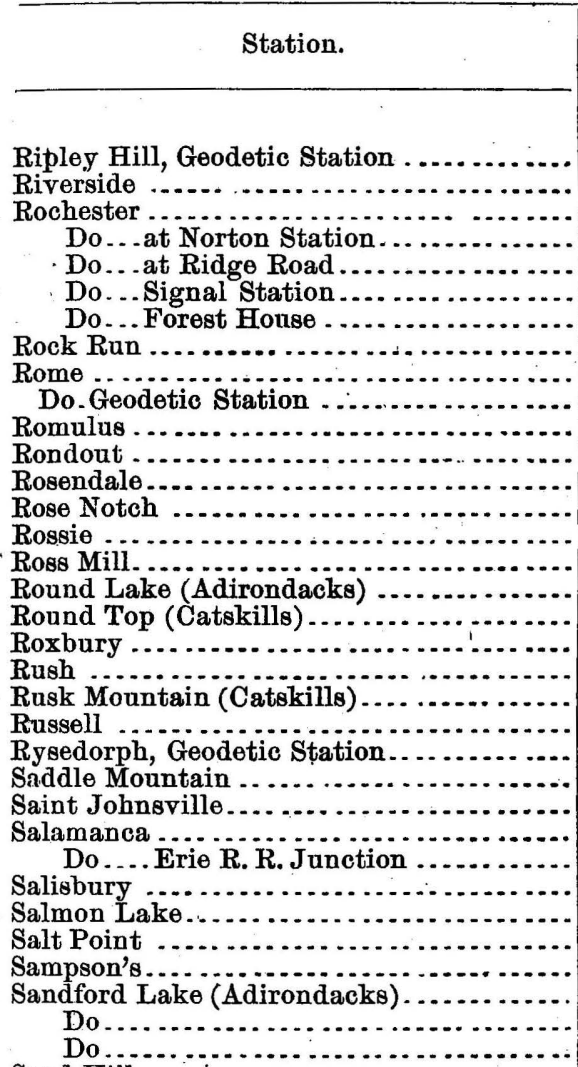

Sand Hill

Sand Lake ...............................

Sandy Creek, Geodetic Station

Do.................

Santanoni Mountain . . . . . . . . . . . . . . . .

Santees

Saranac

Saranac Lake (lower)

Saranac Lake (upper)

Saratoga, station D. \& H. C. R. R

Do............ H. S. \& W. R. R

Do...

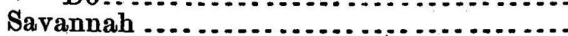

Savona

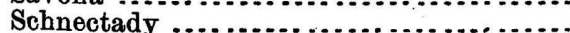

Schenevus

Schodack

Schohari

Schoharie (east peak, Catskills) ..........

Do .... (west peak, Catskills)

Schuyler Lake

Schuyler, Geodetio Stat

Scio

Scoots Ponds

Do ......... No. 2 (Adirondacks)

Scootsville.

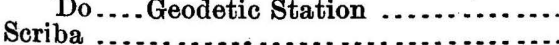

Scroon Mountain

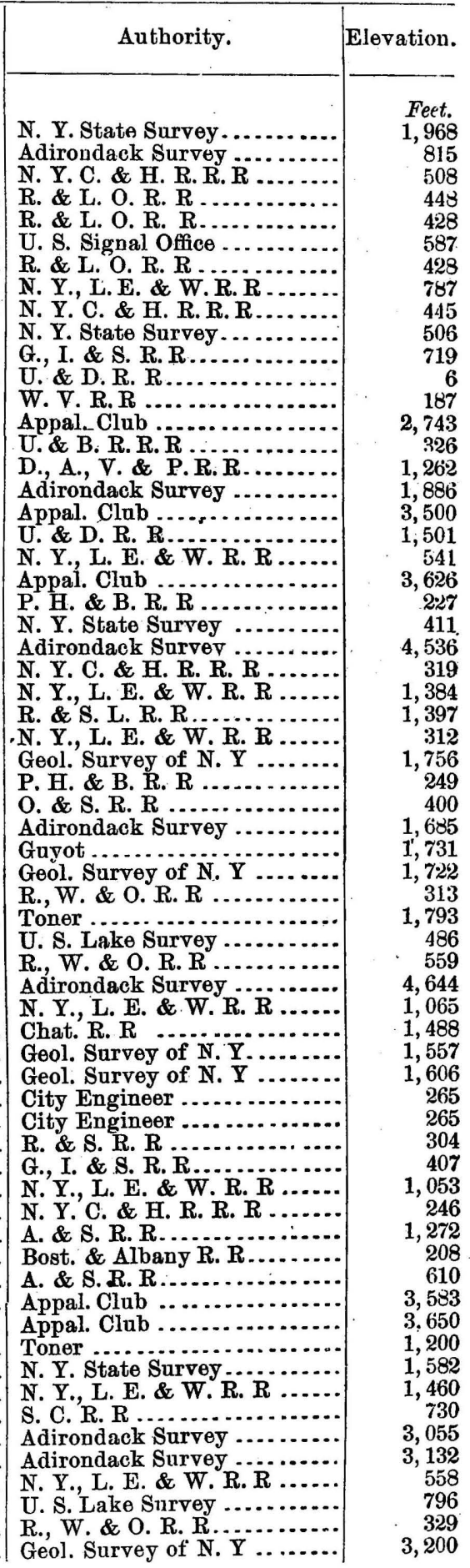




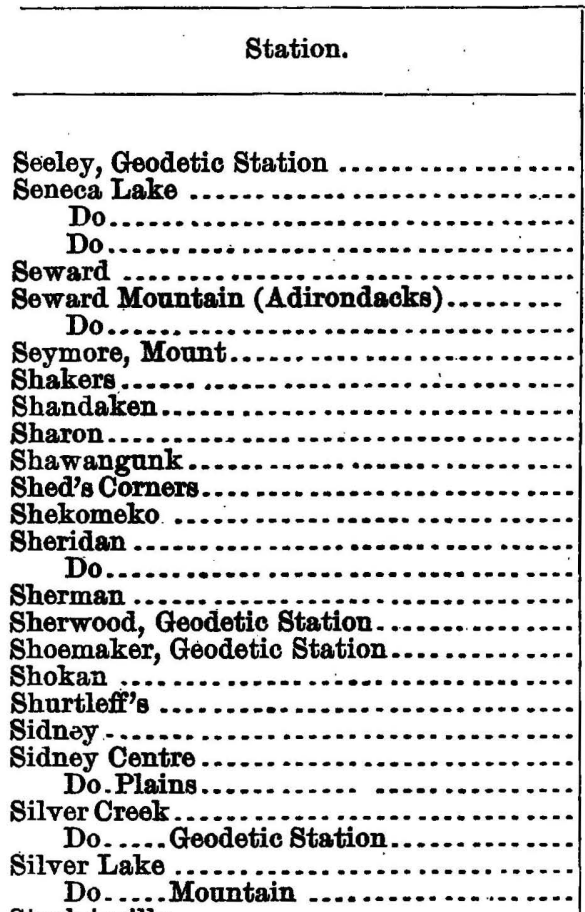

Sinclairville.

Sing Sing

Sister Knob (Catskills)

Skaneateles, Lake

Shidmore

Skylight Mountain

Slide Mountain (Catskilis)

Slingerlands

Sloatsburgh

Smithsborough

Smith's Lake

Do ..... (Adirondacks) ................

Smith's Valley

Smithton (Catskills)

Snowy Mountain Do

(Adirondacks)

Snyder's ...............................

Sodns.

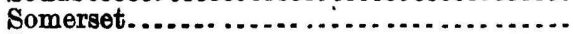

Sonyea

Sorrell Hill, Geodetic Station.............

South Avon

Do.

South Corinth

South Cortland

South Dover.

South Durham

Southfield

South Livonia.

South MeIntyre Mountain

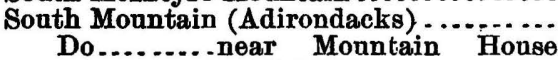

(Catskills) ...............

South Wales

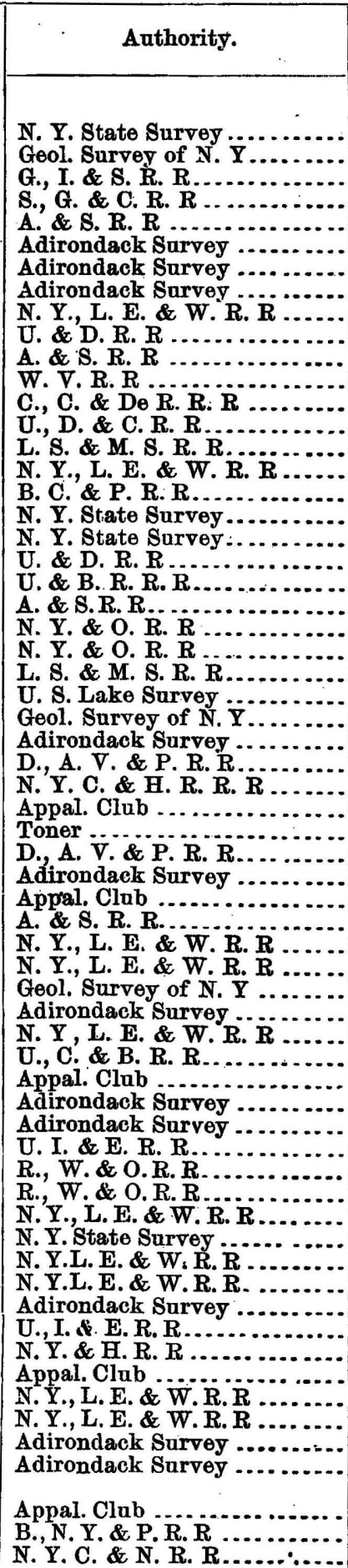

Elevation.

Feet.

1,109

370

445

447

1,177

4,319

4,385

3,929

574

1,072

1,353

277

1,383

667

664

760

1,568

799

1,372

537

416

990

997

1.393

623

726

1,983

2,604

1,330

3, 002

932

1, 317

4,978

4,205

214

350

799

1,775

1,738

1,010

1,140

1,268

3,804

3,860

995

430

332

592

641

620

725

606

1, 151

415

969

491

1,167

4,938

1, 917

2, 497

991

145 


\begin{tabular}{|c|c|c|}
\hline Station. & Authority. & Elevation. \\
\hline & & \\
\hline Speculator Mountain.... & Adirondack Suı & 3,041 \\
\hline Do.................... & Adirondack Survey & 3,005 \\
\hline Spencer . . . . . . . . . & U., I. \& E. R. R .... & 996 \\
\hline 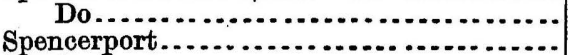 & $\begin{array}{l}\text { G., I. \& S. R. R. } \\
\text { N.Y. C. \& H.R.R.R }\end{array}$ & 1,006 \\
\hline Sprakers............ & N.Y.C.\& H.R.R.R & $\begin{array}{l}501 \\
301\end{array}$ \\
\hline Spring Brook ............ & B., N. Y.\& P.R.R. & 757 \\
\hline 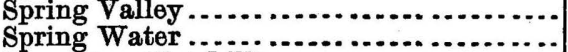 & $\begin{array}{l}\text { N. Y., L. E. \& N. R. R } \\
\text { N. Y., L. E. W. R. R .. }\end{array}$ & $\begin{array}{r}450 \\
1,370\end{array}$ \\
\hline Sprucetop (Catskill) ...... & Appal. Club ........ & 3,567 \\
\hline Stafford................ & N.Y.C. \& H.R.R. R & 894 \\
\hline Do......... & N. Y., L.E.\& W.R.] & 910 \\
\hline Stamford ........ & U. \& D.R.R... & 1,771 \\
\hline Stanfordville .... & P., H. \& B. R. R. & 323 \\
\hline Stanley, junction w & N.C.R.R...... & 904 \\
\hline Starkey & N. C.R.R $R$ & 810 \\
\hline 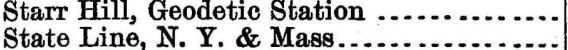 & $\begin{array}{l}\text { N. Y. State Survey .. } \\
\text { P. H. \& B. R. R }\end{array}$ & 1,793 \\
\hline State Line Junction, with extension of & & 784 \\
\hline Tioga R. R. & N.C.R.R R........ & 909 \\
\hline $\begin{array}{l}\text { State Line, Mass. and N. Y } \\
\text { Steamburgh }\end{array}$ & Bost. \& Albany R. R ........ & 914 \\
\hline $\begin{array}{l}\text { Steamburgh } \\
\text { Sterlingville }\end{array}$ & 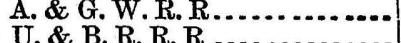 & 1,404 \\
\hline 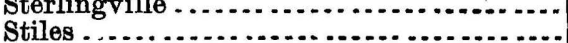 & O.\& S.R.R...... & 580. \\
\hline Stissing . . . . . . & P., H. \& B. R. R... & 388 \\
\hline Do.Junc & U., D.\& C.R.R. & 437 \\
\hline Stittville .. & U.\& B. R. R. . & 560 \\
\hline ockport .... & N. Y., L.E. \& W & 896 \\
\hline Stony Creek. . & Adir. R. R .... & 569 \\
\hline 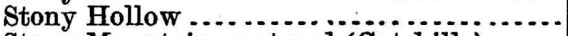 & U. \& D.R.R. & 419 \\
\hline Stony Mountain, east end (Catskills) ... & Appal. Club .. & 3,844 \\
\hline Stony Point, Geodetic Station .......... & U.S. Lake Surve & 276 \\
\hline Stony Ponds ... & Geol. Survey of N. I & 1,536 \\
\hline Strattons $\ldots \ldots \ldots$ & G. I. \& S. R. R ..... & 654 \\
\hline Strykersville (Catskills) ... & Appal. Club ....... & 1,215 \\
\hline Sturgeon Point, Geodetic Station.......... & Lake Surve & 611 \\
\hline Stuyvesant $\ldots \ldots \ldots \ldots \ldots$ & U.S. C. \& G.S .. & 20 \\
\hline Sufferns....... & N. Y., L.E. \& W.R. & 298 \\
\hline Summerdale .. & B., C. \& P. R. R ... & 1,629 \\
\hline Summit....... & N. Y., L. E. \& W. R. R ........ & 1,373 \\
\hline Summit Hill & N. Y., K. \& S. R. R . . . . . . . . . & 1,613 \\
\hline Sunset Rock (Catskills) $\ldots \ldots \ldots \ldots \ldots \ldots$ & Appal. Club $\ldots \ldots \ldots$ & 2,115 \\
\hline Suspension Bridge & N.Y.C.\& H. R.R.R & 580 \\
\hline Sutton Gap, road (Catskills) ................... & Appal. Club .... & 2,235 \\
\hline 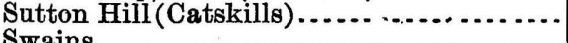 & Appal.Club $\cdots \cdots$ & 2,573 \\
\hline $\begin{array}{l}\text { Swains } \\
\text { Swartwood }\end{array}$ & 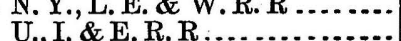 & 1,313 \\
\hline $\begin{array}{l}\text { Sp } \\
\text { Sy }\end{array}$ & 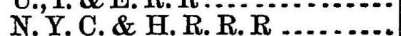 & $\begin{array}{r}1,059 \\
403\end{array}$ \\
\hline $\begin{array}{l}\text { Syracuse... } \\
\text { Table Mountain (Catskills) }\end{array}$ & 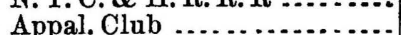 & 3.865 \\
\hline $\begin{array}{l}\text { Table Mountain (Catskills) } \ldots . . . . . . . . \\
\text { Tahawas (Marcy) }\end{array}$ & ondack Survey & $\begin{array}{l}3,865 \\
5,403\end{array}$ \\
\hline Tahawas (Marcy) .......... & N.Y. L. E. \& W.R.R......... & $\begin{array}{r}5,403 \\
480\end{array}$ \\
\hline Tallman ..... & 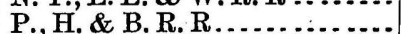 & $\begin{array}{l}480 \\
694\end{array}$ \\
\hline Geodetic Station ........... & N. Y. State Survey. & $\begin{array}{l}694 \\
755\end{array}$ \\
\hline $\begin{array}{l}\text { Tanner Geodetic Station.......... } \\
\text { Tannersville Hotel (Catskills) ... }\end{array}$ & Appal. Club ........ & 1,926 \\
\hline Heights................. & 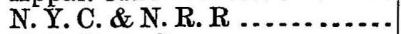 & 387 \\
\hline odetic Station. & N. Y. State Survey ............... & 1,948 \\
\hline ock ............. & 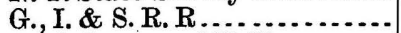 & 411 \\
\hline Taylor's Mountain & of $\mathrm{N} . \mathrm{Y} \ldots \ldots$ & 4,500 \\
\hline Tear of the Clouds, Lake........ & y of $\mathrm{N} . \mathrm{Y} \ldots \ldots$ & 4,327 \\
\hline Teller, Geodetic Station ......... & N. Y. State Survey .......... & 407 \\
\hline Thre & U. \& B.R.R.R . . . . . . & 341 \\
\hline sa Ju & U. \& B. R. R. R ......... & 405 \\
\hline Thirteenth Pond (Adiro & ıck Surrey ........ & 1,652 \\
\hline Thurman .............. & & \\
\hline
\end{tabular}




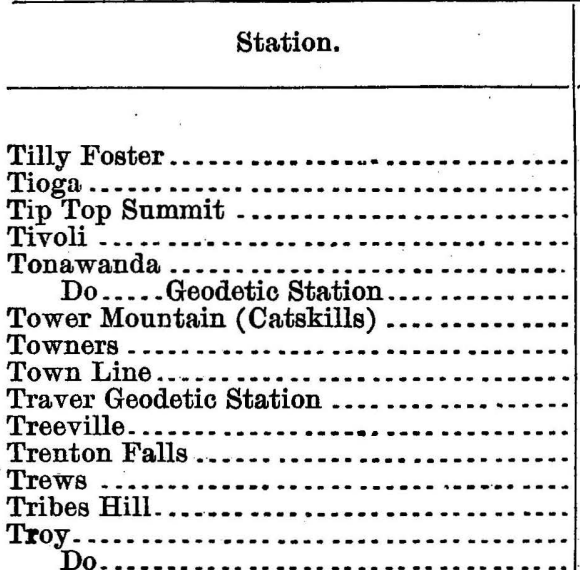

Trumansburg

Truxton

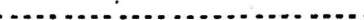

Tully, Lake

Tupper's Lake

Do

Turk's Hill, Geodetic Station

Turners.

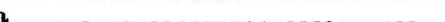

Undercliff (Catskills)

Union

Union Springs

Upper Preston Pond (Adirondacks)

Upper Saranac Lake

Utica

Do

Do

Utsyantha Mt. (Catskills)

Vails Gate

Do.... Junction

Valley

Van Courtland

Vandalia

Van Denburgh, Geodetic Station

Vanetterville

Vanderzee, Geodetic Station

Van Etten

Van Hosen

Van Wagner's

Van Wie, Geodetic Station.

Vedder, Geodetic Station

Verbank

Do.. Village

Vermont

Verona

Verplanck

Victory, Geodetic Station Do

Vienna, Geodetic Station

Vlaie, or Fly Mountain (Catskills)

Walden

Wallace

Do ........... top (Ädirondacks) ....

Wallkill Valley Junction .................

Walton ..................................

Walworth, Geodetic Station ..............

Warner Pass ............................

Warner's

\begin{tabular}{|c|c}
\hline Authority. & Elevation. \\
\hline
\end{tabular}

N. Y. C. \& N. R. R...........

N. Y., L. E. \& W. R. R.

N. Y., L. E. \& W. R. R......

U. S. C. \& G. S..

N. Y. C. \& H. R. R. R.......

U. S. Lake Survey.

Appal. Club ............

N. Y., L. E. \& W. R. R.

N. Y. State Survey .

U., I. \& E. R. R

. \& B. R. R. R...........

N. Y. C. \& H. R. R. R.

N. Y. C. \& H. R. R. R

R.\& S. R. R.

C., C. \& De R. R. R

Geol. Survey of $\mathrm{N}$. $\mathrm{Y}$

Adirondack Survey

U. S. Lake Survey.

N. Y., L. E. \& W. R. R

A. \& S. R. R

W.

Appal. Club

N. Y., L. E. \& W. R. R .

G., I. \& S. R. R ..............

Adirondack Survey

N. Y. C. \& H. R. R. R

U., C. B. R. R..............

rie Canal

Appal. Club

N. Y., L. E. \& W. R. R

N. Y., L. E. \& W. R. R

N. Y., L. E. \& W. R. R

N. Y.C. \& N.R.R....

N. Y., L. E. \& W. R. R

N. Y. State Survey -

G., I. \& S. R. R

G., I. \& S. R. $\mathbf{R}$

U., I. \& E. R. R

Boston \& Albany R. R.

P., H. \& B. R. R

N. Y. State Survey

N. Y. State Survey

U., D. \& C. R. R..

U., D. \& C. R. R .

D., A. V. \& P. R. R

N. Y. C. \& H. R. R. R

U. S. C. \& G. S .

U. S. Lake Survey .

N. Y. State Survey.

N. Y. State Survey

Appal. Club.

W. V. R. R

N. Y., L. E. \& W. R. R

Adirondack Survey

Adirondack Survey

U. \& D. R. R

N. Y. Q O. R. R .

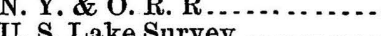

U. S. Lake Surver ...........

N. Y., C. \& H. R. R. R
Feet.

401

805

1,783

9

580

628

2, 931 .

432

742

236

1,030

840

87\%

305

16

30

878

1,135

1,200

1,554

1,504

931

556

1,022

2,200

840

394

2, 170

1, 567

410

446

428

3,203

283

280

300

20

1,415

264

1,010

158

1,012

243

242

28

1, 051

553

522

1, 295

467

34

576

568

568

3,531

351

1, 235

$3,89: 3$

3,857

188

1,239

656

1,963

427 


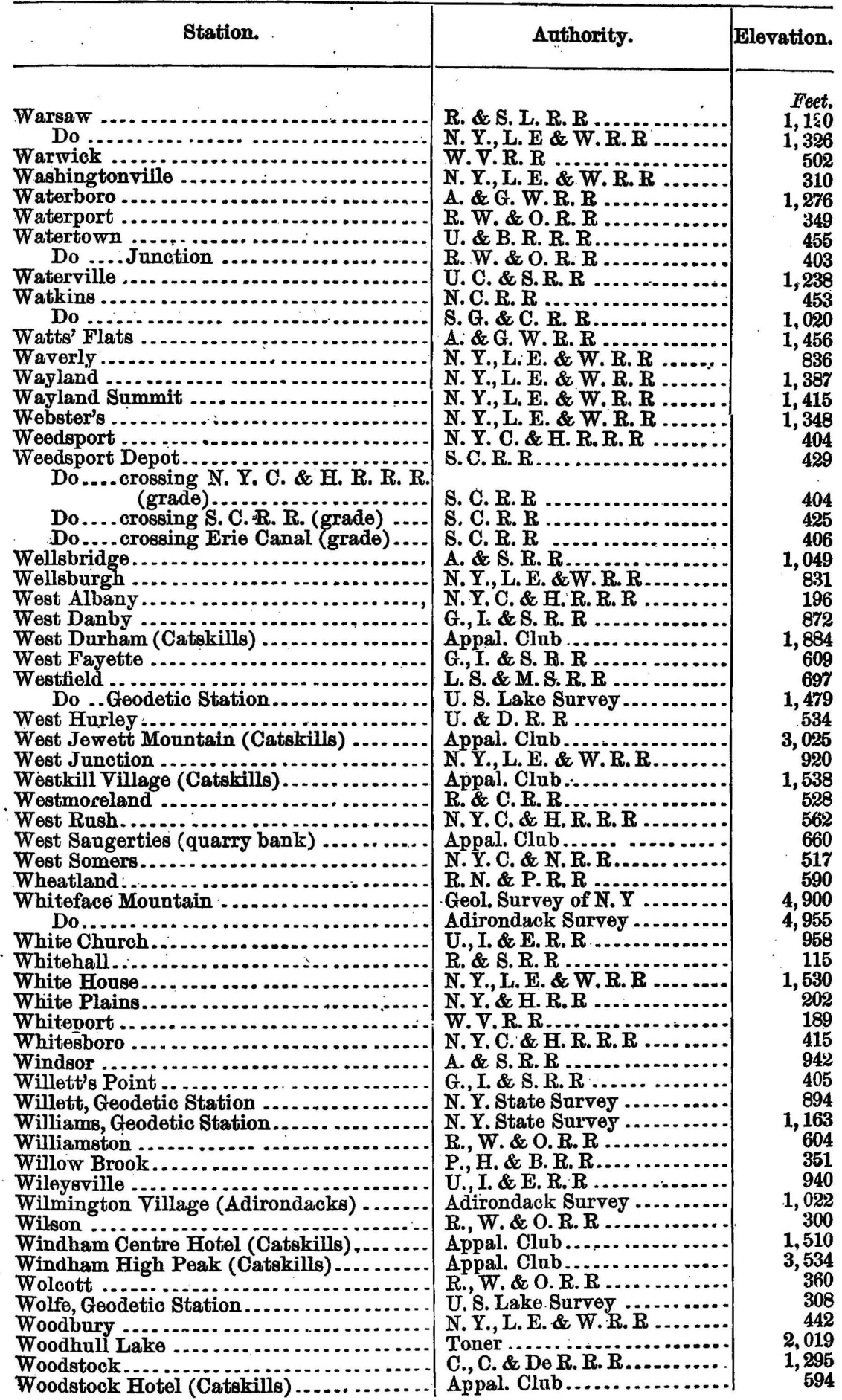




\begin{tabular}{|c|c|c|}
\hline Station. & Authority. & Elevation. \\
\hline $\begin{array}{l}\text { Worcester } \\
\text { Wyckoff's } \\
\text { Wyoming } \\
\text { Wellow Pine, Geodetio Station } \\
\text { Yellow } \\
\text { York Centre } \\
\text { Yorkshire } \\
\text { Yorktown } \\
\text { Yost's } \\
\text { Yule, Geodetic Station }\end{array}$ & 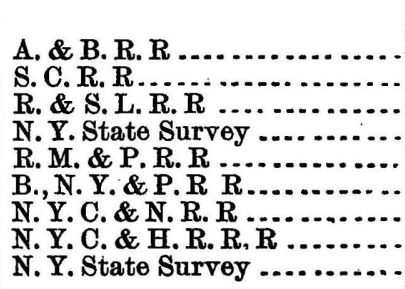 & $\begin{array}{r}\text { Feet. } \\
1,310 \\
726 \\
965 \\
460 \\
780 \\
1,458 \\
439 \\
300 \\
1,683\end{array}$ \\
\hline
\end{tabular}

(346) 
NORTH CAROLINA.

\begin{tabular}{|c|c|c|}
\hline Station. & Authority. & Elevation. \\
\hline & & Feet. \\
\hline $\begin{array}{l}\text { Alexander, Mount } \\
\text { Amos Plott's Balsa }\end{array}$ & Guyot...... & 6,447 \\
\hline Anderson.......... & U.S.C.\& G.S & $\begin{array}{l}6,278 \\
1,547\end{array}$ \\
\hline Apex............. & R.\&.A.A.L.R & 508 \\
\hline ack Creek... & N. Carolina $\mathbf{R}$ & $\begin{array}{l}175 \\
504\end{array}$ \\
\hline aker's Knob.. & U.S.C.\& & 1,812 \\
\hline Bald Mountain. & Guyot........ & 5,550 \\
\hline $\begin{array}{l}\text { Baldwin Depat... } \\
\text { Balsam Cone...... }\end{array}$ & $\begin{array}{l}\text { Car. C. R. R. } \\
\text { Guyot...... }\end{array}$ & $\begin{array}{l}105 \\
6.671\end{array}$ \\
\hline $\begin{array}{l}\text { Balsam Cone........... } \\
\text { Bear Wallow Mountain }\end{array}$ & U.S.C.\& G. & $\begin{array}{l}6,671 \\
4,245\end{array}$ \\
\hline Belton ............ & A. \& F. B. V & 876 \\
\hline Benn.......... & Ü. S. C.\& G & 2,908 \\
\hline Big Craggy Mount & Guyot........ & 6,090 \\
\hline $\begin{array}{l}\text { Do } \\
\text { Black Brother........ }\end{array}$ & $\begin{array}{l}\text { U. S. C. \& G. } \\
\text { Guyot...... }\end{array}$ & $\begin{array}{l}6,068 \\
6,619\end{array}$ \\
\hline Black Rock..... & Guyot.... & $\begin{array}{l}0,019 \\
5,815\end{array}$ \\
\hline Blackstock Kno & U.S.C.\& G & 6,378 \\
\hline Do........ & Guyot...... & 6,380 \\
\hline$\ldots$ & Car. C. R. R.... . & 110 \\
\hline Bowlen's Pyramid.. & Guyot.......... & 6,348 \\
\hline Bristol ................. & A.M.\& O.R.R... & 1,689 \\
\hline Brow & R.\& G. R.R. & 355 \\
\hline Bro & P. A. L.R.R. & 803 \\
\hline eer Plott..... & Guyot....... & 6,246 \\
\hline Broy & C.R.R. & 100 \\
\hline Mount. & Guyot.... & 6,599 \\
\hline Bull's & Guyot...... & 5,935 \\
\hline$\cdots \cdot$ & R.\&A.A.L. & 309 \\
\hline on. & U.S.S & 8 \\
\hline on. & gnal Office & 15 \\
\hline Knob.. & U.S.C.\& G.S.. & 2,284 \\
\hline oline City .... & A.\&N.C.R.R & 10 \\
\hline & R.\&A.A.L.R & 501 \\
\hline Cata & W.N.C.R.R.. & 1,294 \\
\hline Peak.. & Gayo & 6,611 \\
\hline & N. C & 418 \\
\hline Charlotte.. & Charlotte, C.\&.A.R.R. & 747 \\
\hline & 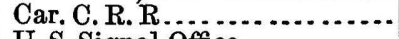 & 725 \\
\hline gnal Sta & Signal Office...... & 838 \\
\hline Peak..... & Guy & 6,234 \\
\hline Top Mor & \& G.S. & 4,573 \\
\hline & $\&$ G.S... & 1,606 \\
\hline & N. Carolina R.R. & 347 \\
\hline Clingman's Mountain & 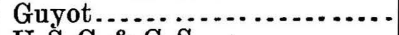 & 6,707 \\
\hline Cold Mountain & U. S. C.\& G.S............ & 4,627 \\
\hline Do......... & Guyot.... & 6,063 \\
\hline Cold Spring Mountain...... & Guyot_............................. & 5,915 \\
\hline Conelly's Gap............... & 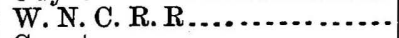 & 1,269 \\
\hline $\mathrm{mb} \ldots \ldots \ldots \ldots$ & Guyot.......................... & 5,426 \\
\hline Craggy Pinnacle.. & Guyot. & 5,945 \\
\hline Dallas........... & C. \& L. N. G. R. R........ & 944 \\
\hline Mountain... & 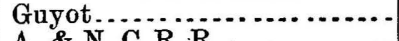 & 6,233 \\
\hline wamp.... & 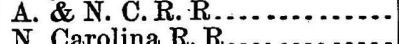 & $\begin{array}{r}66 \\
400\end{array}$ \\
\hline Ea & A. \& F. B. V. R.R............. & $\begin{array}{r}400 \\
1,043\end{array}$ \\
\hline & U. S. C. \&. G. S................. & 2,126 \\
\hline Fodderstack or ? & U. S. C.\& G. S............ & 4,510 \\
\hline
\end{tabular}




\begin{tabular}{|c|c|c|}
\hline Station. & Authority. & Elevation. \\
\hline & & \\
\hline Fort Macon, Sign & U. S. Signal O & \\
\hline $\begin{array}{l}\text { Franklintown } \\
\text { Friendship. }\end{array}$ & R. \& G.R. R. & $\begin{array}{l}417 \\
892\end{array}$ \\
\hline $\begin{array}{l}\text { Frrendsnip.. } \\
\text { Garibaldi... }\end{array}$ & $\begin{array}{l}\text { N. W. N. C. R. } \\
\text { A. \&. A. L. }\end{array}$ & $\begin{array}{l}892 \\
712\end{array}$ \\
\hline . & R. \& G. R. R. & 152 \\
\hline Gastonia. & A. \& R. A. L. I & \\
\hline .... & Guyot.......... & 6,591 \\
\hline oldsboro & N. Carolin & 102 \\
\hline raham.. & N. Carolina R. R & $\begin{array}{r}677 \\
\end{array}$ \\
\hline$\cdots$ & Guyot......... & 5,897 \\
\hline $\begin{array}{l}\text { Graybeard } \\
\text { Great Hog }\end{array}$ & $\begin{array}{l}\text { Guyot... } \\
\text { U. S. C. G. s. }\end{array}$ & $\begin{array}{l}5,448 \\
4,780\end{array}$ \\
\hline Do... & Guyot................ & 4,792 \\
\hline Greenisboro.. & R. \& D. R & 831 \\
\hline Do... & N. Carolin & 430 \\
\hline airy $\mathrm{H}$ & Gayot.... & 6,681 \\
\hline Hal & Guyot .......... & 6,403 \\
\hline Hall & W. N. C. R. R & 1,154 \\
\hline & R. \& A. R. R. & 331 \\
\hline & U. S. C. \& G. \& & 6,102 \\
\hline gnal & ignal of & 20 \\
\hline & N. & 523 \\
\hline & G. R. R. & 505 \\
\hline lount & U. S. C. \& G. S.. & 2,265 \\
\hline & C. \& L. N. G. R. $\mathrm{B}$ & 1,222 \\
\hline $\mathrm{Hig}$ & Guyot.................... & 5,690 \\
\hline Hig & olina R. R. & 943 \\
\hline Statio & Car. C. R. R..... & 1,001 \\
\hline ugh....... & N. Carolina R. R & $\begin{array}{l}539 \\
335\end{array}$ \\
\hline$\ldots$. & t A. R. R... & $\begin{array}{r}335 \\
5,482\end{array}$ \\
\hline$\ldots$ & ot L. N. G.R. I & $\begin{array}{l}5,482 \\
1,321\end{array}$ \\
\hline Jam & rolina $R$. . R. & 821 \\
\hline $\mathrm{Ju}$ & Guy & 6,223 \\
\hline & N. C & 1,016 \\
\hline & A. R. R. & 286 \\
\hline & U. & 1,692 \\
\hline ountain & & 952 \\
\hline & A. & \\
\hline & & 417 \\
\hline Nk, Signal & & 22 \\
\hline 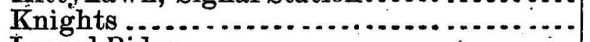 & N. I & 942 \\
\hline & Car & 245 \\
\hline irgh ..... & & 230 \\
\hline & & 1,186 \\
\hline & & 776 \\
\hline Mountain & & 5,707 \\
\hline C. H. & & \\
\hline & & 866 \\
\hline Mountai & & 5,217 \\
\hline & & 4,437 \\
\hline r.... & N:C & 86 \\
\hline & X. R. R . & \\
\hline $\mathrm{m} \ldots .$. & & 5,898 \\
\hline$\theta \ldots . .$. & & 1,194 \\
\hline & R. R & 1,259 \\
\hline n C. H . & R. R..... & 135 \\
\hline s ........... & Car & 238 \\
\hline & G.R.R ....... & 376 \\
\hline (n........... & (n.............. & $\begin{array}{r}450 \\
1.425\end{array}$ \\
\hline - & $\begin{array}{l}\text { W. N.C. } \\
\text { Car. C. I }\end{array}$ & $\begin{array}{r}1,425 \\
65\end{array}$ \\
\hline & $\begin{array}{l}\text { Car. C. }] \\
\text { W.N. } \mathrm{C} .\end{array}$ & 1,647 . \\
\hline Iebanks .......... & N. Carolina R. 1 & \\
\hline
\end{tabular}




\begin{tabular}{|c|c|c|}
\hline Sta & Authority. & Elavation. \\
\hline & & \\
\hline Merry Oaks ... & R. \& A.R.R . & 245 \\
\hline $\begin{array}{l}\text { M.jddleburgh... } \\
\text { Midway ......... }\end{array}$ & $\begin{array}{l}\text { R.\& G. R.R... } \\
\text { R.\& G.R.R.. }\end{array}$ & 461 \\
\hline Mill Brook.. & R.\& G.R.R.. & $\begin{array}{l}200 \\
304\end{array}$ \\
\hline Mitchell, Mount & Guyot....... & $\begin{array}{r}304 \\
6,582\end{array}$ \\
\hline Mitchell's High Pet & U.S. C.\& G.S & 6,688 \\
\hline $\begin{array}{l}\text { Moncure .......... } \\
\text { Monroe }\end{array}$ & R.\& A.R.R . & 145 \\
\hline $\begin{array}{l}\text { Monroe _........... } \\
\text { Morganton ..... }\end{array}$ & W.N.C.R.R. & $\begin{array}{r}586 \\
1,140\end{array}$ \\
\hline Moore........... & U. S. C.\& G. S. & 572 \\
\hline ville & N. Carolina R.R & 308 \\
\hline $\begin{array}{l}\text { Moss Neck Statio } \\
\text { Negro Mountain }\end{array}$ & $\begin{array}{l}\text { Car. C.R.R } \\
\text { U.S.C. \& G. s }\end{array}$ & $\begin{array}{r}159 \\
4597\end{array}$ \\
\hline Neuse ........... & R.\& G.R.R. & $\begin{array}{r}4,597 \\
270\end{array}$ \\
\hline Neuse Rive & N. Carolina R. & 112 \\
\hline n Depot... & A. \& N.C.R. & 12 \\
\hline New Hill.......... & R.\& A.R.R & $\therefore 332$ \\
\hline Newto & C.\& L. N. G.R. & 1,070 \\
\hline $\begin{array}{l}\text { 3nffalo ... } \\
\text { d Mounta }\end{array}$ & $\begin{array}{l}\text { N. Carolina R. I } \\
\text { Guyot ......... }\end{array}$ & 686 \\
\hline Old Field Mou & Guyot $\ldots . . . . . .$. & $\begin{array}{l}5,786 \\
5,100\end{array}$ \\
\hline & R.\& A. R. R. & $\begin{array}{r}0,100 \\
246\end{array}$ \\
\hline Panther Knob. & Guyot $\ldots . . .$. & 5,359 \\
\hline . & P. A. L. R. R & 742 \\
\hline $\begin{array}{l}\text { y Knob...... } \\
\text { cens C. H.... }\end{array}$ & Guyot $\ldots \ldots \ldots$ & 5,026 \\
\hline tose... & $\begin{array}{l}\text { A. \& F. B. R. R. } \\
\text { U. S. C. \& G. S. }\end{array}$ & $\begin{array}{l}1,093 \\
4,910\end{array}$ \\
\hline ntain. & U. S. C. \& G. S. & 2,413 \\
\hline Pineville $\ldots . . .$. & Charlotte C. \& & 575 \\
\hline Mount & U. S. C. \& G. S & 3,832 \\
\hline Pisgah, Mount.... & U. S. C. \& G. S .. & 5,713 \\
\hline Knob... & Guyot & 5,757 \\
\hline & Guyot........... & $\begin{array}{l}2,680 \\
6,393\end{array}$ \\
\hline Princ & N. Carolina $R$. & 160 \\
\hline Raleigh ....... & R. \& A.R.R. & 316 \\
\hline Ded Banks.......... & $\mathrm{R} R$ & $\begin{array}{l}303 \\
176\end{array}$ \\
\hline ch Gap & $\begin{array}{l}\text { Car. C. R. R .. } \\
\text { Car. C. R. R.. }\end{array}$ & $\begin{array}{r}176 \\
2,242\end{array}$ \\
\hline Rei & P. A. L. R.R. & 831 \\
\hline & N. Carolina R. I & 619 \\
\hline Richland Balsa & U. S. C.\& G.S. & 6,370 \\
\hline do & Guyot.......... & 6,425 \\
\hline 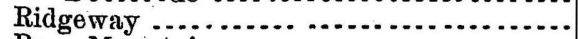 & R.\& G.R.R ... & 415 \\
\hline & Guyot $\ldots \ldots$ & 6,306 \\
\hline Depo & Car. C.R.R.... & 210 \\
\hline $\begin{array}{l}\text { Rockị } \\
\text { Rocks }\end{array}$ & Guyot ................. & 6, 274 \\
\hline & Guyot............ & 6,031 \\
\hline Roc & Guyot .......... & 5,306 \\
\hline Peak & & 6,488 \\
\hline dale ......... & Car. C. R.R.. & 127 \\
\hline & P. A.L.R.R... & 710 \\
\hline (................. & U.S.C.\& G.S & 4,563 \\
\hline epot .. & N. W. N.C. R. R/. & 884 \\
\hline$y \cdots$ & N. Carolina R. R. & $\begin{array}{l}760 \\
320\end{array}$ \\
\hline c. $\mathrm{H}$. & 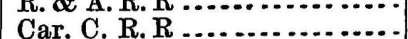 & 875 \\
\hline & Car. C. R.R .................... & 194 \\
\hline ek Knob................ & 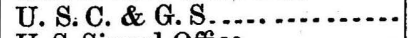 & 2,849 \\
\hline , Signal Station..... & U. S. Signal Office $\ldots . . \ldots \ldots$ & $\begin{array}{r}34 \\
796\end{array}$ \\
\hline (n) & $\begin{array}{l}\text { N.Carolina R. R } \\
\text { Guyot } . . . . .\end{array}$ & $\begin{array}{r}796 \\
6,076\end{array}$ \\
\hline nding lndian $\ldots \ldots \ldots$ & U.S. C.\& G.S ............... & 5,495 \\
\hline
\end{tabular}




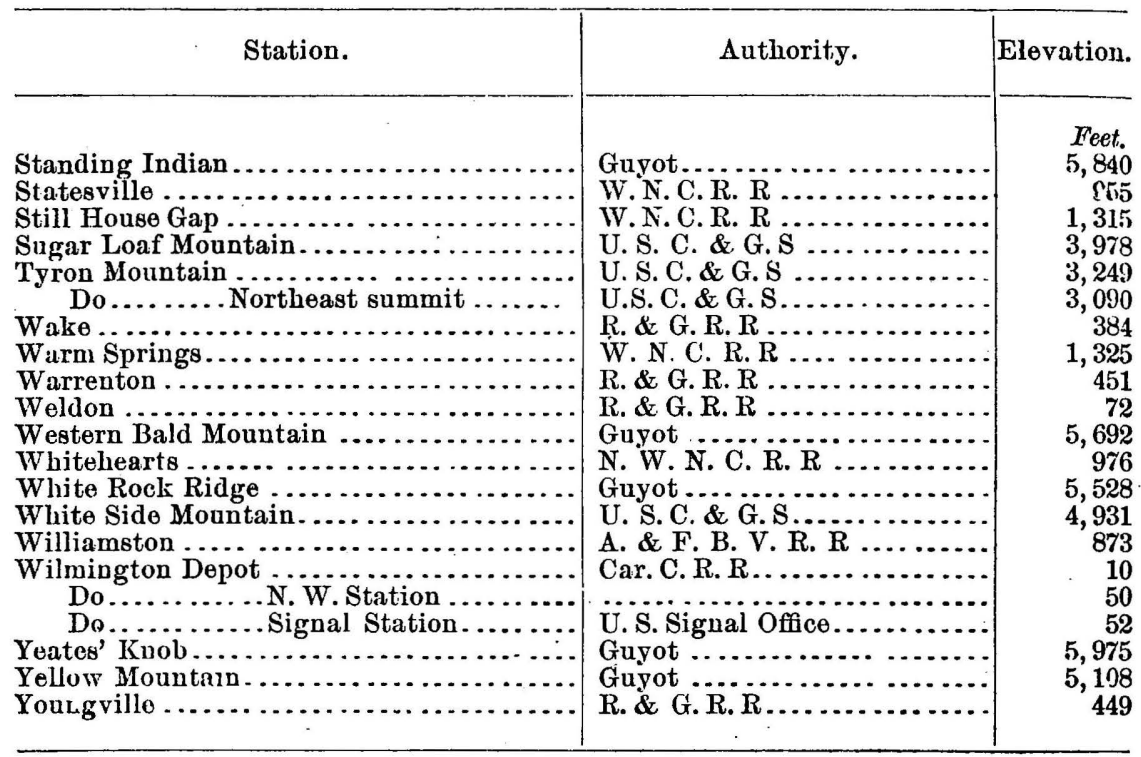




\section{OHIO.}

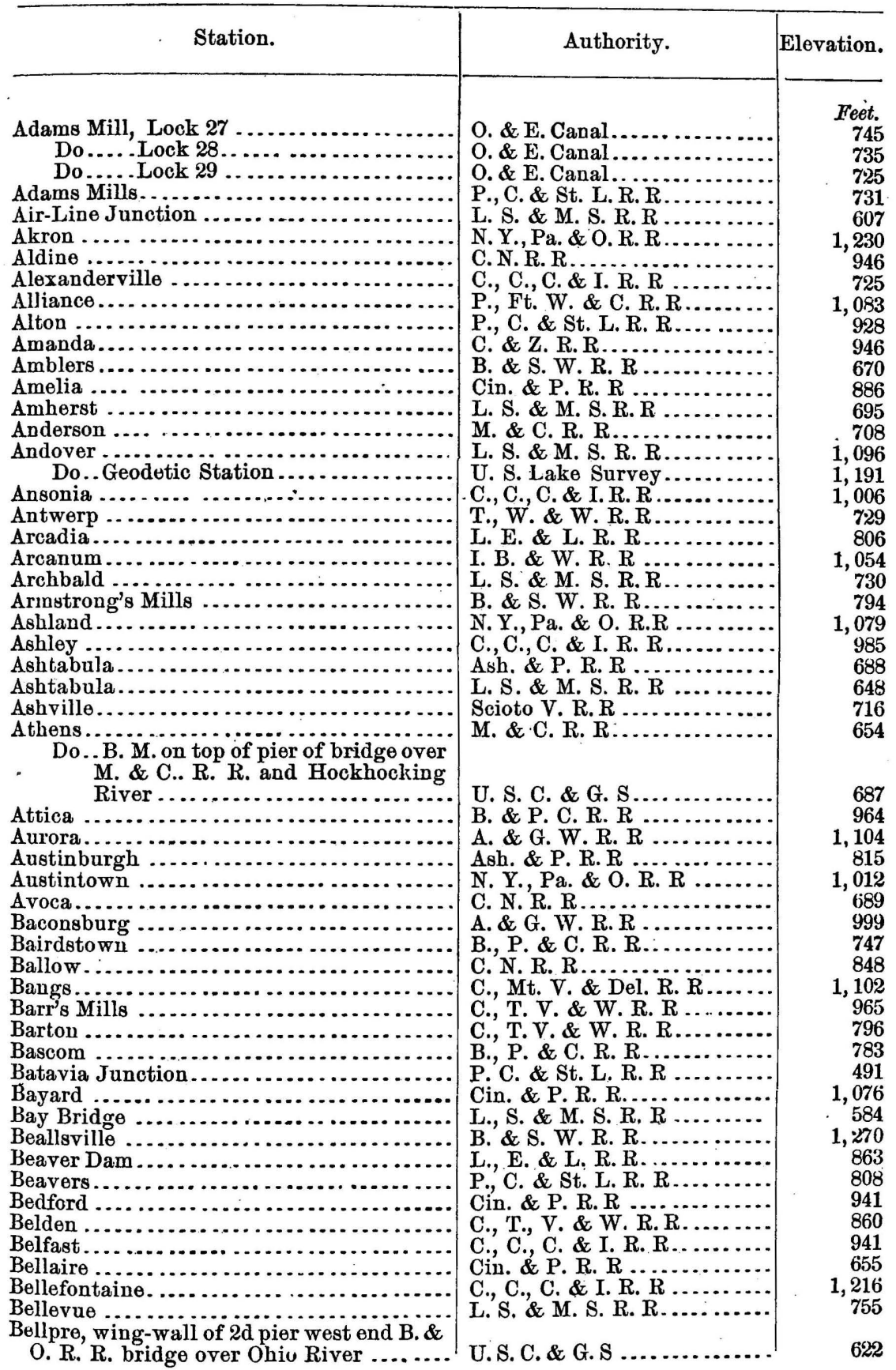




\begin{tabular}{|c|c|c|}
\hline Station. & Authority. & Elevation. \\
\hline & & \\
\hline Belpre, Hotel . & M. \& C. R. R ... & 630 \\
\hline Berea .......... & L. S. \& M. S. R. & \\
\hline Bessemer. & C. \& H. V. R. R & 681 \\
\hline Bethel .... & B. \& S. W. R. & 1,255 \\
\hline Big Poland & M. \& C. R. R. & \\
\hline Big Run..... & Scioto V.R.R. & 555 \\
\hline Big Sand Fur & M. \& C. R.R R & 712 \\
\hline Black Creek. & & 868 \\
\hline Black River ........ & $\begin{array}{l}\text { C.', T. V.\& W. } \\
\text { M.'\& C.R.R... }\end{array}$ & $\begin{array}{l}611 \\
977\end{array}$ \\
\hline $\begin{array}{l}\text { Blanchester ......... } \\
\text { Bloomdale ........ }\end{array}$ & $\begin{array}{l}\text { M. \& C. R. R. } \\
\text { B.\& P. C. R. R. }\end{array}$ & 977 \\
\hline Bloomfield ... & Ash. \& P.R.R... & 905 \\
\hline Blue Ash. & C. N.R.R.... & 859 \\
\hline Bluffton... & L. E.\& L.R.R & 834 \\
\hline (loc & O. \& E. Canal. & 907 \\
\hline Boston ......... & V.R.R................ & 668 \\
\hline Botzur & V. R. R . . . . & 750 \\
\hline Bowlersville. & N. Y., Pa. \& O. R & 966 \\
\hline $\begin{array}{l}\text { Braceville.... } \\
\text { Brachmans... }\end{array}$ & $\begin{array}{l}\text { N. Y., Pa. \& O. R. } \\
\text { Cin. \& P. R. R... }\end{array}$ & $\begin{array}{l}907 \\
713\end{array}$ \\
\hline Branch Hill & P., C.\& St.L.R. & 590 \\
\hline Breme & C. \& M. V.R. R & 831 \\
\hline Sunmit & Miami Canal :- & 960 \\
\hline Bric & V. R.R $\ldots$ & 635 \\
\hline Bridgeport & Cin. \& P. R. R & 659 \\
\hline Brie & A. \& P. R. R.. & 852 \\
\hline Brinley's & P. C. \& St. L. R & 1,124 \\
\hline Bristolville & A. \& P. R. R.. & 927 \\
\hline Broa & N. Y., Pa. \& O. R. & 1,032 \\
\hline Brookv & P. C. \& St. L. R. R & 1,033 \\
\hline Brownh & L. S. \& M. S. R. & 640 \\
\hline Brown's... & Cin. \& P. R. R . & 683 \\
\hline Do.... & P. C. \& St. L. R. R ... & 1,060 \\
\hline $\begin{array}{l}\text { Do........... } \\
\text { Bruce }\end{array}$ & I. B. \& W.R.R.... & 855 \\
\hline Bruce........ & C., T. Va. \& W. R. & 989 \\
\hline Bruss ............ & I. B. \& W. R. R... & 1,092 \\
\hline n & L. S. \& M. S. R. R & 765 \\
\hline tel $\ldots . . . . .$. & $\begin{array}{l}\text { C. } \& \text { H. V.R. R...... } \\
\text { M. \& C. R. R }\end{array}$ & 687 \\
\hline $\sin \ldots \ldots \ldots$ & M. \& C. R. R & 920 \\
\hline us $\ldots . . . . .$. & P., Ft. W. \& C. R. R .. & 930 \\
\hline ink .... & N. Y.,Pa. \& O.R. R... & 958 \\
\hline ill ... & A. \& G. W. R. R $\ldots$ & 1,044 \\
\hline Butler ............. & C., T. V.\&W.R. R & 889 \\
\hline Junction... & P. C. \& St. L. R. R. & 1,091 \\
\hline Caldwell ......... & M. \& P.R.R & 736 \\
\hline & S. V.R.R $\ldots . .$. & 811 \\
\hline & B. \& S. W. R. R.. & 845 \\
\hline Caledonia .... & C., C., C. \& I. R. I & 996 \\
\hline a $\ldots \ldots \ldots$ & C. \& P.R.R... & 540 \\
\hline Goedetic Station...... & U. S. Lake Survey & 891 \\
\hline ennison ... & P. C. \& St. L. R. R & 570 \\
\hline Dover .... & Cin. \& P. R. R.... & 884 \\
\hline Fulton ... & C.,T. V.\& W. R. R .. & 947 \\
\hline old........ & N. Y., Pa. \& O. R.R .. & 1,160 \\
\hline & V.R.R. R & 1,031 \\
\hline (mouth of Rocky Fork). & B. \& S.W.R.R.... & 778 \\
\hline on Hill ..... & C. \& H. V. R. R... & 696 \\
\hline Cardington ..... & C., C., C. \& I. R. R .. & 1,011 \\
\hline $\mathrm{Ca}$ & S. D. \& C. R. R .. & 818 \\
\hline & $\begin{array}{l}\text { C. H. \& D. R. R.... } \\
\text { C. \& H.V.R.R. }\end{array}$ & $\begin{array}{l}696 \\
835\end{array}$ \\
\hline roll...... & $\begin{array}{l}\text { C. \& H. V.R.R..... } \\
\text { C.. C. C. \& I. R. R }\end{array}$ & $\begin{array}{l}835 \\
551\end{array}$ \\
\hline $\begin{array}{l}\text { Carthage } \\
\text { Catawba }\end{array}$ & $\begin{array}{l}\text { C., C., C. \& I. R. R } \\
\text { C., C., C. \& I. R. R } \ldots \ldots \ldots \ldots\end{array}$ & 1,065 \\
\hline (2) & T. W. \& W. R. R.......... & 723 \\
\hline
\end{tabular}




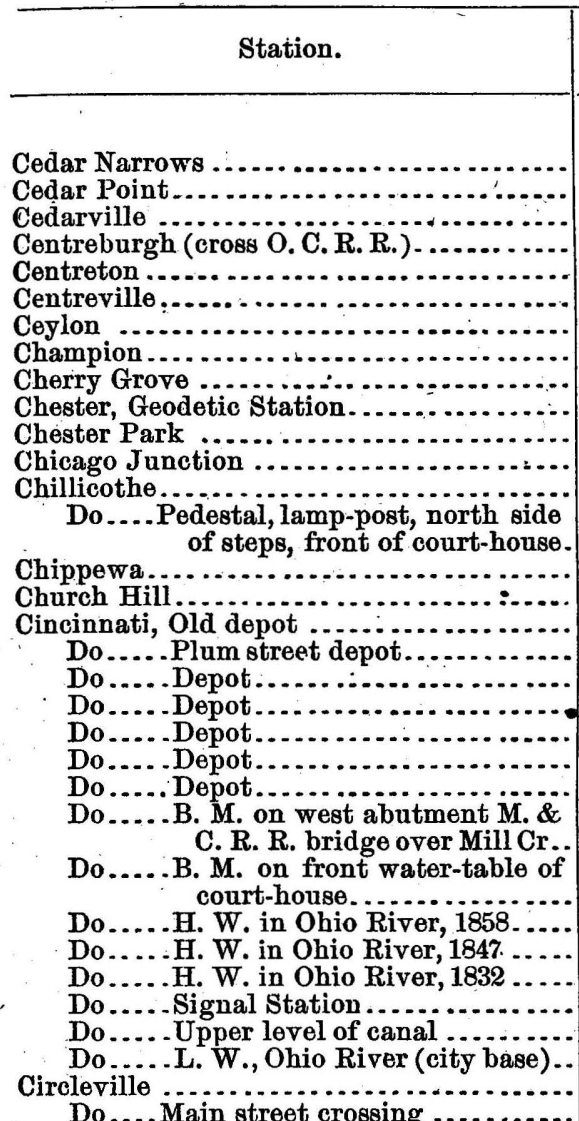

Do.... Main street crossing

Do ....C. \& M. V. R. R

Claridon, Geodetic Station

Clarkesville.

Clarks

Claysville.

Cleveland

Do ..Union Depot...................

Do ... Machine shop ...................

Do ...Euclid avenue ..................

Do .. Newburgh ......................

Do ... Signal Statiou ... ...............

Cleves ..................................

Clinton ...................................

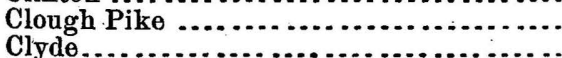

Coburg .................................

College Corner

Collins.

Colnmbia

$$
\text { Do }
$$

Authority.

Elevation.

M. \& P. R. R.

Cin. \& P.R. R ...............

P.C.\& St. L. R. R.

C., Mt. V. \& Del. R. R

B. \& O. R. R

Cin. \& P. R. R

L. S. \& M. S. R. R...........

Ash. \& P.R. R

Cin. \& P. R.R. .

U. S. Lake Survey

C., C., C. \& I. R. R

B.P. \& C. R. R

D.\& S. E. R. R

U. S. C. \& G. S.

C. T. V.\&W.R.R.

N. Y., Pa. \& O. R. R

C. I., St. L. \& C. R. R

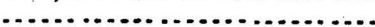

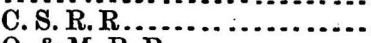

O. \& M. R. R

C. N.R. R

C. C. C. \& $\mathrm{I} \ddot{R} . \mathrm{R}$

U. S. C. \& G. S................

Feet.

638

1,035

1,209

873

870

609

932

847

1,292

508

901 .

638

638

1,056

1,017

501

498

503

494

545

500

509

U. S. C. \& G. S.

495

M. \& C. R. $\mathrm{R}$

M. \& C. R. R

U. S. Signal Office

Miami Canal

M. \& C. R. R.

C. \& M. V. R. R

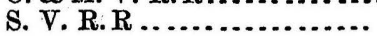

U. s. Lake Survey

C. \& Z. R. R

I., $B \& W$. R. R

P. C. \& St. L. R. R

A. \& G. W. R. R

Cin. \& P. R.R

Cin. \& P. R. R

Cin. \& P. R. R

Cin. \& P. R. R ......................

U. S. Signal Office.

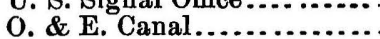

C., I., St. L. \& C. R. R

C., Mt. V. \& Del. R. R.....

C. \& P. R. R . $\ldots \ldots \ldots \ldots \ldots$

L. S. \& M. S. R. R..............

B. P. \& C. R. R........

C. , H. \& D. R

L. S. \& M. S. R. R

C., C., C. \& I. R. R

P. Ft. W. \& $\mathrm{C}, \mathrm{R}, \mathrm{R}$

Columbiana

Columbus, Union Depot.

Do.... Signal Station

Columbus Grove.

Condit.

C. \& H. V. R. R...........

U. S. Signal Office

C., H. \& D. R. R..........

C., Mt. V. \& Del. R. R......

Conesville

P.. C. \& St. L. R. R ........

\section{7}

484

511

511

620

596

440

720

682

690

, 1,366

833

1,080

756

598

583

629

668

797

690

586

497

959

890

691

787

990

879

802

503

1,113

748

805

752

1,086

738

Conneaut

L. S. \& M. S. R. R 


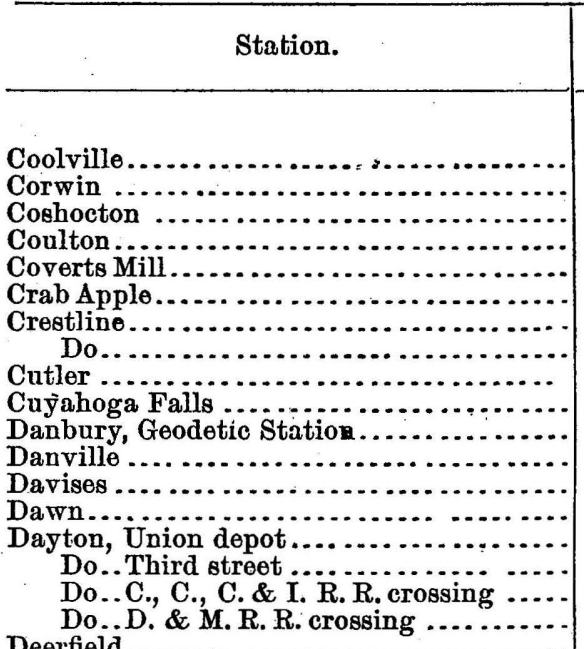

Deerfield

Defiance.

Do...(lock)

Do...(lock)

Do.. Manmee River

De Graff

Delaware

Delhi

Delphos (surface of Miami Canal)

Delta ..(lock) ...........................

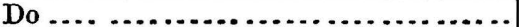

Dennison ................................

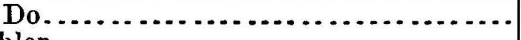

Deshler ........................................

Do..............................

Dexter City.

Dodson's

Dorset

Do

Double Roscoe

Doughtews

Dover

$$
\text { Do... (lock) }
$$

Dresder

Dresden Junction (at connection with P., C. \& St. L. R. R) ....................

Dudley .

Duvall's

Eagleville

Earlville...

East Akron

East Liverpool

Easton

East Orwell.

East Rochester

Eden

Edgerton .....................................

Edinburgh...........................

Edison

Edwards...........................

Eldorado

Elliottsville

Ellis .
Authority.

M. \& C. R. R.

P., C. \& St. L. R. R ........

P., C. \& St. L. R. R..........

D.\& S. E. R. R............ 718

Ash. \& P. R. R ............ $\quad 790$

B. \& S. W. R. R ...........

C., C., C. \& I. R. R ......... 1,155

P., Ft. W. \& C.R.R......... 1,162

M. \& C. R. R .............. 777

C., Mt. V. \& Del. R. R

U. S. Lake Survey.

C., Mt. V.\& Del. R. R

S. V.R.R.

C., C., C. \& I. R. R.

C.,H.\& D. R. R

N.'Y., Pa. \& O.R.R

Miami Canal

C. \& Z.R. R

W., St. L.\& P. R.R

O. \& $\mathrm{E}$ Canal $\mathrm{R}$

O. \& E. Canal .......................

W. \& E. Canal...............

T., W.\& W.R.R ..............

C., C., C. \& I. R.R.

C., C., C.\& I. R. R.

C., I., St. L. \& C. R. R
P., Ft. W. \& C. R. R..

Miami Canal

L., S. \& M. S. R. R.

P., C. \& St. L. R. R
N.Y., Pa. \& O. R. R

B. $\mathrm{P}$ \& $\mathrm{C}, \mathrm{R}, \mathrm{R} \ldots$

C., H. \& D. R. R...........

M. \& P. R. R.

P., C. \& St. L. R. R

L. S. \& M. S. R. R.

L. S. \& M. S. R. R.

O. \& E. Canal G. W. R. R

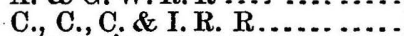

O.\& E. Canal ..................

P., C\&\& St. L. R. R.........

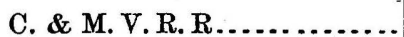

$M, \&$ R

Scioto V.R.R

A. \& P. R. R

C. \& P. R. R

V.R.R.

C. \& P. R. R

C. T. V. $\&$. R.

C., T. V. \& W. R. R.......

Ash. \& P. R. R

Cin. \& P. R. R

C., C., C. \& I. R. R.

L. S. \& M. S. R. R............

Ash. \& P. R. R

C., 'T. V.\& W. R. R ..............

C. \& H. V. R. R .

P. C. \& St.L.R. $\mathbf{R}$

Cin. \& P. R. R

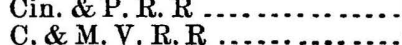

1,011

594

968

567

1,018

743

756

752

739

650

712

697

670

678

667

648

989

903

502

781

784

819

504

1,308

855

721

594

690

1,051

1,018

988

755

973

971

879

735

768

709

714

792

1, 091

967

693

961

937

1,082

975

830

780

689

756

1, 178

638 


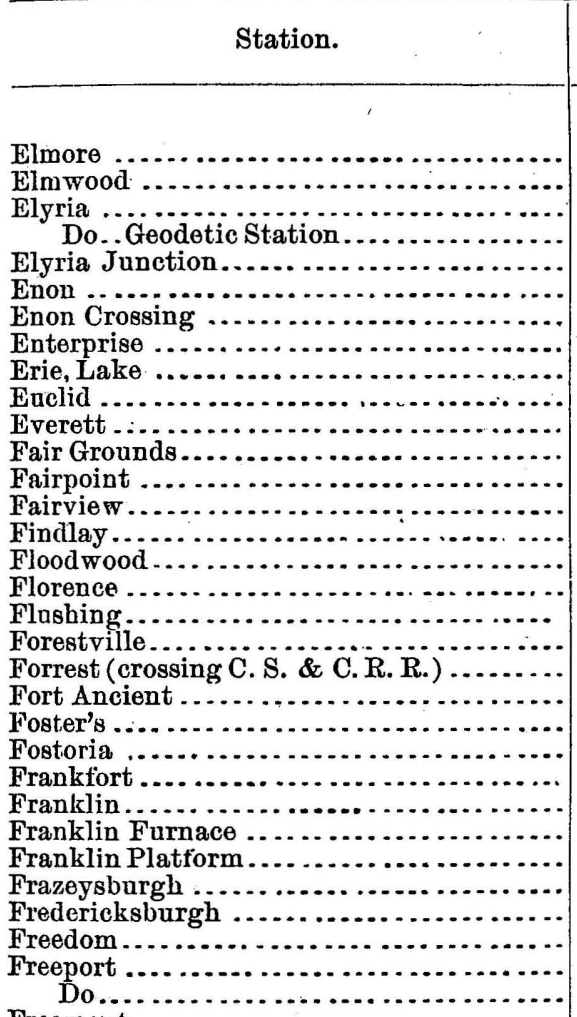

Freemont

Fruit Hill

Galena

Galion

Do..C., C., C. \& I. R. R. crossing

Do..C., C., C. \& I. R. R., Ind'polis Div

Gambier

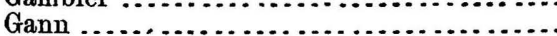

Gano

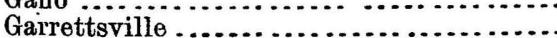

Geneva ......

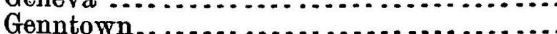

Genoa.

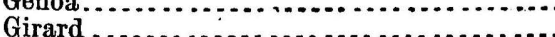

Glade Run . ..........................

Glendalo.

Glen Este ............................

Gnadenhutten

Goes

Gore.

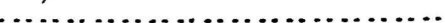

Goshen

Gould's

Grafton

Do

Do..Geodetic Station

Graytown.

Green

Greencamp $(\operatorname{tank})$

Greendale.

Greenfield (crossing of Paint Creek)

Greentown

Greenwich

L. S. \& M. S. R. R

Feet.

Scioto V.R. R

L. S. \& M. S. R. R

U. S. Lake Survey

L. S. \& M. S. R. R

N. Y., Pa.\& O. R. R

P. C. \& St. L. R. R

C. \& H. V.R. R

U. S. Lake Survey

L. S. \& M. S. R. R

V. R.R

Scioto V. R. R

C., T. V. \& W. R. R

P., C. \& St. L. R. R

L. E. \& L. R. R

C. \&, H. V. R. R .

P., C. \& St. L. R. R

C., T. V.\& W. R.R

Cin. \& P. R. R .

P., Ft. W. \& C. R. R

P., C. \& St. L. R. R

P., C. \& St. L. R. R

L. E. \& L. R. R

D. \& S. E. R. R .

C., C., C. \& I. R. R

O.R. R. R

N. Y., Pa. \& O. R. R

P., C. \& St. L. R. R

C., Mt. V. \& Del. R.

N. Y., Pa. \& O. R. R

C., T. V. \& W. R. R

L. S. \& M. S. R. R .

Cin. \& P. R. R

C., Mt. V. \& Del. R. R

C., C., C. \& I. R. R

N.Y.,Pa. \& O.R. R

N. Y., Pa. \& O. R. R

C. Mt. V. \& Del. R. R

C. Mt. V. \& Del. R. R

C. C. C. \& I. R. R

A. \& G. W. R. R

L. S. \& M. S. R. R

C. N. R. R

L. S. \& M. S. R. R

A. \& G. W. R. R

P. C. \& St. L. R. R

C. H. \& D. R. R

Cin \& $\mathrm{P} R \mathrm{R}$

O.\& E. Canal

P. C. \& St. L. R. R

C. \& H. V.R. R

C. T. V.\& W. R. R

P. C. \& St. L. R. R

C., C., C. \& I. R. R

C. T. V. \& W. R. R.

U. S. Lake Survey

L. S. \& M. S. R. R.

N. Y.. Pa. \& O.R. R

N. Y., Pa. \& O.R. R

C. \& H. V. R. R............ $\quad \begin{aligned} & 709 \\ & \text { M }\end{aligned}$

M. \& C. R. R ............. $\quad 1,108$

V. R. R ...................... $\quad 1,1,037$

627

728

769

, 013

782

669

1,140

1,118

940

669

600

782

750

549

1,051

751

1,150

688

882

810

923

1, 168

1,171
1,166

965

874

661
019

668

885

029

865

977

640

830

863

763

853

679

703

801

954

601

1,129

20

09
108
037




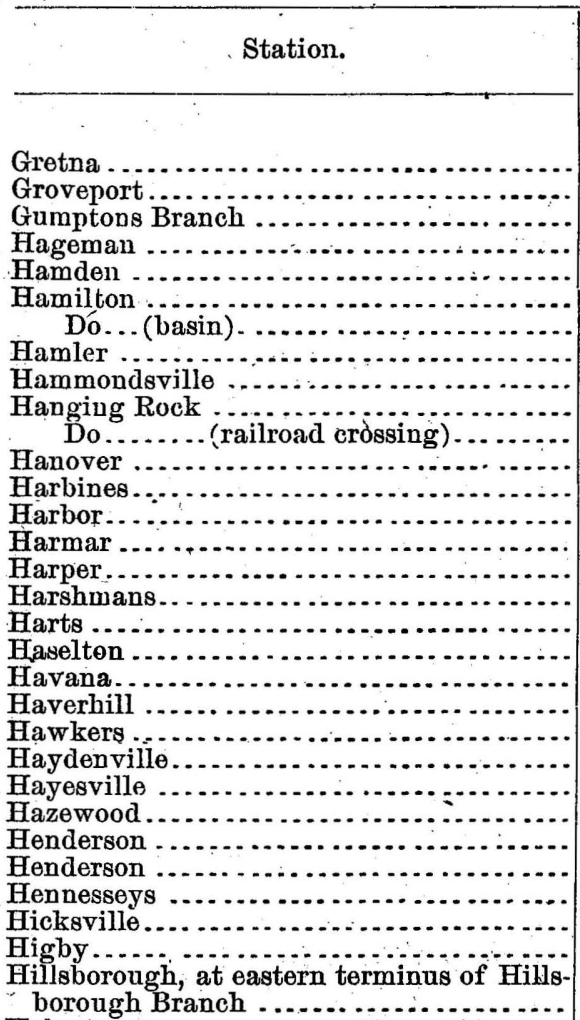

Holgate

Holland

Hollandsburgh

Holloway .

Homeworth

Hookers.

Hopetown

Houston

Howard

Hoyt's Corners

Hubbard

Hudson

Huron

Idlewild

Independence

Do....... (Lock)

Ironton (Ironton R. R., last crossing)

Irwin.

Ivanhoe

Jackšon

Jacobsburgh

Jamestown

Jefferson

Jerusalem

Johnson's

Jones' Station

Judds

Justus

Kansas...

Kelly's Island, Geodetic Station
C., C., C. \& I. R. R

C. \& H. V.R. $R$

C. \& Z. R. R

M. \& C. R. $R$

C. H. \& D. R. R...........

Miami Canal

B. P. \& C.R. R

Cin. \& P.R. R

Scioto V.R. K

Scioto V.R.R

P. C. \& St. L. R. R

P. C. \& St. L. R. R ..............

Ash. \& P. R. R................

M. \& C. R. R

C., C., C. \& I. R. R

C., C., C. \& I. R. R.

C. T. V.\& W. R. R

Ash. \& P. R. R.

B. \& O. R. R.

S. V. R. R

P. C. \& St. L. R. R.

C. \& H. V. R. R

Scioto V.R. R

C. N. R. R

C., C. C. \& I. R.R

C., T. V. \&.W. R. R

P. C. \& St. L. R. R

B., P. \& C. R. R

Scioto V. R. R.

M. \& C. R. R

B., P. \& C. R. R...

L., S. \& M. S. R. R

I., B. \& W. R. R.

C., T. V. \& W

C. \& H. V. R. $\mathrm{R}$

Scioto V. R. R

C., C. C. \& I. R. R

C., Mt. V. \& Del. R. R

B., P. \& C. R. R

A. \& G. W. R. R

Cin. \& P. R. R..

L. S. \&. M. S. R. R
C., C. C. \& I R. R

C. N. R. R

V. R. R

O. \& E. Canal

Scioto V. R. R

C., C. C. \& I. R. R

B. \& S. W. R. R

C. N. R. R.

B. \& S. W. R. R

D. \& S. E. R. R.

L. S. \& M. S. R. R

B. \& S. W. R. R.

Scioto V. R. R.

C., H. \& D. R. R

Cin. \& P. R. R

C., T. V. \& W. R.

U. S. Lake Survey
Elevation.

Feet.

1,086

739

898

686

721

597

610

723

688

557

551

830

806

580

623

1, 299

783

693

831

813

553

915

704

716

842

656

851

1, 031

762

594

1,075

722

641

1,150

911

1,150

83 '

675

959

900

721

937

- 1,053

598

1,156

700

606

662

550

1, 012

932

664

761

1,330

1,071

941

1,300

554

672

884

994

731

614 


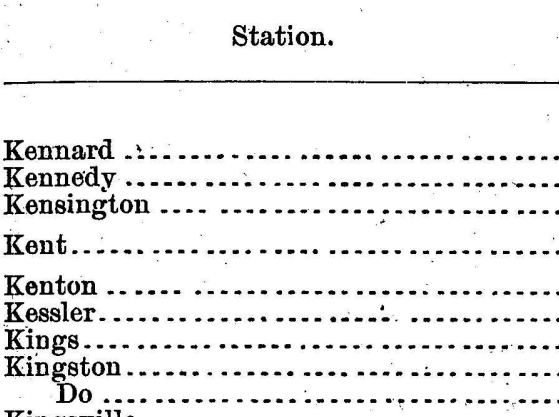

Kingsville.

Kinnikinnick

Kipton

Kneisley's

Krumwoy

Do

Lafferty ..

La Grange

$$
\text { Do ... }
$$

Lancaster

Laughlins

Laura

Lawrence Junction

Leavittsburgh

Do crossing of main line \& Mah Div

Lebanon

Leesburg

Leetonia Do..P., F. W. \& C. crossing

Leipsic

Leon

Levering -

Lexington

Liberty Do. (Tank)

Do. crossing O.\& M. \& P. Ft.W.\& C. R. R

Do .crossing. D. and M. Railroad

Limaville.

Lindsey.

Little Hocking

Little Mountain Geodetic station

Lockbourne

Locke

Lockport (Lock)

Lockville

Locust Switch.

Lodi.

Logan

London

Londonderry

Londonville

Long's Summit

Loveland

Do... B. $\mathrm{M}$. on east abutment $\ddot{M}$. \& $\mathrm{C}$ R. R. bridge over Little Miami

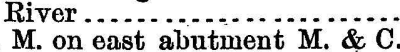
Do...B. M. on east abutment M. \& C.
R. R. bridgo over Little Miami River

N. Y., Pa. \& O.R.R C. N.R. R Civ. \& P. R. R

N. Y., Pa. \& Ohio R. R

S. D. \& C. R. R

I., B. \& W. R. R

P., C. \& St. L. R. R

N.Y., Pa. \& O.R.R

Scioto V. R. R.

L. S. \& M. S. R. R

Scioto Valley R. R

L. S. \& M. S. R. R

C., C. C. \& I. R. R

V.R.R

C., C. C.\& I. R.R

Cin. \& P. R. R.

C., T. V. \& W. R. R

Cin. \& P. R. R

C., C. C. \& I. R. R ..........

C. \& M. V. R. R .............

C., C. C. \& I. R. R ........

Cin. \& P. R. R.................

I., B. \&. W. R. R

Ash. \& P. R. R

A. \& G. W. R. R.

A. \& G. W. R. R

C. N. R. R.

M. \& C. R. R

P., Ft. W. \& C. R. R

N.' Y., Pa. \& O. R. R

C., H. \& D. R. R

L. S. \& M: S. R. R

C., C. C. \& I. R. R

C. \& Z. R. R

T., W. \& W. R. R

N. Y., Pa. \& O. R. R

C. H. \& D. R. R

P., Ft. W. \& C. R. R

Cin. \& P. R. R

L. S. \& M. S. R. R

P., C. \& St. L.R. R

M. \& C. R. R.....

U. S. Lake Surve

Scioto V. R.R.
B. P. \& C. R. R

O. \& E. Canal

C. \& H. V. R. R .

N. Y., Pa. \& O. R. R

S.D. \& C.R.R.

C. \& H. V. R. R .

P., C. \& St.L. R. R

M. \& C. R. R.

P., Ft. W. \& C. R. R

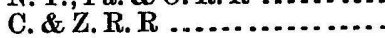

U. S. C. \& G. S.

P. C. \& St. L. R. R............
582

Elevation.

Feet.

1,174

743

1,130

$\{1,050$

1,273

925

612

1,097

770

672

672

853

802

1. 051

718

859

1,027

679

828

854

926

949

970

775

895

907

709

1,030

1,016

1,018

746

1,114

1,056

896

683

1,071

859

885

779

1,098

622

503

635

1,248

720

881

867

797

1,053

855

729

1,049

625

975

883

605

581 


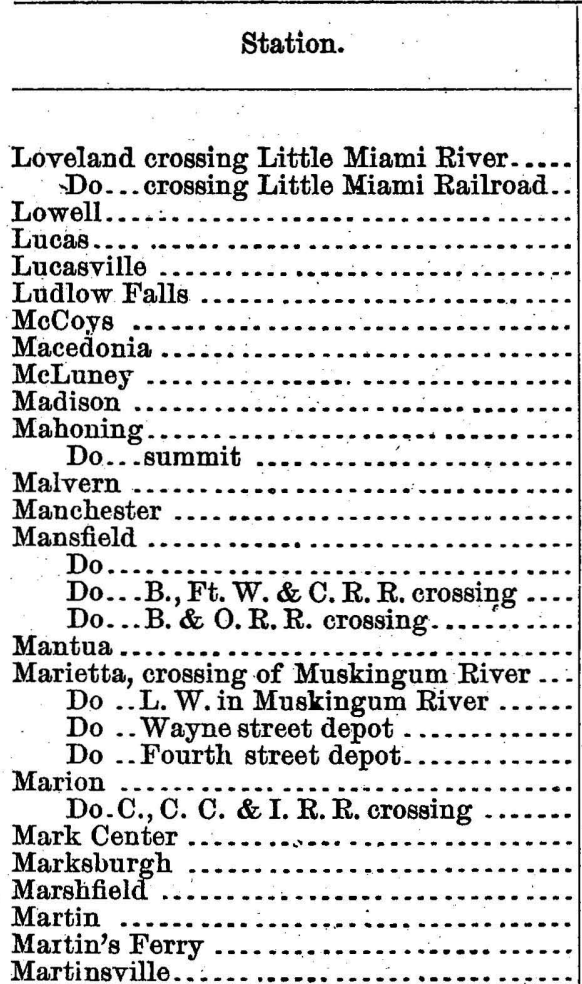

Do..B. M. on east abutment of M. \& C. R. R. bridge

Marysville

Mason

Massillon

Do.

Do.

Do... (Lock 5)

Do... (Lock 5a)

Masterville.

Mauds

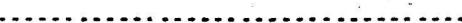

Maumee ..............................

Mechanicsburg .........................

Medina

Melbern

Mentor... . . . . . . . . . . . . .

Mesopotamia, Geodetic Station

Miami City

Miamisburgh

Do

Miamiville

Do...............................

Middle Bass, Geodetic Station

Middleton.

Do

Milford

Milford

$$
\text { Do. }
$$

Millbury Junction

Miller's ...

Millersburg

Millville ....

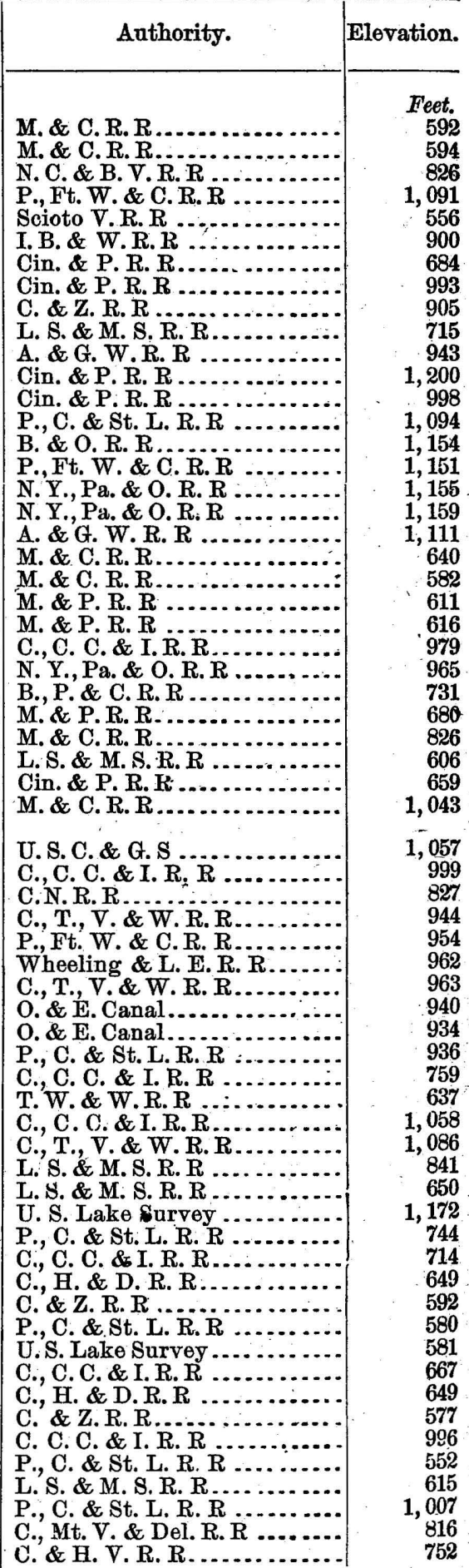




\begin{tabular}{|c|c|c|}
\hline Sta & Authorit & Elevation. \\
\hline & & \\
\hline Milton..... & N. Y., Pa. \& O. & 1,193 \\
\hline $\begin{array}{l}\text { Mineral City .. } \\
\text { Mineral Ridge. }\end{array}$ & M.\&C.R.R. & $\begin{array}{l}726 \\
.070\end{array}$ \\
\hline Minorva ....... & $\begin{array}{l}\text { N. } Y ., P A . \& O . R \\
\text { Cin. \& P.R. R. }\end{array}$ & $\begin{array}{r}970 \\
1,052\end{array}$ \\
\hline Mingo ......... & Cin \& $\mathbf{P}, \mathbf{R} . \mathbf{R}$ & 687 \\
\hline Monday Creok Sta & $\begin{array}{l}\text { N. Y., Pa.\& O. } \\
\text { C.\&. V.R.R. }\end{array}$ & $\begin{array}{r}1,206 \\
689\end{array}$ \\
\hline roo (summit). & D.\& S. E. R. R. & 1,063 \\
\hline Lonroeville ; ; & P., C.\&St.I.R.I & $\begin{array}{l}725 \\
800\end{array}$ \\
\hline Moonvillo & M.C.\& C.R.R. & 723 \\
\hline bridge over Re & U. S. C. \& G. S & \\
\hline Goorfield ...... & C., C. C.\& I.R.R & 1,021 \\
\hline ..... & $\begin{array}{l}\text { C. } \mathrm{N} \cdot \mathrm{R} \cdot \mathrm{R} \\
\text { C.\&M.V.R. }\end{array}$ & $\begin{array}{l}882 \\
666\end{array}$ \\
\hline Monlt & Cin. \& P.R.R.. & 1.103 \\
\hline armel. & Cin. \& P. R. R . & 899 \\
\hline iberty & C.; Mt. V. \& Del & $1 ; 215$ \\
\hline immit & Cin \& P, R. R & 885 \\
\hline Mou & 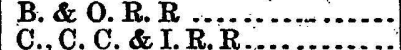 & $\begin{array}{r}991 \\
1.036\end{array}$ \\
\hline Mour & Cin. \& P.R.R..... & $\begin{array}{r}1,036 \\
766\end{array}$ \\
\hline Mun & Ash. \& P. R. R & 866 \\
\hline Nankin & N. Y.Pa. \& O. R. & 1,123 \\
\hline 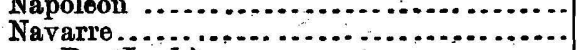 & $\begin{array}{l}\text { T. W. \& W. R. R } \\
\text { C., T. V.\& W. R }\end{array}$ & $\begin{array}{l}680 \\
993\end{array}$ \\
\hline Do.(Lock) ... & O.\&E. Canal ....... & 924 \\
\hline ville......... & $\begin{array}{l}\text { Ash. \& P. R. R } \\
\text { C. \& H. V. R.R }\end{array}$ & $\begin{array}{l}840 \\
6 \pm 2\end{array}$ \\
\hline Newark....... & P. C. \& St. L. 1 & 819 \\
\hline orth and & & 791 \\
\hline $\begin{array}{l}\text { New Baltimore . } \\
\text { New burgh ..... }\end{array}$ & B. P. \& C.R. R & $\begin{array}{l}740 \\
813\end{array}$ \\
\hline Do .... grad & A. \& & 748 \\
\hline $\begin{array}{l}\text { New Carlisle ... } \\
\text { Newcastle (Loci }\end{array}$ & I. B. \& W. R. R & $\begin{array}{r}89 \\
859\end{array}$ \\
\hline Newcomerstow & P., C. \& St. L. R.R & 796 \\
\hline Do......... & O. \&E. Canal . & 800 \\
\hline $\begin{array}{l}\text { New Eng. } \\
\text { New Hav }\end{array}$ & $\begin{array}{l}\text { M. } \\
\text { B. } 8\end{array}$ & $\begin{array}{l}808 \\
918\end{array}$ \\
\hline Now Hol & C. \& & 876 \\
\hline tton. & C. & 897 \\
\hline New Lisbon .... & N. Y., $\mathrm{Pa} . \& \mathrm{O}_{\mathrm{D}} \mathbf{R}$. & 958 \\
\hline New London .... & C., C.C. \& I. R. R .... & 980 \\
\hline $\begin{array}{l}\text { New } \mathbf{L} \\
\text { Now }\end{array}$ & Ash., P. R. R & $\begin{array}{l}906 \\
959\end{array}$ \\
\hline New Par & \& St. I. R. R & 1,020 \\
\hline Now Philadelphia & V. \& W. R. R & 874 \\
\hline $\begin{array}{l}\text { Do } \ldots . . . . . . . . \\
\text { Newport }\end{array}$ & 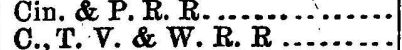 & $\begin{array}{l}804 \\
870\end{array}$ \\
\hline lew Portage .... & N.'Y., Pa. \& O.R. R & 1,204 \\
\hline New Straitsville. & C. \& H.V.R.R & \\
\hline Newtown ......... & P., C.\& St. L. R. R .... & 494 \\
\hline 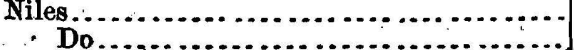 & 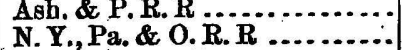 & $\begin{array}{l}864 \\
889\end{array}$ \\
\hline Niles Junction with N. \& N. L. R. R. & A. \& G.W.R.R. R. & 890 \\
\hline North Bend $\ldots \ldots \ldots \ldots \ldots$ & C., I. St. L.\& C. R. R........ & \\
\hline ld Sur & 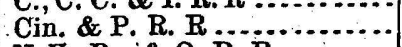 & 1,043 \\
\hline …..... & N. Y., Pa \& O. R. R & 1,082 \\
\hline & L. S. \& M. S. R. R..... & $\begin{array}{l}719 \\
626\end{array}$ \\
\hline & Ash. \& P. R. R . & \\
\hline
\end{tabular}




\begin{tabular}{|c|c|c|}
\hline & Anthority. & Elevation. \\
\hline Oak Harbor & & $\begin{array}{r}\text { Feet. } \\
596\end{array}$ \\
\hline & L. S. \& M. S. R. & $\begin{array}{l}596 \\
815\end{array}$ \\
\hline Odell, Lake & Toner $\ldots . . . . . .$. & 924 \\
\hline ldtown .... & P., C. St. L. \& P & 834 \\
\hline $\begin{array}{l}\text { Old Portage } \\
\text { Olive Branch }\end{array}$ & $\begin{array}{l}\text { O.\& E. Canal -- } \\
\text { Cin.\& P.R. R.- }\end{array}$ & $\begin{array}{l}766 \\
832\end{array}$ \\
\hline Olmstead Fal & L. S. \& M. S. R. & 770 \\
\hline Do.... & U. S. Lake Surve & 794 \\
\hline Imstead .. & C., C. C. \& I.R. K & \\
\hline reida...... & Cin. \& P.R. R & 1,015 \\
\hline ntario.. & N. Y., Pa. \& O. R. & 1,377 \\
\hline$\ldots$ & $\begin{array}{l}\text { A. \& G. W. R. R } \\
\text { C. \& H. V. R. R . }\end{array}$ & $\begin{array}{l}945 \\
698\end{array}$ \\
\hline $\begin{array}{l}\text { Orbiston ... } \\
\text { Oreville... }\end{array}$ & C. \& H. V.R.R & $\begin{array}{l}698 \\
730\end{array}$ \\
\hline Orrville ............ & C., Mt. V. \& Del & 1,056 \\
\hline Do.crossing $\mathrm{C}$ & P., Ft. W. \& C. R. R & 1,058 \\
\hline Orwell ... & N. C. \& B. V. R. & 936 \\
\hline orn.......... & $\begin{array}{l}\text { C., C. C. \& I. R. R } \\
\text { N. Y., Pa. \& O. R. }\end{array}$ & $\begin{array}{l}830 \\
829\end{array}$ \\
\hline trander... & C., C. C.\& I. R. R. & $\begin{array}{l}829 \\
934\end{array}$ \\
\hline & C., H. \& D. R. R & 713 \\
\hline Oxford....... & C., H. \& D. R. R & 918 \\
\hline & P., C. \& St. L. R. & 793 \\
\hline ark & B. \& S. W. R. R & 1,265 \\
\hline alestine.... & $\begin{array}{l}\text { L. S. \& M. S. R. I } \\
\text { P. Ft. W. \& C. R }\end{array}$ & $\begin{array}{r}650 \\
1,014\end{array}$ \\
\hline sco ....... & C., T. V. \& W. R. & $\begin{array}{r}1,014 \\
731\end{array}$ \\
\hline son ... & C., T. V. \& W & 771 \\
\hline$\ldots$ & C., T. V.\& W. I & 439 \\
\hline mberton. & C., C. C. \& I. R. & 1,063 \\
\hline on.. & P. C. \& St. L. R. R . & 519 \\
\hline Peninsula ....... & V. R. R ... & 699 \\
\hline Do ... (Lock) & O. \& E. Cana & 703 \\
\hline Peoria .......... & N. Y., Pa. \& O. I & 1,044 \\
\hline gh & $\begin{array}{l}\text { L. S. \& M. S. R. I } \\
\text { C. H. \& D. R. R. }\end{array}$ & $\begin{array}{l}707 \\
622\end{array}$ \\
\hline $\begin{array}{l}\text { Perrysburgh } \\
\text { Petersburgh Coal Grove I }\end{array}$ & $\begin{array}{l}\text { C. H. \& D. R. R. } \\
\text { Scioto V. R. R... }\end{array}$ & $\begin{array}{l}622 \\
558\end{array}$ \\
\hline urgu Coal & L.S. \& M.S. R & 753 \\
\hline ........... & त. W. R. R. & 918 \\
\hline e's....... & P. C. \& S & 1,029 \\
\hline$\ldots \ldots$. & N. Y., Pa. \& O. R. R.... & 982 \\
\hline on $\ldots \ldots \ldots$ & Sciot & $\begin{array}{l}570 \\
874\end{array}$ \\
\hline r.................. & $\begin{array}{l}\text { M. \& C. R. R } \\
\text { C. H. \& D. R. R }\end{array}$ & $\begin{array}{l}874 \\
918\end{array}$ \\
\hline ille & $\begin{array}{l}\text { C., H. \&. D. R. R .... } \\
\text { C.\& Z. R. R......... }\end{array}$ & $\begin{array}{l}918 \\
519\end{array}$ \\
\hline & P. C. \& St. L. R. R. & 492 \\
\hline Plains. & M. \& C. R. R...... & 902 \\
\hline Ridge... & C. N. R.R. & 658 \\
\hline th ....... & B. \& O.R. & 993 \\
\hline & L. S. \& M. S. R. R..... & 854 \\
\hline .......... & N. Y., Pa. \& O. R. R... & 1,241 \\
\hline & A. \& G. W.R.R... & 1,043 \\
\hline ......... & & 763 \\
\hline aton ... & . R. & 581 \\
\hline & Cin. & 679 \\
\hline uth (S. V. Depot) & Sciot & 533 \\
\hline - junction with M. \& C. R & & 538 \\
\hline 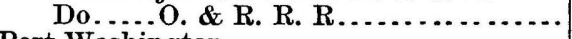 & & 538 \\
\hline Port Washington & P.C.\& St. L.R.R...... & 813 \\
\hline Do...........(Lock) & O. \& E. C & $\begin{array}{r}824 \\
1093\end{array}$ \\
\hline $\begin{array}{l}\text { Pottersbnrgh ......... } \\
\text { Prouts ............. }\end{array}$ & $\begin{array}{l}\text { N. Y., Pa. \& O. R. R. } \\
\text { B. \& O.R. R........ }\end{array}$ & $\begin{array}{r}1,093 \\
708\end{array}$ \\
\hline 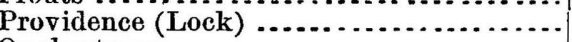 & O.\& E. Canal......... & 637 \\
\hline Quakertown & Ash. \& P. R. R ...... & 817 \\
\hline
\end{tabular}




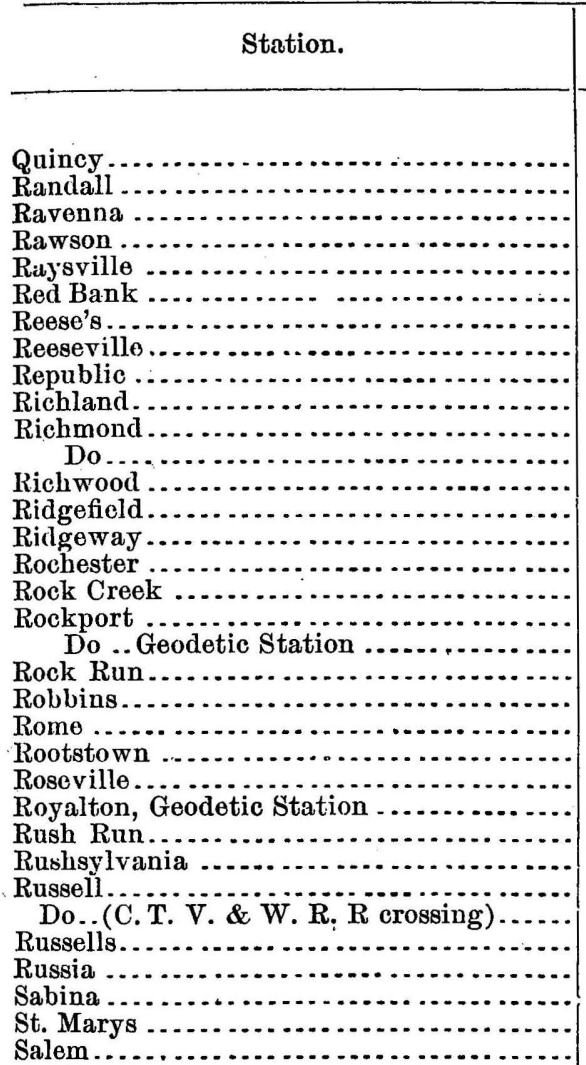
Do.

Salina

Salinesville

Salt Creek

Sandusky, City base

Do..... depot .

Do.... depot

Do depot

Do .. . Geodetic Station

Do... Signal Station

Sargents

Saville.

Saybrook

Schooleys

Sciotoville (junction with $\mathbf{M}$. \& $\mathrm{C}$. R. R)

Selma

Seymours

Shakerton

Sharon.

Sharonville

Shawville.

Shelby . Do.Junction

Shiloh

Shoups.

Sidney

Silver Creek

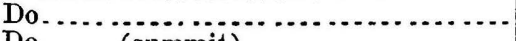
Do...... (summit)

Silverton.
C., C. C. \& I. R. R A. \& G. W. R. R Cin. \& P. R. K. P. C. \& St, L R. R Scioto V.R. R C. \& Z.R.R

B., P. \& C. R. R P., Ft. W. \& C. R. R P. C. \& St. L. R. R L. S. \& M. S. R. R. N. Y., Pa. \& O.R. R B., P. \& C. R. R C., C. C. \& J.. R. R C., C. C. \& I. R.R Ash. \& P. R. R . L. S. \& M. S. R. R U. S. Lake Survey . P., C. \& St. L. R. R N. Y., Pa. \& O.R. R Ash. \& P. R. R

Cin. \& P. R. R

C. \& Z. R. R

U. S. Lake Survey

Civ. \& P. R. R

C., C. C. \& I. R. R

C., T. V. \& W.R. R

N. Y., Pa. \& O. R. R P., C. \& St. L. R. R

C., C. C. \& I. R. R C. \& M. V. R. R

Miami Canal

M. \& P. R. R.

P., Ft. W. \& C. R. R C. \& H. V.R. R

Cin. \& P. R. R

D. \& S. E. R. R

City Engineer

L. S. \& M. S. R. R

S., D.\& C. R. R

B.\& O.R. R

U. S. Lake Survey

U. S, Signal Office.

Scioto V. R. R.

C., T. V. \& W. R. R ............

L. S. \& M. S. R. R

M. \& C. R. R

Scioto V. R. R

P., C. \& St. L. R. R

Ash. \& P. R.R

C. N. R. R

C., C., C. \& I. R. R

Scioto V. R. R....

L. S. \& M. S. R. R.

B. \& O.R. R

B. \& O.R. R

C., C., C.\& I. R. R

P., C. \& St. L. R. R

C., C., C. \& I. R. R

C., T. V. \& W. R. R

N.Y., Pa. \& O. R. R

D. \& S. E.R.R

C. N. R. R
Elevation.

Feet.

1,050

1,046

1,103

821

636

501

735

1,086

885

1,201

968

1,114

948

779

1, 059

935

846

884

781

758

982

884

1,123

, 783

1,272

679

1, 236

969

973

501

971

1,066

864

682

1,173

659

879

588

587

593

598

609

632

639

577

986

651

666

546

1,083

797

745

601

597

744

1,088

1,086

1,080

831

969

948

1,392

1,118

825 


\begin{tabular}{|c|c|c|}
\hline & Author & Elevation. \\
\hline relleys Station & & \\
\hline & C. \&. Z. R. R... & $\begin{array}{l}841 \\
901\end{array}$ \\
\hline ld... & P., C. \& St. L. I & $\begin{array}{r}773 \\
192\end{array}$ \\
\hline $\begin{array}{l}\text { thville } \\
\text { der.... }\end{array}$ & $\begin{array}{l}\text { P., Ft. W. \& C. R. R } \\
\text { N.Y., Pa. \& O. R.R }\end{array}$ & $\begin{array}{r}1,122 \\
900\end{array}$ \\
\hline & A. \&G. W. R.R. & 1,041 \\
\hline harleston. & $\begin{array}{l}\text { P., C. \& St. L. R } \\
\text { P., C. \& St. L. I }\end{array}$ & $\begin{array}{l}1,039 \\
1,126\end{array}$ \\
\hline ebanon... & 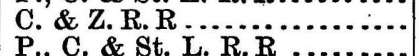 & $\begin{array}{r}652 \\
. \quad 628\end{array}$ \\
\hline affo & C., C., C. \& I. I & 1,033 \\
\hline vill & $\begin{array}{l}\text { Miami Canal } \\
\text { M. \& C. R. R . }\end{array}$ & $\begin{array}{l}8+7 \\
827\end{array}$ \\
\hline ld & P., C. \& St. L. H & 991 \\
\hline & $\begin{array}{l}\text { B. \& O. R.R } \\
\text { P., C. \& St. L. R. }\end{array}$ & $\begin{array}{r}\mathbf{1 , 1 7 6} \\
\mathbf{7 5 8}\end{array}$ \\
\hline & B., P.\& C. R. R & 731 \\
\hline & M. \& P. R.R $\ldots$ & $\begin{array}{l}629 \\
904\end{array}$ \\
\hline & Cin. \& P. R.R.R & 679 \\
\hline Do.......Wash & P., C. \& St. L. R. & 728 \\
\hline $\begin{array}{l}\text { tillwater................. } \\
\text { Donction }\end{array}$ & $\begin{array}{l}\text { C., T. V. \& W. R } \\
\text { P., C. \& St. L. R }\end{array}$ & $\begin{array}{l}863 \\
791\end{array}$ \\
\hline tiles .......... & Cin. \& P.R. R.. & 50 \\
\hline . & Scioto V. R. R . . & \\
\hline & C.\& Z. R. R.... & 1,008 \\
\hline$\cdots$ & $\begin{array}{l}\text { P., Ft. W. \& C. R. R } \\
\text { C., T. V. \& W. R. R }\end{array}$ & $\begin{array}{r}1,187 \\
917\end{array}$ \\
\hline .......... & Ash. \& P.R.R & 836 \\
\hline r, surfac & $\begin{array}{l}\text { L.S. \& M.S.R.R } \\
\text { N.Y., Pa. \& O.R.R. }\end{array}$ & $\begin{array}{r}715 \\
1,169\end{array}$ \\
\hline .... & O. \& H. V.R. R.... & 769 \\
\hline 's . & & 834 \\
\hline 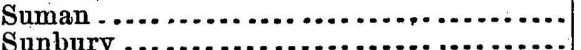 & $\begin{array}{l}\text { B., P. \& C. R } \\
\text { C., Mt. Y. \& }\end{array}$ & $\begin{array}{l}747 \\
970\end{array}$ \\
\hline & L., S. \& M. S. R. I & $\begin{array}{l}97 \\
68\end{array}$ \\
\hline & L. S. \& M. S. & 65 \\
\hline & N. Y., Pa. \& & 1,33 \\
\hline re.......... & N. Y. & 1,043 \\
\hline Park.... & $\begin{array}{l}\text { P., C. } \\
\text { O.\& }\end{array}$ & $\begin{array}{l}555 \\
662\end{array}$ \\
\hline $\begin{array}{l}\text { ock) } \\
\text { n, Geodetic station }\end{array}$ & $\begin{array}{l}\text { U. \& } \\
\text { U. S. }\end{array}$ & $\begin{array}{r}662 \\
1,307\end{array}$ \\
\hline & & 869 \\
\hline cks .... & & 617 \\
\hline & & 75 \\
\hline$\theta \quad \therefore \ldots \ldots \ldots$ & R. R... & 843 \\
\hline . & O.' & 583 \\
\hline & $\begin{array}{l}\text { L. } \mathbf{S} \\
\text { L. }\end{array}$ & \\
\hline & L. \& P: R. R & \\
\hline (Lock 1 , sur & $0 . \&$ & \\
\hline (Look 2) $\ldots \ldots \ldots \ldots$ & & \\
\hline ock 3) .... & & \\
\hline ock 4) $\ldots$. & & \\
\hline water of $\mathrm{k}^{5}$ aumee $\mathrm{Ri}$ & O. \& & \\
\hline 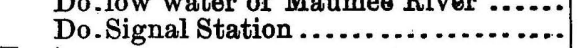 & U.S. Signal 0 & 651 \\
\hline 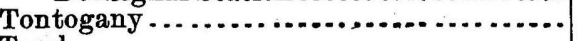 & C., H. \& D. R. & $65 ?$ \\
\hline & M. \& C. R. R... & \\
\hline Geodetic Station.... & U. S. Lake Survey & \\
\hline rento & P., C. \& St. L. R.R... & \\
\hline & O. \& E. Canal... & \\
\hline
\end{tabular}




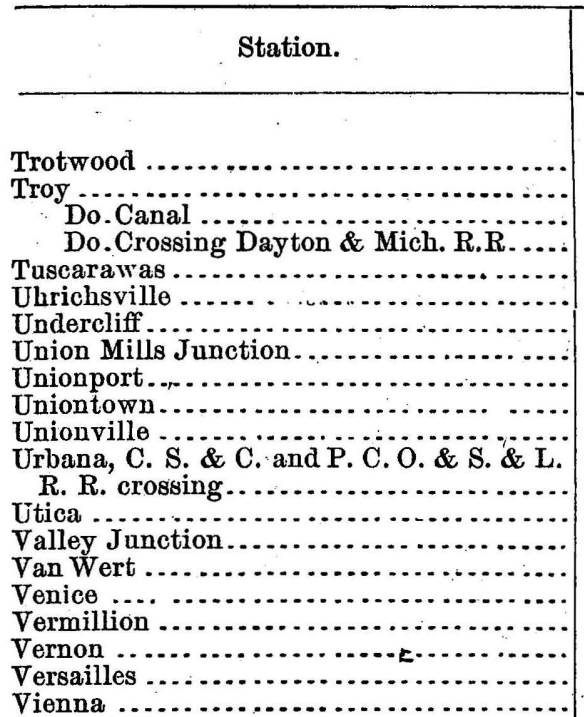

Vienna Junction

Vincent.

Wade.

Wadsworth

Wakeman

Walkerton Junction

Wapakoneta

Warren

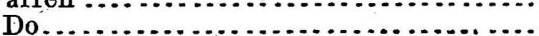

Warrensville, Geodetic Station.

Warwick.

Washington C. $\mathrm{H}$

$$
\text { Do. }
$$

Washingtonville

Wauseon

Waverly Depot.

Wayne

Waynesburgh

Weavers

Wegeo

Wellston.

Wellsville. Do.

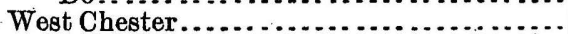

Westerville

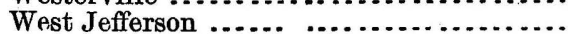

West Lafayetto

West Liberty

Weston

West Salem

West Wheoling Do ...... low water in Ohio River. .

Wetmore

\section{Wheeler}

Wheelersburgh

Whipple

White House

Whites Mill

Wickliffe

Williamsport

Williugton

Willoughby

P., C. \& St. L. R. R

C., H. \& D. R. R

Miami Canal

I., B. \& W. R. R.

C., T. V. \& W. R. R

P., C. \& St. L. R. R

P., C. \& St. L. R. R

B., P. \& C. R. R

V.R.R.

L. S. \& M. S. R. $\ddot{R}$

N. Y., Pa. \& O. R. R

C. N.R. R.

C., I., St. L. \& C. R. R

P., Ft. W. \& C. R. R

L.'S. \& M. S. R. R

L. S. \& M. S. R. R

C., C., C. \& I. R. R.

C., C., C. \& I. R. R

M. \& C. R. R

N. Y., Pa. \& O. R. R

M. \& C. R. R.

Cin. \& P. R. R

N. Y., Pa. \& O. R. R

L. S. \& M. S. R. R

B., P. \& C. R. R.

C. H. \& D. R.

Ash. \& P. R. R

M. \& C. R. R.

U. S. Lake Survey .

C., T. V. \& W. R. R

C. \& Z. R. R .

T. W. \& W. R. R.

N. Y., Pa.\& O. R. R

L. S. \& M. S. R. R.

Scioto V. R. R

C. \& Z. R. R .

Cin. \& P. R. $\mathrm{R}$

P., C. \& St. L. R . R

B. \& S. W. R. R

D. \& S. E. R. R

Cin. \& P. R. R

C., C., C. \& I. R. R.........

C., Mt. V. \& Del. R. R......

P., C., \& St. L. R. F .......

P., C. \& St. L. R. R.

S. D. \& C. R. R.

C., H. \& D. R. R .

N. Y., Pa. \& O. R. R

C., T. V. \& W. R. R

C., T. V. \& W. R. R .........

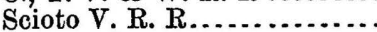

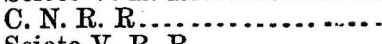

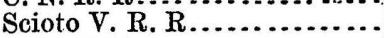

M. P. R. R.

T., W. \& W. R. R

B., P. \& C. R. R............

L. S. \& M. S. R. R.............

C. \& M. V. R. R ..........

C., C., C. \& I. R. R

L. S. \& M. S. R. R.

U. S. Lake Survey
Elevation.

Feet.

844

850

830

847

827

863

495

759

946

1,073

704

1,030

974

499

784

585

596

1,127

977

1, 130

869

774

495

1,349

862

719

876

875

671

1,214

958

983

660

1,059

768

574

764

981.

977

645

738

688

845

745

875

908

803

1, 099

666

1, 095

648

618

558

875

540

656

653

717

657

783

855

635

613 


\begin{tabular}{|c|c|c|}
\hline Station. & Authority. & Elevation. \\
\hline & & Feet. \\
\hline Wilmington $\ldots \ldots \ldots \ldots$ & C. \& M. V.R. R........... & 1,017 \\
\hline Winchester $\ldots \ldots \ldots \ldots \ldots \ldots \ldots \ldots \ldots \ldots \ldots$ & C. \& H. V. R. R . . . . . . & 771 \\
\hline Windham...$\ldots \ldots \ldots \ldots \ldots \ldots$ & N. Y., Pa. \&. O. R. R....... & 964 \\
\hline 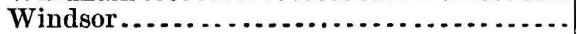 & N. Y.,Pa. \& O.R. R........ & 1,067 \\
\hline Winona...$\ldots \ldots \ldots \ldots \ldots \ldots \ldots$ & C. \&. H. V. R. R ........ & 743 \\
\hline Woodburn...$\ldots \ldots \ldots \ldots \ldots \ldots \ldots \ldots$ & P. E. \& St. L. R. R......... & 533 \\
\hline 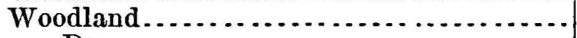 & N. Y., Pa. \& O.R. R....... & 950 \\
\hline Do........... & P., Ft. W. \& C. R. R........ & 1,245 \\
\hline Woodsfield $\ldots \ldots \ldots \ldots \ldots \ldots \ldots \ldots \ldots$ & B. \& S. W. R. R ........... & 1,173 \\
\hline Xenia $\ldots \ldots \ldots \ldots \ldots \ldots \ldots$ & P., C. \& St. L. R. R ......... & 928 \\
\hline Yellow Creek..$\ldots \ldots \ldots \ldots \ldots \ldots \ldots$ & O. \& E. Canal ................ & 758 \\
\hline 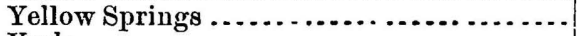 & P., C. \&. St. L. R. R....... & 978 \\
\hline York $\ldots \ldots \ldots \ldots \ldots \ldots \ldots \ldots \ldots \ldots \ldots$ & C., T. V.\& W. R. R........ & \\
\hline Youngstown...$\ldots \ldots \ldots \ldots \ldots \ldots$ & A. \& G. W. R. R . . . . . . . & 863 \\
\hline Zaleski........... & M. \& Co. R. R $\ldots \ldots \ldots \ldots$ & 721 \\
\hline Zanesville ....... & C. \& M. V. R. R ......... & 742 \\
\hline Zoar $\ldots \ldots \ldots \ldots \ldots \ldots \ldots \ldots \ldots \ldots \ldots \ldots \ldots$ & Cin. \& P. R. R . . . . . . . . & 887 \\
\hline Zoar Mills (lock) $\ldots \ldots \ldots \ldots \ldots \ldots \ldots \ldots$ & O. \& E. Canal ................ & 893 \\
\hline
\end{tabular}




\section{OREGON.}

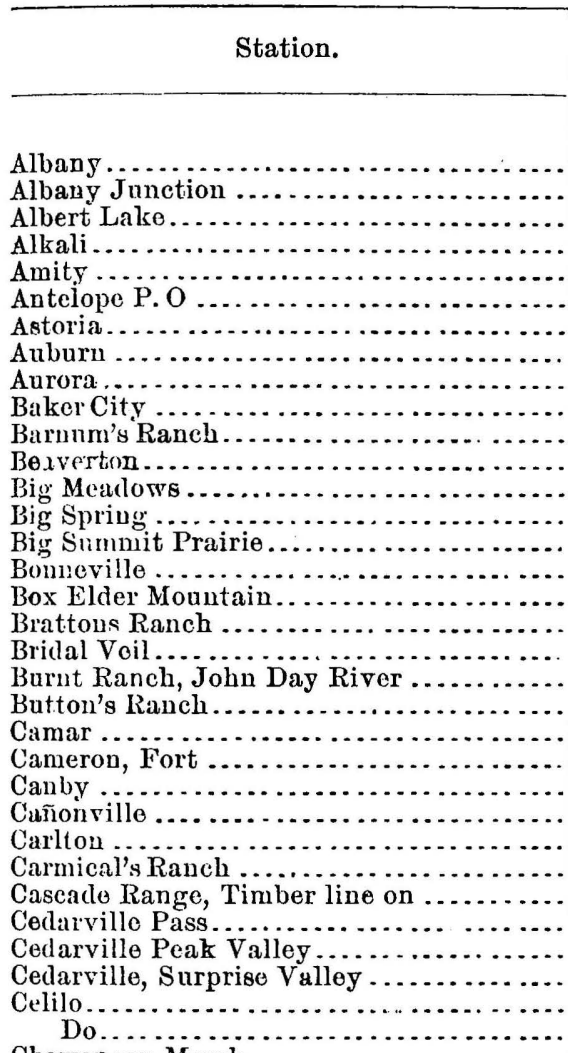

Chewancan Marsh.

Clackamas.

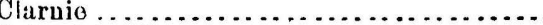

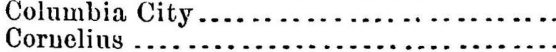

Corral Springs .......................

Corvallis.

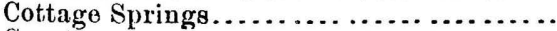

Coyote

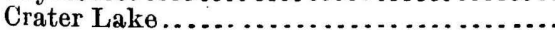

Creswell

Cross Hollows... . . . . . . . . . . . . . . . . . .

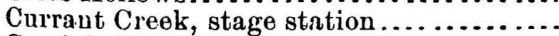

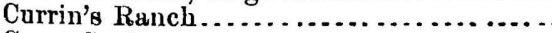

Curry Spring...

Dallas

Dalles, Tho

$$
\text { Do.................. }
$$

Dayton Junction

Derry ..........................

Deschutte's River Bridge

Diamond Peak ..........

Dillard \& Renshaw's Ranch Dilley

Authority. Elev

Oregon \& Cal R R Feet.

A. \& L. R. R ..............

Wheeler.................... 4,209

N. P.R. R ................. $\quad \begin{array}{r}232 \\ 232\end{array}$

W. \& O. \& O. C. R. R....... 183

Wheeler .................... $\quad 2,845$

Med. Dept. U. S. A .......... $\quad 50$

Smithsonian Inst ........... $\quad 3,30$ n

Oreg. \& Cal. R. R............ 218

Oreg. R. R. \& Nav. Co....... $\quad 3,418$

Wheeler ................... $\quad 1,795$

W. \& O. \& O. C. R. R ....... $\quad 212$

Wheeler .................. 4,124

Wheeler .................... 4,553

Wheeler ................. 5,124

N. P. R. R ...............

Wheeler .................... $\quad 9,541$

Wheeler $\ldots \ldots \ldots \ldots \ldots \ldots \ldots \ldots . .4, \quad 4,495$

N. P. R. R ................. 46

Wheeler ................... $\quad 1,561$

Whooler .................. 4, 237

Powell ................... 44

Wheeler .................. 6,057

Oreg. \& Cal. R. R ........... 175

Pacific R. R. Reports ........ $\quad 516$

W. \& O. \& O. C. R. R....... 222

Wheeler ................... $\quad 2,736$

....................... 7,000

Wheeler ................. 6,356

Wheeler................. $\quad 8,308$

Wheeler................. $\quad 4,675$

Oreg. R. R. \& Nav. Co...... 142

N.P.R.R.,............... 160

Wheoler................. 4,336

Oreg. \& Cal. R. R . ......... 134

N.P.R.R............... $\quad 214$

N.P.R.R.............. 90

W. \& O. \& O. C. R. R ...... 200

Wheeler.................. $\quad 4,569$

W. \& O. \& O. C. R. R ...... 253

Wheeler................. $\quad 5,718$

Powell .................... 257

Wheeler.................. $\quad 7,143$

Oreg. \& Cal. R. R . . . . . . . . $\quad 565$

Wheeler................. $\quad 3,197$

Wheeler................... $\quad 2,142$

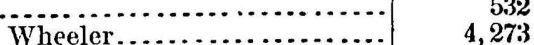

Wheeler................. 135

N. P.R. R................ 106

Oreg. R. R. \& Nav. Co....... 77

Oreg. R. R. \& Nav, Co....... 1,141

W. \& O. \& O. C. R. R ....... 214

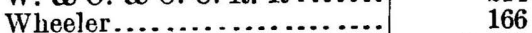

Wheeler................. $\quad 2,563$

Wheeler ................ 8,807

Wheeler................. 4,657

W. \& O. \& O. C. R. R...... 


\begin{tabular}{|c|c|c|}
\hline Stat & Authority. & Elevation. \\
\hline & & Febt. \\
\hline $\begin{array}{l}\text { Drains } \\
\text { Drew Valley Post-Office }\end{array}$ & $\begin{array}{l}\text { Oreg: \& Cal. R. R ... } \\
\text { Wheeler............. }\end{array}$ & $\begin{array}{r}318 \\
4,953\end{array}$ \\
\hline Duke ........................................... & U.S.C.\& G.S....... & \\
\hline Durand's Ranc & Wheeler......... & 4,312 \\
\hline ast Portland & Oreg. \& Cal. R. R & 52 \\
\hline bla.......... & Toner $\ldots \ldots \ldots$ & 500 \\
\hline Empire ........ & U. S. C. \& G. S... & 49 \\
\hline Eugene .......... & Oreg. \& Cal. R. R & $\begin{array}{r}451 \\
., 143\end{array}$ \\
\hline an's Rar & $\begin{array}{l}\text { Wheeler........... } \\
\text { Wheeler......... }\end{array}$ & 2,143 \\
\hline $\begin{array}{l}\text { Fish Lake ........ } \\
\text { Flet cher Ranch Vail }\end{array}$ & $\begin{array}{l}\text { Wheeler........... } \\
\text { Wheeler........... }\end{array}$ & $\begin{array}{l}3,155 \\
4,819\end{array}$ \\
\hline Forest Grove....... & $\mathrm{W} . \& \mathrm{O} \& \mathrm{O} . \mathrm{C} . \mathrm{I}$ & 193 \\
\hline Ranc & ler............. & 4,174 \\
\hline Froman...... & R. R. & 263 \\
\hline Fry.. & L.R.R R... & 287. \\
\hline$\cdots . .$. & W. \& O. \& O. C. I & 206 \\
\hline$\cdots$ & . \& Cal. R. R & 210 \\
\hline & L. R...... & 306 \\
\hline Moun & Wh & 8,990 \\
\hline & & 180 \\
\hline Lanc & & 3.36 \\
\hline Ranc & W] & 4,470 \\
\hline $\mathrm{Ha}$ & & 3,287 \\
\hline & Cal. R. R & 307 \\
\hline s Lal & Wallen .............. & 4,150 \\
\hline & Oreg. \& Cal. R. R & 332 \\
\hline $\mathrm{H}$ & & i, 187 \\
\hline & U. & 39 \\
\hline & O. \& O. C.I & 198 \\
\hline & & 260 \\
\hline $\mathbf{H}$ & Wh & 4,139 \\
\hline & Cal. R. R .... & 206 \\
\hline enc & O.\& O. C.R.R & 195 \\
\hline & $\mathrm{R} R \ldots \ldots$ & 336 \\
\hline & ¿ Cal. R. R ... & 264 \\
\hline ach, Hon & $r$ & 4,466 \\
\hline & al. R. R & 345 \\
\hline & $\&$ G. S...... & 100 \\
\hline Klam & Wh & 4,108 \\
\hline & R. Reports & 4,131 \\
\hline MaI & 的 & 4,371 \\
\hline & Wh & 4,825 \\
\hline & & 1,200 \\
\hline s Ranch & er. .. & 5,162 \\
\hline & & 1,000 \\
\hline & $\&$ Cal. R. R ..... & 657 \\
\hline 8 Pass. & \& O.C.R.R. & 302 \\
\hline & R. R ......... & 371 \\
\hline adows, on E. Fork of De Chute's. & & 4,258 \\
\hline 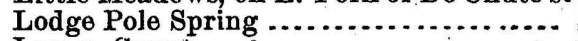 & & 1,648 \\
\hline & nian Inst ..... & 5,600 \\
\hline wis Mountain. & $r \ldots \ldots \ldots \ldots . .$. & 7,957 \\
\hline & & 2,443 \\
\hline & & 191 \\
\hline $\mathbf{M}$ & \& O. C. R.R. & 178 \\
\hline $\mathbf{M}$ & $\&$ Cal. R. R .... & 322 \\
\hline (n) $>0$ & C. \& G. S........ & \\
\hline & R. R. \& Nav. Co.. & 3,161 \\
\hline & & 4,420 \\
\hline 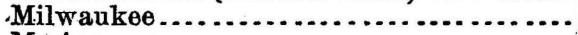 & Oreg. \& Cal. R. R ........ & 117 \\
\hline & N.P.R.R.................... & 100 \\
\hline ......... & er. & $\begin{array}{l}4,828 \\
9\end{array}$ \\
\hline & & 2,443 \\
\hline
\end{tabular}




\begin{tabular}{|c|c|c|}
\hline Station. & Authority. & Elevation. \\
\hline$-a_{0}$ & & \\
\hline Mule Spring .... . & Wheeler & 4,739 \\
\hline Mutton, Mount... & Wheeler. & 3,693 \\
\hline Neenee Spring . . . . . . & Wheeler. & 2,914 \\
\hline North End Mountain. & Wheeler. & 8,471 \\
\hline North Twin-lake........ & Wheeler. & 5,117 \\
\hline North Sands .............. & U. S. C. & \\
\hline North Yamhill. & W. \& 0 . & 204 \\
\hline Oak Grovo .... & Wheeler. & 2,415 \\
\hline Oakland ....... & Oreg. \& C & 450 \\
\hline Okanagon, Fort ... & Toner .... & 810 \\
\hline Oregon City & Oreg. \& $\mathrm{C}$ & 99 \\
\hline Orford, Fort ...................... & Med. Dep & 50 \\
\hline Parker ........... & W. \& 0 & 234 \\
\hline Parrish Ranch.... & Wheeler. & 3,330 \\
\hline Parton's Ranch & Wheeler. & 4,498 \\
\hline Pauline Peak.. & Wheeler... & 7,387 \\
\hline Pendleton..... & Oreg. R. R. \& Nav. Co.... & 1,043 \\
\hline Pigeon $\ldots . . . . . .$. & U.S.C.\&.G.S. . & 24 \\
\hline Pigeon Point..... & U.S. C. \&. G. S. & \\
\hline Pitt, Mount .... & Wheeler. . & 9,818 \\
\hline Portland ......... & Institution ... & 150 \\
\hline Do...................... & W.\& O.\& O.C.R.R ...... & $\mathbf{5 3}$ \\
\hline Do.................... & N. P. R. R................ & 43 \\
\hline Do .. (opposite) & Pacific R. R & 66 \\
\hline Do ... Fourth street bridge .............. & & 190 \\
\hline Do ... Signal Station ................... & Office........... & -67 \\
\hline 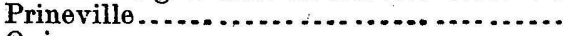 & Wheeler. & 2,899 \\
\hline Quinns .. & N. P. R. R & 236 \\
\hline Reedville .... & W. \& O.\& O. C. R. R & 253 \\
\hline 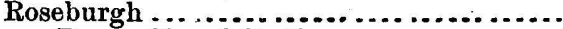 & Oreg. \& Cal. R. R.... & 485 \\
\hline 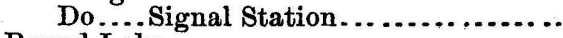 & U. S. Signal Office... & 537 \\
\hline Round Lake .............................. & & 4,671 \\
\hline St. Joseph . . . . . . . . . . & W. \& $0 . \&$ & 158 \\
\hline Salem $\ldots \ldots \ldots \ldots \ldots \ldots \ldots \ldots \ldots \ldots$ & Oreg. \& Cal & 187 \\
\hline Scott, Mount ....................... & Wheeler... & 9,016 \\
\hline Shivar's Bridge, Deschutes River ..... . & Wheeler...... & 702 \\
\hline Sikan Marsh $\ldots \ldots \ldots \ldots \ldots \ldots \ldots \ldots \ldots$ & 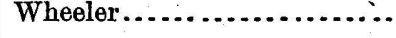 & 4,960 \\
\hline Six Bit House....................... & 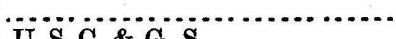 & 1,151 \\
\hline Skiff .............. & U. S. C. \& G & 37 \\
\hline 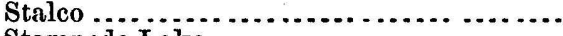 & 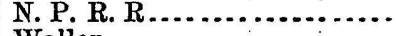 & 48 \\
\hline 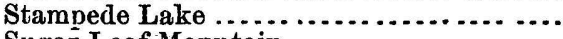 & Wallen............. & 4,196 \\
\hline Sugar Loaf Mountain . . . . . . . . . . . . . . . . . & 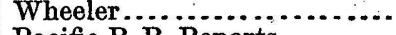 & 8,415 \\
\hline Summit Pass...................... & Pacific R. R. Reports.......... & 5,595 \\
\hline Tangent ...... & Oreg. \& Cal & 269 \\
\hline Timber, Mount & Wheeler... & 7,519 \\
\hline Troutdale ..... & N. P. R. R. & 60 \\
\hline Turner ......... & Oreg. \& Cal. R. R.. & 310 \\
\hline Tygh Valley .. & Wheeler... & 1,089 \\
\hline 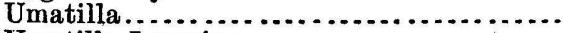 & Oreg. R. R. \& Nav. R. R...... & 277 \\
\hline Umatilla Junction . . . . . . . . . . . . . . . . . & 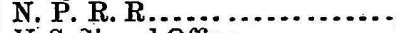 & 302 \\
\hline Umatilla, Signal Station.................. & U. S. Signal Office. & 384 \\
\hline Umpqua, Fort. . . . . . . . . . . . . . . . . . . & Med. Dept., U. S. A............ & \\
\hline Union $\ldots \ldots \ldots \ldots$ & Oreg. R. R. \& Nav. Co....... & 2,793 \\
\hline Union Peak ........................... & 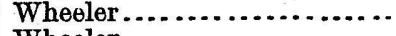 & 7,298 \\
\hline Upper Cascades ...................... & Wheeler... & 127 \\
\hline Upper Klamath ..... & & 4,131 \\
\hline Vancouver (opposite) $\ldots \ldots \ldots \ldots \ldots \ldots \ldots$ & Pacific R. R. Reports......... & 105 \\
\hline Vicuto $\ldots \ldots \ldots \ldots \ldots \ldots$ & 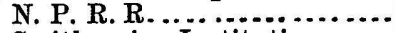 & 104 \\
\hline Wallamet ........ & Smithsonian Institution ..... & 120 \\
\hline Warm Spring Agency $\ldots \ldots \ldots \ldots \ldots \ldots$ & Wheeler..................... & 1,574 \\
\hline Warm Spring Indian Agency. ............ & 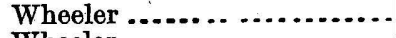 & 1,513 \\
\hline 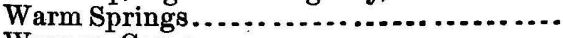 & Wheeler.......................... & 1,529 \\
\hline 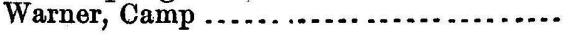 & Wheeler........................ & 5,730 \\
\hline
\end{tabular}




\begin{tabular}{|c|c|c|}
\hline Station. & Authority. & Elevation. \\
\hline 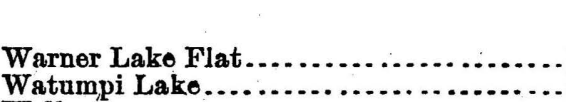 & 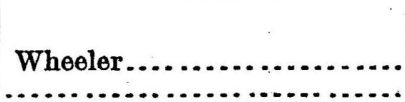 & $\begin{array}{l}\text { Feet. } \\
4,544 \\
4,287\end{array}$ \\
\hline $\begin{array}{l}\text { Wells } \\
\text { Willows } \\
\text { Woodland }\end{array}$ & 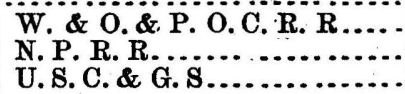 & $\begin{array}{r}247 \\
234 \\
107\end{array}$ \\
\hline
\end{tabular}


PENNSYEVANIA.

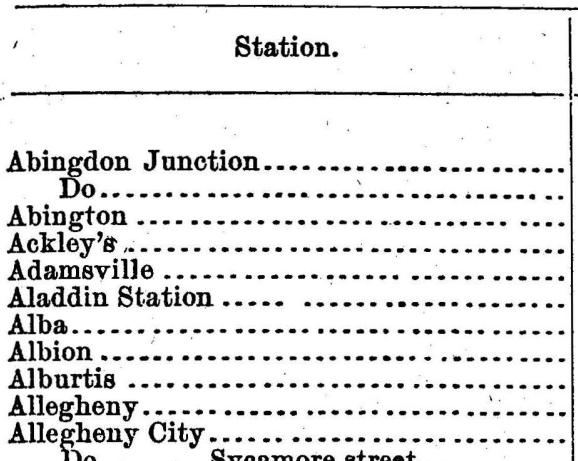

Do ......... Sycamore street

Do .......... East Lane.

Allentown, $\mathbf{B}$.

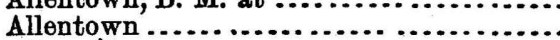

Allenville

Allenwood ..................................

Alligripus

Alpsville

Alton

Alton Summit.

Altoona

Do. W. line of ticket office

Do.front door of ticket office

Ambler .....

Anderson's ..............................

Do .... water station ...................

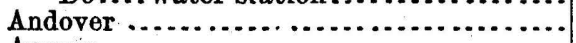

Angora .

Annville.

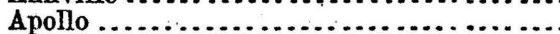

Apple's brick house.

Aqueduct

Ararat Summit

Archbald

Ardmore

Argyle.

Armstrong's Coal Mines

Armstrong Station

Arnold's Station.

Arnot

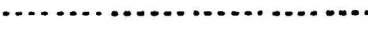

Ashland Depot

Ashley

Ashtabula

Athens Bridge

Athens Mills

Auburn

Auburn Junction

Austinburgh

Avery

Avon

Avondale

Do.................

Do...opposite center of coal office

Babcock

Baden

Bailey's Run

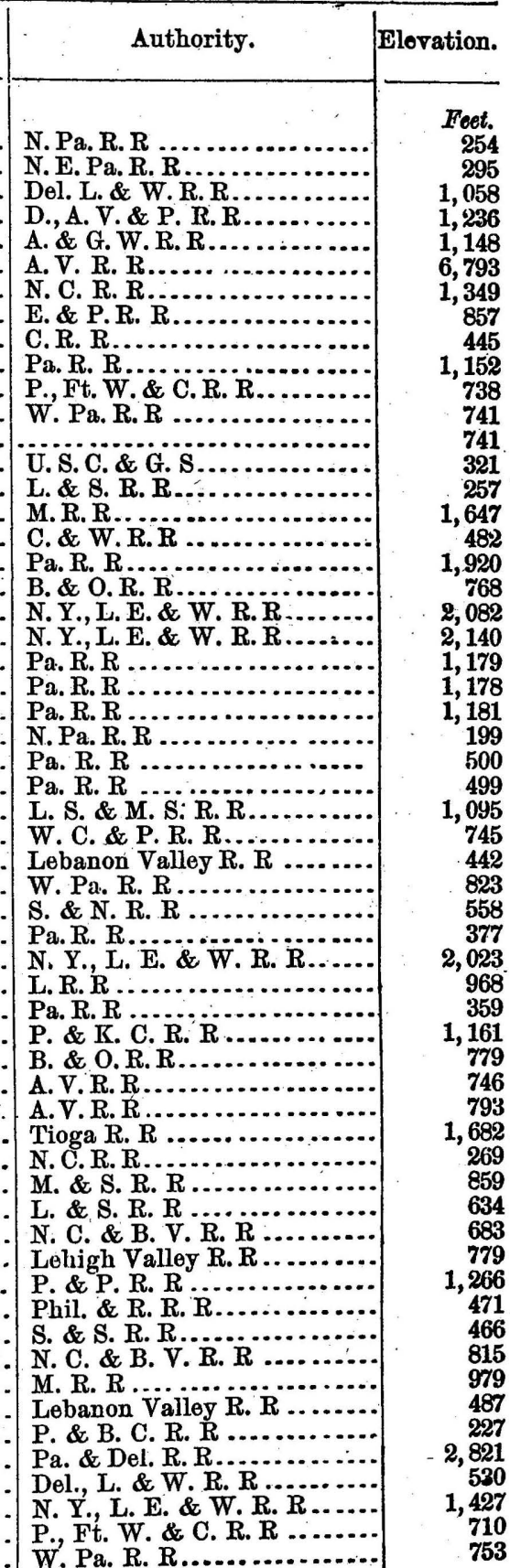




\begin{tabular}{l} 
Station. \\
\hline
\end{tabular}

Beaver Co. Collieries, New Castle .......... Do .............. Brier Hill, Mahon-

Do ing Valley Hattenluargh, Lower

Do Vein

Do Sandy Lake

Beaver Dam Sandy Lake, Lower Vein

Beaver Falls

Beaver Valley water-tank

Bechtelsville

Bedford

Beechwood

Beersville

Bellefonte.

Belle Valley

Bellevue

Bell's Mills

Bell's Mills Junction.

Belmont

Benezette

Bennett.

Bennett Station

Bennington Furnace

Berlin

Bernice

Berwick

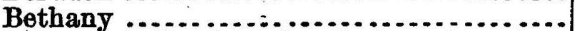

Bethayer's Station, if mile from junction.

Bethlehem

Bethlehem Junction

Biohl

Bigler

Big Shanty

Bird-in-Hand

Birdsborough

Birmingham

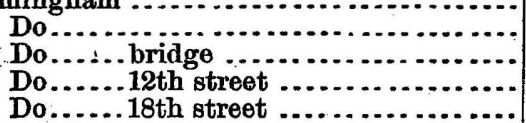

\begin{tabular}{|l|l}
\hline Authority. & Elevation \\
\hline
\end{tabular}

Pa. R. R

Feet.

387

$P a, R$. $R$

S. \& M. P. R. R........... 1,889

B. \& S. S. R. R

T. \& C. R. R ............. $\quad 989$

Chester Valley R. R ......... 299

B. \& O. R. R .............. 804

E. B. \& W. R. R ......... 486

C. \& W. R. R.............. 1,064

H. \& B. T. R. R ........... 1, 388

Pa. R. R

S. C. R.R.

L. \& L. R. R

L. \&. Q. R. R

Bennett's Br. R. R

Del., L. \& W. R. R

Berlin R. R.

A. \& G. W. R.R

Pa. R. R …....

Cleve. \& P.R.R

N. C. \& B. V. R. R

N. C. \& B. V.R.R

724

804

493

384

1,206

530

2,010

1,550

1,073

711

666

793

929

N. C. \& B. V.R.R

N. C. \& B. V.R.R

1,093

1,313

W. \& R.R.R . . . . .

1,113

603

771

924

C. \& W. R. R .................

401

Bed. \& Bridge. R. R

P. \& E. R. $\mathbf{R}$

E. Broadtop R. R

R.

1,062

1,244

658

744

1,007

P. \& E. R. R ...............

P., Ft. W. \& C. R. R . . . .

Bennett's Br. R. R

B. G.R.R

Phil. \& R. R. R.

Bennett's Br. R. R.

W. Pa. R. R ....

Pa. R. R .................

Berlin R. $\mathrm{R}$

S. L. \& S. R. R

Del., L. \& W. R. R

S. W. Pa. R. R

N. Pa. R. R

Lehigh Valley R. R

L.\& $\mathrm{L}$. $\mathrm{R}$.

L. C: \& S. C. R. R

T. \& C. R. R

N. Y., L. E. \& W. R. R

$\mathrm{Pa}, \mathrm{R}, \mathrm{R}$ \&a $\mathrm{W}$

Phila. \& R. R. $\mathrm{R}$

Pa. R. R.

P., C. \& St. $\mathrm{L} . \dddot{R} . \ddot{R}$

B. \& O. R. R

P. V.\& C.R. R.......................

P. V. \& C. R. R................
728

1,268

1,060

49

1,040

741

553

2,038

2,176

1,858

505

1,051

144

235

240

518

1,662

1,730

359

173

866

766

751

802

795 


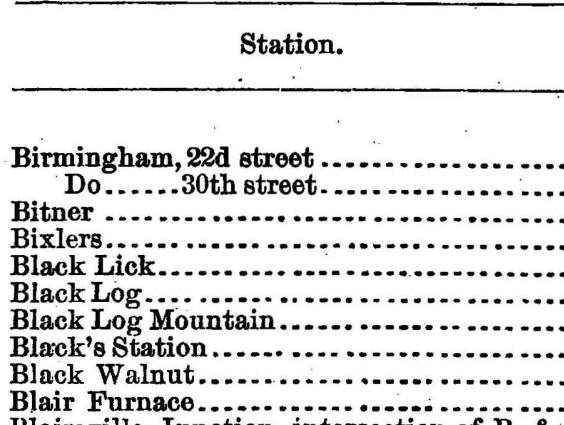

Blairsville Junction, intersection of $\mathrm{B} . \ddot{\&}$

I. B. of Pa. R. R. with main line.........

Blairsville, Market-street Station

Blandon

Bloody Run Summit.

Bloomfield, Iron Mines and Furnace.

Bloomsburgh

Blossburgh

Blue Ball

Bolivar

Bonnaffon

Borie

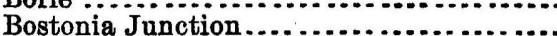

Bowers .................................

Bowmansville...........................

Bnyce's

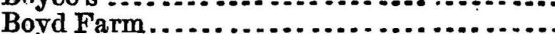

Boyertown

Braddock's

Braddock's Station

Bradford

Bradleys ................................

Brady's Bend Station

Brallier's Summit

Branch

Brandon's Station.......................

Brandonville.

Brandts

Brandy wine

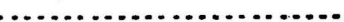

Briar Creek.

Bridgeport, Bedford Coun̂ty

Do .... Bedford County $. . . \ldots \ldots \ldots \ldots . .$.

Do.... Montgomery County

Do.... Cumberland County

Do.... Cumberland County, north end

Susquehanna Bridge

Do.... Clearfield County

Bridgeton, bench-mark

Bridgeville.

Brilliant Station.

Brinton's Station

Bristol, middle of Market street

Broad Ford

Broad Top City

Brockville

Brockwayville

Brodbead

Brogueville, bench-mark

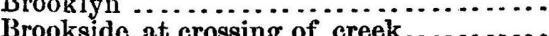

Brook Tuunel ............................

Brookville Station.

Brown

Authority. Elev

P. V. \& C. R. R ... ...........

P. V.\& $C_{.} R . R \ldots . . . . . .$.

B. V. R. R ....................

Pa. R. R.........................

Pa. R. R

Pa. R. R.....................

Levels

A. V. R. R.....................

Lehigh Valley R. R...........

Pa. R. R......................

Pa. R. R.

Pa. R. R.

E. Pa. R. R.

H. \& B. T. R. R

Pa. $R, R$

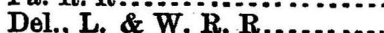

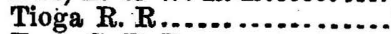

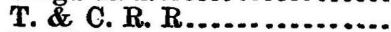

Pa. R. R

P., W. \& B. R. R...............

Phil. \& T. R. R.

Bennett's Br. R. R

E. Pa. R. R.

L. \& S. R. R.

Chartiers R. R.

P., T. \& B. R. R

C. R. R.

B. \& O. R. R......................

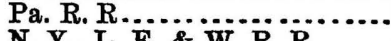

N. Y., L. E. \& W. R. R.....

E. \& C. R. R..............

A. V. R. R.

H. \& B. T. R. R.

L. S. \& M. S. R. R

A. V.R. R.

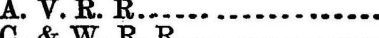

C. \& W. R. R................

N. Y., L. E. \& W. R. R

W. \& R. R. R.

Del $\mathrm{L}$ \& $\mathrm{R} \mathrm{R}$

Bed. \& Bridge R. R...........

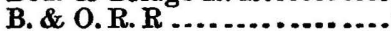

Phil. \& R. R. R.............

N. C. R. R .

Cumberland Valley R. R.....

T. \& C. R. R...............

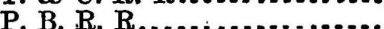

Chartiers R. R .................

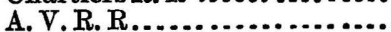

Pa.R. R

Phil.\& T. R.R......................

P. \& C. R. R.

Levels

Schuylkill Valley R. R......

N. Y., R. \& P. R. R

L. \& L. R. R ...............

P. B. R. R ..................

E. B. \& W. R. $R$............

Mt. A. R. R .................

B. \& O, R. R

Bennett's Br. R. R...........

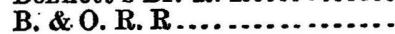

Elevation.

Feet.

$78 \mathrm{C}$

761

$2,04 \xi$

482

963

462

1,616

922

649

1,114

1,113

1,011

418

1,234

1,460

489

1,348

1,547

1, 033

60

35

1,074

454

436

865

1,086

389

769

828

1,464

2,118

856

1,108

1,200

961

1,285

1,047

556

273

502

930

941

76

355

357

1,190

305

822

747

757

21

873

1, 983

778

1,448

313

478

331

707

1,558

1, 235

757 


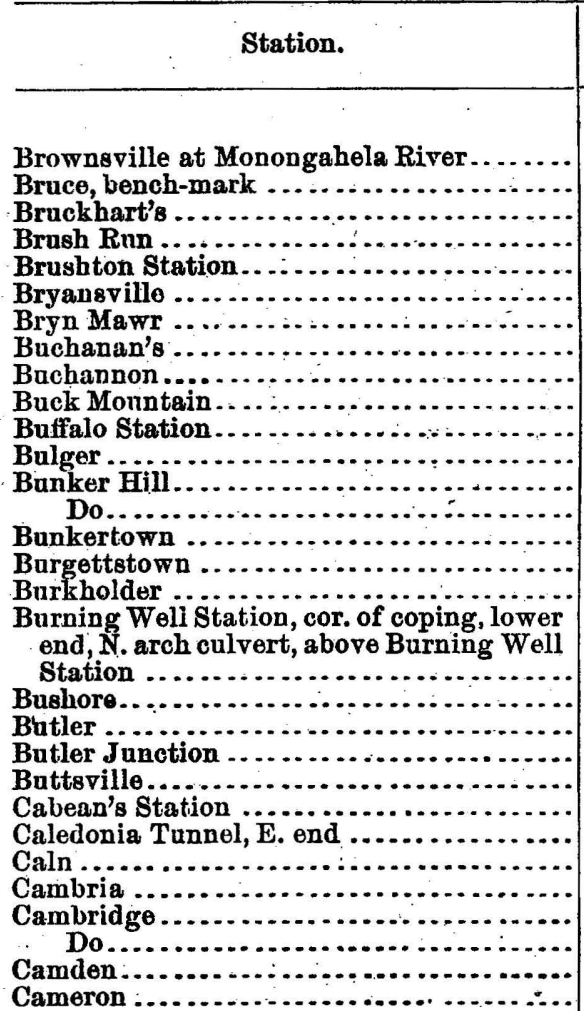
Do.

Campbelltown

Camp Ground Station

Camp Hill.

Camp Hummel

Canaan

Cannansburg

Cantner.

Canton

Carbondale

Do.....Coal Brooke Breaker

Do.....Canal level.

Do.B. M. on base of column west side of jail entrance

Carlton

Carpenter's

Carpenter's Station

Castleman.

Catasauqua

Catawissa Do.

Catfish

Catfish Station, door sill telegraph office, sonth side..

Cedar Hollow

Central Point

Centre Hall

Centreville.$$
\text { Do }
$$

Chadd's Ford

\begin{tabular}{|c|c|}
\hline Authority. & Elevation. \\
\hline 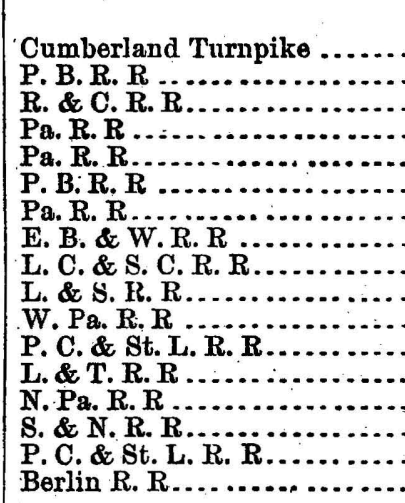 & $\begin{array}{r}\text { Feet. } \\
873 \\
331 \\
425 \\
\mathbf{9 3 3} \\
9222 \\
241 \\
416 \\
672 \\
1,059 \\
1,620 \\
\mathbf{7 6 6} \\
1,156 \\
437 \\
528 \\
706 \\
1,011 \\
1,992\end{array}$ \\
\hline
\end{tabular}

A. V. R. $R$

S., L. \& S. R. R .................. W. Pa. R. R

W. Pa. R. R

N. Y., R. \& P. R. R

L. \& Q. R. R.................. Bennett's Br. R. R ...........

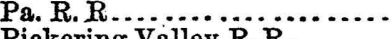
Pickering Valley R. R........ A. \& G. W. R. $\mathbf{R}$ P. \& P. R. R.

P. V.\& C. R. $R$ P. \& E. R. R.

Del.,L. \& W. R. R J. S., P. C. \& B. R. R

A. V.R. R .

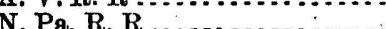

T. \& C. R. R

Pa. R. R.

Chartiers R. R

S. \& M.P. R. R

N. C. R. R $\because \dddot{W} \dddot{R}$

N. Y., L. E. \& W. R. R........

L. R. R..................

Cumberland Valley R. R....

U. S. C. \& G. S...

A. \&. G. W. R. R

N. C. R. R

Pa. R. R.

B. \& O. R. R.

Lehigh Valley R. R

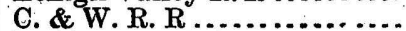

D.,H. \& W. R. R

Pa. R. R.

A. V. R. R

945

1,593

1,008

769

1,996

389

1,148

359

322

1,163

1,158

754

964

458

700

789

178

1,682

1,066

935

2,108

1,261

1,079

1,086

965

477

473

1,047

1,201

854

1,757

288

477

470

968

Chester Valley R. R

859

P. \& K. C. R. R.

L. C. \& S: C. R. R.

Chester Valley R. R..........

P. F. \& B. R. R

P. \& B. C. R. R.................

W. \& R. R. R

3

5

.

6

6

.

$2 \%$




\begin{tabular}{|c|c|c|}
\hline Statio & Authority. & Elevation. \\
\hline & & \\
\hline Chambersburg .. & Cumberland Valley & 618 \\
\hline Do.......... M. on base & & \\
\hline 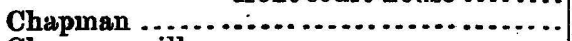 & C. \& F. R. R. & 541 \\
\hline $\begin{array}{l}\text { Chapmansvi } \\
\text { Chartier's St }\end{array}$ & L. \& L.R.R & 576 \\
\hline Cheney .... & W. C. \& P. R & $\begin{array}{l}765 \\
240\end{array}$ \\
\hline .. & P. \& B. C. R. I & 234 \\
\hline Chester SpI & Pickering Valley & 278 \\
\hline$\ldots . .$. & Phil. \& R.R. & 410 \\
\hline $\begin{array}{l}\text { Childs....... } \\
\text { Chiques.... }\end{array}$ & $\begin{array}{l}\text { B., C. \& P. R. } \\
\text { Pa. R. R..... }\end{array}$ & $\begin{array}{l}1,474 \\
255\end{array}$ \\
\hline Do.Br & Pa. R. R. & 351 \\
\hline hristiana. & Pa. R.R... & 491 \\
\hline Chulask & Del., L. \& V & 455 \\
\hline - & Phil. \& N. & 184 \\
\hline$\ldots$ & B. \& O.R. R & 761 \\
\hline$\ldots$ & P.\&E. R. R. & 1,396 \\
\hline arion Summit ....... & P.\&E.R.R... & 2,024 \\
\hline Clark's & L.S. \& M. S. R & 1,164 \\
\hline Clarksville & E. \& P.R. J & 894 \\
\hline .. & B. \& 0.R. R & 1,143 \\
\hline Clez & T. \& & 1,103 \\
\hline Clearfield Creek . & T. $\&$ & 1,140 \\
\hline Clermont, Bishop's Sum & McK. \& B. I & 2,074 \\
\hline ........ & W.C.\& P.R.R. & 109 \\
\hline$\ldots$ & N. C. \& B. V. R. R & 900 \\
\hline c........ & L. \& L. R. R.. & 362 \\
\hline $\begin{array}{l}\text { Coal Branch ... } \\
\text { Do...... Cre }\end{array}$ & $\begin{array}{l}\text { L.S.\&.M. S } \\
\text { N. C.\&. F. }\end{array}$ & 1,199 \\
\hline 8ing - & II. \& B.T.R. & 1,126 \\
\hline & H. \& B.T.R. & $\mathbf{1}, 110$ \\
\hline Coal Port $\ldots \ldots \ldots \ldots \ldots \ldots \ldots \ldots \ldots$ & L. \& S.R.R.... & $\mathbf{5 8 5}$ \\
\hline $\begin{array}{l}\text { Coatesville } \\
\text { Do } \ldots \text { intersection of } \mathrm{W}, \& \mathrm{R}, \mathrm{R}, \mathrm{R}\end{array}$ & $\begin{array}{l}\text { W.\&.R.R.R ... } \\
\text { Pa.R.R....... }\end{array}$ & \\
\hline 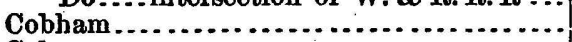 & P. T. \& B. R. R & 1,13 \\
\hline & L. C. \& & 1,026 \\
\hline anton ............ & A. \& G.W.R.R & 1,064 \\
\hline Run $\ldots \ldots \ldots . . . .$. & H. \& B. T. R. R & \\
\hline ink ............. & $\begin{array}{l}\text { N.Pa. R.R.R. } \\
\text { W.\&R.R.R... }\end{array}$ & $\because \quad \mathbf{5 2 5}$ \\
\hline pring...... & S. \& S.R. R .... & \\
\hline kdale........... & C. R. R.......... & 316 \\
\hline Coledale ........ & L. \& S.R.R.... & $\begin{array}{r}962 \\
1543\end{array}$ \\
\hline tation .... & A. V.R.R. & $\begin{array}{r}1,543 \\
746\end{array}$ \\
\hline ation ........... & E. Broadtop R. & 1,359 \\
\hline eville ............ & men R. R. & 155 \\
\hline $\begin{array}{l}\text { Collie } \\
\text { Collin }\end{array}$ & $\begin{array}{l}\text { B. G.R.R ...... } \\
\text { Pa. R.R........ }\end{array}$ & 1,642 \\
\hline Columbia ........... & P.T.\& B.R. R . & 1,06 \\
\hline o...Cross Roads. & & $1 ; 148$ \\
\hline Columbia & 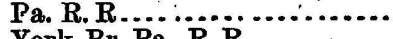 & 251 \\
\hline 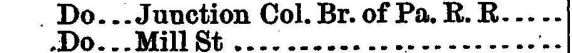 & $\begin{array}{l}\text { York Br. Pa., R.R ....... } \\
\text { R \& C.R. R.......... }\end{array}$ & $\begin{array}{l}251 \\
264\end{array}$ \\
\hline Do....Col. Depot.... & R.\& C.R.R.... & 25 \\
\hline Do...Pa. R. R. Depot & C.\& P. D. R.R . & 251 \\
\hline Do... Pa. R. R. track in front of passen- & ...... & 241 \\
\hline Columbus ............... & W.R. R ........ & 1,427 \\
\hline o........... & & $\begin{aligned} 1,401 \\
237\end{aligned}$ \\
\hline & & 1,361 \\
\hline
\end{tabular}




\begin{tabular}{|c|c|c|}
\hline & Authority. & Elevation. \\
\hline oncord.. & P.\& E.R.R. R. & \\
\hline onemaugh & Pa. R. R....... & $\begin{array}{l}1,304 \\
1,225\end{array}$ \\
\hline Do..... & Pa. R. R & 1,135 \\
\hline onestog: & W. \& R. R. R. & 647 \\
\hline $\begin{array}{c}\text { onewago .............. } \\
\text { Doridge. }\end{array}$ & $\begin{array}{l}\text { Pa. R. R } R . . . \\
\text { Pa. R. R....... }\end{array}$ & $\begin{array}{l}429 \\
546\end{array}$ \\
\hline onfluence ........ & B. \& O.R.R. & \\
\hline nnant & E. \& P. R. R.. & 1,066 \\
\hline onnellsy & Levels............ & 1,082 \\
\hline $\begin{array}{c}\text { Connellsville } \\
\text { Do ...... }\end{array}$ & $\begin{array}{l}\text { P. \& C. R. R } \\
\text { s. W. Pa. R. R }\end{array}$ & $\begin{array}{l}894 \\
915\end{array}$ \\
\hline Do...s. W & B. \& O.R.R & 894 \\
\hline $\begin{array}{l}\text { onsliohocken ... } \\
\text { okt's ............ }\end{array}$ & $\begin{array}{l}\text { Phil. \& R. R. R } \\
\text { Chartier's R. R }\end{array}$ & $\begin{array}{r}59 \\
1,003\end{array}$ \\
\hline ok's Mill & B. \& O.R. R .. & 774 \\
\hline $0 \mathbf{k} \mathbf{8} \mathrm{nu}$ & P. \& E. R. R . & 709 \\
\hline & $\begin{array}{l}\text { E. Broadtop R: } \\
\text { M. R. R ........ }\end{array}$ & $\begin{array}{l}1,541 \\
1,547\end{array}$ \\
\hline Spri & S. \& A.R.R.. & 1,127 \\
\hline & N. Pa. R. R... & 549 \\
\hline$\cdots$ & Pa. R. R....... & \\
\hline & Lehigh Valley $R$. & 296 \\
\hline .. & & 763 \\
\hline$\cdots \cdot \cdot$ & E., B.\&W. R. I & 361 \\
\hline & L.\& C.R.R $\ldots$ & $\begin{array}{r}603 \\
30\end{array}$ \\
\hline all's ..... & Phil.\& T.R.R.. & $\begin{array}{r}39 \\
131\end{array}$ \\
\hline crossing & P. \& E.R.R.... & 1,431 \\
\hline Corry & $\begin{array}{l}\text { A.\& G. W. R. R . } \\
\text { B., C. \& P. R. R. }\end{array}$ & $\begin{array}{l}1,428 \\
1,423\end{array}$ \\
\hline Do.j & P., T. \& B. R. R. & $\begin{array}{l}1,423 \\
1,433\end{array}$ \\
\hline Do.ju & P., T. \& B. R. R & 1,445 \\
\hline Do.c & \& B.R.R. R & 1,439 \\
\hline Do.c & P., T & 1,434 \\
\hline Do.c & P., T. \& B. R. R & 1,431 \\
\hline$\& \mathrm{P}$ & $\begin{array}{l}\text { A. \& G. W. R. R } \\
\text { A. \& G. W. R. R }\end{array}$ & 1,431 \\
\hline$\cdots$ & $\begin{array}{l}\text { A. \& G. W. R. R } \\
\text { J. S., P. C. \& B. }\end{array}$ & $\begin{array}{l}1,443 \\
1,661\end{array}$ \\
\hline & N. C. \& F. R. R. & $\begin{array}{l}1,661 \\
1,277\end{array}$ \\
\hline c & R. R ... & 768 \\
\hline & H. & 921 \\
\hline & B..V.R & 790 \\
\hline & Tio & 1,208 \\
\hline ck Station . & R. $\mathbf{R}$ & 809 \\
\hline (............... & St. L & 879 \\
\hline & H. \& B. T. R. R & 1,242 \\
\hline & N. Y., L. E. \& W & 2,098 \\
\hline ilie.......... & G. W. R. R .............. & 893 \\
\hline ille ...... & Phil. \& N. R. R. & \\
\hline $\mathrm{n} \ldots \ldots \ldots \ldots \ldots$ & $\begin{array}{l}\text { Pa. R. R } \\
\text { E. \& C. R. R }\end{array}$ & 2,017 \\
\hline $\begin{array}{l}\text { Do.R. R. Junction } \\
\text { Do.switch to E. \& } \mathrm{C} . \mathrm{R}\end{array}$ & 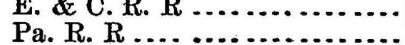 & $\begin{array}{l}2,028 \\
2,628\end{array}$ \\
\hline & $\& \mathrm{~B} . \mathrm{R} \mathrm{R}$ & $\begin{array}{l}2,020 \\
1,540\end{array}$ \\
\hline & P. R. R ................ & 765 \\
\hline$\ldots$ & & 662 \\
\hline C & ill Valley R. R.. & .668 \\
\hline & W.R.R.......... & 565 \\
\hline ville.... & & 1,141 \\
\hline lahonda ... & $\&$ E. R. R $\quad \ldots \ldots \ldots \ldots$ & 1,480 \\
\hline$\ldots \ldots \ldots$ & $\underset{B}{\mathbb{E} \text { E. R. R R }} \mathrm{R}, \ldots \ldots \ldots$ & $\begin{array}{r}1,605 \\
657\end{array}$ \\
\hline (.......... & $\mathrm{R}$ & $\begin{array}{r}657 \\
1,960\end{array}$ \\
\hline & $\mathrm{R} R$ & 628 \\
\hline 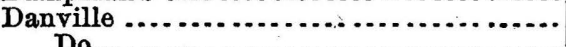 & C. \& W. R. R... & 494 \\
\hline & & \\
\hline
\end{tabular}




\begin{tabular}{|c|c|c|}
\hline Station. & Authority. & Elevation. \\
\hline & ( & Feet. \\
\hline Danville, N. E. end depot, top of near rail. . & Del., L. \& & 457 \\
\hline 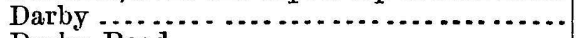 & P., W. \& B. & 45 \\
\hline 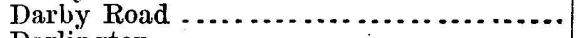 & W. C. \& P. & 103 \\
\hline Darlington $\ldots \ldots \ldots \ldots$ & P., Ft. W. & 981 \\
\hline Do $\ldots \ldots \ldots \ldots$ & W. C. \& P. & 143 \\
\hline st end R. R. bridge & N. C. R. R. & 349 \\
\hline Do _... center of passenger & N. C. R. R. & 349 \\
\hline $\begin{array}{l}\text { Davidson } \ldots \ldots \ldots \\
\text { Dawson }\end{array}$ & S. W. Pa. R. & 898 \\
\hline 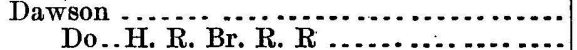 & P., T. \& B. B & 1,076 \\
\hline De Golias ........ & $\begin{array}{l}\text { B. } \& \text { U. R. R } \\
\text { N. Y., L. E. }\end{array}$ & $\begin{array}{r}864 \\
1,523\end{array}$ \\
\hline Delano Station ............ & W. Pa. R. R. & 1,224 \\
\hline Delaware Water Gap....... & Del., L. \& & 319 \\
\hline$\ldots \ldots \ldots \ldots . . . . .$. & P. B. R. R. & 435 \\
\hline Dent's Run Station........... & Bennett's Br & 926 \\
\hline 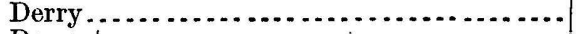 & Pa. R. R.. & 1,172 \\
\hline$\ldots \ldots \ldots \ldots, \ldots$ & P. \& E. R. ] & 485 \\
\hline Dilk's Station ................. & W. Pa.R. & 1,307 \\
\hline Dilleville Junction, Col. Bran & Pa.R. R.. & 359 \\
\hline 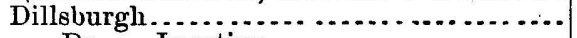 & M. \& D. R. I & 542 \\
\hline Do.....Junction... & Cumberland V & 427 \\
\hline 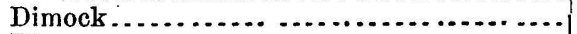 & M. R. R.... & 1,507 \\
\hline Dinsmore.. & P., C. \& St. & 1,059 \\
\hline ( & P., Ft. W. \& & 722 \\
\hline . & Pá. \& Del. R. & 374 \\
\hline Donaldson................. & L. \& T. R. R. & 910 \\
\hline 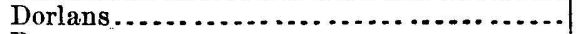 & E. B. \& W. I & 280 \\
\hline$\ldots \ldots \ldots \ldots$. & M. C. R. R.. & 647 \\
\hline Station.............. & A. V. R. R. & 915 \\
\hline $110 . . . . . . . . . .$. & Phil. \& R. R & 161 \\
\hline Forge.... & E. B. \& W. I & 274 \\
\hline Downington...$\ldots \ldots \ldots$ & Pa. R. R.... & 266 \\
\hline Do....terminus junction with $N$. & & \\
\hline r of Pa. R. R $\ldots \ldots \ldots$ & $\because \cdots \cdots$ & $\begin{array}{l}256 \\
\mathbf{3 4 7}\end{array}$ \\
\hline 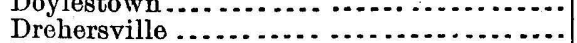 & (n......... & $\begin{array}{l}347 \\
494\end{array}$ \\
\hline 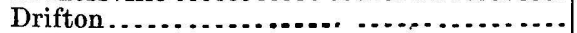 & L. \& S. R. R. & 1,633 \\
\hline 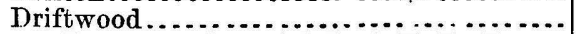 & P. \& E. R. R. & 816 \\
\hline Do.. -junction with P. \& & Bennett's Br. F & 813 \\
\hline Duubar.... & P. \& C. R. R. & 1,011 \\
\hline Do....... & T. \& C. R. R. & 1,453 \\
\hline Duncan ... & L. C. \& S. C. I & 1,078 \\
\hline Dun & Pa. R. R.... & 356 \\
\hline isville... & Pa. R. R... & 990 \\
\hline hing's ........ & Del., L. \& W. R. & 1,400 \\
\hline a's Eddy........... & P., T. \& B. R. R. & 1,156 \\
\hline 1esne Borough.......... & W. P.R. R ... & 741 \\
\hline Durbin ..... & E. \& C.R. R. & 1,927 \\
\hline e Hill... & Schuylkill Vall & 661 \\
\hline Eagle Rock................. & P.,T. \& B. R. R & 1,046 \\
\hline . . . . . & B. E. Valley R. & 635 \\
\hline$\cdots$ & N. C. \& F. R. R & 906 \\
\hline t Falls. & Phil. \& R. R. R & 119 \\
\hline Ea & S. W. Pa. R. R. & 1,062 \\
\hline y Statio & Pa. R. R... & 918 \\
\hline East Mahanoy Junc & E. M. R. R . . . . $\ldots \ldots \ldots$ & 1,109 \\
\hline Easton. & Lehigh Valley R. R.......... & 210 \\
\hline Do..... & L. \& S. R. R... & 215 \\
\hline Do. B. M. on W. corner jail, ......... & U. S. C. \& G. S.. & \\
\hline Do. window sill E. side C. H......... & U. S. C. \& G. S... & 364 \\
\hline 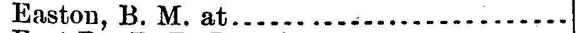 & U. S. C. \& G. S & 214 \\
\hline 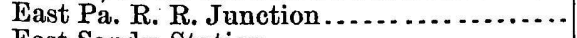 & Lehigh Valley $\mathbf{R}$ & 251 \\
\hline ast Sandy Station ........... & A. V.R.R... & 975 \\
\hline & & \\
\hline
\end{tabular}




\begin{tabular}{|c|c|c|}
\hline Station. & Anthority. & Elevation. \\
\hline & & \\
\hline Ebensburgh ........ & E. \& C. R.R. & $\begin{array}{l}2,022 \\
1,663\end{array}$ \\
\hline $\begin{array}{l}\text { Eckley } \\
\text { Economy } \ldots \ldots \ldots \ldots\end{array}$ & P.,Ft. W. \& C. R. R. & $\begin{array}{r}1,663 \\
715\end{array}$ \\
\hline Eddington, Dunk's Ferr & Phil. \& T. R. R...... & 42 \\
\hline Edge Hill ............... & N. Pa. R. R ... & 293 \\
\hline $\begin{array}{l}\text { Edgewater Station } \\
\text { Edgewood Station }\end{array}$ & $\begin{array}{l}\text { A. V. R. R.... } \\
\text { Pa. R. R..... }\end{array}$ & $\begin{array}{l}761 \\
923\end{array}$ \\
\hline Edgeworth....... & P., Ft. W. W. \& C. R. R & 725 \\
\hline Edinborongh & P. \& P. R. R ............ & 1,212 \\
\hline $\begin{array}{r}\text { Do...Geodetic S } \\
\text { Edinburgh .......... }\end{array}$ & $\begin{array}{l}\text { U. S. Lake Surv } \\
\text { N. C. \& B. V. R }\end{array}$ & $\begin{array}{r}1,603 \\
802\end{array}$ \\
\hline Egypt ............. & B. \& O.R.R.... & 1,302 \\
\hline Eldorado. & Pa.R.R......... & ], 093 \\
\hline & B., N.Y.\&P. I & 1,443 \\
\hline Elizabeth ........... & P.V.\& C.R. R & 747 \\
\hline Elizabeth Furnace & Pa. R. R....... & 1,079 \\
\hline htown ...... & Pa. R. R .... & \\
\hline Ellrod & B. \& O.R.R & 768 \\
\hline Ellwood. & S. \& S.R.R. & 673 \\
\hline$\cdots \ldots \ldots$. & Pa.R.R..... & 285 \\
\hline nction... & Perkiomen R. ] & 391 \\
\hline tion. & E. Pa.R.R. & 434 \\
\hline $9 . . .$. & W.\&R.R.R. & 231 \\
\hline Emig & N. C. R. R .... & 376 \\
\hline Station. & A. V.R.R. & 905 \\
\hline Empori & B., N. Y.\&P. & 1,024 \\
\hline & P.\& E.R.R. & 1,033 \\
\hline Emsworth... & W.\& C & 725 \\
\hline Enon & W. \& C & 994 \\
\hline Enterprise ...... & P. B. R. R $\ldots$ & 531 \\
\hline Do & Pitho & 1,261 \\
\hline Enz... & t's Br. R. R. & 964 \\
\hline Station & A. V.R.R ... & 982 \\
\hline Eph & R. $d$ & 384 \\
\hline Erie, De & L. & 686 \\
\hline Do.Chestnut st., at 20 & City Levels.... & 643 \\
\hline esunt and $26 \mathrm{th}$ st .. & City Levels.... & 763 \\
\hline Do.Water in reservoir, & & \\
\hline works... & City Levels.... & 808 \\
\hline $\begin{array}{l}\text { Do.Centre of State st., at } \\
\text { Do.Geodetic Station...... }\end{array}$ & $\begin{array}{l}\text { P.\&. E. R. R ..... } \\
\text { U.S. Lake Surve }\end{array}$ & 593 \\
\hline Do.Signal Station ............................ & U. S Signal Office .... & 681 \\
\hline Espy & Del., L. \& W. R. R. & 490.25 \\
\hline & E. \& P.R.R.. & 1,088 \\
\hline & R. R... & 1,009 \\
\hline $\operatorname{gh} . . .$. & & 1,284 \\
\hline & H. & 1,118 \\
\hline en Water Station & tt's Br. R. R & 1,398 \\
\hline$\ldots \ldots \ldots$ & S. W. & 1,034 \\
\hline s Mills ... & $\mathrm{Ch}$ & \\
\hline ..... & R. R & 193 \\
\hline Ext & $\mathrm{Ch}$ & 324 \\
\hline le $\ldots . . . \ldots$. & Del., L. \& I & \\
\hline , junction of coal ro & W. Pa. R. R.. & 933 \\
\hline & B. \& O.R.R. & 1,380 \\
\hline it Station & t's Br. R. R. & 1,086 \\
\hline$x+\ldots \ldots \ldots \ldots \ldots \ldots$ & P., Ft. W. \& C. R. R ........ & 715 \\
\hline & Lehigh Valley R. R....... & 1,673 \\
\hline $\begin{array}{l}\text { Fairview... } \\
\text { Do.... }\end{array}$ & E. \& P.R. R $\ldots \ldots \ldots$ & 735 \\
\hline Do.... & $\begin{array}{l}\text { Pa. R. R........ } \\
\text { Pa. }\end{array}$ & 385 \\
\hline Fairville $\ldots . .$. & P \& B. C. R. R. & 255 \\
\hline $\begin{array}{l}\text { Fall Brook ........ } \\
\text { Falls .............. }\end{array}$ & $\begin{array}{l}\text { Tioga R. R ............ } \\
\text { Lehigh Valley R.R. }\end{array}$ & $\begin{array}{r}1,842 \\
587\end{array}$ \\
\hline
\end{tabular}




\begin{tabular}{|c|c|c|}
\hline Station. & Authority. & Elevation. \\
\hline & & Feet. \\
\hline Farmer's Valley.. & McK. \& B & 1,476 \\
\hline Farrandeville .... & P. \& E. R. & 583 \\
\hline Fayetto........... & P. \& C. R. & 921 \\
\hline Felton ... & P. B. R. R & 536 \\
\hline Fenmore. & P. B.R.R. R & 435 \\
\hline …............ & S. W. Pa. Ex & 1,132 \\
\hline 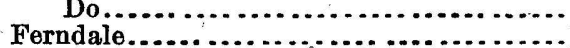 & P. \& C. R. R & 1,138 \\
\hline Ferney & P. \& E. R. R & 594 \\
\hline Fernwood & W. C. \& P. F & 90 \\
\hline Figarts ... & B. G. R. R. & 2,108 \\
\hline Finstman's........ & P. \& C. R. R & 1,076 \\
\hline Fishers Ferry $\ldots \ldots \ldots \ldots \ldots \ldots$ & N. C. R. R.. & 433 \\
\hline Fishers Summit ................. & H. \& B. T. R & 974 \\
\hline 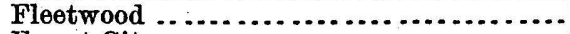 & E. Pa. R. R. & 449 \\
\hline Forest City ................. & N. Y., L. E. & 1,460 \\
\hline Forge $\ldots . . . \ldots \ldots \ldots \ldots \ldots \ldots$ & S. \& S. R. R. & 435 \\
\hline 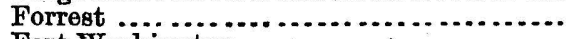 & E. B. \& W. R & 576 \\
\hline Fort Washington................. & N. Pa. R. R...... & 170 \\
\hline 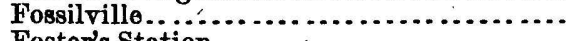 & Bed. \& Bridge & 1,091 \\
\hline Foster's Station .. & A. V. R. R: & 970 \\
\hline Fosterville ................. & S. W. Pa. R. & $\begin{array}{r}967 \\
1.029\end{array}$ \\
\hline ain Mills $\ldots \ldots \ldots \ldots \ldots \ldots \ldots \ldots \ldots \ldots \ldots$ & Pa. R. R.... & $\begin{array}{l}1,029 \\
1,042\end{array}$ \\
\hline Irgh Station $\ldots \ldots \ldots \ldots \ldots \ldots \ldots$ & A. V. R. R. & 897 \\
\hline Foxchas & Phil. \& N. R & 200 \\
\hline Frackville & M. C. R. R. & 1,479 \\
\hline Franklin & A. \& G. W. & 987 \\
\hline Do.. Branch Junction at head block of & & \\
\hline$\ldots \ldots \ldots \ldots \ldots \ldots$ & A. \& G. W. R & 1,074 \\
\hline Srankstown & B. \& O. R. R R . R & $\begin{array}{r}1,018 \\
783\end{array}$ \\
\hline Do... & 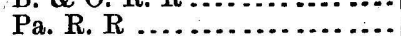 & 918 \\
\hline Fraser .. & Pa. R. R .... & 490 \\
\hline Fredoni & S. \& A. R. R. & 1,177 \\
\hline Freeburgh & S. \& N. R. R & 509 \\
\hline Freedon & P., Ft. W. \& C. I & 703 \\
\hline Freemar & Lehigh Valley R. R .. & 225 \\
\hline Do... & L. \& S. R. R ...... & 224 \\
\hline Freeport, Second Station ... & W. Pa. R. R & 772 \\
\hline Frenchtown............. & Lehigh Valley $\mathbf{R}$. & 690 \\
\hline$\ldots \ldots \ldots \ldots \ldots \ldots$ & MeK. \& B. R. R. & 1,465 \\
\hline$\ldots \ldots \ldots \ldots \ldots \ldots \ldots . . .6$. & R. \& C. R. R .. & 472 \\
\hline Fuller's $\ldots \ldots \ldots \ldots \ldots \ldots \ldots \ldots \ldots \ldots \ldots \ldots$ & Bennett's Br. R. R. & 1,327 \\
\hline Gaines' (Water in Pine Creek) . ........... & J. S., P. C. \& B & 1,259 \\
\hline Gallaghersville...................... & Pa. R. $\mathbf{R} \ldots \ldots$. & 298 \\
\hline . & Pa. R. R $\ldots \ldots$....... & 2,161 \\
\hline$\ldots \ldots \ldots \ldots \ldots$ & 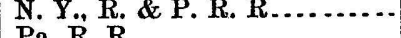 & 1,432 \\
\hline$\ldots \ldots, \ldots \ldots \ldots \ldots$ & Pa. R. R & 559 \\
\hline (n) & Chester Valley R. R ....... & $\begin{array}{l}225 \\
469\end{array}$ \\
\hline 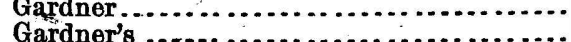 & C. \& R. R . & 1,568 \\
\hline Garland.. & 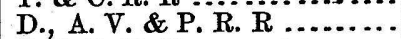 & 1,293 \\
\hline 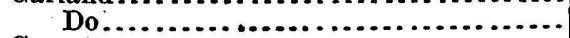 & P. \& E. R. R . . . . . . . & 1,308 \\
\hline Gorrett & B. \& O. R. R $\ldots \ldots \ldots \ldots$ & 1,948 \\
\hline 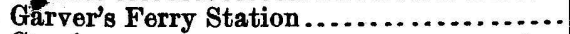 & A. V. R. R... & 785 \\
\hline Garvin...$\ldots$ & N. C. \& F. R. R ............ & 1,327 \\
\hline Gaysport, B. M. on step of ladies' waiting & & \\
\hline & W. \& R. R. $\ddot{\mathrm{R}}$. & 432 \\
\hline Genera ... & 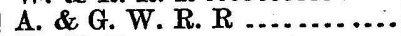 & 1,069 \\
\hline 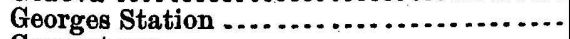 & Pa. R. R. & 1,196 \\
\hline 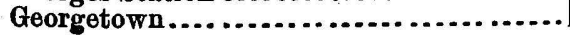 & & \\
\hline
\end{tabular}




\begin{tabular}{|c|c|c|}
\hline & Aut & Elevation. \\
\hline & & \\
\hline Germantown Depot & Phil. \& R. R.R. & 215 \\
\hline $\begin{array}{l}\text { Gilesville } \ldots . . . . . . . \\
\text { Gillett's.......... }\end{array}$ & N.Y., L. E.\& \& & 2,016 \\
\hline Girard .... & L.S. \& M. $\ddot{\mathrm{s}}$ & $\begin{aligned} 1,187 \\
717\end{aligned}$ \\
\hline Girard Ju & E. \& P. R. R & $\begin{array}{r}697 \\
1407\end{array}$ \\
\hline $\begin{array}{l}\text { Girard Manor St } \\
\text { Girard ville...... }\end{array}$ & M. \& S.R.R R... & $\begin{array}{l}1,407 \\
1,021\end{array}$ \\
\hline (a) & C. R. R $R$ & 165 \\
\hline len Carbon ... & $\begin{array}{l}\text { N. C. R. R. } \\
\text { M. H. \& S. H. R. R.. }\end{array}$ & $\begin{array}{r}335 \\
1,124\end{array}$ \\
\hline & B. \& O.R. R & 1,623 \\
\hline $\begin{array}{l}\text { le ... } \\
\text { on... }\end{array}$ & P., Ft. W. \& C & 721 \\
\hline$\ldots$ & L. \& S. R. R.. & 215 \\
\hline ock & $\begin{array}{l}\text { W. ER.R.H } \\
\text { Pa.R.R.... }\end{array}$ & $\begin{array}{l}218 \\
453\end{array}$ \\
\hline & W. C.\&P.R & 199 \\
\hline$\cdots$ & Lehigh Valley $\mathrm{R}$. & 591 \\
\hline$\cdots$ & $\begin{array}{l}\text { W.C.\&.P.I } \\
\text { N.C.R.R. }\end{array}$ & $\begin{array}{l}160 \\
551\end{array}$ \\
\hline$\cdots$ & P.\& E.R.R. & $\begin{array}{l}001 \\
605\end{array}$ \\
\hline ndo & P., T. \& B. R. & $1 ; 348$ \\
\hline.. & $\begin{array}{l}\text { S. \& S. R. R. } \\
\text { N. C. R. R... }\end{array}$ & $\begin{array}{l}799 \\
304\end{array}$ \\
\hline & Pa. R.R.... & 1,006 \\
\hline$\ldots . . . .$. & Pa.R.R.... & 385 \\
\hline & $\begin{array}{l}\text { D.,H.\&W.R.I } \\
\text { N.C.\&F. R. R }\end{array}$ & $\begin{array}{l}999 \\
907\end{array}$ \\
\hline & D., A.V.\& P.R.R & 1,341 \\
\hline$\cdots . .$. & Bennett's Br. R. R & 975 \\
\hline $\begin{aligned} \text { Granv } \\
\text { D }\end{aligned}$ & $\begin{array}{l}\text { N. C. R. R... } \\
\text { Pa.R.R..... }\end{array}$ & $\begin{array}{r}1,368 \\
498\end{array}$ \\
\hline Yrape & & 1,059 \\
\hline & aen $\mathrm{F}$ & 149 \\
\hline & $\operatorname{men} R \cdot R$ & $\begin{array}{r}426 \\
970\end{array}$ \\
\hline ;n....... & $\underset{R}{\&}$ B. R. R. & 1,279 \\
\hline Great Be & W. Pa & $\begin{array}{r}1,260 \\
879\end{array}$ \\
\hline $\begin{array}{r}\text { Great Be } \\
\text { Do.. }\end{array}$ & N. Y., L. E. \& W. R.. & 884 \\
\hline Greencastle $\ldots \ldots$. & Cumberland Valley R. R... & 585 \\
\hline Do....... M. on & & \\
\hline on Land & Perki & 246 \\
\hline & S. W. Pa & 1,070 \\
\hline & Pa. R. R & 1,091 \\
\hline & Pa. R.R... & 543 \\
\hline Greer & Del., L. \& & 1,185 \\
\hline I & $\mathrm{G}_{\mathrm{P}} \mathbf{W}, \mathbf{R}$ & 984 \\
\hline о00 & $\begin{array}{l}\text { E.\&P.R. } \\
\text { B.R.R... }\end{array}$ & 823 \\
\hline & W. C.\&P & 218 \\
\hline Gree & R.R. & 903 \\
\hline Ferry Bridge ... & P., W & 37 \\
\hline & 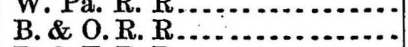 & $\begin{array}{l}8 \% 7 \\
784 \\
\end{array}$ \\
\hline & P.\& E.R.R... & 772 \\
\hline tump.................. & $\begin{array}{l}\text { N.C. R.R. R.... } \\
\text { B.\&S.S.R.R. }\end{array}$ & $\begin{array}{l}1,2,00 \\
1,020\end{array}$ \\
\hline Guth's & C. \& F. R.R... & 491 \\
\hline Guyer, Half Moon Gap. & L. C. \& S.R.R. & 1,144 \\
\hline $\begin{array}{l}\text { Gwynedd } \\
\text { Hadley }\end{array}$ & 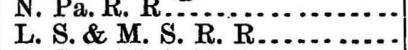 & 1,071 \\
\hline Hadley's ................. & L. S. \& M. S. R. R. & 1,070 \\
\hline $\begin{array}{l}\text { Halif } \\
\text { Halls }\end{array}$ & $\begin{array}{l}\text { N. C. R.R...... } \\
\text { C. \& W. R. R... }\end{array}$ & $\begin{array}{r}381 \\
513\end{array}$ \\
\hline & & \\
\hline
\end{tabular}




\begin{tabular}{|c|c|c|}
\hline Station. & Authority. & Elevation. \\
\hline Halls Station...on Ca & & Feet. \\
\hline bank of river.............. & M. C. R. R ...... & 513 \\
\hline $\begin{array}{l}\text { Harrisburg } \\
\text { Hamlin } . . .\end{array}$ & $\begin{array}{l}\text { Phil. \& R. R. R.......... } \\
\text { McK \& B. R. R }\end{array}$ & $\begin{array}{r}375 \\
1,557\end{array}$ \\
\hline Hammersville...... & W. Pa. R. R............... & 743 \\
\hline 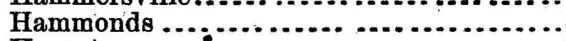 & Pa.R. R........ & 1,133 \\
\hline $\begin{array}{r}\text { Hampton } \\
\text { Do....... }\end{array}$ & 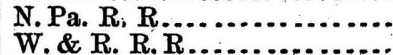 & $\begin{array}{l}276 \\
223\end{array}$ \\
\hline 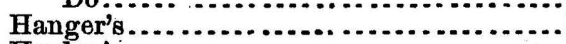 & B. V.R. R ................. & 2,073 \\
\hline a's $\ldots \ldots \ldots \ldots \ldots \ldots \ldots \ldots \ldots \ldots$ & P., C. \& St. L. R.R.... & 942 \\
\hline 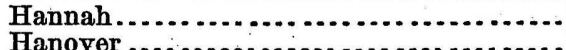 & B. E. Valley R. R. R...... & 1,057 \\
\hline (n) & 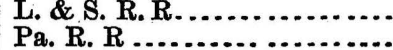 & $\begin{array}{l}654 \\
599\end{array}$ \\
\hline [anover Junction........................... & F.C.R.R.................. & 442 \\
\hline 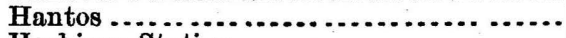 & L. \& S. R. R .... & 1,005 \\
\hline 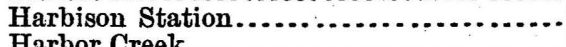 & W.P.R.R R $R$ & 802 \\
\hline $\begin{array}{l}\text { Harbor Creek } \\
\text { Harnish's Station }\end{array}$ & 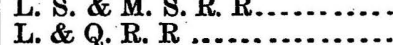 & $\begin{array}{l}731 \\
309\end{array}$ \\
\hline 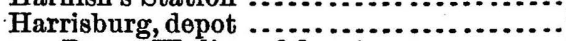 & Pa. R. R .................. & 320 \\
\hline 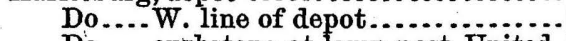 & Pa. R. R. & 321 \\
\hline $\begin{array}{r}\text { Do ..... curbstone at famp post, United } \\
\text { States Hotel.......................... }\end{array}$ & Pa. R. R & 321 \\
\hline Do..... W. line of Lebanon Valley & Pa. R. R. & 322 \\
\hline Do.... W. line of State street & Pa. R. R & 326 \\
\hline Do.... junc. with Pa. R. R. \& N. C.R.R. & Cumberland Valley R. R & 322 \\
\hline Do.... top of N. rail, main east bound & & \\
\hline Do.... top' of N. rail, W. line of State st. & Cum & 321 \\
\hline 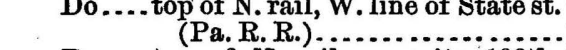 & Cumberland Valley R. $\mathbf{R}$ & 327 \\
\hline $\begin{array}{l}\text { Do..... top of N. rail, opposite } 106 \mathrm{th} \\
\text { mile post (Pa. R.R.).............. }\end{array}$ & & 330 \\
\hline $\begin{array}{l}\text { Do.... top of N. rail at weighing seales, } \\
\text { W. line of house, near 106th } \\
\text { mile post }\end{array}$ & 20 & 334 \\
\hline Do....top of north witness stone to & & 004 \\
\hline 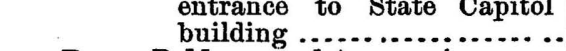 & Cumberland Valley R. R & 264 \\
\hline $\begin{array}{l}\text { Do..... B. M. on sandstone coping, near } \\
\text { base of column, } \mathrm{S} \text {. E. corner } \\
\text { of vestibule, east entrance } \\
\text { to State Capitol building }\end{array}$ & $\cdot$ & 368 \\
\hline $\begin{array}{l}\text { Do.... B. M. on slaty limestone rock } \\
\text { in river bed, about } 20 \mathrm{ft} \text {. S. from } \mathrm{S} \text {. line }\end{array}$ & Cumberiand & .200 \\
\hline $\begin{array}{l}\text { of C. V. R. R. bridge, and } 60 \text { feet S. fr. } \\
\text { 1st pier, } 2 \text { ft. above low-water mark. }\end{array}$ & Cumberland Valley R.R. & 292 \\
\hline $\begin{array}{l}\text { Harrisburg, top of } \mathrm{S} . \text { rail, } \mathrm{E} . \text { end of } \mathrm{C} . \\
\text { V. R. R. bridge }\end{array}$ & Cumberland Valley R. R.... & 332 \\
\hline $\begin{array}{l}\text { Harrisburg, top of } \mathrm{S} . \text { rail, W. end of C. } \\
\text { V.R. R. bridge }\end{array}$ & Cuxnberland Valley R.R & 353 \\
\hline $\begin{array}{l}\text { Harrisburg, B. M. on projecting course of } \\
\text { stone work at base of lst pier, S. W. cor. } \\
\text { W. end of C. V.R. R. bridge.................. }\end{array}$ & Cumberland Valley R. R & 297 \\
\hline $\begin{array}{l}\text { Harrisburg, B. M. on center of top surface } \\
\text { of monument in capitol grounds ......... }\end{array}$ & U.S. C. \& G. S .. & 357 \\
\hline $\begin{array}{l}\text { Harrisburg, B. M on base pillar at S. E. cor- } \\
\text { ner capitol building }\end{array}$ & U.S & 367 \\
\hline & S. \& A. I & 1,340 \\
\hline & 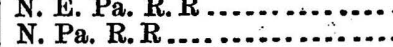 & 242 \\
\hline & & 229 \\
\hline
\end{tabular}




\begin{tabular}{|c|c|c|}
\hline Station. & Authority. & Elevation. \\
\hline & & Feet. \\
\hline $\begin{array}{l}\text { Haugus....... } \\
\text { Hawk Eye... }\end{array}$ & $\begin{array}{l}\text { Berlin R. R. } \\
\text { S. W. P. A. R. R. }\end{array}$ & \\
\hline Hawkins Stati & Pa.R.R ............... & \\
\hline Hawk Switch & N. Valley R. R. & 1,221 \\
\hline Hawley ....... & Del. \& Hudson C & 880 \\
\hline Do & N. Y., L. E. \& W. R. R & 899 \\
\hline $\begin{array}{l}\text { Haysville } \ldots . . . . \\
\text { Hazardville .... }\end{array}$ & $\begin{array}{l}\text { P., Ft. W. \&. C. } \\
\text { L. \& S. R. R ... }\end{array}$ & $\begin{array}{l}721 \\
417\end{array}$ \\
\hline Hazelwood..... & B. \& O. R. R & 789 \\
\hline Heathville. & Bennett's Br. R. & 1,161 \\
\hline Hecla ...... & L. Schuyl. R. R. & 590 \\
\hline Heidelburg & Lebanon Valley & 379 \\
\hline Heilmansd & L. \& T.R.R.... & 515 \\
\hline Heist & York Branch P & 437 \\
\hline Heler & W.Pa. R.R .... & 1,017 \\
\hline Hell & York Branch P. I & 348 \\
\hline Hellertown & N.Pa. R. R ..... & 276 \\
\hline Hemlock ... & P.\& E. R.R... & 1,464 \\
\hline & W., C. \& P.R.R & 318 \\
\hline Hende & Chester Valley R. & 165 \\
\hline Henr & Pa.R.R............ & 1,391 \\
\hline Henr & P., T.\& B. R. R & 1,035 \\
\hline Henryville & Del., L. \& W.R. R & 596 \\
\hline Herm & W. Pa. R.R R..... & 1,301 \\
\hline Her & M. \& S. R. R.... & 431 \\
\hline Herr & N. Y., L. E. \& W. & 1,770 \\
\hline $\bar{H}$ & S. \& N. B. R. R .. & 415 \\
\hline Hes & L. \& Q.R.R ... & 424 \\
\hline Hest & Pa.R. R ..... & 143 \\
\hline & U. \& N. F. R.R & 1,415 \\
\hline Hil & W. \& R.R.R..... & 530 \\
\hline Hicks & P., T. \& B. R. R ... & 1,091 \\
\hline חו & Lehigh Va & 1,016 \\
\hline Hicl & Del., L. \& & 521 \\
\hline Higl & N. Y. \& N. R.R R & \\
\hline & P., Ft. W. \& C. R. R & 1,043 \\
\hline $\mathbf{M}$ & P. B. R. R .......... & 383 \\
\hline & Pa. R.R......... & 300 \\
\hline & iers R. R... & 880 \\
\hline $\operatorname{lgh} . .$. & berland 'T'p'k & 1,750 \\
\hline & Pa.R.R........... & 1,129 \\
\hline ills..... & W. Pa.R.R... & 780 \\
\hline & N. Pa. R.R..... & 555 \\
\hline & N.C.\& B. V.R.R. & 799 \\
\hline tation.. & A. V.R.R & 865 \\
\hline & P.\& C.R.R. & 978 \\
\hline Mill . & T. Pa. Extension & 961 \\
\hline & igh Valley R.R & 295 \\
\hline & C., C.\& A.R. R ... & 1,151 \\
\hline sburg $\ldots \ldots \ldots \ldots \ldots$ & Pa. R. R. & 953 \\
\hline ... draw-bridge ..... & Pa.R.R ........ & 942 \\
\hline$d \ldots \ldots \ldots \ldots$ & P.,V.\& C.R. R.... & 761 \\
\hline 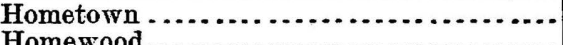 & N.'Valley R. R ..... & 1,176 \\
\hline Homewood . . . & Pa.R.R R & 923 \\
\hline $\begin{array}{l}\text { Do...... N. C. \& B. V. R.R. } \\
\text { Do...P. Ft. W. \& C.R.R. }\end{array}$ & P., Ft. W. \& C. R. R ......... & $\begin{array}{l}949 \\
950\end{array}$ \\
\hline Honesdale ........... & Del. \& Hudson Canal.......... & \\
\hline 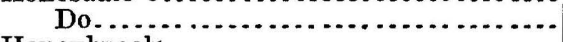 & N. Y., L.E. \& W. R.R....... & 966 \\
\hline & W. \& R.R.R. R & 596 \\
\hline & M. \& C. Co. R. R & 647 \\
\hline Hood's Road $\ldots \ldots \ldots \ldots \ldots \ldots \ldots \ldots \ldots \ldots$ & W. C. R.R R..... & 595 \\
\hline $\begin{array}{l}\text { Hopbottom } \\
\text { Hope Mills }\end{array}$ & $\begin{array}{l}\text { D., L. \& W.R.R. } \\
\text { N.C. \& F, R, R. }\end{array}$ & $\begin{array}{r}893 \\
1,107\end{array}$ \\
\hline $\begin{array}{l}\text { Hope } \\
\text { Hopes }-1118\end{array}$ & L.\&S.R.R ..... & $\begin{aligned} 1,100 \\
219\end{aligned}$ \\
\hline
\end{tabular}




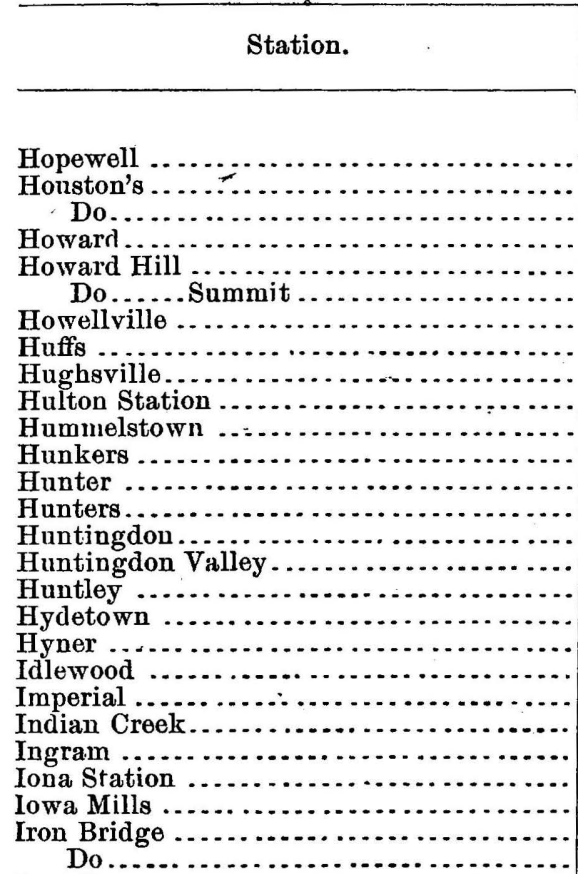

Irou Stone

Irvineton

Do.

Irvineton Junction, with P. \& E. R. R

Irving

Irwins Station

Isabella.

Jackson

Jackson Centre

Jackson's

Jack's Run

Jackstown .

Jacob's Creek

Do.

Jacob's Mill.

Jamestown.

Do... crossing E. \& P. R. R

Do.... crossing Franklin Division, $\mathbf{L}$. S. \& M. S. R. R

Jamison

Jeddo...

Jefferson.

Jenkintown Junction

Jermyn

Jersey Mills.

Jersey Shore

Do.

Main street

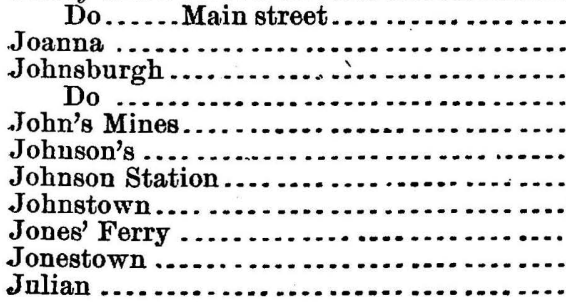

H. \& B. T. R. R

Chartiers R. R

Pa. R. R

B. F. Valley R. R

N. Y., L. E. \& W. R. R

N. Y., R. \& P. R. R

Chester Valley R. R

S. W. Pa. R. R

M. C. R. R.

A. V. R. R

Lebanon Valley $R . R$

S. W. Pa. R. R.

P., T. \& B. R. R

M. R. R

H. \& B. T. R. R

Phil. \& N. R. R

P. \& E. R. R . .

P., 'T. \& B. R. R

P.\& E. R. R

P., C. \& St. L. R. R

P., T. \& B. R. R

B. \& O.R. R

P., C. \& St. L. R. R

A. V. R. R .........

Pa. R. R .

P. \& C. R. R

C. R. R

D., A. V. \& P.R. R

P. \& E. R. R .

P., T. \& B. R. R

L. \& T. R. R .

Pa. R. R

W.R.R.R

D., A. V. \& P. R. R

N.C. \& F. R. R

P. \& E. R. R

P., Ft. W. \& C. R. R

Pa. R. R

B. \& O.R.R .

S. W.Pa. R. R

Pa. R. R

L. S. \& M. S. R. R

L. S. \& M.S. R. R

E. \& P. R. R

P., T. \& B. R. R

L. \& S. R: R

S. \& S. R. R

N. Pa. R. R

L. R. R

J. S., P. C. \& B. R. R.......

P. \& E. R. R.

J. S., P. C. \& B. R. R ........

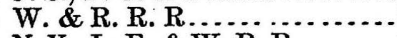

N. Y., L. E. \& W. R. R . . . .

P. \& E. R. R.

M. C. R. R

Chartiers R. R

A. V. R. R

Pa. R. R

P., C. \& St. L. R. R.

B. E. Valley R. R ............
Eleration.

Freet.

898

949

1,056

679

2,230

2,235

221

1,001

600

778

376

945

1,061

1,547

621

117

861

1,252

644

847

1,008

990

871

748

1,299

496

1,051

312

1,164

1,172

1,171

499

884

639

1,181

1,257

1,230

728

595

797

1,034

504

990

990

979

1,073

1,618

531

203

971

653

593

548

627

1,461

1,442

805

971

760

1,184

757

422

851 


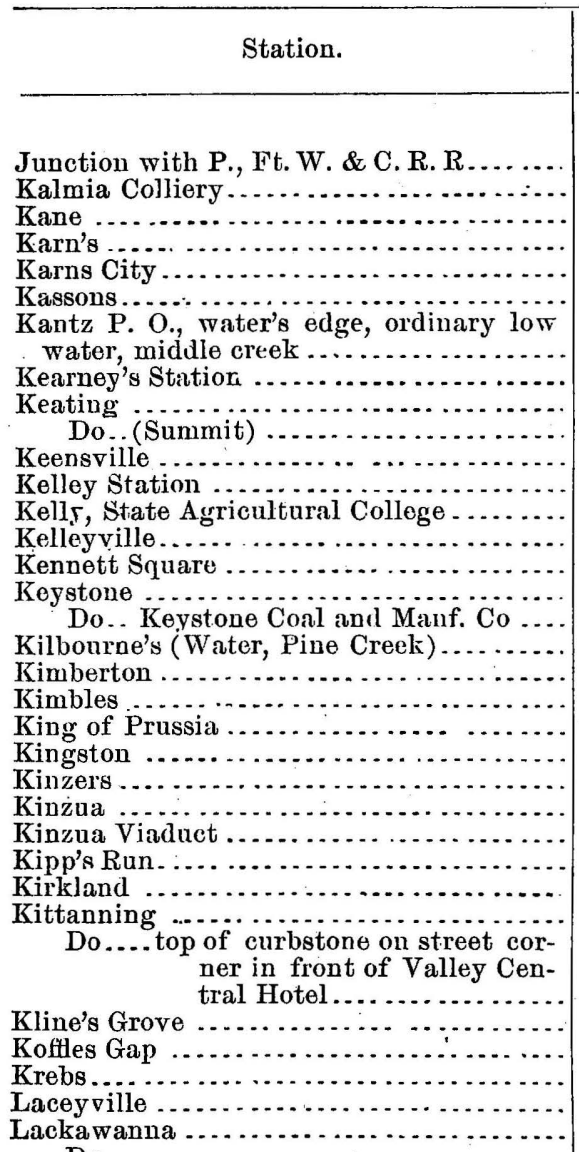

Lackawanna

Do..............

La Fayette.

Lagrange

Lakeville

Lamoka

Lamokin Junction

Lancaster Junction Do

B. M. on stone wall Lancaster Locomotive Works........

Lancaster Pike.

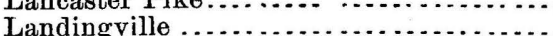

Landisburgh

Landisville

Do .... R. \& Col. R. R. Crossing

Lanes Mill

Langdons . . . . . . . . . . . . . . . . . . . . .

Lanigan Furnace.

Lansdale Junction

Larabee.

Larimer's Station

Latrobe.

Laubachs

Laughlin

Laurel

Do
W. Pa. R. R

L. \& T. R. R

P. \& E. R. R

W. Pa. R. R

P. \& K. C. R.

E. \& P. R. R.

S. \& N.R. R

Pa. R. R

P. \& E. R. R

B., N. Y. \& P. R. R

S. \& N. B. K. R.

A. V.R. R

W.. C. \& P. R. R

P. \& B. C. R. R

M. \&. S. R. R

S. R. R

J. S., P. C. \& B. R. R

Pickering Valley R. R

N. Y., L. L. \& W. R. R

Chester Valley R. R

Del., L. \& W. R. R

Pa. R. R.

N. Y., R. \& P. R. R

N. Y., L. E. \&. W. R. R

D., H. \& W. R. R ...........

W. C. R. R......................

Pa. R: R

A. V. R. R

D., H. \& W.

C. \&.W. R. R

Lehigh Valley R. R

Del., L. \& W. R. R.

N. Y., L. E. \& W. R. R

Lehigh Valley R. R........

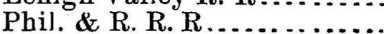

Lehigh Valley R. R.

U. \& T. R. R

B. R. R..

P., W. \& B. R. R

R. \& C. R. R.

Pa. R. R

E. B. \& W. R. K

Phil. \& R. R. R

Levels

R. \& C. R. R

N. Y., R. \& P. R. R

P. \& E. R. R.

M. C. R. R

N. Pa. R. R

B., N. Y. \& P. R. R

Pa. R. R

Pa. R R

L. \& S. R. R

B. \& O. R. R.

P. B. R. R...
Elevation.

Feet.

739

1,237
2,014

768

1,204

1,111

448

1,048

718

1,881

447

781

1,111

102

260

1,033

1,976

1,301

211

851

190

562

46

1,956

2,110

463

548

1,594

810

445

530

1,225

658

637

648

569

53

598

1,412

1,042

37

378

359

696

503

740

404

405

$\cdot 1,487$

1,135

717

368

1,481

866

1,006

304

770

412

241 


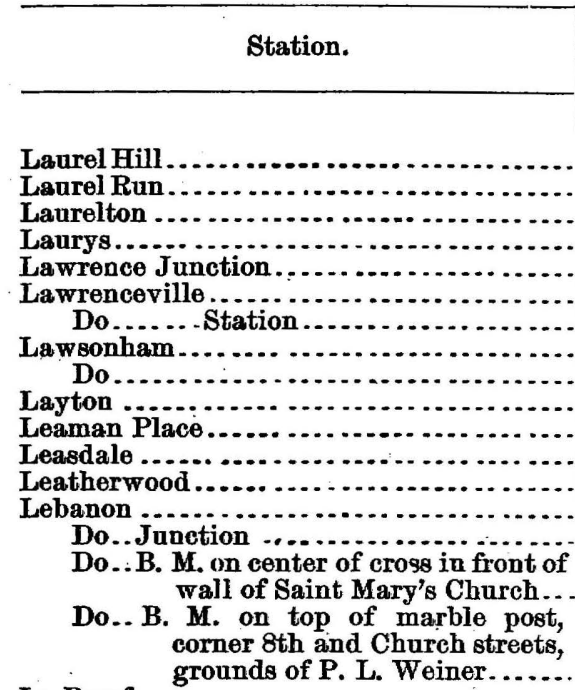

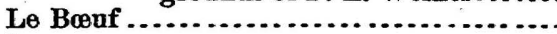

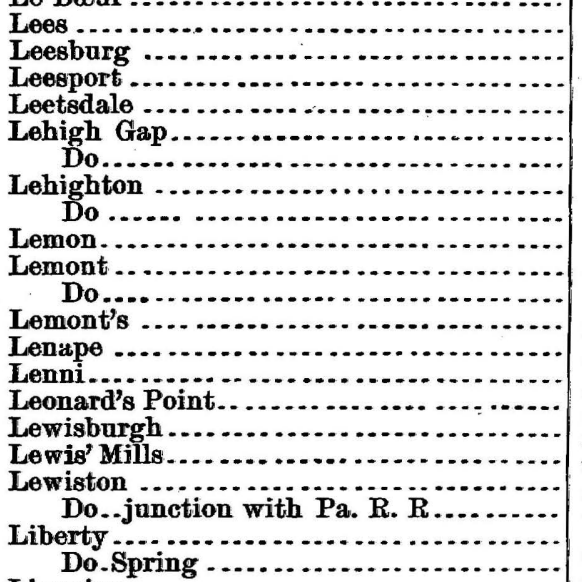

Ligonier

Lilly

Lillys.

Limerick

Lime Ridge

Lime Valley Station

Lincoln ville.

Linden

Do. Surface of canal

Lindorff's Summit

Linesville

Linwood

Lititz

Little Cooley

Littletown

Livengood's Mill

Livermore

Liverpool

Do... Center of Market street

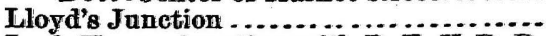

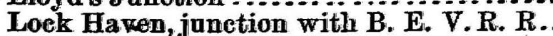
Lockport

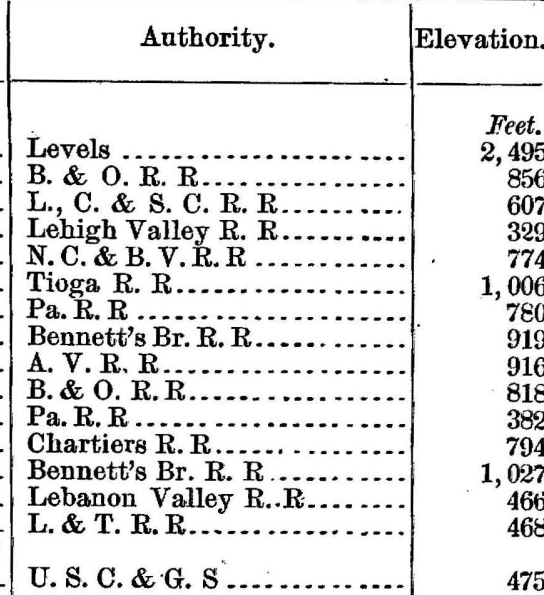

U. S. C. \& G. S

\section{P.\& E. R. R}

Chester Valley R. R

N.C.\& F.R.R.

Phil. \& R. R. R

P., Ft. W. \& C. R. R.......

L. \& S. R. R................

Lehigh Valley R. R..........

L. \& S. R. R

Leigh Valley $\mathrm{R} . \mathrm{R}$.............

M. R. $\mathbf{R}$.

L., C. \& S. C. R. R

S. W. Pa. Extension

P. \& C. R. R

W. \& R. R. R................

W., C. \& P. R. R

T. \& C. R. R

L., C. \& S. C. R. R

E. B. \& W. R. R

Mifflin \& Center Co. R. R

B., N. Y. \& P. R. R

T. \& C. R. R .........

Ligonier Vall

Pa. R. $\mathrm{R}$

Phil. \& R. R. R .

Del., L. \& W. R. R

L. \& Q. R. R

U. \& T. R. R

P. \& E. R. R

J. S., P. C. \& B R. R

Pa. R. R

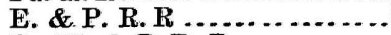

P., W. \& B. R. R

R. \& C. R. R.

P. \& P. R. R

Pa. R. R

S. R. R.

W. Pa. R.R

N. C. R. R

S. \& N. R. R

B. G. R. R

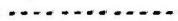

L.\& S. R. R
Feet.

856

607

329

1,006

780

919
916

818

382

794

466

468

475

466

1,217

279

1,045

298

715

393

389

494

466

1,041

1,002

1,030

1,084

183

136

1,:306

466

542

498

499

1,646

1, 103

1,748

2,030

1, 887

138

509

384

1,382

533

528

1,185

31

375

1,203

619

2,001

945

396

409

2,167

556

356
1,033 


\begin{tabular}{|c|c|c|}
\hline & Auth & Elevation. \\
\hline & & Feet. \\
\hline $\begin{array}{l}\text { Lockport....... } \\
\text { Lock Ridge ... }\end{array}$ & C.\& F. R. R. & $\begin{array}{r}1,054 \\
440\end{array}$ \\
\hline ocust Gap Ju & M. \& S.R.R. & 1,037 \\
\hline Logan ......... & M. \& C. Co. R. & 512 \\
\hline ogan's Ferry & A. V.R. R. . & 757 \\
\hline $\begin{array}{l}\text { Logausport S } \\
\text { Long Run }\end{array}$ & A.V.RR & $\begin{array}{l}785 \\
765\end{array}$ \\
\hline $\begin{array}{l}\text { Long Run.. } \\
\text { Lovells .... }\end{array}$ & P.\& E.R.R.. & 1,364 \\
\hline r Cata & L. \& S. R. R. & \\
\hline Lowrie, at Wa & L.C.\& S. C.R. & 1,109 \\
\hline$\cdots$ & P. \& E.R.R... & 1,603 \\
\hline Lutzville ...... & $\begin{array}{l}\text { Bed. \& Bridge. } \\
\text { C. \& W. R. R... }\end{array}$ & $\begin{array}{r}1,045 \\
529\end{array}$ \\
\hline $\begin{array}{l}\text { Lykenstown } \\
\text { Lykee }\end{array}$ & S. B.R.R..... & 677 \\
\hline ykens V. $\mathrm{H}$ & N. C. R. R. & 380 \\
\hline Lynn ......... & M.R.R.... & 1,032 \\
\hline on (Pa. Furna & L. C. \& S. C.R. & 1,074 \\
\hline Lyons ......... & E. Pa. R. R ...... & $\begin{array}{l}471 \\
661\end{array}$ \\
\hline ierville. & $\begin{array}{l}\text { S. \& N.R.R R.. } \\
\text { C. \&W.R.R.. }\end{array}$ & $\begin{array}{l}661 \\
759\end{array}$ \\
\hline $\mathrm{McCa}$ & W.C.R.R.... & 463 \\
\hline$\cdots$ & C. \& P.D. R. & 169 \\
\hline & A. V.R.R.... & \\
\hline $\mathrm{MeCl}$ & P., T. \& B. R. R & 1,058 \\
\hline$\ldots$ & H. \& B. T.R.R. & 674 \\
\hline & P., C. \& St. L. R & \\
\hline .... & P. \& P.R.R ... & 1,053 \\
\hline$\ldots$ & B.R. R .... & \\
\hline$\cdots$ & B. \& O.R.R R & \\
\hline McKune's & Lehigh Valley R. R. & $\begin{array}{r}598 \\
615\end{array}$ \\
\hline nmit .. & $\begin{array}{l}\text { N. Y., R.\& P.R. R. } \\
\text { Pa. R.R.......... }\end{array}$ & $\begin{array}{r}1,615 \\
522\end{array}$ \\
\hline $\begin{array}{l}\text { McVeytown .... } \\
\text { Magee .......... }\end{array}$ & $\begin{array}{l}\text { Pa. R.R......... } \\
\text { P., T. \& B.R.R.. }\end{array}$ & $\begin{array}{r}5 \% 2 \\
1,131\end{array}$ \\
\hline Magee's........... & Phila. \& R. R. R & \\
\hline$\cdots$ & Bennett's Br. R. R.. & 1,387 \\
\hline$\cdots$ & 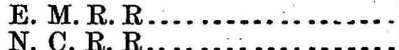 & $\begin{array}{l}1,343 \\
405\end{array}$ \\
\hline$\ldots$ & $\begin{array}{l}\text { N. C. R. R. } \\
\text { N. C. \& B. V. R. R }\end{array}$ & $\begin{array}{l}405 \\
769\end{array}$ \\
\hline & D., H. \& W. R. R & \\
\hline & Del., L. \& W. R. I & \\
\hline$\cdots$ & Pa. R. R . . . . . . & \\
\hline Mana & 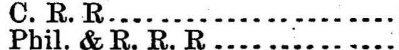 & $\begin{array}{r}192 \\
57\end{array}$ \\
\hline $\begin{array}{r}\text { Manay } \\
\text { Do }\end{array}$ & Pa.R. R........ & 519 \\
\hline Manc & $\&$ P.R.R... & \\
\hline & R. R... & \\
\hline & Co. R.R.... & \\
\hline & Bridge. R. R.. & 1,136 \\
\hline Mawor & W. \& R. R. R. ......... & 572 \\
\hline & Pa. R & \\
\hline 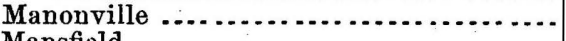 & $R$ & 798 \\
\hline$\ldots \ldots$ & St. $\mathrm{R}, \mathrm{R} R$ & $\begin{array}{l}1,140 \\
773\end{array}$ \\
\hline $\begin{array}{l}\text { Do } \\
\text { Mantua }\end{array}$ & $\begin{array}{l}\text { P., C. \& St. L. R. R } \\
\text { Pa. R. R }\end{array}$ & \\
\hline Mapl & Pa. R. R ....... & \\
\hline $\mathbf{M}$ & $R . \ldots \ldots \ldots \ldots$ & \\
\hline & $R \ldots \ldots \ldots \ldots \ldots \ldots$ & \\
\hline urg - & B. T.R.R... & \\
\hline Martha $\ldots$ & B. E. Valley R.R ................. & 912 \\
\hline & Pa. R. R & 1,054 \\
\hline Martinsburg .. & P.\& K. C. R. R. & \\
\hline Do......... & Pa. R. R $\quad \ldots . . .$. & $\begin{array}{l}1,366 \\
1,344\end{array}$ \\
\hline D & & \\
\hline
\end{tabular}




\begin{tabular}{|c|c|c|}
\hline Station. & Authority. & Elevation. \\
\hline & & \\
\hline $\begin{array}{r}\text { Marysville. W. end of R. R. bridge } \ldots . . . . . \\
\text { Do ..... B. M. on hard sandstone, per- } \\
\text { pendicular from S. line of } \\
\text { bridge, about } 40 \text { feet W. of }\end{array}$ & N. C. R. R... & Feet. \\
\hline 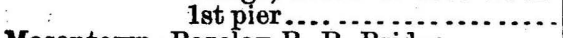 & N. C. R. R & 312 \\
\hline Masontown, Barclay R. R. Bridge ......... & B. R. R & 794 \\
\hline Mauch Chunk $\ldots \ldots \ldots \ldots \ldots \ldots \ldots \ldots \ldots \ldots$ & Lehigh Valley & 544 \\
\hline 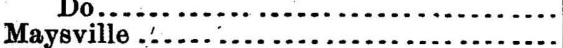 & 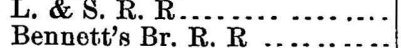 & $\begin{array}{r}532 \\
1,108\end{array}$ \\
\hline Meadville ..................... & A. \& G. W. R. R & 1,080 \\
\hline Mechanicsburgh $\ldots \ldots \ldots$ & Cumberland & 436 \\
\hline Do.......... junc. with C. Valley R. R & M. \& D. R. R & 427 \\
\hline $\begin{array}{l}\text { Mediadic's Run Bridge, B. M. on top of cap- } \\
\text { ston, N. E. cor., W. }\end{array}$ & W. C. \& P. R. & 210 \\
\hline abutment......... & Bennett's Br. & 1,097 \\
\hline 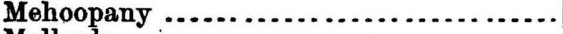 & Lehigh Valley & 634 \\
\hline 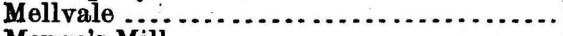 & N. C.R.R... & 168 \\
\hline 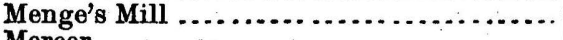 & Pa. R. R ....... & 455 \\
\hline 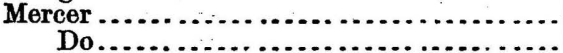 & $\begin{array}{l}\text { N. C. \& F. R. R... } \\
\text { S. \& A. R. R }\end{array}$ & $\begin{array}{l}1,097 \\
1,108\end{array}$ \\
\hline Merion....... & Pa. R. R.... & $\begin{array}{r}147 \\
247\end{array}$ \\
\hline Do $\ldots \ldots \ldots \ldots \ldots \ldots$ & Phil. \& R. R. R & 81 \\
\hline Mertztown ................ & E. Pa. R. R ... & 455 \\
\hline Meshopper .......... & Lehigh Valley R. ] & 644 \\
\hline Mexico....... & Pa: R. R....... & 433 \\
\hline Mejersdale $\ldots \ldots \ldots \ldots$ & S. R. R..................... & 1,964 \\
\hline $\begin{array}{l}\text { Do..... L. \& B. R. R... } \\
\text { Meyer's Switch............. }\end{array}$ & B. \& O.R.R R.... & 2,065 \\
\hline Middaughs....... & P.', \& B. R. $\mathrm{R}, \ldots \ldots \ldots$ & $\begin{array}{r}1,243 \\
615\end{array}$ \\
\hline Middlebury & 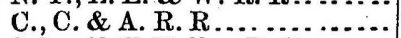 & 1,178 \\
\hline 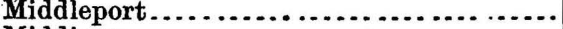 & Schuylkill Valley R. R....... & 724 \\
\hline Middlesex................. & E. \& P.R. R.............. & 833 \\
\hline Middletown . $\ldots . . . . . . . .$. & Pa. R. R...... & 314 \\
\hline Middletown Ferry $\ldots \ldots \ldots \ldots \ldots \ldots$ & N. C. R. R.... & 307 \\
\hline Middletown Junction, Col. Br........... & Pa. R. R......... & 314 \\
\hline 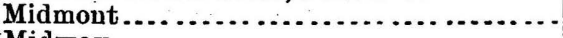 & N. Y., L. E. \& W. R. R ...... & 2,101 \\
\hline Midway $\ldots \ldots \ldots \ldots \ldots \ldots \ldots$ & L. S. \& M. S. R. R.............. & 996 \\
\hline 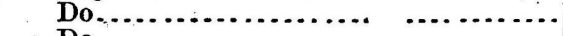 & Pa. R. R... & 394 \\
\hline (1) & P., C. \& St. L. R & 1,106 \\
\hline Mifflin .... & L. \& T. R. R... & 490 \\
\hline Do....... & Pa. R. R ........ & 441 \\
\hline Mifflinburgh ......... & L., C. \& S. C. R. R ........... & 565 \\
\hline Mifflin Cross-roads ........ . & D., H. \& W. R. R ........... & 811 \\
\hline Mile Post . . . . . . . . . . . . & A. V.R.R........ & 746 \\
\hline Milesburgh ...... & ranch R.R....... & 700 \\
\hline Milford & S. \& M. P.F & 1,932 \\
\hline Mill Creek . & Pa. R. R.. & 604 \\
\hline I Creek Junction ... & M. C. R. R. & 633 \\
\hline Miller, Logan's Run . . . . . . . & L., C. \& S. C. R. R .......... & 1,070 \\
\hline Millers............... & 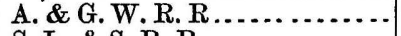 & 1,169 \\
\hline Do....... & S. L. \& S. R. R .............. & 1,330 \\
\hline Millersburgh ... & S. B. R. R . . . . . . . . . . & 397 \\
\hline Do......junction with L. V.C.R. R... & N. C. R. R $\ldots \ldots \ldots \ldots \ldots$ & 396 \\
\hline Miller's Farm ....... & 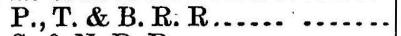 & 1,131 \\
\hline Miller's Mill..... & S. \& N. R. R...... & 487 \\
\hline Miller's Station & Bennett's Br. R. R ........... & 905 \\
\hline Millerstown .... & E. Pa. R. R . . . . . . & 383 \\
\hline Do..... & 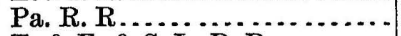 & 408 \\
\hline Millerton . & T. \& E. \& S. L. R. R ........ & 1,246 \\
\hline Millgrove... & Yough. R. R . . . . . . . . . . & 867 \\
\hline Mill Hall . . . . . . . . . . . . . & B. \& E. Valley R. R ............ & 573 \\
\hline Mill Lane................. & Chester Valley R. R......... & 315 \\
\hline Millmont $\ldots . \ldots \ldots \ldots$ & & 585 \\
\hline
\end{tabular}




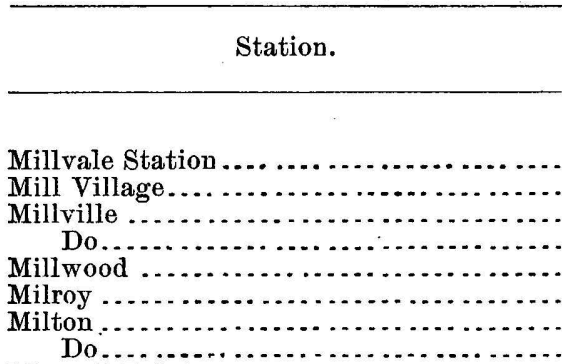

Mineral Point.

Do...... junction with B.\& O.R.R., Pittsburgh Division...

Do....... S. \&. M. P. R. R. junction.

Minersville..............................

Mingo ............................

Minnequa.

Mintzer's

Modena.

Mohrsville

Monckton

Monocacy

Monongahela City

Monroe Station

Monroeton, junction with S., L. \& E. R. R .

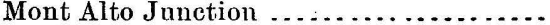

Do . .......... . near the furnace......

Montandon, junction of L. C. \& S. C. R. R.

Monterey Station

Montgomery

$$
\text { Do. }
$$

Montoursville

Montrose

Montrose Station

Mooreheads

Mooresburgh

Moore's

Moorestown

Moravia

Moredale

Morgans

Morganza

Morning Side Station

Morrisville, N. E side of Wash stret ...

Morton

Mortonville

Moscow

Mount Bartow

Mount Braddock

$$
\text { Do }
$$

Mount Carbon

Mount Carmel

Mount Dallas

Mount Eagle.

Mount Joy, east side of R. R. hotel

Mount Pleasant

Do

Do

Mount Union, junction of E. B. T. R. R. (narrow gauge) -.............

Mountville.

Mount Wolf

Muddy Creek Forks, B. M

Mud Pike

Muncy

Do

Authority. Eleva

Pa. R. R.

A. \& G. W. R. R

N. Y., L. F. \& W. R. R

Bennett's Br. R. R.

Pa. R. R.

M. \& C. Co. R. R

P. \& E. R. R.

C. \& W. R. R .

Pa. R. R.

S. \& M. P. R. R

B. \& O.R. R.

M. H. \& S. H. R. R

Pbil. \& R. R. R

N. C. R. R

C. \& W. R. R

W. \& R. R. R

Phil. \& R. R. R

Phil. \& R. R. R

P. V. \& C. R. R

W. Pa. R. R

B. R. R

Cumberland Valley $\mathbf{R} . \ddot{R}$

Mt. A. R. R :

P. \& E. R. R

A. V.R.R

C. \& W. R. R

P. \& E. R. R.

C. \& W. R. R

M. R. R .

Del., L. \& W. R. R

L. S. \& M. S. R. R

C. \& W. R. R

P., W. \& B. R. R

E. B. \& W. R. R

N. C. \& B. V. R. R

H. \& B. T. R. R

P. \& C. R. R .

Chartiers R. R

A. V.R. R

Phil. \& T. R. R

W. C. \& P. R. R

W. \& R.R.R

Del., L. \& W. R. R

C. R. R

S. W. Pa. Extension

P. \& C. R. R .

Phil. \& R. R. R

N. C. R. R

Bed. \& Bridge. R. R

B. E. Valley R. $R$

Bennett's Branch R. R

T. \& C. R. R

P.\& C. R. R

Pa. R. R

Pa. R. R.

N. C.R.R

P. B. R. R

S. \& M. P. R. R

C. \& W. R. R

P.\& E. R. R

\section{Feet. \\ 833 \\ 1,216 \\ 780 \\ 1,093 \\ 1,155 \\ 746 \\ 473 \\ 465 \\ 1,414}

1,824

1,825

700

116

1,230

964

278

302

344

162

753

840

762

714

968

462

875

486

490

525

1,656

1,053

768

618

94

443

806

1,680

944

938

747

34

121

260

1,558

469

1,203

1,175

606

1,054

i, 053

662

366

999

1,784

1,086

597

404

376

367

2,016

495

519 


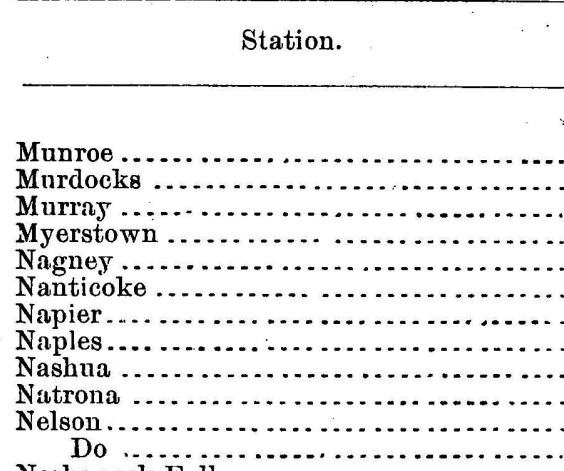

Neshanock Falls

Nesquehoning

New Albany

New Berlin

Newberry

New Bethlehem

New Bridge ...

New Brighton

New Buffalo, water in mill-race

New Castle

Do ....junction with N. C. Br. of P.,Ft W. \& C. R. R

Do .... junction with E. \& P. R. R ...

Do ....junction with N. C. \& B.V.R.R. half mile south of city

New Castle Pool

New Columbia

New Cumberland

New Florence

New Freedom

New Gallilee.

New Hanburgh

New Haven

Newkirk

New Milford

New Philadelphia

Newport

$$
\text { Do }
$$

Do.

New Providence Station

Newton

Newton Hamilton

Newton's Mills

Newtown

Newville

New York Junction

Nicetown

Nicholson

Nigger Hill

Niles Valley

Nineveh

Do.junction

Noble's

Noblestown

Norristown

Northbrook

North East

North Pa. R. R. junction

North Point.

Northumberland, intersection with Bloomsburgh Division D., L. \&.

Cumberland T'p'k

Pa. R. R

L. \& T. R. R

Lebanon Valler $\mathrm{R} . \mathrm{R}$

M. \& C. Co. R. R ...........

Del., L. \& W. R. R

Bed. \& Bridge. R. R

L. S. \& M. S.R.R

E. \& P.R. R

W. Pa. R. R

C., C. \& A. R. R

N.C. \& F. R. R

N. C. \& F. R. R

N. Valley R. $R$

S. L. \& S. R. R

C. R. R

P. \& E. R. R

Bennett's Branch R. R

H. \& B. T. R. R

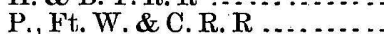

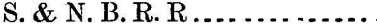

M. C. R. R

E. \& P.R. R

N. C. \& B. Y. R. R

N. C. \& F. R. R

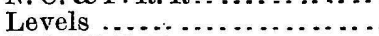

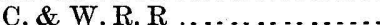

N. C. R. R.

Pa.R.R

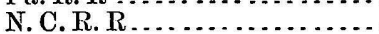

P., Ft. W. \& C. R. R

S. \& A. R. R

S. W. Pa. Extension

S. Valley R. R

Del., L.\& W. R. R

S. Valley R. R

Lehigh Valley R. R

N. C. \& B. V. R. R

Pa. R. R.

L. \& Q. R. R

D., A. V. \& P. R. R

Pa. R. R.

P. \& P. R. R

Pliil. \& N. R. R

Cumberland Valley R. R....

Phil. \& R. R. R.............

Phil. \& R. R. R............

Del., L. \& W. R. R .........

N. Pa. R. R . . . . . . . . . .

C., C. \& A. R. R..........

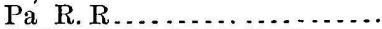

N. Y., L. E. \& W. R. R .....

U. \& T. R. R . . . . . . . . .

P., C. \& St L. R. R.........

Phil. \& R. R. R ............

W. \& R. R. R.................

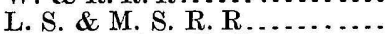

Lehigh Valley R. R........

P. \& E. R. R ...............

S. \& N. B. R. R
Elevation.

Feet.

1,065

1,626

456

474

678

538

1,108

1, 165

821

768

1, 186

1,060

992

801

1,197

364

529

1,080

831

750

377

876

809

803

793

799

477

312

1,076

827

957

1, 158

893

912

1,087

690

1,023

812

395

401

1,411

599

1,258

145

533

105

139

769

452

1, 192

1, 121

981

1, 298

926 


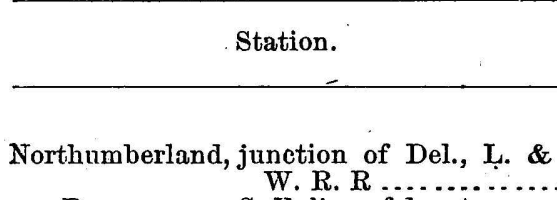
Do North Warren S. E. line of depot.

North West

Oakdale Do

Oak Hill Station

Oakland Do

Oakley'

O'Harra

Ohio Pyle

Oil City

Do.

Do. Centre street crossing

Do. east, connects with A. V. R. R ...

Do.conneets with P., T. \& B. R. R. \& with Frankliu Br. of the A. \& G. W. R. $R$

Oleopolis

Do.

Olyphant

Ore Valley, B. M

Osceola .

Paintersville.

Palmyra

Paoli

Paoli Road

Paradise

Pardoe

Paris Road

Parker Junction with A. V. R. R

Parker Station

Parkesburgh

Parkton

Parnassus Station

Parryville

Paschall

Patton's

Do. bench on top of parapet coping. of arch culvert, W. side of road ......

Paxton

Peach Bottom

$$
\text { Do... }
$$

Pecopson

Pencord

Pennsylvauia House

Peullinn

Penntielıl

Penn Haven

Do $\therefore$... juuction

Do...... Do

Penningtonville

Penn Junction

Peunock's Summit

Peun Station

$$
\text { Do }
$$

Pennsville

Penn Valley

Pequen Station.

Perkiomen Junction

Perryville.

\begin{tabular}{r|r} 
Authority. & Elevation. \\
\hline Feet.
\end{tabular}

P. \& E. R. R .............. 454

Del, L. \& W. R. R .........

D., A. V. \& P. R. R......... 1, 216

W. Pa. R. R ................

B. \& O. R. R .............. 849

P., C. \& St. L. R. R ........ 906

Pa. R. R.................. $\quad 750$

Chester Valley R. R ........ 301

Del., L. \& W. R. R ......... 1,011

Del., L. \& W. R. R .......... 945

Perkiomen R. R ............ 130

E. \& C. R. R ..............

B. \& O. R. R

L. S. \& M. S. R. R..., ....

P., T. \& B. R. R

P., T. \& B. R. R

L. S. \& M. S. R. R

L. S. \& M. s. R. R

P., T. \& B. R. R

Pitbole Valley R. R

P. B. R R

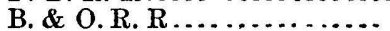

S. W. Pa. R. R...............

Lebanon Valley R. R .......

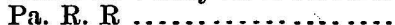

Chester Valley R. R

Del., L. \& W. R. R.

S. \& A. R. R .

P., C. \& St. L. R. R

P. \& K. C. R. R . . . . . . .

A. V.R. R

Pa. R. R.

N. C. R. R

A. V. R. R

L. \& S. R. R

P., W. \& B. R. R

W. C. R. R

2,015

1,237

1,010

1,008

1,110

1,010

1,017

1,032

1,019

810

570

768

952

455

534

238

1,521

1,205

835

888

889

537

430

763

443

58

505

Bennett's Br. R. R

Lebanon Valley $R$. $\mathrm{R}$

C. \&.P.D. R. R

P. B.R.R.

W. \& R. R. R .

Phil, \& R. R R

P., T. \& B. R. R

N.Pa. R. R ........

..............

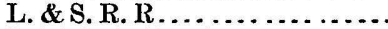

Lehigh Valley R. R

L. \&. S. R. R.

Pa. R. R

E.Pa. R.R.

Pa. \& Del. R.

P. \& B. C. R. R.

Pa. R. R

S. W. Pa. R. R.

Phil. \& T. R. R

L. \& Q. R. R

Phil. \& R. R. R

Pa. R. R
1,131

376

99

118

180

61

1,153

239

1,289

724

705

708

500

257

463

506

974

1,054

21

300

109

441 
\begin{tabular}{l|l}
\hline Authority. & Elevation.
\end{tabular}

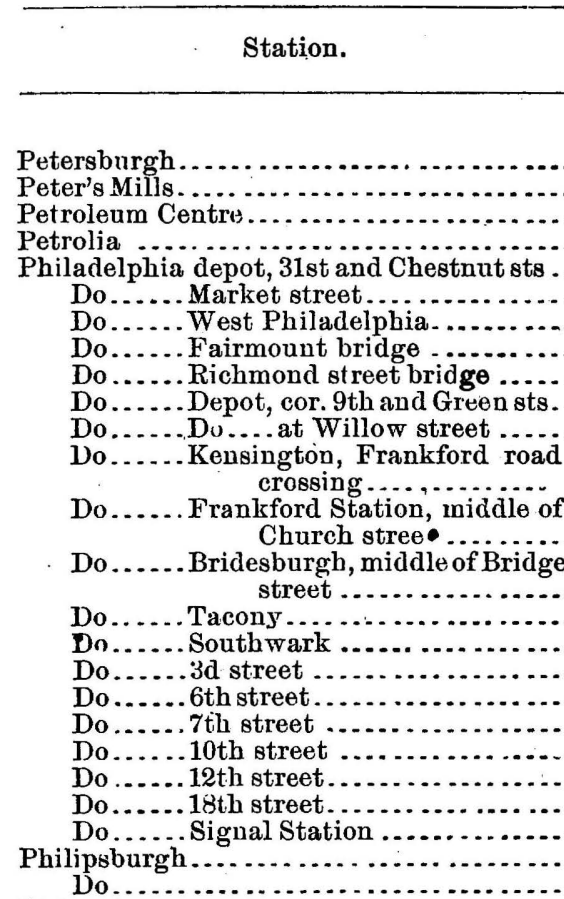

Philson's

Phonixville.

Picture Rock

Pikeland

Pine

Pine Creek

Pinegrove.

Pine Grove.

Do

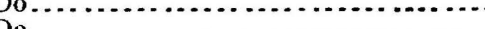

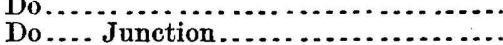

Pine Hill.

Pinkerton.

Pioneer .

Piper's Run

Pithole City

Pittsluurgh.. Union Depot

Do.... on window-sill of Monongahela

Incline Plane, Check House..

Do .... on Belt course of Union Depot,

maiu entrance ...............

Do.... on East end door-sill of Point Breeze Hotel, at intersection of Penn. and Fifth avenues..

Do.... on Belt course of Munshall's distillery, corner Penn. ave. and Water street

N. Pa. R. R

Phil: \& T. R. R

City levels .................

Reservoir, on Bedford ave....
Do.... on embankment of Upper (old) Reservoir, Bedford ave ......

Do.... on flow-line of Highland ave.

(new) Reservoir.

Do .... on flow-line of Herron Hili (new) Reservoir
Pa. R. R

L. \& L. R. R . . . . . . . . . . P., T. \& B. R. R

P. \& K. C. R. R

W., C. \& P.R.R

Pa.R. R

Pa. R. R

Pa. R. R ..

Phil. \& R. R. R

Phil. \& R. R. R...............

Phil. \& T. R. R

Phil. \& T. R. R

Phil. \& T. R. R

P., W. \& B. R. R

P., W. \& B. R. R

P., W. \& B. R. R

P., W. \& B. R. R

P., W. \& B. R. R

P., W. \& B. R. R

P., W. \& B. R. R

U. S. Signal Office

T. \& C. R. R

A. V. R. R

B. \& O.R. R. .

Phil. \& R. R. R

M. C. D. R

Pickering Valley R. $\mathbf{R}$

P. \& E. R. R. . . . . . . . . . .

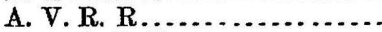

B. \& O.R. R

L., C. \& S: C. R. R

S. \&.A. R. R

N. Y., L. E. \& W. R. R

S. \& S. R. R

B. V. R. R.

B. \& O. R. R $\mathbf{R} \ldots \ldots \ldots \ldots$

P., T. \& B. R. R

H. \& B. T. R. R .............

Pithole Valley R. R..........

P., Ft. W. \&. C. R. R

City levels ..................

City levels

973

City levels ..................

City levels

City levels

751

865

Feet.

678

1, 085

1,175

14

32

51

28

47
28

32

32

32

34

7

3:3

34

32

27

36

1,425

855

1,820

110

668

272

565

812

1,874

1,236

1,250

668

520

1,649

1, 099

1,309

745

1,106

746

City levels

1, 101

City levels

1,064

City Levels.

1,259 


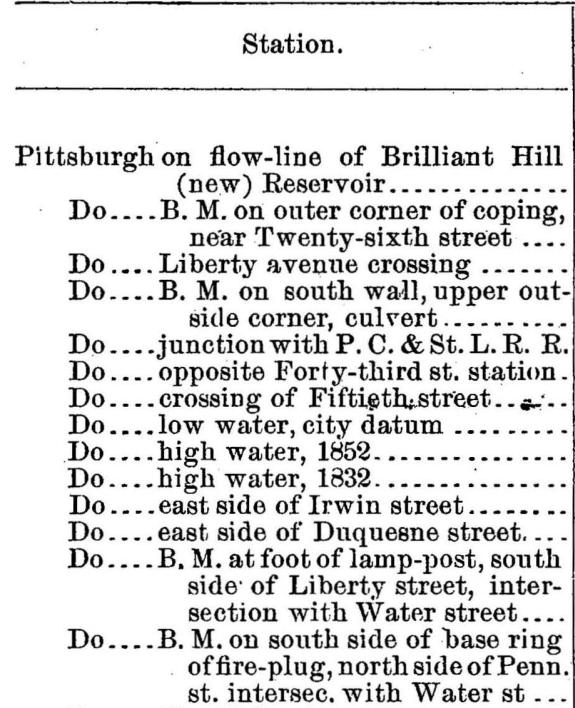

Do.... Sigual Station .. .............

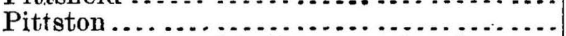

Do - west end bridge, crossing Susquehanna .....................

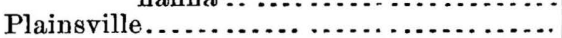

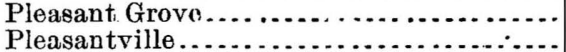

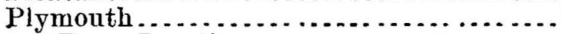

Do... Junction . . . . . . . . . . .

Point Lookout . . . . . . . . . . . . . . . . .

Polk.................................

Pomeroy, E. intersec. of P. \& D. R. R...

Do... W. intersec. of P. \& D. R. R...

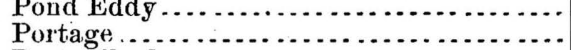

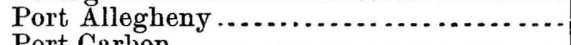

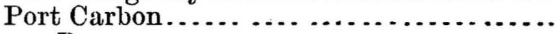

Do ....................................

Port Clinton ................................

Do....

Port Jervis . .............................

Port Kennedy

Portland.

Port Matilda, Main st.........................

Port Perry Junction.

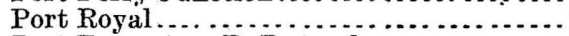

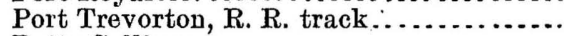

Potts Colliery .........................

Pottsgrove ..............................

Pott's Landing.

Pottstown.

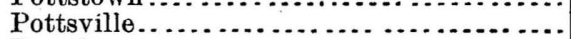

Powelton ........................................

Pulaski.............................

Prather.

Prescott. . . . .

President. . . . . . . . . .

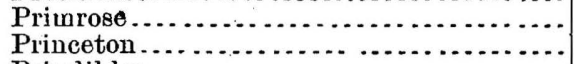

Prindibles.

Quakake Station

Quakertown

\begin{tabular}{|c|c|}
\hline Authority. & Elevation. \\
\hline Feet.
\end{tabular}

City levels ................. 934

A. V. R. R................ 745

A. V. R. R .............. 743

A. V. R. R ............... 728

P., V.\& C. R. R.........

P., V. \& C. R. R ........... 732

P., V. \& C. R. R ............ 739

Levels ......................

Levels ................... $\quad 730$

Levels .................... 733

Penn. R. R ................ 737

Penn. R. R ............... 732

Penn. R. R ............... 721

Penn. R. R ................. 726

U. S. Signal Office............. $\quad 762$

D., A. V. \& P. R. R......... 1,245

Del., L. \& W. R. R .......... 572

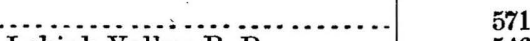

Lehigh Valley $\mathrm{R}, \mathrm{R} . \ldots . . .$.

H. \& B. T. R. R........... 748

Pithole Valley R. R........... $\quad 1,634$

Del., L. \& W. R. R..........

Del., L. \& W. R. R..........

B. G. R. R................. $\quad 1,915$

L. S. \& M. S. R. R.......... 1,084

Pa. R. R............... $\quad 483$

Pa. R. R................... 489

N. Y., L. E. \& W. R. R...... $\mathbf{5 7 0}$

Pa. R. R... .............. $\quad 1,675$

B., N. Y., \& P. R. R.......... $\quad 1,482$

S. Valley R. R...............

M. C. R. R ................ 634

Del. \& Hudson Canal..........

Phil. \& R. R. R ............. $\quad 410$

Del. \& Hudson Canal:........ 455

Phil. \& R. R. R........... 87

Del., L. \& W. R. R..........

B. E. Valley R. R........... $\quad 1,007$

B. \& O. R. R..............

B. \& O. R. R ............ 792

S. \& N. B. R. R.............. 428

N. H. \& S. H. R. R........ 1,094

C. \& W. R. R.............. 489

Phil. \& R. R. R............

Phil. \& R. R. R........... 150

Phili \& R. R. R............. 614

T. C. R. R .............. $\quad 1,798$

E. \& P. R. R ............. 826

Pithole Valley R. R............ 1,251

Lebanon Valley R. R........ 501

P., T. \& B. R. R ............. . $\quad 1,048$

P., U. \& St. L.R.R........ $\quad 1,021$

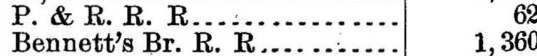

L. R. R................... $\quad{ }_{703}$

C. \& W. R. R................ 1,353

N. C. \& B. V. R. R......... 817 


\begin{tabular}{|c|c|c|}
\hline Station. & Authority. & Elevation. \\
\hline & & Feet. \\
\hline Quakertown & N. Pa. R. R. & 496 \\
\hline 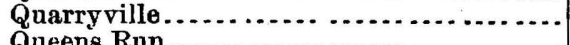 & L. \& Q.R.R & 488 \\
\hline Queens Run & P. \& E. R. R & 584 \\
\hline Radebaughs Station $\ldots \ldots \ldots$ & Pa. R. R. ... & 1,150 \\
\hline $\begin{array}{l}\text { Radnor....... } \\
\text { Rahn's...... }\end{array}$ & $\begin{array}{l}\text { Pa. R. R.... } \\
\text { Perkiomen } \mathrm{R} .\end{array}$ & 409 \\
\hline $\begin{array}{l}\text { Rahn's............. } \\
\text { Ralston........... }\end{array}$ & $\begin{array}{l}\text { Perkiomen R } \\
\text { N. C. R. R... }\end{array}$ & $\begin{array}{l}138 \\
860\end{array}$ \\
\hline Ransom & Lehigh Valley & $\begin{array}{l}860 \\
579\end{array}$ \\
\hline Rarigs Station................ & C. \&W.R.R & 1,340 \\
\hline Rathbon $\ldots . . . \ldots \ldots \ldots \ldots \ldots \ldots$ & P. \& E. R. R & 1,317 \\
\hline 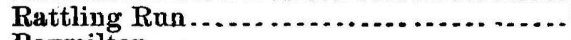 & S. \& S.R.R. & 692 \\
\hline Raymilton $\ldots \ldots \ldots \ldots \ldots \ldots \ldots \ldots$ & L. S. \& M. S. R & 1,138 \\
\hline $\begin{array}{l}\text { Reading } \\
\text { Do. B. M. on coping stone easternmost }\end{array}$ & Phil. \& R. R. & 268 \\
\hline 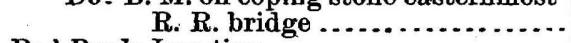 & U.S. C.\& G. & 264 \\
\hline Red Bank Junction. ....................... & Bennett's Br. R. I & 851 \\
\hline Redington & Lehigh Valley & 211 \\
\hline Red Lion & C. \& F.R.R ..... & 793 \\
\hline Do & P. B. R. R........... & 900 \\
\hline 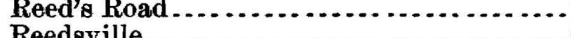 & E., B.\& W. R & 309 \\
\hline Reedsville $\ldots \ldots \ldots$ & M. \& C.Co.R & 593 \\
\hline tion $\ldots . .$. & Pa. K. R.. & 903 \\
\hline $\begin{array}{l}\text { Reeseville............ } \\
\text { Reimerton }\end{array}$ & $\begin{array}{l}\text { Pa. R. R . } \\
\text { A. V.R.R. }\end{array}$ & $\begin{array}{l}495 \\
837\end{array}$ \\
\hline $\begin{array}{l}\text { Reimerton } \ldots \ldots \ldots \ldots \\
\text { Reinhold's } \ldots \ldots \ldots \ldots\end{array}$ & R.\& C.R. R. & 449 \\
\hline Reiter...................... & L. \& L.R.R.. & 299 \\
\hline Remington $\ldots \ldots \ldots \ldots \ldots$ & P., Ft. W. \& C.R.R........ & 710 \\
\hline Reno.............. & 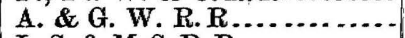 & 1,011 \\
\hline Do. & L.S.\& M.S.R.R & 1,018 \\
\hline Renovo... & P. \& E. R.R . & 672 \\
\hline Reynolds. : & L. Schuyl. R.R.............. & 663 \\
\hline $\begin{array}{l}\text { Reynoldsville, at crossing of Brookville } \\
\text { pike }\end{array}$ & & \\
\hline Rheems & Pa. R. R ..... & $\begin{array}{r}1,377 \\
439\end{array}$ \\
\hline Riceville $\ldots \ldots \ldots \ldots \ldots$ & U. \& T.R.R & 1,369 \\
\hline 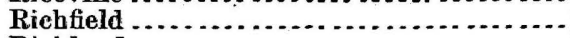 & S. \& N.R.R. & 765 \\
\hline Riebland $\ldots \ldots \ldots \ldots \ldots$ & Lebanon Valley $I$ & 491 \\
\hline Riddlesburgh $\ldots \ldots \ldots \ldots \ldots \ldots \ldots$ & H. \& B.T. R. R .. & 865 \\
\hline Ridgeway...$\ldots \ldots \ldots \ldots \ldots \ldots \ldots$ & P.\& E.R.R & 1,393 \\
\hline Do $\ldots \ldots \ldots \ldots \ldots$ & N. Y., R. \& P. & 1,376 \\
\hline Ridley Park . . . . . . . . . . . . . . . . . . . . . & P., W. \& B. R. & 73 \\
\hline 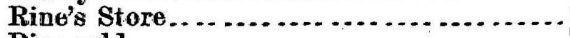 & S. \& N. R. R.... & 412 \\
\hline Ringgold $\ldots \ldots \ldots \ldots \ldots \ldots \ldots$ & 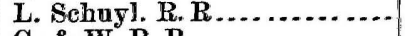 & 558 \\
\hline Ringtown & 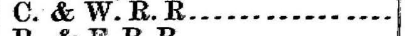 & 1,130 \\
\hline 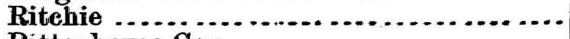 & P. \& E.R.R & 632 \\
\hline Rittenhouse Gap & C. \& F.R.R $R$ & 940 \\
\hline n & $\begin{array}{l}\text { B. \& U.R.R.... } \\
\text { T. \& C.R.R... }\end{array}$ & 1,466 \\
\hline $\begin{array}{r}\text { Roaring Run } \\
\text { Do }\end{array}$ & W.Pa. R. R . & $\begin{array}{r}1,426 \\
830\end{array}$ \\
\hline Roaring Spring Junction . . & 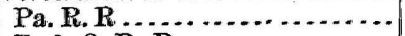 & 1,196 \\
\hline 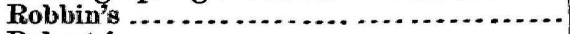 & B. \& O.R. R... & 768 \\
\hline 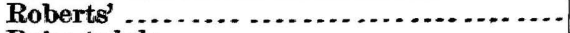 & S. \& M. P.R. R ... & 2,042 \\
\hline Robertadale ......... & 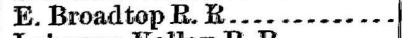 & 1,785 \\
\hline nia.......... & Lebanon Valley R. R......... & 441 \\
\hline tion & 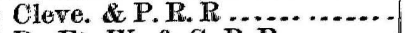 & 711 \\
\hline Do .. junction with C. \& P. R. R .... & P., Ft. W. \& C. R.R........ & 706 \\
\hline 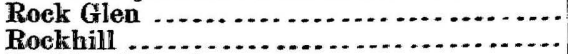 & D., H. \& W. R.R R........... & 921 \\
\hline Rockhill $\ldots \ldots \ldots$ & E. Broadtop R. R . $\ldots \ldots \ldots$ & 624 \\
\hline Rockland Station................. & A. V. R.R R & 926 \\
\hline Rockport $\ldots \ldots \ldots \ldots \ldots$ & Lehigh Valley R. R ............ & 906 \\
\hline $\begin{array}{r}\text { Do } \\
\text { Rockvifle }\end{array}$ & L. \& S.R. R & 882 \\
\hline 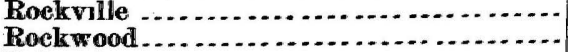 & P. $\mathrm{S}_{\mathrm{T}} \mathrm{R} \cdot \mathrm{R}, \ldots$ & 1016 \\
\hline Rockwood... & P.,T.\& B.R.K.. & $\begin{array}{r}1,016 \\
352\end{array}$ \\
\hline & & \\
\hline
\end{tabular}




\begin{tabular}{|c|c|c|}
\hline Station. & Authority. & Elevation. \\
\hline & & \\
\hline Romain ........ & S.R.R R & $\begin{array}{l}1,974 \\
1,222\end{array}$ \\
\hline Rootsedale..... & $\begin{array}{l}\text { B.G. R.R. } \\
\text { P.\&B. R. R }\end{array}$ & $\begin{array}{r}1,222 \\
312\end{array}$ \\
\hline Rosemont ..... & Pa. R. R ... & 395 \\
\hline Ross ............. & W.Pa.R.R & $\begin{array}{r}745 \\
788\end{array}$ \\
\hline Rough and Read & H. \& B. T. R. I & 889 \\
\hline $\begin{array}{l}\text { Roulette } \\
\text { Round Island.... }\end{array}$ & J. S.,P. C., \& B.R.R & 1,537 \\
\hline Roups Station... & Pa. R. R.... & 882 \\
\hline Rouseville ..... & P., T. \& B. R.R & 1,039 \\
\hline $\begin{array}{l}\text { Rowlands .......... } \\
\text { Royer's Ford .... }\end{array}$ & $\begin{array}{l}\text { N. Y., L. E. \& } \\
\text { Phil. \& R.R.R }\end{array}$ & 704 \\
\hline Roy Stone......... & P.\& E.R.R. & 1,415 \\
\hline Run ...... & L. S. \& M. S. & 996 \\
\hline Runmerfield . & Lehigh Valley & 696 \\
\hline Rupert .......... & & 495 \\
\hline $\begin{array}{l}\text { Do.............. } \\
\text { Russelburg .... }\end{array}$ & $\begin{array}{l}\text { Del., L. \& W. I } \\
\text { D., A. V. \& P. I }\end{array}$ & $\begin{array}{r}482 \\
1,233\end{array}$ \\
\hline utherford's & Lebanon Val & 429 \\
\hline Rynd Farm ... & P.,T.\& B. R & 1,043 \\
\hline town' ..... & A. \& G. W. I & 1,116 \\
\hline $\begin{array}{l}\text { Safe Harbor........ } \\
\text { St. Clair ............ }\end{array}$ & C. \& P. D. R. & 198 \\
\hline t. Clair ... & $\begin{array}{l}\text { M. C. R.R.. } \\
\text { Pa.R.R .... }\end{array}$ & $\begin{array}{r}752 \\
1,09 \%\end{array}$ \\
\hline St. George Station & A. V.R.R $\mathbf{R}$ & 935 \\
\hline $\begin{array}{l}\text { St. Mary's...... } \\
\text { Salem }\end{array}$ & P.\& E. R.R.. & 1,667 \\
\hline Salina - & $\begin{array}{l}\text { L. S. \& M. S. K. } \\
\text { W. Pa. R. R.... }\end{array}$ & $\begin{array}{l}998 \\
955\end{array}$ \\
\hline Salisbiury ....... & S. R. R ............ & 2,032 \\
\hline Saltil & E. Broadtop I & 781 \\
\hline $\begin{array}{l}\text { Saltsburgh....... } \\
\text { Salt Works .... }\end{array}$ & B. \& O. R. R $\ldots$ & $\begin{array}{r}765 \\
766\end{array}$ \\
\hline Saltzburgh, Marke & $\begin{array}{l}\text { B. } \\
\text { W. Pa. R. R......... }\end{array}$ & \\
\hline Salunga ........... & Pa.R.R....... & 403 \\
\hline 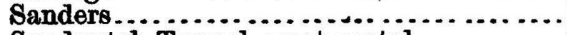 & E. \& C. R. R . & 2,019 \\
\hline Sandpatch Tunnel, & B. \& O.R.R. . & 2,283 \\
\hline $\begin{array}{r}\text { Do.............. } \\
\text { Sand Works }\end{array}$ & $\begin{array}{l}\text { B. \& O.R.R. R } \\
\text { B. \& O.R.R. }\end{array}$ & ¿, 235 \\
\hline reek Stat & A. V.R.R.... & 746 \\
\hline Lake........ & L. S. \& M. S. R. R & 1,165 \\
\hline Lick Station & W.Pa.R.R....... & 887 \\
\hline 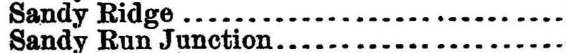 & $\begin{array}{l}\text { T. \& C. R. R ..... } \\
\text { L. \& S. R. R ..... }\end{array}$ & $\begin{array}{l}1,912 \\
1,025\end{array}$ \\
\hline Sang H & Pa. R. R ........... & 1,143 \\
\hline Sanner's ............. & S. \& M. P. R. R ..... & 1,839 \\
\hline $\begin{array}{l}\text { Sarah Furnace Sta } \\
\text { Sartwell: }\end{array}$ & 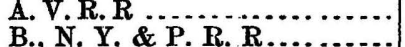 & $\begin{array}{r}861 \\
1,452\end{array}$ \\
\hline & W. Pa. R. R . . . . . & $\begin{array}{l}1,406 \\
1,027\end{array}$ \\
\hline & W. Pa. R. R & 1,201 \\
\hline $\mathbf{S}$ & H. \& B. T. R. R......... & 849 \\
\hline ....... & Lehigh Valley R. R...... & 774 \\
\hline ghonda ..... & P. \& E. R.R R $\ldots \ldots \ldots \ldots$ & $1, \frac{522}{108}$ \\
\hline & Phila. \& R. R. R .. & \\
\hline sill Bridge & Lebanon Valley $\mathbf{R}$. $\mathbf{R}$ & 274 \\
\hline huylkill Haven & Phila. \& R. R. R ............... & 526 \\
\hline Schwenkville ..... & Perkiomen R. R .............. & 152 \\
\hline otch Valley . & $\begin{array}{l}\text { D., H. \& W. R. R . . . . . . } \\
\text { S.W.Pa. R. R }\end{array}$ & $\begin{array}{l}1,017 \\
1,042\end{array}$ \\
\hline & E. Broadtop R. R & 717 \\
\hline ranton, Union D & Del., L. \& W. R. R.... & 739 \\
\hline Do... Hyde Park & City Engineer ............. & 746 \\
\hline & City Engine & 716 \\
\hline
\end{tabular}




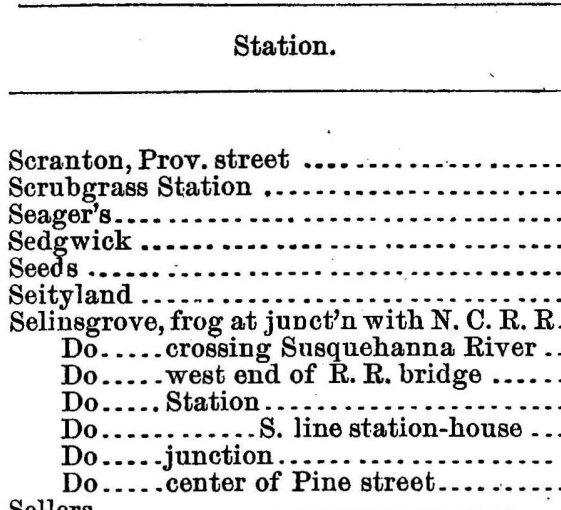

\section{Sellers ...}

Sellersville.

Sewickley

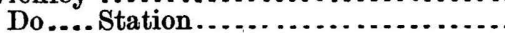

Do.... Y. Branch of Pennsylvania R. $R$

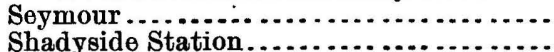

Shaeffer's

Shaffer.

Shafton Station

Shainlines

Shamokin

Shamrock

Shanghai Station

Sharon

Sharon Hill

Sharpsburgh, Main street

Sharpsburgh Station

Sharpsville

Shaw mont

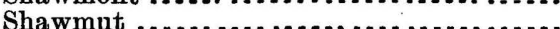

Shaw's Landing

Shaw's Run.

Sheffield

Shelmires

Shenandoah, City Depot................

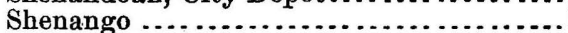
Do... Grade crossing S. \& A. R. R

Shenk's Ferry

Sheridan Do

Shimer's

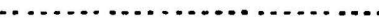

Shimmels .............................

Ship Bridge

Shippen...

Shippensburgh

Shippensburgh, B. M. on water table, N.

W. corner Main \& Railroad sts

Shirleysburgh

Shock's Mill

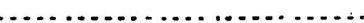

Shoemakersville.

Shohola.

Shoo-Fly Tumel

Shugart? Tunnel

Shuman's Bridge, bench-mark on W. end..

Shuman's Tunnel

Sinking Springs.

Sinnemahouing

Skippack

Slab Run

\begin{tabular}{|r|} 
Authority. \\
Elevation. \\
Feet.
\end{tabular}

City Engineer ...............

A. V. R. R .............. 945

C. \& W. R. R ........... 512

B. \& O. R. R .............. 868

W. \& R. R. R .............. 195

N. C. R. R................ 611

Sunbury \& Lewistown R. R.. 438

Sunbury \& Lewistown R. R.. $\quad 439$

Sunbury \& Lewistown R. R.. 439

Sunbury \& Lewistown R. R.. $\quad \mathbf{4 4 0}$

N. C. R. R.............. 438

N. C. R. R................... 438

S. \& N. B. R. R........... 441

R. \& C. R. R............... 382

N. Pa. R.R............ 331

P., Ft. W. \& C. R. R ....... 736

Yough. R. R .............. $\quad 780$

B. \& O. R. R .............. 779

N. C. \& B. V.R. R.......... 797

Pa.R.R ................. 856

Bennett Branch R. R........ 1, 425

P., T. \& B. R. R ............. $\quad 1,133$

Pa. R. R ................. 905

Chester Valley R. R.......... 136

N. C.R.R.............. $\quad 738$

E.Pa. R.R............... 433

Pa. R. R .................. $\quad 1,173$

A. \& G. W. R. R .......... 859

P.,W.\& B.R.R........... 65

W. Pa. R. R .............. 739

A. V.R. R .............. $\quad 745$

E. \& P.R. R ............. 948

Phil.\& R. R. R............. $\quad 69$

P. \& E. R.R ............. 1,427

A. \& G. W. R.R........... 1,092

T. \& C. R. R ............... 1,112

P. \& E. R. R .............. 1,337

E. B. \& W. R. R............ 246

M. \& S. R. R . . . . . . . . . . . . . $\quad 1,252$

E. \& P. R. R .............. 941

A. \& G.W.R.R ........... , 936

C. \& P. D.R.R............

Lebanon Valley R. R......... 459

P., C. \& St. L. R. R.......... $\quad 846$

Del., L. \& W. R. R.......... 521

L. \& L. R. R ............... $\quad 289$

T. \& C. R. R ............. $\quad 1,641$

Pa. R. R................. 411

B., N. Y. \& P. R. R......... 1, 206

Cumberland Valley R. R .....

U. S. C. \& G. S ..............

E. Broadtop R. R........... 572

Pa. R. R ................. 269

Phil. \& R. R. R............. 314

N. Y., L. E. \& W. R. R....... 648

B. \& O. R. $R \ldots \ldots \ldots \ldots \ldots \ldots$

L. C. \& S. C. R. R......... 1,131

Pa. R. R .................. 699

C. \& W. R. R ............ 803

R. \& C. R. R............. $\quad 348$

P. \& E. R. R.............

Perkiomen R. R............ 145

Bennett's Br. R. R .......... 1, 405 


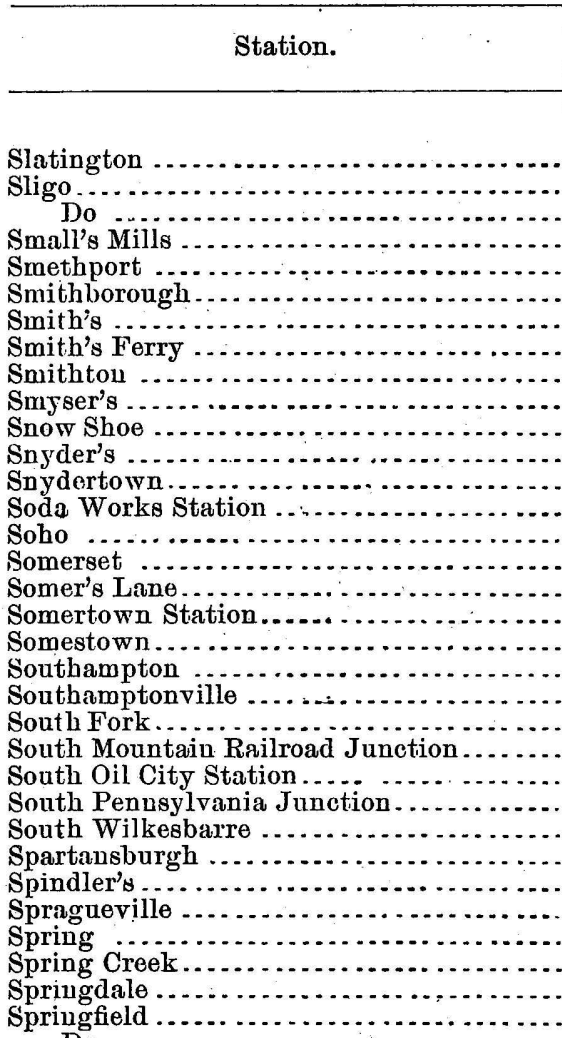

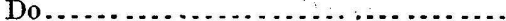

Do..................................

Do....Raiiroad Junction

Spring Forge

Spring Garden, bench-mark

Springhill Do

Spring Mill

Spring Run Village

Spring vale

Springville$$
\text { Do }
$$

Standing Stone

Stanhope

Star

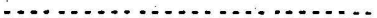

State Line

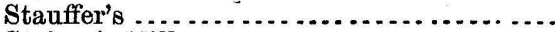

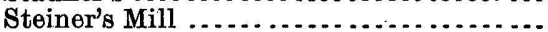

Sterling

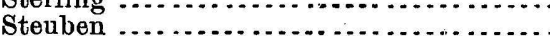

Steven's Point

Stewart

Stewart's Station

Stewart's Switch

Stillwater

Stoneboro'

Stoneboro's, junction with $\mathrm{F}$ division of $\mathrm{L}$.

S. \& M. S. R. R

Authority. Elev

Lehigh Valley R. R

A. V.R. R

W. Pa. R. R

P. B. R. R.

McK. B. R. R

S. C. R.R

Lebanon Valley R. R

Cleve. \& P. R. R.

B. \& O.R. R

N. C. R. R

B. \& S. S. R.R

B. \& O.R. R

N. C. R.R.

A. V.R. R .

B. \& O.R.R.

S. \& M. P. R. R

Tioga R. R

N. Pa.R.R

M. C. R. R

B. \& O. R. R ..

Phil. \& N. R. R

Pa.R.R

Cumberland Valley R.R

A. V. R. R

Cumberland Valley R. $R$

Lehigh Valley R. R

P., T. \& B. R. R

Pa. R. R

Del., L. \& W. R. R

E. \& P. R. R.

P. \& E. R. R

W. Pa. R. R

Phil. \& R. R. R

L. S. \& M. S. R. R

W. \& R. R. R

Pa. R. R

Pa. R. R

P. B. R. R

W. C. \& P. R. R

Pa. R. R .......

Phil \& R. R.

Phil. \& R. R. R ..................

E. B. \& W. R. $\mathrm{R}$

P. B. R. R

M. R. R

Pa. R. R

Pa. R. R

Lehigh Valley R. R

S. \& S. R. R

D., A. V. \& P. R. R.

N. Y., L. E. \& W. R. R

B., N. Y. \& P. R. R

P. \& C. R. R

T. \& C. R. R

P. \& E. R. R

L. \& L. R. R

N. Y., L. E. \& W. R. R

P., T. \& B. R. R

Pa. $R$

P. T. \& B. R. R.

N. Y., L. E. \& W. R. R

L. S. \& M, S. R. R .....

N. C. \& F. R. R ...........
Elevation.

Freet.

366

1,115

775

434

1,493

794

472

698

792

389

1,572

788

497

761

769

2,128

1,018

156

946

1,567

240

1,485

458

1,009

632

546

1,457

404

490

961

1,395

749

69

664

645

876

455

432

128

749

53

877

405

734

1, 257

390

777

702

555

1,376

1,470

1,443

1,057

1, 428

916

333

1,120

1,047

791

1,473

1,560

1,171

1,171 


\begin{tabular}{|c|c|c|}
\hline Station. & Authority. & Elevation. \\
\hline 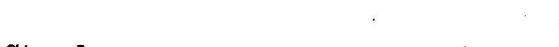 & & Feet. \\
\hline Stoneham .............. & P. \& E. R. R. & 1,347 \\
\hline Stone Honse .......... & P. \& K. C. R. R & 1,089 \\
\hline Street Road .......... & W.C. \& P.R.R & 252 \\
\hline Stroudsburgh .......... & Del., L. \& W. R. & 403 \\
\hline Sugar Creek ............ & A. \& G. W. R. R & 1,014 \\
\hline Sugar Grove ......... & A. \& G. W. R. R & $\overrightarrow{1}, 040$ \\
\hline Sugar Noteh ........... & Lehigh Valley $\mathrm{K}$ & 666 \\
\hline Do ..................... & L. \& S. R. R .. & 660 \\
\hline Sullivan $\ldots \ldots \ldots \ldots . . . .$. & P., Ft. W. \& C. & 765 \\
\hline Summerhill .......... & Pa. R. R ....... & 1,557 \\
\hline Sunbury & N. C. R. R. & 444 \\
\hline 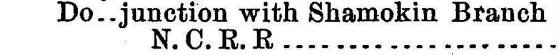 & N. C. R. R. & \\
\hline Do..north line of depot building ..... & N. C. R. R & 444 \\
\hline Do..junction with D. W.\& H.R.R & N. C. R. R. & 451 \\
\hline Do..Junction & D., H. \& W. R. & 443 \\
\hline $\begin{array}{r}\text { Do.-junction of Shamokin Branch of } \\
\text { N. C. R. } R \ldots \ldots \ldots \ldots \ldots \ldots\end{array}$ & & 444 \\
\hline Susquehanna ......... & N. Y., L. E. \& W. R. R, & 914 \\
\hline Do ................... & P. \& E. R. R . . . . . . & 534 \\
\hline$\ldots \ldots \ldots$ & Pa. R. R ................. & 342 \\
\hline$\ldots . . .6$. & Pa. R. R ....... & 350 \\
\hline Susquehanna Junction & N. Y., L. E. \& V & $98: 2$ \\
\hline Swansville ........... & E. \& P. R. R & 735 \\
\hline$\ldots \ldots \ldots \ldots \ldots$ & W. C. \& P. R. R & 125 \\
\hline $\begin{array}{l}\text { Swatara Gap } \ldots . . . . \\
\text { Swede Furnace } \ldots . . .\end{array}$ & $\begin{array}{l}\text { L. \& T. R. R ... } \\
\text { Phil, \& R. R. R. }\end{array}$ & $\begin{array}{r}447 \\
66\end{array}$ \\
\hline Swiss Vale Station .... & Pa. R. R ...... & $92 * 2$ \\
\hline 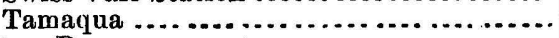 & C. \& W. R. R & 804 \\
\hline Do ................ & T. B. R. R ... & 787 \\
\hline Tamenend ......... & N. Valley R. R & 1,287 \\
\hline Tarentum. & W. Pa. R. R... & 757 \\
\hline Do & A. V.R.R... & 778 \\
\hline Tarr Farm . . ........... & P., T. \& B. R. R & 1,062 \\
\hline 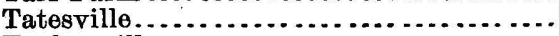 & H. \& B. T. R. R. & 1,096 \\
\hline Taylorsville......................... & B. \& O. R. R ... & 1,008 \\
\hline Taylorville $\ldots . . . \ldots \ldots \ldots \ldots \ldots \ldots \ldots$ & Del., L. \& W. R. & 683 \\
\hline Teeple Town $\ldots \ldots \ldots \ldots \ldots \ldots$ & P. \&. P. R. R ... & 1,204 \\
\hline Temperanceville....... & P., C. \& St. L. R. R......... & 767 \\
\hline 'Temple . . ........... & 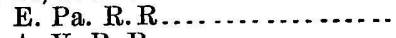 & 387 \\
\hline Templeton Station ..................... & A. V. R. R ..... & 824 \\
\hline Thompson ........... & N. Y., L. E. \& W. R. R ..... & 1,703 \\
\hline Do ............ & P., V. \& C. R. R.......... & 765 \\
\hline Thompson's ...... & 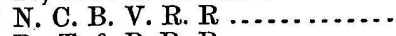 & 860 \\
\hline 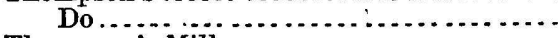 & P., T.\& B. R. R............ & 1,143 \\
\hline Thompson's Mills .... . . . . . . . . . . . . & 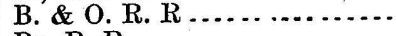 & 1,108 \\
\hline Thompsontown $\ldots \ldots \ldots \ldots \ldots \ldots$ & Pa. R. R ...... & 419 \\
\hline Thorndale $\ldots \ldots \ldots \ldots \ldots \ldots \ldots \ldots$ & Pa. R. R ...... & 313 \\
\hline Thurlow ..... & P., W. \& B. R. R ............... & $\begin{array}{r}34 \\
110\end{array}$ \\
\hline Tidioute .... & 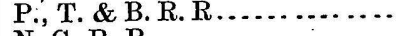 & $1, \mathbf{1 1 2}$ \\
\hline Timonium.... & 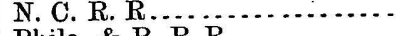 & 395 \\
\hline 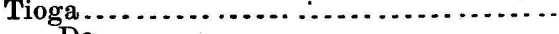 & Phila. \& R. R. R $\ldots \ldots$ & 129 \\
\hline Do - & S. C. R. R..... & $\begin{array}{r}896 \\
042\end{array}$ \\
\hline $\begin{array}{l}\text { Do } \\
\text { Tioga Ju }\end{array}$ & T.\& E. \& S. L. R. R...... & $\begin{array}{l}1,042 \\
1,021\end{array}$ \\
\hline $\begin{array}{l}\text { Tloga Junction } \\
\text { Tioga Village . }\end{array}$ & 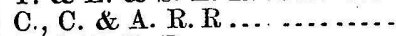 & 1,052 \\
\hline Tiona ......... & 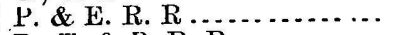 & 1,359 \\
\hline Tionesta & 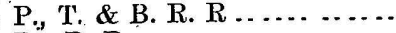 & 1,060 \\
\hline Tipton .. & 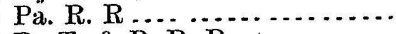 & 990 \\
\hline Titusville & P., T. \& B. R. R $\ldots \ldots \ldots$ & 1,194 \\
\hline Do..... & Pithole Valley R. R........ & 1,181 \\
\hline Do.. Franklin street crossing .......... & 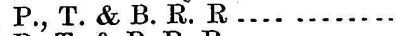 & 1,194 \\
\hline Do.. Washington street crossing ..... & P., T. \& B, R. R . . . . . . . & 1,194 \\
\hline & & 1,195 \\
\hline
\end{tabular}




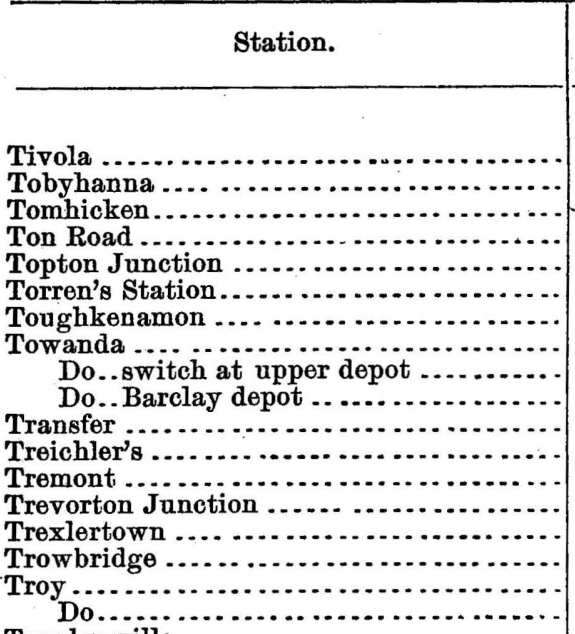

Trunkey ville.

Tryonville

Tryonville Junction

Tuckerton

Tullytown

Tunkhannock

Tunkhannock, Junction with L. V. $\mathrm{R}$. $\mathrm{R}$

Turner

Turner's

Tuscarora

Do............................................

Tuscarora Mountain

W. corner of water station...

'Tussey's Mountain

Tyler's Station

Tylersville

Tyrone

....................................

Tyrone Water Station

Ulster

Union

$$
\text { Do. }
$$

Union City

Do $\ddot{P} \&$ E. Junction

Uniondale

Union Forge

Union Furnace

Uniontown

$$
\text { Do. }
$$

Unionville

Upper Catasauqua

Upper Lehigh.

Upton

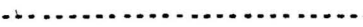

Ursina Junction, U. \& N. F. R. R.Junc

Utica ................................

Valley Coal Mines

Valley Forge.

Valley Store

Valley Works

Vanscoyoc

$$
\text { Do... }
$$

Venango .......
M. C. R. R …. L. \& W. R.

D., H. \& W. R. R

W. C. R. R

E. Pa. R. R.

Pa. R. R

P. \& B. C. R. R.

Lehigh Valley R. R

B. R. R.

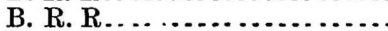

A. \& G. W. R. R

L. \& S. R. R.

L. \& T. R. R.................

N. C. R. R .

C. \& F. R. $\mathrm{R}$

T. \& E. \& S. L. R. R Bennett's Br. R. R

N. C. R. R

P., T. \& B. R. R

P., T. \& B. R. R.

U.' \& T. R. R .

Phila. \& R. R. R

Phila. \& T. R. R . . . . . . .

Lehigh Valley R. R........

M. R. R

L. S. \& M. S. R. R ....... N. C. \& F. R. R.

Schuylkill Valley R. R......

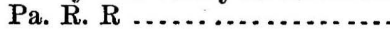

Pa. R. R

Levels

Levels

Bennett's Br. R. R

M. R. $R \ldots \ldots \ldots \ldots \ldots$

L., C. \& S. C. R. R . . . . .

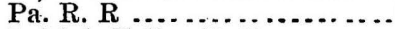

Lehigh Valley R. R..........

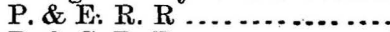

R. \& C. R. R

A. \& G. W. R. R........

U. \& T. R. R.......................

N. Y., L. E. \& W. R. R ..... L. \& T. R. R ............

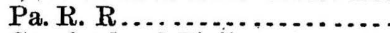
Cumberland $T^{\prime} p \mathbf{k}^{\mathbf{k}} \ldots \ldots \ldots \ldots$

P. \& C. R. R . . . . . . . .

S. W. Pa. Extension ..............

P. \& E. Valley R. R ........

L. \& S. R. R

L. \& S. R. R .............

Pa. R. R.

U. \& N. F. R. R . . . . . . .

B. \& O.R. R.

A. \& G. W. R. R..........

P. \& C. R. R ...............

Phil. \& R. R. R ..............

Chester Valley R. R .........

S. W. Pa. R. R

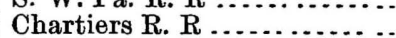

B. G. R. R...

T. \& C. R. R .

A. \& G. W. R. R.
Elevation.

Feet.

708

1,932

1,228

577

485

920

283

738

738

725

993

344

766

428

411

1,440

1,186

1,148

1,098

1,318

1,320

298

20

611

611

1,060

1,137

750

909

429

424

1,926

1,984

1, 232

1,400

907

896

743

1, 270

399

1, 301

1,270

1,670

432

799

952

981

990

782

284

1,802

430

1,342

1,406

1,035

1,035

98

295

1, 075

1, 995

1,427

1, 163

746 


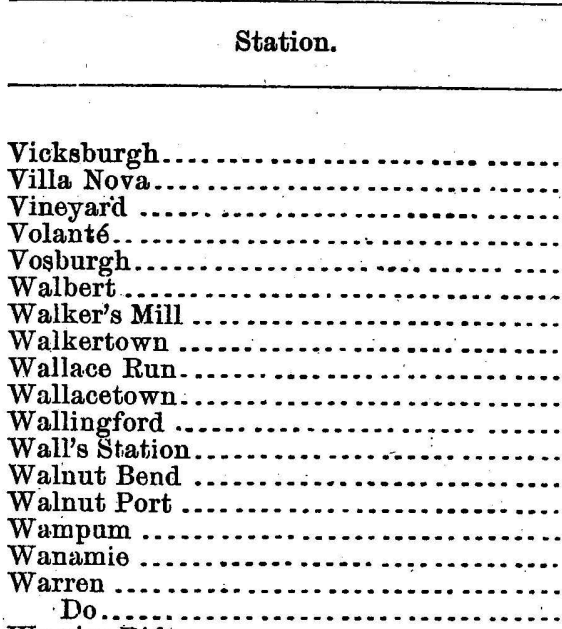

Warrior Ridge

Warrior Run.

Washington

..................................

Do:

Do...

Waterford

Watersville (surf of Little Pine Cr.

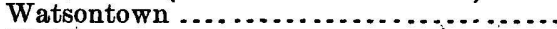

Watt's

Waverly .............................

Waverly Junction.

Wayne

Do.

Do............................................

Waynesburgh Station

Webster's Mill

Weissport.

Wells

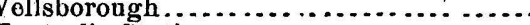

Wentzel's Station

Wernersville.

Wernway.

West Alexandria Do

West Brownsville, in street in front of hotel.

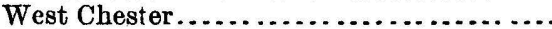

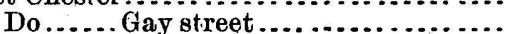

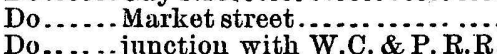

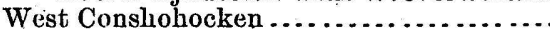

West Falls.

West Grove

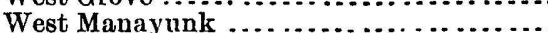

West Newton

West Overton

West Pittston

Westport

(1).

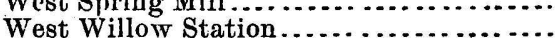

Westwood Junction

Wetmore.

Wheatland

Bull. 5-18

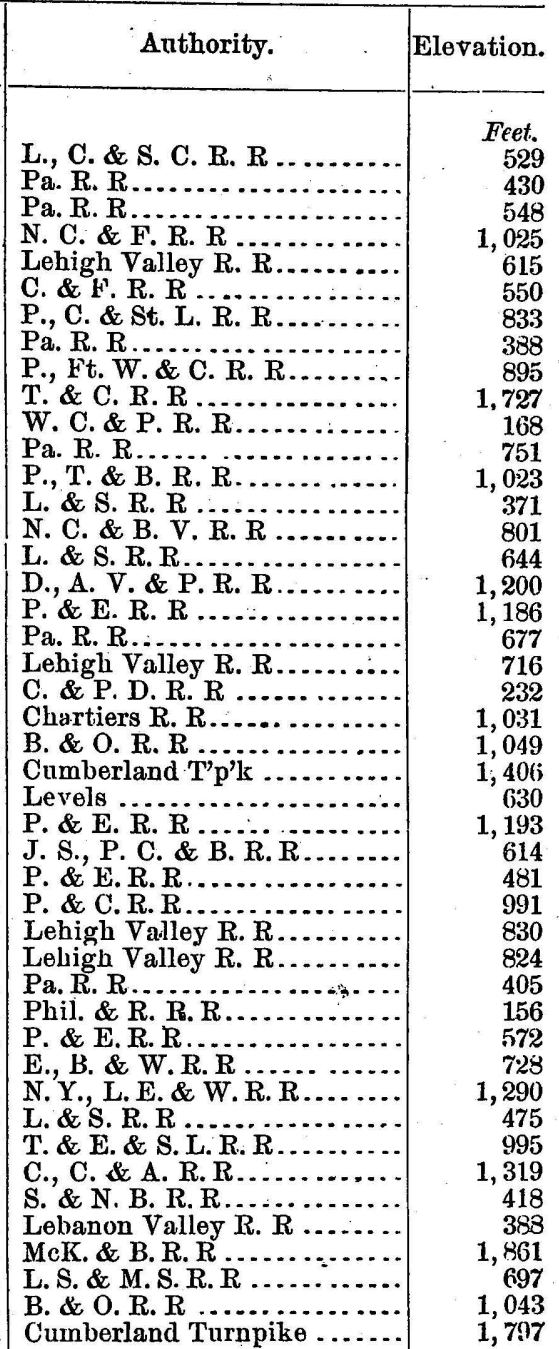

P. V. \& C. R. R............ 774

W. C. \& P. R. R ............ 406

W. C. R. R ............... 420

W. C. R. R

P. \& B. C. R. R ............. 133

Phil \& R. R.R ............. 61

Phil. \& R. R. R ........... 61

P. \& B. C. R. R .............. 444

Phil. \& R. R. R ............ 61

B. \& O. R. R . ............ 782

P. \& C. R. R.............. 1,045

Del., L. \& W. R. R .......... $\quad 579$

P. \& E. R. R............... 691

Phil. \& R. R. R............ 61

L. \& Q. R. R.............. 449

M., H. \& S. H. R. R......... 671

P. \& E. R. R .............. 1,804

E. \& P. R. R ........... 841 


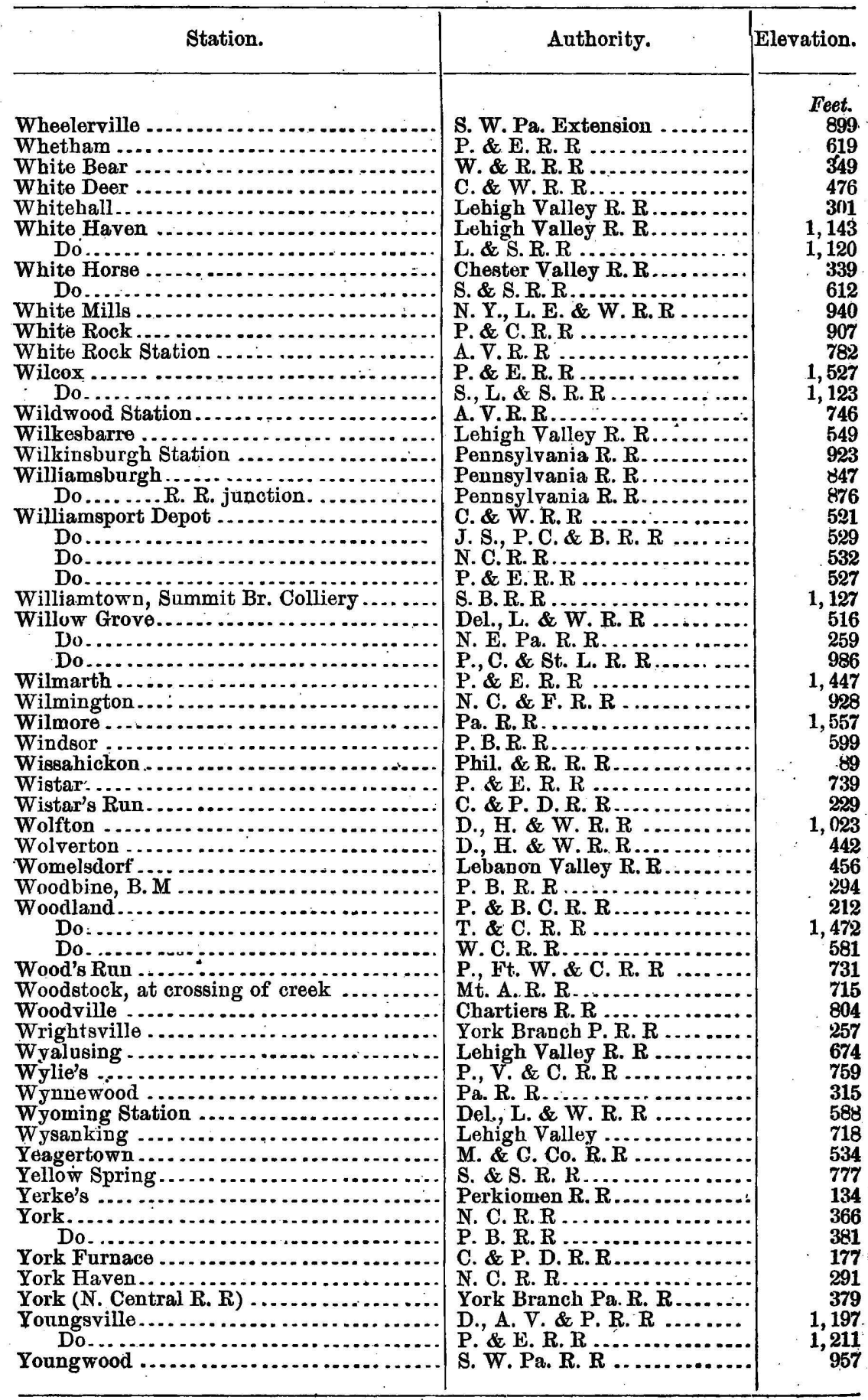


RHODE ISLAND.

\begin{tabular}{|c|c|c|}
\hline Station. & Authority. & Elevation. \\
\hline & & Feet. \\
\hline Albion .... & Prov. \& Worces. R. R & 88 \\
\hline Allendale. & Prov. \& Spring. R. R & 104 \\
\hline 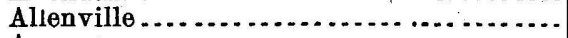 & Prov. \& Spring. R: R & 134 \\
\hline 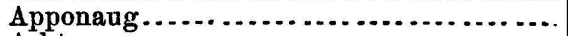 & N. Y., Prov. \&. Bost. R & 25 \\
\hline Asliton..... & Prov. \& Worces. R. R .. & \\
\hline Auburn ... & N. Y., Prov. \& Boston R. R ... & 43 \\
\hline Barrington & Prov., Warren \& Bristol R. R. & 22 \\
\hline Berkley ... & Prov. \& Worces. R. R....... & 69 \\
\hline Bristol .... & Prov., Warren \& Bristol $\mathrm{R}$ & 17 \\
\hline Carolina . & N. Y., Prov. \& Bost. R. R. & 89 \\
\hline 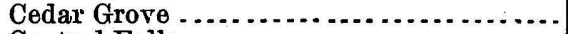 & Prov., Warren \& Brisiol R. & 36 \\
\hline Central Falls............ & Prov. \& Worces. R. R..... & 70 \\
\hline Centredale $\ldots . . \ldots \ldots \ldots \ldots \ldots \ldots$ & P. \& S. R. R......... & 104 \\
\hline Cowesett ............... & N. Y., Prov. \& Bost. R. & 29 \\
\hline Davisville. & N. Y., Prov. \& Bost. R. R & 55 \\
\hline Drownville & Prov., Warren \& Brist. R. & 30 \\
\hline Dyerville ......... & Prov. \& Spring. R. R .... & 74 \\
\hline East Providénce... ... & Prov., Warren \& Brist. $\mathrm{R}$ & 20 \\
\hline Georgiaville ...... & Prov. \& Spring. R. R .... & 164 \\
\hline Graystone.. & Prov. \& Spring. R. R.... & 114 \\
\hline Greenwich . ............. & N. Y., Prov. \& Bost. R. R.... & 32 \\
\hline Hamlet $\ldots \ldots \ldots \ldots$ & Prov. \& Worces. R. R ... & 146 \\
\hline Harrisville. . . . . . & Prov. \& Spring. R. R. & 326 \\
\hline Hills Grove ..... & N. Y., Prov. \& Bost. R. R & 55 \\
\hline 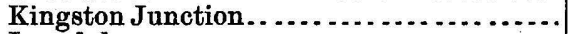 & N. Y., Prov. \& Bost. R. R & 111 \\
\hline Lonsdale. . . . . . . . . . . . . . . . . . . & Prov. \& Worces. R. R.... & 66 \\
\hline Manton ... & Prov. \& Spring. R. R ... & 74 \\
\hline Manville.. & Prov. \& Worces. R. R....... & 105 \\
\hline Nayatt... & Prov., Warren \& Bristol R. R. & 24 \\
\hline Newport, Signal Station .................. & U. S. Signal Office............ & 44 \\
\hline New Shoreham, Signal Station ............ & U. S. Signal Office......... & 27 \\
\hline 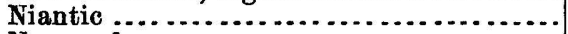 & N. Y., Prov. \& Bost. R. R & 44 \\
\hline Norwood. & N. Y., Prov. \& Bost. R. R .... & 58 \\
\hline Oakland... & Prov. \& Spring. R. R ....... & 308 \\
\hline Olneyville & Prov. \& Spring. R. R ........ & 49 \\
\hline (n................... & Prov. \& Spring. R. R ....... & 396 \\
\hline Pawtucket & Bost. \& Prov. R. R ......... & 79 \\
\hline Do. & Prov. \& Worces. R. R...... & 73 \\
\hline Providence & Bost. \& Prov. R. R ...... & 10 \\
\hline Do.... & N. Y., Prov. \& Bost. R. R. & 12 \\
\hline Richmond... & N. Y., Prov. \& Bost. R. R.... & 87 \\
\hline River Point & H., P. \& F. R. R.......... & 93 \\
\hline Slocumville & N. Y., P. \& B. R. R . . . . . . & 136 \\
\hline Smithfield ....... & Prov. \& Spring. R. R ........ & 262 \\
\hline Tar Kiln ...... & Prov. \& Spring. R. R ... & 364 \\
\hline Valley Falls....... & Prov. \& Worces. R. R.... & 68 \\
\hline Warren ........... & Prov., Warren \& Bristol R. R. & 24 \\
\hline Westerly $\ldots \ldots \ldots \ldots \ldots \ldots \ldots \ldots$ & N. Y., P. \& B. R.R..... & 33 \\
\hline Wickford Junction $\ldots \ldots \ldots \ldots \ldots \ldots$ & N. Y., P.\& B. R.R ..... & 89 \\
\hline Woodlawn $\ldots \ldots \ldots \ldots$ & Prov. \& Worces. R. R ...... & 56 \\
\hline 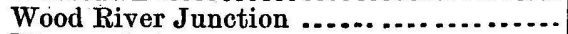 & N. Y, Prov. \& Bost. R. R .... & 56 \\
\hline 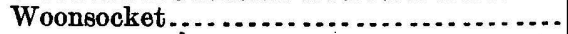 & Prov. \& Worces. R. R....... & 159 \\
\hline
\end{tabular}


SOUTH CAROLINA.

\begin{tabular}{|c|c|c|}
\hline Station. & Authórity. & Elevation. \\
\hline & & \\
\hline $\begin{array}{l}\text { Abbeville ... } \\
\text { Adjers . . }\end{array}$ & C. \& G. R. R. ... & 535 \\
\hline $\begin{array}{l}\text { Adjers ........ } \\
\text { Aiken...... }\end{array}$ & C., C.\& A. R. R. & $\begin{array}{l}540 \\
520\end{array}$ \\
\hline Allendale ... & P.R.R.R...... & 192 \\
\hline Alston ...... & C. \& G. R. R. . & 259 \\
\hline Anderson .. & C. \& G. R. R. . & 764 \\
\hline Appleton ........... & P.R.R.R R.... & 262 \\
\hline $\begin{array}{l}\text { Augusta Bridge ... } \\
\text { Barr's........... }\end{array}$ & $\begin{array}{l}\text { S. Carolina R. R. } \\
\text { C. C.\& A.R. R. }\end{array}$ & $\begin{array}{l}185 \\
458\end{array}$ \\
\hline Batesburg........ & C., C.\&A.R. R. & 657 \\
\hline Beaufort Wh & U.S. C. \& G.S & 7 \\
\hline Beaufort ... & P.R.R.R.... & 20 \\
\hline Beldoc .... & P.R. R.R... & 116 \\
\hline Belton ..... & C. \& G. R. R . & 896 \\
\hline Birds Crossin & C. \& G.R.R . & 760 \\
\hline Blacks ....... & A. \& R.A. L.R & 372 \\
\hline Blackstock & C., C. \& A. R. R. & 621 \\
\hline Blackville.. & S. Carolina R. & 301 \\
\hline & C. \& G.R. R.. & 293 \\
\hline Blytheweod & C., C. \& A.R. I & 505 \\
\hline Bookman's . & C. \& G.R.R.. & 204 \\
\hline Branchville & S. Carolina R. & 140 \\
\hline Brunson's ... & P.R.R.R... & 139 \\
\hline Cæsar's Head & U.S.C.\& G.S & 3,118 \\
\hline Cars & S. Carolina R. R & 370 \\
\hline Chappell's . & C. \& G.R.R.... & 402 \\
\hline Charlestor & S. Carolina R. R & 16 \\
\hline Do..... & S. \& C. R. R ... & 9 \\
\hline Do... Signal & U. S. Signal Offic & 52 \\
\hline Chester ..... & C., C. \& A.R.R & 543 \\
\hline Clinton .... & C. \& G. R. R. & 687 \\
\hline Columbia ........ & C. \& G.R. R. & 227 \\
\hline Do... Blading 8 & C., C. \& A. R.R & 296 \\
\hline Do....S. C. R. R. & C., C.\& A. R.R & 233 \\
\hline Do... Old Junetio & C., C. \& $A . R . R$. & 195 \\
\hline C'ornwalls........ & C., C. \& A.R. R & 638 \\
\hline Dankins ... & C. \& G. R. R. & 286 \\
\hline Dead Fall:. & C., C. \& A. R. R & 156 \\
\hline Don & C. \& G.R.R . & 760 \\
\hline Dyso & C. \& G.R.R .. & 420 \\
\hline Earl & P.R.R.R...... & 79 \\
\hline Early Depot..... & U.S. C.\& G.S & 1,131 \\
\hline Eilenton ......... & P.R.R.R.... & 149 \\
\hline Enon & A. \& R.A.L.R.R. & 987 \\
\hline Fish & C. \& G. R. R .... & 498 \\
\hline Fort & C., C. \& A. R. R.. & 336 \\
\hline \& Mill ....... & C. \& G.R.R.. & 179 \\
\hline Furman University... & U.S.C.\&G.S.. & 1,074 \\
\hline Garey's Lane......... & C. \& G. R. R .. & 580 \\
\hline Glassy $\ldots . . . \ldots \ldots$ & U. S. C. \& G. S. & 1,699 \\
\hline Glassy Rock ..... & U. S. C. \& G. S .......... & 3,076 \\
\hline lale's..... & C. \& G. R.R .... & 708 \\
\hline Golden Grove. & C. \& G. R. R ............... & 900 \\
\hline Grantsville....... & C., C. \& A.R.R R & 243 \\
\hline (1) & 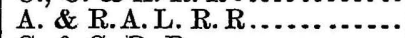 & 976 \\
\hline & C. \& G. R. R $\ldots \ldots \ldots$ & 989 \\
\hline Do .... Episcopal Church...$\ldots \ldots \ldots \ldots$ & U. S. C. \& G.S $\ldots \ldots \ldots$ & 1,105 \\
\hline & & \\
\hline
\end{tabular}




\begin{tabular}{|c|c|c|}
\hline Station. & Authority. & Elevation. \\
\hline & & Feet. \\
\hline Greenwood .... & C. \& G. R. R & 671 \\
\hline Gum Tree.... & U. S. C. \& G. & 17 \\
\hline Helena ....... & C. \& G. R. R . & 532 \\
\hline Hudges .... & C. \& G. R. R & 714 \\
\hline Hogback ... & U. S. C. \& G. & 3,230 \\
\hline . $\ldots \ldots \ldots \ldots \ldots$ & C. \& G. R. R. & 810 \\
\hline Hopes................ & C. \& G. R. R. & 297 \\
\hline Jacksonboro Depot.. & S. \& C. R. R. & 17 \\
\hline 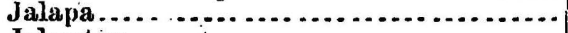 & C. \& G. R. R .. & 576 \\
\hline Johnston ............ & C., C. \& A. R. R & 670 \\
\hline Jonesville.......... & C. \& G. R. R ... & 583 \\
\hline $\begin{array}{l}\text { Keislers...... } \\
\text { Killians..... }\end{array}$ & C., C. \& A.R. I & $\begin{array}{l}546 \\
391\end{array}$ \\
\hline $\begin{array}{l}\text { Kunjans..... } \\
\text { Kinards..... }\end{array}$ & $\begin{array}{l}\text { C., C. \& A. R. } \\
\text { C. \& G. R. R. }\end{array}$ & 331 \\
\hline Langleys.... & C., C. \& A. R.j & $\begin{array}{l}602 \\
200\end{array}$ \\
\hline Lawrens :.. & C. \& G. R. R. & 589 \\
\hline Leesville... & C., C. \& A. R. & 645 \\
\hline Lewis.... & C., C. \& A. R. & 574 \\
\hline Lexington & C., C. \& A. R. & 370 \\
\hline Limestone & A. \& R. A. L. I & 787 \\
\hline Martins .. & C. \& G. R. R & 619 \\
\hline 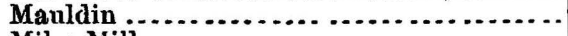 & U. S. C. \& G. & 1,328 \\
\hline Miles Mill ............................. & C., C. \& A. R. & 443 \\
\hline Millet.............. & P. R. R. R ... & 135 \\
\hline Montgomery's .... . & C. \& G. R. R & 199 \\
\hline . & C. \& G. R. R & 502 \\
\hline 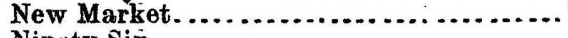 & C. \& G. R. R. & 630 \\
\hline 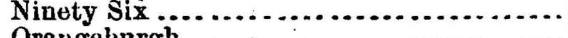 & C. \& G. R. R. & 570 \\
\hline 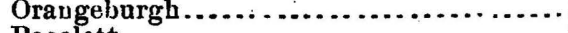 & S. Carolina R & 265 \\
\hline Pacolett $\ldots$ & C. \& G. R. R & $\begin{array}{r}718 \\
29\end{array}$ \\
\hline 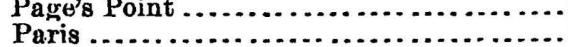 & $\begin{array}{l}\text { U. S. C. \& G. } \\
\text { U.S. C. \& G. S }\end{array}$ & $\therefore 2,054$ \\
\hline Parkes, $\mathrm{T} . \dot{0}$ & C. \& G. R. R. & 704 \\
\hline 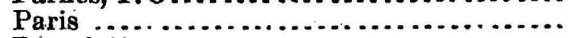 & C. \& G. R. R & 291 \\
\hline 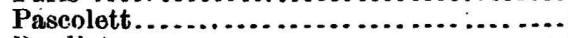 & A. \& R. A. L. & 857 \\
\hline Pendleton... & A. \& R. A. L. & 879 \\
\hline Do................... & C. \& G. R. R & 817 \\
\hline Pickens C. H ........ & U. S. C. \& G. & 1,162 \\
\hline Pickensville.......................... & A. \& R. A. L. & 1,094 \\
\hline Piedmont . . & C. \& G. R. R & 794 \\
\hline Piunacle.............................. & U. S. C. \& G.S & 3,436 \\
\hline 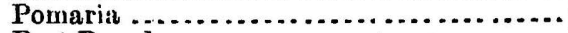 & C. \& G. R. R .. & 330 \\
\hline Port Royal . . . . . . . . . . . . . . . . . . . . & P. R. R. R ... & 11 \\
\hline Prosperity $=\ldots \ldots \ldots \ldots$ & C. \& G. R. R. & 551 \\
\hline Rich Mountain . . . . . . . . . . . . . . . . . & U. S. C. \& G. & 3,569 \\
\hline Ridge Spring ...... & C., C. \& A. R. & 647 \\
\hline Ridgeway & $\cdots \cdots \cdots$ & 626 \\
\hline 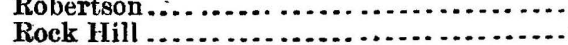 & 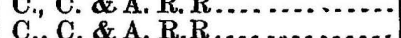 & 562 \\
\hline Rocky Mountain & 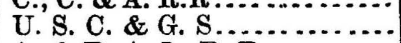 & 1,029 \\
\hline 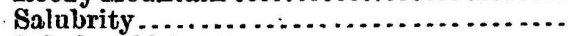 & A. \& R. A. L. R. & 1,006 \\
\hline old town $. . \ldots . . . . . . . . . . .$. & G. R. R & 386 \\
\hline & & 512 \\
\hline 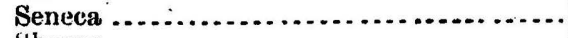 & $R . . . . . .$. & 954 \\
\hline & $R \ldots \ldots \ldots . . . . .$. & 444 \\
\hline 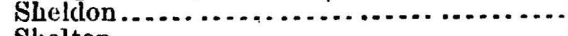 & P. R. R. R . & 21 \\
\hline (n).................... & C. \& G. R. R. & 315 \\
\hline 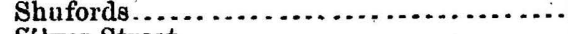 & C. \& G. R. R. & 1,000 \\
\hline Silver Street $\ldots \ldots \ldots \ldots \ldots \ldots \ldots \ldots$ & C. \& G. R. R . & 470 \\
\hline 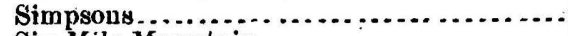 & C., C.\&.A. R. R. & 604 \\
\hline Six-Mile Mountain...$\ldots \ldots \ldots \ldots \ldots \ldots$ & U. S. C. \& G. S . $\ldots \ldots$ & 1,595 \\
\hline Smiths $\ldots \ldots \ldots \ldots, \ldots$ & C., C. \& A. R. R & 545 \\
\hline Shurch... & & 887 \\
\hline
\end{tabular}




\begin{tabular}{|c|c|c|}
\hline Station. & Authority. & Elevation. \\
\hline 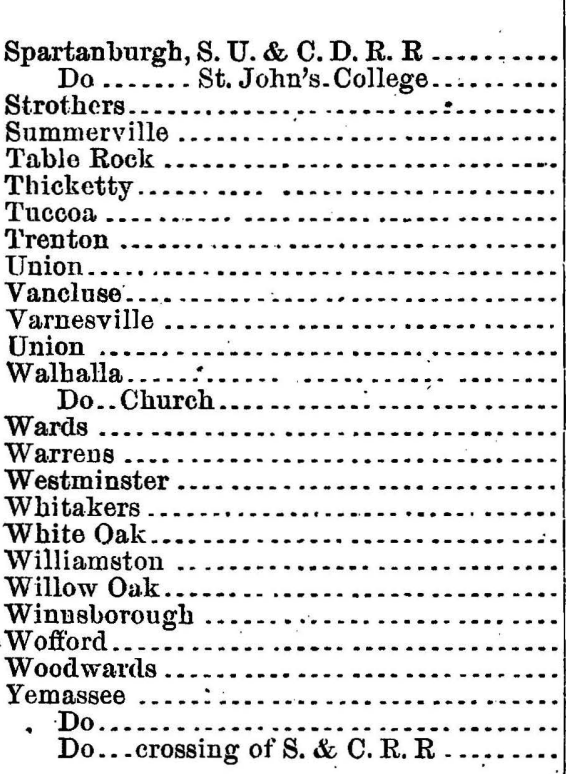 & 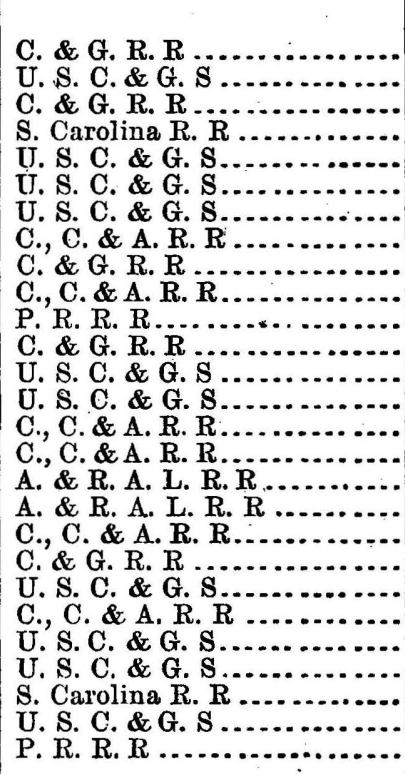 & $\begin{array}{r}\text { Freet. } \\
693 \\
875 \\
292 \\
68 \\
\mathbf{3}, \mathbf{1 2 4} \\
\mathbf{1}, 271 \\
\mathbf{1 , 7 7 8} \\
\mathbf{6 2 0} \\
\mathbf{5 7 9} \\
.299 \\
101 \\
\mathbf{5 7 9} \\
\mathbf{9 8 5} \\
\mathbf{1 , 1 1 8} \\
\mathbf{6 7 3} \\
\mathbf{5 4 5} \\
\mathbf{9 1 9} \\
\mathbf{9 0 7} \\
\mathbf{5 4 8} \\
840 \\
\mathbf{1 3 5} \\
\mathbf{5 4 3} \\
\mathbf{8 7 8} \\
\mathbf{5 8 2} \\
\mathbf{2 5} \\
\mathbf{2 3} \\
\mathbf{2 5}\end{array}$ \\
\hline
\end{tabular}




\section{TENNESSEE.}

\begin{tabular}{|c|c|c|}
\hline Station. & Authorits. & Elevation. \\
\hline & & Feet. \\
\hline Albany ...... & L., N., S. \& N. A. R. & 564 \\
\hline Allens........ & L., N., S. \& N. A. R. R. & 353 \\
\hline ; & W. \& A. R. R......... & 695 \\
\hline Annadell.... & C. S.R.R.............. & 1,249 \\
\hline$\ldots \ldots \ldots \ldots \ldots \ldots \ldots \ldots \ldots \ldots \ldots$ & L., N., S. \& N. A. R. R. & 648 \\
\hline Athens...... & E.T., Va. \& Ga. R. R & 933 \\
\hline 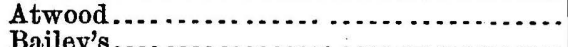 & L., N., S. \& N. A. R. & 428 \\
\hline 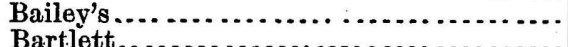 & L., N., S. \& N. A. & 664 \\
\hline 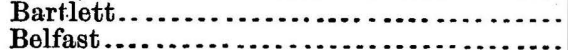 & L., N., S. \& N. A. & 263 \\
\hline 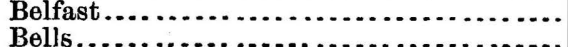 & D. R. V. R. R $\ldots$ & 814 \\
\hline $\begin{array}{l}\text { Bells } \\
\text { Benton Switch }\end{array}$ & L., N., S. \& N. A. R. R & 320 \\
\hline Bethel.......... & 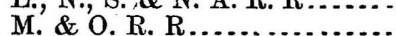 & $\begin{array}{l}574 \\
463\end{array}$ \\
\hline Big Hatchie & L., N., S. \& N. A. R. & 286 \\
\hline Big Sandy $\ldots \ldots \ldots \ldots \ldots \ldots \ldots \ldots \ldots \ldots \ldots$ & L., N., S. \& N. A. R. & 345 \\
\hline 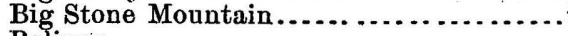 & 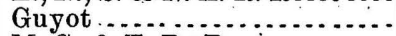 & 5,614 \\
\hline 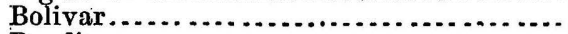 & M. C. \& T. R. R. & 430 \\
\hline 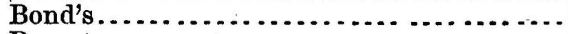 & L., N. S. \& N. A. R. & 314 \\
\hline 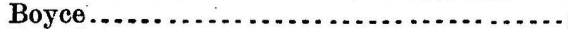 & C. S. R. R........... & 694 \\
\hline 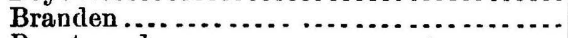 & L., N., S. \& N. A. R. & 307 \\
\hline 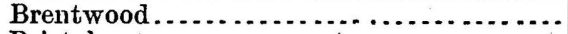 & L., N., S. \& N. A. I & 698 \\
\hline Bristol...... & N.\&W & 1,689 \\
\hline Brown's Road............. & L., N. S. \& N. A. R. R & 476 \\
\hline 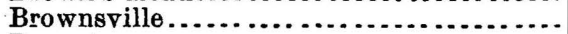 & L., N., S. \& N. A. R. F & 333 \\
\hline 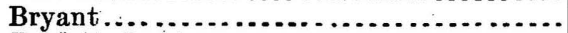 & D. R. V.R. R......... & 671 \\
\hline 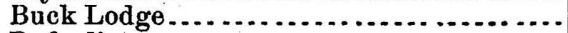 & L., N., S. \& N. A. R. R....... & 711 \\
\hline 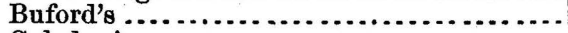 & L., N., S. \& N. A. R. R....... & 702 \\
\hline $\ldots \ldots \ldots \ldots \ldots \ldots \ldots \ldots \ldots \ldots$ & L., N., S. \& N. A. R. R ..... & 421 \\
\hline$\ldots \ldots \ldots \ldots \ldots \ldots \ldots$ & L., N., S. \& N. A. R. R ...... & 686 \\
\hline . $\ldots \ldots \ldots \ldots \ldots \ldots \ldots$ & L., N., S. \& N. A. R. R ..... & 362 \\
\hline ........................ & L., N., S. \& N. A. R. R ...... & 246 \\
\hline Carroll ................. & M. \& O. R. R......... & 375 \\
\hline 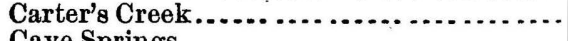 & L., N., S. \& N. A. R & 602 \\
\hline Cave Springs $\ldots \ldots \ldots \ldots$ & $\cdots \cdots$ & 697 \\
\hline $\begin{array}{c}\text { Chattanooga } \\
\text { Do }\end{array}$ & & $\begin{array}{l}681 \\
783\end{array}$ \\
\hline Cherry's............ & L., N., S.\& N. A. R. R & 535 \\
\hline Che walla.......... & Memphis \& C. R. R.. & 409 \\
\hline Chickamanga............ & W. \& A. R. R ...... & 685 \\
\hline Chilhowee Mountain ..................... & Guyot.. & 2,452 \\
\hline 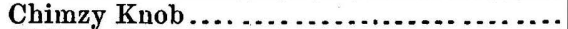 & ...... & 5,588 \\
\hline Chitwood...... & C. S. $\mathrm{F}$ & 1,332 \\
\hline$\ldots \ldots \ldots$ & L., N., S. \& N. A. R. R ..... & 392 \\
\hline Cleveland.... & E. T., Va. \& Ga. R. R........ & 878 \\
\hline$\ldots \ldots . . . .$. & L., N., S. \& N. A. R. R ...... & 353 \\
\hline Clingman's Dome. & Guyot............. & 6,660 \\
\hline Colliersville... & Memphis \& C. R. R... & 379 \\
\hline Collins, Mount .... & Gay & 6,188 \\
\hline Columbia........... & L., N., S. \& N. A. R. R ..... & 644 \\
\hline Corner Knob. & Guyot....................... & 5,246 \\
\hline Corraine .... & 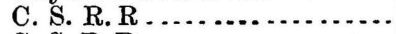 & $81: 3$ \\
\hline Coulterville.... & C. S. R.R ... & 712 \\
\hline Crockett........ & M. \& O. R.R . & 296 \\
\hline (n. & Guyot............... & 5,931 \\
\hline . & L., N., S. \& N. A. R. R. . . . ... & $35 \overline{5}$ \\
\hline 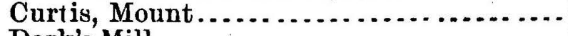 & Guyot..... & 6,568 \\
\hline Dark's Mill . . . . . . . . . . . . . . . . . . . . . . . . . & L., N., S. \& N. A. R. R........ & 574 \\
\hline & & 767 \\
\hline
\end{tabular}




\begin{tabular}{|c|c|c|}
\hline & Authority. & Elevation. \\
\hline & & Feet. \\
\hline $\begin{array}{l}\text { Dayton } . . . . . . . \\
\text { Dodsen's ......... }\end{array}$ & $\begin{array}{l}\text { C. S. R. R } \\
\text { L., N., S. \& N.A.R.R }\end{array}$ & $\begin{array}{l}715 \\
804\end{array}$ \\
\hline Duck River.... & L., N., S. \& N. A. R. R & 578 \\
\hline udley's ....... & L., N., S.\& N. A. R. R & 494 \\
\hline yer............ & M. \& O. R. R .......... & 365 \\
\hline 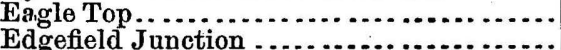 & $\begin{array}{l}\text { Guyot. } \\
\text { L., N., S. \& A.R.R.. }\end{array}$ & $\begin{array}{r}5,433 \\
414\end{array}$ \\
\hline k Ridge Summi & D.R.V.R.R.............. & $\begin{array}{l}414 \\
789\end{array}$ \\
\hline mory Gap....... & C. S. R. R & 840 \\
\hline 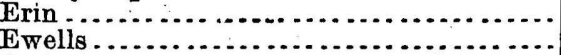 & $\begin{array}{l}\text { L., N., S. \& N. A. R.R. R. } \\
\text { L., N., S. \& N. A. R.R.. }\end{array}$ & $\begin{array}{l}404 \\
747\end{array}$ \\
\hline vings . & 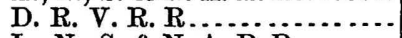 & 730 \\
\hline ounds. & L., N., S.\& N. A. & 439 \\
\hline$\ldots \ldots$ & M. \& O. R. R............ & 433 \\
\hline Ige Peak & $\begin{array}{l}\text { D. R. V.R.R R.... } \\
\text { Gurot_............. }\end{array}$ & $\begin{array}{r}656 \\
5,087\end{array}$ \\
\hline & L., N., S.\& N.A. R & 778 \\
\hline aklin........ & L., N., S. \& N. A. R. R & 617 \\
\hline & L., N., S. \& N.A. R. & $\begin{array}{l}406 \\
494\end{array}$ \\
\hline$\cdots$ & L., N., S. \& N. A. R. R. & $\begin{array}{l}494 \\
277\end{array}$ \\
\hline in ... & Memphis \& C. R. R ... & 378 \\
\hline$\cdots$ & L., N., S. \& N. A.R.R & 378 \\
\hline lice..... & $\begin{array}{l}\text { L., N., S.\& N. A. R. } \\
\text { C. S. R. R........ }\end{array}$ & $\begin{array}{l}344 \\
826\end{array}$ \\
\hline & C. S. R. R .......... & 1,289 \\
\hline ction & Memphis \& C. R.R .. & 575 \\
\hline & L., N., S. \& N. A. R. R & 360 \\
\hline & C. S. R. R ........... & 737 \\
\hline our & Guyot............... & 4,922 \\
\hline Moun & & 4,226 \\
\hline .... & E. T., Va. \& Ga. R. R & 1,581 \\
\hline ant. & Guyot......................... & 6,636 \\
\hline & L., N., S. \& N. A. R. R & 513 \\
\hline & & 5,600 \\
\hline & D. R. V. R. R ....... & 579 \\
\hline & L., N., S. \& N. A. R. R. & \\
\hline & C.S. R. R ........... & $1,40 !$ \\
\hline & M. \& O. R. R ........ & 427 \\
\hline ille.. & \&. N.A.R & 446 \\
\hline Henry & L., N., S. \& N. A. R.R. & 518 \\
\hline Do & & 6,373 \\
\hline Hills & 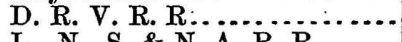 & \\
\hline & L., N., S. \& N. A. R. R. & 329 \\
\hline $\operatorname{ane} . . . . .$. & L., N., S. \& N. A. R. R ....... & 630 \\
\hline$\ldots$ & M. \& O.R.R R & 425 \\
\hline & E. T., Va. \& Ga. R. R. & 1,643 \\
\hline & N. \& N. R. R.......... & \\
\hline Jo & L., N., S. \& N.A. R. R & 314 \\
\hline & E. T., Va. \&. Ga. R. R... & 1,734 \\
\hline & D. R. V. R. R.............. & \\
\hline & M. \& O. R. R......... & 309 \\
\hline & C. S. R. R.. & 696 \\
\hline Kuox & R........ & 900 \\
\hline Signal Station...... & & 980 \\
\hline . & Memphis \& C. R. R... & 531 \\
\hline & C. S. R. R... & 1,197 \\
\hline eak....... & Guyot....... & 5,922 \\
\hline e, Mount... & Guyot.... & 6,612 \\
\hline $8 \ldots \ldots \ldots$ & L., N., S. \& N. A. R. R.... & \\
\hline burgh ...... . & D.R.V.R.R.............. & \begin{tabular}{l}
727 \\
\hdashline 00
\end{tabular} \\
\hline at $\ldots \ldots \ldots$ & C. S. R. R. & \\
\hline Love, Mount........ & 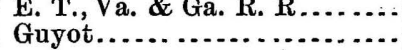 & 6,443 \\
\hline
\end{tabular}




\begin{tabular}{|c|c|c|}
\hline Station. & Authority. & Elevation. \\
\hline & . & Feet. \\
\hline 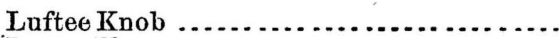 & Guyot... & 6,232 \\
\hline Lynnville...... & L., N., S. \& I & 734 \\
\hline McElweo......... & C. S. R. R.. & 788 \\
\hline McKenzio . . . . . . . . . . . . . . . . . . . . . & L., N., S. \& N. & 470 \\
\hline 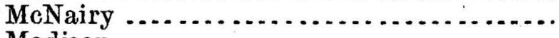 & M. \& O. R. R...... & 454 \\
\hline 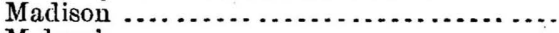 & L., N., S. \& N. A. R. R & 466 \\
\hline 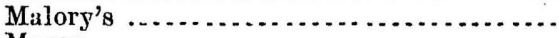 & L., N., S. \& N. A. R. R & 674 \\
\hline Mason $\ldots \ldots \ldots \ldots \ldots \ldots \ldots$ & L., N., S. \& N. & 296 \\
\hline 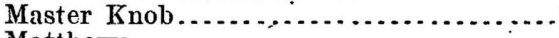 & Guyot $\ldots \ldots \ldots$ & 6,013 \\
\hline 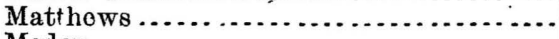 & L., N., S. \& N. A. R. R & 398 \\
\hline . & M. C. \& T. R. R.... & 420 \\
\hline$\ldots \ldots \ldots \ldots \ldots \ldots$ & C. S. R. R ........ & 833 \\
\hline . & C. S. R. R .... & 711 \\
\hline 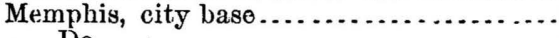 & City engineer $\ldots \ldots$ & 117 \\
\hline Do & L., N., S. \& N. A. R. R & 227 \\
\hline . & Miss. \& Tenn. R. R.. & 258 \\
\hline Do....... & M. \& C. R. R..... & 244 \\
\hline 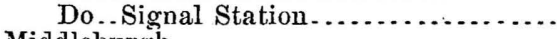 & U. S. Signal Office. & 321 \\
\hline 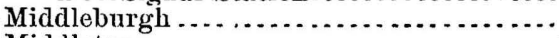 & M. C.\&T.R.R..... & 537 \\
\hline Midldleton........ & Memphis \& C. R. R . & 408 \\
\hline Milan ............ & L., N., S. \& N. A. R. R & 408 \\
\hline 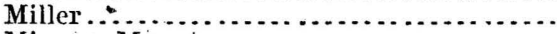 & C. S. R. R .......... & 847 \\
\hline 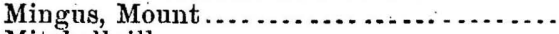 & Gryot..................... & 5,694 \\
\hline . & L., N., S. \& N. A. R. R & 748 \\
\hline 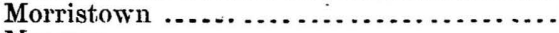 & E.T., Va. \& Ga. R. R & 1,283 \\
\hline Moscow & Memphis \& C. R. R. & 352 \\
\hline 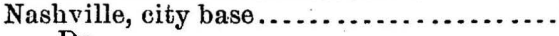 & City ongineer $\ldots \ldots \ldots$ & 232 \\
\hline Do $\ldots \ldots \ldots \ldots \ldots \ldots \ldots \ldots \ldots \ldots \ldots$ & L., N., S. \& N. A. R. & 409 \\
\hline Do ..... & N.\& N. R. R... & 430 \\
\hline$\ldots \ldots \ldots \ldots \ldots \ldots \ldots$ & N. \& D. R. R...... & 445 \\
\hline Do...H. W. Cumberland R........... & City engineer....... & 397 \\
\hline Do... L. W. Cumberland R........... & Ciry engineer... & 340 \\
\hline 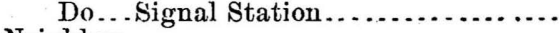 & U. S. Signa.l of & 507 \\
\hline 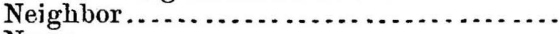 & Gujot..... & 5,771 \\
\hline . & C. S. R. R.... & 917 \\
\hline . . & E. T., Va. \& Ga. R. R & 1,057 \\
\hline New River........ & C.S.R.R...... & 1,215 \\
\hline North Bald Mountain . . . . . . . . . . . . . . . . . . & Guyot....... & 4,711 \\
\hline Oakclale Junction . . . . . . . . . . . . . . . . . . & C. S.R.R..... & 812 \\
\hline Oak Hill .............................. & L., N., S. \& N. A. R. R & 355 \\
\hline Oakland, top of ridge.............. & E., H. \& N. R. R .... & 880 \\
\hline Oconee Mountain $\ldots \ldots \ldots \ldots \ldots \ldots \ldots \ldots \ldots$ & Guyot ........... & 6,135 \\
\hline 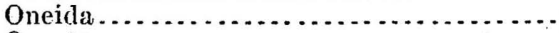 & C. S. R. R .. & 1,454 \\
\hline 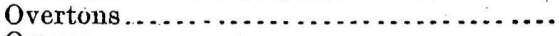 & L., N., S. \& N. A. R.R & 533 \\
\hline$\ldots \ldots \ldots \ldots$ & L., N., S. \& N. A. R. R & 677 \\
\hline 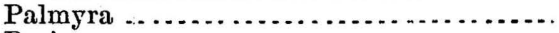 & L., N., S. \& N. A. R. R & 367 \\
\hline 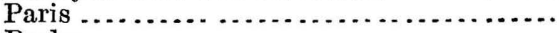 & L., N. S. \& N., A. R. R....... & 447 \\
\hline Parks $\ldots . . . \ldots \ldots \ldots \ldots \ldots \ldots \ldots \ldots$ & 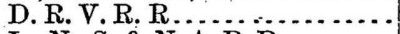 & 650 \\
\hline 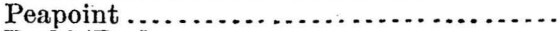 & L., N., S. \& N. A. R. R......... & 256 \\
\hline - & Guyot.................... & 6,232 \\
\hline$\ldots \ldots \ldots \ldots \ldots \ldots \ldots$ & D.R. V.R.R...... & $72 \%$ \\
\hline Pilot Knol........ & L., N., S. \& N. A. R. R ....... & 447 \\
\hline Pilot Mountain .... & C. S. R. R . . . . . . . . . & 1,348 \\
\hline Pinson ............ & M. \& O.R.R......... & 384 \\
\hline Pleasant Grove... & L., N., S. \& N., A. R. R........ & 719 \\
\hline Pocahontas ...... & Memphis \& C. R. R......... & 394 \\
\hline . & L., N., S. \& N. A. R. R . . . . . . . & 352 \\
\hline 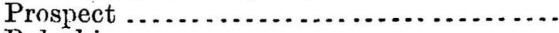 & L., N., S. \& N. A. R. R........ & 588 \\
\hline$\ldots \ldots \ldots \ldots, \ldots, \ldots$ & L., N, S. \& N. A. R. R. . . . . . . & 641 \\
\hline 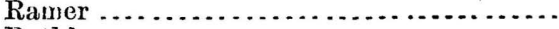 & M. \& O.R. R. . . . . . . . . . & 416 \\
\hline ........................... & C. S.R.R............ & 788 \\
\hline Raven Knob ..... & 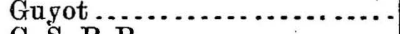 & 6,230 \\
\hline Retrio $\ldots \ldots \ldots$ & C. S. R. R & 747 \\
\hline & & 724 \\
\hline
\end{tabular}




\begin{tabular}{|c|c|c|}
\hline Station. & Authority. & Elevation. \\
\hline & & Feet. \\
\hline Richland......... & L., N., S. \& N. & 774 \\
\hline Rives ............. & M. \& O.R. R. & 301 \\
\hline Robbins.......... & C. S. R. R... & 1,382 \\
\hline Rock Creek ....... & C. S. R. R.. & 753 \\
\hline 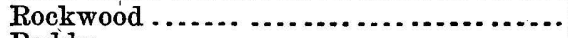 & C. S. R. R.. & 885 \\
\hline Roddy,$\ldots \ldots \ldots \ldots \ldots \ldots \ldots$ & C. S. R. R... & 784 \\
\hline Rosboro' & D. R. V. R. R. & 708 \\
\hline 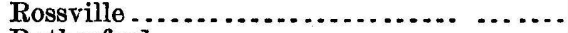 & Memphis \&. C. & 316 \\
\hline 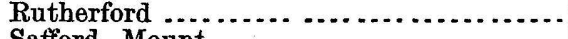 & M. \& O. R. R ... & 321 \\
\hline Safford, Mount $\ldots \ldots \ldots \ldots \ldots \ldots \ldots$ & Guyot................ & 6,535 \\
\hline $\begin{array}{l}\text { Sailor's Rest. . . . . . . . . . . . . } \\
\text { Saulsbury }\end{array}$ & L., N., S. \& N. A. R & 364 \\
\hline $\begin{array}{l}\text { Saulsbury } \ldots \ldots \ldots \ldots \ldots \ldots \\
\text { Sanndersville } \ldots \ldots \ldots \ldots \ldots\end{array}$ & Mernphis \& C. R. & $\begin{array}{l}536 \\
545\end{array}$ \\
\hline Sedgemore ............... & C. S. R. R... & 1,401 \\
\hline Shelby's ..................... & L., N., S. \& N. & 249 \\
\hline ( & L., N., S. \& N. & 279 \\
\hline 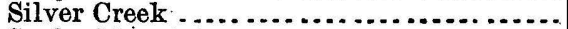 & D.R. V.R. R ... & 702 \\
\hline 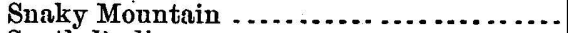 & Gupot......... & 5,195 \\
\hline 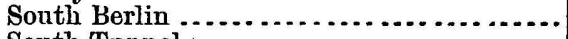 & D. R. V.R. R & 775 \\
\hline South Tunnel .................. & L., N., S. \& I & 804 \\
\hline Spring City ....... & C. S. R. R... & 781 \\
\hline Springdale . . . . . . . . . . . . & L., N., S. \& N. A & 256 \\
\hline Springville. . . . . . & L., N., S. \& N. A & 340 \\
\hline Staunton $\ldots \ldots \ldots \ldots \ldots \ldots \ldots$ & L., N., S. \& N. A. & 303 \\
\hline Steele's $\ldots \ldots \ldots \ldots$ & L., N., S. \& N. A. & 365 \\
\hline 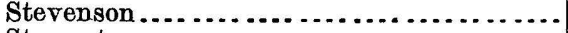 & L., N., S. \& N. A & 709 \\
\hline 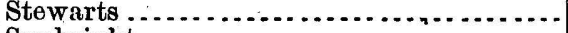 & L., N., S. \& N. & 464 \\
\hline Sunbright. . . . . . . . & 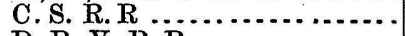 & 1,359 \\
\hline 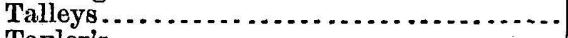 & 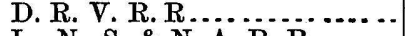 & 789 \\
\hline Taylor's. ............... & L., N., S. \& N. A. & 307 \\
\hline Tennessee Ridge ..................... & L., N., S. \& N. A & 720 \\
\hline 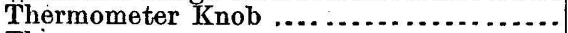 & Guyot........... & 6,157 \\
\hline Thompsons...$\ldots \ldots$ & L., N., S. \& N. A. I & 777 \\
\hline Three Brothers (highest) . . . . . . . . . . . . . . . & Guýot .............. & 5,907 \\
\hline Thunderhead. $\ldots \ldots \ldots \ldots \ldots \ldots \ldots \ldots$ & Guyot........... & 5,520 \\
\hline Thunderknob ............................. & Guyot.................. & 5,682 \\
\hline Trenton $\ldots \ldots \ldots \ldots$ & M. \& O. R. R & 321 \\
\hline Trezerant ........ & L., N., S. \& N. & 443 \\
\hline Tricorner Knob. . & Guyot......... & 6,188 \\
\hline Turkey Knob.... & Guyot. & 4,740 \\
\hline Union ........... & E. T., Va. \& Ga. R. R & 1,457 \\
\hline Union City . . . . . . . . . . . . . & M. \& O. R. R ......... & 346 \\
\hline Vernon Furnace................. & L., N., S. \& N. A. R. R ..... & 367 \\
\hline 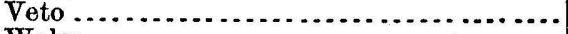 & L., N., S. \& N. A. R. R ...... & 619 \\
\hline ( & L., N., S. \& N. A. R. R ...... & $\cdot 668$ \\
\hline Ward $\ldots \ldots \ldots \ldots \ldots \ldots \ldots \ldots \ldots \ldots \ldots$ & D. R. V. R. R ... & 732 \\
\hline 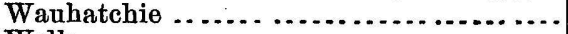 & Alá. G. S. R. R . . . . . . & 690 \\
\hline Wells ............ & L., N., S. \& N. A. R. R... & 242 \\
\hline West Harpeth.... & L., N., S. \& N. A. R. R... & 670 \\
\hline 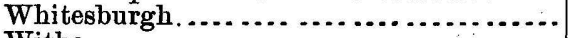 & E. T., Va. \& Ga. R. R....... & 1,214 \\
\hline 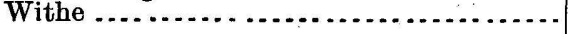 & L., N., S. \& N. A. R. R....... & 271 \\
\hline
\end{tabular}


TEXAS.

\begin{tabular}{|c|c|c|}
\hline Station. & Authority. & Elevation. \\
\hline & & \\
\hline Albany..... & T. C. R. R.. & \\
\hline Alexander. & T. C. R. R.......... & 1,141 \\
\hline Allen ........ & H. \& T. C. R. R . & 669 \\
\hline $\begin{array}{l}\text { Alleyton ..... } \\
\text { Ames ...... }\end{array}$ & G., H. \& S. A. R. I & $\begin{array}{r}193 \\
78\end{array}$ \\
\hline Angus ...... & H. \& T. C. R. R. & 448 \\
\hline Aquilla ... & T. C. R. R........ & 531 \\
\hline Arcola $\ldots . .$. & I. \& G. N. R.R. & 66 \\
\hline Arroyo Escondid & Toner ............. & 2,115 \\
\hline Austin....... & I. \& G. N. R. R. & 477 \\
\hline Do.(Freight De & H. \& T.C. R. R. & 501 \\
\hline Do.(Passenger & H. \& T. C. R.R.. & 513 \\
\hline 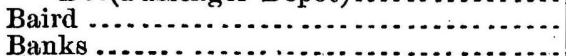 & $\begin{array}{l}\text { T. \& P.R.R } \\
\text { G., C. \& S. F. R. }\end{array}$ & $\begin{array}{r}1,524 \\
702\end{array}$ \\
\hline $\begin{array}{l}\text { Banks ........... } \\
\text { Beaumont...... }\end{array}$ & 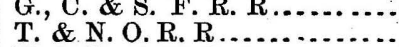 & $\begin{array}{r}702 \\
26\end{array}$ \\
\hline Belknap, Fort... & Med. Dept. U. S. A & 1,600 \\
\hline Belleville .... & G., C.\& S. F.R. R & 262 \\
\hline Bells........... & Mo., Kans. \& Tex. & 675 \\
\hline Belmont Farm . & Toner ............ & 722 \\
\hline Beltou & G., C. \& S. F. R. & 620 \\
\hline Benuh & H. \& T. C. R. R. & 306 \\
\hline ineral ...... & & 1,228 \\
\hline Big Sandy, cross. T: & T. \& P.R. R .... & 336 \\
\hline Bliss, Fort ........... & Med. Dept. U. s. A & 3,830 \\
\hline Do.....Astronon & Wheeler............ & 3,623 \\
\hline Bluff............... & Toner $\ldots . . . . .$. & 180 \\
\hline Blum ................... & G., C.\& S.F.R. R. & 592 \\
\hline Bonham .... & T.\& P.R.R ...... & 582 \\
\hline Borden.. & G., H. \& S. A.R.R & 303 \\
\hline Boston .............. & Smithsonian Inst. & 600 \\
\hline Brackettville, Sign & U. S. Signal Office. & 1,137 \\
\hline Brazc & G., C.\& S.F.R.R & 70 \\
\hline nd......... & H. \& T. C.R. R.... & 467 \\
\hline Brenham....................... & G., C. \& S. F. R. R & 301 \\
\hline Briar Creek & H. \& T. C. R.R.... & 350 \\
\hline Briar Creek ..... & Toner & 378 \\
\hline Brown, Fort. & Mexican Bounda & 165 \\
\hline Browns .......... & L.\& S. V.R.R .... & 286 \\
\hline Brownsville ....... & R. G. R. R......... & 33 \\
\hline $\begin{array}{r}\text { Do... Signal Station..... } \\
\text { Bryan }\end{array}$ & U. S. Signal Office ........... & \\
\hline (n) & H. \& T. C. R. R . . . . . . . & 371 \\
\hline to $\ldots \ldots \ldots \ldots$ & 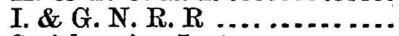 & 387 \\
\hline ulo Springs ... & Smithsonian Inst .. & 1,800 \\
\hline Burton $\ldots . . . . . .$. & H. \& T. C. R. R ... & 436 \\
\hline Caldwell.......... & G., C.\& S.F.R.R. & 411 \\
\hline Calvert.... & H. \& T. C. R. R... & 337 \\
\hline Camden ............. & L. \& S. V.R. R ............... & 234 \\
\hline Cameron........... & G., C.\& S. F. R. R .......... & 407 \\
\hline .............. & T. \& P.R. R ................ & 1,015 \\
\hline & T. C.R.R.................. & 1,592 \\
\hline Castroville......... & Mexican Boundary Survey... & 672 \\
\hline Do..... Signal Station........ & U. S. Signal Office ............. & 778 \\
\hline Cedar Grove $\ldots \ldots \ldots \ldots \ldots \ldots$ & Toner ............................... & 60 \\
\hline 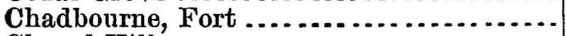 & Med. Dept. U. S. A ........... & 2,120 \\
\hline Chapel Hill....................... & H. \& T. C. R R $R \ldots$ & 337 \\
\hline 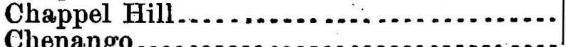 & Smithsonian Inst ............. & 542 \\
\hline$m$ & I. \& G. N. & \\
\hline
\end{tabular}




\begin{tabular}{|c|c|c|}
\hline Sta & Authorit5. & Elevation. \\
\hline & & Feet. \\
\hline $\begin{array}{l}\text { China Grove ... } \\
\text { Chocolate..... }\end{array}$ & $\begin{array}{l}\text { I. \& G. N. R. R } \\
\text { G., C. \& S. F. R. R }\end{array}$ & $\begin{array}{l}50 \\
56\end{array}$ \\
\hline Cisco (T. \& P. & T. C. R. R.............. & 1,611 \\
\hline Clark, Fort........ & Med. Dept. U. S. A & 1,000 \\
\hline $\begin{array}{l}\text { Clarksville.......... } \\
\text { Clear Lake....... }\end{array}$ & $\begin{array}{l}\text { T. \& P. R. R R.... } \\
\text { G., C. \& S. F.R.R. }\end{array}$ & $\begin{array}{r}464 \\
68\end{array}$ \\
\hline Cleburne .......... & G., C. \& S.F.R. R : & 933 \\
\hline Clifton $: . . . . .$. & G., C. \&. S. F. R. R & 670 \\
\hline Columbia ........ & I. \& G. N. R. R... & $\begin{array}{r}34 \\
213\end{array}$ \\
\hline $\begin{array}{l}\text { Columbus ....... } \\
\text { Comanche Spring }\end{array}$ & 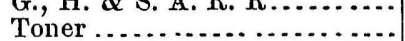 & $\begin{array}{r}213 \\
2,874\end{array}$ \\
\hline Concho, Signal S & U. S. Signal Office. & 1,888 \\
\hline $\mathrm{C}$ & Smithsonian Inst & 3,600 \\
\hline Concrete........... & G., W. T.\& P.R. R & 246 \\
\hline Cone............... & G., C. \& S. F. R. R & 375 \\
\hline Converse............ & G., H. \& S. A. R. R & 721 \\
\hline Corpus Christi ..... & Med. Dept. U. S. A & 20 \\
\hline Corsicana & H. \& T.C.R.R. & 427 \\
\hline Do... Signal St & U. S. Signal Offic & 448 \\
\hline Courtney ........... & H. \& T. C. R. R . & 201 \\
\hline Crabb .............. & G., C. \& S. F. R. R & 91 \\
\hline Crawford ........ & G., C. \& S. F.R.R. & 687 \\
\hline $\begin{array}{l}\text { Crockett.......... } \\
\text { Croghan, Fort .... }\end{array}$ & I. \& G.N. R.R ..... & 350 \\
\hline an, Fort $\ldots . .$. & Med. Dept. U.S. A. & 1,000 \\
\hline $\begin{array}{l}\text { Crosby ................... } \\
\text { Cross-Roads......... }\end{array}$ & $\begin{array}{l}\text { T. \& N. O. R. R..... } \\
\text { Smithisonian Inst .. }\end{array}$ & $\begin{array}{r}50 \\
672\end{array}$ \\
\hline Cross Timbers............. & I. \& G. N.R.R .... & $\begin{array}{r}072 \\
75\end{array}$ \\
\hline & T.\&N.O.R.R... & 52 \\
\hline Cuero....... & G., W.T.\& P. R. R & 177 \\
\hline 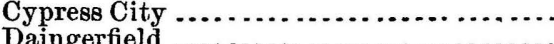 & H.\& 'I. C. R. R ... & 155 \\
\hline Daingerfield & E. L.\& R.R.R.R . & 403 \\
\hline 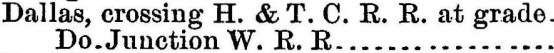 & T.\&P.R.R $\ldots \ldots$ & $\begin{array}{l}466 \\
439\end{array}$ \\
\hline 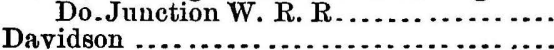 & T.\&P.R.R & $\begin{array}{l}432 \\
255\end{array}$ \\
\hline $\begin{array}{l}\text { Davidson ..... } \\
\text { Davis, Fort ... }\end{array}$ & Med. Dept. U. S. A.. & 4,700 \\
\hline Signal Stati & U.S. Signal Office.. & 4,918 \\
\hline$\ldots$. & N. O.R.R. & 89 \\
\hline & T. \& P.R.R ... & 422 \\
\hline Delo & Toner $\ldots \ldots \ldots$ & 960 \\
\hline Deniso & Mo., Kans. \& Tex. R. R....... & 722 \\
\hline Do.low water Re & & \\
\hline $\begin{array}{l}\text { Do.Junction H. \& } \mathrm{T} \text {. } \\
\text { Do.Signal Station... }\end{array}$ & $\begin{array}{l}\text { Mo., Kans. \& Tex. R. R } \\
\text { U. S. Signal Office..... }\end{array}$ & $\begin{array}{l}723 \\
767\end{array}$ \\
\hline Deveres........... & T.\& N.O.R.R ........... & 66 \\
\hline & I. \& G.N.R.R ... & 409 \\
\hline Do & & 3,824 \\
\hline Dor & N.R.R. & 307 \\
\hline Du & R. R ......... & 1,449 \\
\hline Fort .. & ept. U. S. A & 1,460 \\
\hline Dye & G., C. \& S. F. R. R & 116 \\
\hline & G., H. \& S. A. R. R & 203 \\
\hline Signal Station... & U. S. Signal Office ..... & 800 \\
\hline (n................ & Mex. Boundary Survey & 4,536 \\
\hline & G., H. \& S. A. R. R...... & 123 \\
\hline & T. \& P.R ......... & 1,299 \\
\hline Clara & G., H. \& S. A. R. R & 633 \\
\hline & H. \& T. C. R. R......... & 395 \\
\hline & Mex. Boundary Survey ...... & 422 \\
\hline Elcia & 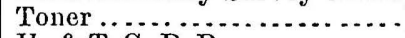 & 3,607 \\
\hline & H. \& T. C. R.R.. & 600 \\
\hline & I. \& G. N. R. R. & 390 \\
\hline $\begin{array}{l}\text { Elmo } \\
\text { El Pas }\end{array}$ & T.\& P. R. R & $\begin{array}{r}501 \\
3720\end{array}$ \\
\hline Do.Signal Station... & U. S. Signal Office... & $\begin{array}{l}3,720 \\
3,370\end{array}$ \\
\hline
\end{tabular}




\begin{tabular}{|c|c|c|}
\hline Station. & Authority. & Elevation. \\
\hline & & Feet. \\
\hline 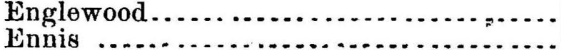 & I. \& G. N. R. R & 420 \\
\hline $\begin{array}{l}\text { Ennis } \\
\text { Escondido }\end{array}$ & 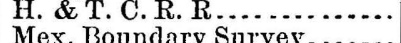 & 547 \\
\hline 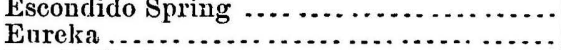 & 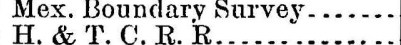 & 2,806 \\
\hline (n) & 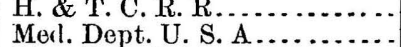 & $\begin{array}{r}69 \\
200\end{array}$ \\
\hline Ferris............. & 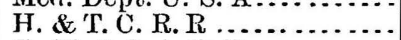 & 472 \\
\hline Flatonia .......... & G., H. \& S. A. R. R .......... & 473 \\
\hline Footes .......... & 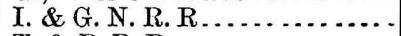 & 273 \\
\hline Foruey .......... & T. \& P. R. R ... & 469 \\
\hline Fort Worth $\ldots . . . \ldots \ldots \ldots$ & T. \& P.R.R . . . . . . . . & 623 \\
\hline 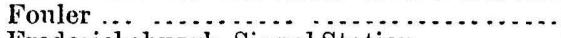 & T. C. R. R. . . . . . . . & 581 \\
\hline Fredericlisburgh, Signal Stati & U. S. Signal Office ........... & 1,742 \\
\hline Frontera $\quad \ldots \ldots \ldots \ldots \ldots \ldots$ & Mex. Boundary Survey....... & 3,796 \\
\hline Fulton...... & To & 242 \\
\hline Station & G., C.\& S. F. R. & \\
\hline al Station $\ldots \ldots \ldots \ldots \ldots$ & H. \& T.C.R.R. & $\begin{array}{r}40 \\
563\end{array}$ \\
\hline $\begin{array}{l}\text { Garrett ......... } \\
\text { Gates, Fort .... }\end{array}$ & 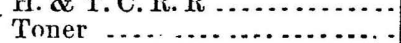 & $\begin{array}{r}563 \\
1.000\end{array}$ \\
\hline 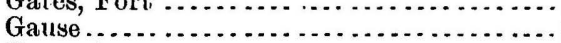 & I. \& G. N.R.R. & $\begin{array}{r}1,000 \\
376\end{array}$ \\
\hline . & I. \& G. N.R.R. & 753 \\
\hline (n................. & H. \& T. C.R.R & 536 \\
\hline . & T. \& P.R. R ... & 324 \\
\hline 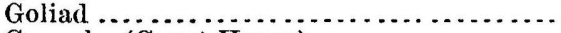 & Smithsinian Inst ............ & 50 \\
\hline 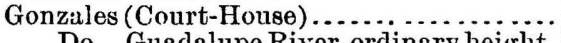 & G., W.T.\& P. R. R........... & 276 \\
\hline 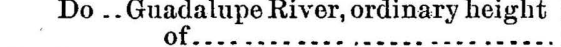 & G. W. T. \& P. R. R..... & 232 \\
\hline Gordon & T. \& P.R.R... & 822 \\
\hline Grabam, Fort .... & Med. Dept. U.S. A ............ & 900 \\
\hline Grand Saline....... & 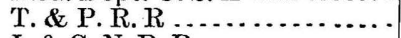 & 400 \\
\hline Grapeland ......... & I. \&. G. N. R. R & 480 \\
\hline 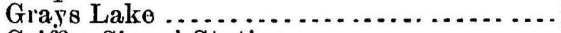 & I. \& G. N.R. R ..... & 136 \\
\hline Griffin, Signal Station .................... & U.S. Signal Office.. & 1,243 \\
\hline$\ldots \ldots \ldots \ldots \ldots$ & II. \& T.C.R.R ............. & 481 \\
\hline 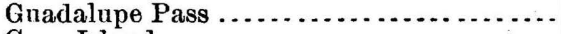 & P. R. R. Reports ............. & 5,717 \\
\hline$\ldots \ldots \ldots \ldots$ & 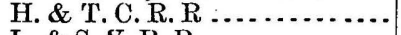 & 96 \\
\hline Gum Springs..... & 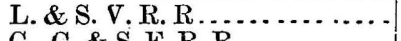 & 301 \\
\hline 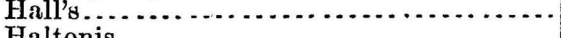 & 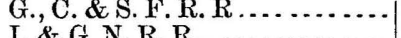 & $\begin{array}{r}33 \\
141\end{array}$ \\
\hline (n) & $\cdots \cdots \cdots$ & 141 \\
\hline$\ldots \ldots \ldots \ldots \ldots \ldots \ldots$, & H.\& T.C.R.R.. & $\begin{array}{r}415 \\
38\end{array}$ \\
\hline (1) & roner $\& \mathrm{~T}, \mathrm{R}, \mathrm{R}$ & $\begin{array}{r}38 \\
467\end{array}$ \\
\hline 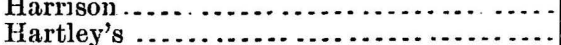 & 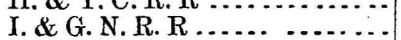 & $\begin{array}{l}407 \\
120\end{array}$ \\
\hline Harwood $\ldots \ldots \ldots \ldots$ & G., H. \& S. A. R. R......... & 463 \\
\hline . & I. \& G. N. R. R ........... & 305 \\
\hline$\ldots \ldots \ldots \ldots \ldots$ & G., C. \& S. F.R.R.......... & 546 \\
\hline$\ldots \ldots \ldots \ldots \ldots$ & Smithsonian Inst ............ & 600 \\
\hline ostead $\ldots \ldots \ldots \ldots \ldots \ldots \ldots \ldots \ldots$ & H. \& T. C.R.R .. & 245 \\
\hline Henrietta, Signal Station ................. & U.S. Sigual Office............ & 915 \\
\hline 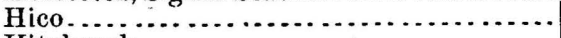 & 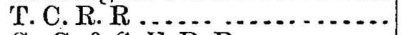 & 1,007 \\
\hline$\ldots \ldots \ldots \ldots \ldots \ldots$ & G., C. \& S. F. R. R.............. & 17 \\
\hline Hocheim, top of divide above ............. & G., W. T.\& P.R.R......... & 355 \\
\hline 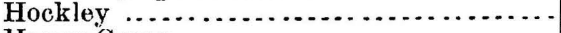 & H. \& T. C. R. R ............. & 225 \\
\hline$\ldots \ldots \ldots \ldots \ldots$ & T. \& P.R. R .... & 682 \\
\hline (n) & G., C. \& S. F. R. I & 63 \\
\hline (n) & H.\& T.C.R.R. & 37 \\
\hline ( & 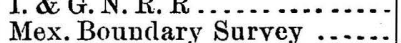 & $\begin{array}{r}53 \\
2,054\end{array}$ \\
\hline Howe. & 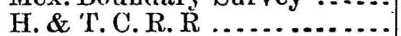 & $\begin{array}{r}2,054 \\
859\end{array}$ \\
\hline Howta................. & H. \& T. C. R. R ........... & 281 \\
\hline Hubbard Timbers $\ldots \ldots \ldots \ldots \ldots \ldots \ldots$ & T. \& P. R. R . . . . . . . & 1,874 \\
\hline Hueco Pass . . . . . . . . . . . . . . . . . . & 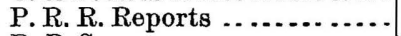 & 5,450 \\
\hline & 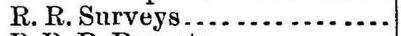 & 5,027 \\
\hline & P. R. R. Reports . ............. & 3,935 \\
\hline & & 887 \\
\hline
\end{tabular}




\begin{tabular}{|c|c|c|}
\hline Sta & Authority. & Elevation. \\
\hline & & Feet. \\
\hline $\begin{array}{l}\text { Hurd's Pass ....... } \\
\text { Hutchins ....... }\end{array}$ & $\begin{array}{l}\text { R.R. Surveys } \\
\text { H.\& T. C. R.R }\end{array}$ & $\begin{array}{r}4,419 \\
472\end{array}$ \\
\hline Independence Sp & & 4,778 \\
\hline $\begin{array}{l}\text { Indianola, Sig } \\
\text { Inge, Fort... }\end{array}$ & $\begin{array}{l}\text { U. S. Signal Office. } \\
\text { Med. Dept. U. S. A. }\end{array}$ & $\begin{array}{r}26 \\
845\end{array}$ \\
\hline Iredell..... & T. C.R.R............. & $\begin{array}{l}840 \\
881\end{array}$ \\
\hline Jackass Prairi & R.G.R.R ..... & 19 \\
\hline Jacksborough, & U. S. Sigual Office. & 1,133 \\
\hline Jacksonville. & I. \& G. N.R.R... & 525 \\
\hline $\begin{array}{l}\text { Jefferson, Junct } \\
\text { Jewett......... }\end{array}$ & $\begin{array}{l}\text { T. \& P. R.R R... } \\
\text { I. \& G. N. R. R } \ldots\end{array}$ & $\begin{array}{l}221 \\
496\end{array}$ \\
\hline Junction, I. R. R & G., W.'T.\& P.R. & 25 \\
\hline Keechi ......... & I. \& G. N. R. R.. & 282 \\
\hline Kelley's....... & I. \& G. N. R. R. & 381 \\
\hline Kilgore ....... & I. \& G. N. R. R... & 371 \\
\hline Kingsbury ... & G., H. \& S. A. R. & 616 \\
\hline Kinney ....... & G., C. \& S. F. R. & 399 \\
\hline Kiowa Peak.. & Petermann $\ldots \ldots$ & 2,895 \\
\hline Kopperl....... & G., C. \& S. F. R. & 574 \\
\hline Kosse ......... & H. \& T. C.R.R. & 504 \\
\hline Lake ......... & I. \& G. N. R. R.. & $\begin{array}{r}314 \\
011\end{array}$ \\
\hline $\begin{array}{l}\text { Lambert ........ } \\
\text { Lancaster.... }\end{array}$ & Med. Dept. U. S. A & $\begin{array}{l}1,011 \\
2,350\end{array}$ \\
\hline $\begin{array}{l}\text { Lancaster........ } \\
\text { Laredo.......... }\end{array}$ & Toner............ & $\begin{array}{l}2,580 \\
806\end{array}$ \\
\hline Do.Signal Sta & U.S. Sigual Office. & 401 \\
\hline Lariss & Isouian Inst & 755 \\
\hline Lavas & Toner ............... & 17 \\
\hline ter... & H. \& T. C. R. R. & 464 \\
\hline Leon $\ldots . . . .$. & T. C. R. R ....... & 1,269 \\
\hline Leon Spring & Mex. Boundary Si & $2,80 \pi$ \\
\hline Liberty & T. \& N. O.R. R... & \\
\hline Lincoln, Fort ... & Med. Dept. U. S. A & 900 \\
\hline Lindale.......... & I. \& G. N. R. R... & \\
\hline Llano Estacado ... & n.......... & $\begin{array}{r}3,200 \\
\text { to } 4,700\end{array}$ \\
\hline Long Point . & Toner & 400 \\
\hline sss. I. \& G. I & T. \& P. R. R ... & 336 \\
\hline $\operatorname{ads} \ldots$ & I. \& G. N. R. R... & 300 \\
\hline Lulin & G., H. \& S. A. R. F & 418 \\
\hline & G., C. \& S. F. R. P & 315 \\
\hline Mc] & H. \& T. C. R. R .. & 589 \\
\hline sh, For & Med. Dept. U. S. A & 806 \\
\hline McKavet & pt. U. S. A & 2,060 \\
\hline Do.... Signal Station & U. S. Signal Office & 1,217 \\
\hline McKinney ..................... & H. \& T.C. R. R... & 615 \\
\hline & H. \& T. C. R. R... & 557 \\
\hline rion $\ldots . . . \ldots \ldots$ & G., H. \& S. A. R. R .. & 566 \\
\hline Falls....... & H. \& T. C. R. R...... & 394 \\
\hline & I. \& G. N. R. R.. & \\
\hline $11, J$ unction of J. \& S. D & T. \& P. R. R... & 371 \\
\hline Ścott, Fort & t. U. S. A & 1,300 \\
\hline , Fort ... & Med. Dept. U. S. A & 1,200 \\
\hline 's Creek .... & L. \& S. V.R.R ... & 241 \\
\hline Signal Stat & Sigual Office. & 1,620 \\
\hline & I. \& G. N. R. R . & 132 \\
\hline & H. \& T. C. R. R & 692 \\
\hline & G., C. \& S. F. R. R .... & 791 \\
\hline Fort . & Med. Dept. U. S. A..... & 150 \\
\hline & T. \& P. R. R . & 494 \\
\hline Mexia $\ldots \ldots \ldots \ldots \ldots$ & H. \& T. C. R. R ... & 537 \\
\hline $\begin{array}{l}\text { Milano } \\
\text { Do Junction }\end{array}$ & I. \& G. N. R. R.... & $\begin{array}{r}475 \\
500\end{array}$ \\
\hline о. & H. \& T. C. R. R........... & 426 \\
\hline 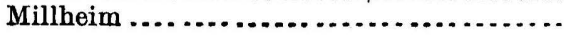 & G., C. \& S. F. R. R .... & 169 \\
\hline
\end{tabular}




\begin{tabular}{|c|c|c|}
\hline Station. & Authority. & Elevation. \\
\hline & & Feet. \\
\hline ( & H. \& T. C. R. & 300 \\
\hline Mine Creek... & Smithsouian Ins & 600 \\
\hline 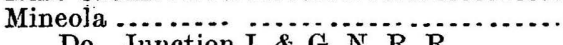 & I. \& G. N. R. R. & 402 \\
\hline Do.. Junction I. \& G. N. R. R.......... & T. \& P. R. R . & \\
\hline 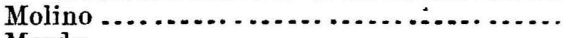 & P. R. R. Repo & 3,830 \\
\hline 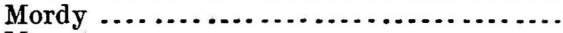 & G., C. \& S. F. & 783 \\
\hline . & T.C. R. R.. & . 734 \\
\hline Mount Airy . . . . . . . . . . . . . . . . . . . . . . . . & T. C. R. R... & 1,439 \\
\hline 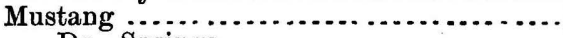 & G., C. \& S. F. R. R .......... & 47 \\
\hline 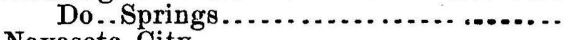 & Toner $\ldots \ldots \ldots$ & 1,982 \\
\hline 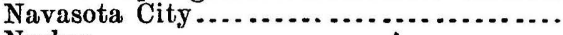 & H. \& T. C. R. I & 219 \\
\hline Neches ... & I. \& G. N. R. R & 411 \\
\hline Neuwied ......... & Toner ........... & 800 \\
\hline New Boston ..... & T. \& P. R. R .. & 387 \\
\hline North, Fort $\ldots \ldots \ldots \ldots$ & Toner ......... & 1,100 \\
\hline North Franklin Mountain . ................ & Wheeler.. & 7,069 \\
\hline Nueces $\ldots \ldots \ldots \ldots \ldots \ldots \ldots \ldots \ldots \ldots \ldots \ldots$ & Mex. Boundar & 932 \\
\hline 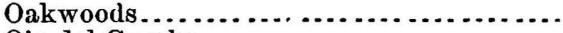 & I. \& G. N. R. $\stackrel{R}{R}$ & 280 \\
\hline del Cuerbo ........................... & P. R. R. Repo & 3,893 \\
\hline Ojo Escondido..... & Toner ......... & 2,311 \\
\hline Orange........... & T. \& N. O. R. R & 10 \\
\hline tow $\ldots \ldots \ldots \ldots \ldots$ & I. \& G. N. R. R & 507 \\
\hline Oyster Creek................ & I. \& G. N. R. R & 38 \\
\hline Paige ..... & H. \& T. C. R. R & 573 \\
\hline 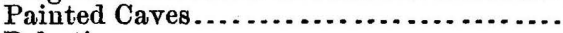 & Mex. Boundary S & 952 \\
\hline … & I. \& G. N. R. & 495 \\
\hline (n) & H. \& T. C. R. R & 471. \\
\hline Paris.... & T. \& P. R. R ... & 592 \\
\hline Peach Creek....... & ........... & 206 \\
\hline Pecos Spring . .................. & .......... & 3,098 \\
\hline 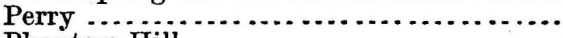 & H. \& T. C. R. R ............ & 483 \\
\hline 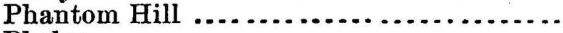 & Med. Dep.t. U. S. A .......... & 2,300 \\
\hline Phelps & I. \& G. N.R. R $\ldots \ldots \ldots \ldots \ldots$ & 377 \\
\hline 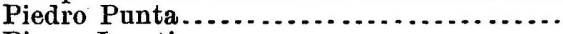 & Toner ......... & 744 \\
\hline 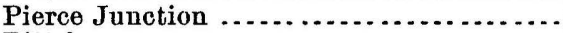 & G., H. \& S. A. R & 63 \\
\hline Pittsburg & E. L.\& R.R.R. R & 402 \\
\hline 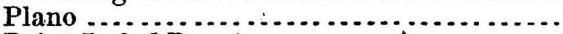 & H.\& T. C. R.R. . & 667 \\
\hline Point Isabel Depot $\ldots \ldots \ldots \ldots \ldots \ldots \ldots$ & R. G. R. R.... & 8 \\
\hline Polk, Fort ... . . . . . . . . . . . . . . . . . & $\ldots \ldots \ldots$. & 15 \\
\hline Prairie.$\ldots \ldots \ldots \ldots \ldots \ldots \ldots \ldots \ldots \ldots \ldots \ldots$ & I. \& G. N.R.R. & 87 \\
\hline Presidio del Norte $\ldots \ldots \ldots \ldots \ldots \ldots \ldots \ldots$ & .......... & 2,779 \\
\hline ton $\ldots \ldots \ldots \ldots \ldots \ldots \ldots \ldots \ldots \ldots$ & P. R. R. Reports. & 790 \\
\hline 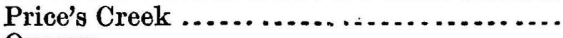 & G., W.T.\& P. R. R.... & 105 \\
\hline 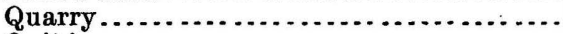 & G., C. \& S. F. R. R . & 235 \\
\hline & Mex. Boundary Survey ....... & 856 \\
\hline aan, Fort. $\ldots \ldots \ldots \ldots \ldots \ldots$ & Med. Dept. U. S. A ........... & 3,710 \\
\hline 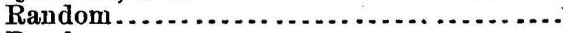 & Toner $\ldots \ldots \ldots \ldots \ldots \ldots \ldots$ & 147 \\
\hline Randon...$\ldots \ldots \ldots \ldots \ldots \ldots \ldots \ldots \ldots \ldots \ldots$ & G., H. \& S. A. R. R........ & 93 \\
\hline (n, & 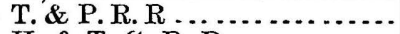 & 1,294 \\
\hline 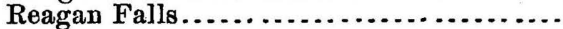 & H. \& T. C. R. R & 385 \\
\hline 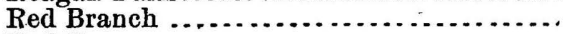 & I. \& G. N.R.R . & 226 \\
\hline & T. \& P.R.R. & 1,539 \\
\hline 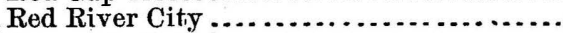 & Toner.... & 572 \\
\hline Resaca de la Palma ...................... & R. G.R.R .... & 27 \\
\hline 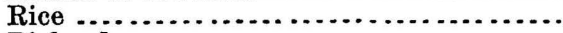 & H. \& T. C.R. R ........... & 473 \\
\hline 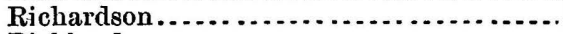 & H.\& T. C.R. R ............ & 643 \\
\hline 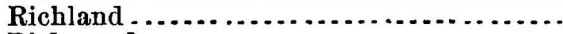 & H. \& T. C. R. R .............. & 377 \\
\hline 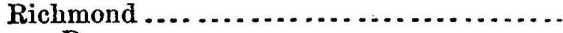 & G.,H. \& S. A. R. R........... & 73 \\
\hline Do $\ldots \ldots \ldots \ldots \ldots \ldots$ & G., C.\& S. F. R. R........... & 105 \\
\hline Ringgold Barracks...$\ldots \ldots \ldots \ldots$ & Med. Dep., U. S. A . . . . . . . . . & 521 \\
\hline 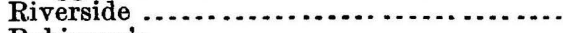 & I. \& G. N.R. R $\ldots \ldots \ldots \ldots$ & 169 \\
\hline Robinson's ......... & L. \& S. V. R. R $\ldots \ldots \ldots \ldots$ & 256 \\
\hline & & 469 \\
\hline
\end{tabular}




\begin{tabular}{|c|c|c|}
\hline Station. & Authority. & Elevation. \\
\hline & & Feet. \\
\hline Roger's ............... & G., C. \& S. F. R. & 538 \\
\hline Rosenberg Junction & G., C. \& S. F. R. & 109 \\
\hline Ross ................ & H. \& T. C. R. R. & 576 \\
\hline Round Rock.. & I. \& G. N. R. R. & 720 \\
\hline San Antonio.. & G., H. \& S. A. R.] & 683 \\
\hline 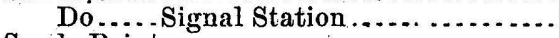 & U. S. Signal Offic & 676 \\
\hline Sandy Point......... & I. \& G. N.R.R. . & 58 \\
\hline San Ëlciario. & Mex. Boundary S & 3,607 \\
\hline . & Mex. Boundary Survey....... & 818 \\
\hline n................. & G., H. \& S. A. R. R & 341 \\
\hline Sealy....... & G., C. \& S. F. R. R & 189 \\
\hline Seco ................. & Toner $\ldots . .$. & 689 \\
\hline $\begin{array}{l}\text { Sequin } \ldots . . . \\
\text { Shelden ..... }\end{array}$ & $\begin{array}{l}\text { G., H. \&.S. A. R. } \\
\text { T. \& N O.R.R. }\end{array}$ & 559 \\
\hline $\operatorname{sing~H}$ \& T. C. R. R. at grade. & T. \& P.R.R... & $\begin{aligned} 57 \\
747\end{aligned}$ \\
\hline 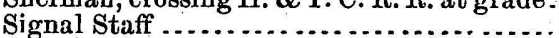 & 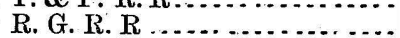 & 7 \\
\hline isterdale ... & Smithsonian Inst & 1,320 \\
\hline Sour Lake. & T. \& N.O.R.R.. & 47 \\
\hline$g . . .$. & I. \& G. N.R.R. & 126 \\
\hline ngfield ...... & Smithsonian Ins & 4,500 \\
\hline ston, Fort, Signal Station ...... & U. S. Signal Offic & 3,050 \\
\hline tork's ........................... & I. \& G.N.R.R. & 385 \\
\hline ur Springs..... & E. L. \& R. R. R. F & 462 \\
\hline ait Ridge....... & H. \& T. C. R. R. . & 285 \\
\hline$\ldots \ldots \ldots$ & H. \& T. C. R. R... & 372 \\
\hline Tehuacana ............ & Toner ............ & 482 \\
\hline Temple Junction ......................... & G., C. \& S. F. R. R & 695 \\
\hline 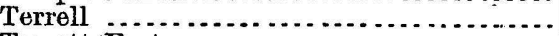 & T. \& P.R. R..... & 514 \\
\hline Terrett, Fort . . . . . . . & Med. Dep. U. S. A & 1,320 \\
\hline Terry ........ & T. \& N. O.R.R. & 23 \\
\hline Thompson .. & G., C. \& S. F. R. R & 68 \\
\hline Do .... & H. \& T. C. R. R.. & 122 \\
\hline Thornton ... & 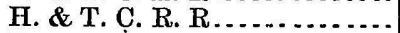 & 496 \\
\hline Tinge....... & Toner ....................... & 892 \\
\hline Trinity ... & I. \& G. N. R. R......... & 234 \\
\hline Troupe............ & 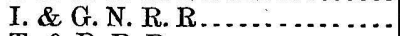 & 467 \\
\hline ................. & T. \& P.R.R .... & 1,469 \\
\hline$\ldots \ldots \ldots . . . . .$. & I. \& G. N. R.R.. & 531 \\
\hline Union Hill & Med. Dept.U.S. A. & 542 \\
\hline$\ldots \ldots \ldots \ldots \ldots \ldots \ldots$ & G., H. \&'S. A. R. R. & 688 \\
\hline Uvalde, Signal Station .............. & U.'S. Signal Office. & 891 \\
\hline Valley Mills...................... & G.C. \& S. F. R.R. & 592 \\
\hline 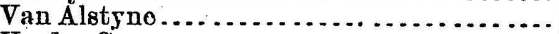 & H. \& R. C.R.R.. & 803 \\
\hline 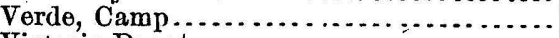 & Mea. Dept. U. S. & 1,400 \\
\hline$\ldots \ldots \ldots . . .6$. & G., W.T.\& P.R.R. & 87 \\
\hline t....... & G., C. \& S. F. R. R. & \\
\hline Wa & G., H. \& S. A. R. R. . . . . . . & 378 \\
\hline er Waller....... & H. \& T. C. R. R . . . . . . . & 252 \\
\hline Wal & G., C.\& S. F. R. R & 131 \\
\hline it Springs.$\ldots \ldots \ldots \ldots \ldots \ldots$ & T.C.R.R . & 901 \\
\hline 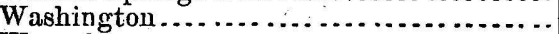 & Smithsonian Inst. & 360 \\
\hline estos & I. \& G. N. R. R.... & 365 \\
\hline erford $\ldots \ldots \ldots \ldots \ldots$ & 'T. \& P. R. R... & 864 \\
\hline erville, Parson's Seminary .......... & Inst ............. & 394 \\
\hline & G., H. \& S. A. R. R . . . . . . & 420 \\
\hline & H.\&'T. C. R. R . . . . . . & 324 \\
\hline ; Bernard.. & G., H. \& S. A. R. & 163 \\
\hline Creek .......... & G. W. T. \& P.R.R... & 126 \\
\hline Westfield ........... & I. \& G. N.R.R....... & 114 \\
\hline Wheelock $\ldots \ldots \ldots \ldots \ldots \ldots \ldots$ & Smithsonian Inst ............. & 450 \\
\hline White Cloud $\ldots . \ldots \ldots \ldots \ldots \ldots \ldots$ & Toner ....................... & 255 \\
\hline Whitehouse $\ldots \ldots \ldots \ldots \ldots \ldots \ldots \ldots \ldots \ldots$ & I. \& G. N.R. R. . . . . . . . & 471 \\
\hline Whitewright .......................... & Mo., Kans, \& Tex. R. R ...... & 748 \\
\hline
\end{tabular}




\begin{tabular}{|c|c|c|}
\hline Station. & Authorit.y. & Elevation. \\
\hline 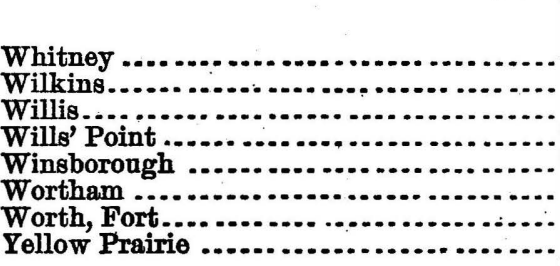 & $\begin{array}{l}\text { T. C. R. R. } \\
\text { L. \& S. V. R. R } \\
\text { I. \& G. N. R. R } \\
\text { T. \& P. R. R. } \\
\text { E. L. \& R. R.R. R. } \\
\text { H. \& T. C. R. R } \\
\text { Toner } \\
\text { G., C. \& S. F. R. R } \\
\end{array}$ & $\begin{array}{r}\text { Feet. } \\
586 \\
271 \\
381 \\
530 \\
532 \\
482 \\
629 \\
458\end{array}$ \\
\hline
\end{tabular}

Bull. 5-19 


\section{UTAH.}

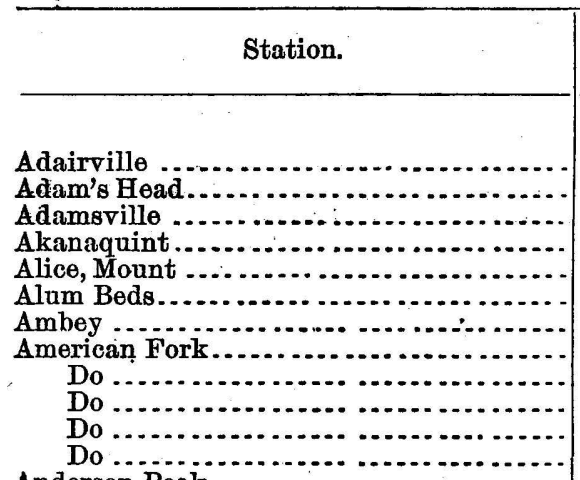

Anderson Peak

Antelope Island

Antelope Spring

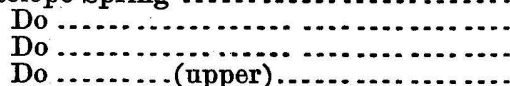

Aquarius Platean

Aspen Lake

Atkinson

Averitt Spring

Awapa Platean

Bald Head

Bald Mountain .

Bald Monntains

Baldy Peak

Bangs, Mount

Bare Bush Point (Wasatch Plateau)

Bartels, Mount

Barton Mountain

Bean Spring

Bear Lake

Bear River Bridge

Bear Valley

Do

Beaver

Do astronomical station

Beaver Dam Mountains

Beaver Valley

Bechler, Mount

Belknap, Mount

Do.........

Berry's Springs

Big Dam Springs

Big Narrows

Big Springs

Black Cap Butte

Black Rock

Do ....................

Blacktail Peak

Blue Creek

\begin{tabular}{|c|c|}
\hline & Elevation \\
\hline & \\
\hline owell .. & \\
\hline Powell ... & $\begin{array}{r}10,3 \\
5,6\end{array}$ \\
\hline $\begin{array}{l}\text { Wheeler.. } \\
\text { P.R.R.Re }\end{array}$ & \\
\hline Powell ... & 9,54 \\
\hline $\begin{array}{l}\text { D. \& R.G. } \\
\text { C.P.R.R. }\end{array}$ & \\
\hline U.C.R.R & \\
\hline P.R.R. Report & \\
\hline $\begin{array}{l}\text { Wheeler. } \\
\text { Powell .. }\end{array}$ & \\
\hline U.S & \\
\hline Powel & 10,71 \\
\hline Wh & \\
\hline Pow & \\
\hline Por & \\
\hline & \\
\hline Whe & \\
\hline Whe & \\
\hline Powell .. & \\
\hline Powe & \\
\hline $\begin{array}{l}\text { Sumn } \\
\text { Summ }\end{array}$ & \\
\hline Powell ... & 8,000 \\
\hline Powe & \\
\hline & \\
\hline & 11,97 \\
\hline ler. & $\begin{array}{r}11,73 \\
7,95\end{array}$ \\
\hline & \\
\hline Pow & 10,25 \\
\hline Pov & $\begin{array}{r}10,05 \\
9,85\end{array}$ \\
\hline & \\
\hline Pow & \\
\hline $\mathrm{Has}$ & $\begin{array}{l}5,91 \\
4,19\end{array}$ \\
\hline & 4,19 \\
\hline Wheeler... & 7,071 \\
\hline Powell ... & \\
\hline$\ldots$ & \\
\hline & \\
\hline & \\
\hline G. .1 & \\
\hline $\mathrm{H}$ & $\begin{array}{r}9,716 \\
12,200\end{array}$ \\
\hline Po & 12,200 \\
\hline WI & 11,89 \\
\hline & 2, \\
\hline U.S & \\
\hline Wheeler & 4,315 \\
\hline $\begin{array}{l}\text { D. \& R.G.R. } \\
\text { D.\& R.G.R. }\end{array}$ & $\begin{array}{l}6,0,20 \\
4,234\end{array}$ \\
\hline $\begin{array}{l}\text { D. \& R. G.R. R } \\
\text { Powell ........ }\end{array}$ & $\begin{array}{l}4,234 \\
6,530\end{array}$ \\
\hline U. W.R.R & $\begin{array}{l}0,250 \\
4,263\end{array}$ \\
\hline U. C. R. R . & 4,799 \\
\hline $\begin{array}{l}\text { U. S } \\
\text { Vin. }\end{array}$ & \\
\hline & $4,37 !$ \\
\hline
\end{tabular}

(414) 


Station.
Blue Gate Mesa $\ldots \ldots \ldots \ldots \ldots \ldots \ldots \ldots \ldots \ldots$
Blue Gate Plateau, north $\ldots \ldots \ldots \ldots \ldots \ldots \ldots$

Do. south

Blue Mountain

Blue Spring

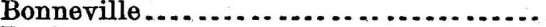

Bovine

Bowl Valley

Box Elder Peak

Bingham Junction

Brian, Mount

Brian's Head

Brigham City

Do

Brown's Park

Brown's Peak

Bruin Point.

Buckhorn Flats

Buckhorn Junction

Buck Horn Spring

Buckhorn Spring

Bullionville Road Crossing

Burro Peak

Cachill

Call's Fort

Cameron, Fort

Camp Floyd Pass

Carmel, Mount

Castle Gate

Castle Rock

Castle Valley

Castle Valley Junction

Cedar Cify.

Cedar Mountain Pass

Cedar Ridge Summit.

Cedar Spring

Cedar Summit

Cedar Valley

Centerville

Cherry Creek

Circle Valley

Do

Circleville

City of Rocks

Clarkson

Clarkston

Clayton's Peak

Clear Creek

Clear Creek Mountain

Coal Point

Coalville

Colob Plateau

Copenhagen

Corinne.

Do.signal station

Corn Creok Settlement

\begin{tabular}{|c|c|}
\hline Authority. & Elevation. \\
\hline
\end{tabular}

Powell

Powell

$5,94 i$.

6,800

Powell

Powell

Wheeler

C. P. R. R

Powell

Wheeler

D. \& R. G. R. R

Powell

Powell

C. P. R. R .

U. \& N. R. $\mathbf{R}$

Powell

Powell

Powell

D. \& R. G. R. R

D. \& R. G. R. R

Powell .

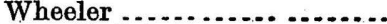

D. \& R.G.R.R

King

U. \& N. R. R

U. \& N. R. R

Wheeler

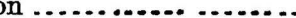

Powell

D. \& R. G. R. R

U. P. R. R

Powell

D. \& R. G. R. R

Wheeler.

P. R. R. Reports

D. \& R. G. R. R

Wheeler .......

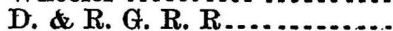

Powell

U. C. R. R

D. \& R. G. R. R

D. \& R. G. R. R

Powell

Wheeler

Wheeler.

Hayden

Powell

King

D. \& R. G. R. R

Wheeler.

Powell

Summit Co. R. R

Powell

Hayden

C. P. R. R

U. S. Signal Office

Powell
$\{6,800$

$\{7,300$

11,071

7,131

4,310

4,347

$\{7,600$

$\{7,700$

9,542

4,366

11,178

11,260

4, 226

4,321

4,476

$\{5,500$

$\{5,800$

5,392

10,150

5,697

5,692

5,770

5,688

5,548

12,834

4,971

4,255

6,058

5,234

5,241

6,322

6,249

$\{5,000$

$\{6,500$

5,795

5,726

6,364

6,228

5,100

4,984

$\{5,300$

$\{6,000$

4,253

5,299

6,055

$\{6,000$

$\{6,300$

5,624

6,078

4,800

5,930

11, 889

6,229

9,132

6,760

5,596

$\{8,000$

$\{9,000$

4,999

4, 232

4,249

4,800 


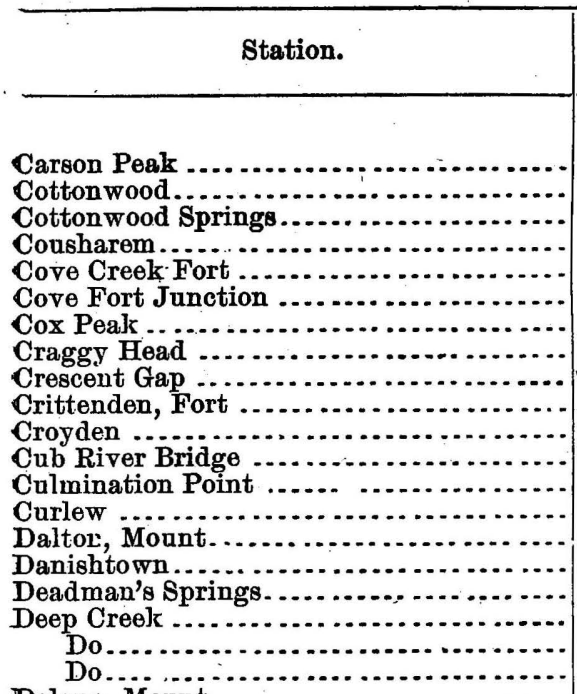

Delano, Mount

Dellenbaugh, Mount

Deseret

$$
\text { Do }
$$

Desert Mountain

Desert Spring

Desert Springe

Deweyville

$$
\text { Do. }
$$

Diamond City

Diamond Valley.

Dilley's Ranch, Hansel Spring

Dodoquiba Spring

Douglas, Camp ..

$$
\text { Do................................... by level }
$$

Drape

$$
\text { Do... }
$$

East Fork Sevier

East Salient.

East Tavaputs Plateau

Echo.

Echo Park (mouth Yampa River)

Ellen, Mount.

Ellenore, Mount

Ellen Spring

Ellsworth, Mount

El Vado de los Padres (Colorado River) Do

Emma's Park

Emmons, Mount

Ephraim

Ephraim Point

Epley Butte

Escalante City

Escalante Desert

E. T. City

Eureka City

Eyrię Peak.

Fairfield

Farmington

Faust

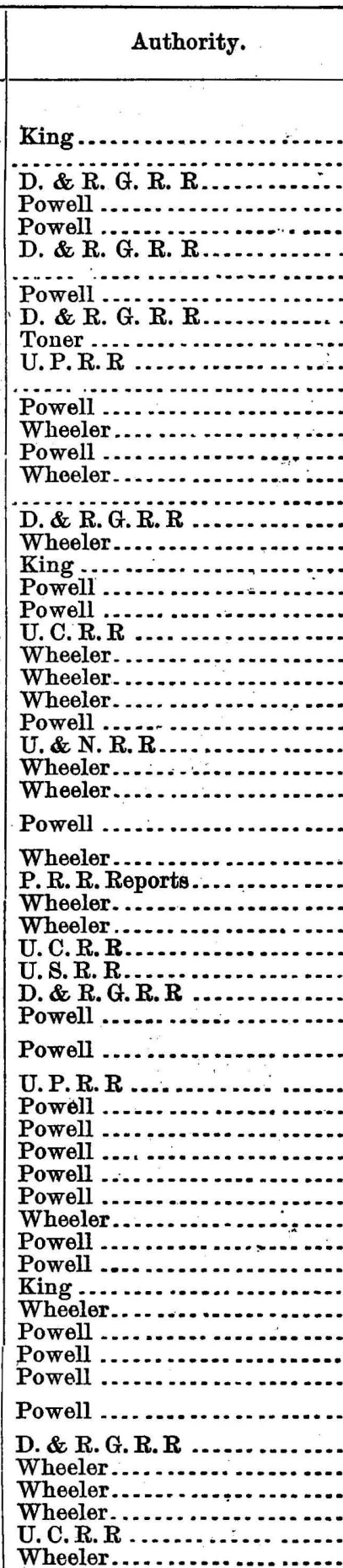

Elevation.

Feet.

9,630

4,200

4,661

6,850

6,000

5,477

13,250

8,250

$4,7: 20$

4,860

5,250

4,542

9,700

4,397

10,480

4,400

6,782

5,025

5,237

5,127

12,240

6,650

4, 541

4,642

8,175

5,887

5,610

4,328

4,415

6,369

$\{4,300$

$\{4,800$

5,043

4,659

5,024

4,905

4,443

4,513

7,714

7,490

$\{5,500$

$\left\{\begin{array}{l}9,700 \\ 5,480\end{array}\right.$

5,480

5,080

11,410

7,690

9,940

8,150

3,194

3,220

7,370

13,694

5,633

8,690

5,860

5,700

55,000

$\{5,500$

4,272

6,400

9,458

4,866

4,261

5,296 


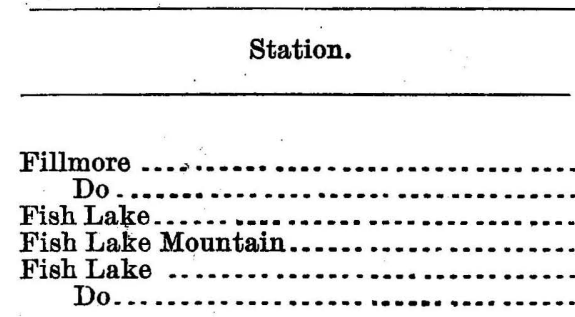

Fish Lake Platean

Fish Lake Valley

Fish Spring .

Flaming Gorge .

Floyd, Camp (now Fairfield)

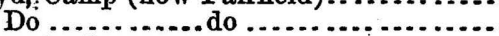

Fountain Green

Frances Point

Frisco

Germania

$$
\text { Do..... }
$$

Gilbert's Peak

Do. ......timber-line on

Glencoe

$$
\text { Do. Bridge }
$$

Glenwood

Goshen

Gooseberry Valley (south end)

Gould's Ranch

Granite Rock

Grantville

Grass Valley

$$
\text { Do }
$$

Graves Valley

Great Sage Plain (mean elevation)

Great Salt Lake (May 16, 1873)

Green River.

Gunnison

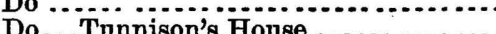

Gunnison's Crossing (Green River)

Gunnison Plateau

$$
\text { Dó............ (highest point) }
$$

Gunnison Point

Gunnison Valley

Gunsight Mountain

Hague Spring .

Half-way House.

Hampton's Bridge .

Do.... Station

Hanging Rock

Hansel Pass.

Hardy's.

Harry, Mount.

Hawawah Spring.

Hay Patch Spring.

Hay Spring .....

Heber Mountain

Do.

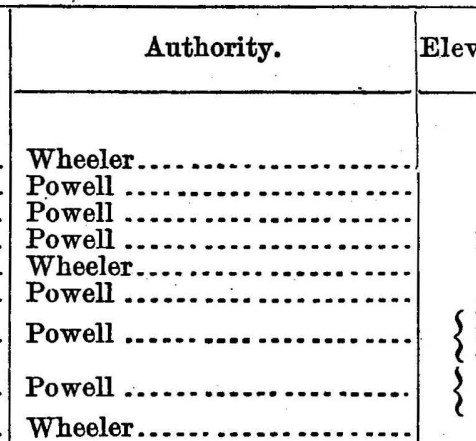

Powell

Med. Dept., U. S. A

Wheeler

Wheeler

Powell .................................

U. C. R. R

D. \& R. G. R. $\mathbf{R}$

U. C. R. R

King

King ....

Wheeler

Powell

Powell

Powell

Powell

Powell

D. \& R, G. R. R

D. \& R. G. R. R

Summit Co. R. $R$

Powell

Wheeler

Powell

Powell

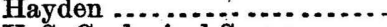

U. S. Geological Survey

D. \& R. G. R. R

Powell

Powell

Powell

Powell

\section{Powell}

Powell

Powell

Powell

Haydon

Powell

U. W. R. R

Hayden

Wheeler

\section{Toner.}

Wheeler

D. \& R. G. R. R

Powell

Wheeler

Wheeler

Wheeler

King
Elevation.

Feet.

6,025

5,100

8,790

11, 578

8,763

$8,79 \%$

S 10,500

$\{11,600$

8,750

8,850

6,269

5,820

4,860

4,867

5,874

10,430

6,315

4,296

4,242

13,687

11,100

5,221

5,237

5,300

4, 482

$\{8,600$

$\{8,700$

4,052

4,356

4,251

5,520

$\{6,200$

$\{7,500$

6,857

4,300

9,640

6,500

4,218

4,021

5,118

5,144

5,120

5, 134

4,083

$\{7,000$

$\{9,800$

9,864

6,000

$\{4,100$

$\{4,500$

8,306

5,450

4,326

4,300

4, 700

5,974

5,138

5,487

11, 300

5, 455

5,590

5, 092

10,138

9,850 


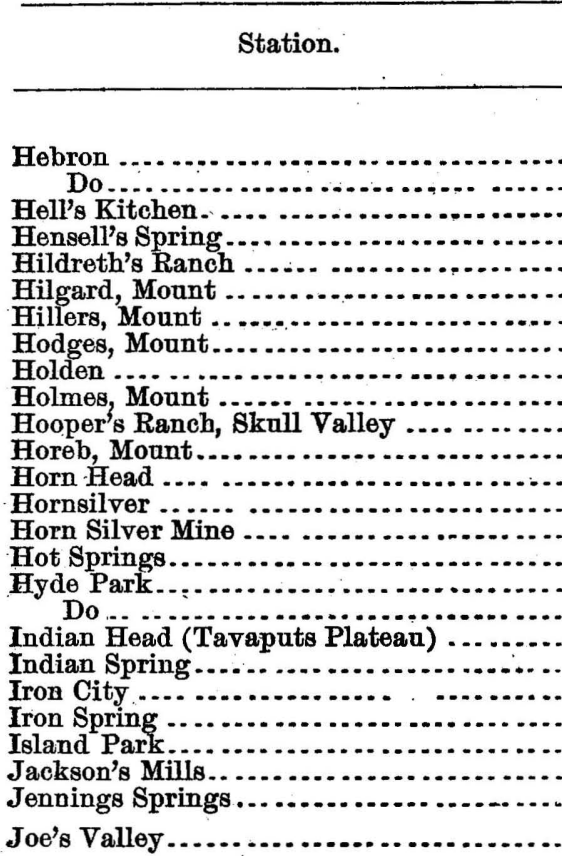

$$
\text { Do }
$$

Johnson's Pass

Johnson Spring

Juab

Juab Valley

Kaibab Plateau

Kaiparowits Peak

Kaiparowits Plateau

Do

Kang

.(east end)

Do... Hamblin's)

Do..(Adam's) ........................

Do...(Farnsworth's)

Kanab Plateau

Kanab Springs

Kanara

$$
\text { Do }
$$

Kansa

Kansas Prairi

$$
\text { Do }
$$

Katharine's Peak

Kaysville

Kelton

Do

Key

Kimball, Mount

King's Meadows

Knowlton

Lake

Lake Butte, near Laketown

Lake Range (highest peak)

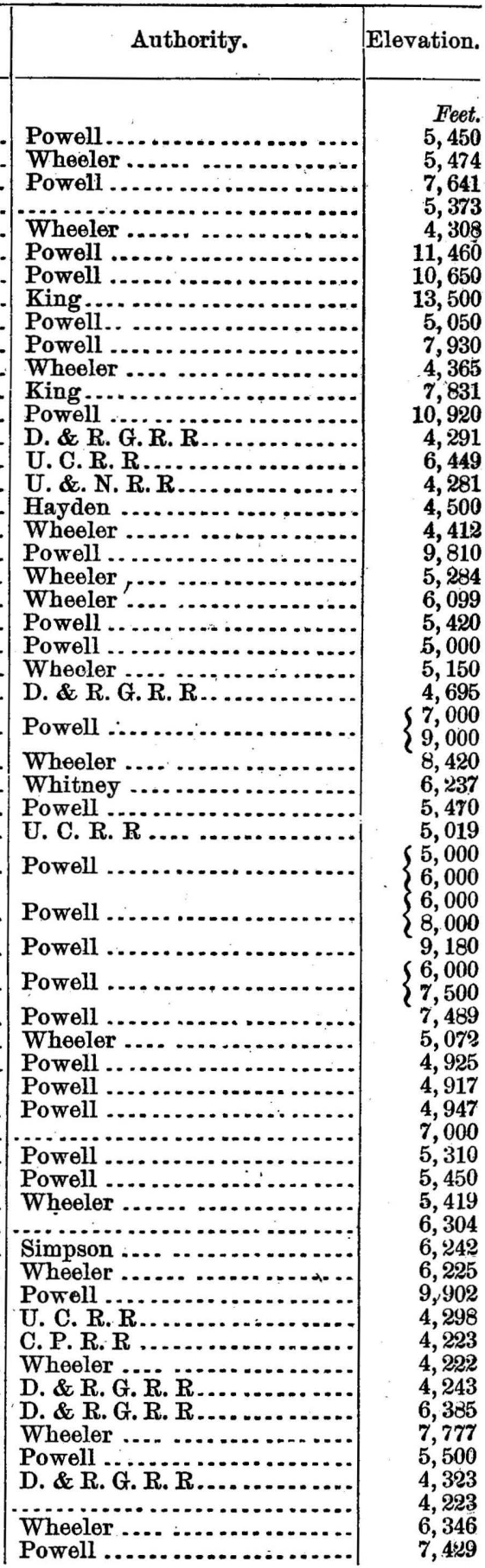




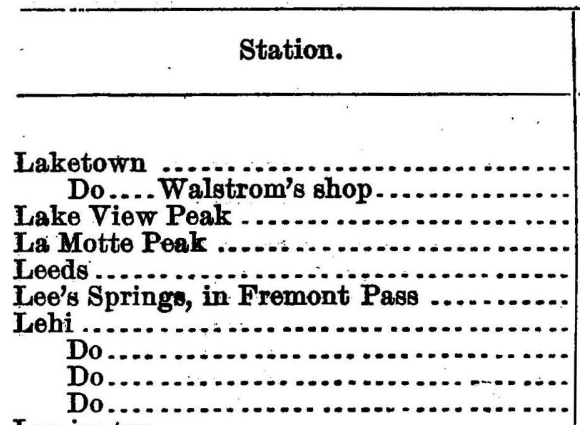

Lemington.

Leonora Peak

Lewiston Peak

Lime Pass.

Little Bitter Creek Springs

Little Cottonwood

$$
\text { Do.... do }
$$

Little Creek Peak

Little's Ranch

Logan

$$
\text { Do }
$$

$$
\text { Do }
$$

Logan Peak

$$
\text { Do...do }
$$

Lone Peak

Lone Rock Valley

Long Valley .

Lost Creek Vạlley

Lovendahls

Lucin

McCready's Meadow

Mammoth Mill

Mansard Point

\section{Markagunt Plateau}

Maroni Peak

Marsh's Peak

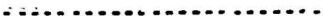

Marysvale

$$
\text { Do .... }
$$

Marysvale Poak

Masuk Plateau

Matlin

Meadow Creek

Meadow ville

Mendon

$$
\text { Do }
$$

Midget Crest

Milford

Mill Creek Station

Miller Creek

Mill Fork

Mills

Mill Spring

Mill Station

\begin{tabular}{|c|c|}
\hline Authority. & Elevation. \\
\hline & Feet. \\
\hline Hayden & 6,000 \\
\hline Wheeler. & 5,993 \\
\hline Hayden . & 7,795 \\
\hline King _....... & 12,892 \\
\hline Powell .... & 3,400 \\
\hline $\begin{array}{l}\text { Wheeler.: } \\
\text { U.C.R.R. }\end{array}$ & $\begin{array}{l}6,883 \\
4,517\end{array}$ \\
\hline U.S.R.R. & 4,586 \\
\hline Wheeler & 4,596 \\
\hline D. \& R. G. & $\begin{array}{l}4,544 \\
4,674\end{array}$ \\
\hline Powell .... & $\begin{array}{l}4,014 \\
8,900\end{array}$ \\
\hline Wheeler. & 10,623 \\
\hline King ..... & 10,740 \\
\hline Powell .. & 6,858 \\
\hline U.S.R.R. & 4,288 \\
\hline Wheoler. . & 4,359 \\
\hline Powell ... & 10,010 \\
\hline Powell ... & 7,000 \\
\hline Hayden ${ }_{\mathrm{p}} \mathrm{p}_{\mathrm{p}}$ & 4,509 \\
\hline $\begin{array}{l}\text { U. \& N. R. R } \\
\text { Wheeler. } \ldots \ldots \ldots \\
\end{array}$ & $\begin{array}{l}4,507 \\
4,533\end{array}$ \\
\hline Hayden ... & 10,004 \\
\hline$\therefore \ldots . . . .$. & 7,950 \\
\hline$\ldots \ldots \ldots$ & 11,295 \\
\hline P.R.R.Re & $\begin{array}{l}4,400 \\
5,400\end{array}$ \\
\hline Powell ... & $\begin{array}{l}5,000 \\
6,000\end{array}$ \\
\hline Powell .. & 5,500 \\
\hline U. C. R. I & 4,277 \\
\hline C. P. R. R & 4,498 \\
\hline n & 6,240 \\
\hline . & 6,947 \\
\hline$\ldots \ldots \ldots \ldots$ & 6,290 \\
\hline (n) & 11,000 \\
\hline Powell. & 8,010 \\
\hline King ..... & 12,410 \\
\hline$\ldots \ldots \ldots$ & 11,600 \\
\hline $\mathbf{R} \ldots \ldots \ldots$ & 5,808 \\
\hline$\ldots \ldots \ldots$ & 5,810 \\
\hline (n) & 10,359 \\
\hline 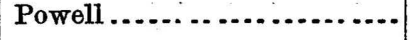 & 8,200 \\
\hline C. P. R. R & 4,597 \\
\hline Wheeler. & 5,992 \\
\hline ( & 6,200 \\
\hline $\mathbf{R} \ldots \ldots \ldots$ & 4,459 \\
\hline$\ldots \ldots \ldots \ldots$ & 4,553 \\
\hline 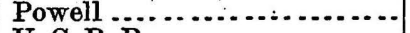 & 11,414 \\
\hline$\therefore \ldots \ldots \ldots \ldots \ldots \ldots$ & 4, 908 \\
\hline Wheoler & 6,504 \\
\hline $\begin{array}{l}\text { D. \& R. G. R. R. } \\
\text { D. \& R. G. R. R... }\end{array}$ & 5,505 \\
\hline 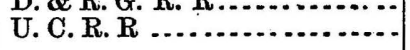 & 4,852 \\
\hline Wheeler... & $\begin{array}{l}6,504 \\
6,504\end{array}$ \\
\hline U. C. R. R. & 4,859 \\
\hline Powell .... & 4,900 \\
\hline Powell ... & 5,380 \\
\hline 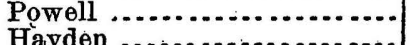 & 11,240 \\
\hline Hayden............ & 5,793 \\
\hline
\end{tabular}

Mona

$$
\text { Do. }
$$

Monroe

Monroe Peak

Montpelier 


\begin{tabular}{|c|c|c|}
\hline Station. & Authority. & Elevation. \\
\hline & & \\
\hline Monument . & C.P. R. R & 4,227 \\
\hline Moraine Valley & Powell . & $\begin{array}{r}9,000 \\
10,000\end{array}$ \\
\hline Morgan ......... & U. C.R.R & 4,220 \\
\hline $\begin{array}{l}\text { Moses, Mount ... } \\
\text { Mounds House . }\end{array}$ & $\begin{array}{l}\text { Wheeler. } \\
\text { Powell .. }\end{array}$ & $\begin{array}{l}8,725 \\
4,940\end{array}$ \\
\hline Mountain City & Powell ... & 5,760 \\
\hline $\begin{array}{l}\text { Mountain Meador } \\
\text { Mountain Spring }\end{array}$ & $\begin{array}{l}\text { Wheeler... } \\
\text { Powell ... }\end{array}$ & $\begin{array}{l}5,741 \\
5,741\end{array}$ \\
\hline Mount Carmel .. & Powell ... & 5,250 \\
\hline $\begin{array}{l}\text { Mount Pleasant } \\
\text { Do......... }\end{array}$ & $\begin{array}{l}\text { Wheeler. } \\
\text { Powell .. }\end{array}$ & $\begin{array}{l}5,875 \\
5,900\end{array}$ \\
\hline nsinia Peak .. & Powell ... & 10,940 \\
\hline $\begin{array}{l}\text { Naor } \\
\text { Narr }\end{array}$ & Wheiler..- & $\begin{array}{l}9,951 \\
4,498\end{array}$ \\
\hline Navajo Well $\mathbf{P}$ & $\begin{array}{l}\text { D. } \\
\text { Powell .... }\end{array}$ & $\begin{array}{l}4,498 \\
6,460\end{array}$ \\
\hline ebo, Mount... & Wheeler.. & 11,992 \\
\hline eels ...... & $\begin{array}{l}\text { Powell } \\
\text { U.C.R. }\end{array}$ & $\begin{array}{r}11,680 \\
4,356\end{array}$ \\
\hline Nephi......... & Powell $\because$ & 5,130 \\
\hline Do & $\begin{array}{l}\text { U.C.R.R } \\
\text { P.R.R.R }\end{array}$ & 5,056 \\
\hline & Wheeler... & 4,920 \\
\hline in $\ldots \ldots \ldots \ldots$ & Wheeler... & 7,045 \\
\hline Deville Summ & $\begin{array}{l}\text { Powell } \\
\text { D.\& R. G. }\end{array}$ & $\begin{array}{l}5,120 \\
6,605\end{array}$ \\
\hline Logan Peal & Hayd & 10,004 \\
\hline Nort & Wheeler. & 4,509 \\
\hline ain $: \ldots \ldots \ldots \ldots$ & & 9,695 \\
\hline Promontory & Wheeler.. & 7,134 \\
\hline $\begin{array}{l}\text { North Willard } \\
\text { Oak Creek .... }\end{array}$ & $\begin{array}{l}\text { Wheeler.. } \\
\text { Wheeler.. }\end{array}$ & $\begin{array}{r}4,276 \\
\cdot \quad 5,158\end{array}$ \\
\hline Oak & Simpson. & 7,200 \\
\hline$\ldots \ldots \ldots \ldots \ldots \ldots$ & $2+$ & 6,790 \\
\hline (n) & Wh & 9,589 \\
\hline Ogden, junc. with U & C. 1 & $\begin{array}{l}0,010 \\
4,303\end{array}$ \\
\hline Do ........... & & 4,374 \\
\hline nmit .. & D. & 7,731 \\
\hline (........ & Wheele & 6,273 \\
\hline Do.................... & & \\
\hline Panguitch Lake ................. & Por & 8 \\
\hline Panguitch Hayfields.............. & Powell & 7,000 \\
\hline Paradise ................, & Wheeler.. & 4,863 \\
\hline $\begin{array}{l}\text { Paragoonah } \\
\text { Pana }\end{array}$ & eler.......... & 6,222 \\
\hline $\begin{array}{l}\text { Pana } \\
\text { Park } \text { City }\end{array}$ & Summit Co $\mathrm{R}$ R & $\begin{array}{l}4,562 \\
6.851\end{array}$ \\
\hline Parowan............. & Powell ............... & 5,970 \\
\hline & & \\
\hline Parowan Valley........... & Powell ... & \\
\hline Patmos Head ................ & Powell ........ & 9,830 \\
\hline Paunsagunt Plateau.................. & Powell ... & 8,000 \\
\hline & R. Reports.. & \\
\hline Do............ & Wheeler........... & \\
\hline Do............ & U.C.R.R.......... & 4,548 \\
\hline & Powell & \\
\hline Pennell, Mount... & Powell... & 11,320 \\
\hline len Pass ..................... & Powell ........... & 7,730 \\
\hline 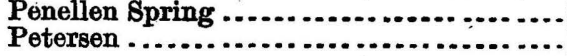 & $\begin{array}{l}\text { Powell } \\
\text { U.P.R. R........ }\end{array}$ & $\begin{array}{l}7,730 \\
4,907\end{array}$ \\
\hline
\end{tabular}




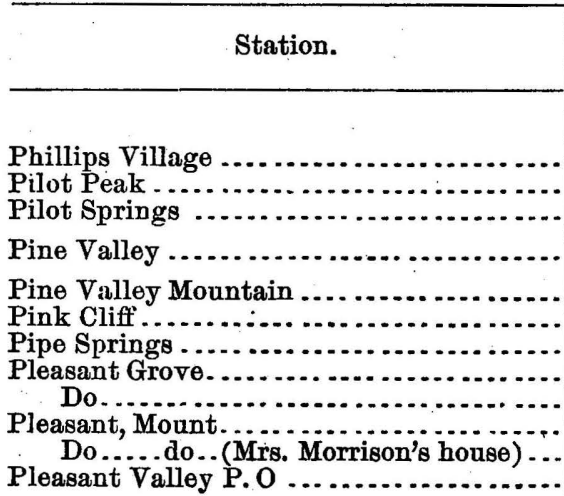

Pleasant Valley

Plymouth

Point Carbon

Point Nelson

Portage

Potato Valley

Prattville.

Promontory

Do..... Mount

Provo.

Do.

Do

Do................

Provo Peak (Wasatch Mountains)

Do.

do

Rabbit Valley

Randolph

Rattlesnake Point

Red Cañon Summit

Red Plateau

Richey's Ranch

Richfield.

Richmon

Do.

Do.

Riverside

Rockfield

Rocky Rest Spring

Round Lake Valley

Round Prairio

Round Valley

Rush Valley

Do.

Do.

\begin{tabular}{|l|l}
\hline Authority. & Elevation. \\
\hline
\end{tabular}

........................ $\quad \begin{aligned} & \text { Feet. } \\ & 4,32 \gamma\end{aligned}$

Wheeler.................... 10,900

Wheeler................. 4,704

Powell ..................... $\{\quad 5,200$

Powell ...................... \{ to 6,000

Powell ..................... $\quad 9,260$

Powell .................... 4,890

U.C.R.R . . . .

U.S.R.R................. 4,557

Wheeler.................. 5,875

Powell ..................... 5, 5, 898

Wheeler. ................... 6,064

Powell .................... \& 7,500

to 8,200

Hayden .................... 4,500

King ........................ $\quad 11,443$

Powell ........................
Hayden ..................

Wheeler................... $\left\{\begin{array}{l}5,700 \\ \text { Po }\end{array}\right.$

Powell ...................... $\{$ to 7,000

C.P.R.R................ 4,905

Wheeler.................. $\quad 7,459$

D. \& R. G. R. R ............. 4,517

U.C.R.R ................ 4, 4, 456

U.S.R.R................. 4, 4,

Wheeler................... 4,567

Powell .................... 4,547

Wheeler...................... 11,066

Powell .................... 11,000

Powell .................. $\quad 6,800$

Hayden ..................... $\{$ to 7,500

D. \& R. G.R.R R.......... 5,701

D. \& R. G.R.R ............. $\quad 7,910$

Powell

6,000

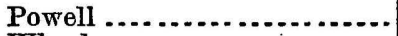

to 7,500

6,169

5,283

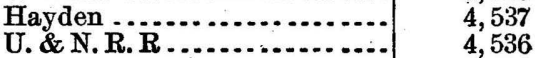

Wheeler.................. 4,656

U. C. R. R .............. 4, 583

D. \& R.G. R. R ............

Powell .................... $\quad 4,510$

Powell $\ldots \ldots . . . . . . . .5,500$

to 6,000

Simpson .................... 5,571

Powell ..................... $\left\{\begin{array}{r}5,300 \\ \text { to } 6,000\end{array}\right.$

Simpson ...................

Wheeler .................... 5,234

Powell .... 4.500

5,300

Powell ...................... 4,500

Sahara Plateau.

Pow

Saint George .

Salem (Pondtown)

Salina

Salina Summit

Salt Lake City

Do

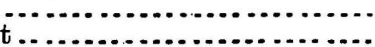

Toner

Powell

D. \& R. G. R. R

D. \& R. G. R. R

D. \& R.G.R. R

Powell

5,200

2,880

6,200

4,580

5,182

7, 927

4, 228

4,354 


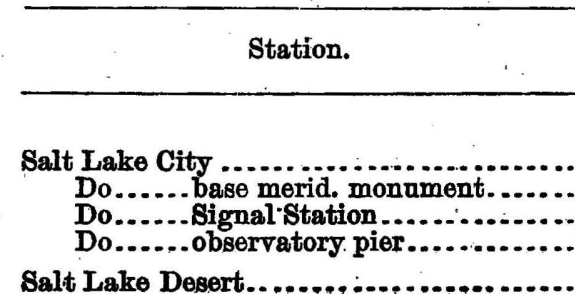

Salt Lake Valley.

Salt Spring Ranch

Salt Wells

Sandy

$$
\text { Do }
$$

San Francisco Spring

San Pete Valley

San Rafael River.

San Rafael Valley

Santa Claxa Settlement

Santaquin

Do

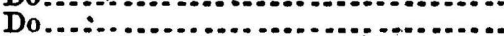

Santaquin Mountains (Wasatch Range)

Saw Tooth Narrows

Scipio

$$
\text { Do }
$$

Scofield

Section Ridge

Session Settiement

Sevier Cañon

Sevier Desert

Sevier Lake

Sevier Lake Desert

Sevier Pass (west side)

Sevier Platean

Sevier River Bridge

Do ...........

Sheep Salient

Sheep Trough Spring

Shinarump

Short Cat Poes.

Sknell Vill ....................................... Do

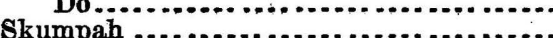

Skutumpa or Clarkston..................

Smithfield

Do $\ldots \ldots \ldots \ldots \ldots$

Do

Soldier Summit

Solitude Peak.

(1)

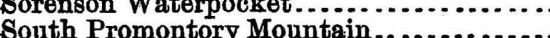

South Tent (Wasatch Plateau)

Spanish Fork$$
\text { Do }
$$$$
\text { Do. }
$$

Spanish Fork Peak

Spanish Valley

Split Mountain

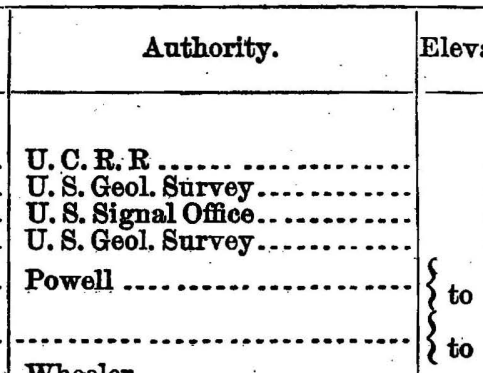

\section{Wheeler}

Wheeler.

U.S.R. R

Wheeler.

Powell

D. \& R. G.R. R

Powell

Powell

Powell

U. C.R. $\mathbf{R}$

U. S. R. R

Powell

D. \& R.G.R. $\mathrm{R}$

Powell.

Wheeler.

D.\& R.G. R. R

King

P.R.R. Reports

D.\& R. G.R.R

Powell

Wheeler

Wheeler

Wheeler

Powell

Powell

Wheeler

Poweil

Powell

Powell

Wheeler

Simpson

Simpson

Wheeler

Wheeler

Hayden

U.\& N.R. R

Wheeler

D. \& R. G. R. R

Powell

Powell .

Powell

D. \& R.G. R. R

U. C. R. $R$

U. S: R. $R$

Powell

Powell

King
Elevation.

Freet.

4,261

4,334

4,346

4,334

4,200

5,000

4,200

to 4,500

4,634

4,203

4,399

4,457

6,527

5,200

to 6,200

5,331

4, 100

to 4,200

2, 900

5,030

4,813

4,884

9,670

6,527

5,260

5,115

7,832

8,268

4,183

5,482

5,000

to 5,400

4,600

4,874

4,768

9,000

to 10,300

4,888

5,283

5,560

4,600

5,330 .

3,921

5,347

6,369

4,850

4,356

6,142

5,930

4,565

4, 562

4,623

7,465

8,800

5,630

7,460

11,240

4,865

4,493

4,556

9,970

3,900

to 5,300 8,865 


\begin{tabular}{|c|c|c|}
\hline Stat & & Elevation. \\
\hline & & \\
\hline pringville ....... & D. \& R. & \\
\hline Do.................... & $\begin{array}{l}\text { U. C. R. R } \\
\text { U. S. R. R. }\end{array}$ & $\begin{array}{l}4,451 \\
4,525\end{array}$ \\
\hline tevenson, Camp. & & 5,930 \\
\hline 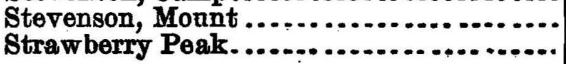 & $\begin{array}{l}\text { Powell } \ldots . . . \\
\text { Powell } . . . .\end{array}$ & $\begin{array}{r}10,840 \\
9,020\end{array}$ \\
\hline Strawberry Valley ......................... & Powell ..... & $\begin{array}{r}7,400 \\
\text { to } 8,000\end{array}$ \\
\hline Sulphur Springs & D. \& R. G & 6,493 \\
\hline Do ............. (I & Powell ... & 5,101 \\
\hline $\begin{array}{l}\text { Do } \\
\text { Summit (Bradbury's) }\end{array}$ & 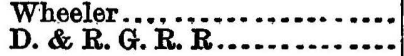 & $\begin{array}{l}5,400 \\
7,190\end{array}$ \\
\hline Summit Valley.................,$\ldots$ & Powell $\ldots . . . \ldots \ldots \ldots \ldots$ & $\begin{array}{r}6,500 \\
\text { to } 7,200\end{array}$ \\
\hline $\begin{array}{l}\text { Park..... } \\
\text { iff .......... }\end{array}$ & $\begin{array}{l}\text { Powell .. } \\
\text { Powell .. }\end{array}$ & $\begin{array}{r}6,400 \\
10,070\end{array}$ \\
\hline Table Cliff Plateau & Powell .. & \\
\hline Point (Aq & Pov & 10,670 \\
\hline$\ddot{. .}$ & $\begin{array}{l}\text { Whoeler.: } \\
\text { U. W.R. }\end{array}$ & \\
\hline & C. P. R. R & 4,544 \\
\hline Countains & King & 6,900 \\
\hline idge .... & Po & $\begin{array}{r}11,380 \\
5,660\end{array}$ \\
\hline & & \\
\hline Pag & Po & \\
\hline Thistle Valley ... & Powell ... & 06 \\
\hline Thor & & 11,240 \\
\hline$\ldots$ & & $\begin{array}{l}5,380 \\
6,990\end{array}$ \\
\hline Mount & & 11,957 \\
\hline k. & & 10,260 . \\
\hline$\because$ & $\underset{P}{\mathbf{W}}$ & $\begin{array}{l}4,833 \\
7,650\end{array}$ \\
\hline na Poak. & & $\begin{aligned} 13,458 \\
13,4\end{aligned}$ \\
\hline Monnt. & & $12,27 \cdot 1$ \\
\hline ient.. & & 9,980 \\
\hline & & 10,396 \\
\hline neh. & & 4,583 \\
\hline Monnt & Po & 3,100 \\
\hline (n) & Reports & $\begin{array}{l}8,187 \\
4,487\end{array}$ \\
\hline kavatz, Mount & Hayden. & 10,815 \\
\hline Tununk Plateau ... & Powell .. & \\
\hline Twin & 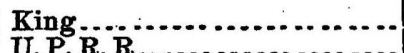 & 11, \\
\hline & & \\
\hline ta River (mouth of) & Powell ............. & \\
\hline Uinta Valley .......... & Powell .... & \\
\hline Un-k & Por & \\
\hline & $\ldots \ldots \ldots$ & 6 \\
\hline e Valley .. & Simpson & $\begin{array}{l}4,49 \\
4,54\end{array}$ \\
\hline Utah Valley ...... & Powell .... & $\left\{\begin{array}{r}4,50 \\
\text { to } 5,20\end{array}\right.$ \\
\hline Ute Peak $\ldots . . . . . . .$. & King $\ldots . . .$. & \\
\hline & Powell ..................... & \\
\hline $\begin{array}{l}\text { Virgi } \\
\text { Waas }\end{array}$ & en & \\
\hline & & \\
\hline
\end{tabular}




\begin{tabular}{|c|c|c|}
\hline Stz & Anthority. & Elevation. \\
\hline 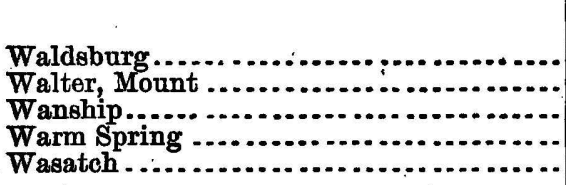 & 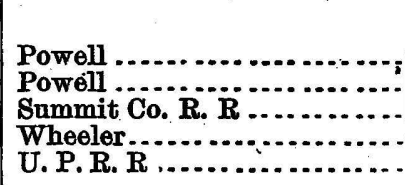 & $\begin{array}{r}\text { Feet. } \\
\mathbf{5 , 6 0 0} \\
\mathbf{9}, 860 \\
\mathbf{5}, 864 \\
\mathbf{3}, 807 \\
6,833\end{array}$ \\
\hline Wasatch Plateau..................... & Powell ..... & 3 to 11,000 \\
\hline $\begin{array}{l}\text { Washi-pah-ghum Spring. } \\
\text { Washington } \\
\text { Do............................. }\end{array}$ & 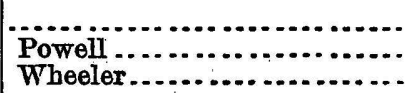 & $\begin{array}{r}4,412 \\
2,720 \\
2,906\end{array}$ \\
\hline Waterpocket Fold................ & Powell ..................... & $\left\{\begin{array}{r}6,000 \\
\text { to } 7,500\end{array}\right.$ \\
\hline 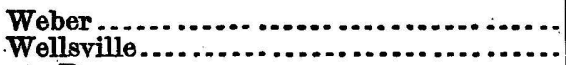 & $\begin{array}{l}\text { U.P. R. R ........ } \\
\text { Hayden }\end{array}$ & \\
\hline 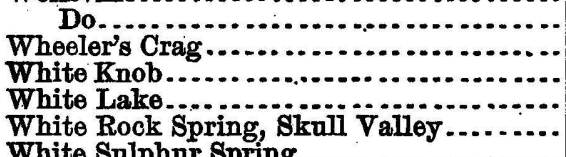 & $\begin{array}{l}\text { Wheeler... } \\
\text { Powell } \\
\text { Powell } \\
\text { Powell } \\
\text { King } . . . . . \\
\end{array}$ & $\begin{array}{l}4,600 \\
7,850 \\
6,800 \\
7,950 \\
5,542\end{array}$ \\
\hline 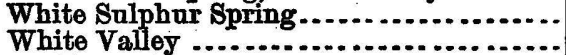 & Simpison & $\begin{array}{l}5,201 \\
4,360\end{array}$ \\
\hline 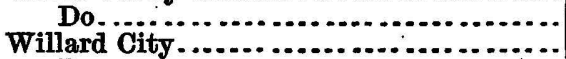 & $\begin{array}{l}\text { Wheeler } \\
\text { U. \& N. R. R ......... }\end{array}$ & $\begin{array}{l}4,436 \\
4,352\end{array}$ \\
\hline Do..................... & 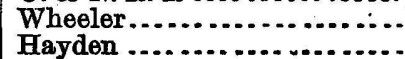 & $\begin{array}{l}4,288 \\
4,350\end{array}$ \\
\hline $\begin{array}{l}\text { Wilson's Peak } \\
\text { Wilson Spring } . . .\end{array}$ & $\begin{array}{l}\text { King } \\
\text { D. \&. G. R. R. }\end{array}$ & $\begin{array}{r}13,235 \\
4,330\end{array}$ \\
\hline Wonsits Valley ............................ & Powell ................... & $\left\{\begin{array}{r}4,700 \\
\text { to } 5,000\end{array}\right.$ \\
\hline 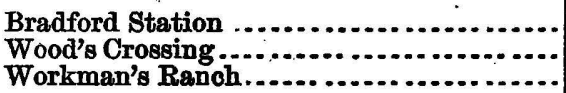 & $\begin{array}{l}\text { Conn. \& Pass. R. R. R... } \\
\text { U. C. R. R . } \\
\text { Powell }\end{array}$ & $\begin{array}{r}410 \\
4,299 \\
4,080\end{array}$ \\
\hline Yampa Platean .. & Powell . & $\left\{\begin{array}{r}6,000 \\
\text { to } 7,000\end{array}\right.$ \\
\hline $\begin{array}{l}\text { York } \\
\text { Do } \\
\text { Young Point } \\
\text { Young's Ranch }\end{array}$ & 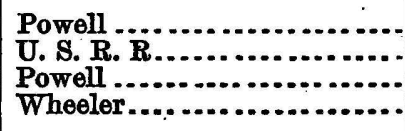 & $\begin{array}{r}4,980 \\
4,957 \\
8,880 \\
5,642\end{array}$ \\
\hline
\end{tabular}




\section{VERMONT.}

\begin{tabular}{|c|c|c|}
\hline Station. & Anthority. & Elevation. \\
\hline & & Feet. \\
\hline Alburgh ........ & Cent. Vt. R. R. & 127 \\
\hline Alburgh Springs & Cent. Vt. R. R. & 119 \\
\hline Anthony, Mount & Geol. Survey of & 2,505 \\
\hline Ascutney, Mount & Geol. Survey of I & 3,320 \\
\hline Bald Mountain .. & Geol. Survey of & 3,124 \\
\hline Barnet.......... & Conn. \& Pass. $R$. & 466 \\
\hline Barre Junction. & M. \& W. R. R. F & 515 \\
\hline Barton & Conn. \& Pass. & 959 \\
\hline $\begin{array}{l}\text { Bartonsville } \\
\text { Bellows Falls }\end{array}$ & Cent. Vt. R. R . & 469 \\
\hline $\begin{array}{r}\text { Bellows Falls ... } \\
\text { Do .......... }\end{array}$ & $\begin{array}{l}\text { Cheshire R. R .. } \\
\text { Cent. Vt. R. R. }\end{array}$ & $\begin{array}{l}305 \\
276\end{array}$ \\
\hline Bethel ....... & Cent. Vt. R. R. & $\begin{array}{l}270 \\
576\end{array}$ \\
\hline Bolton ....... & Cent. Vt. R. R. & 345 \\
\hline Boltonville... & M. \& W. R. R. & 624 \\
\hline Bradford Station. & Conn. \& Pass. R. & 410 \\
\hline Braintree .......... & Cent. Vt. R. R.. & 784 \\
\hline Brandon ...... & Cent. Vt. R. R. & 353 \\
\hline Brattleboro .......... & Vt. Valley R. R. & 228 \\
\hline Brookville ........... & Cent. Vt. R. R. . & 301 \\
\hline Burke, Mount.... & Petermann .... & 3,500 \\
\hline Burlington ........... & Cent. Vt. R. R. & 109 \\
\hline Do..... Signal & U. S. Signal Office. & 268 \\
\hline Cambridge Centre & Port. \& Ogden R. R & 473 \\
\hline Camel's Hump .... & Guyot............ & 4,088 \\
\hline Cavendish ....... & Cent. Vt. R. R. & 921 \\
\hline Charlotte ....... & Cent. Vt. R. R. & 161 \\
\hline Chester ............. & Cent. Vt. R. R. & 501 \\
\hline ................ & Cent. Vt. R. R. & 639 \\
\hline Cole & Cent. Vt. R. R & 326 \\
\hline Connecticut River Bridge, g & Cent. Vt. R. R. & 352 \\
\hline Cuttingsville & Cent. Vt. R. R... & 1,011 \\
\hline Dauville ....... & Port. \& Ogden R. R & 1,375 \\
\hline Derbyline... & Conn. \& Pass. R. R. & $\overrightarrow{1}, 056$ \\
\hline Duwm & Vt. Valley R. R. & 263 \\
\hline East Berkshire.. & Missisquoi R. R.... & 441 \\
\hline East Fairfield .... & Port. \& Ogden R. R. & 421 \\
\hline East Hardwick .. & Port. \& Ogden & 1,087 \\
\hline Hiohoate & Port. \& Ogden & 365 \\
\hline East Montpelier. & M. \& W. R. R. R & 720 \\
\hline East Putney .... & Vt. Valley R. R. & 296 \\
\hline 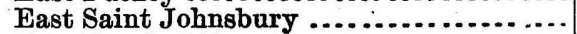 & P. \& O.R. R ... & 795 \\
\hline 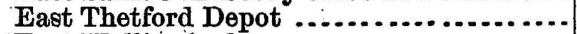 & Conn. \& Pass. $\mathbf{R}$ & 413 \\
\hline East Wallingford ............... & Cent. Vt. R. R.. & 1,195 \\
\hline burg Falls .. & isquoi $\mathbf{R} . \mathbf{R}$. & 436 \\
\hline Mount ... & tt............. & 3,148 \\
\hline x Mountain. & Guyot.............. & 3,872 \\
\hline $\mathrm{E}$ & t. Vt. R. R. .... & 350 \\
\hline Fairfield ......... & Port. \& Ogden R. R ......... & 369 \\
\hline$\ldots \ldots \ldots \ldots$ & n. \& Pass. R. R. R ....... & 438 \\
\hline Fletcher ........ & Port. \& Ogden R. R......... & 431 \\
\hline Gassetts ... & Cent. Vt. R. R . . . . . . . . . & 715 \\
\hline ria ........ & Cent. Vt. R. R .. & 365 \\
\hline eensborough. & Port. \& Ogden R. R......... & 1,166 \\
\hline on . & M. \& W. R.R.R. & 773 \\
\hline on Pond & M. \& W.R.R.R . . . . & 1,094 \\
\hline Hardwick....... & Port. \& Ogden R. R........... & 881 \\
\hline 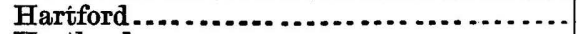 & 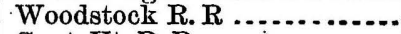 & 485 \\
\hline Hartland .. & Cent. Vt. R. R ....... & 423 \\
\hline
\end{tabular}




\begin{tabular}{|c|c|c|}
\hline Station. & Authority. & Elevation. \\
\hline & & \\
\hline Healdville ..... & Cent. Vt. R. R . & 1,379 \\
\hline Ta & Geol. Survey of & \\
\hline & Guyot.......... & 2.692 \\
\hline Highgate Centre & Port. \& Ogden $\mathrm{R} . \mathrm{R}$ & 312 \\
\hline D:- o & Ashuelot R. R & \\
\hline ogback .... & Guyot.............. & 3,648 \\
\hline Moant & Cent. Vt. R. R . & 1,359 \\
\hline yde Park ... & Port. \& Ogden R & 586 \\
\hline & Grand Trunk R. & 1,181 \\
\hline ond (West I & Port. \& Ogden I & 1,553 \\
\hline a Peak ........... & Geol. Survey of $\nabla$ & $\begin{array}{r}4,018 \\
541\end{array}$ \\
\hline on Peak. & $\begin{array}{l}\text { Port. \& Ogden } \mathbf{R} \\
\text { Guyot............. }\end{array}$ & $\begin{array}{r}541 \\
4,221\end{array}$ \\
\hline Lake & Port. \& Ogde & 101 \\
\hline & M.\&W.R.R. & 1,347 \\
\hline ction & Cent. Vt. R. R & 351 \\
\hline ount .. & Guyot......... & \\
\hline & Cent. Vt. R. R . & 1,061 \\
\hline $\mathrm{I}$ & $\& \mathrm{~Pa}$ & 741 \\
\hline & Conn. \& P & \\
\hline & Conn. \& P & 455 \\
\hline Mount & Gus & 4,430 \\
\hline & M. \& V & 1,140 \\
\hline & Cent. I & 341 \\
\hline & & 541 \\
\hline & Cent. & 361 \\
\hline & M. \& V & 484 \\
\hline unc & Cer & 529 \\
\hline & Port & 657 \\
\hline $\mathrm{N}$ & Cony & 426 \\
\hline & & 291 \\
\hline $\mathbf{N}$ & Cen & 131 \\
\hline & Cen & 739 \\
\hline & & 387 \\
\hline & Mis & 393 \\
\hline & Geo & 915 \\
\hline $\mathrm{N}$ & & 402 \\
\hline & \& Pass. R. R. R & 406 \\
\hline & Guy & 3,954 \\
\hline & Co & 440 \\
\hline & & 327 \\
\hline & M. & 752 \\
\hline & & 3,986 \\
\hline & & 921 \\
\hline $\operatorname{Pr}$ & Gec & 2,690 \\
\hline & & 257 \\
\hline & Wo & 650 \\
\hline & Cen & 698 \\
\hline & & 473 \\
\hline$\ldots$ & & 328 \\
\hline a..... & & 333. \\
\hline & & 120 \\
\hline$\ldots .$. & & 1,016 \\
\hline & & 517 \\
\hline & & 519 . \\
\hline Centre .. & Cent. Vt. R. R . & 490 \\
\hline pot pl & \& Pass. R. R. R & 472 \\
\hline & Cent. Vt. R. R ............ & 390 \\
\hline Johnsbury ........... & Conn. \& Pass. R. R. R.... & 591 \\
\hline & Cent. Vt. R. R & 346 \\
\hline 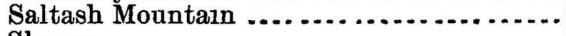 & Geol. Survey of Vt.. & 2,850 \\
\hline 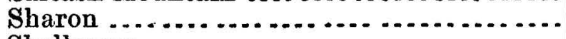 & Cent. Vt. R. R...... & 507 \\
\hline - & Cent. Vt. R. R.............. & 151 \\
\hline & Port. \& Ogden R. R ........ & 374 \\
\hline
\end{tabular}




\begin{tabular}{|c|c|c|}
\hline Station. & Authority. & Elevation. \\
\hline 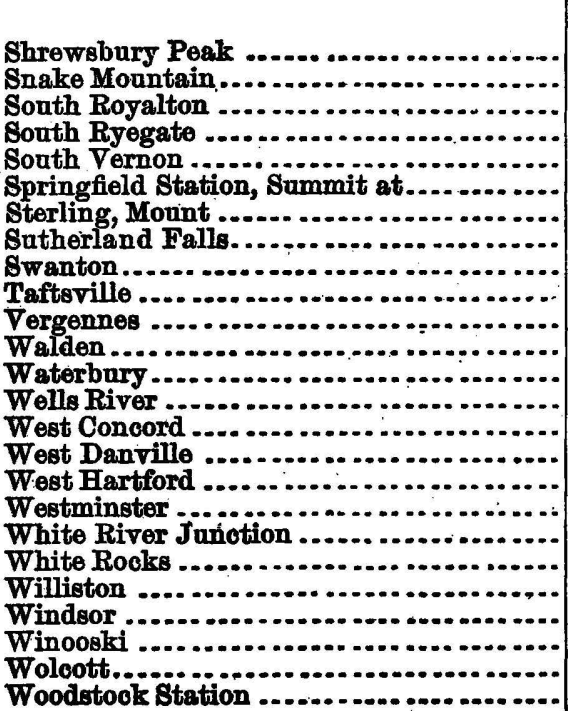 & 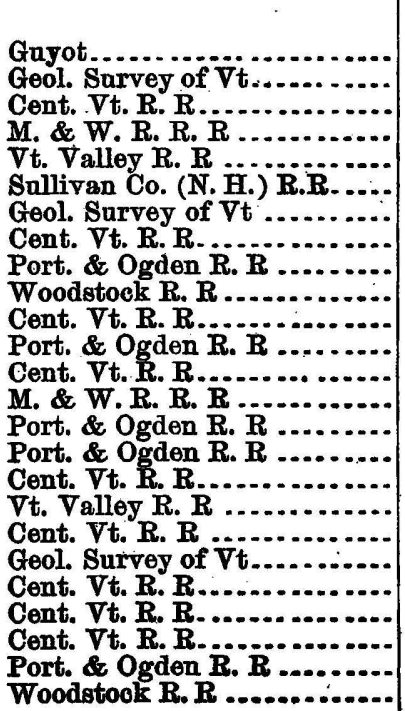 & $\begin{array}{r}\text { Feot. } \\
3,845 \\
\mathbf{1 , 3 1 0} \\
508 \\
724 \\
261 \\
.374 \\
3,700 \\
433 \\
160 \\
657 \\
201 \\
1,673 \\
434 \\
443 \\
851 \\
\mathbf{1 , 5 5 8} \\
\mathbf{4 3 1} \\
\mathbf{2 6 4} \\
\mathbf{3 6 9} \\
\mathbf{2 , 5 3 2} \\
\mathbf{3 1 2} \\
\mathbf{3 3 1} \\
\mathbf{1 9 0} \\
\mathbf{7 0 5} \\
\mathbf{6 9 7}\end{array}$ \\
\hline
\end{tabular}


VIRGINIA.

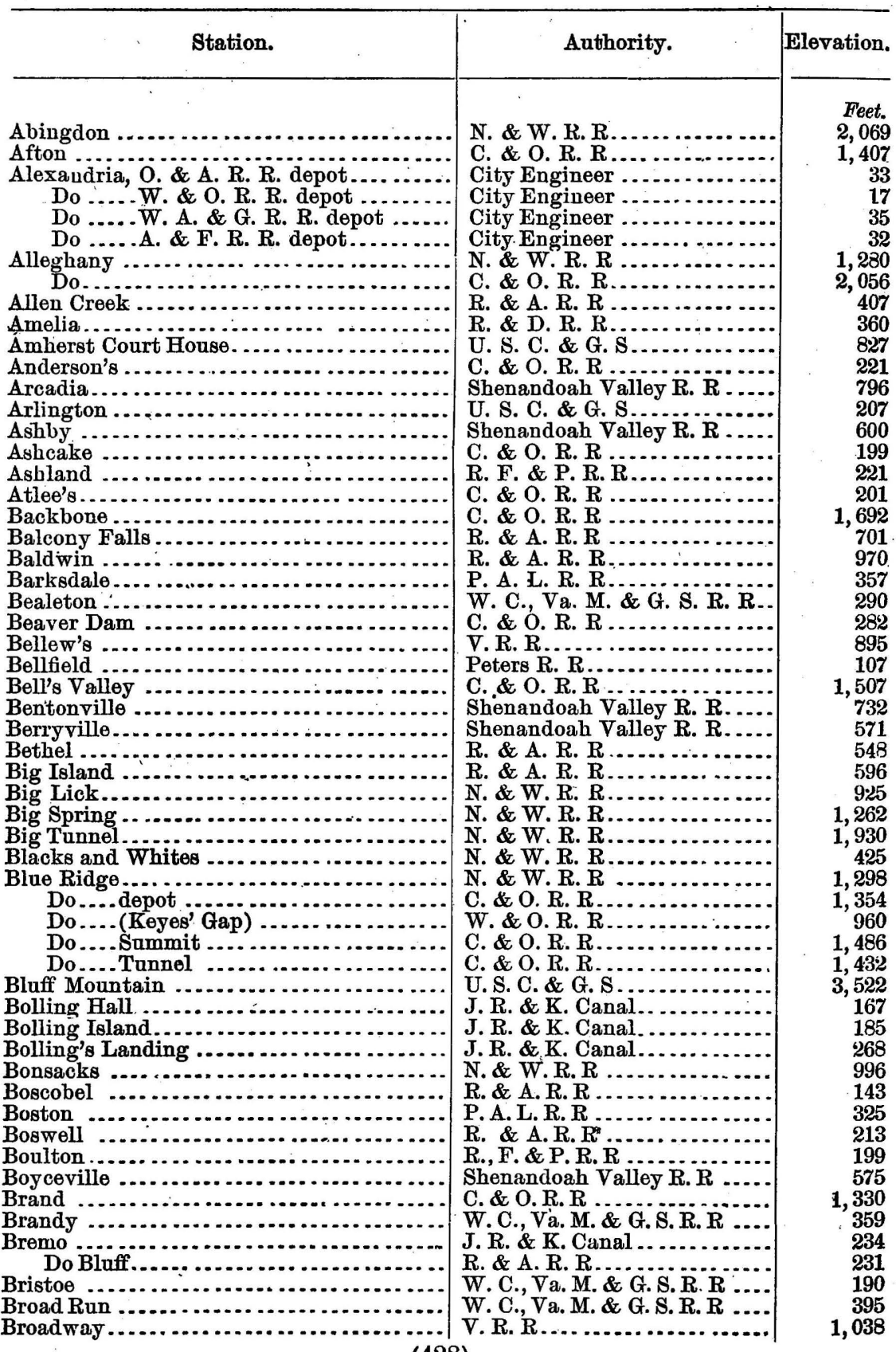




\begin{tabular}{|c|c|c|}
\hline Stati & Authority. & Elevation. \\
\hline & & Feet. \\
\hline Brooke..... & R., F. \& P.R. R.. & \\
\hline rown's... & R. \& A.R.R..... & 291 \\
\hline $\begin{array}{l}\text { Buchanan.. } \\
\text { Buckuer ... }\end{array}$ & Shenandoah Valley $\mathrm{I}$ & 837 \\
\hline $\begin{array}{l}\text { Buckuer ... } \\
\text { Buckton ... }\end{array}$ & W.C. Va.M. \& G. & 328 \\
\hline Buffalo Forg & Shenandoah Valley i & 755 \\
\hline Do.Gap. & C.\& O.R.R ........ & \\
\hline Do.Moun & U. S. C. \& G. & 3,971 \\
\hline Buford's ..... & N. \& W. R. R & 1,014 \\
\hline Bull Mounta & U.S.C.\& G.S & 3,216 \\
\hline Bull Run M & U.S.C.\& G.S & 1,375 \\
\hline Bumpass .. & C.\& O.R. R. & 329 \\
\hline Burke's .... & W. C., Va. M. \& G. S & 258 \\
\hline Burkeville & R.\& D.R.R. & 523 \\
\hline Cady ....... & C. \& O.R.R. & 106 \\
\hline Cahas Moun & U.S.C.\& G.S ... & 3,571 \\
\hline Callaghan & C.\& O.R.R R... & 1,428 \\
\hline & C. \& O.R.R ........ & 356 \\
\hline $\begin{array}{l}\text { Cape } \\
\text { Capo }\end{array}$ & $\begin{array}{l}\text { U. S. Signal Office... } \\
\text { V.R.R ........... }\end{array}$ & $\begin{array}{r}16 \\
701\end{array}$ \\
\hline Catlett & W.C., Va.M.\&G.s.R. & 250 \\
\hline Ceda & R.\& A.R.R . . . . . & 159 \\
\hline Cedarsville & Shenandoah Valley $\mathrm{R}$ & 569 \\
\hline Dan & N. \& W.R.R ......... & 1,785 \\
\hline Charlottesville... & C.\& O.R.R..... & 450 \\
\hline Chatham ....... & W.C., Va.M. \& G & 624 \\
\hline Hill & $\begin{array}{l}\text { B.\& P.R.R . . . . } \\
\text { R.\& P.R.R........ }\end{array}$ & $\begin{array}{r}7 \\
143\end{array}$ \\
\hline $\begin{array}{l}\text { Ches } \\
\text { Chicl }\end{array}$ & $\begin{array}{l}\text { R.\& P.R.R. } \\
\text { C.\& O.R.R. }\end{array}$ & $\begin{array}{r}143 \\
94\end{array}$ \\
\hline Chin & U. S. Signal Office.. & 18 \\
\hline $\mathrm{Ch}$ & N.\&W.R.R .. & 2,012 \\
\hline Chul & P. A. L.R.R. & 280 \\
\hline & N. \& W.R.R & 303 \\
\hline & N.\& W.R.R . & 1,766 \\
\hline Clarl & U.S.C.\& G.S & 1,097 \\
\hline Clar & W.\&O.R.R. & 578 \\
\hline Clay & N.\& W.R.R $\ldots \ldots \ldots$ & 863 \\
\hline Clitto & W.C., Va.M. \& G.S.R.R & 170 \\
\hline Forge & C.\& O.R. R ............. & 1,052 \\
\hline Clov & P.A.L.R.I & 491 \\
\hline Cloy & Shenandoah Valley R. R & 1,125 \\
\hline & P.A. L.R. R . & \\
\hline Cobl & W. C., Va. M. \& G. S.R. R & 401 \\
\hline Coho & R., Y.R.\& C.R.R ...... & \\
\hline Colem & 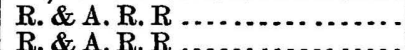 & 578 \\
\hline $\begin{array}{l}\text { Colum } \\
\text { Conco }\end{array}$ & $\begin{array}{l}\text { R. \& A.R.R...... } \\
\text { N.\& W.R.R . }\end{array}$ & $\begin{array}{l}206 \\
833\end{array}$ \\
\hline $\begin{array}{l}\text { Con } \\
\text { Con }\end{array}$ & J.R.\& K. Canal .. & 143 \\
\hline d... & C.\& O.R.R......... & 1,246 \\
\hline on . & C. \& & 1,245 \\
\hline & V.R.R.................... & 1,107 \\
\hline Cow Pasture Bridge. & C. \& O.R.R.............. & 1,135 \\
\hline ille .............. & ...... & 1,515 \\
\hline$\ldots \ldots \ldots \ldots$ & C.\&O.R.R............. & 1,366 \\
\hline & Shenandoah Valley R. R ..... & 1,242 \\
\hline$\ldots$ & $\mathrm{s} R$ & 718 \\
\hline Culpeper & U. S. C. \& G. S............ & $\begin{array}{l}403 \\
534\end{array}$ \\
\hline Do....Court-House & U. S. C. \& G. S............ & 512 \\
\hline anville $\ldots \ldots \ldots \ldots \ldots \ldots$ & W. C., Va. M. \& G. S.R. R.... & 413 \\
\hline & 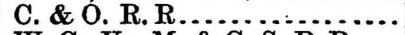 & 324 \\
\hline ans & W. C., Va. M. \& & 455 \\
\hline Disparic . & $\begin{array}{l}\text { R., Y.R. \& C. } \\
\text { N. \& W. R. R.. }\end{array}$ & $\begin{array}{r}67 \\
117\end{array}$ \\
\hline & & \\
\hline
\end{tabular}




\begin{tabular}{|c|c|c|}
\hline & Authori & Elevation. \\
\hline & & \\
\hline $\begin{array}{l}\text { Dover ........ } \\
\text { Drewry Blufi }\end{array}$ & R. \& A. R. R . & \\
\hline $\begin{array}{l}\text { Drewry Bluf } \\
\text { Drummond, I }\end{array}$ & R.\& P.R.R. & 119 \\
\hline $\begin{array}{l}\text { Drummond } \\
\text { Dry Fork. }\end{array}$ & Toner ............. & 21 \\
\hline $\begin{array}{l}\text { Dry Fork } \\
\text { Dublin }\end{array}$ & & $\begin{array}{r}624 \\
266\end{array}$ \\
\hline ublin .... & $\begin{array}{l}\text { N.\&W.R.R. } \\
\text { C. \& O.R.R... }\end{array}$ & $\begin{array}{l}2,066 \\
1,484\end{array}$ \\
\hline $\begin{array}{l}\text { Do..... } \\
\text { agle Rock }\end{array}$ & R.\& \& A. R. R & $\begin{array}{r}1,404 \\
936\end{array}$ \\
\hline St & Shenandoah Val & 946 \\
\hline linburgh & V.R.R R & 845 \\
\hline $\begin{array}{l}\text { Elba } \\
\text { Elizabeth Fu }\end{array}$ & $\begin{array}{l}\text { R., F.\&P. R. R } \\
\text { C.\&O.R.R... }\end{array}$ & $\begin{array}{r}185 \\
1,812\end{array}$ \\
\hline Flk Creek M & J. R. \& K. Cana & 415 \\
\hline & R.\&A.R.R... & 198 \\
\hline Elk Island & R. \& A.R.R.. & 198 \\
\hline$\ddot{*}$ & adoah Val & 958 \\
\hline Elle & $\begin{array}{l}\text { J.R. \& K. Cana } \\
\text { C. \& O.R. R... }\end{array}$ & $\begin{array}{l}143 \\
106\end{array}$ \\
\hline $\mathbf{E l}$ & U. S. C.\& G. S & 4,473 \\
\hline mor & N. \&W.R.R.. & 2,096 \\
\hline & W. C., Va. M. \& & 724 \\
\hline$\ldots$ & W. C.. Va. M. \& & 382 \\
\hline (n.... & $\begin{array}{l}\text { Sheuandoah Va } \\
\text { R., Y. R. \& C. R }\end{array}$ & $\begin{array}{l}522 \\
163\end{array}$ \\
\hline & U.'s. c. \& G.S. & 364 \\
\hline & W.C., Va. M. \& & 535 \\
\hline$\ldots \ldots \ldots \ldots \ldots$ & N. \& W. R.R. & 316 \\
\hline$\cdots \cdot$ & W. \& O.R. R. & 320 \\
\hline Ferrol... & C. \& O.R.R... & 1,810 \\
\hline$\ldots$ & C. \& O.R.R. R & 1,320 \\
\hline n................ & $\begin{array}{l}\text { R., Y.R.\& C.R } \\
\text { N.\&W. R.R... }\end{array}$ & $\begin{array}{r}44 \\
307\end{array}$ \\
\hline $\begin{array}{l}\text { Ford's } \\
\text { Forest }\end{array}$ & $\begin{array}{l}\text { N. \& W.K.K... } \\
\text { N.\&W.R.R. }\end{array}$ & $\begin{array}{l}307 \\
877\end{array}$ \\
\hline & V.R.R ... & 953 \\
\hline Fork Mor & . \& G. S & 3,851 \\
\hline & V.R.R ....... & 1,247 \\
\hline & B. & 27 \\
\hline$\cdots \cdot \ldots$ & R. R . . . & 50 \\
\hline ....... $>$ > & P. R. R & 234 \\
\hline tall & R., F.\& P.R.R. & \\
\hline $\begin{array}{l}\text { Frede } \\
\text { Fron }\end{array}$ & C.\& O.R.R & $\begin{array}{l}347 \\
495\end{array}$ \\
\hline $\begin{array}{r}\text { Front } \\
\text { D }\end{array}$ & S.R.R. & $\begin{array}{l}495 \\
546\end{array}$ \\
\hline & $\begin{array}{l}\text { W.C., Va.M. \& G. S.R.R. } \\
\text { W.C., Va.M. \& G.S.R.R.. }\end{array}$ & $\begin{array}{l}546 \\
357\end{array}$ \\
\hline & 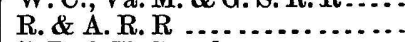 & $\begin{array}{l}357 \\
936\end{array}$ \\
\hline & J.R. & 208 \\
\hline & J.I & 463 \\
\hline & A. $R . R$. & 455 \\
\hline & O.R.R. & 111 \\
\hline & P.\& R. . & 145 \\
\hline G & & $\begin{array}{r}922 \\
2.088\end{array}$ \\
\hline 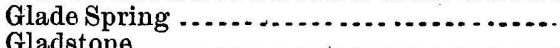 & A., & $\begin{array}{r}2,088 \\
399\end{array}$ \\
\hline (n............ & A.R.R R. R R & $\begin{array}{l}399 \\
855\end{array}$ \\
\hline (1) & $\mathrm{R} . \mathrm{R}$ & 1,522 \\
\hline Gle & & 715 \\
\hline & aandoah Valley R.R ... & 760 \\
\hline Gordonsville .... & 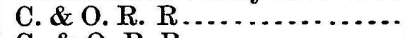 & 495 \\
\hline & & 1,410 \\
\hline & C. \& O. R.R.. & $\begin{array}{l}1,410 \\
589\end{array}$ \\
\hline $\begin{array}{l}\text { Greenba } \\
\text { Green S }\end{array}$ & $\begin{array}{l}\text { P., A. L.R.R } \\
\text { C.\&O.R.R }\end{array}$ & $\begin{array}{l}589 \\
529\end{array}$ \\
\hline $\begin{array}{l}\text { Green Sprngs... } \\
\text { Greenville ..... }\end{array}$ & doah Valley R. R... & 1,550 \\
\hline 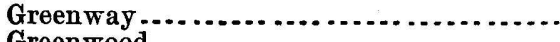 & R. \&A.R.R R & 383 \\
\hline & & 1,068 \\
\hline
\end{tabular}




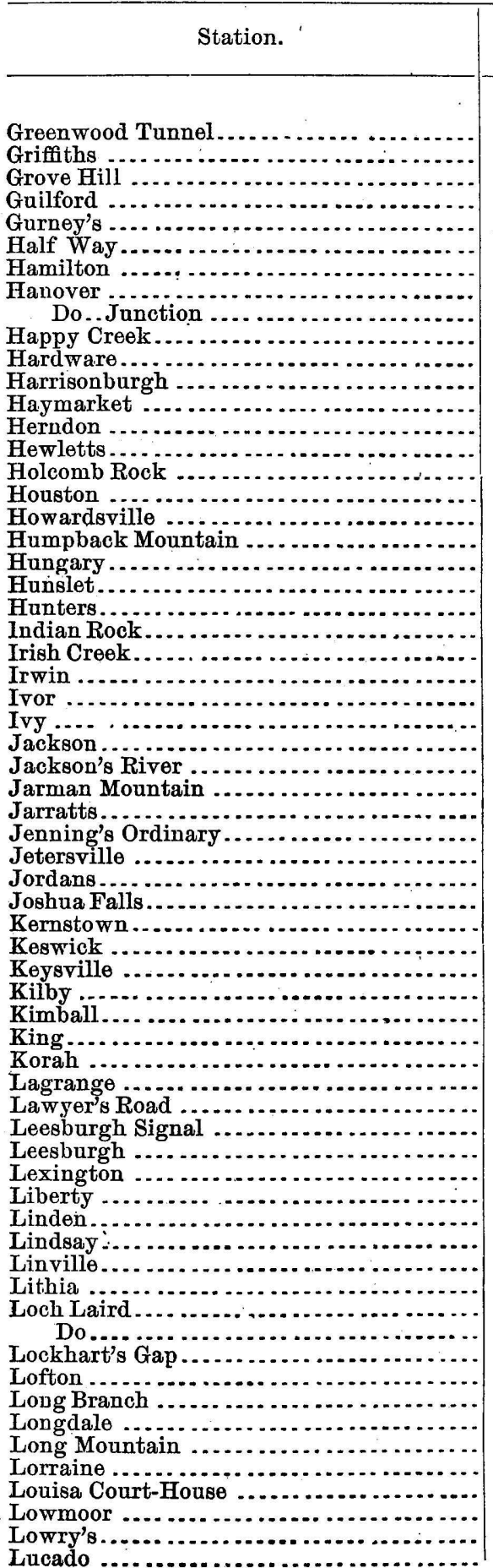

C. \& O. R. R

C. \& O.R. R . ................ Shenandoah Valley $\mathbf{R} . \mathbf{R}$.... W. \& O. R. R . R., F. \& P. R. R .......... R. \& P: R. R. W. \& O.R. R C. \& O.R. R. C. \& O. R. R. W. C., Va. M. \& G. S. R. R... R. \& A. R. R V. R. R. W. C., Va. M. \& G. S. R. R. W. \& O.R. C. \& O.R.R R. \& A. R. R Shenandoah Valley R. R R. \& A. R. R. U. S. C. \& G. S R., F. \& P. R. R C. \& O.R. R W.\& O.R.R R. \& A. R. R Shenandoah Valley R. R R. \& A. R. R. N. \& W. R. R C. \& O. R. R R. \& A. R. R. C. \& O.R. R. U. S. C. \& G. S Peters. R. R P. A. L. R. R F., O. \& C. R. R.................. R. \& A. R. R. . . . . . . V.R. R.....

W. C., Va. M. \& G. S. R. R... P. A. L. R. R

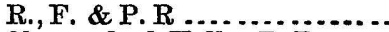
Shenandoah Valley $\mathbf{R} . \mathbf{R}$

C. \& O. R. R

R. \& A. R. R

C. \& O. R. R W. C., Va. M. \& G. S. R. R... U. S. C. \& G. S..............

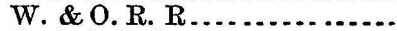

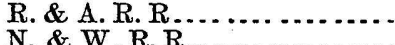

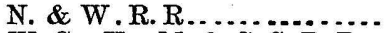
W. C., Va. M. \& G. S. R. R.. W. C., Va. M. \& G. S. R. R...

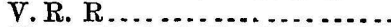
Shenandoah Valley R. R..... R. \& A. R. R................ Shenandoah Valley $\mathbf{R} . \mathbf{R}$.... W. \& O. R. R...................... B. \& P. R. R C. \& O. R. R U. S. C.\& G. S R. \& A. R. R C. \& O.R. R

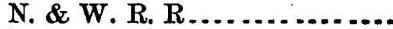
W. C.; Va. M. \& G. S. R. R ...
Elevation.

Feet.

1,072

1,165

966

415

121

114

454

82

134

790

266

1, 338

337

395

278

562

1,348

315

3,645

214

100

345

780

1,010

159

87

545

845

1,136

3,161

154

498

446

455

455

744

436

625

212

895

1, 511

106

1,618

739

683

321

958

959

916

477

1,242

968

- 784

800

896

1,785

82

1,166

1,436

142

452

1,156

791

833 


\begin{tabular}{|c|c|c|}
\hline Sta & Authority. & Elevation. \\
\hline & & Feet. \\
\hline uray .......... & $\begin{array}{l}\text { Shenandoah Valley R. R } \\
\text { W. C. Va, M. \& G.S.R. I }\end{array}$ & $\begin{array}{l}822 \\
529\end{array}$ \\
\hline Do......... & U. S. C.\&G. S... & 796 \\
\hline Do...... Sigr & U. S. Signal Office & 652 \\
\hline ynch's........ & W.C., Va. M. \& & 730 \\
\hline yndhurst .. & Shenandoah Va & 1,340 \\
\hline Madison ........ & W.C., Va. M. \& & 395 \\
\hline Maiden Adventur & R.\&A.R.R... & 143 \\
\hline Manakin .......... & R.\& A.R.R.. & 141 \\
\hline assas Junc & W. C., Va. M. \& & 317 \\
\hline ester .... & P.A.L.R.R. & 38 \\
\hline Manchester Cros & R. \& P.R.R. & 102 \\
\hline Manor .......... & Shenandoah $\mathrm{Va}$ & 497 \\
\hline $0 .$. & R.\&A.R.R.. & 324 \\
\hline (.......... & N. \& W.R.R. & 2,136 \\
\hline 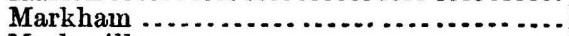 & W. C., Va. M.s & 552 \\
\hline ille.. & Shenandoah Va & 1,066 \\
\hline & Shenandoah Va & 1,165 \\
\hline 11 Moun & U.S.C.\& G.S & 3,374 \\
\hline s ...... & N.\&W.R.R. & 1,919 \\
\hline & F., O.\& C.R. I & 485 \\
\hline Mason's Tunnel & C.\& O.R. R.. & 1,592 \\
\hline Do......... & C. \& O.R.R. & 1,551 \\
\hline Mattoaca... & R. 8 & 375 \\
\hline x ........... & P. A. L. R. R. & 223 \\
\hline town .... & $\mathrm{V} . \mathrm{R} . \mathrm{R} \ldots \ldots$ & 788 \\
\hline adows.. & N. \& & 2,028 \\
\hline .......... & R., Y.R. \& C. $\mathrm{B}$ & \\
\hline l's Rive & C. \& O.R.R.. & 550 \\
\hline in ........ & P. A. L.R.R. & 589 \\
\hline & C. \& O.R. R... & 519 \\
\hline 's Ferry. & J. R. & 143 \\
\hline ton Mills. & R.\& A. R. R .. & 231 \\
\hline Do........... & J. R. \& K. Can & 234 \\
\hline fown. & V. R. R ............. & 660 \\
\hline Midland ....... & W. C., Va. M. \& G., S.R. R.. & 321 \\
\hline Do...Juncti & R. \& A. R. R . & 504 \\
\hline Mills & A. R. R. & 340 \\
\hline Millboro'.... & C. \& O.R.R. & 1,679 \\
\hline Do .. Bridge & C. \& O.R.R ... & 1,642 \\
\hline Milfo & R., F. \& P. R. R. & 100 \\
\hline & R. \& A. R. R.. & 725 \\
\hline 1 is & W. C., Va. M. \& G. S. R. R & 350 \\
\hline Ford .... & 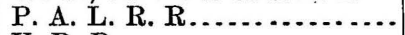 & 360 \\
\hline ford. & V. $\mathrm{R}$ & 1,171 \\
\hline & $R$ & 916 \\
\hline & P.R.R.. & \\
\hline & V. $\mathrm{R}$ & 1,258 \\
\hline 's Hill & U. S. C.\& G. S . & 383 \\
\hline & N. \& W.R.R... & 1,546 \\
\hline Bridge... & A. R. R.. & 736 \\
\hline & B. 8 & 30 \\
\hline & J. R.\& K. Canal. .. & 223 \\
\hline New Market ........ & R.\& A.R. R..... & 350 \\
\hline & & 971 \\
\hline River Depot... & N.\& W.R.R ................... & 1,757 \\
\hline Ferry .......... & 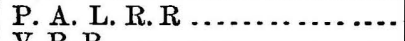 & 340 \\
\hline wn $\ldots \ldots \ldots$ & V.R.R $\ldots \ldots \ldots$ & 731 \\
\hline $\begin{array}{l}\text { Noel's } \\
\text { Nokesville } \ldots \ldots \ldots \ldots \ldots \ldots\end{array}$ & 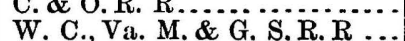 & $\begin{array}{l}254 \\
270\end{array}$ \\
\hline Norfolk, Signal Station....... & U. S. Signal Office........... & 30 \\
\hline North Anna $\ldots \ldots \ldots \ldots$ & 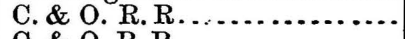 & 244 \\
\hline $\begin{array}{l}\text { North Mountain ....... } \\
\text { North Mountain Sumn }\end{array}$ & C. \& O.R.R...... & 2,064 \\
\hline & & \\
\hline
\end{tabular}




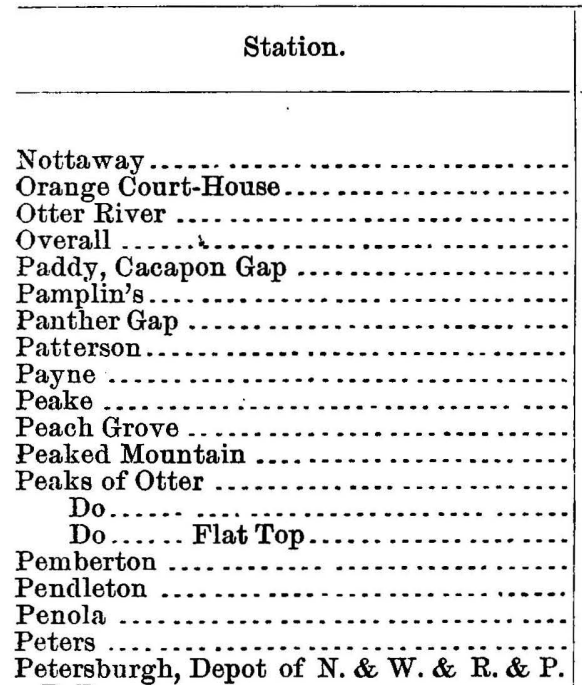

R. $\mathbf{R} \ldots \ldots \ldots \ldots \ldots \ldots \ldots \ldots \ldots \ldots \ldots \ldots . \ldots \ldots$ Do ..... Depot of P. R. R

Peters Mountain

Plains

Pleasant Hill .

Pleasant Valley

Pocahontas Do

Pond Gap

Port Republic

Port Walthall

Potomac Run

Powhatan

Prospect

Purcellville.

Quantice

Ragged Mountain

Rapid Anne.

Rappahannock

Ream's

Rein's Island, River Lock

Reitsville

Rice's

Richmond, depot of $\mathbf{R} ., \mathrm{F} . \&$ P. R. R

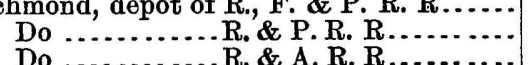

R. \& A. R. R

Do ............R. \& D. R. R........

Do ............ R., Y. R. \& C. R. R

Do .............. \& O. R. $\mathrm{R}$

Rileysville

Ripplemead.

River

Riverside

Riverton.

Riverville

Roanoke...

Rock Castle Do

Rolling Mills

Romancoke

Rope Ferry

\begin{tabular}{|l|l} 
Authority. & Elevation \\
\hline
\end{tabular}

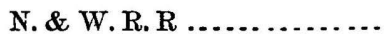

Feet.

W. C. Va M \& G.S.R R

W. C., Va. M. \& G. S. R. R....

Shenandoah Valley R. R ..... 662

W. \& O.R. R . . . . . ......... $\quad 2,009$

N. \& W.R. R, ............ 678

C. \& O. R. R .............. 1,594

Shenandoah Valley R. R..... $\quad 1,135$

R. \& A. R. R ............. 266

C. \& O. R. R ............... 193

U. S. C. \& G. S............ 522

U. S. C. \& G. S............ $\quad 2,929$

Guyot...................... $\quad 3,993$

U. S. C. \& G. S............ 4, 001

U. S. C. \& G. S............ $\quad 3,875$

R. \& A. R. R ............... 190

C. \& O. R. R ............. 472

R., F. \& P. R. R......... 94

C. \& O. R. R ............ 1,175

City Engineer

9

City Engineer.

70

U. S. C. \& G.S

W. C., Va. M: \& G,,$\vec{S} . \mathbf{R} . \mathbf{R}$

Peters. R. R.

V. R. R

R. \& P. R. R

N. \& W. R. R

C. \& O.R. R.

Shenandoah Valley R. $\mathrm{R}$

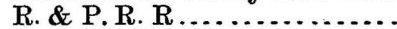

R., F. \& P. R. R . . . . . .

P. A. L. R. R.

N. \& W. R. R

W. \& O. R. R

B. \& P. R. R

...........

W. C., Va. M. \& G. S. R. R.

W. C., Va. M. \& G. S. R. R.

Peters R. R.

W. C., Va. M. \& G. S. R. R

J. R.\& K. Canal ............

R. \& D. R. R.

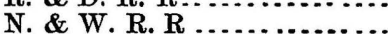

R., F. \& P. R. R

City Engineer

$\ldots . . . . .$.

City Engineer

City Engineer

City Engineer

City Engineer

Shenandoah Valley R. R....

N. \& W. R. R .

W. C., Va. M. \& G. S. R. R

Shenandoah Valley R. R

Shenandoah Valley R. R

R. \& A. R. R .

Shenandoah Valley $\mathbf{R} . \mathbf{R}$

J. R. \& K. Canal .

R. \& A. R. R .

R. \& A. R. R ..............

R., Y. R. \& C. R. R

R. \& A. R. R

W. \& O. R. R
1,824

565

119

1,248

17

2,312

1,682

1,096

87

320

575

553

16

3,298

306

275

162

444

115

831

396

10

181

84

83

28

18

28

726

1,607

493

938

497

423

907

177

175 


\begin{tabular}{|c|c|c|}
\hline Station. & Authority. & Elevation. \\
\hline & & \\
\hline Rural Retrea & A., M. \& O.R. F & 2,515 \\
\hline $\begin{array}{l}\text { Rutherglen . } \\
\text { Sabbot Hill.: }\end{array}$ & $\begin{array}{l}\text { R., F. \& P. R. R } \\
\text { J. R. \& K. Canal. }\end{array}$ & $\begin{array}{l}203 \\
143\end{array}$ \\
\hline St. Asaph Ju & B. \& P. R. R ... & $\begin{array}{r}140 \\
45\end{array}$ \\
\hline Salem ..... & W. C. Va. M. \& G. S. R. R... & 633 \\
\hline Salisbury ..: & R. \& A. R. R & 894 \\
\hline $\begin{array}{l}\text { Saltpeter Ca } \\
\text { Saltville }\end{array}$ & $\begin{array}{l}\text { R. \& A. R.R.... } \\
\text { N. \& W. R. R... }\end{array}$ & $\begin{array}{r}892 \\
1.724\end{array}$ \\
\hline Scottsburg.. & P. A. L. R. R... & 342 \\
\hline Scottsville ... & R. \&A. R. R. & 275 \\
\hline Seven Mile Fo & N. \& W.R.R. & 1,988 \\
\hline Shadwell & W. C., Va. M. \& G. S. & 303 \\
\hline $\begin{array}{l}\text { Shenandoah Iron } \mathrm{Y} \\
\text { Do .....R. (Sni }\end{array}$ & $\begin{array}{l}\text { Shenandoah Valley R. } \\
\text { W. \& O. R. R ........ }\end{array}$ & $\begin{array}{l}940 \\
360\end{array}$ \\
\hline Sherando ........ & Shenandoah Val & 1,380 \\
\hline Smith Lock .. & R. \& A.R. R... & 516 \\
\hline Smith Mounts & U. S. C. \& G. \& & 2,043 \\
\hline hickersville. & W. \& O.R.R & 680 \\
\hline South Anna De & C. \& O.R.R. & 70 \\
\hline South Branch & W.\& O.R.R. & 2,148 \\
\hline South River. & R.\&A.R.R . & 850 \\
\hline Spear Mounta & U. S. C. \& G. S . & 1,616 \\
\hline Spout Spring. . & N.\& W.R.R & 848 \\
\hline Springfield & W. C., Va. M. \& G & 240 \\
\hline $\begin{array}{l}\text { Staples Mills... } \\
\text { Stapleton ...... }\end{array}$ & $\begin{array}{l}\text { J.R. \& K. Canal. } \\
\text { R.\& A. R. R ..... }\end{array}$ & $\begin{array}{l}455 \\
447\end{array}$ \\
\hline Staunton ....... & C.\& O.R.R.... & 1,387 \\
\hline Do $\ldots . .$. . & V.R.R...... & 1,379 \\
\hline Staytide. & N.\&W.R.R. & 1,640 \\
\hline Steel $\ldots \ldots \ldots \ldots$ & C. \& O.R.R ... & 1,209 \\
\hline Stephenson's ....... & 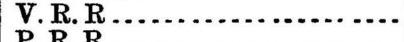 & 499 \\
\hline $\begin{array}{l}\text { Stony Creek - } \\
\text { Strasburg }\end{array}$ & P.R.R R & $\begin{array}{r}74 \\
637\end{array}$ \\
\hline $\begin{array}{l}\text { Strasburg } \ldots . . . . \\
\text { Do }\end{array}$ & $\begin{array}{l}\text { W. C., Va. M. \& G. S.R.R R } \\
\text { W. C. Va. M. \& G.S.R.R }\end{array}$ & 637 \\
\hline $\begin{array}{r}\text { Do ...Junction } \\
\text { Stuart Draft ..... }\end{array}$ & $\begin{array}{l}\text { W. C., Va. M. \& G. S. R. R } \\
\text { Shenandoah Valley R: R. }\end{array}$ & $\begin{array}{r}694 \\
1.388\end{array}$ \\
\hline & $\begin{array}{l}\text { Shenandoah Valley R: } R \text {. } \\
\text { N.\& W. R. R ............ }\end{array}$ & $\begin{array}{r}1,388 \\
58\end{array}$ \\
\hline $\begin{array}{l}\text { Suffolk } \\
\text { Sugarloaf Mounta }\end{array}$ & U.s.c.\&G.S... & $\begin{array}{r}58 \\
2,292\end{array}$ \\
\hline $\begin{array}{l}\text { Sugarloaf Mounta } \\
\text { Sweet Hall ....... }\end{array}$ & 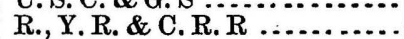 & 2,290 \\
\hline & C.\& O.R.R & 1,650 \\
\hline Sycamore. & W. C., Va. M. \& G. S.R. R. & 733 \\
\hline & C. \&O.R.R. R & 160 \\
\hline rsville... & R., F.\& P.R.R. & 119 \\
\hline raph Ro & B. \& P.R.R... & 82 \\
\hline Temple & & \\
\hline Thax & V.R.R... & 962 \\
\hline Thompson & Shenandoah Valle & 790 \\
\hline & W. C., Va. M. \&\& G. S. R. R. & 399 \\
\hline Timberville .. & V.R.R... & 1,018 \\
\hline Tinker Creek & Shenandoah Valle & 961 \\
\hline Row Moun & U. S. C. \& G. S... & 2,938 \\
\hline Toler & C. \& O.R.R.... & 463 \\
\hline hawk ......... & P. A. L. R. R .. & 257 \\
\hline Tonis Brook ........ & V.R.R............ & 745 \\
\hline Trevilian ..... & C.\& O.R.R.... & 523 \\
\hline Trice & O.R.R..... & 1,816 \\
\hline dale.... & andoah Valley R. R. & 1,400 \\
\hline Tuckahoo ....... & J. R. \& K. Canal_........... & 143 \\
\hline Tunstalls ......................................... & R., Y.R.\& C. R. R......... & \\
\hline nvillo & C. \& O.R.R.R. R & 1,420 \\
\hline prings & 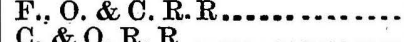 & $\begin{array}{r}500 \\
1905\end{array}$ \\
\hline $\begin{array}{l}\text { Variety Springs .............. } \\
\text { Verdiersville.............. }\end{array}$ & $\begin{array}{l}\text { C. \&O.R.R } . . . \\
\text { F. O.\&C.R.R. }\end{array}$ & $\begin{array}{r}1,905 \\
514\end{array}$ \\
\hline $\begin{array}{l}\text { Verdiorsville } \\
\text { Verdon }\end{array}$ & C. \& O.R. R ................ & 220 \\
\hline & & , 272 \\
\hline
\end{tabular}




\begin{tabular}{|c|c|c|}
\hline Station. & Authority. & Elevation. \\
\hline & & \\
\hline Vesuvius ........ & Shenandoeh Valley R. R & 1,420 \\
\hline 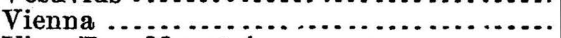 & W. \& O.R.R ........... & 395 \\
\hline View 'Tree Mountain..... & U.S.C.\& G. S & 1,087 \\
\hline Vinita & R. \& A. R. R. & 142 \\
\hline Virgin's Mills ........... & J.R. \& K. C. R.R. & 251 \\
\hline Wadesville ..... & V.R.R & 495 \\
\hline Wakefield .... & N. \& W. R. R & 100 \\
\hline Walker Ford:.......... & R. \& A. R. R. & 431 \\
\hline 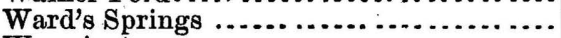 & W. C., Va. M. \& & 797 \\
\hline Warminster..$\ldots \ldots \ldots$ & R. \& A. R. R. & 332 \\
\hline Warren $\ldots . . . \ldots \ldots \ldots \ldots$ & R. \& A. R. R. & 299 \\
\hline Warrenton Junction ................... & W. C., Va. M. \& G. S. R. R. & 265 \\
\hline Water Lick .............. & W. C., Va. M. \& G. S. R.R. & 550 \\
\hline Waterloo & B. \& P. R. R............... & 50 \\
\hline Waverly ......... & N. \& W. R. R & 114 \\
\hline 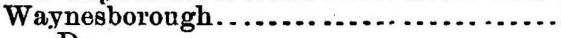 & C. \& O.R.R........ & 1,302 \\
\hline Do ........... & Shenandoah Valley $\mathbf{R}$ & 1,298 \\
\hline Weldon ........... & Peters. R. R ... & 105 \\
\hline Wellville ........ & N.\& W. R. R. & 420 \\
\hline . & N. \& W. R. R. & 1.559 \\
\hline Westham ... & R. \& A.R.R . & 116 \\
\hline .................... & R., Y.R.\& C. R.R. & \\
\hline Westview ....... & R. \& A. R. R....... & 183 \\
\hline Weyers Cave ... & Shenandoah Valley $\mathrm{R}$ & 1,123 \\
\hline White House..... & R., Y. R. \& C.R. R .. & 18 \\
\hline White Post ....... & Shenandoah Valley R. R.... & 610 \\
\hline White Top....... & 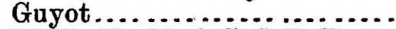 & 5,530 \\
\hline Whittle's ........ & W. C., Va. M. \& G. S. R. R ... & 812 \\
\hline Wickham ........ & C. \& O. R. R. & 75 \\
\hline Williamsons ...... & C. \& O.R. R.. & 1,053 \\
\hline 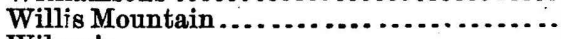 & U. S. C. \& G. S. & $\mathbf{1}, \mathbf{1 5 9}$ \\
\hline Wilson's $\ldots \ldots \ldots \ldots \ldots \ldots \ldots \ldots \ldots \ldots$ & N. \& W.R.R. . & 367 \\
\hline Wilton..$\ldots \ldots \ldots \ldots \ldots \ldots \ldots \ldots$ & R. \& A. R. R & 996 \\
\hline Winchester $\ldots \ldots \ldots \ldots \ldots \ldots$ & V.R.R...... & 717 \\
\hline$\ldots \ldots \ldots \ldots \ldots \ldots$ & N. \& W. R.R. . & 84 \\
\hline Wingina ...... & R. \& A. R. R ... & 350 \\
\hline Woel ........... & C. \& O. R. R... & 257 \\
\hline Wolf Trap .... & P.A.C. R.R. & 349 \\
\hline Wood Bridge . & B. \& P. R. R . . . . . & \\
\hline 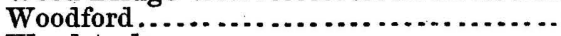 & R., F.\& P. R. R.......... & 125 \\
\hline Woodstock . . . . & 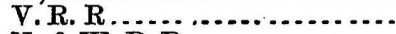 & 820 \\
\hline 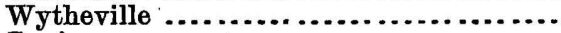 & N. \& W. R.R . . . . . . . . . & 2,242 \\
\hline 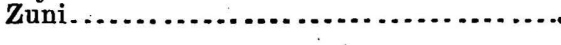 & & \\
\hline
\end{tabular}


WASHINGTON TERRITORY.

\begin{tabular}{|c|c|c|}
\hline $\mathbf{S}$ & Authority. & Elevation. \\
\hline & & \\
\hline $\begin{array}{l}\text { Aiks, Mount..... } \\
\text { Ainsworth ..... }\end{array}$ & N. Trans. Survey.. & 7,623 \\
\hline $\begin{array}{l}\text { Ainsworth ....... } \\
\text { Baker, Mount .. }\end{array}$ & N.S. C.\&G.S... & $\begin{array}{r}351 \\
10,827\end{array}$ \\
\hline & Petermann & $\begin{array}{l}10,027 \\
11,100\end{array}$ \\
\hline Bellingham, For & Med. Dept. U. S. A & 88 \\
\hline dy's Pass ..... & N.P. R. R. Surveys & 6,147 \\
\hline lispell Peak . & N. Trans. Survey... & 6,905 \\
\hline $\begin{array}{l}\text { ispelur Lake } \\
\text { tonment Ster }\end{array}$ & N. Trans. Survey .. & 2,110 \\
\hline ment $\mathrm{s}$ & P. R. R. Rep & $\begin{array}{l}3,412 \\
6,080\end{array}$ \\
\hline $\begin{array}{l}\text { Cariton, Mour } \\
\text { Castle Rock.. }\end{array}$ & $\begin{array}{l}\text { N. Trans. Sur } \\
\text { N.P.R.R... }\end{array}$ & $\begin{array}{r}0,080 \\
83\end{array}$ \\
\hline eville .. & N.P.R.R. & 207 \\
\hline Chehalis........ & 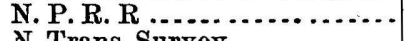 & 204 \\
\hline Chewelah Peak & N. Trans. Survey .. & 5,748 \\
\hline Colfax $\ldots \ldots \ldots$ & Oreg. R. R . . . . . & $\begin{array}{l}1,941 \\
1,917\end{array}$ \\
\hline $\begin{array}{l}\text { Colville } \ldots . . . . . \\
\text { Do.Fort .... }\end{array}$ & $\begin{array}{l}\text { N. Trans. Survey .. } \\
\text { P. R. R. Reports .. }\end{array}$ & $\begin{array}{l}1,917 \\
1,215\end{array}$ \\
\hline Do...do .... & Smithsonian Inst. & 1,963 \\
\hline Do.Mount .... & N. Trans. Survey & 5,667 \\
\hline Constance, Mount . & Petermann & $\begin{array}{l}7,777 \\
4,210\end{array}$ \\
\hline & N.P. R. R. Sv & $\begin{array}{l}4,210 \\
1,360\end{array}$ \\
\hline $\begin{array}{l}\text { Dayton .............. } \\
\text { Ellensburg....... }\end{array}$ & $\begin{array}{l}\text { Oreg. R.R ........ } \\
\text { N.P.R.R ....... }\end{array}$ & $\begin{array}{l}1,360 \\
1,510\end{array}$ \\
\hline Elorka 1 & N. Trans. Survey.. & 1,831 \\
\hline Eltopia $\ldots . . . \ldots \ldots$ & N. P. R. R ......... & 600 \\
\hline Grange City ........ & Oreg. R. R........ & $\begin{array}{l}100 \\
502\end{array}$ \\
\hline Great Plain of the C & 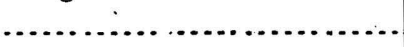 & \\
\hline Harriston .. & N. P.R.R ... & 1,950 \\
\hline & Williamson......... & 11,225 \\
\hline $\begin{array}{l}\text { Horseshoe Bend. } \\
\text { Ike's Mountain . }\end{array}$ & N.P.R.R R........ & \\
\hline Intes & Pacific R. R. Reports. & $\begin{array}{l}5,100 \\
2,750\end{array}$ \\
\hline , Mount . & Humboldt.. & 15,500 \\
\hline $\begin{array}{l}\text { Junipe } \\
\text { Kachis }\end{array}$ & N.P.R.R R ........ & 318 \\
\hline $\begin{array}{l}\text { Kachiss, Lak } \\
\text { Kalama ...... }\end{array}$ & $\begin{array}{l}\text { Pacific R. R. Reports. } \\
\text { N.P. R. R . . }\end{array}$ & $\begin{array}{r}2,158 \\
33\end{array}$ \\
\hline & Toner ................ & \\
\hline $\begin{array}{l}\text { Kechelus, Lake } \\
\text { Kuthas, Lake }\end{array}$ & $\ldots \ldots \ldots \ldots$ & 2,388 \\
\hline $\begin{array}{l}\text { Kuthas } \\
\text { Lake V }\end{array}$ & N.P.R.R & 1,953 \\
\hline $\begin{array}{l}\text { Lake } \mathrm{Vi} \\
\text { Lind... }\end{array}$ & $\begin{array}{l}\text { N.P.R.R } \\
\text { N.P.R.R.......... }\end{array}$ & $\begin{array}{r}324 \\
1,363\end{array}$ \\
\hline & N.P.R.R R............ & 2,134 \\
\hline Pass ...... & ns. Survey ........... & 3,248 \\
\hline & N: Trans. Survey ........... & 2,034 \\
\hline Moscow ............. & Oreg. R. R......................... & 2,536 \\
\hline 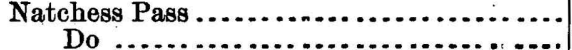 & $\begin{array}{l}\text { N. Trans. Servey ................ } \\
\text { N. Trans. Survey }\end{array}$ & $\begin{array}{l}4,900 \\
4,900\end{array}$ \\
\hline 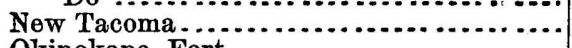 & N.P.R.R & 31 \\
\hline $\begin{array}{l}\text { Okinokane, Fort .... } \\
\text { Do .... Lake... }\end{array}$ & Pacific R. R. Reports & 779 \\
\hline Signal St & U. S. Signal Office........... & $\begin{array}{l}1,105 \\
36\end{array}$ \\
\hline & Wilkes............................. & 8,138 \\
\hline Palouse Junction & N.P.R.R $\mathbf{R} \ldots \ldots$ & \\
\hline $\begin{array}{l}\text { Puyallup } \\
\text { Pioket, Camp } . . .\end{array}$ & 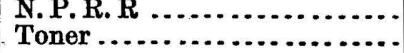 & $\begin{array}{r}07 \\
150\end{array}$ \\
\hline & & 1,811 \\
\hline
\end{tabular}




\begin{tabular}{|c|c|c|}
\hline Station. & Authority. & Elevation. \\
\hline mo $:=$ & & Feet. \\
\hline Pluvin's Point... . & N.P.R.R & 950 \\
\hline Pomeros Prairie. & N.P.R.R & 1,806 \\
\hline Ranïer, Mount (Tac & U. S. C. \& & 14,444 \\
\hline Ritzville .......... & N.P.R.R. & 1,825 \\
\hline Ratcheen Lake... & 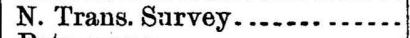 & 2,214 \\
\hline Saint Helena, Mour & Petermann $\ldots \ldots \ldots \ldots$ & 9,750 \\
\hline Sheets Pass ........ & urvey ............ & 3,606 \\
\hline$\ldots \ldots \ldots$ & . Reports....... . & 3,327 \\
\hline Sister Lake ....... & N. Trans. Survey . . ............. & 2,449 \\
\hline Skalahum, Mount & Petermann ................... & 7,400 \\
\hline Skomegan, Mount & Petermann & 8,400 \\
\hline Snake River...... & N.P.R.R. & 328 \\
\hline Snoqualmie Pass . & Reports ........ & 3,468 \\
\hline .................. & urvey.......... & 3,110 \\
\hline Spokane Falls.... & N.P.R.R = & 1,910 \\
\hline Spokane House.. & Pacific R. R. Reports......... & 1,200 \\
\hline : & 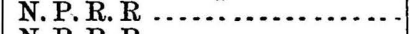 & 1,908 \\
\hline Stampede Pass, summit 9, 100 foot tunnel .. & N.P.R.R $R \ldots \ldots$ & 2,885 \\
\hline Do......... summit of Cascade Range. & N.P.R.R ...... & 3,980 \\
\hline 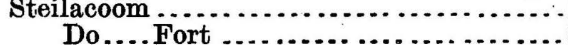 & Med. Dept, U. & 1,300 \\
\hline Stenger's Pass...... & N. Trans. Survey.. & 3,105 \\
\hline nger's Peak ..... & N. Trans Survey .. & 6,299 \\
\hline 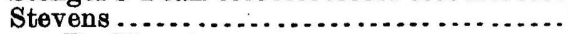 & 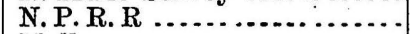 & 2,282 \\
\hline Mount & Mullan ..................... & 6,900 \\
\hline Striped Peak... & Mullan... & 6,000 \\
\hline Stuck Junction... & N.P.R.R & 110 \\
\hline ualunl Pass... & N. Trans. Survey.............. & 3,110 \\
\hline$\ldots \ldots \ldots$ & N. Trans. Survey ............. & 3,430 \\
\hline 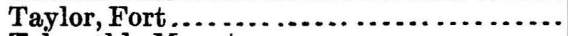 & Toner ....................... & 135 \\
\hline Tchopáhk, Monnt ..................... & Peter & 7,200 \\
\hline$\ldots \ldots \ldots \ldots$ & N.P. R. R & 315 \\
\hline 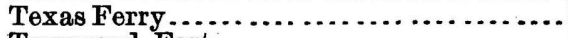 & Oreg. R. R & 519 \\
\hline usend, Fort & Toner.... & 135 \\
\hline Trent ............ & N.P.R.R & 1,989 \\
\hline Cañon. & N.P.R.R & 1,750 \\
\hline ver, Fort...................... & Med. Dept. U.S. A ........... & 50 \\
\hline Wait's Lake. & N. Trans. Survey .............. & 1,816 \\
\hline Walla Wall & Oreg. R. R................... & 908 \\
\hline$\ldots \ldots \ldots \ldots$ & 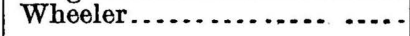 & 1,034 \\
\hline Walla Walla, Fort... & & 1,396 \\
\hline Walla Walla Missior & & 2,100 \\
\hline Wallula .......... & Oreg. R. R & 308 \\
\hline Wallula Junction...$\ldots \ldots \ldots \ldots \ldots \ldots$ & 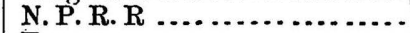 & 326 \\
\hline lootzas Lake . . . . . . . . . . . . . . . . . . . . & Toner..... & 2,940 \\
\hline Winlock ............. & N.P.R.R. & \\
\hline City ... & N.P.R.R . . . . . . & 990 \\
\hline Yakix & Pacific R. R. Reports......... & 3,468 \\
\hline & N.Trans. Survey ............. & 3,625 \\
\hline & N.P.R.R ........ & \\
\hline
\end{tabular}


WEST VIRGINIA.

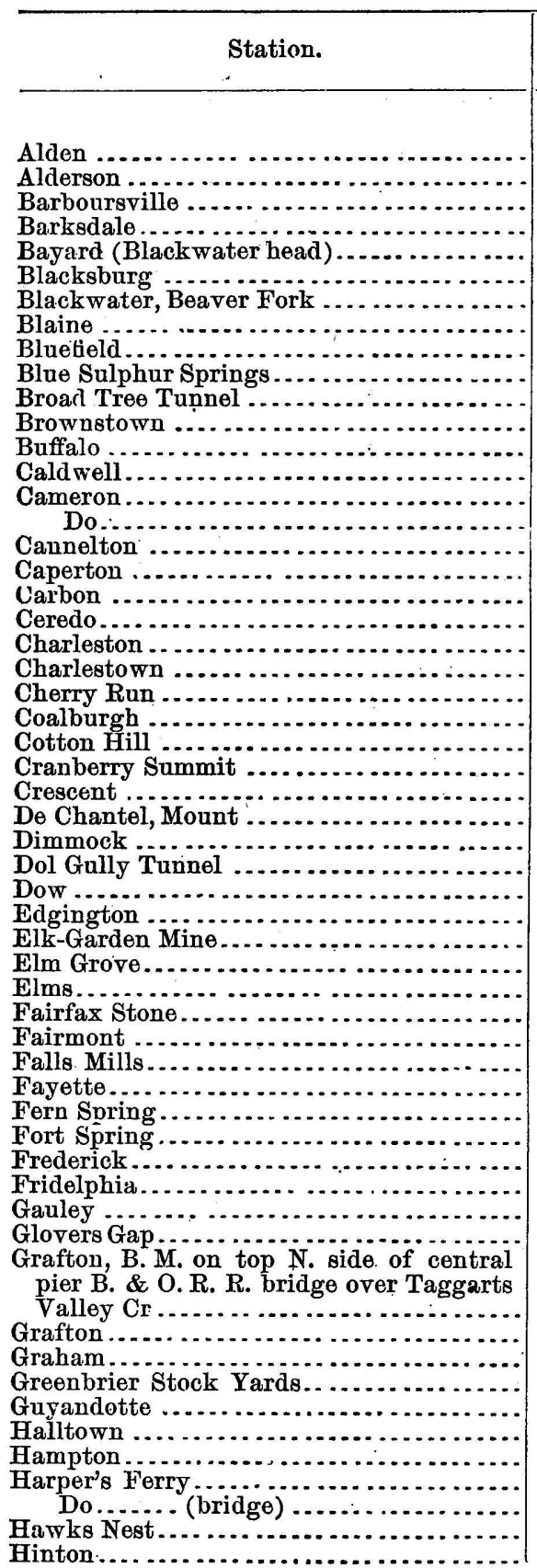

C. \& O.R. R

C. \& O.R. R

C. \& O.R. R

C.\& O.R. R

W. Va., C. \&

W. Va., C. \& P.R.R

W. Va., C. \& P. R. R.

N. \& W.R.R

C.\& O.R. R

B.\& O.R. R

C. \& O.R. R

C. \& O.R. R

C. \& O. R. R

B. \& O.R. R

Valley R. $R$

C. \& O.R. R

C. \& O.R. R

B. \& O.R. R

C. \& O.R. R

C. \& O.R. R

Shenandoah Valley $\mathbf{R} . \mathbf{R}$

B. \& O. R. R

C. \& O. R. R

C. \& O. R. R

B. \& O. R. R

C. \& O. R. R

B. \& O. R. R

C. \& O. R. R

B. \& O. R. R

C. \& O. R. R

P., C. \& St. L. R. R

W. Va., C. \& P. R. R

B. \& O.R. R.

C. \& O.R.R.

W. Va., C. \& P. R. R

B. \& O. R. R

N. \& W. R. R

C. \& O. R. R

C. \& O. R. R

C. \& O. R. R

C. \& O. R. R

B. \& O.R. R.

C. \& O. R. R

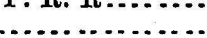

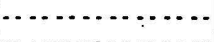

(n...............

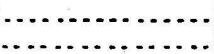

. n.

U. S. C. \& G. S

B. \& O. R. R

N. \& W. R. R

C. \& O. R. R

C. \& O. R. R

Valley R. R.

C. \& O. R. R

B. \& O. R. R

Valley R. R.

C. \& O. R: R

C. \& O. R. R
Elevation

605

1,548

578

1,338

3,150

$6: 20$

3,080

1,689

2,552

598

1,104

608

1,109

1,766

1,049

547

638

984

667

545

603

517

398

625

792

2,551

638

$6 \% 2$

1,045

545

1,432

704

2,300

681

860

3,100

888

2, 325

900

914

1,626

640

735

707

1, 150

997

985

2,387

1,530

560

339

540

272

277

827

137 


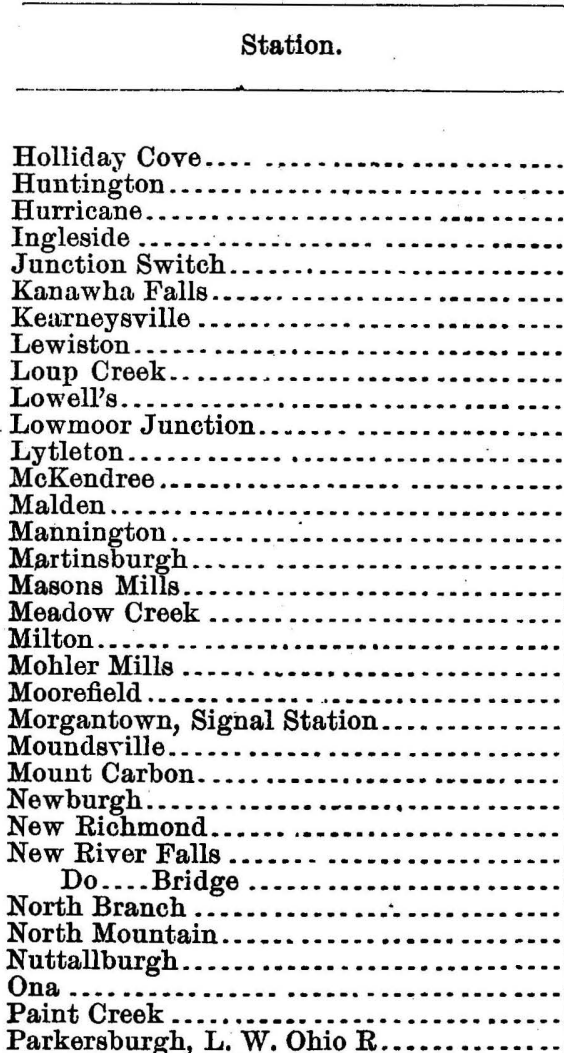

Do $\mathrm{H}$. W. Ohio $\mathrm{R}$

Do ...... Bridge

Do ...... B. M. on $S$. front of water table of P. O. \& C H.

Pawpaw

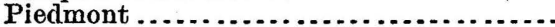

Point Mills.

Prince

Quinnimont

Ripon

River View.

Ronceverte

Roney's Point.

Rowlesburg, B. M. on base of center pillar W. end B. \& O. R. R. bridge over Cheat

\section{River}

Saint Albans

Salton

Scary

Scott.

Shaw

Shenandoah Junction

Shepherdstown.

Siding.

Steeles

Stone Cliff

Summit Point.

Talcott

\begin{tabular}{|c|c|}
\hline Authority. & Elevation. \\
\hline & Feet. \\
\hline P., C. \& St. L. R. I & 721 \\
\hline C. \& O. R. R..... & 566 \\
\hline C. \& O.R. R...... & 6,837 \\
\hline N. \&W. R. R...... & 1,937 \\
\hline C. \& O. R. R.... & 560 \\
\hline C. \& O.R. R. & 669 \\
\hline B. \& O. R. R. & 589 \\
\hline C. \& O. R. R. & 616 \\
\hline C. \& O. R. R. & 647 \\
\hline C. \& O.R. R. & 1,512 \\
\hline C. \& O. R. R. & 1,155 \\
\hline B. \& O. R. R. & 930 \\
\hline C. \& O.R. R. & 1,141 \\
\hline C. \& O.R. R & 606 \\
\hline B. \& O. R. R. & . 967 \\
\hline B. \& O. R. R. & 435 \\
\hline C. \& O.R. R. & 1,527 \\
\hline C. \& O.R. R. & 1,265 \\
\hline C. \& O. R. R. & 586 \\
\hline C. \& O.R.R. & 1,540 \\
\hline W. \& O.R. R & 806 \\
\hline U. S. Signal Office. & 963 \\
\hline B. \& O. R. R . . . . & 640 \\
\hline C. \& O. R.R. & 639 \\
\hline B. \& O.R. R & 1,215 \\
\hline C. \& O.R.R. & 1,289 \\
\hline C. \& O. R. R & 1,290 \\
\hline C. \& O.R. R... & 827 \\
\hline B. \& O.R. R. & 605 \\
\hline B. \& O. R. R. & 547 \\
\hline C. \& O.R.R. & 946 \\
\hline C.'\& O: R. R. & 622 \\
\hline C. \& O.R.R. & 622 \\
\hline M. \& C. R. R. & 574 \\
\hline M. \& C. R. R. & 624 \\
\hline 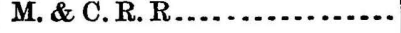 & 664 \\
\hline U.S. C. \& G. & 616 \\
\hline C. \& O.R.R. & 1,237 \\
\hline B. \& O.R.R. & 925 \\
\hline B. \& O.R.R. & 896 \\
\hline C. \& O.R.R.. & 1,192 \\
\hline C. \& O.R.R ... & 11,965 \\
\hline Shenandoah Valley R.R.... & 519 \\
\hline C. \& O.R.R R & 1,072 \\
\hline $\begin{array}{l}\text { C. \& O R. R R.R.R. } \\
\text { B. \& }\end{array}$ & $\begin{array}{r}1,663 \\
829\end{array}$ \\
\hline
\end{tabular}

U. S. C. \& G. S

C.\& O.R.R

C. \& O.R. R

C. \& O.R.R

C. \& O.R.R

C. \& O.R. R

W. Va., C. \& P. R. R .......

Shenandoah Valley R. R

Shenandoah Valley R. R.

C. \& O. R. R

C. \& O.R. R

C. \& O.R. R

C. \& O.R. R.

Valley R. R

C. \& O.R. R

(439)
Feet.

566

6,837

937

669

589

616

547

1,155

930

606

967

435

, 265

586

, 540

963

640

1,215

, 289

890

605

547

622

622

574

664

616

, 237

896

1,192

1,965

519
1,072

829

1,402

592

608

591

683

1, 003

1,290

515

405

1,150

597

1,210

1,076

623

1,512 


\begin{tabular}{|c|c|c|}
\hline Station. & Authority. & Elevation. \\
\hline 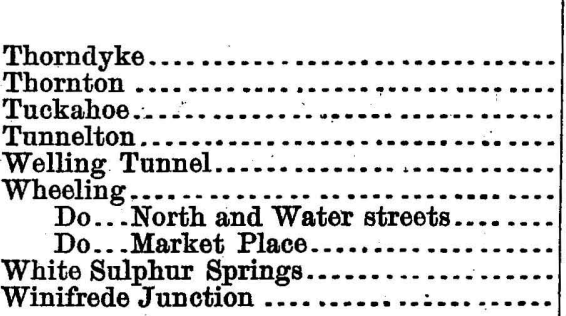 & 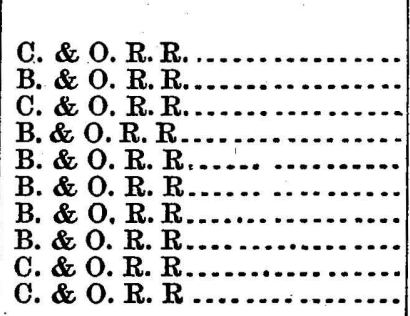 & $\begin{array}{r}\text { Feet. } \\
640 \\
1,038 \\
2,035 \\
1,820 \\
1,193 \\
637 \\
645 \\
662 \\
1,922 \\
616\end{array}$ \\
\hline
\end{tabular}




\section{WISCONSIN.}

\begin{tabular}{|c|c|c|}
\hline & Authority. & Elevation. \\
\hline & & \\
\hline $\begin{array}{l}\text { Abbotsford ....... } \\
\text { Ableman's ...... }\end{array}$ & $\begin{array}{l}\text { Wis. Cent. R.R. } \\
\text { C. \& N. W.R.R. }\end{array}$ & $\begin{array}{r}1,425 \\
878\end{array}$ \\
\hline Amherst .......... & Wis. Cent. R. R. & 1,089 \\
\hline 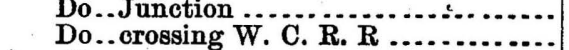 & G. B.\& Minn. R. & $\begin{array}{l}1,171 \\
1,130\end{array}$ \\
\hline 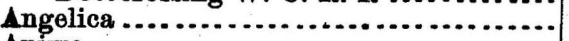 & Mil. \& N.R.R .. & 867 \\
\hline Aniwa .......... & M., L. S. \& W. R.R & 1,418 \\
\hline $\begin{array}{l}\text { Appleton ............. } \\
\text { Do..Fox Rive }\end{array}$ & M., L. S. \& W. R. R . & $\begin{array}{ll}715 \\
718\end{array}$ \\
\hline Do...Junotion & M. L. S.\&W.R. & 801 \\
\hline Ashland.... & Wis. Cent. R. R. & 678 \\
\hline $\begin{array}{l}\text { Auburndale .... } \\
\text { Angusta ...... }\end{array}$ & $\begin{array}{l}\text { Wis. Cent.R.R. } \\
\text { C.\&. N.W.R.R. }\end{array}$ & $\begin{array}{r}1,217 \\
965\end{array}$ \\
\hline Baldwin ........ & C. \&N.W.R.R. & 1,132 \\
\hline Baraboo.... & C.\& N.W.R.R & 861 \\
\hline Barton ............. & N.W.U.R.R ... & 910 \\
\hline Bear Creek . ........ & M., L.S.\&W.R.R .. & 817 \\
\hline $\begin{array}{l}\text { Beaver........... } \\
\text { Belgium ........ }\end{array}$ & $\begin{array}{l}\text { Wis. Val.R.R........ } \\
\text { M.L.S.\&W.R.R. }\end{array}$ & $\begin{array}{c}963 \\
755\end{array}$ \\
\hline Belơit, crossing $\mathrm{C}$. & C.\& N.W.R.R... & 740 \\
\hline $\begin{array}{r}\text { Do, B. M. on I } \\
\text { tank...... }\end{array}$ & C.\& N. W.R.R. & 741 \\
\hline Do, level of & C.\& N.W.R.R. & 729 \\
\hline Birnamwood .... & M., L.S.\& W. R. & 1,293 \\
\hline Black River Sta & C.\& N.W.R.R. & 802 \\
\hline Blooms ..... & C. \& N.W.R.R & 881 \\
\hline Boyer's Bluff, Geod & U. S. Lake Surv & 712 \\
\hline Branch & M., L. S. \& W. R & 729 \\
\hline , Geodetic Stat & U. S. Lake Surve & 767 \\
\hline Buckbee. & M., L.S.\& W.R.R... & 831 \\
\hline ista......... & Wis. Cent. R. R . & 1,074 \\
\hline Butte De Morts Lak & Mil. \& N.R.R R..... & 735 \\
\hline $\begin{array}{l}\text { aledonia, Geodetic Station......... } \\
\text { Camp Douglas Junction, crossing } \mathrm{C} \text { \& }\end{array}$ & U. S. Lake Survey.. & 791 \\
\hline 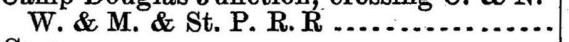 & C. \& N.W.R.R & -929 \\
\hline Cao $\ldots \ldots . . . . . . . .$. & M., L. S.\&.W.R. & 844 \\
\hline & Mil.\& N.R.... & 773 \\
\hline ar Grove & M., L.S.\& W.R.R.. & 697 \\
\hline Centralia..... & Wis. Val. R.R..... & 1,015 \\
\hline Centreville.. & $\begin{array}{l}\text { M., L. S. \& W. R. R. } \\
\text { Mil. \& N.R.R...... }\end{array}$ & 837 \\
\hline $\begin{array}{l}\text { Chilton .............. } \\
\text { Clayton......... }\end{array}$ & $\begin{array}{l}\text { Mil.\& N.R.R } \\
\text { M., L.S. \& W.R. R }\end{array}$ & $\begin{array}{r}851 \\
1,347\end{array}$ \\
\hline $\begin{array}{l}\text { Clayton........... } \\
\text { Clintonville..... }\end{array}$ & M., L.S.\& W. R. R. & $\begin{aligned} 1,347 \\
824\end{aligned}$ \\
\hline Colby .............. & Wis. Cent. R. R ......... & 1,315 \\
\hline Col & Wis. Cent. R. R. & 1,042 \\
\hline Dane $\ldots \ldots \ldots \ldots \ldots$ & C. \& N.W. R. R & 1,058 \\
\hline Davidson's Farm .................. & Mil. \& N. R. R .. & 747 \\
\hline Day's Mill........... & Mil. \& N. R. R $\ldots \ldots \ldots \ldots$ & 720 \\
\hline 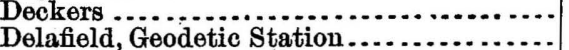 & 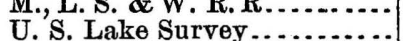 & $\begin{array}{r}756 \\
1,242\end{array}$ \\
\hline $\begin{array}{l}\text { Delafield, Geodetic } \mathrm{s} \\
\text { De Pere............. }\end{array}$ & Mil. \& N. R. R ... & $\begin{aligned} 1,242 \\
591\end{aligned}$ \\
\hline Dexter & G. B. \& Minn. R. R...... & 1,001 \\
\hline Dillma & M., L. S. \& W. R. R . . & 668 \\
\hline & Wis. Cent. R. R ... & 1,466 \\
\hline Dover, Geodetic Station ................... & U. S. Lake Survey - ............ & 861 \\
\hline 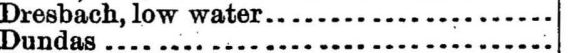 & $\begin{array}{l}\text { U. S. Engr. Corps. } \ldots \ldots \ldots \\
\text { M., L. S. \& W. R. R } \ldots\end{array}$ & \\
\hline Eau Claire........ & C. \& N. W.R. R.. & 836 \\
\hline
\end{tabular}




\begin{tabular}{|c|c|c|}
\hline Station. & Authority. & Elevation. \\
\hline & & Feet. \\
\hline Eden ....... & N. W. U. R. R & 1,019 \\
\hline Eland Junction .. & M., L. S. \& W & 1,244 \\
\hline Elkhart Lake . . . . . . . . . . . & Mil. \& N. R. R & 944 \\
\hline Elk Mound .............. & C. \& N. W. R. & 926 \\
\hline 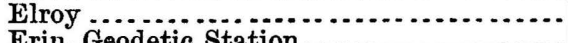 & C. \& N. W. R. R & 955 \\
\hline $\begin{array}{l}\text { Erin, Geodetic Station .... } \\
\text { Evansville, B. M. on fou }\end{array}$ & U. S. Lake Survey & 1,358 \\
\hline water tank ................... & C. \& N. R. R & 893 \\
\hline Fairchild $\ldots \ldots \ldots$ & C. \& N. R. R .. & 1,066 \\
\hline Fall Creek Station ..... & C. \& N. W..R. I & 929 \\
\hline Fifield $\ldots \ldots \ldots \ldots \ldots$ & Wis. Cent. R. R. & 1,458 \\
\hline Fond du Lac & N. W. U. R. R ... & 769 \\
\hline 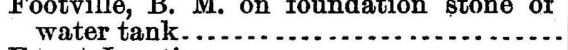 & C. \& N. W. R. F & 816 \\
\hline Forest Junntion .......... & M., L. S. \& W. I & 830 \\
\hline 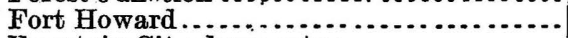 & Mil. \& N. R. R . & 588 \\
\hline Fountain City, low water. & U. S. Engr. Cor & 637 \\
\hline Fredonia.................... & Mil. \& N. R. R. & 788 \\
\hline Goodhope .................. & Mil. \& N. R. R. & 694 \\
\hline Graftion .................. & Mil. \& N. R. R..... & 752 \\
\hline Grand Rapids ..... & G. B. \& Minn. R. R. & 1,024 \\
\hline Granville .......... & N. W. U.R. R ... & 738 \\
\hline Green Bay $\ldots . . . . . . . . . .$. & Mil. \& N. R. R . . . . & 588 \\
\hline reenville....... & $\begin{array}{l}\text { Mil. \& N. R. R . } \\
\text { M., L. S. \& W. R }\end{array}$ & $\begin{array}{l}582 \\
812\end{array}$ \\
\hline Grimins........... & M., L. S. \& W. I & 845 \\
\hline Hammond ....... & C. \& N. W. R. R & 1,100 \\
\hline Hancock ..................... & Wis. Cent. R. R. & 1,102 \\
\hline Hanover, cross. C. \& N. W. R. R. \& Monroo & & \\
\hline $\begin{array}{r}\text { Br, of M. \& St. P. R. R. } \\
\text { Hartland. }\end{array}$ & C. \& N. W. R. R & 780 \\
\hline $\begin{array}{l}\text { Hartland } \ldots \ldots \ldots \ldots \ldots \ldots \\
\text { Hayton } . . . \ldots \ldots \ldots \ldots \ldots \ldots\end{array}$ & Mil. \& N. R. R. & 839 \\
\hline $\begin{array}{l}\text { Hayton } \ldots . . . . . . . . \\
\text { Herr Cassel ........ }\end{array}$ & Mil. \& N. R.R... & 822 \\
\hline $\begin{array}{l}\text { Herr Cassel ....... } \\
\text { Hersey ............... }\end{array}$ & N. W. U. R. R $\cdots$ & 1,049 \\
\hline Horicon, Geodetic Station & U. A N. W. K. K . & 1,168 \\
\hline Horicon Lake ............. & Toner............ & 1,118 \\
\hline Hortonville & M., L. S. \& W. & 808 \\
\hline Do....Jun & M., L. S. \& W. & 789 \\
\hline udson. & C. \& N. W. R.R. & 700 \\
\hline Humbird & C. \& N. W.R.R & 1,018 \\
\hline Hunting & M., L. S. \&.W. I & 940 \\
\hline Jackson............. & N.W. U.R.R .. & 897 \\
\hline Junction City ..... . & Wis. Cent. R. R ... & 1,145 \\
\hline Kaukauna .. & M., L. S. \& W. R. R ........ & 655 \\
\hline Kelleys ...... & M., L. S. W. R. R. . .......... & 1,248 \\
\hline Kewaskum... & N.W. U.R.R.... & 959 \\
\hline$\ldots \ldots . .$. & Mil. \& N.R.R ... & 915 \\
\hline pp & C. \& N. W. R. R & 919 \\
\hline Knowlton & Wis. Val. R. R .... & 1,131 \\
\hline La Crosse, L. W & U. S. Engr. Corps . & 626 \\
\hline Do... C. M. \& St. P. R. R. depot..... & City Engineer .... & 698 \\
\hline Do... Signal Station............... & U. S. Signal Office & 708 \\
\hline kke Shore Junction ........ & M., L. S. \& W. R. R & 642 \\
\hline Lavalle . . . . . . . . & C. \& N. W. R. R... & 897 \\
\hline atic Station $\ldots \ldots \ldots \ldots$ & U. S. Lake Survey... & 1,019 \\
\hline Lindivern..... & N. W.U.R.R .. & 638 \\
\hline Lisbon, Geodet & U. S. Lake Survey & 1,054 \\
\hline Little Chute & 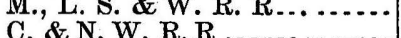 & 707 \\
\hline Lowrie & C. \& N. W. R, R & 848 \\
\hline Madison, cross. C. \& N. W. \& P. du C.R.R. & C. \& N. W. R. R... & 848 \\
\hline Do Signal Station & & 949 \\
\hline Aanitowoc... & $\&$ W. R. R......... & 593 \\
\hline & & 832 \\
\hline
\end{tabular}




\begin{tabular}{|c|c|c|}
\hline Station. & Authority. & Elevation. \\
\hline & & Feet. \\
\hline Mannville. & Wis. Cent. R. R & 1,292 \\
\hline Marion $\ldots$ & M., L. S. \& W. & 860 \\
\hline Marshfield & Wis. Cent. R. R & 1,289 \\
\hline Marshland, crossing of G. B. \& M. R. R. & C. \& N. W. R. R & 659 \\
\hline 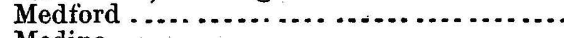 & Wis. Cent. R. R & 1,413 \\
\hline 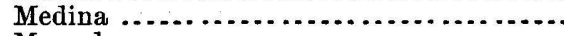 & M., L. S. \& W. I & 813 \\
\hline 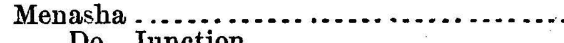 & Wis. Cent. R. R & 756 \\
\hline $\begin{array}{l}\text { Do...Junction } \ldots \ldots \ldots \ldots \\
n_{1}\end{array}$ & Mil. \& N. R. R... & 832 \\
\hline Menomonee $\ldots \ldots \ldots$ & C. \& N. W. R. R & 878 \\
\hline Merrilon & C. \& N. W. R. R & 934 \\
\hline 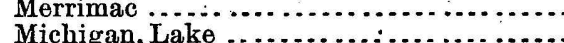 & C. \& N. W.R.R & 796 \\
\hline $\begin{array}{l}\text { Michigan, Lake .... } \\
\text { Mignon ............. }\end{array}$ & Mil. \& N. R. R. & $\begin{array}{l}582 \\
673\end{array}$ \\
\hline Milladore & Wis. Cent. R. R & 1,197 \\
\hline Millston . & C. \& N.W.R.R & 915 \\
\hline Milwaukee, depot L. S. \& M. S. R. R. & City Engineer .. & 592 \\
\hline 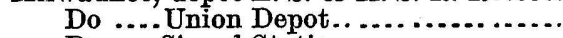 & City Engineer & 584 \\
\hline 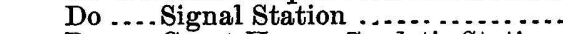 & U. S. Signal Offi & 697 \\
\hline Do .... Court-House, Geodetic Station. & U. S. Lake Surv & 838 \\
\hline Minerva $\ldots \ldots \ldots \ldots \ldots \ldots \ldots \ldots$ & G. B. \& Minn. R & 832 \\
\hline Minnesota Junction, Geodetic Station..... & U. S. Lake Surv & 998 \\
\hline 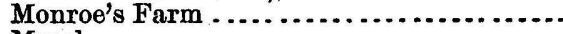 & Mil. \& N. R.R. . & 669 \\
\hline Mosel ............... & M., L. S. \& W. R & 639 \\
\hline Nagowicka...... & 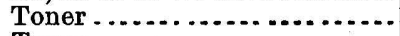 & 882 \\
\hline Nemahbin, Lake . . . . . . . . . & Toner $\ldots \ldots \ldots$ & 867 \\
\hline New Berlin, Geodetic Station ... & U. S. Lake Surve & 1,073 \\
\hline 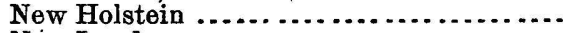 & Mil. \& N. R. R. - & 933 \\
\hline New London . ............... & M., L. S. \& W. R. R. & 756 \\
\hline 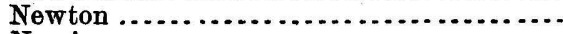 & M., L. S. \& W. R. R. & 657 \\
\hline Norris . & M., L. S. \& W. R. R. & 1,298 \\
\hline Northport & G. B. \& Minn. R. R & 777 \\
\hline Norwalk ... & C. \&.N. W. R. R. & 1,020 \\
\hline Geodetic Station ................. & U. S. Lake Surve & 1,168 \\
\hline oc, Lake. & Toner .......... & 860 \\
\hline Ogdensburgh........ & G. B. \& M. R. R... & 865 \\
\hline Ogenia. & Wis. Cent. R. R .............. & 1,565 \\
\hline Oregon Station, B. M. on S. W. cor. of abut- & & \\
\hline highway, S. of....... & C. \& N. W. R. R & 952 \\
\hline Oshkosh ........ & M., L. S. \& W. R & 753 \\
\hline Otsburg & M., L. S. \& W. R. & 698 \\
\hline .......... & Wis. Cent. R. R . & 784 \\
\hline Per & Wis. Cent. R. R & 1,285 \\
\hline Pewaukee, & Toner.......... & 1,891 \\
\hline Phillips & Wis. Cent. R. R. & 1,454 \\
\hline Plainfield & Wis. Cent. R. R & 1,113 \\
\hline Plover ... & Wis. Cent. R. R. & 1,078 \\
\hline $\mathrm{Ply}$ & Mil. \& N. R.R . & 844 \\
\hline . & Wis. Cent. R. R & 792 \\
\hline ds ...... & Wis. Val. R. R . & 972 \\
\hline Port Washington & M.,L. S. \& W.R.R & 669 \\
\hline Pra & M.;L. S. \& W. R. R. & 777 \\
\hline ott, low water. & U. S. Eng'r. Corps .. & 664 \\
\hline Random Lake .................. & Mil. \& N.R.R .. & 877 \\
\hline Reedsburg ....... & C. \& N. W. R.R. & 877 \\
\hline berts & C. \& N.W. R. R... & 1,036 \\
\hline field. & N.W. U.R.R ... & 890 \\
\hline land. & Mil. \& N. R. R & 643 \\
\hline Iton ......... & G., B. \& Minn. R. R....... & \\
\hline $\mathrm{Ru}$ & C. \& N. W. R.R... & 974 \\
\hline lolph .. & 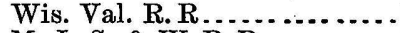 & 1,146 \\
\hline Rudville.. & M., L. S. \& W. R. R ......... & \\
\hline 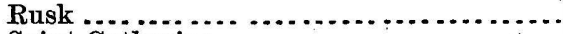 & C. \& N. W. R. R . . . . . . . & 901 \\
\hline Saint Catherine .................. & N. W. U.R. R . . . . . . . . . . & 765 \\
\hline Saukville ................... & Mil. \& N. R. R . ............ & \\
\hline
\end{tabular}




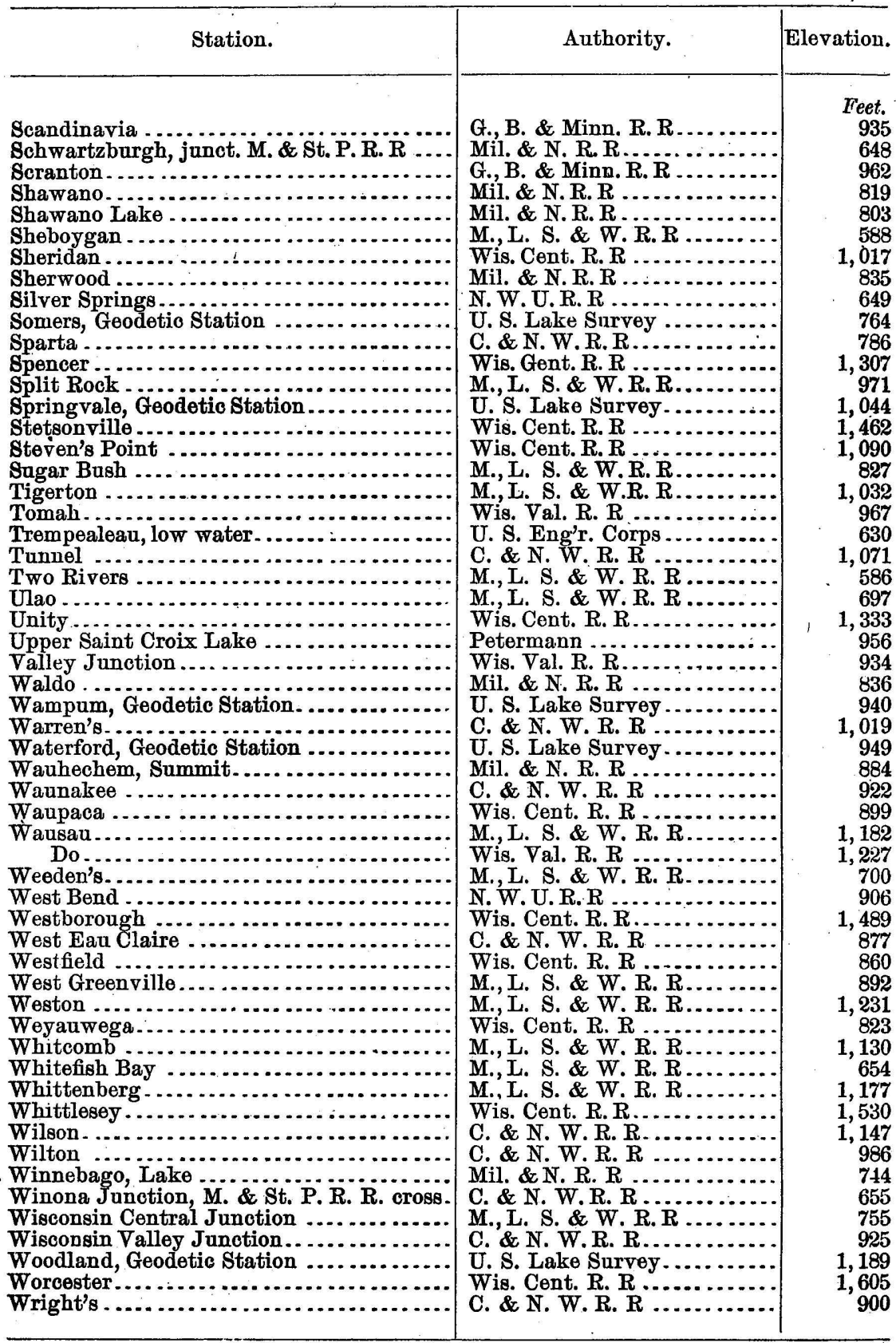


WYOMING.

\begin{tabular}{|c|c|c|}
\hline Station. & Authority. & Elevation, \\
\hline & & Feet. \\
\hline Amethyst Mountai & & \\
\hline Arrow Peak ...... & $\begin{array}{l}\text { Hayden } \\
\text { King ... }\end{array}$ & $\begin{array}{l}9,423 \\
8,683\end{array}$ \\
\hline spen....... & U.P.R.R & 7,405 \\
\hline tlantic Cit & Hayden - & 7,850 \\
\hline Atlantic Peak. & Hayden & 12,794 \\
\hline Aurora & U.P.R.R . & 6,719 \\
\hline $\begin{array}{l}\text { Baird, Mount ....... } \\
\text { Bald Mountain... }\end{array}$ & $\begin{array}{l}\text { Hayden ........ } \\
\text { Jones ....... }\end{array}$ & $\begin{array}{l}9,990 \\
9,897\end{array}$ \\
\hline $\begin{array}{c}\text { Do........Wind } \\
\text { ber-line on .... }\end{array}$ & & 10,760 \\
\hline $\begin{array}{l}\text { Baronette's Bridge, I } \\
\text { Park }\end{array}$ & Havden & 5,978 \\
\hline $\begin{array}{l}\text { Baronette's Peak, } \\
\text { Park }\end{array}$ & nayden & 10.459 \\
\hline Barrel Spring ..... & Toner... & $\begin{array}{r}10,459 \\
6,854\end{array}$ \\
\hline Bastion $\mathrm{M}$ & King ....... & 6,991 \\
\hline Battle Mounta & King ........ & 8,991 \\
\hline Baxter .... & Ton & 6,300 \\
\hline Beaver Lak & Hayden & 7,415 \\
\hline Bellevue Peak ...... & King ..... & 9,820 \\
\hline Beulah Lake, Yellor & Hayden. & 7,530 \\
\hline Big Horn Mountains & Raynolds. & $\begin{array}{r}8,000 \\
12,000\end{array}$ \\
\hline Big Pond & Toner... & 6,541 \\
\hline Bison Peak, Yellows & Hayden ... & 9,038 \\
\hline Bitter Creek ........ & U. P.R.R ... & 6,705 \\
\hline Black Butte..... & Hayden..... & 8,170 \\
\hline $\begin{array}{r}\text { Do............... } \\
\text { Boleter ........ }\end{array}$ & $\begin{array}{l}\text { King } . . . \\
\text { Toner .......... }\end{array}$ & $\begin{array}{l}8,176 \\
4,325\end{array}$ \\
\hline $\begin{array}{l}\text { Boleter } \\
\text { Bradley's Peak }\end{array}$ & $\begin{array}{l}\text { Toner........ } \\
\text { Hayden.... }\end{array}$ & $\begin{array}{l}4,325 \\
9,500\end{array}$ \\
\hline Bridger............. & U.P.R.R.... & $6 ; 637$ \\
\hline 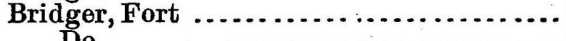 & Dont Ti & 6,753 \\
\hline Do_................... & Med. Dept., U. S. A...... & $\begin{array}{r}6,656 \\
11,410\end{array}$ \\
\hline $\begin{array}{l}\text { Bridger's Pass.. } \\
\text { Brown, Camp .. }\end{array}$ & Jones ..................... & $\begin{array}{r}11,410 \\
5,498\end{array}$ \\
\hline $\begin{array}{r}\text { Brown, Ca } \\
\text { Do.... }\end{array}$ & Jones $\ldots \ldots \ldots \ldots \ldots$ & $\begin{array}{l}5,498 \\
5,447\end{array}$ \\
\hline Brown's Park ..... & Powell ................ & 5,409 \\
\hline Brya & U.P.R.R. & 6,196 \\
\hline & U.P.R.R .... & 7,785 \\
\hline Bunsen Peak, Yellowstone National Park . & Hayden .. & 8,775 \\
\hline Carbon & U.P.R.R & 6,830 \\
\hline Cast & Pre. R. R. Levels & 4,999 \\
\hline & King ........... & 8,774 \\
\hline Mount & Hayden. & 13,000 \\
\hline Cheyenne & U.P.R.R R........... & 6,059 \\
\hline Do ...Signa & U. S. Signal Office....... & 6,089 \\
\hline Cheyenne Pass. & King ........... & 8,791 \\
\hline & U.P. R. R. Surveys .... & 8,650 \\
\hline Chimney Peak .... & King $(. . . \ldots \ldots \ldots \ldots \ldots$ & 8,168 \\
\hline 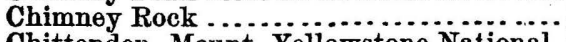 & Jones & 11,853 \\
\hline Chittenden, Mount, Yellowstone National & & \\
\hline 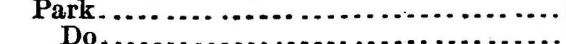 & & $\begin{array}{l}10,190 \\
10.027\end{array}$ \\
\hline Church Buttes........ & U.P.R.R & 6,368 \\
\hline Coffin Mou & Hayden ....... & 11,376 \\
\hline & & 6,314 \\
\hline & $\mathrm{D} D$ & 7,078 \\
\hline
\end{tabular}

Bull. 5-21 


\begin{tabular}{|c|c|c|}
\hline Station. & Authority. & Elevation. \\
\hline & & Feet. \\
\hline 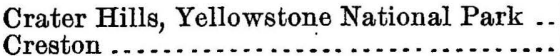 & Hayden . & $\begin{array}{l}7,820 \\
7,048\end{array}$ \\
\hline Deer Creek Agen & Smithsonian & 5,000 \\
\hline Deer Mour & King ....... & 8,377 \\
\hline Delham Pe & King .... & 11,524 \\
\hline Devil's Gat & Petermann & 6,028 \\
\hline Doane, Mount, Yellowistone N & Hayden .. & 10,713 \\
\hline Dunraven Peak, Yellowstone & Hayden & 9,988 \\
\hline Edson...... & U.P.R.R & 6,772 \\
\hline Elephant's Back & Hayden : & 8,884 \\
\hline Elk Mount & King ... & 11,511 \\
\hline Encamp & King .... & 8,171 \\
\hline Essez & Has & 8,750 \\
\hline Eva & U.P.R.R. & 8,424 \\
\hline Evansto & Hayden ... & 6,835 \\
\hline Do. & R. R & 6,768 \\
\hline Mount, Yellowstone Natic & Has & 7,600 \\
\hline Fetterm & mann & 4,973 \\
\hline Do. & 'Ton & 5,012 \\
\hline Flamir & & 5,925 \\
\hline Flat Mo & & 9,200 \\
\hline Fremont's & Fremont & 13,570 \\
\hline Do. & Hayden. & 13,790 \\
\hline $\begin{array}{l}\text { Gardner's River Springs, Yellows } \\
\text { tional Park ............................ }\end{array}$ & Hayden. & $\left\{\begin{array}{r}6,100 \\
\text { to } 6,500\end{array}\right.$ \\
\hline Garnet Hill, Yellowstone National & Hayden ... & 7,177 \\
\hline Genie, Mo & Haj & 12,546 \\
\hline 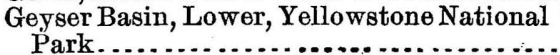 & & 7.250 \\
\hline Geyser Basin, Upper, Yellows & & 7,250 \\
\hline & Hayden . & 7,400 \\
\hline Gibbon Geyser Basin, Yellowstone Na & & \\
\hline Gibbon Lake, Yellowstone National Park.. & & $\begin{array}{l}7,527 \\
7,838\end{array}$ \\
\hline W & sonian Ins & $\ddot{7}, 400$ \\
\hline Gra & & 11,003 \\
\hline Grand T6to & $\mathrm{Ha}$ & 13,691 \\
\hline & U. $\stackrel{P}{P}$ & 6,289 \\
\hline & U.P. & 7,319 \\
\hline Green Rive & U.P.R.R & 6,088 \\
\hline 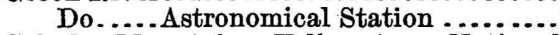 & Wheeler & 6,097 \\
\hline Grizzly Mountain, Yellowstone National & & \\
\hline Park $\ldots \ldots \ldots$ & Hay & 9,982 \\
\hline eak ....... & $n \ldots . .$. & 11,570 \\
\hline (............ & ......... & 7,800 \\
\hline (n. & (n.................... & $\begin{array}{l}6,590 \\
6,500\end{array}$ \\
\hline Mount & Hayden ................. & 10,235 \\
\hline Hayder & Hayden ... & 13,691 \\
\hline ..... timber $]$ & Hayden ....... & 11,000 \\
\hline Haystack Mountain, Yellowstone National & & \\
\hline & King $\ldots . . . \ldots \ldots \ldots . . . . . . . . .$. & 7,689 \\
\hline $\begin{array}{l}\text { Heart Lake, Yellow } \\
\text { Hell Roaring Mou }\end{array}$ & Hayden .................. & 7,475 \\
\hline $\begin{array}{l}\text { Hell Roaring Mou } \\
\text { tional Park ..... }\end{array}$ & Hayden .... & \\
\hline Lake, Yellowstone National Park & & 7,530 \\
\hline & Pre. R. R. Levels. & 3,634 \\
\hline & U.P.R.R ... & 7,236 \\
\hline & Hay & 10,818 \\
\hline Count, Yellowstone National Park. & & 10,528 \\
\hline 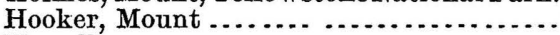 & Hay & 12,900 \\
\hline & Ton & 7,090 \\
\hline & Petermann ... & 6,187 \\
\hline Index I & Hayden & 11,702 \\
\hline
\end{tabular}




\begin{tabular}{|c|}
\hline Station. \\
\hline 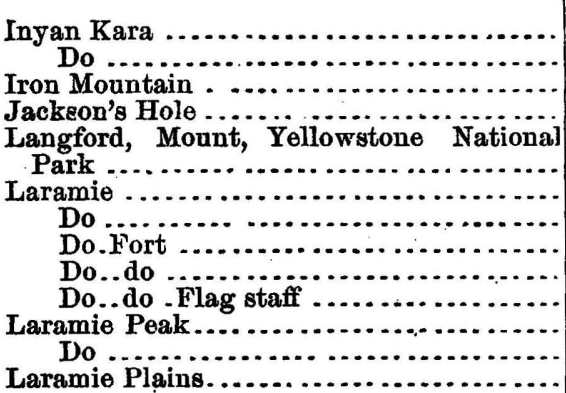 \\
\hline
\end{tabular}

Latham

Lawrence.

Leidy, Mount

Lewis, Lake, Yellowstone National Park.

Lookout

McDougal's Gap

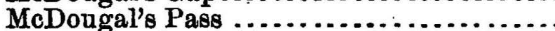

Madison Lake, Yellowstone National Park

Mammoth Hot Springs, at Cabins, Yellowstone National Park

Marston ...........................

Mary's Lake, Yellowstone National Park.

Medicine Bow

Medicine Bow Mountains

Medicine Butte (Pill Hill)

Medicine Peak..............................

Miller ...................................

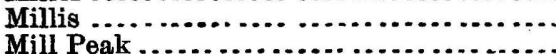

Miner's Delight ........................

Moran, Mount

Mountain City

Muddy Mountain

Mud Geysers on Yellowstone River, Yellowstone National Park

Do.

Norris, Mount, Yellowstone National Park.

North Twin Butte, Lower Basin, Yellowstone National Park

Owl Creek Pass

Pacific Springs

Pelican Hill, Yellowstone National Park

Percy

Peshen's Store.

Phil. Kearney, Fort

Phlox Mountain

Piedmont

Pierre's Hole

Pilot Butte

Pine Bluffs

$$
\text { Do. }
$$

Piney, Fort

Point of Rocks.

Promontory Top, Yellowstone National

Park

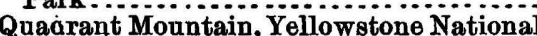

Park.

Quaking Aspen Mountain

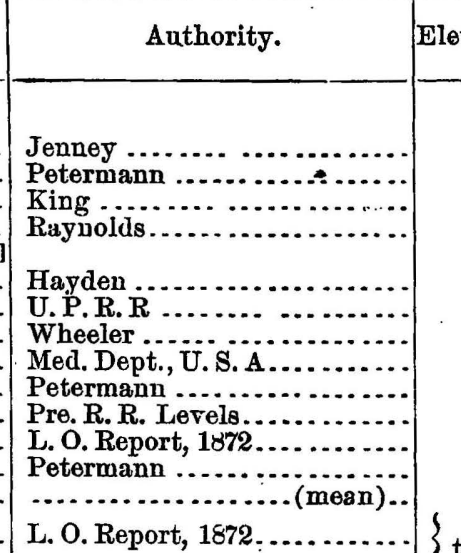

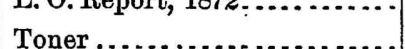

Toner

Hayden

Hayden

U.P.R. R

Hayden

Hayden

Hayden

Hayden

Toner

Hayden .

U. P. R. R

L. O. Report,1872

Hayden

King ........................

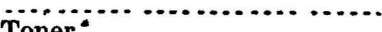

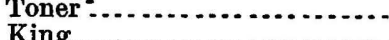

Hayden .......................

Hayden

Pre. R. R. Levels ............

King................

Hayden

Ludlow ...........................

Hayden

Hayden

Jones ........................

Toner

Hayden

U. P. R. R

King

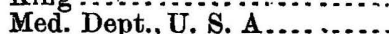

Jones ..........................

U. P. R.R

Raynolds

Hayden

King

U.P. R. R

Lander

Lander.

Hayden

Hayden

King
Elevation.

Feet.

6,700

6,600

6,908

6,000 .

$10,77 \dot{y}$

7.158

7,123

4,519

4,470

4,274

11,000

10,000

7,000

s 7,000

to 9,000

6,900

6,200

11,177 .

7,800

7,177

8,343

9,300

8,300

6,387

6,245

8,336

6,571

8,000

12,000

8,769

12, 231

5,908

6,790

10,506

8,419

12,800

3,589

8,012

7, 725

7,626

10,019

7,976

7,836

7,144

9,580

6,971

6,500

6,000

9,136

7,0002

6,400

7,900

8,878

5,047

7,580

6,517

8,706

10,127

8,688 


\begin{tabular}{|c|c|c|}
\hline Station. & $\mathrm{Au}$ & Elevation. \\
\hline & & \\
\hline Quien Hornet Mou & King ...... & 9,300 \\
\hline $\begin{array}{l}\text { Raw Hide Peak... } \\
\text { Rawlins ......... }\end{array}$ & $\begin{array}{l}\text { Petermann } \\
\text { U. P. R. R }\end{array}$ & $\begin{array}{l}6,370 \\
6,753\end{array}$ \\
\hline Red Buttes ....... & U.P. R. R & 7,309 \\
\hline Red Desert..... & U. P. R.I & 6,722 \\
\hline Red Mountain, Yellor & Hayden .. & 9,777 \\
\hline Red Rock Pass ....... & $\begin{array}{l}\text { Hayden } \\
\text { U.P. R. R }\end{array}$ & $\begin{array}{l}7,271 \\
5,850\end{array}$ \\
\hline Riddle Lake, Yellows & Hayden ....... & 8,000 \\
\hline River Butte......... & King ..... & 7,872 \\
\hline Rock Creek ..... & U. P.R.R & 6,713 \\
\hline Rock Nountain ... & King $\ldots . . .$. & 6,885 \\
\hline $\begin{array}{l}\text { Rock Springs ..... } \\
\text { Russell Fort }\end{array}$ & $\begin{array}{l}\text { U. P. R. R. } \\
\text { Pre. R. R. }\end{array}$ & $\begin{array}{l}6,270 \\
6,159\end{array}$ \\
\hline $\begin{array}{l}\text { Russell, Fort (........ } \\
\text { Do..... (flag-st }\end{array}$ & $\begin{array}{l}\text { Pre. } \mathrm{R} . \mathbf{R} . \\
\text { Wheeler.. }\end{array}$ & $\begin{array}{l}6,159 \\
6,041\end{array}$ \\
\hline Sailor Mountain.... & Jones .... & 10,046 \\
\hline Do.... & Jones ..... & 9,746 \\
\hline Saint Mary's Peak... & King ........... & 7,722 \\
\hline Saint Vrain's Pass... & Petermann $\ldots$ & 8,000 \\
\hline $\begin{array}{l}\text { Salt Welìs ............ } \\
\text { Sanders Fort }\end{array}$ & $\begin{array}{l}\text { U. P.R.R. } \\
\text { U. P. R.R. }\end{array}$ & $\begin{array}{l}6,381 \\
7,180\end{array}$ \\
\hline $\begin{array}{l}\text { Sanders, Fort ........ } \\
\text { Do ....... (flag-sta }\end{array}$ & Wheeler, R. R. Le & 7,168 \\
\hline Sander's Peak...... & King ............. & 9,077 \\
\hline eak. & & 9,930 \\
\hline Separation & U.P. R.R. & 6,677 \\
\hline Peak & Hayden ... & 8,500 \\
\hline 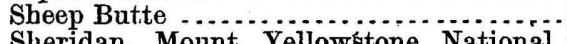 & King .... & 9,722 \\
\hline 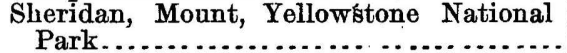 & & \\
\hline & Hon & 10,150 \\
\hline 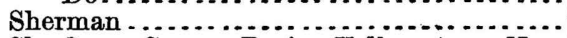 & U. P. R.R & 8,256 \\
\hline Shoshone Geyser Basin, Yellowstone & & \\
\hline one National & $\begin{array}{l}\text { Hayden . } \\
\text { Hayden. }\end{array}$ & $\begin{array}{l}7,837 \\
7,830\end{array}$ \\
\hline (n) & Wheeler & 4,106 \\
\hline Smoothface Mountain (north), Yellowstone & & \\
\hline Nationa & Hayden .. & 10,500 \\
\hline $\begin{array}{l}\text { south), } \mathbf{Y} \\
\text { Nationa }\end{array}$ & Hayden... & 10,417 \\
\hline Soda Hill, Yellowst & & 9,518 \\
\hline uth & & 7,526 \\
\hline & Reports & 7,490 \\
\hline & R. Surveys. & 7,470 \\
\hline South Pass City & Hayden .................... & 7,873 \\
\hline & Petermann ............... & 7,857 \\
\hline $\begin{array}{l}\text { South Twin Butte, Lower Geyser Basin, } \\
\text { Yellowstone National Park ................... }\end{array}$ & & \\
\hline ................ & Pre. R. R. Levels ... & 3,739 \\
\hline Ridge (top), Yellowstone Na- & & \\
\hline & ............. & 8,806 \\
\hline Split & & 8,260 \\
\hline Staml & $\ldots \ldots \ldots \ldots$ & 8,012 \\
\hline & ( & 7,767 \\
\hline Steel, Fort (flag-staff)...$\ldots \ldots \ldots \ldots \ldots$ & Wheeler $\ldots . . . .$. & 6,850 \\
\hline (n) & U. P. R. R............... & 6,515 \\
\hline $\begin{array}{r}\text { Steel, Mount }{ }_{1} \\
\text { Do }{ }_{1} \ldots\end{array}$ & 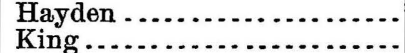 & $\begin{array}{l}7,770 \\
7,771\end{array}$ \\
\hline Stevenson; Mount, Yellowstone National & & \\
\hline $\begin{array}{l}\text { Park } \\
\text { Sulphur Springs, near Bridger's Pass }\end{array}$ & Hayden ...................... & 10,420 \\
\hline $\begin{array}{l}\text { Sulphur Springs, n } \\
\text { Sweeetwater Bridg }\end{array}$ & $\begin{array}{l}\text { Hayden } \\
\text { Smithsonian Inst }\end{array}$ & $\begin{array}{l}7,008 \\
7,000\end{array}$ \\
\hline & U. P.R. R. Surveys & 7,020 \\
\hline 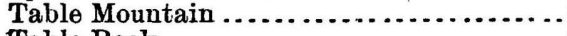 & Hayden ............................ & 7,728 \\
\hline & King.................... & 7,551 \\
\hline
\end{tabular}




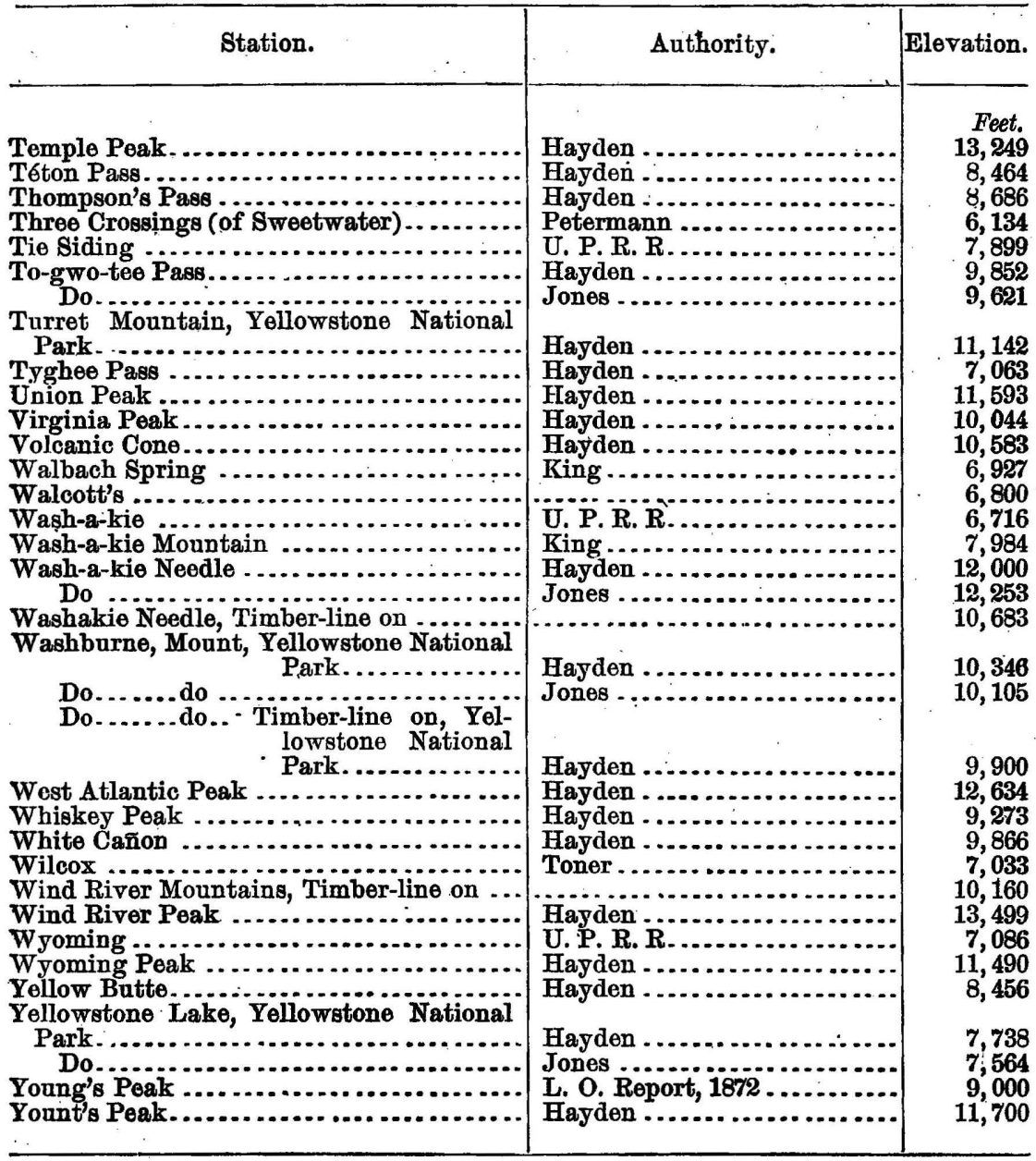




